\title{
Labels of aberrant Clusters of Differentiation gene expression in a compendium of systemic lupus erythematosus patients
}

\author{
Trang T. Le, Ph.D., Nigel O. Blackwood, Matthew K. Breitenstein, Ph.D.* \\ Department of Biostatistics, Epidemiology, \& Informatics, Perelman School of Medicine, \\ University of Pennsylvania, Philadelphia, PA, USA \\ * correspondence to: mkbreit at pennmedicine dot upenn dot edu
}

\begin{abstract}
Background
This author manuscript serves as an extended annotation of gene expression for all known clusters of differentiation (CD) within a compendium of systemic lupus erythematosus (SLE) patients. The overarching goal for this line of research is to enrich the perspective of the $\mathrm{CD}$ transcriptome with upstream gene expression features.

\section{Introduction}

CDs are cell surface biomarkers that denote key biological differences between cell types and disease state. For each of the $>400$ known $\mathrm{CDs}^{1}$, distinct monoclonal antibodies (mABs) enable robust immunophenotyping and serve as scalable biomarkers for translational research. Annotation of CD molecules have been organized through a series of international meetings known as the Human Leucocyte Differentiation Antigens (HLDA) Workshops, affiliated with the Human Cell Differentiation Molecules (HCDM) organization.
\end{abstract}

CD nomenclature <http://www.hcdm.org/>

\section{Methods}

A compendium containing human SLE gene expression data was previously collected, aggregated, and normalized by collaborators in the Greene Lab at the University of Pennsylvania. <https://github.com/greenelab/rheum-plierdata/tree/master/sle-wb $>$ This compendium was slightly modified to include basic demographic information and exclude patients not belonging to classifications of healthy control, treatment naïve SLE, or SLE with exposure to various treatments - the modified dataset represents our 'SLE Compendium'. Entrez gene ID to CD mapping was provided by HCDM ${ }^{1}$. The SLE Compendium dataset and R code corresponding to data pre-processing can be found on the Breitenstein Lab Github page.

\section{SLE Compendium: <https://breitensteinlab.github.io/SLE-Compendium-2018/>}

Within our SLE Compendium, all known CDs gene expression of , were categorized as 'aberrant' or 'non-aberrant' based on the following criteria: $i$ ) two-tailed normalization at $20^{\text {th }}$ and $80^{\text {th }}$ percentile of relative gene expression. Specifically, the two tails encompassed 'aberrant' CD expression, whereas the middle distribution served as 'nonaberrant'. Following visual inspection of all histograms, specific CDs were deemed to require manual adjustments. ii) Manually adjustment of two-tail normalization was applied when CDs followed apparent normal distribution but would require slight modification of thresholds to characterization feature variation. iii) Binarization CD features was applied when an obvious non-normal distribution was observed. Thresholds separated the expression values into low/ high groups (instead of non-aberrant/aberrant) to capture apparent patterns in the expression distributions.

In future research, CDs will be enriched with perspective of interdependent gene expression features using the integrated machine learning pipeline for aberrant biomarker enrichment (i-mAB pipeline).

i-mAB pipeline: < https://breitensteinlab.github.io/i-mAB/>

\section{Results}

Within the original study cohorts, multiple observations were generated for most patients. Observation characteristics of the SLE Compendium, including PMID for the 6 original studies ${ }^{2,3,4,5,6,7}$, can be found in Table 1. Additional sample/cohort can be ascertained from the Gene Expression Omnibus via GSE or GEO accession ID.

Gene Expression Omnibus: <https://www.ncbi.nlm.nih.gov/geo/query/acc.cgi>

(C) 2018 The Authors. Open Access chapter published by World Scientific Publishing Company and distributed under the terms of the Creative Commons Attribution Non-Commercial (CC BY-NC) 4.0 License 
Table 1. SLE Compendium characteristics as ascertained from study of origin

\begin{tabular}{|c|c|c|c|c|c|c|c|}
\hline & $\underline{\text { Cohort }}^{2}$ & $\underline{\text { Cohort }}^{3}$ & $\underline{\text { Cohort }}^{4}$ & $\underline{\text { Cohort }}^{5}$ & $\underline{\text { Cohort }}^{6}$ & $\underline{\text { Cohort } 6}^{7}$ & Overall \\
\hline Study PMID & 18631455 & 23203821 & 24644022 & 25736140 & 27040498 & 26138472 & --- \\
\hline Study GEO identifier & GSE11907 & GSE39088 & GSE49454 & GSE61635 & GSE65391 & GSE78193 & --- \\
\hline Healthy control* & 0 & 46 & 0 & 30 & 72 & 12 & 160 \\
\hline median age (range) & --- & $\begin{array}{c}34.5 \\
(19-50)\end{array}$ & --- & --- & $\begin{array}{c}12 \\
(6-21)\end{array}$ & --- & $\begin{array}{c}16 \\
(6-50)\end{array}$ \\
\hline gender - female/male & --- & 34 & --- & --- & 57 & --- & 91 \\
\hline SLE-treatment naïve* & 37 & 21 & 177 & 99 & 924 & 32 & 1290 \\
\hline median age (range) & $\begin{array}{c}14 \\
(8-17)\end{array}$ & $\begin{array}{c}43 \\
(20-50)\end{array}$ & $\begin{array}{c}40 \\
(18-71)\end{array}$ & --- & $\begin{array}{c}15 \\
(6-19)\end{array}$ & --- & $\begin{array}{c}16 \\
(6-71)\end{array}$ \\
\hline gender - female & 35 & 21 & 148 & --- & 817 & --- & 1021 \\
\hline $\begin{array}{l}\text { SLE-various } \\
\text { treatments }\end{array}$ & $\mathbf{0}$ & 57 & $\mathbf{0}$ & $\mathbf{0}$ & $\mathbf{0}$ & 69 & 126 \\
\hline median age (range) & --- & $\begin{array}{c}36 \\
(19-50)\end{array}$ & --- & --- & --- & --- & $\begin{array}{c}36 \\
(19-50)\end{array}$ \\
\hline gender - female/male & --- & 57 & --- & --- & --- & --- & 57 \\
\hline
\end{tabular}

*observation characteristics include multiple observations per patient

By default, CD gene expression thresholds were labeled as 'aberrant' or 'non-aberrant', with 'aberrant' being further delineated as 'low' or 'high'. Gene expression was stratified at 20 and 80 percentiles, with low expression being between 0 and 20, average between 20 and 80, and high between 80 and 100. Descriptive statistics of CD gene expression with corresponding Entrez ID can be found in Supplement 1, Table 2.

Overall the default two-tailed thresholds provided satisfactory characterization of 'aberrant' (including 'low' and 'high') vs. 'non-aberrant' normal gene expression distributions. However, some CDs required manual adjustment, including shifting of thresholds $(\mathrm{n}=3)$ and binary transformation for non-normal distributions $(\mathrm{n}=85)$ (Supplement 2, Table 3). Amongst CD genes requiring binary transformation, no clear data-driven hypothesis of 'aberrant' vs 'average' was practical so gene expression was labeled simply as 'low' or 'high' (i.e. no clear baseline comparison is available). Consensus review by the research team determined manual revisions of thresholds. Detailed labelling of all CD features $(n=351)$ identified within the SLE Compendium can be found in Supplement 3, Figures 1-290. Included are histograms of CD gene expression distribution and descriptive statistics of expression and variation.

\section{References}

1. Clark G, Stockinger H, Balderas R, van Zelm MC, Zola H, Hart D, Engel P. Nomenclature of CD molecules from the tenth human Leucocyte differentiation antigen workshop. Clinical \& Translational Immunology. 2016; 5(1).

2. Chaussabel D, Quinn C, Shen J, Patel P, Glaser C, Baldwin N, et al. A modular analysis framework for blood genomics studies: application to systemic lupus erythematosus. Immunity. 2008 18;29(1): 150-64. (GSE11907)

3. Lauwerys BR, Hachulla E, Spertini F, Lazaro E, Jorgensen C, Mariette X, et al. Down-regulation of interferon signature in systemic lupus erythematosus patients by active immunization with interferon $\alpha$-kinoid. Arthritis \& Rheumatology. 2013; 65(2):447-56. (GSE39088)

4. Chiche L, Jourde-Chiche N, Whalen E, Presnell S, Gersuk V, Dang K, et al. Modular transcriptional repertoire analyses of adults with systemic lupus erythematosus reveal distinct type I and type II interferon signatures. Arthritis \& Rheumatology. 2014; 66(6):1583-95. (GSE49454)

5. Carpintero MF, Martinez L, Fernandez I, Romero AG, Mejia C, Zang Y, et al. Diagnosis and risk stratification in patients with anti-RNP autoimmunity. Lupus. 2015; 24(10):1057-66. (GSE61635)

6. Banchereau R, Hong S, Cantarel B, Baldwin N, Baisch J, Edens M, et al. Personalized immunomonitoring uncovers molecular networks that stratify lupus patients. Cell. 2016; 165(3):551-65. (GSE65391)

7. Welcher AA, Boedigheimer M, Kivitz AJ, Amoura Z, Buyon J, Rudinskaya A, et al. Blockade of interferon- $\gamma$ normalizes interferon-regulated gene expression and serum CXCL10 levels in patients with systemic lupus erythematosus. Arthritis \& Rheumatology. 2015; 67(10):2713-22. (GSE78193) 
bioRxiv preprint doi: https://doi.org/10.1101/277145; this version posted March 8,2018 . The copyright holder for this preprint (which was not

Supplement 1. Table 2. CD expression characteristics with the SLE Compendium

\begin{tabular}{|c|c|c|c|c|c|c|}
\hline $\begin{array}{c}\text { Cluster of } \\
\text { Differentiation }\end{array}$ & $\begin{array}{l}\text { Entrez } \\
\text { ID }\end{array}$ & $1^{\text {st }}$ quartile & Median & Mean & $\begin{array}{l}\text { Standard } \\
\text { error }\end{array}$ & $\begin{array}{c}3^{\text {rd }} \\
\text { quartile }\end{array}$ \\
\hline CD1A & 909 & 0.2877 & 0.4131 & 0.4306 & 0.0049 & 0.5418 \\
\hline CD1B & 910 & 0.0429 & 0.2810 & 0.2859 & 0.0067 & 0.4699 \\
\hline CD1C & 911 & 0.4298 & 0.4847 & 0.5165 & 0.0046 & 0.6191 \\
\hline CD1D & 912 & 0.4725 & 0.5790 & 0.5669 & 0.0047 & 0.6692 \\
\hline CD1E & 913 & 0.0808 & 0.3933 & 0.3550 & 0.0072 & 0.5690 \\
\hline CD2 & 914 & 0.5507 & 0.6660 & $\begin{array}{l}0.6479 \\
\end{array}$ & 0.0045 & 0.7567 \\
\hline CD3D & 915 & 0.5816 & 0.6579 & 0.6517 & 0.0037 & 0.7393 \\
\hline CD3E & 916 & 0.3478 & 0.4621 & 0.4756 & 0.0050 & 0.5963 \\
\hline CD3G & 917 & 0.4454 & 0.5510 & 0.5513 & 0.0045 & 0.6593 \\
\hline CD4 & 920 & 0.4148 & 0.5369 & 0.5325 & 0.0055 & 0.6743 \\
\hline CD5 & 921 & 0.4914 & 0.6062 & 0.5890 & 0.0047 & 0.7053 \\
\hline CD6 & 923 & 0.5397 & 0.6628 & 0.6438 & 0.0048 & 0.7728 \\
\hline CD7 & 924 & 0.4072 & 0.5400 & 0.5347 & 0.0052 & 0.6661 \\
\hline CD8A & 925 & 0.4365 & 0.5553 & 0.5594 & 0.0049 & 0.6907 \\
\hline CD8B & 926 & 0.3612 & 0.4399 & 0.4622 & 0.0044 & 0.5634 \\
\hline CD9 & 928 & 0.2634 & 0.4078 & 0.4108 & 0.0056 & 0.5510 \\
\hline CD10 & 4311 & 0.3475 & 0.4486 & 0.4754 & 0.0053 & 0.5955 \\
\hline CD11A & 3683 & 0.3848 & 0.4876 & 0.4934 & 0.0047 & 0.5992 \\
\hline CD11B & 3684 & 0.4461 & 0.5705 & 0.5660 & 0.0049 & 0.6893 \\
\hline CD11C & 3687 & 0.3237 & 0.4352 & 0.4567 & 0.0051 & 0.5685 \\
\hline CD13 & 290 & 0.4166 & 0.5281 & 0.5234 & 0.0048 & 0.6361 \\
\hline CD14 & 929 & 0.4937 & 0.6117 & 0.5974 & 0.0047 & 0.7128 \\
\hline CD15 & 2526 & 0.3056 & 0.3946 & 0.4032 & 0.0041 & 0.4802 \\
\hline CD16B & 2215 & 0.6375 & 0.7064 & 0.6900 & 0.0039 & 0.7744 \\
\hline CD18 & 3689 & 0.2797 & 0.3487 & 0.3843 & 0.0047 & 0.4451 \\
\hline CD19 & 930 & 0.3390 & 0.4880 & 0.4796 & 0.0057 & 0.6270 \\
\hline CD20 & 931 & 0.4250 & 0.5417 & 0.5368 & 0.0045 & 0.6543 \\
\hline CD21 & 1380 & 0.4267 & 0.5343 & 0.5282 & 0.0051 & 0.6581 \\
\hline CD22 & 933 & 0.2415 & 0.3850 & 0.3967 & 0.0051 & 0.5316 \\
\hline CD23 & 2208 & 0.3318 & 0.3686 & 0.4186 & 0.0052 & 0.5378 \\
\hline CD25 & 3559 & 0.3142 & 0.3952 & 0.4048 & 0.0042 & 0.4792 \\
\hline CD26 & 1803 & 0.3800 & 0.4833 & 0.4955 & 0.0048 & 0.6179 \\
\hline
\end{tabular}




\begin{tabular}{|c|c|c|c|c|c|c|}
\hline CD27 & 939 & 0.4434 & 0.5544 & 0.5574 & 0.0049 & 0.6732 \\
\hline CD28 & 940 & 0.5163 & 0.6076 & 0.5988 & 0.0042 & 0.6912 \\
\hline CD30 & 943 & 0.4002 & 0.5314 & 0.5164 & 0.0049 & 0.6416 \\
\hline CD31 & 5175 & 0.5092 & 0.6208 & 0.6047 & 0.0047 & 0.7196 \\
\hline CD33 & 945 & 0.4531 & 0.5563 & 0.5536 & 0.0045 & 0.6564 \\
\hline CD34 & 947 & 0.0888 & 0.2385 & 0.3193 & 0.0072 & 0.5643 \\
\hline CD35 & 1378 & 0.2969 & 0.3960 & 0.4141 & 0.0048 & 0.5101 \\
\hline CD36 & 948 & 0.5503 & 0.6644 & 0.6425 & 0.0046 & 0.7539 \\
\hline CD37 & 951 & 0.4015 & 0.5194 & 0.5212 & 0.0048 & 0.6362 \\
\hline CD38 & 952 & 0.2643 & 0.3496 & 0.3615 & 0.0042 & 0.4382 \\
\hline CD39 & 953 & 0.4219 & 0.5449 & 0.5388 & 0.0048 & 0.6571 \\
\hline CD40 & 958 & 0.4295 & 0.5178 & 0.5264 & 0.0049 & 0.6269 \\
\hline CD41 & 3674 & 0.2975 & 0.4246 & 0.4302 & 0.0051 & 0.5517 \\
\hline CD42A & 2815 & 0.4343 & 0.5542 & 0.5424 & 0.0049 & 0.6606 \\
\hline CD42B & 2811 & 0.2818 & 0.4168 & 0.4206 & 0.0055 & 0.5492 \\
\hline CD43 & 6693 & 0.4208 & 0.5204 & 0.5248 & 0.0046 & 0.6187 \\
\hline CD44 & 960 & 0.4447 & 0.5468 & 0.5461 & 0.0044 & 0.6526 \\
\hline CD45 & 5788 & 0.4831 & 0.5932 & 0.5897 & 0.0049 & 0.7097 \\
\hline CD46 & 4179 & 0.3236 & 0.4766 & 0.4776 & 0.0056 & 0.6164 \\
\hline CD47 & 961 & 0.3421 & 0.4422 & 0.4758 & 0.0051 & 0.6049 \\
\hline CD48 & 962 & 0.5302 & 0.6346 & 0.6206 & 0.0045 & 0.7340 \\
\hline CD49B & 3673 & 0.0505 & 0.0692 & 0.2157 & 0.0057 & 0.3656 \\
\hline CD49C & 3675 & 0.0874 & 0.1118 & 0.2535 & 0.0065 & 0.4513 \\
\hline CD49D & 3676 & 0.4888 & 0.5827 & 0.5825 & 0.0044 & 0.6847 \\
\hline CD49E & 3678 & 0.4647 & 0.5786 & 0.5710 & 0.0044 & 0.6751 \\
\hline CD49F & 3655 & 0.4701 & 0.5373 & 0.5076 & 0.0043 & 0.5757 \\
\hline CD50 & 3385 & 0.4232 & 0.5380 & 0.5340 & 0.0050 & 0.6507 \\
\hline CD51 & 3685 & 0.4443 & 0.5668 & 0.5565 & 0.0046 & 0.6670 \\
\hline CD52 & 1043 & 0.5706 & 0.6869 & 0.6614 & 0.0046 & 0.7794 \\
\hline CD53 & 963 & 0.4696 & 0.5772 & 0.5712 & 0.0045 & 0.6843 \\
\hline CD54 & 3383 & 0.4393 & 0.4668 & 0.4858 & 0.0041 & 0.5453 \\
\hline CD55 & 1604 & 0.3661 & 0.4807 & 0.4874 & 0.0048 & 0.5927 \\
\hline CD56 & 4684 & 0.4322 & 0.5026 & 0.4613 & 0.0043 & 0.5338 \\
\hline CD57 & 27087 & 0.0730 & 0.1057 & 0.2654 & 0.0066 & 0.4719 \\
\hline CD58 & 965 & 0.4668 & 0.5758 & 0.5660 & 0.0045 & 0.6744 \\
\hline
\end{tabular}




\begin{tabular}{|c|c|c|c|c|c|c|}
\hline CD59 & 966 & 0.3650 & 0.4031 & 0.4285 & 0.0038 & 0.4862 \\
\hline CD61 & 3690 & 0.3290 & 0.4558 & 0.4583 & 0.0051 & 0.5751 \\
\hline CD62E & 6401 & 0.0770 & 0.0991 & 0.2399 & 0.0067 & 0.4248 \\
\hline CD62L & 6402 & 0.7253 & 0.8035 & 0.7461 & 0.0049 & 0.8507 \\
\hline CD62P & 6403 & 0.3499 & 0.3783 & 0.4157 & 0.0043 & 0.4860 \\
\hline CD63 & 967 & 0.3594 & 0.4536 & 0.4675 & 0.0049 & 0.5648 \\
\hline CD66A & 634 & 0.2915 & 0.3878 & 0.4013 & 0.0045 & 0.5012 \\
\hline CD66B & 1088 & 0.1388 & 0.2813 & 0.3267 & 0.0057 & 0.4510 \\
\hline CD66C & 4680 & 0.1392 & 0.2528 & 0.2982 & 0.0058 & 0.4030 \\
\hline CD66D & 1084 & 0.4487 & 0.5544 & 0.5490 & 0.0047 & 0.6591 \\
\hline CD66E & 1048 & 0.0563 & 0.0711 & 0.1684 & 0.0054 & 0.2331 \\
\hline CD66F & 5669 & 0.0613 & 0.0806 & 0.2017 & 0.0063 & 0.3510 \\
\hline CD68 & 968 & 0.3514 & 0.4872 & 0.4973 & 0.0055 & 0.6275 \\
\hline CD69 & 969 & 0.3740 & 0.4842 & 0.4911 & 0.0048 & 0.6100 \\
\hline CD70 & 970 & 0.2230 & 0.2760 & 0.3463 & 0.0050 & 0.4596 \\
\hline CD71 & 7037 & 0.3510 & 0.4346 & 0.4396 & 0.0038 & 0.5131 \\
\hline CD72 & 971 & 0.3064 & 0.4160 & 0.4328 & 0.0049 & 0.5519 \\
\hline CD73 & 4907 & 0.4912 & 0.5631 & 0.5156 & 0.0051 & 0.5975 \\
\hline CD74 & 972 & 0.4180 & 0.5258 & 0.5255 & 0.0048 & 0.6352 \\
\hline CD79A & 973 & 0.3382 & 0.4927 & 0.4903 & 0.0055 & 0.6265 \\
\hline CD79B & 974 & 0.3125 & 0.4823 & 0.4761 & 0.0058 & 0.6293 \\
\hline CD80 & 941 & 0.0514 & 0.1602 & 0.2415 & 0.0063 & 0.4027 \\
\hline CD81 & 975 & 0.5014 & 0.6132 & 0.6063 & 0.0045 & 0.7234 \\
\hline CD82 & 3732 & 0.3397 & 0.4747 & 0.4727 & 0.0051 & 0.5940 \\
\hline CD83 & 9308 & 0.3623 & 0.4244 & 0.4407 & 0.0040 & 0.5118 \\
\hline CD84 & 8832 & 0.3429 & 0.4340 & 0.4523 & 0.0045 & 0.5422 \\
\hline CD85A & 11025 & 0.6931 & 0.7578 & 0.7279 & 0.0038 & 0.8070 \\
\hline CD85C & 10990 & 0.0850 & 0.4020 & 0.3630 & 0.0067 & 0.5531 \\
\hline CD85D & 10288 & 0.3225 & 0.4431 & 0.4598 & 0.0053 & 0.5779 \\
\hline CD85F & 353514 & 0.3677 & 0.5059 & 0.5027 & 0.0053 & 0.6397 \\
\hline CD85G & 23547 & 0.4090 & 0.4471 & 0.4459 & 0.0041 & 0.4722 \\
\hline $\mathrm{CD} 85 \mathrm{H}$ & 11027 & 0.5010 & 0.6070 & 0.5915 & 0.0044 & 0.7014 \\
\hline CD85J & 10859 & 0.3159 & 0.4139 & 0.4257 & 0.0046 & 0.5250 \\
\hline CD85K & 11006 & 0.4451 & 0.5721 & 0.5530 & 0.0049 & 0.6757 \\
\hline CD85M & 79166 & 0.0732 & 0.0925 & 0.1941 & 0.0058 & 0.2385 \\
\hline
\end{tabular}




\begin{tabular}{|c|c|c|c|c|c|c|}
\hline CD86 & 942 & 0.4575 & 0.5648 & 0.5551 & 0.0046 & 0.6661 \\
\hline CD87 & 5329 & 0.3865 & 0.5033 & 0.5014 & 0.0048 & 0.6157 \\
\hline CD88 & 728 & 0.4475 & 0.5733 & 0.5605 & 0.0048 & 0.6804 \\
\hline CD89 & 2204 & 0.2910 & 0.3992 & 0.4156 & 0.0049 & 0.5191 \\
\hline CD90 & 7070 & 0.4564 & 0.5648 & 0.5543 & 0.0046 & 0.6762 \\
\hline CD91 & 4035 & 0.3538 & 0.3758 & 0.4351 & 0.0045 & 0.5273 \\
\hline CD92 & 23446 & 0.2598 & 0.3575 & 0.3815 & 0.0048 & 0.4715 \\
\hline CD93 & 22918 & 0.5621 & 0.6614 & 0.6459 & 0.0043 & 0.7482 \\
\hline CD94 & 3824 & 0.3531 & 0.4708 & 0.4715 & 0.0051 & 0.5947 \\
\hline CD95 & 355 & 0.2904 & 0.3998 & 0.4154 & 0.0050 & 0.5267 \\
\hline CD96 & 10225 & 0.4582 & 0.5713 & 0.5583 & 0.0046 & 0.6695 \\
\hline CD97 & 976 & 0.6236 & 0.6971 & 0.6633 & 0.0046 & 0.7619 \\
\hline CD98 & 6520 & 0.3606 & 0.4524 & 0.4941 & 0.0051 & 0.5967 \\
\hline CD99 & 4267 & 0.4504 & 0.5680 & 0.5712 & 0.0050 & 0.6912 \\
\hline CD100 & 10507 & 0.3385 & 0.4490 & 0.4607 & 0.0050 & 0.5779 \\
\hline CD101 & 9398 & 0.2724 & 0.3138 & 0.3420 & 0.0047 & 0.3551 \\
\hline CD102 & 3384 & 0.4566 & 0.5730 & 0.5620 & 0.0049 & 0.6810 \\
\hline CD103 & 3682 & 0.4387 & 0.5648 & 0.5500 & 0.0050 & 0.6722 \\
\hline CD104 & 3691 & 0.0816 & 0.1027 & 0.2067 & 0.0057 & 0.2799 \\
\hline CD105 & 2022 & 0.3528 & 0.3820 & 0.4359 & 0.0046 & 0.5444 \\
\hline CD106 & 7412 & 0.0866 & 0.1180 & 0.2716 & 0.0065 & 0.4658 \\
\hline CD107A & 3916 & 0.4480 & 0.5595 & 0.5639 & 0.0050 & 0.6827 \\
\hline CD107B & 3920 & 0.4848 & 0.6087 & 0.5944 & 0.0050 & 0.7172 \\
\hline CD108 & 8482 & 0.0407 & 0.0579 & 0.2029 & 0.0058 & 0.3577 \\
\hline CD109 & 135228 & 0.0000 & 0.0000 & 0.1030 & 0.0052 & 0.1858 \\
\hline CD110 & 4352 & 0.3177 & 0.4492 & 0.4495 & 0.0054 & 0.5842 \\
\hline CD111 & 5818 & 0.0928 & 0.1236 & 0.2683 & 0.0066 & 0.4653 \\
\hline CD112 & 5819 & 0.3224 & 0.4273 & 0.4002 & 0.0044 & 0.4934 \\
\hline CD113 & 25945 & 0.0681 & 0.0913 & 0.2426 & 0.0064 & 0.4338 \\
\hline CD114 & 1441 & 0.5753 & 0.6686 & 0.6467 & 0.0043 & 0.7422 \\
\hline CD115 & 1436 & 0.5198 & 0.6335 & 0.6169 & 0.0047 & 0.7290 \\
\hline CD116 & 1438 & 0.4898 & 0.6032 & 0.5869 & 0.0048 & 0.7041 \\
\hline CD117 & 3815 & 0.3513 & 0.4393 & 0.4171 & 0.0048 & 0.5119 \\
\hline CD118 & 3977 & 0.0659 & 0.0853 & 0.1629 & 0.0050 & 0.1796 \\
\hline CD119 & 3459 & 0.4095 & 0.5120 & 0.5233 & 0.0048 & 0.6231 \\
\hline
\end{tabular}




\begin{tabular}{|c|c|c|c|c|c|c|}
\hline CD120A & 7132 & 0.5005 & 0.6198 & 0.6110 & 0.0047 & 0.7361 \\
\hline CD120B & 7133 & 0.4559 & 0.5577 & 0.5536 & 0.0043 & 0.6504 \\
\hline CD121A & 3554 & 0.0190 & 0.0244 & 0.1232 & 0.0049 & 0.1807 \\
\hline CD121B & 7850 & 0.2424 & 0.3407 & 0.3743 & 0.0052 & 0.4773 \\
\hline CD122 & 3560 & 0.3886 & 0.5172 & 0.5126 & 0.0052 & 0.6399 \\
\hline CD123 & 3563 & 0.3700 & 0.4103 & 0.3952 & 0.0043 & 0.4318 \\
\hline CD124 & 3566 & 0.2042 & 0.2736 & 0.3085 & 0.0043 & 0.3769 \\
\hline CD125 & 3568 & 0.3568 & 0.4262 & 0.4325 & 0.0041 & 0.5189 \\
\hline CD126 & 3570 & 0.3755 & 0.4928 & 0.4965 & 0.0051 & 0.6100 \\
\hline CD127 & 3575 & 0.5461 & 0.6411 & 0.6247 & 0.0040 & 0.7205 \\
\hline CD130 & 3572 & 0.3375 & 0.4164 & 0.4160 & 0.0041 & 0.5015 \\
\hline CD131 & 1439 & 0.4445 & 0.5347 & 0.5372 & 0.0041 & 0.6284 \\
\hline CD132 & 3561 & 0.3418 & 0.4780 & 0.4704 & 0.0053 & 0.5944 \\
\hline CD133 & 8842 & 0.0488 & 0.0621 & 0.1466 & 0.0050 & 0.1944 \\
\hline CD134 & 7293 & 0.3926 & 0.4894 & 0.4966 & 0.0045 & 0.5970 \\
\hline CD135 & 2322 & 0.0291 & 0.0373 & 0.1480 & 0.0057 & 0.2172 \\
\hline CD136 & 4486 & 0.0000 & 0.0000 & 0.1381 & 0.0070 & 0.1647 \\
\hline CD137 & 3604 & 0.3478 & 0.4435 & 0.4429 & 0.0045 & 0.5422 \\
\hline CD138 & 6382 & 0.3120 & 0.3694 & 0.3835 & 0.0044 & 0.4721 \\
\hline CD140A & 5156 & 0.0972 & 0.1263 & 0.2032 & 0.0053 & 0.2115 \\
\hline CD140B & 5159 & 0.3238 & 0.3561 & 0.4020 & 0.0049 & 0.4690 \\
\hline CD141 & 7056 & 0.2608 & 0.2839 & 0.3207 & 0.0043 & 0.3411 \\
\hline CD142 & 2152 & 0.0865 & 0.1460 & 0.2883 & 0.0067 & 0.4879 \\
\hline CD143 & 1636 & 0.0946 & 0.1203 & 0.2143 & 0.0056 & 0.2871 \\
\hline CD144 & 1003 & 0.1160 & 0.1465 & 0.2313 & 0.0054 & 0.2808 \\
\hline CD146 & 4162 & 0.0703 & 0.0887 & 0.1900 & 0.0058 & 0.2591 \\
\hline CD147 & 682 & 0.3429 & 0.4740 & 0.4832 & 0.0055 & 0.6138 \\
\hline CD148 & 5795 & 0.4195 & 0.5279 & 0.5285 & 0.0045 & 0.6324 \\
\hline CD150 & 6504 & 0.4758 & 0.6168 & 0.5923 & 0.0048 & 0.7180 \\
\hline CD151 & 977 & 0.3240 & 0.4176 & 0.4358 & 0.0045 & 0.5333 \\
\hline CD152 & 1493 & 0.5168 & 0.5735 & 0.5624 & 0.0046 & 0.6629 \\
\hline CD153 & 944 & 0.2956 & 0.3934 & 0.3992 & 0.0045 & 0.4975 \\
\hline CD154 & 959 & 0.3712 & 0.4881 & 0.4943 & 0.0053 & 0.6246 \\
\hline CD155 & 5817 & 0.4646 & 0.5331 & 0.5162 & 0.0043 & 0.5983 \\
\hline CD156A & 101 & 0.4237 & 0.5389 & 0.5380 & 0.0050 & 0.6521 \\
\hline
\end{tabular}




\begin{tabular}{|c|c|c|c|c|c|c|}
\hline CD156C & 102 & 0.4647 & 0.5321 & 0.5452 & 0.0039 & 0.6233 \\
\hline CD157 & 683 & 0.3466 & 0.4645 & 0.4743 & 0.0051 & 0.5949 \\
\hline CD158E & 3811 & 0.3143 & 0.4080 & 0.4189 & 0.0043 & 0.5066 \\
\hline CD158I & 3809 & 0.0000 & 0.0000 & 0.1134 & 0.0060 & 0.1442 \\
\hline CD158K & 3812 & 0.3227 & 0.4613 & 0.4677 & 0.0053 & 0.6060 \\
\hline CD160 & 11126 & 0.3611 & 0.4916 & 0.4939 & 0.0048 & 0.6118 \\
\hline CD161 & 3820 & 0.4143 & 0.5328 & 0.5259 & 0.0049 & 0.6424 \\
\hline CD162 & 6404 & 0.3945 & 0.5122 & 0.5080 & 0.0047 & 0.6193 \\
\hline CD163 & 9332 & 0.2490 & 0.3195 & 0.3529 & 0.0043 & 0.4156 \\
\hline CD164 & 8763 & 0.3328 & 0.4739 & 0.4945 & 0.0050 & 0.6267 \\
\hline CD166 & 214 & 0.3513 & 0.4532 & 0.4597 & 0.0048 & 0.5668 \\
\hline CD167A & 780 & 0.3657 & 0.4349 & 0.4419 & 0.0043 & 0.5196 \\
\hline CD167B & 4921 & 0.0842 & 0.1105 & 0.2371 & 0.0063 & 0.3681 \\
\hline CD168 & 3161 & 0.0473 & 0.1676 & 0.2173 & 0.0056 & 0.3415 \\
\hline CD169 & 6614 & 0.0290 & 0.1402 & 0.2449 & 0.0072 & 0.4275 \\
\hline CD170 & 8778 & 0.4018 & 0.5207 & 0.5186 & 0.0049 & 0.6382 \\
\hline CD171 & 3897 & 0.0649 & 0.0819 & 0.2025 & 0.0061 & 0.3430 \\
\hline CD172A & 140885 & 0.4620 & 0.5746 & 0.5634 & 0.0045 & 0.6771 \\
\hline CD172G & 55423 & 0.3642 & 0.4814 & 0.4895 & 0.0049 & 0.6088 \\
\hline CD174 & 2525 & 0.0000 & 0.0000 & 0.1298 & 0.0063 & 0.2475 \\
\hline CD177 & 57126 & 0.0226 & 0.0311 & 0.1232 & 0.0047 & 0.1642 \\
\hline CD178 & 356 & 0.4686 & 0.5054 & 0.5078 & 0.0044 & 0.5625 \\
\hline CD179A & 7441 & 0.0409 & 0.0523 & 0.1288 & 0.0045 & 0.1056 \\
\hline CD179B & 3543 & 0.5017 & 0.5881 & 0.5847 & 0.0044 & 0.6935 \\
\hline CD180 & 4064 & 0.0896 & 0.3274 & 0.3306 & 0.0066 & 0.5309 \\
\hline CD181 & 3577 & 0.5136 & 0.6410 & 0.6177 & 0.0049 & 0.7477 \\
\hline CD182 & 3579 & 0.5253 & 0.6211 & 0.6064 & 0.0041 & 0.7002 \\
\hline CD183 & 2833 & 0.4208 & 0.5164 & 0.5323 & 0.0046 & 0.6433 \\
\hline CD184 & 7852 & 0.4024 & 0.4948 & 0.5076 & 0.0045 & 0.6019 \\
\hline CD185 & 643 & 0.3929 & 0.5298 & 0.5033 & 0.0047 & 0.6156 \\
\hline CD186 & 10663 & 0.4005 & 0.4329 & 0.4296 & 0.0042 & 0.4769 \\
\hline CD191 & 1230 & 0.4444 & 0.5803 & 0.5620 & 0.0051 & 0.6919 \\
\hline CD193 & 1232 & 0.4625 & 0.5722 & 0.5543 & 0.0049 & 0.6712 \\
\hline CD194 & 1233 & 0.0459 & 0.0605 & 0.1963 & 0.0064 & 0.3592 \\
\hline CD195 & 1234 & 0.3732 & 0.4295 & 0.4471 & 0.0042 & 0.5207 \\
\hline
\end{tabular}




\begin{tabular}{|c|c|c|c|c|c|c|}
\hline CD196 & 1235 & 0.3381 & 0.4530 & 0.4578 & 0.0050 & 0.5753 \\
\hline CD197 & 1236 & 0.4787 & 0.6108 & 0.5969 & 0.0050 & 0.7204 \\
\hline CD199 & 10803 & 0.3392 & 0.4020 & 0.3840 & 0.0038 & 0.4403 \\
\hline CD200 & 4345 & 0.0567 & 0.0819 & 0.2404 & 0.0062 & 0.4174 \\
\hline CD201 & 10544 & 0.2714 & 0.3164 & 0.3688 & 0.0048 & 0.4618 \\
\hline CD202B & 7010 & 0.0674 & 0.2322 & 0.2739 & 0.0064 & 0.4369 \\
\hline CD203C & 5169 & 0.0818 & 0.1037 & 0.1722 & 0.0046 & 0.1822 \\
\hline CD204 & 4481 & 0.0663 & 0.0921 & 0.2563 & 0.0064 & 0.4345 \\
\hline CD205 & 4065 & 0.4357 & 0.5313 & 0.5450 & 0.0040 & 0.6345 \\
\hline CD206 & 4360 & 0.0625 & 0.0808 & 0.1978 & 0.0054 & 0.3369 \\
\hline CD207 & 50489 & 0.0724 & 0.0917 & 0.2102 & 0.0060 & 0.3352 \\
\hline CD208 & 27074 & 0.2642 & 0.3410 & 0.3676 & 0.0043 & 0.4326 \\
\hline CD209 & 30835 & 0.0000 & 0.0000 & 0.1070 & 0.0056 & 0.1425 \\
\hline CD210 & 3587 & 0.4240 & 0.5205 & 0.5219 & 0.0044 & 0.6222 \\
\hline CDW210B & 3588 & 0.5186 & 0.6042 & 0.5920 & 0.0041 & 0.6832 \\
\hline CD212 & 3594 & 0.4045 & 0.5213 & 0.5310 & 0.0049 & 0.6489 \\
\hline CD213A1 & 3597 & 0.4451 & 0.5561 & 0.5489 & 0.0048 & 0.6578 \\
\hline CD213A2 & 3598 & 0.0658 & 0.0828 & 0.1504 & 0.0045 & 0.1479 \\
\hline CD215 & 3601 & 0.0744 & 0.0945 & 0.1943 & 0.0057 & 0.2438 \\
\hline CD217A & 23765 & 0.3543 & 0.4847 & 0.4848 & 0.0053 & 0.6106 \\
\hline CD218A & 8809 & 0.2310 & 0.3237 & 0.3634 & 0.0053 & 0.4489 \\
\hline CD218B & 8807 & 0.3551 & 0.4772 & 0.4696 & 0.0049 & 0.5795 \\
\hline CD220 & 3643 & 0.0558 & 0.0707 & 0.1690 & 0.0054 & 0.2508 \\
\hline CD221 & 3480 & 0.3040 & 0.3717 & 0.3908 & 0.0041 & 0.4642 \\
\hline CD222 & 3482 & 0.3865 & 0.4883 & 0.4928 & 0.0047 & 0.5964 \\
\hline CD223 & 3902 & 0.3208 & 0.4433 & 0.4631 & 0.0048 & 0.5783 \\
\hline CD225 & 8519 & 0.6660 & 0.7433 & 0.7008 & 0.0047 & 0.7958 \\
\hline CD226 & 10666 & 0.4493 & 0.5699 & 0.5684 & 0.0046 & 0.6877 \\
\hline CD227 & 4582 & 0.2539 & 0.2935 & 0.3209 & 0.0038 & 0.3633 \\
\hline CD228 & 4241 & 0.0977 & 0.1279 & 0.2904 & 0.0067 & 0.5091 \\
\hline CD229 & 4063 & 0.4910 & 0.6255 & 0.6065 & 0.0049 & 0.7315 \\
\hline CD230 & 5621 & 0.4669 & 0.5790 & 0.5668 & 0.0047 & 0.6783 \\
\hline CD231 & 7102 & 0.0694 & 0.1267 & 0.1527 & 0.0038 & 0.2059 \\
\hline CD232 & 10154 & 0.3927 & 0.5165 & 0.5161 & 0.0050 & 0.6311 \\
\hline CD233 & 6521 & 0.3742 & 0.4941 & 0.4807 & 0.0052 & 0.5946 \\
\hline
\end{tabular}




\begin{tabular}{|c|c|c|c|c|c|c|}
\hline CD234 & 2532 & 0.2298 & 0.2971 & 0.3469 & 0.0051 & 0.4538 \\
\hline CD235A & 2993 & 0.0264 & 0.0336 & 0.0949 & 0.0040 & 0.1100 \\
\hline CD235B & 2994 & 0.2290 & 0.3563 & 0.3744 & 0.0056 & 0.5058 \\
\hline CD236 & 2995 & 0.4735 & 0.5625 & 0.5534 & 0.0043 & 0.6455 \\
\hline CD238 & 3792 & 0.2072 & 0.2995 & 0.3301 & 0.0045 & 0.4292 \\
\hline CD239 & 4059 & 0.1152 & 0.1459 & 0.2366 & 0.0059 & 0.2638 \\
\hline CD240CE & 6006 & 0.1861 & 0.2264 & 0.2542 & 0.0041 & 0.2675 \\
\hline CD240D & 6007 & 0.0252 & 0.0322 & 0.1400 & 0.0050 & 0.2184 \\
\hline CD241 & 6005 & 0.0282 & 0.0361 & 0.1449 & 0.0051 & 0.2316 \\
\hline CD242 & 3386 & 0.3286 & 0.3667 & 0.3986 & 0.0041 & 0.4481 \\
\hline CD243 & 5243 & 0.3582 & 0.4697 & 0.4731 & 0.0050 & 0.5995 \\
\hline CD244 & 51744 & 0.5086 & 0.5471 & 0.5625 & 0.0044 & 0.6383 \\
\hline CD246 & 238 & 0.0540 & 0.0687 & 0.1963 & 0.0064 & 0.3157 \\
\hline CD247 & 919 & 0.5804 & 0.6672 & 0.6598 & 0.0039 & 0.7547 \\
\hline CD248 & 57124 & 0.3734 & 0.4714 & 0.4798 & 0.0047 & 0.5894 \\
\hline CD249 & 2028 & 0.0632 & 0.0860 & 0.2344 & 0.0064 & 0.4235 \\
\hline CD252 & 7292 & 0.2576 & 0.3412 & 0.3685 & 0.0047 & 0.4500 \\
\hline CD253 & 8743 & 0.3552 & 0.4537 & 0.4613 & 0.0046 & 0.5625 \\
\hline CD254 & 8600 & 0.0778 & 0.1061 & 0.2277 & 0.0060 & 0.3639 \\
\hline CD257 & 10673 & 0.4844 & 0.6047 & 0.5812 & 0.0048 & 0.6959 \\
\hline CD258 & 8740 & 0.3544 & 0.5111 & 0.5044 & 0.0057 & 0.6435 \\
\hline CD262 & 8795 & 0.3919 & 0.5091 & 0.5013 & 0.0049 & 0.6212 \\
\hline CD263 & 8794 & 0.3718 & 0.4614 & 0.4833 & 0.0048 & 0.5685 \\
\hline CD264 & 8793 & 0.0537 & 0.0768 & 0.2575 & 0.0069 & 0.4421 \\
\hline CD265 & 8792 & 0.0607 & 0.0770 & 0.1617 & 0.0049 & 0.2216 \\
\hline CD266 & 51330 & 0.2305 & 0.2770 & 0.3496 & 0.0051 & 0.4592 \\
\hline CD267 & 23495 & 0.3001 & 0.3167 & 0.3391 & 0.0042 & 0.3625 \\
\hline CD269 & 608 & 0.2179 & 0.3244 & 0.3582 & 0.0049 & 0.4692 \\
\hline CD270 & 8764 & 0.3937 & 0.5311 & 0.5315 & 0.0053 & 0.6555 \\
\hline CD271 & 4804 & 0.0484 & 0.0626 & 0.1994 & 0.0059 & 0.3569 \\
\hline CD272 & 151888 & 0.3200 & 0.4516 & 0.4583 & 0.0049 & 0.5837 \\
\hline CD273 & 80380 & 0.0000 & 0.0000 & 0.0919 & 0.0048 & 0.1376 \\
\hline CD274 & 29126 & 0.0346 & 0.0441 & 0.1299 & 0.0049 & 0.1768 \\
\hline CD276 & 80381 & 0.0618 & 0.0801 & 0.2286 & 0.0069 & 0.3964 \\
\hline CD277 & 11119 & 0.3237 & 0.4063 & 0.4270 & 0.0044 & 0.5089 \\
\hline
\end{tabular}




\begin{tabular}{|c|c|c|c|c|c|c|}
\hline CD278 & 29851 & 0.4065 & 0.5303 & 0.5204 & 0.0051 & 0.6423 \\
\hline CD279 & 5133 & 0.0390 & 0.0499 & 0.1709 & 0.0061 & 0.2742 \\
\hline CD280 & 9902 & 0.0455 & 0.2031 & 0.2347 & 0.0058 & 0.3882 \\
\hline CD281 & 7096 & 0.3368 & 0.4997 & 0.4942 & 0.0060 & 0.6577 \\
\hline CD282 & 7097 & 0.2973 & 0.4055 & 0.4288 & 0.0050 & 0.5405 \\
\hline CD283 & 7098 & 0.0974 & 0.1225 & 0.2435 & 0.0059 & 0.3783 \\
\hline CD284 & 7099 & 0.3382 & 0.4540 & 0.4687 & 0.0051 & 0.5907 \\
\hline CD286 & 10333 & 0.3518 & 0.4914 & 0.4930 & 0.0054 & 0.6335 \\
\hline CD288 & 51311 & 0.4240 & 0.5298 & 0.5305 & 0.0048 & 0.6437 \\
\hline CD289 & 54106 & 0.3317 & 0.3715 & 0.3772 & 0.0042 & 0.3984 \\
\hline CD290 & 81793 & 0.4269 & 0.5439 & 0.5341 & 0.0046 & 0.6416 \\
\hline CD292 & 657 & 0.0928 & 0.1299 & 0.2889 & 0.0065 & 0.4903 \\
\hline CDW293 & 658 & 0.0771 & 0.1042 & 0.2290 & 0.0054 & 0.3880 \\
\hline CD294 & 11251 & 0.2721 & 0.2885 & 0.3274 & 0.0045 & 0.3827 \\
\hline CD295 & 3953 & 0.4433 & 0.5012 & 0.5038 & 0.0041 & 0.5759 \\
\hline CD296 & 417 & 0.3463 & 0.3758 & 0.4412 & 0.0044 & 0.5359 \\
\hline CD297 & 420 & 0.0485 & 0.0637 & 0.2164 & 0.0062 & 0.3780 \\
\hline CD298 & 483 & 0.3326 & 0.4407 & 0.4449 & 0.0047 & 0.5547 \\
\hline CD299 & 10332 & 0.0339 & 0.0427 & 0.1953 & 0.0067 & 0.3262 \\
\hline CD300A & 11314 & 0.4993 & 0.6021 & 0.5909 & 0.0045 & 0.6974 \\
\hline CD300C & 10871 & 0.4161 & 0.5457 & 0.5341 & 0.0051 & 0.6624 \\
\hline CD301 & 10462 & 0.4865 & 0.5319 & 0.5278 & 0.0044 & 0.6119 \\
\hline CD304 & 8829 & 0.0821 & 0.1085 & 0.2568 & 0.0065 & 0.4547 \\
\hline CD305 & 3903 & 0.3634 & 0.4618 & 0.4744 & 0.0044 & 0.5776 \\
\hline CD306 & 3904 & 0.2039 & 0.2878 & 0.3070 & 0.0044 & 0.3783 \\
\hline CD307A & 115350 & 0.0862 & 0.1091 & 0.1929 & 0.0053 & 0.2121 \\
\hline CD307B & 79368 & 0.3870 & 0.5031 & 0.4953 & 0.0047 & 0.6056 \\
\hline CD307C & 115352 & 0.4221 & 0.5118 & 0.5191 & 0.0044 & 0.6154 \\
\hline CD307D & 83417 & 0.0523 & 0.0663 & 0.1390 & 0.0046 & 0.1500 \\
\hline CD307E & 83416 & 0.2651 & 0.3560 & 0.3732 & 0.0044 & 0.4562 \\
\hline CD308 & 2321 & 0.1455 & 0.1845 & 0.2555 & 0.0054 & 0.2298 \\
\hline CD309 & 3791 & 0.0792 & 0.1003 & 0.1694 & 0.0045 & 0.1944 \\
\hline CD312 & 30817 & 0.4273 & 0.5262 & 0.5254 & 0.0046 & 0.6323 \\
\hline CD315 & 5738 & 0.0863 & 0.1089 & 0.1899 & 0.0050 & 0.2524 \\
\hline CD316 & 93185 & 0.1475 & 0.5214 & 0.4480 & 0.0070 & 0.6486 \\
\hline
\end{tabular}




\begin{tabular}{|c|c|c|c|c|c|c|}
\hline CD317 & 684 & 0.4043 & 0.5385 & 0.5355 & 0.0054 & 0.6746 \\
\hline CD318 & 64866 & 0.0909 & 0.2574 & 0.3190 & 0.0070 & 0.5427 \\
\hline CD319 & 57823 & 0.3282 & 0.4635 & 0.4620 & 0.0048 & 0.5813 \\
\hline CD320 & 51293 & 0.4975 & 0.5343 & 0.5618 & 0.0042 & 0.6432 \\
\hline CD321 & 50848 & 0.3659 & 0.4622 & 0.4825 & 0.0047 & 0.5838 \\
\hline CD322 & 58494 & 0.0578 & 0.0736 & 0.2095 & 0.0065 & 0.3546 \\
\hline CD324 & 999 & 0.0830 & 0.1046 & 0.1786 & 0.0048 & 0.1851 \\
\hline CD325 & 1000 & 0.1798 & 0.2115 & 0.2514 & 0.0044 & 0.2504 \\
\hline CD326 & 4072 & 0.0374 & 0.0473 & 0.1227 & 0.0043 & 0.1631 \\
\hline CD327 & 946 & 0.1152 & 0.4171 & 0.3868 & 0.0067 & 0.5691 \\
\hline CD328 & 27036 & 0.3945 & 0.4399 & 0.4761 & 0.0041 & 0.5506 \\
\hline CD329 & 27180 & 0.3735 & 0.4845 & 0.4874 & 0.0050 & 0.5985 \\
\hline CD331 & 2260 & 0.0623 & 0.0815 & 0.1848 & 0.0057 & 0.2825 \\
\hline CD332 & 2263 & 0.1078 & 0.1379 & 0.1910 & 0.0046 & 0.1685 \\
\hline CD333 & 2261 & 0.0938 & 0.1198 & 0.2726 & 0.0064 & 0.4759 \\
\hline CD334 & 2264 & 0.1030 & 0.1317 & 0.2100 & 0.0055 & 0.1767 \\
\hline CD335 & 9437 & 0.0930 & 0.1176 & 0.2136 & 0.0056 & 0.3205 \\
\hline CD336 & 9436 & 0.0406 & 0.0514 & 0.1626 & 0.0057 & 0.2566 \\
\hline CD337 & 259197 & 0.3529 & 0.4894 & 0.4933 & 0.0046 & 0.6105 \\
\hline CD338 & 9429 & 0.0000 & 0.0000 & 0.0981 & 0.0052 & 0.0917 \\
\hline CD339 & 182 & 0.0478 & 0.0622 & 0.1937 & 0.0061 & 0.3276 \\
\hline CD340 & 2064 & 0.4351 & 0.4842 & 0.4856 & 0.0044 & 0.5603 \\
\hline CD344 & 8322 & 0.0000 & 0.0000 & 0.1506 & 0.0071 & 0.3500 \\
\hline CD349 & 8326 & 0.0453 & 0.0572 & 0.1384 & 0.0049 & 0.1556 \\
\hline CD350 & 11211 & 0.0368 & 0.0508 & 0.2128 & 0.0066 & 0.3945 \\
\hline CD351 & 8395 & 0.2458 & 0.3268 & 0.3550 & 0.0044 & 0.4425 \\
\hline CD353 & 56833 & 0.0388 & 0.0496 & 0.1923 & 0.0060 & 0.3306 \\
\hline CD354 & 54210 & 0.4307 & 0.5610 & 0.5460 & 0.0052 & 0.6796 \\
\hline CD355 & 56253 & 0.0568 & 0.0724 & 0.1858 & 0.0061 & 0.2610 \\
\hline CD357 & 8784 & 0.0684 & 0.0893 & 0.2214 & 0.0061 & 0.3834 \\
\hline CD358 & 27242 & 0.3184 & 0.3443 & 0.3893 & 0.0049 & 0.4793 \\
\hline CD360 & 50615 & 0.5752 & 0.6418 & 0.6174 & 0.0045 & 0.7088 \\
\hline CD361 & 2124 & 0.2721 & 0.3649 & 0.3831 & 0.0047 & 0.4680 \\
\hline CD362 & 6383 & 0.2068 & 0.2533 & 0.3137 & 0.0050 & 0.3991 \\
\hline CD363 & 1901 & 0.4069 & 0.5292 & 0.5368 & 0.0051 & 0.6612 \\
\hline
\end{tabular}




\begin{tabular}{|c|c|c|c|c|c|c|}
\hline CD364 & 221476 & 0.4220 & 0.4661 & 0.4906 & 0.0047 & 0.5900 \\
\hline CD366 & 84868 & 0.4126 & 0.5178 & 0.5167 & 0.0046 & 0.6249 \\
\hline CD367 & 50856 & 0.5184 & 0.6120 & 0.6118 & 0.0042 & 0.7098 \\
\hline CD369 & 64581 & 0.4805 & 0.5836 & 0.5811 & 0.0049 & 0.6980 \\
\hline
\end{tabular}

By default, CD gene expression was stratified at 20 and 80 percentiles, with low expression being between 0 and 20, average between 20 and 80, and high between 80 and 100. Two-tailed thresholds were labeled as 'aberrant' or 'nonaberrant', with 'aberrant' being further delineated as 'low' or 'high'. 
bioRxiv preprint doi: https://doi.org/10.1101/277145; this version posted March 8,2018 . The copyright holder for this preprint (which was not certified by peer review) is the author/funder, who has granted bioRxiv a license to display the preprint in perpetuity. It is made available under aCC-BY-NC 4.0 International license.

Supplement 2. Table 3. Clusters of differentiation gene expression requiring. Feature transformation included threshold shifting $(n=3)$ or binary dichotomization $(n=85)$.

\begin{tabular}{|c|c|c|c|c|}
\hline $\begin{array}{c}\text { Cluster of } \\
\text { Differentiation }\end{array}$ & Entrez ID & Feature transformation & Threshold & Figure \# \\
\hline CD34 & 947 & Dichotomization & 0.18 & 38 \\
\hline CD49B & 3673 & Dichotomization & 0.1 & 54 \\
\hline CD49C & 3675 & Dichotomization & 0.18 & 55 \\
\hline CD57 & 27087 & Dichotomization & 0.2 & 66 \\
\hline CD62E & 6401 & Dichotomization & 0.2 & 70 \\
\hline CD62L & 6402 & Dichotomization & 0.7 & 71 \\
\hline CD66E & 1048 & Dichotomization & 0.12 & 78 \\
\hline CD66F & 5669 & Dichotomization & 0.15 & 79 \\
\hline CD72 & 971 & First threshold shifted & 0.35 & 84 \\
\hline CD80 & 941 & Dichotomization & 0.1 & 89 \\
\hline CD85C & 10990 & Dichotomization & 0.2 & 95 \\
\hline CD85M & 79166 & Dichotomization & 0.2 & 102 \\
\hline CD91 & 4035 & Dichotomization & 0.45 & 108 \\
\hline CD104 & 3691 & Dichotomization & 0.2 & 121 \\
\hline CD105 & 2022 & First threshold shifted & 0.3 & 122 \\
\hline CD106 & 7412 & Dichotomization & 0.2 & 123 \\
\hline CD108 & 8482 & Dichotomization & 0.15 & 126 \\
\hline CD111 & 5818 & Dichotomization & 0.2 & 129 \\
\hline CD113 & 25945 & Dichotomization & 0.15 & 131 \\
\hline CD118 & 3977 & Dichotomization & 0.18 & 136 \\
\hline CD121A & 3554 & Dichotomization & 0.1 & 140 \\
\hline CD133 & 8842 & Dichotomization & 0.1 & 151 \\
\hline CD135 & 2322 & Dichotomization & 0.1 & 153 \\
\hline CD140A & 5156 & Dichotomization & 0.2 & 157 \\
\hline CD142 & 2152 & Dichotomization & 0.2 & 160 \\
\hline CD143 & 1636 & Dichotomization & 0.2 & 161 \\
\hline CD144 & 1003 & Dichotomization & 0.25 & 162 \\
\hline CD146 & 4162 & Dichotomization & 0.2 & 163 \\
\hline CD154 & 959 & Dichotomization & 0.35 & 170 \\
\hline CD167B & 4921 & Dichotomization & 0.2 & 185 \\
\hline CD168 & 3161 & Dichotomization & 0.15 & 186 \\
\hline CD169 & 6614 & Dichotomization & 0.1 & 187 \\
\hline CD171 & 3897 & Dichotomization & 0.2 & 189 \\
\hline CD179A & 7441 & Dichotomization & 0.15 & 195 \\
\hline CD180 & 4064 & Dichotomization & 0.2 & 197 \\
\hline
\end{tabular}




\begin{tabular}{|c|c|c|c|c|}
\hline CD194 & 1233 & Dichotomization & 0.1 & 206 \\
\hline CD200 & 4345 & Dichotomization & 0.2 & 211 \\
\hline CD202B & 7010 & Dichotomization & 0.15 & 213 \\
\hline CD203C & 5169 & Dichotomization & 0.18 & 214 \\
\hline CD204 & 4481 & Dichotomization & 0.2 & 215 \\
\hline CD206 & 4360 & Dichotomization & 0.15 & 217 \\
\hline CD207 & 50489 & Dichotomization & 0.15 & 218 \\
\hline CD213A2 & 3598 & Dichotomization & 0.15 & 225 \\
\hline CD215 & 3601 & Dichotomization & 0.2 & 226 \\
\hline CD220 & 3643 & Dichotomization & 0.15 & 230 \\
\hline CD225 & 8519 & Dichotomization & 0.63 & 234 \\
\hline CD228 & 4241 & Dichotomization & 0.2 & 237 \\
\hline CD231 & 7102 & First threshold shifted & 0.05 & 240 \\
\hline CD235A & 2993 & Dichotomization & 0.07 & 244 \\
\hline CD239 & 4059 & Dichotomization & 0.2 & 248 \\
\hline CD240D & 6007 & Dichotomization & 0.1 & 250 \\
\hline CD241 & 6005 & Dichotomization & 0.1 & 251 \\
\hline CD246 & 238 & Dichotomization & 0.2 & 255 \\
\hline CD249 & 2028 & Dichotomization & 0.2 & 258 \\
\hline CD254 & 8600 & Dichotomization & 0.2 & 261 \\
\hline CD264 & 8793 & Dichotomization & 0.15 & 266 \\
\hline CD265 & 8792 & Dichotomization & 0.15 & 267 \\
\hline CD271 & 4804 & Dichotomization & 0.15 & 272 \\
\hline CD274 & 29126 & Dichotomization & 0.1 & 275 \\
\hline CD276 & 80381 & Dichotomization & 0.2 & 276 \\
\hline CD279 & 5133 & Dichotomization & 0.1 & 279 \\
\hline CD280 & 9902 & Dichotomization & 0.15 & 280 \\
\hline CD283 & 7098 & Dichotomization & 0.2 & 283 \\
\hline CD292 & 657 & Dichotomization & 0.2 & 289 \\
\hline CD297 & 420 & Dichotomization & 0.2 & 290 \\
\hline CD299 & 10332 & Dichotomization & 0.1 & 294 \\
\hline CD304 & 8829 & Dichotomization & 0.2 & 296 \\
\hline CD307A & 115350 & Dichotomization & 0.2 & 300 \\
\hline CD307D & 83417 & Dichotomization & 0.1 & 303 \\
\hline CD309 & 3791 & Dichotomization & 0.2 & 306 \\
\hline CD315 & 5738 & Dichotomization & 0.2 & 309 \\
\hline CD316 & 93185 & Dichotomization & 0.2 & 311 \\
\hline CD318 & 64866 & Dichotomization & 0.2 & 312 \\
\hline CD322 & 58494 & Dichotomization & 0.15 & 314 \\
\hline
\end{tabular}


bioRxiv preprint doi: https://doi org/10.1101/277145 this version posted March 8 2018. The copyright holder for this preprint (which was not certified by peer review) is the author/funder, who has granted bioRxiv a license to display the preprint in perpetuity. It is made available under aCC-BY-NC 4.0 International license.

\begin{tabular}{|c|c|c|c|c|}
\hline CD324 & 999 & Dichotomization & 0.2 & 318 \\
\hline CD326 & 4072 & Dichotomization & 0.1 & 319 \\
\hline CD331 & 2260 & Dichotomization & 0.2 & 321 \\
\hline CD333 & 2261 & Dichotomization & 0.2 & 325 \\
\hline CD334 & 2264 & Dichotomization & 0.2 & 327 \\
\hline CD335 & 9437 & Dichotomization & 0.2 & 328 \\
\hline CD336 & 9436 & Dichotomization & 0.1 & 329 \\
\hline CD339 & 182 & Dichotomization & 0.1 & 330 \\
\hline CD349 & 8326 & Dichotomization & 0.1 & 333 \\
\hline CD350 & 11211 & Dichotomization & 0.1 & 336 \\
\hline CD353 & 56833 & Dichotomization & 0.1 & 337 \\
\hline CD355 & 56253 & Dichotomization & 0.15 & 339 \\
\hline CD357 & 8784 & Dichotomization & 0.15 & 341 \\
\hline CDW293 & 658 & Dichotomization & 0.2 & 342 \\
\hline
\end{tabular}


bioRxiv preprint doi: https://doi.org/10.1101/277145; this version posted March 8, 2018. The copyright holder for this preprint (which was not certified by peer review) is the author/funder, who has granted bioRxiv a license to display the preprint in perpetuity. It is made available under aCC-BY-NC 4.0 International license.

Supplement 3. Figures 1 - 351. Distributions Clusters of Differentiation and labels of aberrant gene expression. 
Figure 2 - CD1B

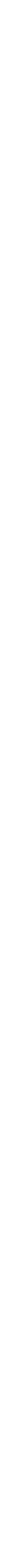


Figure 4 - CD1D

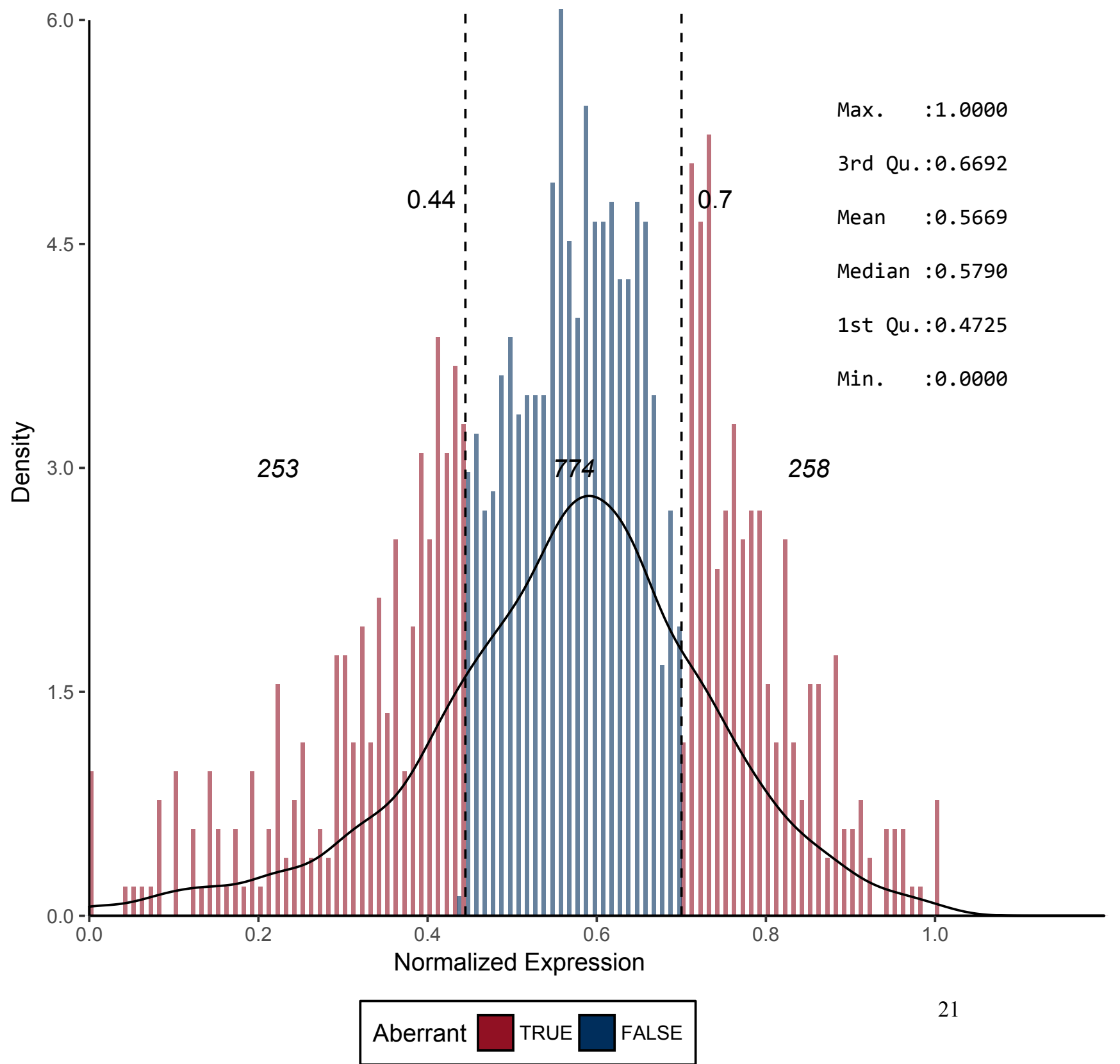


Figure 5 - CD1E

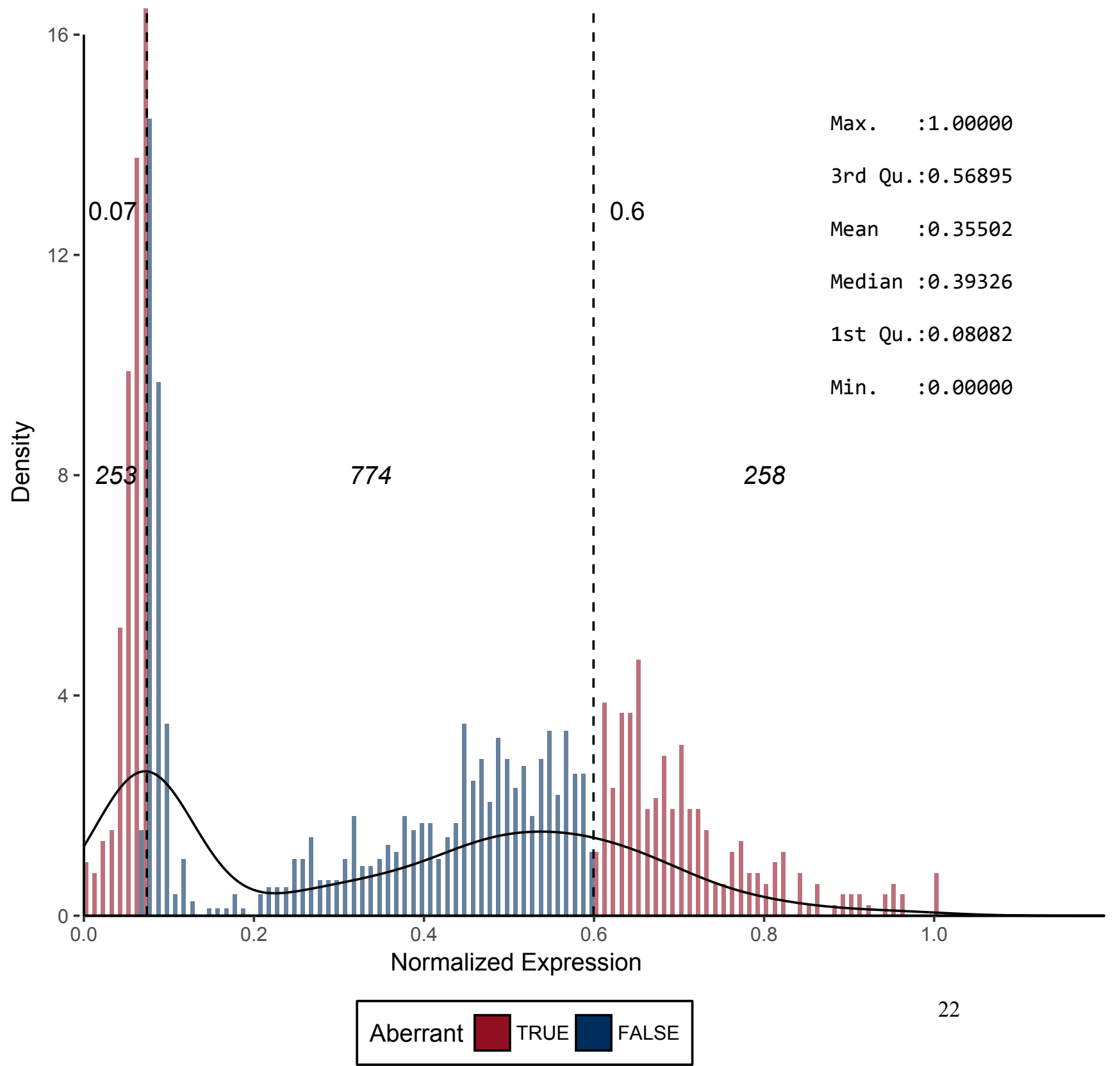


Figure 6 - CD2

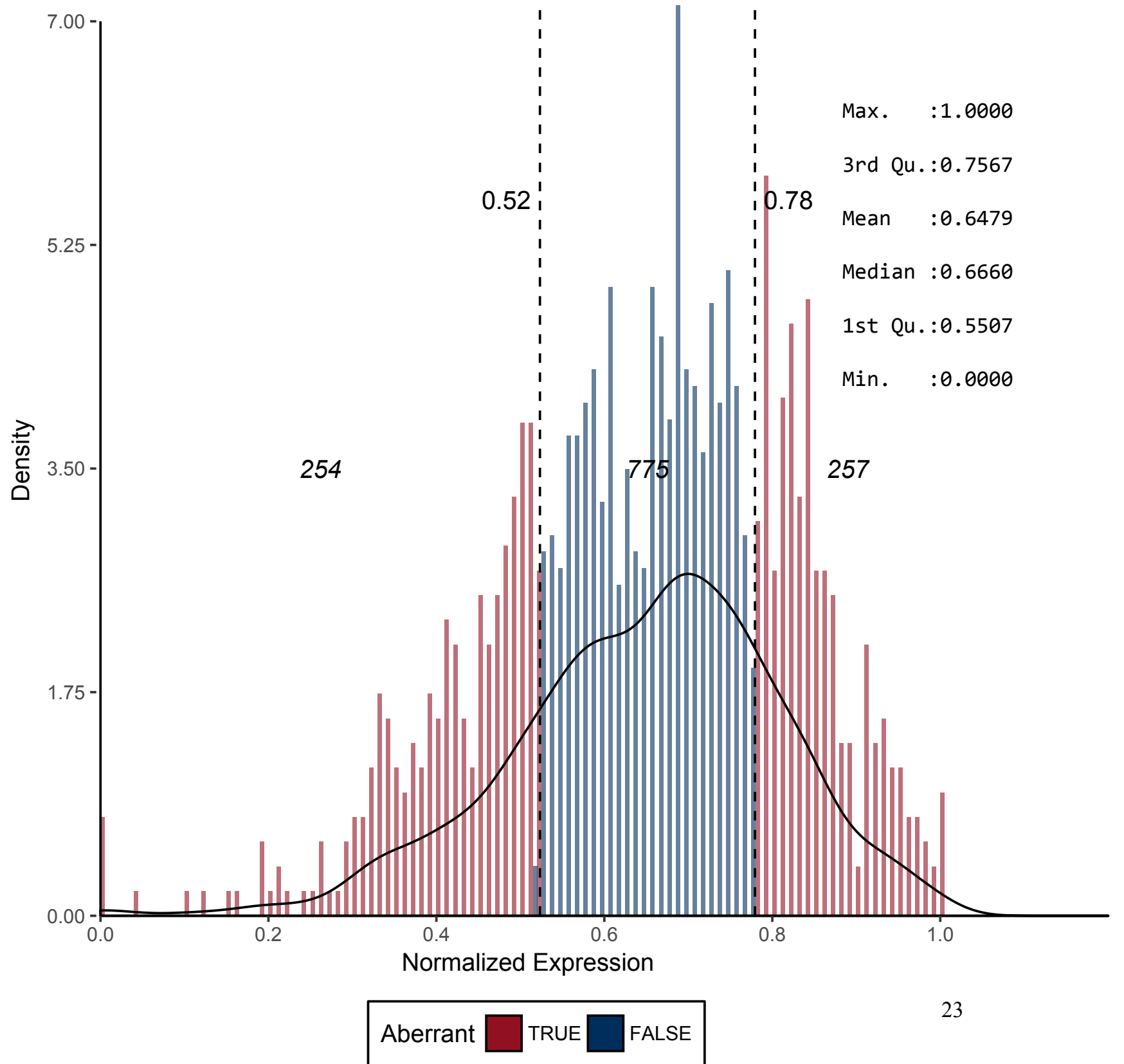


Figure 7 - CD3D
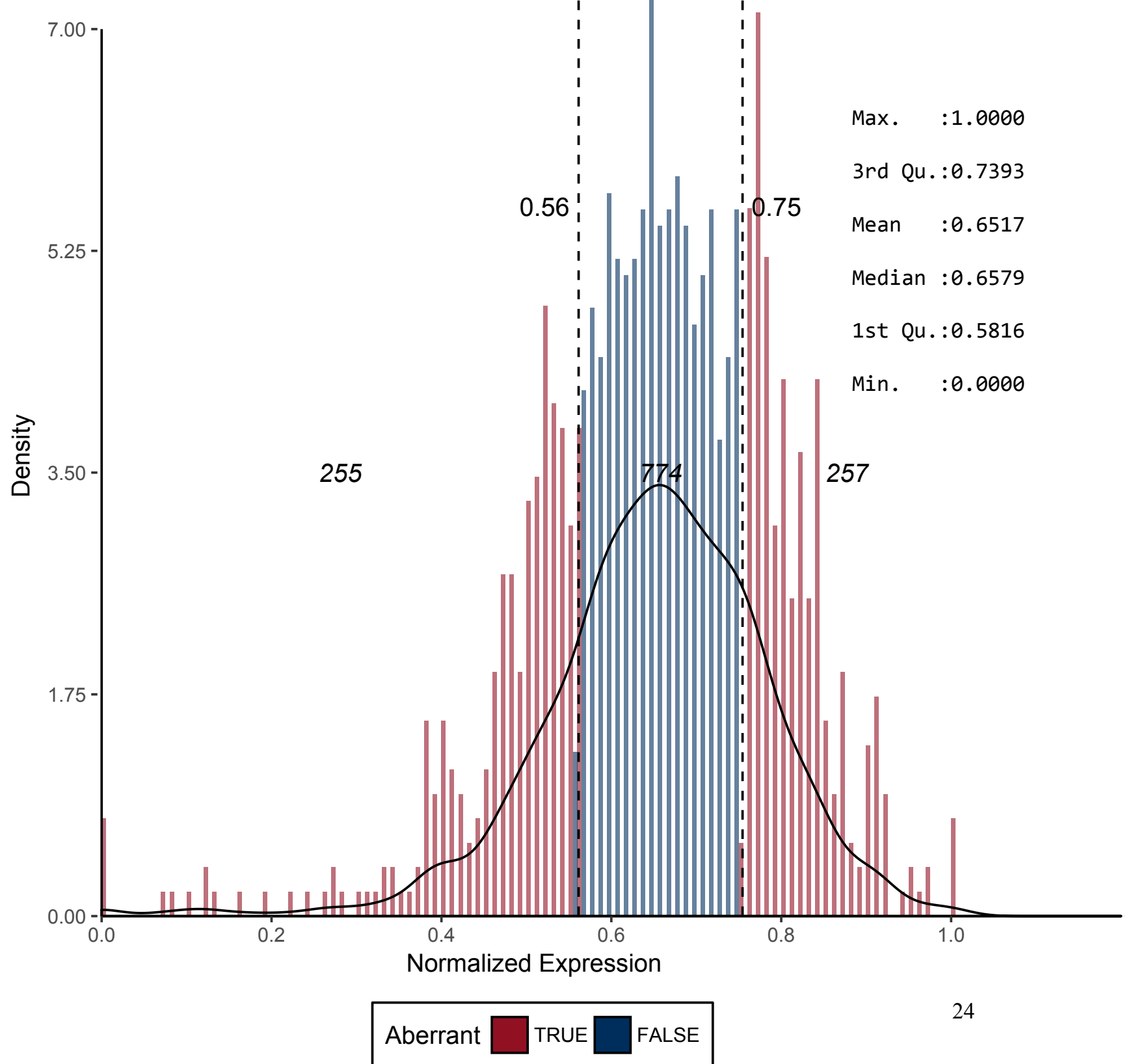
Figure 10 - CD4

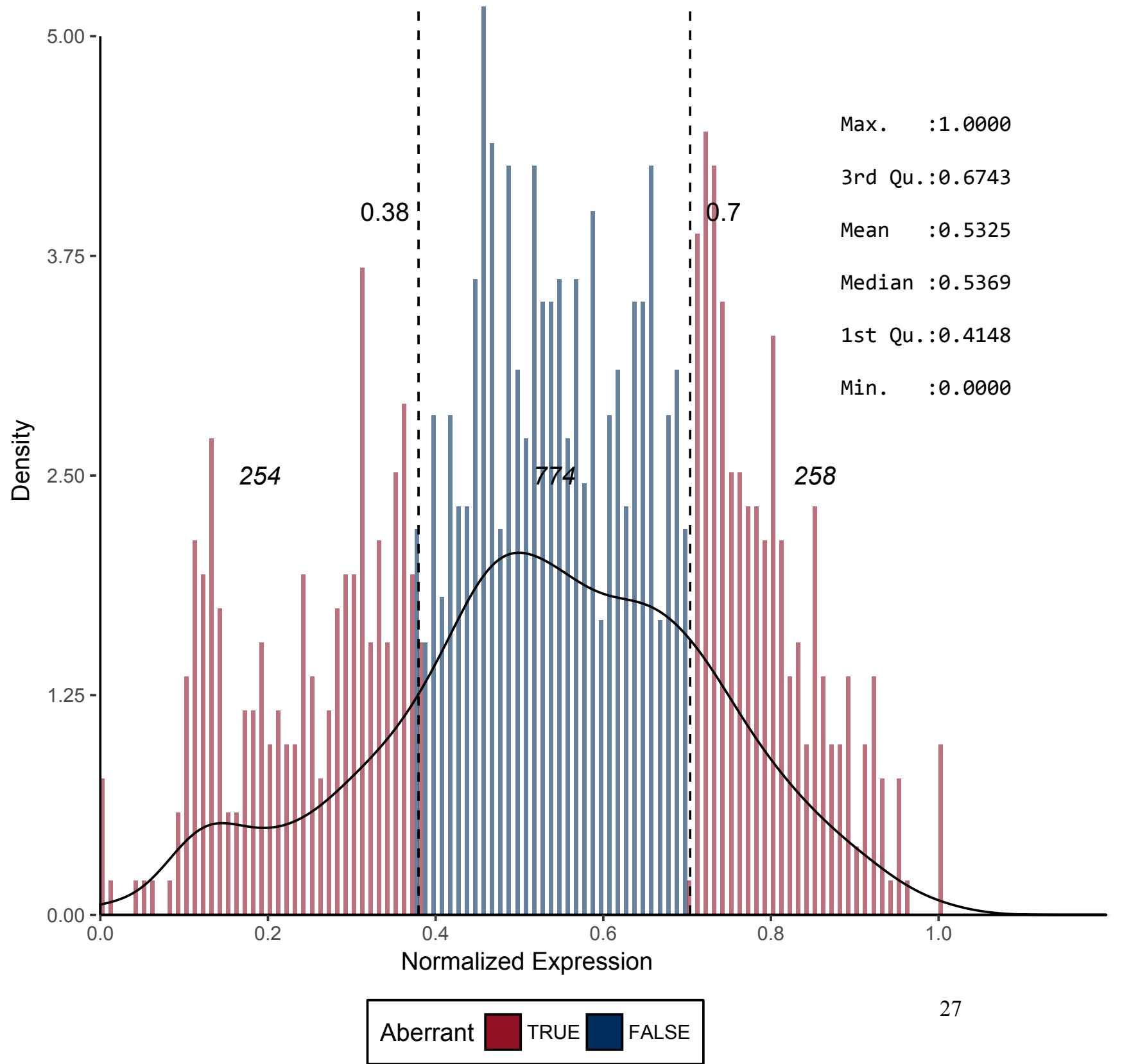


Figure 12 - CD6

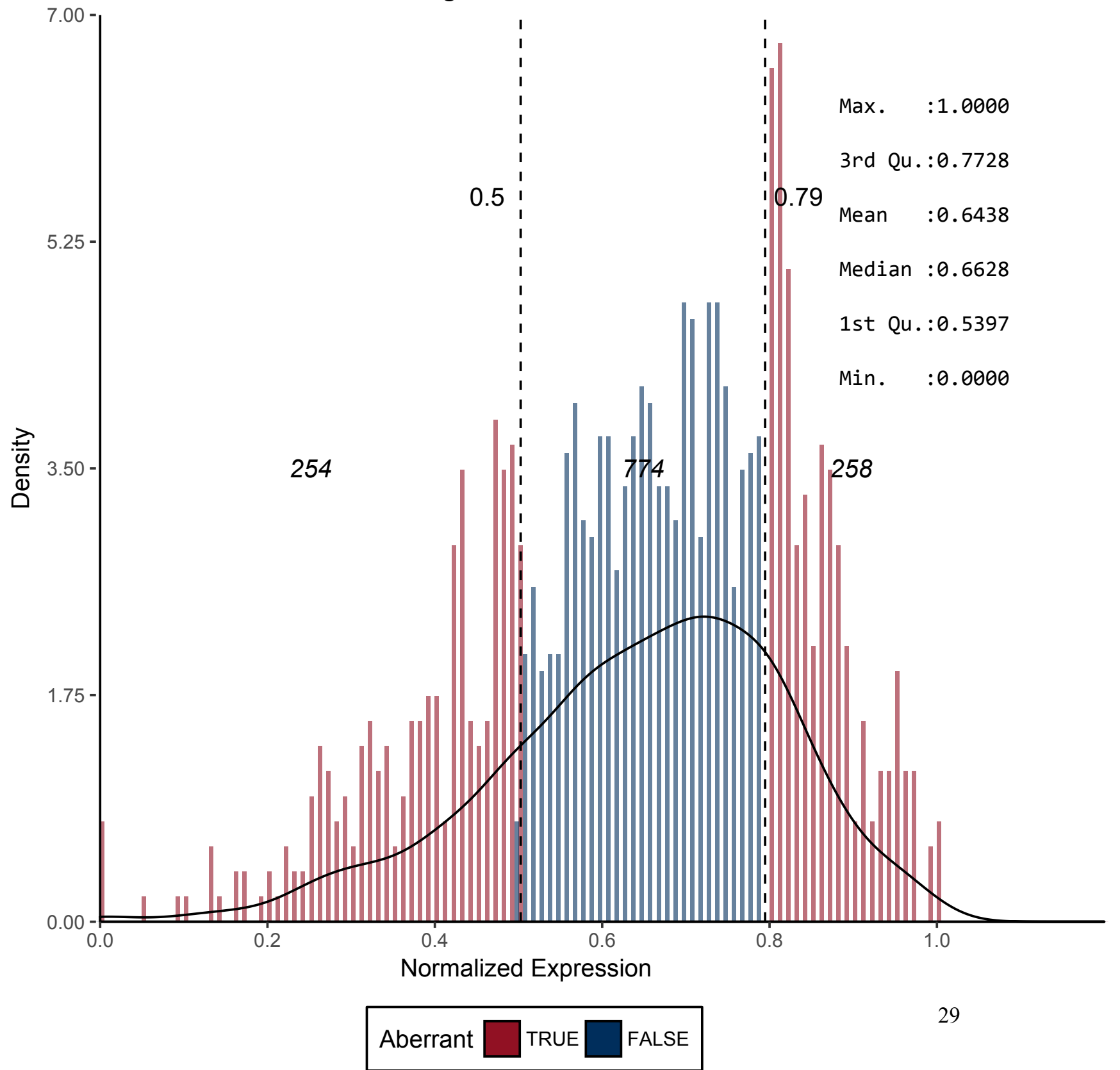


Figure 13 - CD7

-
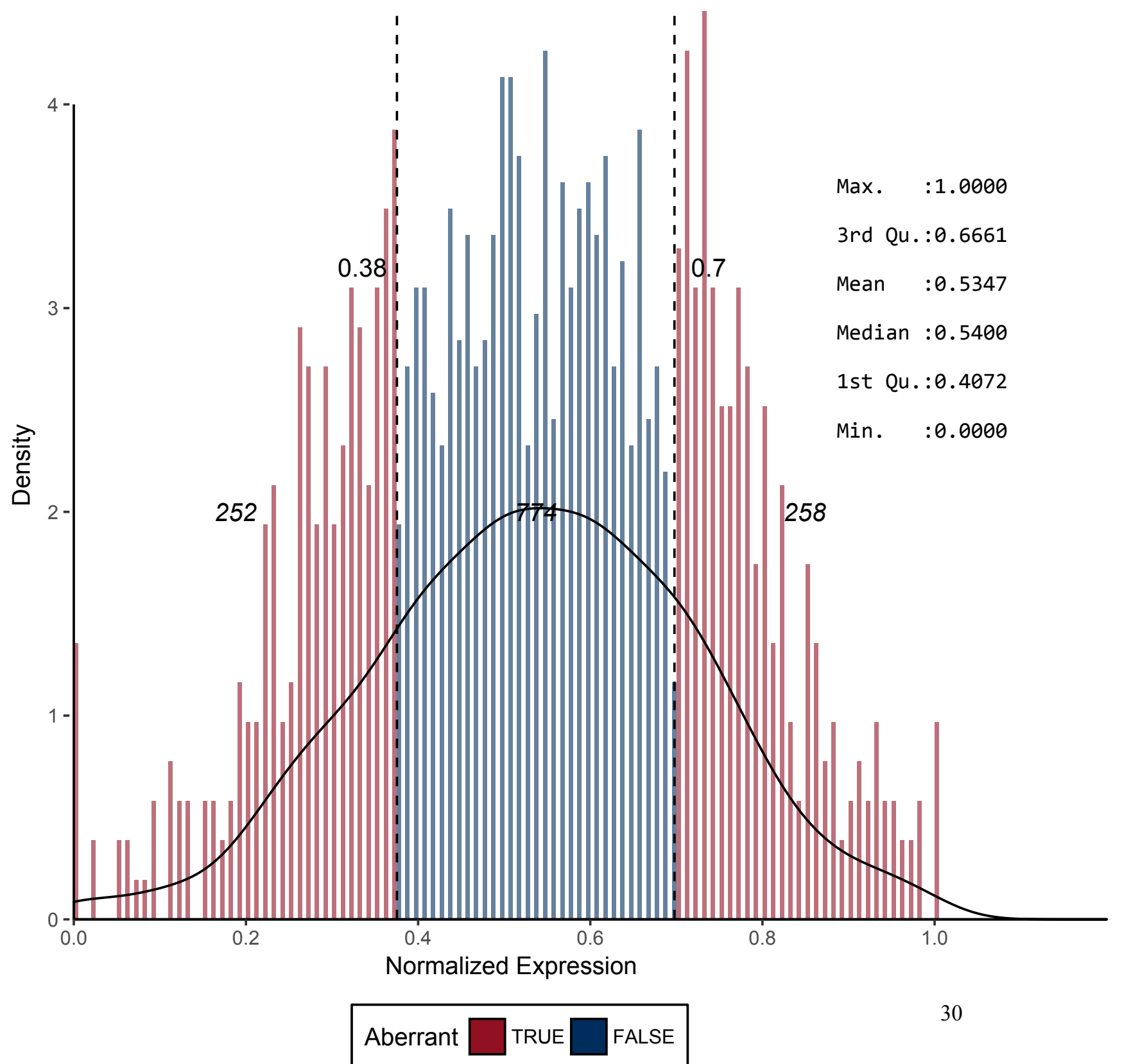
Figure 14 - CD8A
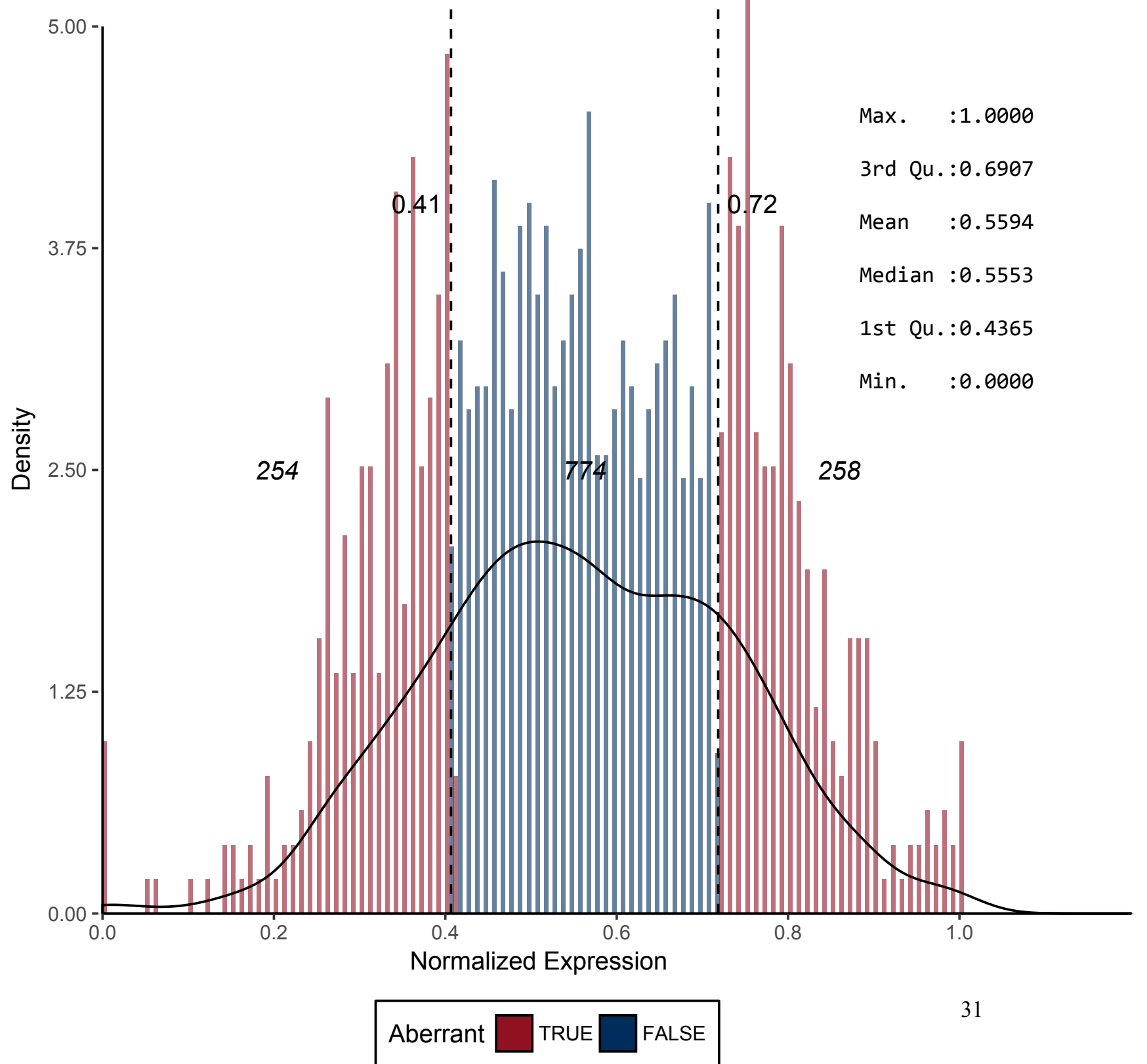
Figure 15 - CD8B

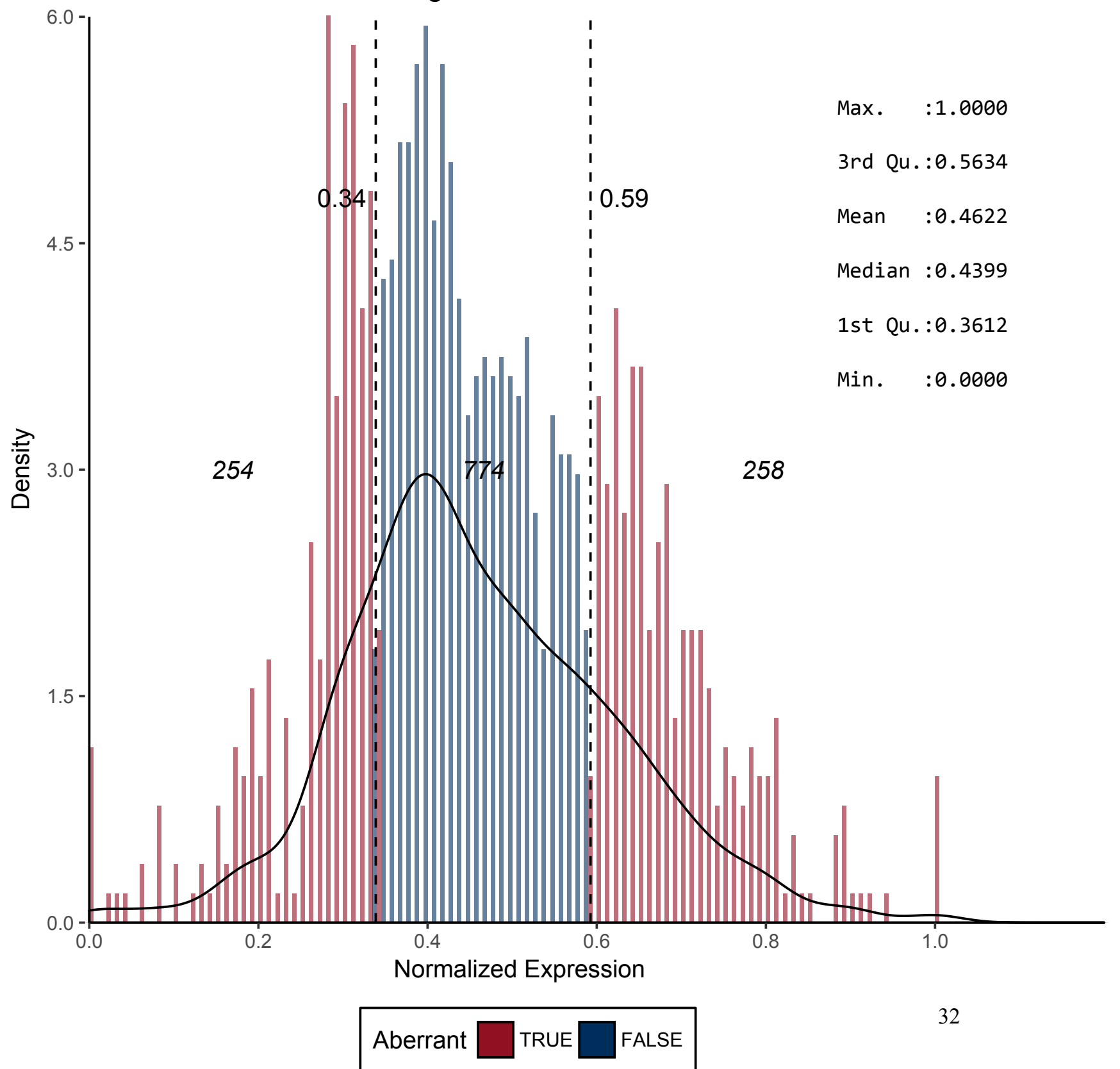


Figure 18 - CD11A

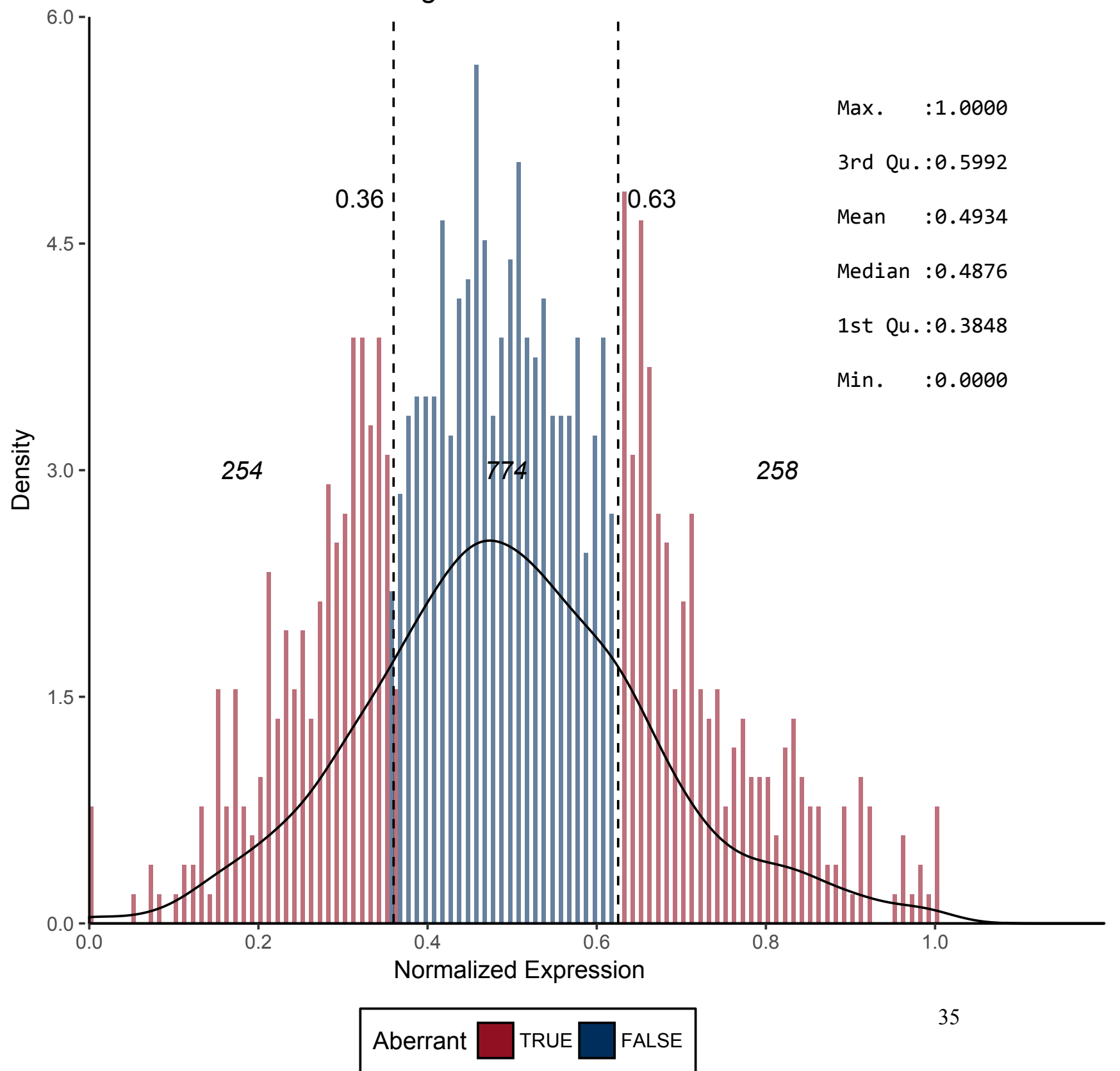


Figure 19 - CD11B
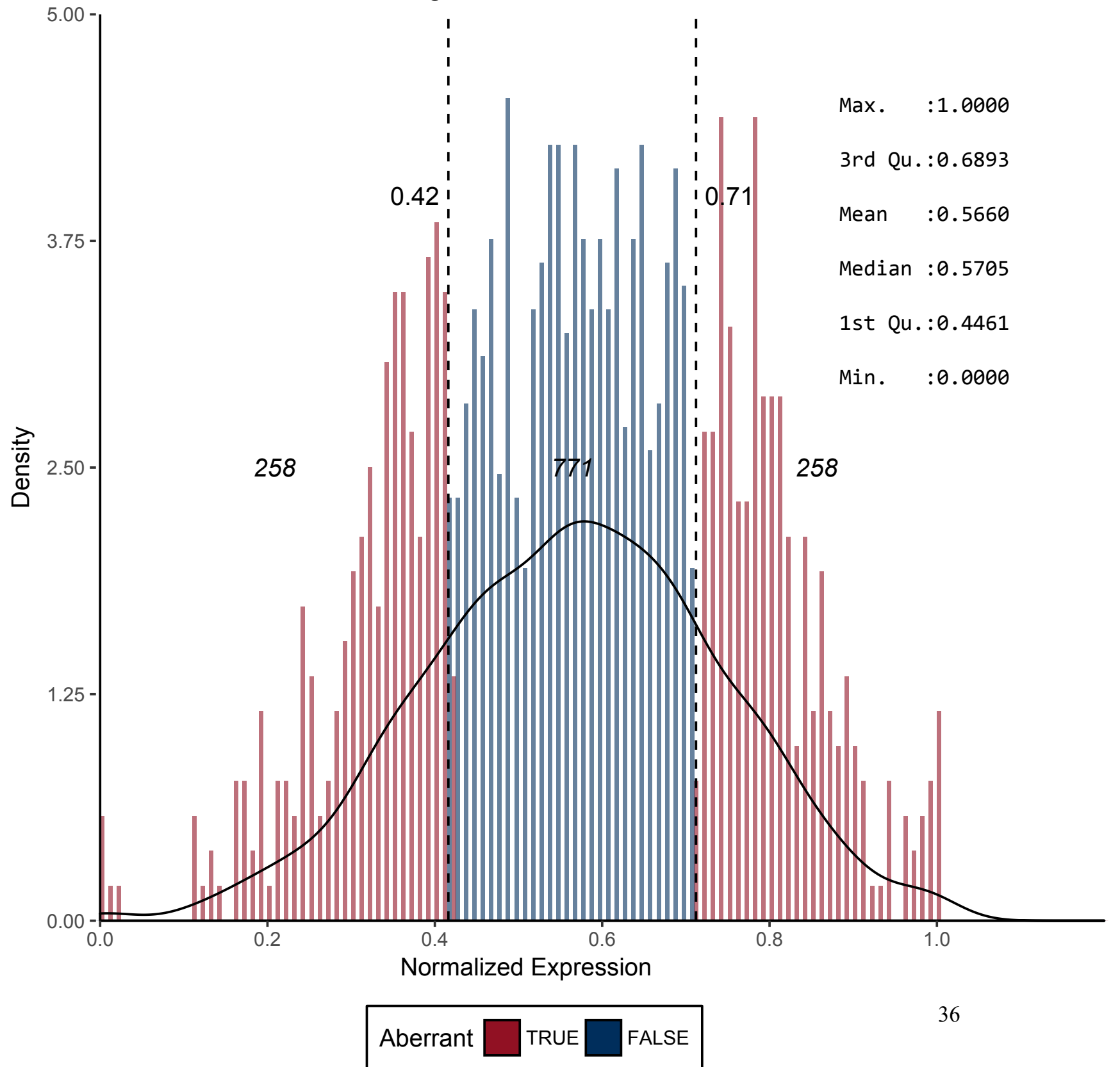
Figure 20 - CD11C

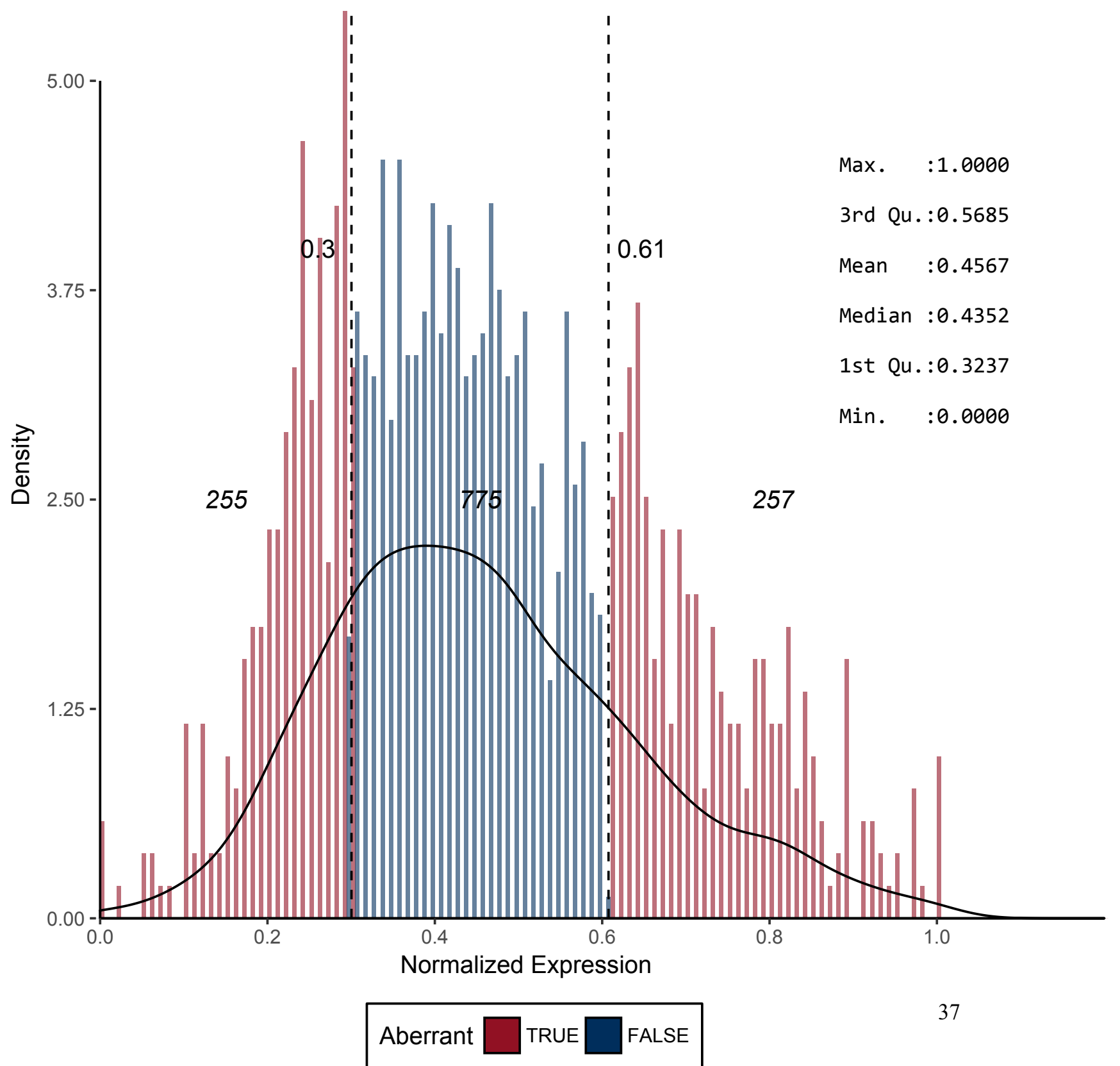


Figure 21 - CD13

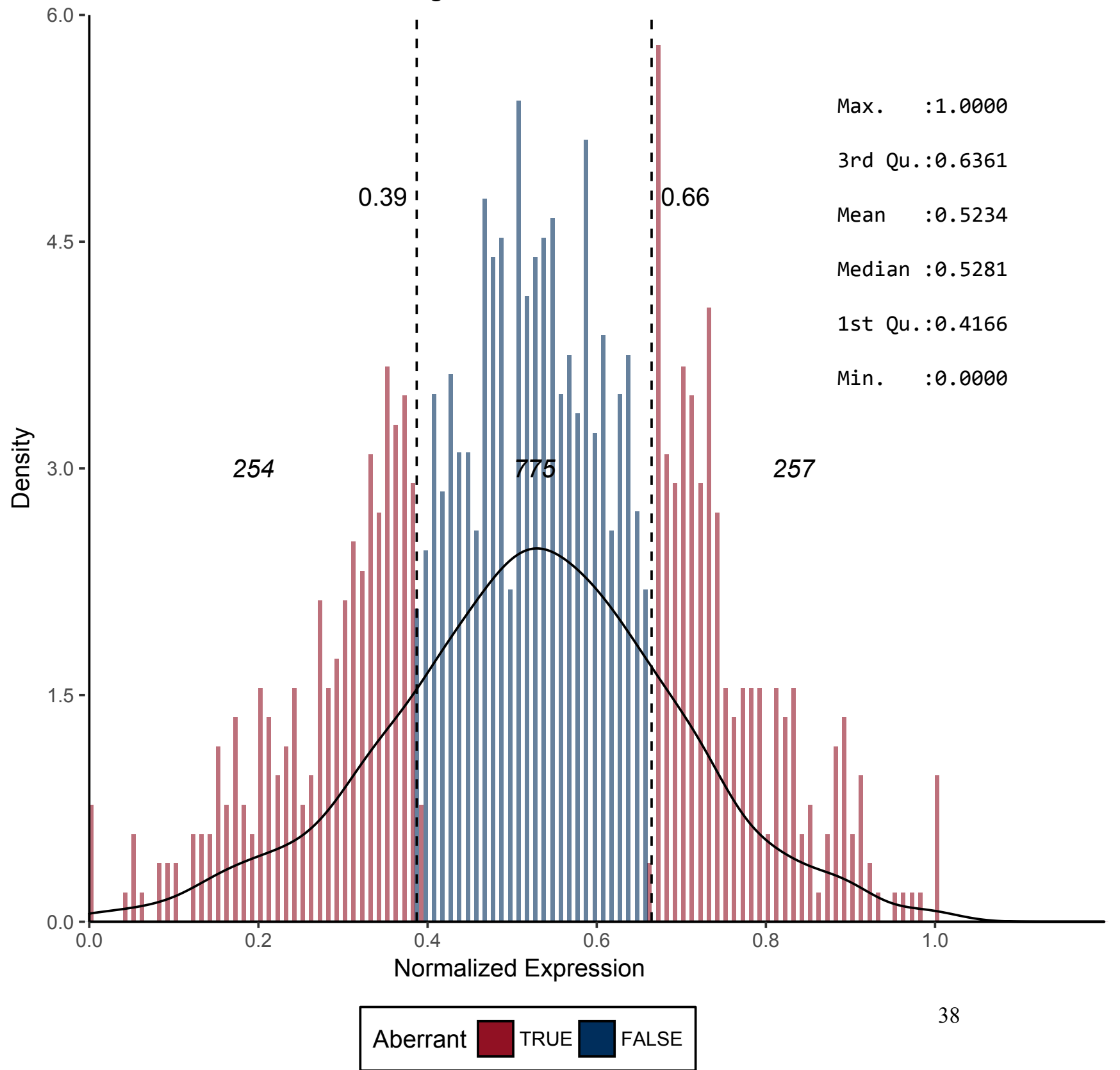


Figure 23 - CD15

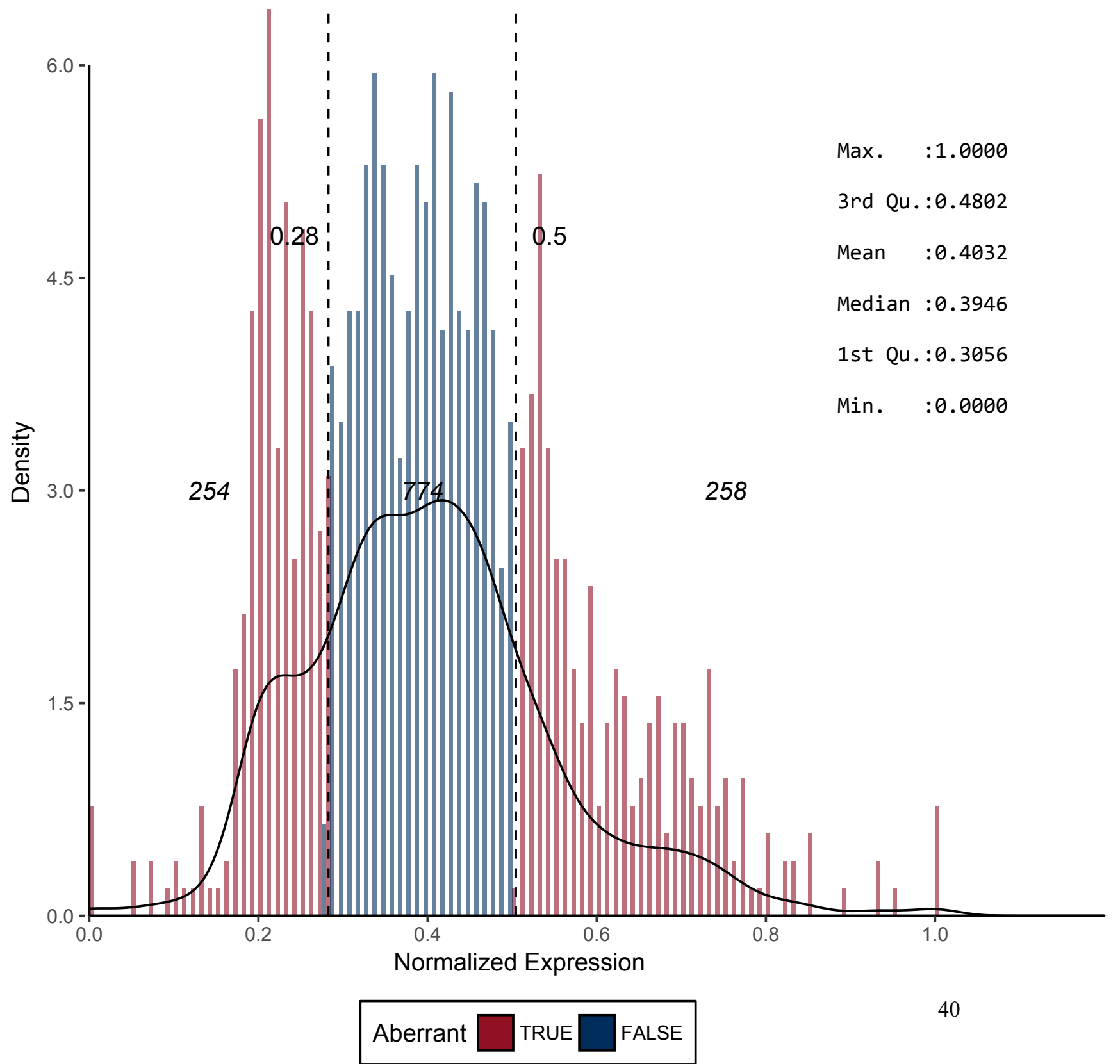


Figure 24 - CD16B

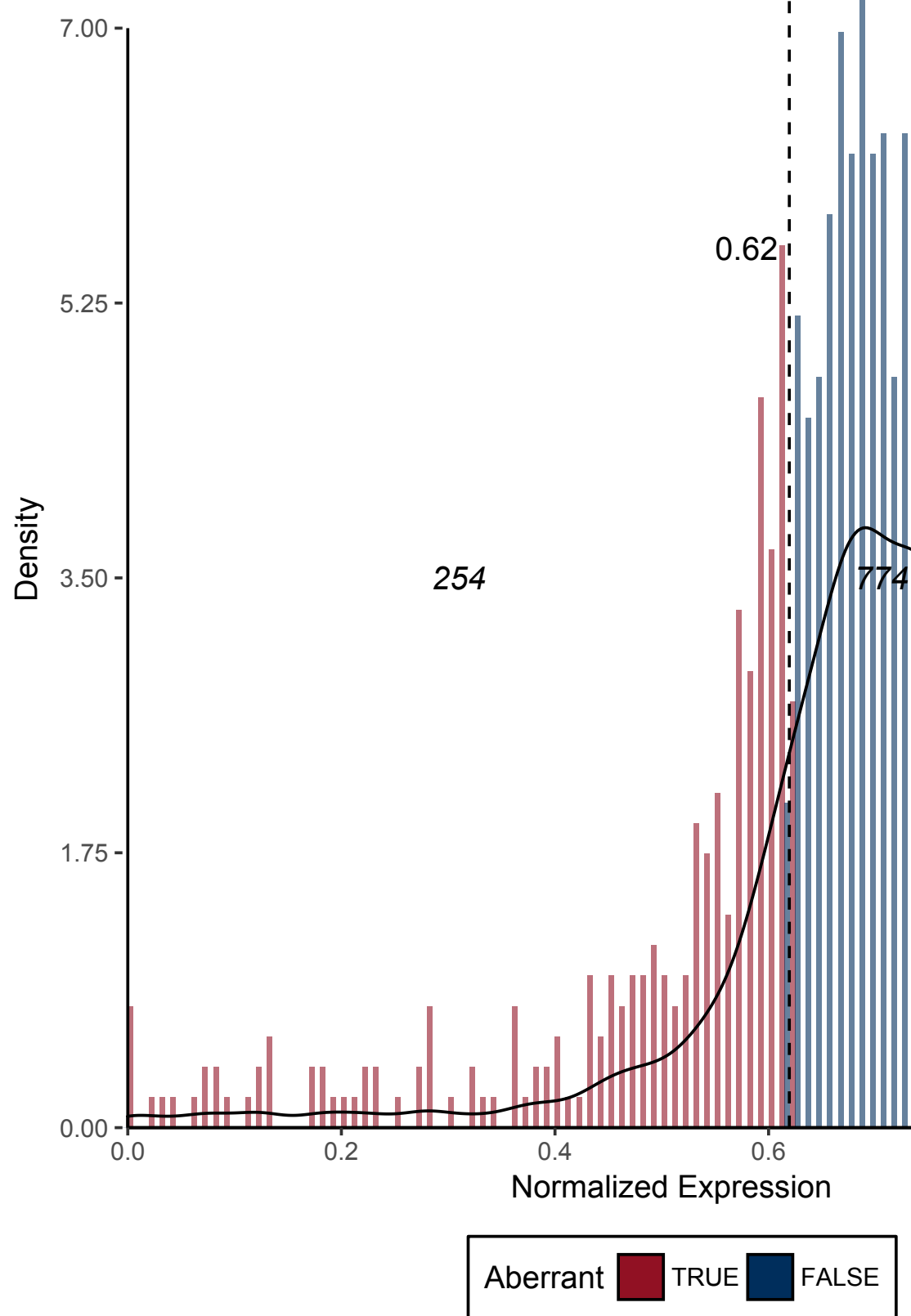

Max. $: 1.0000$

3rd Qu.:0.7744

Mean $\quad: 0.6900$

Median :0.7064

1st Qu.:0.6375

Min. $\quad: 0.0000$

258 
Figure 25 - CD18

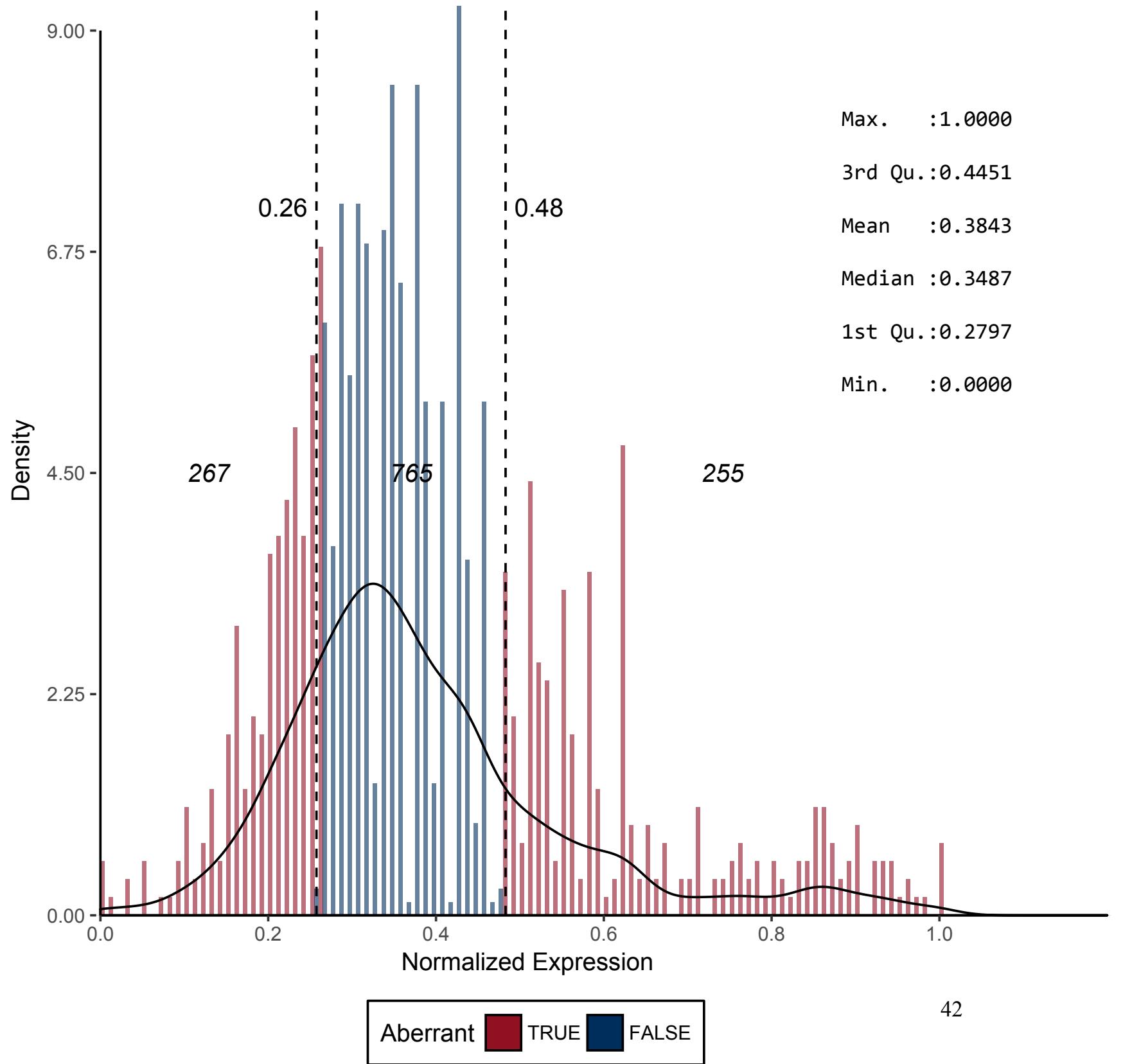


Figure 27 - CD20

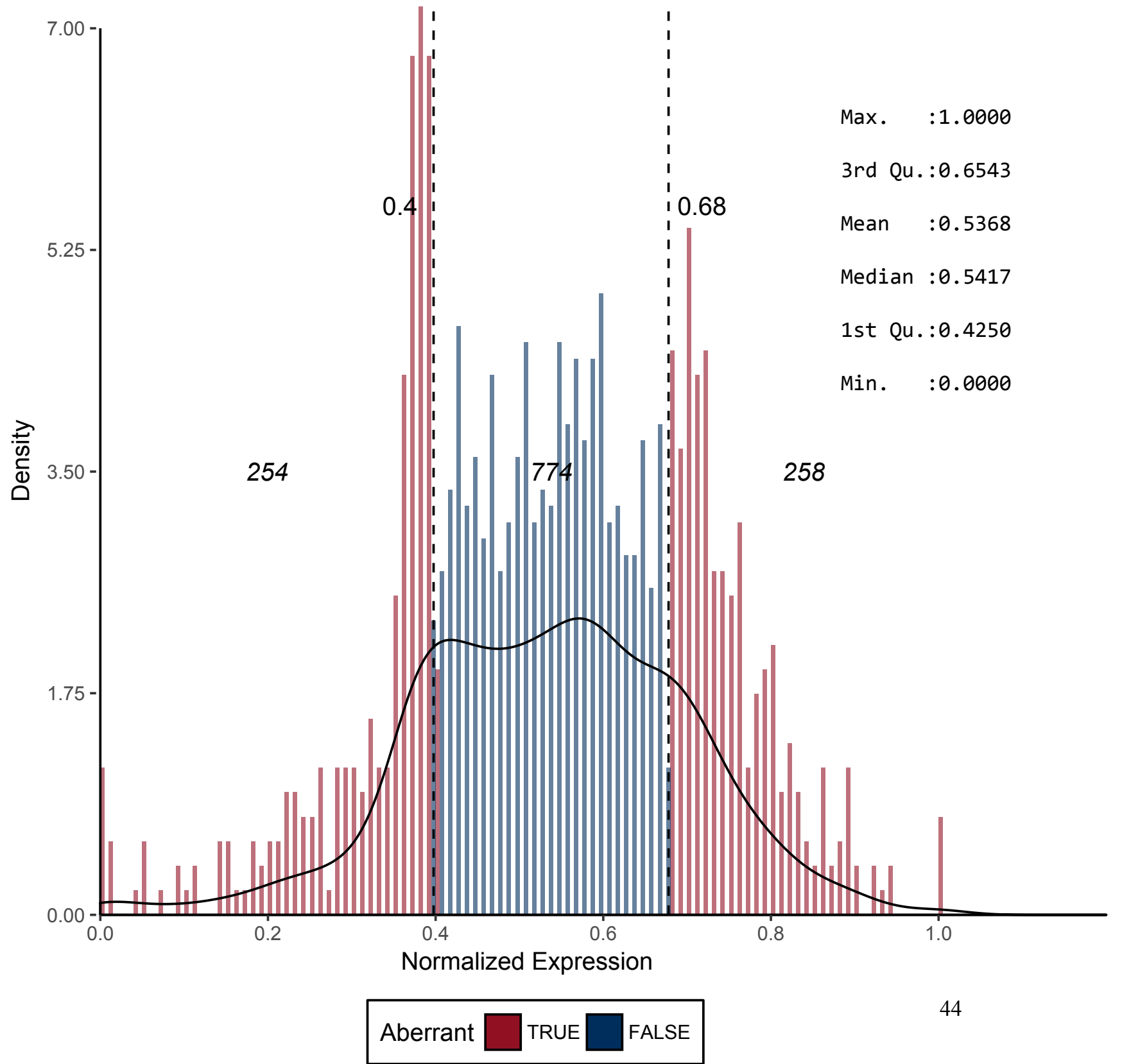


Figure 28 - CD21

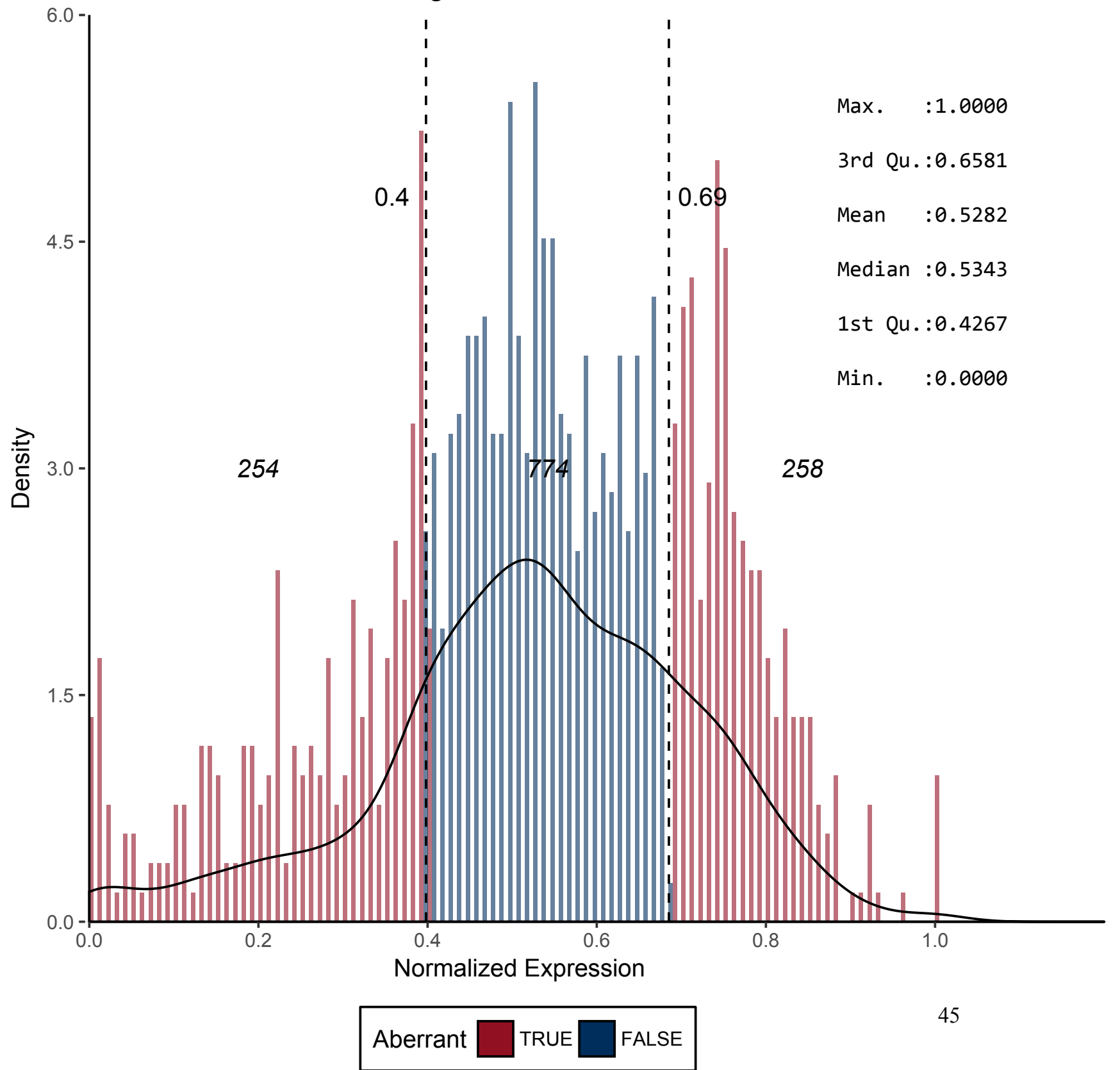


Figure 29 - CD22

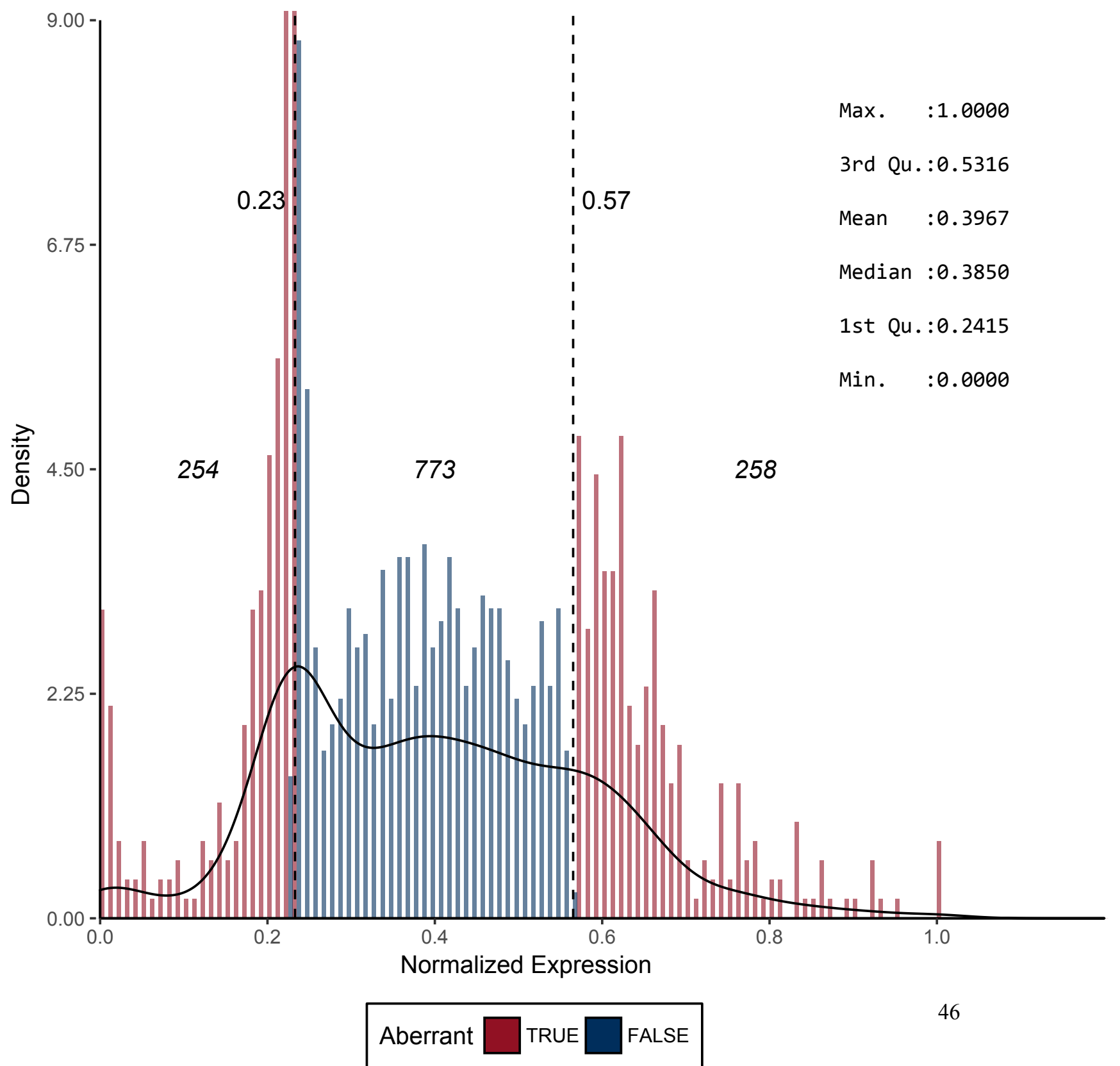


Figure 31 - CD25

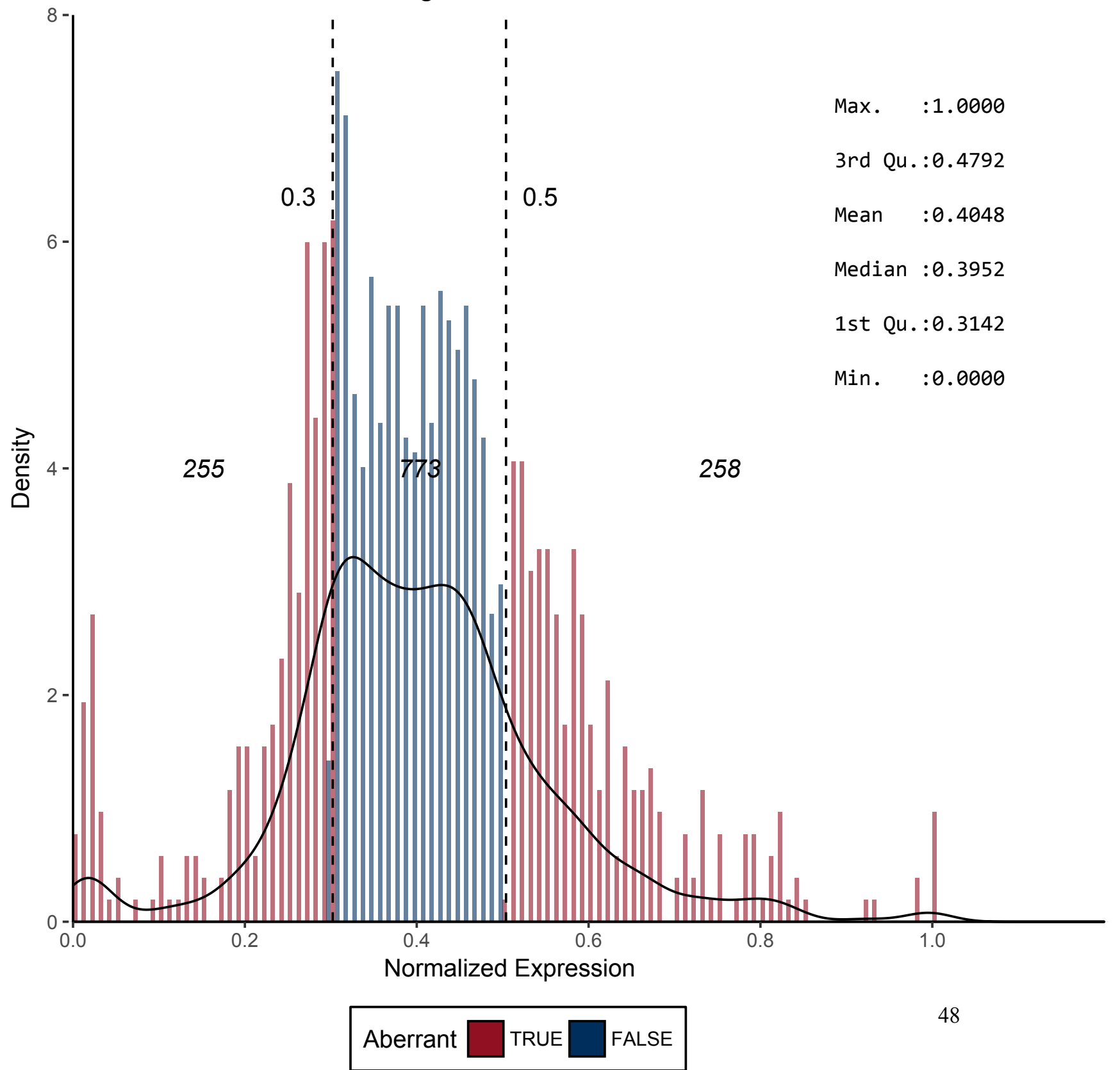


Figure 33 - CD27

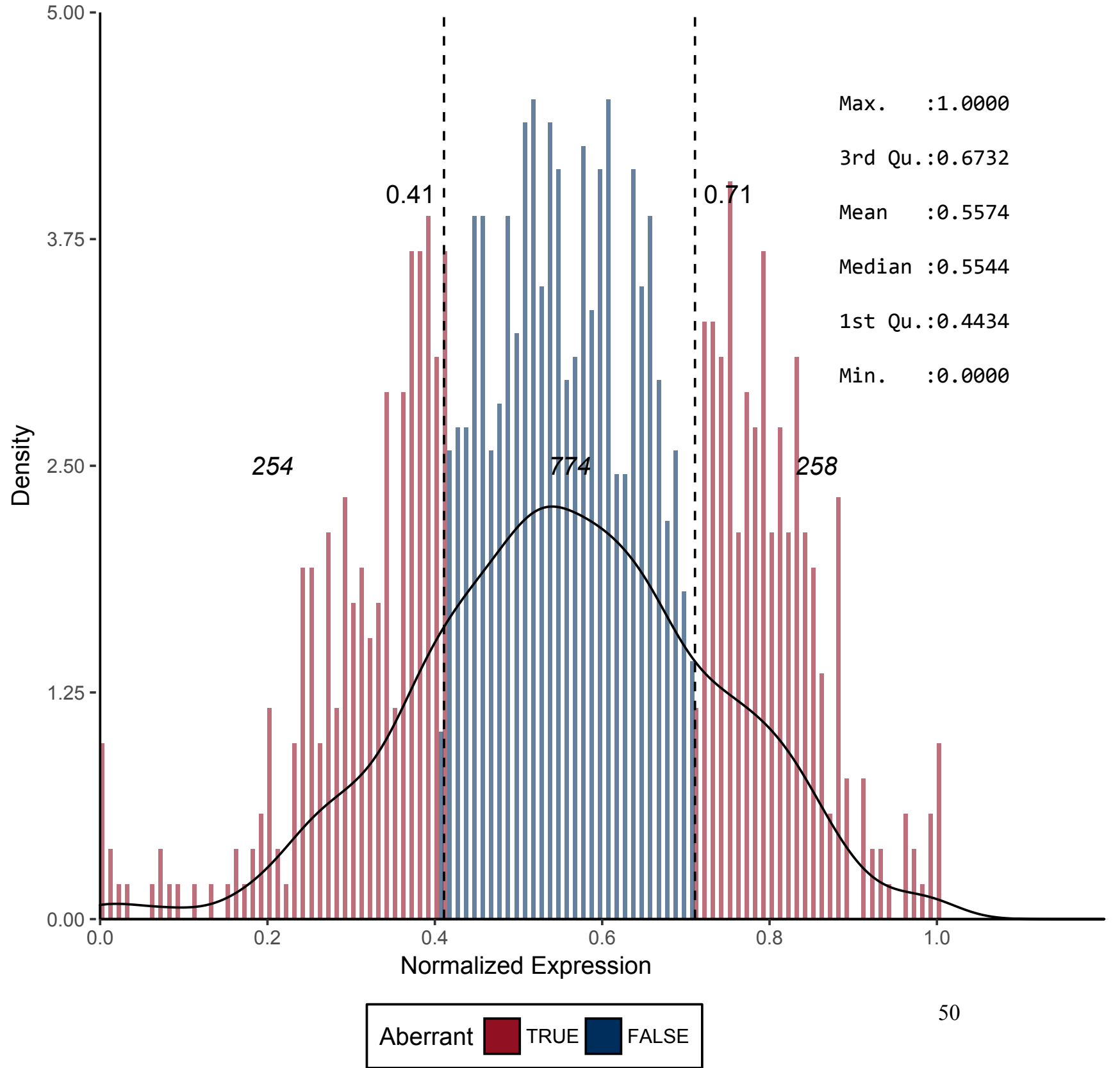


Figure 35 - CD30
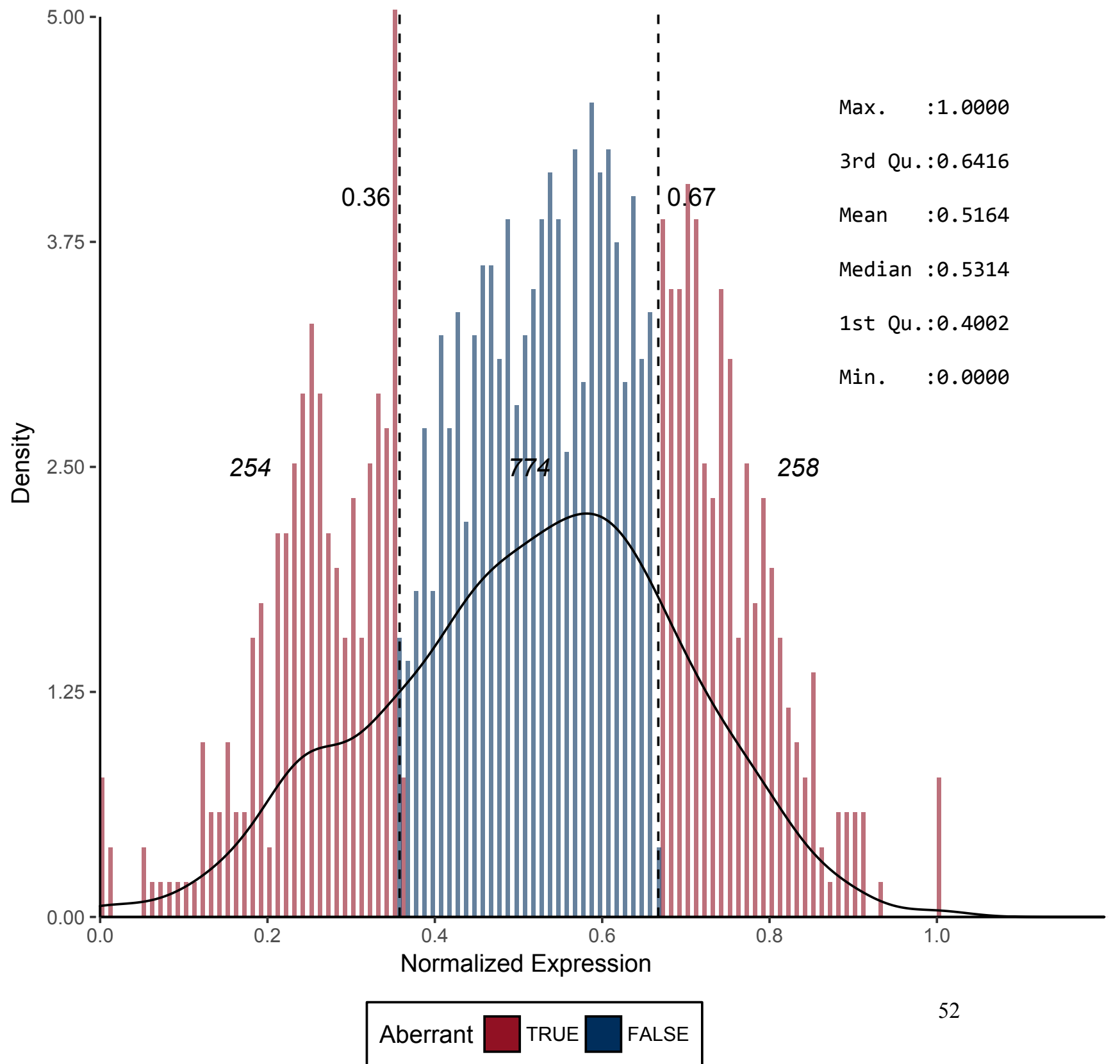
Figure 37 - CD33

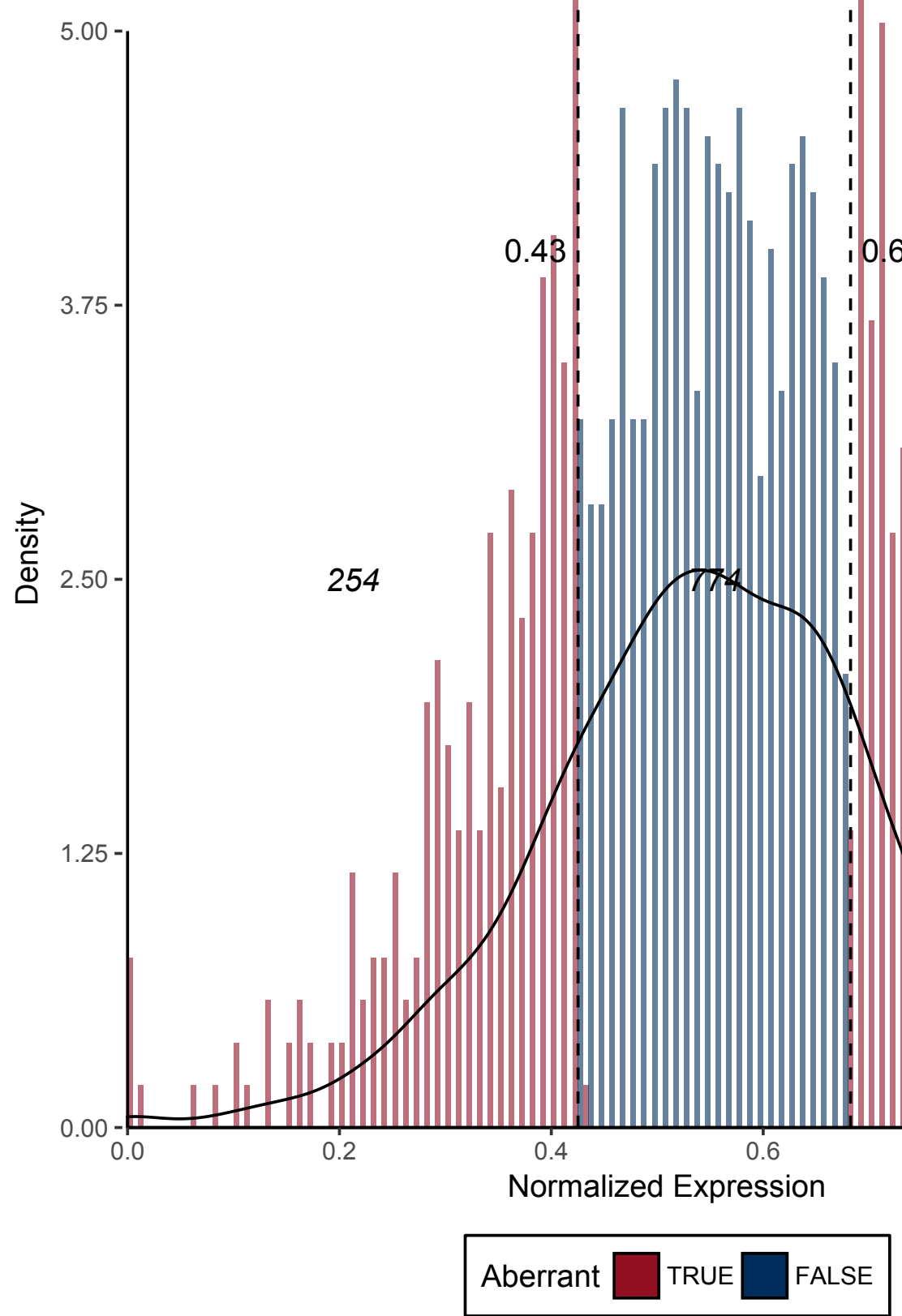

Max. $: 1.0000$

3rd Qu.:0.6564

Mean $\quad: 0.5536$

Median :0.5563

1st Qu.:0.4531

Min. $\quad: 0.0000$

258 
Figure 38 - CD34*

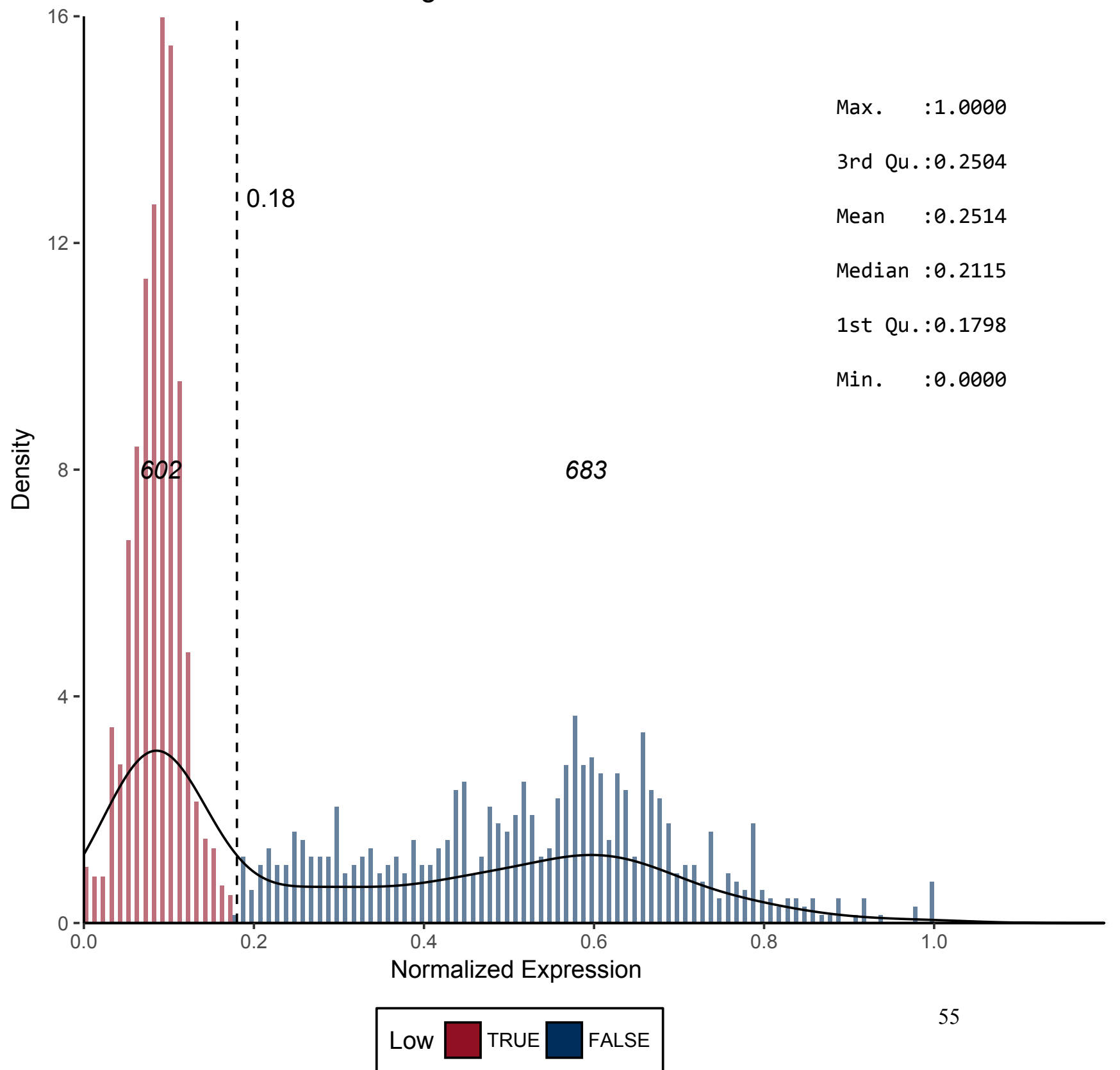


Figure 39 - CD35

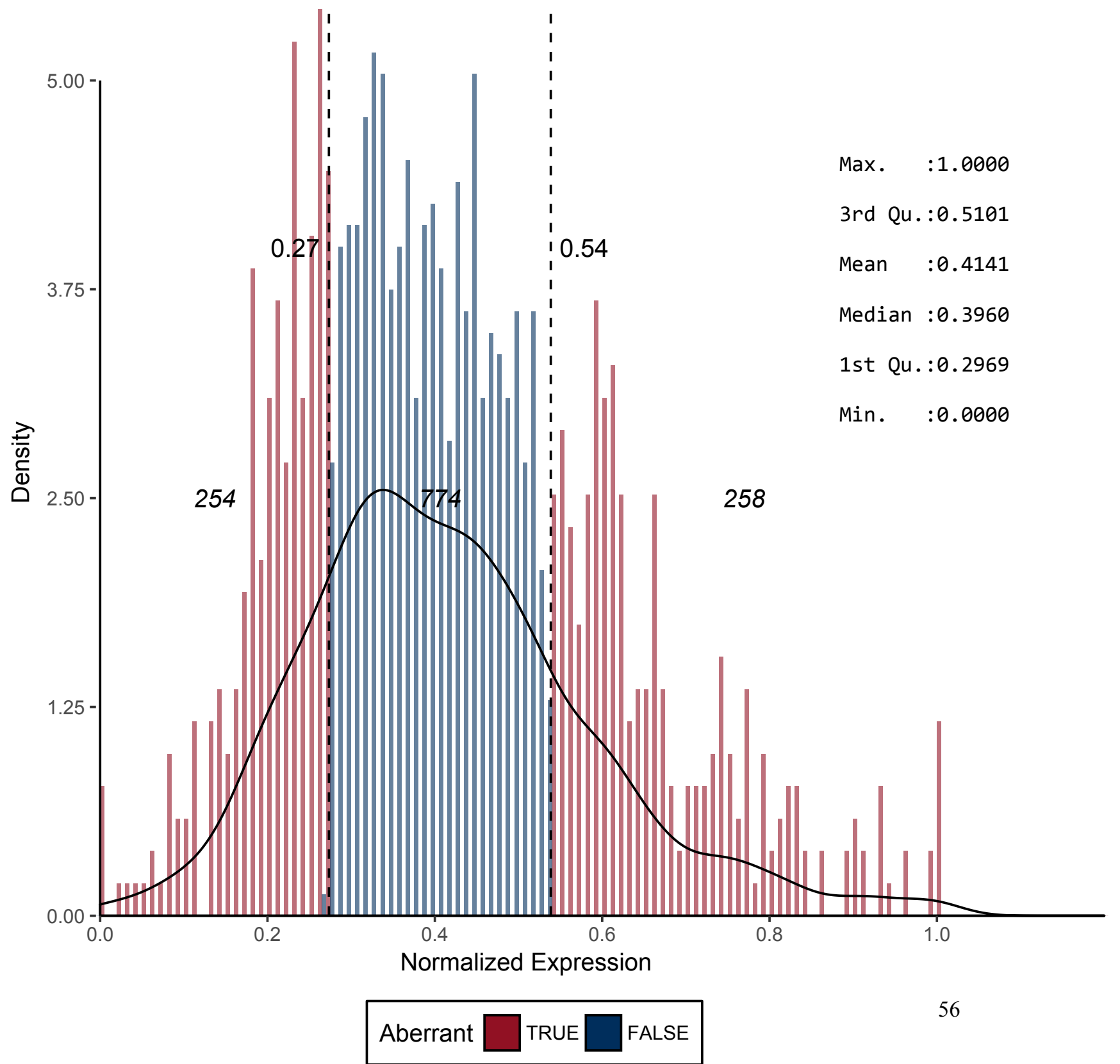


Figure 40 - CD36

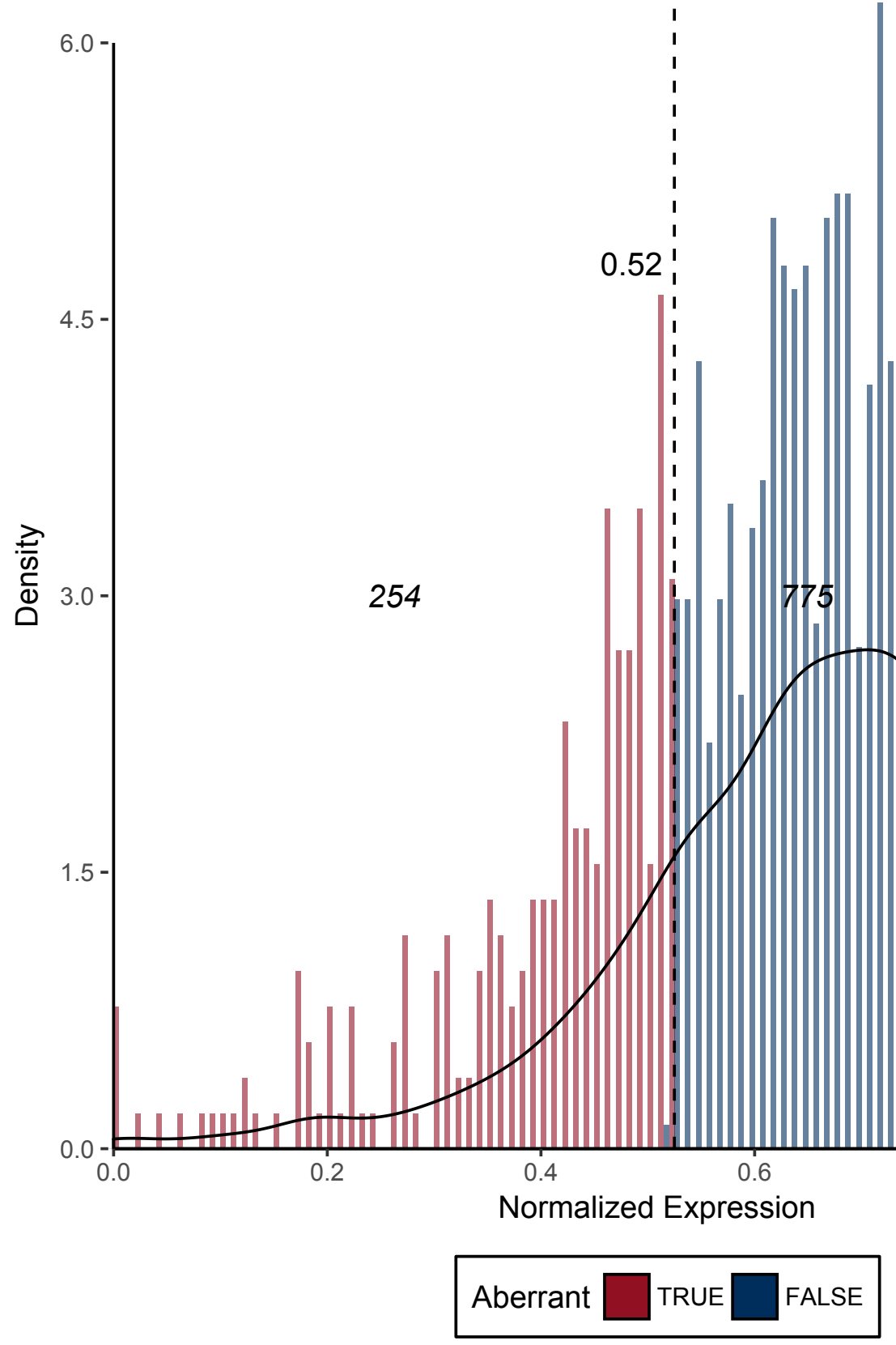

Max. $: 1.0000$

3rd Qu.:0.7539

Mean :0.6425

Median :0.6644

1st Qu.:0.5503

Min. $\quad: 0.0000$

257

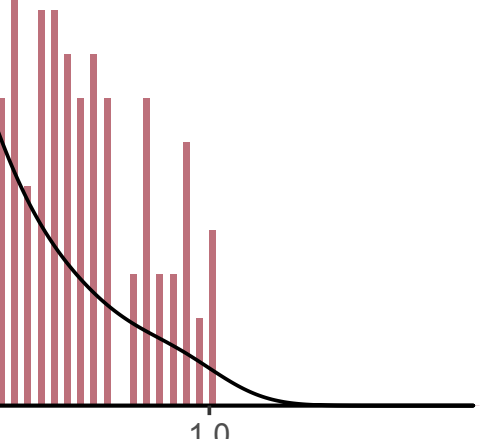


Figure 41 - CD37

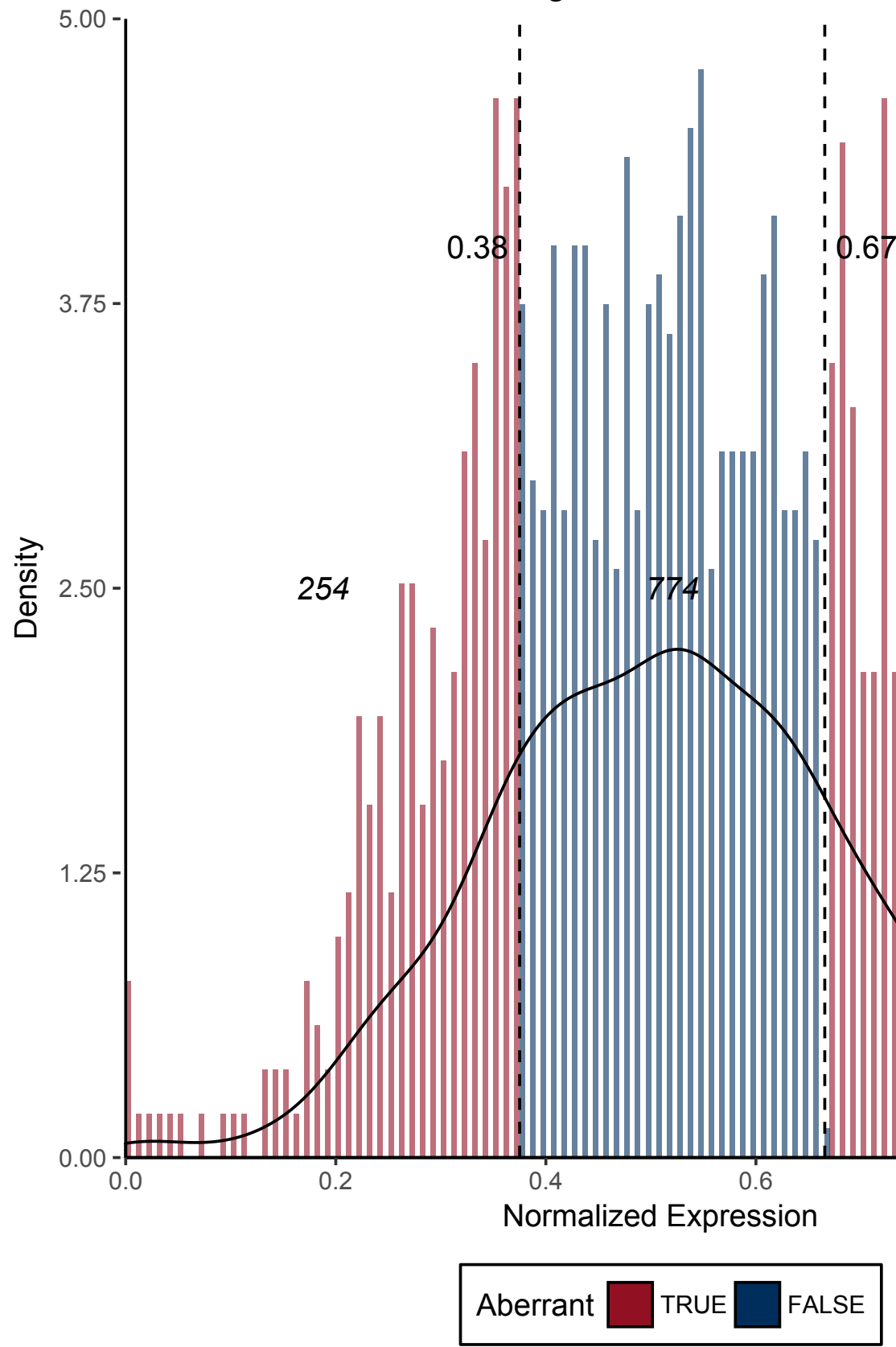

Max. $: 1.0000$

3rd Qu.:0.6362

Mean $\quad: 0.5212$

Median :0.5194

1st Qu.:0.4015

Min. $\quad: 0.0000$

\section{8}


Figure 42 - CD38

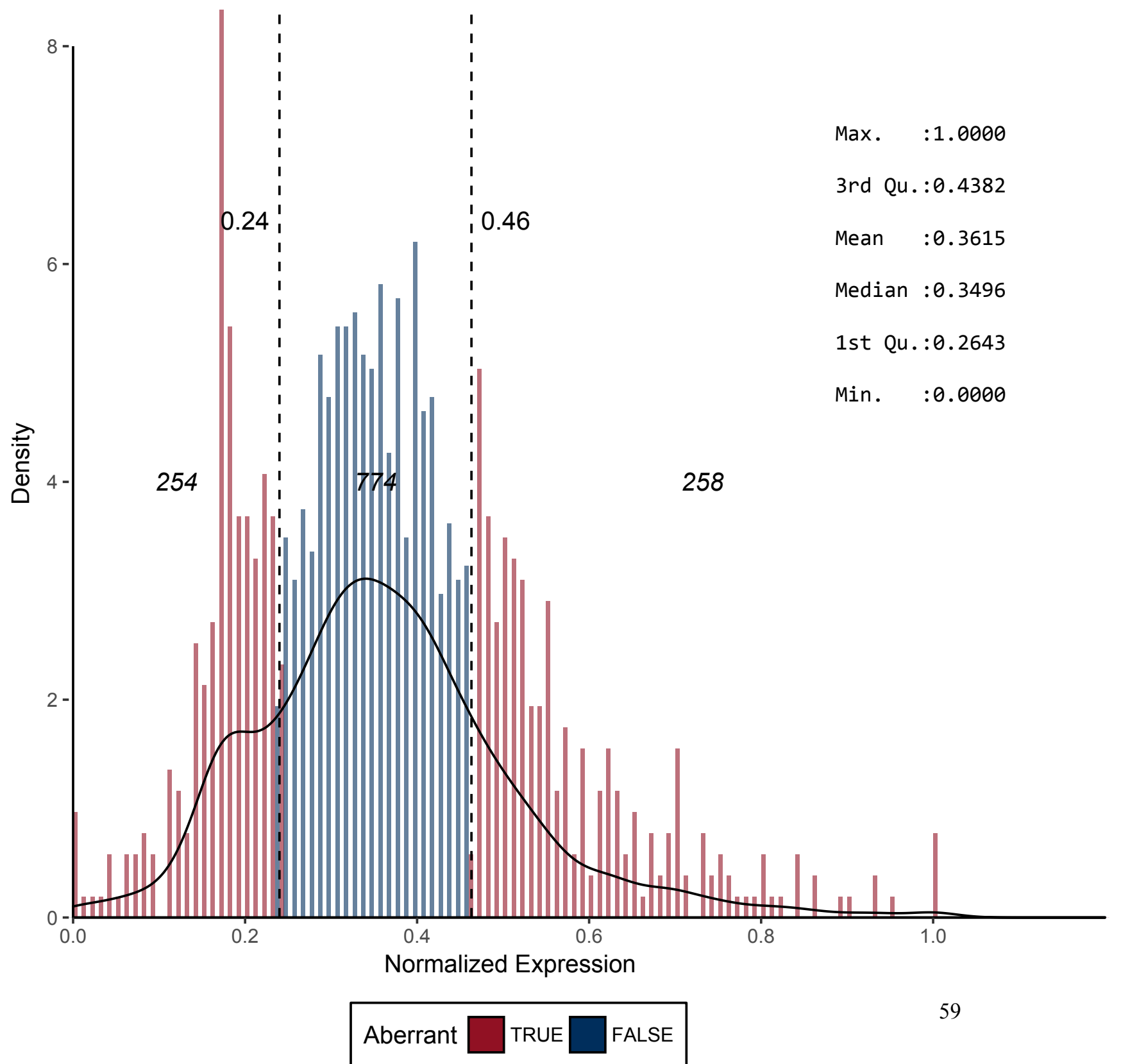


Figure 44 - CD40

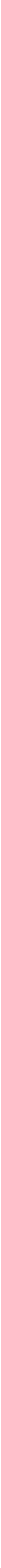


Figure 46 - CD42A

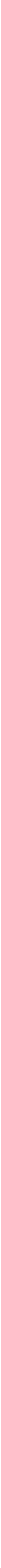


Figure 48 - CD43

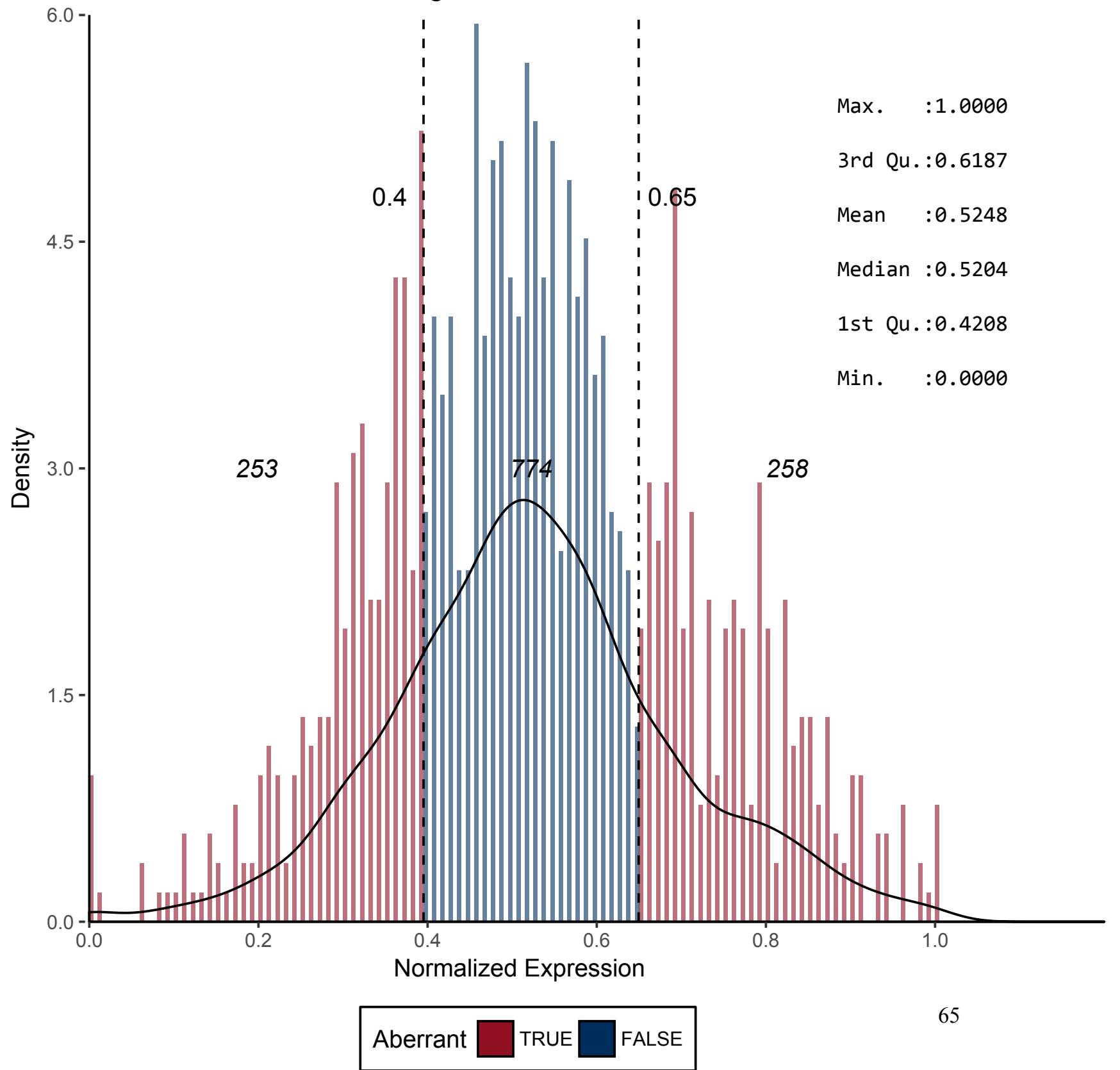


Figure 50 - CD45

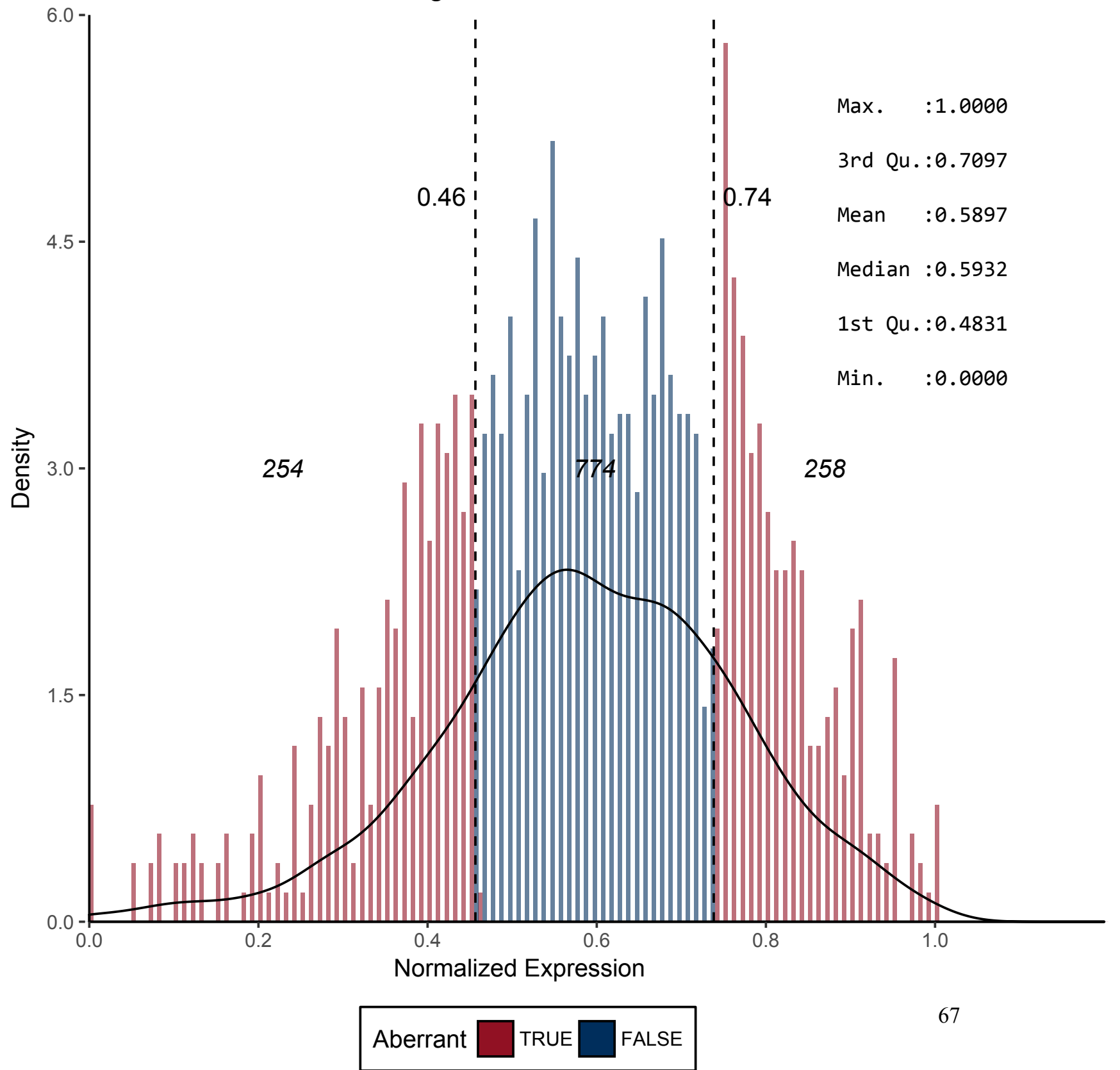


Figure 51 - CD46
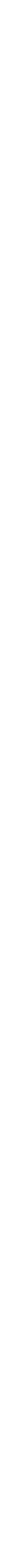
Figure 52 - CD47
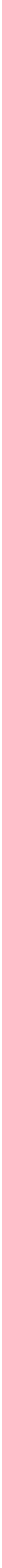
Figure 53 - CD48

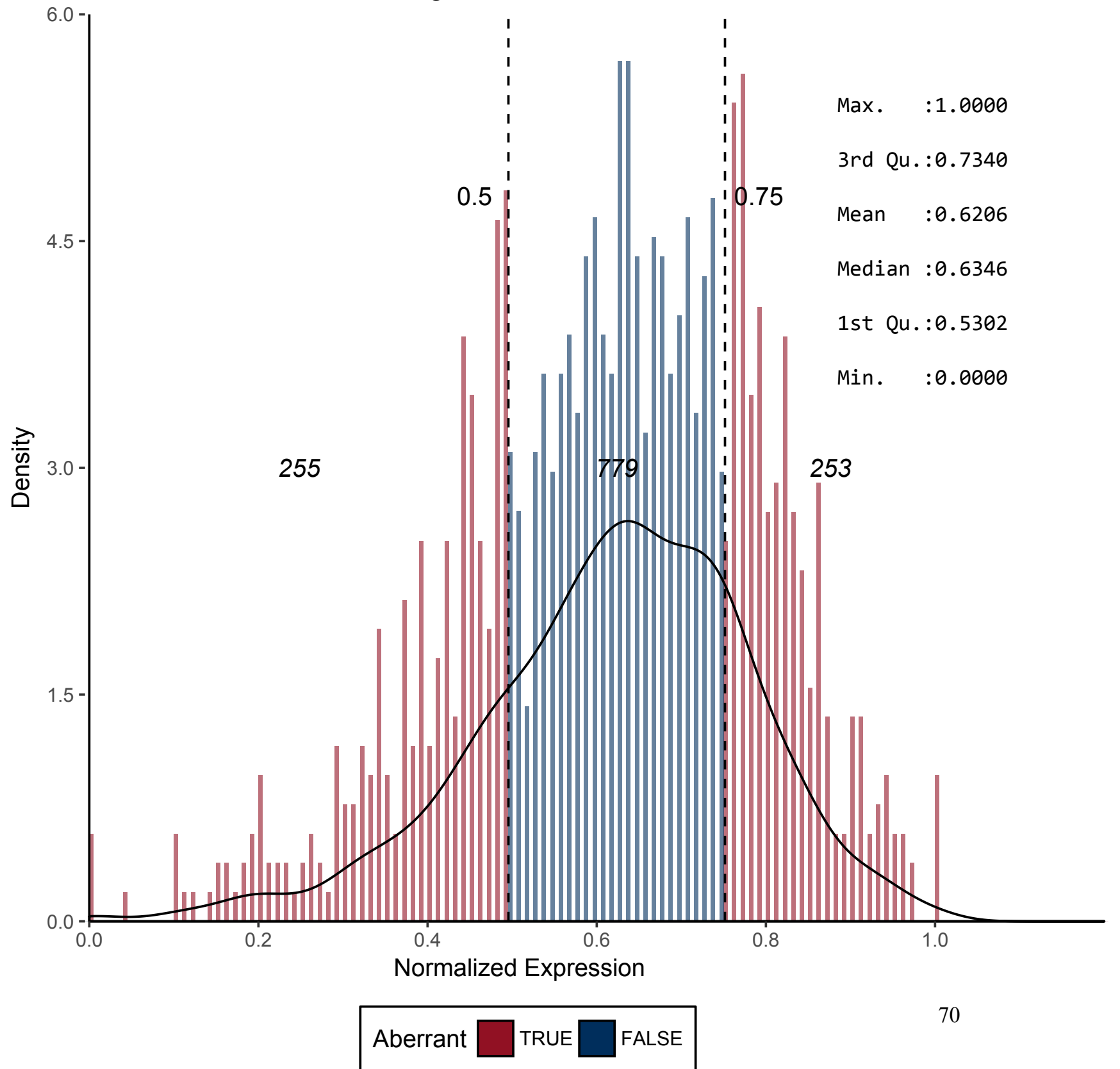


Figure 54 - CD49B*

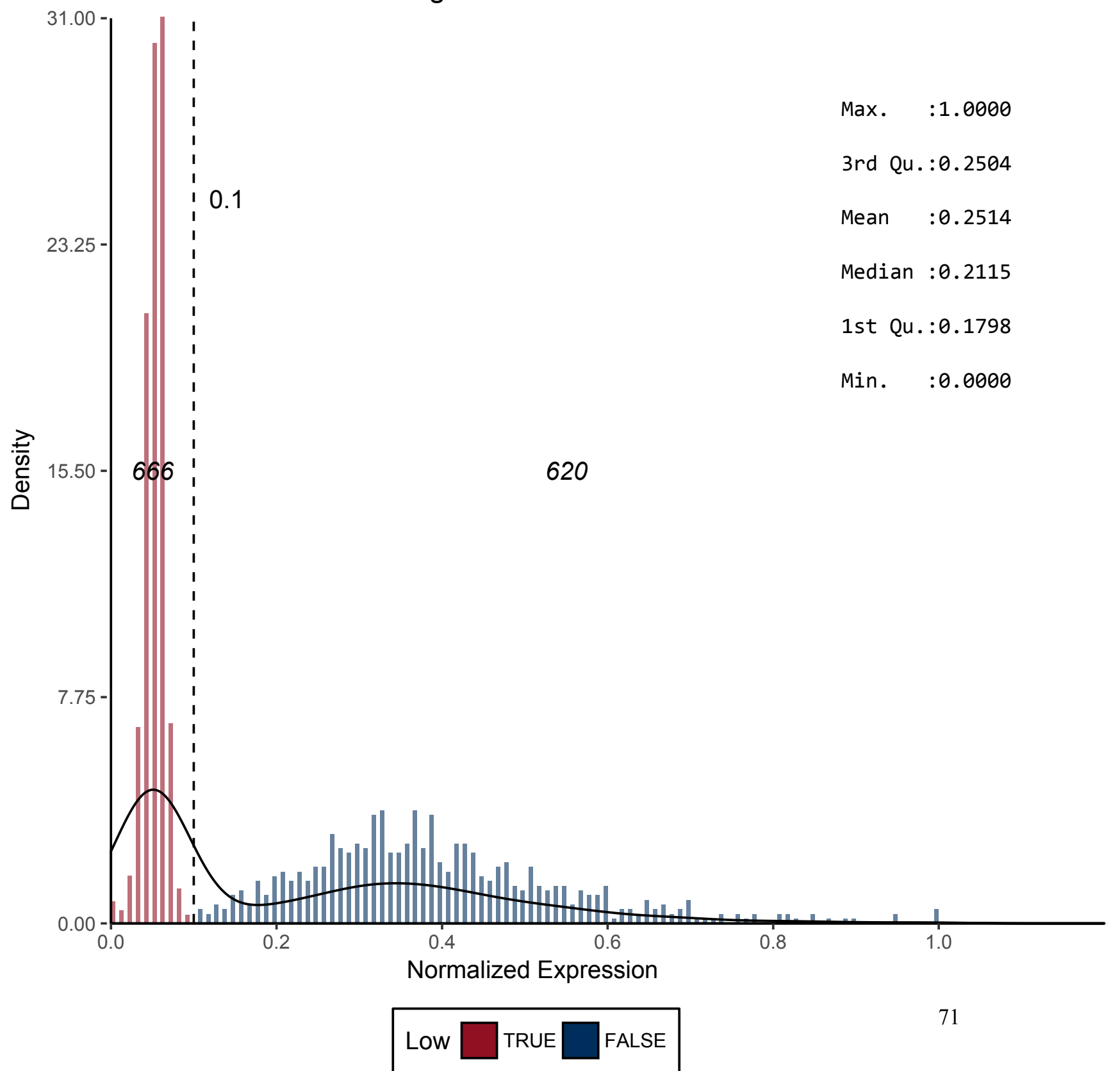


Figure 56 - CD49D
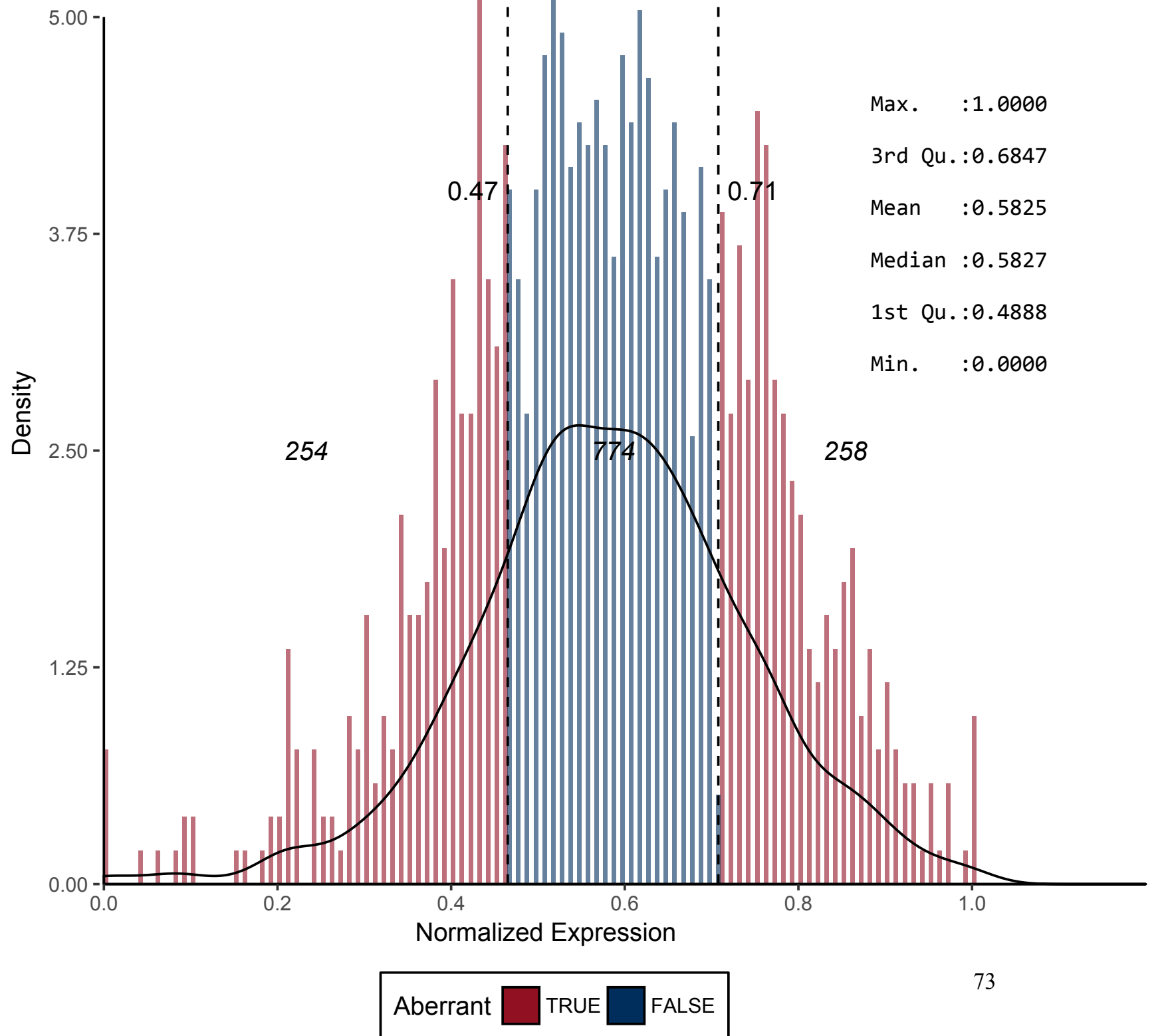
Figure 57 - CD49E
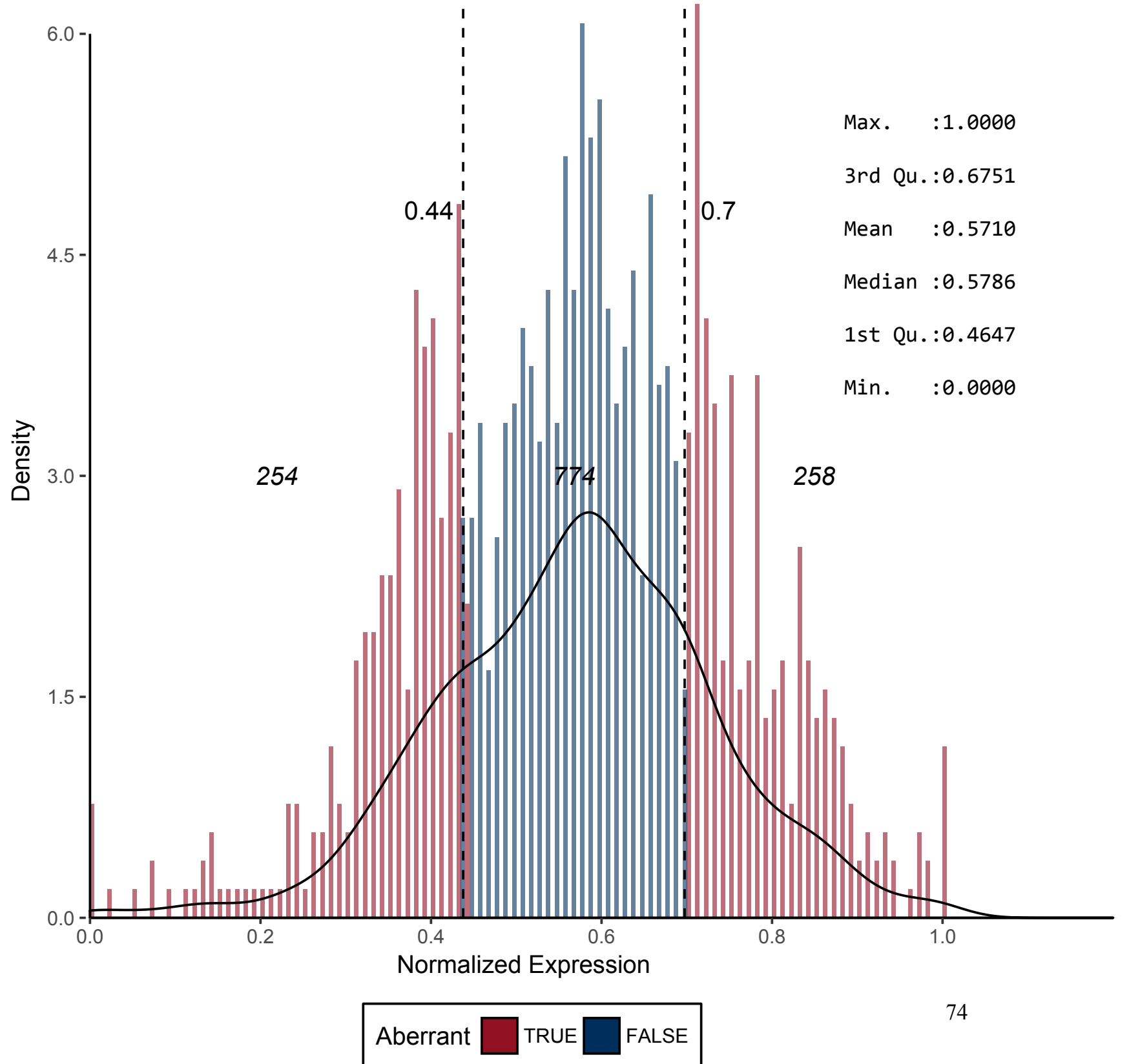
Figure 58 - CD49F
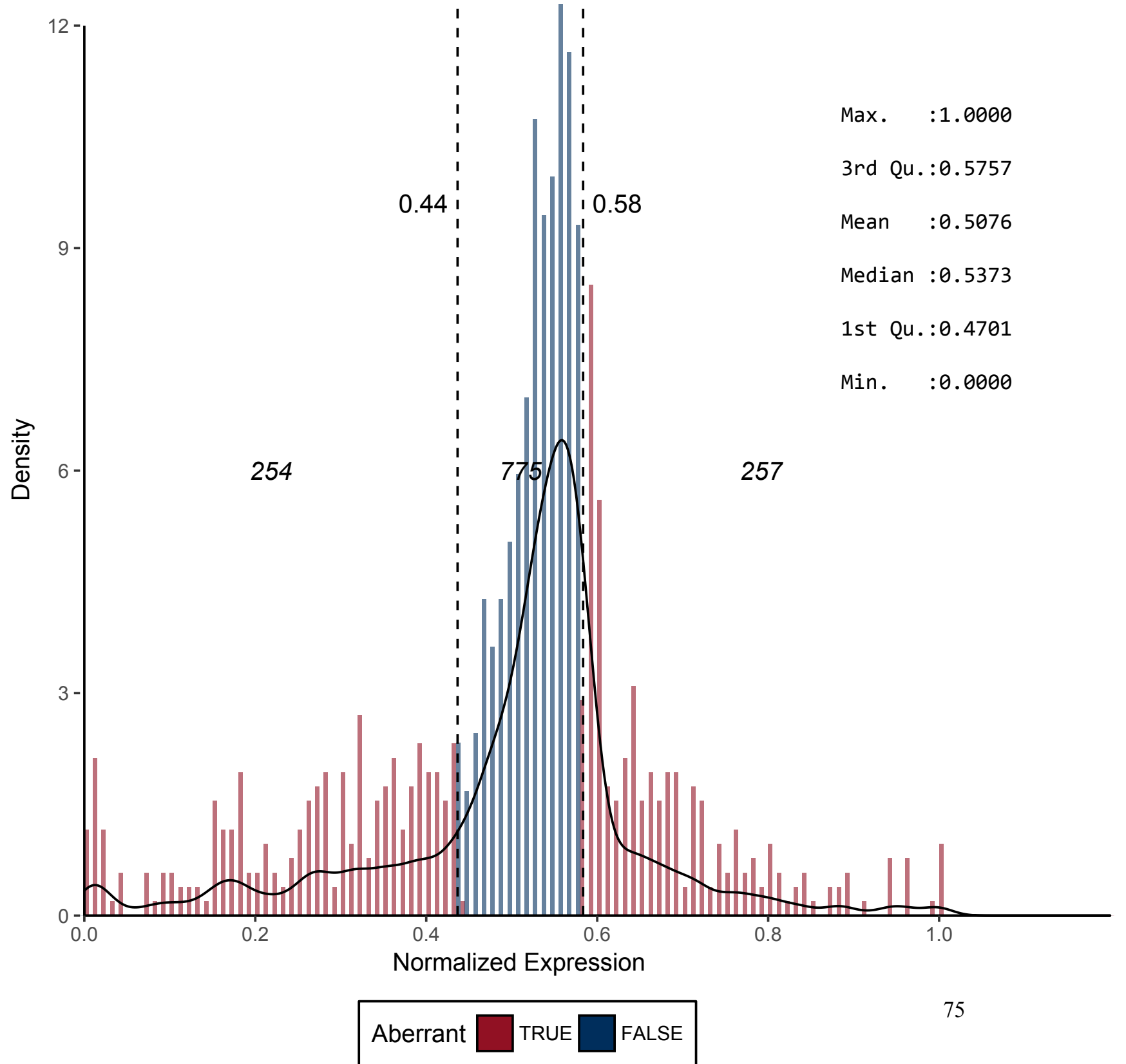
Figure 59 - CD50

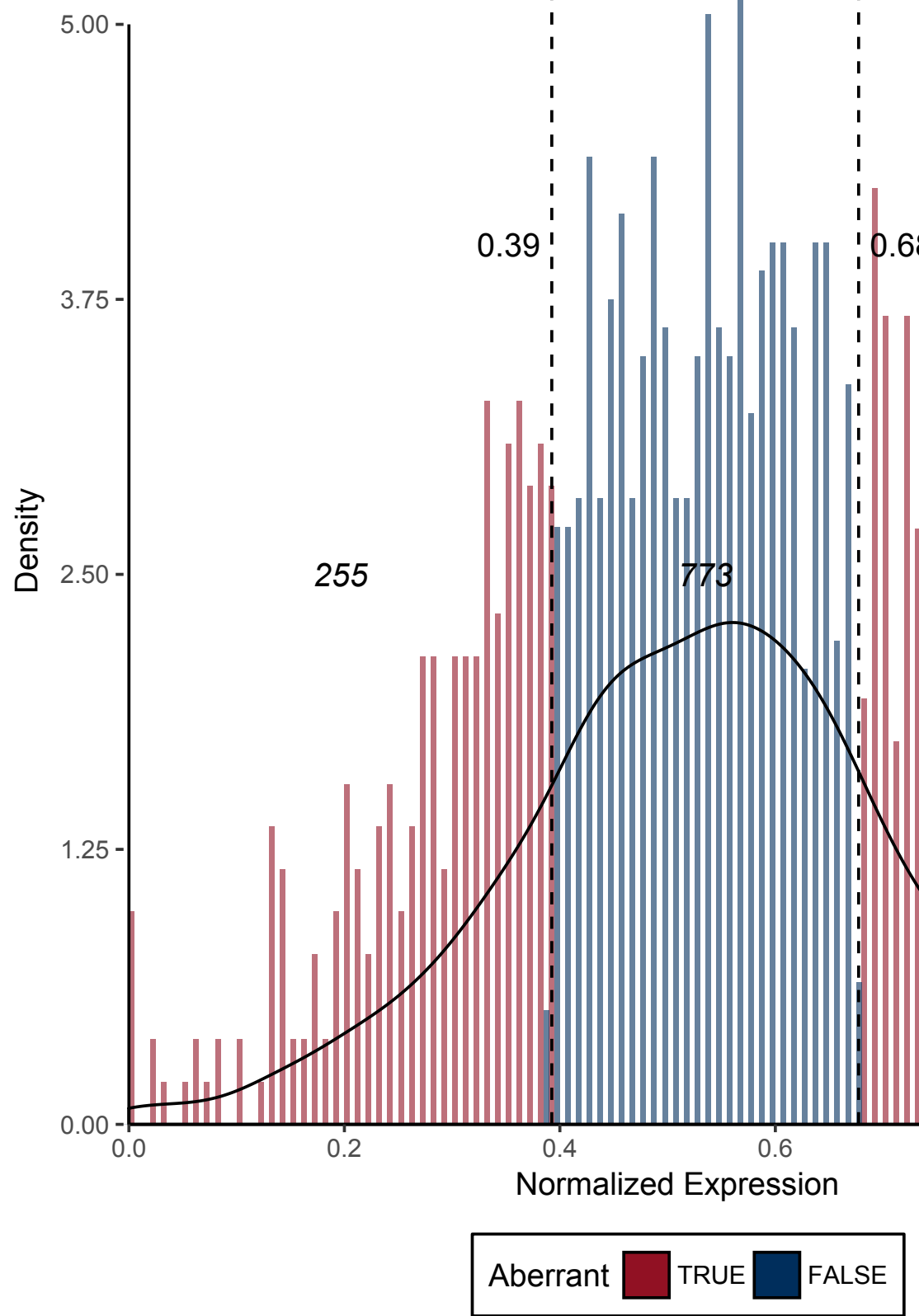

Max. $: 1.0000$

3rd Qu.:0.6507

Mean $\quad: 0.5340$

Median $: 0.5380$

1st Qu.:0.4232

Min. $\quad: 0.0000$ 
Figure 61 - CD52

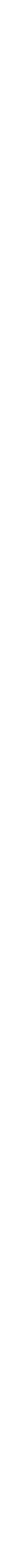


Figure 62 - CD53

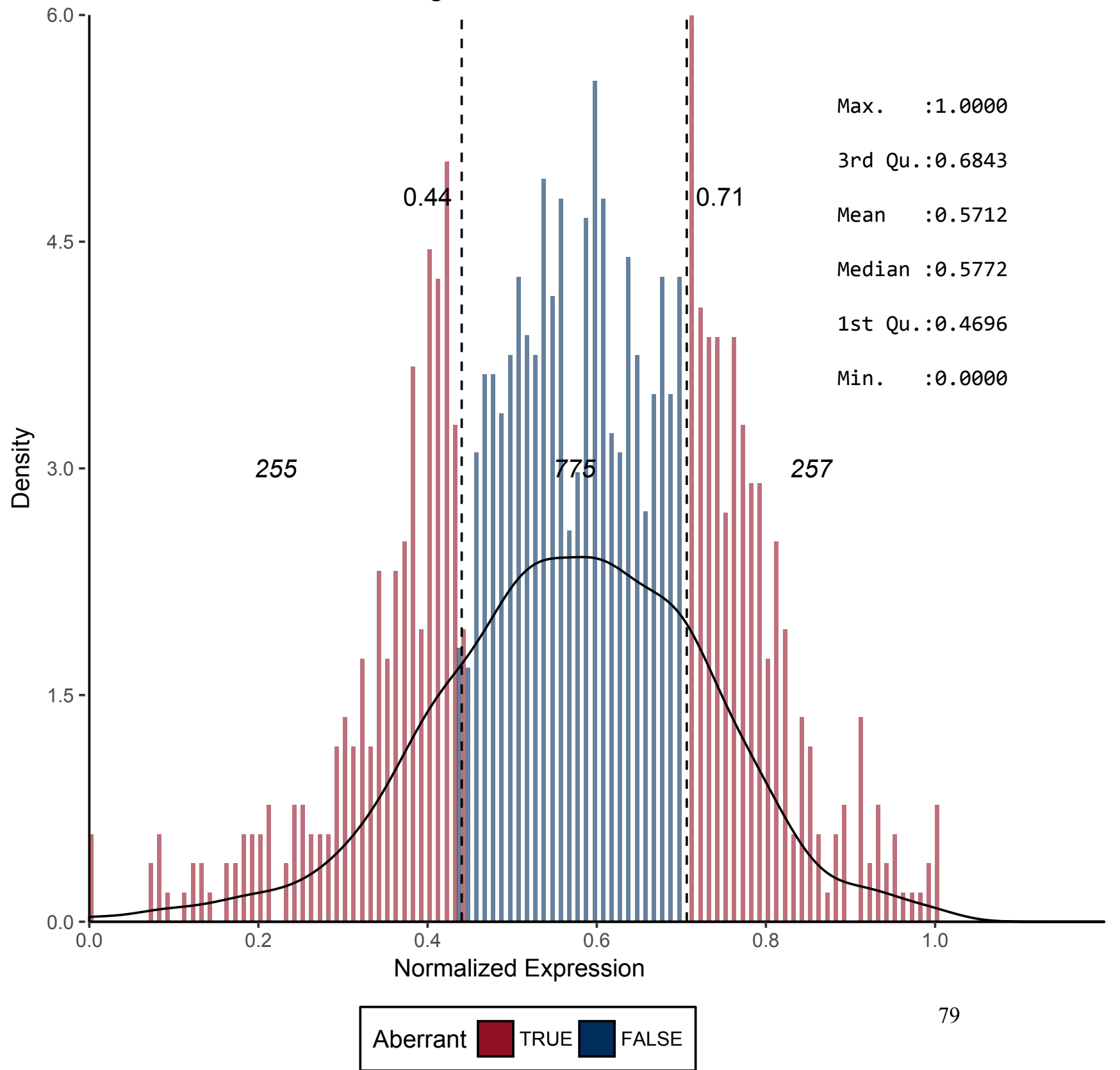


Figure 63 - CD54

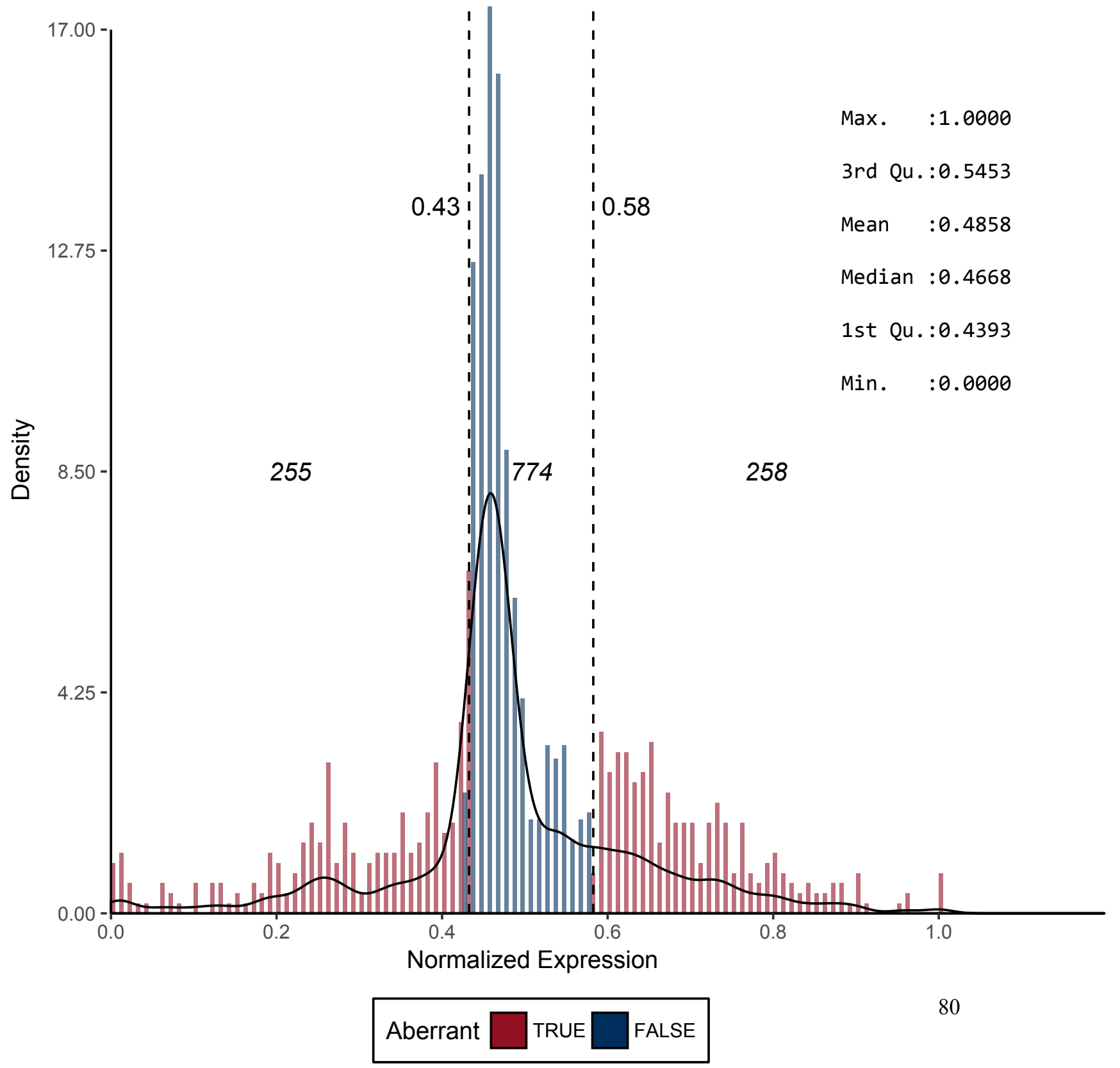


Figure 64 - CD55

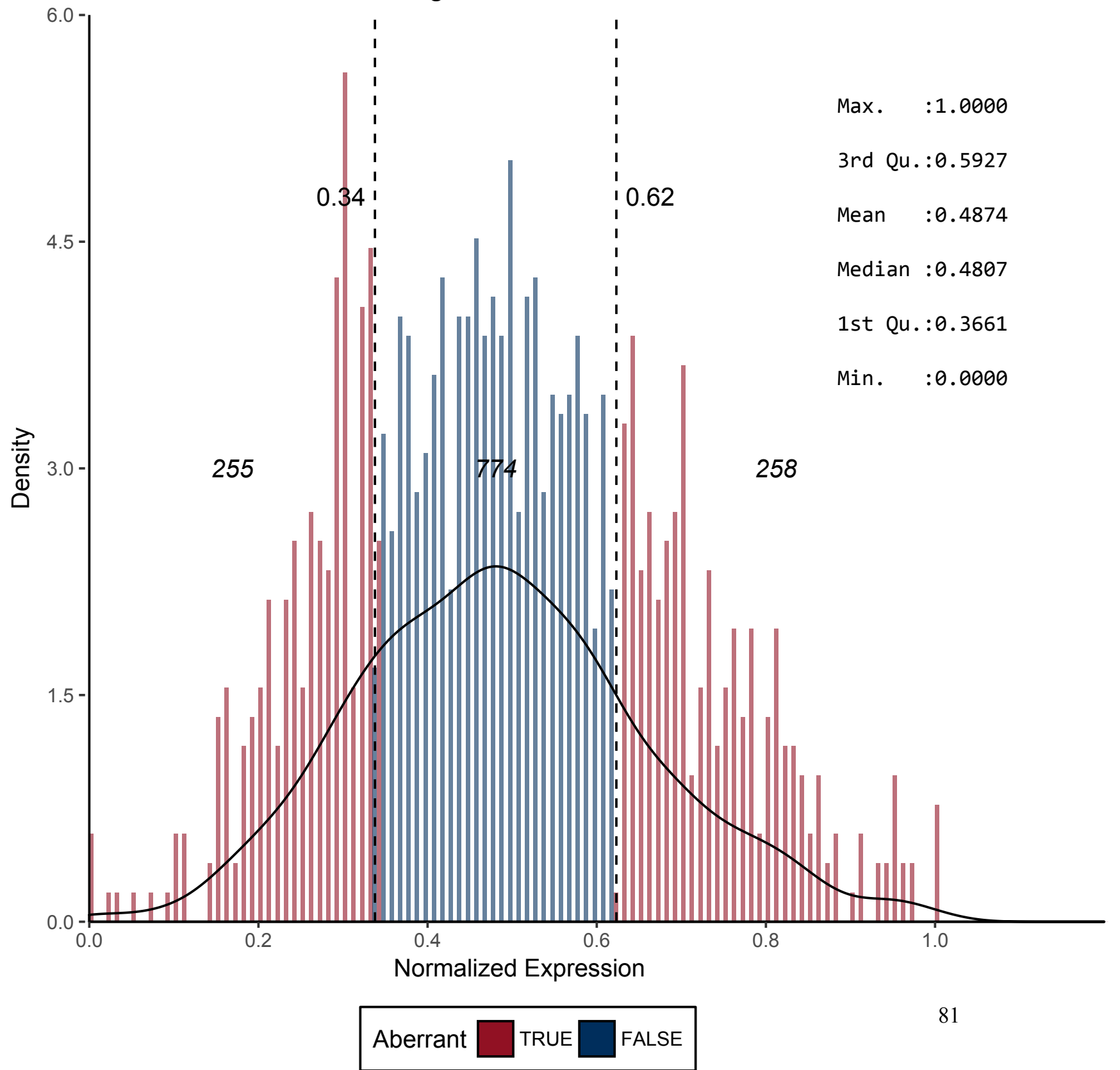


Figure 65 - CD56

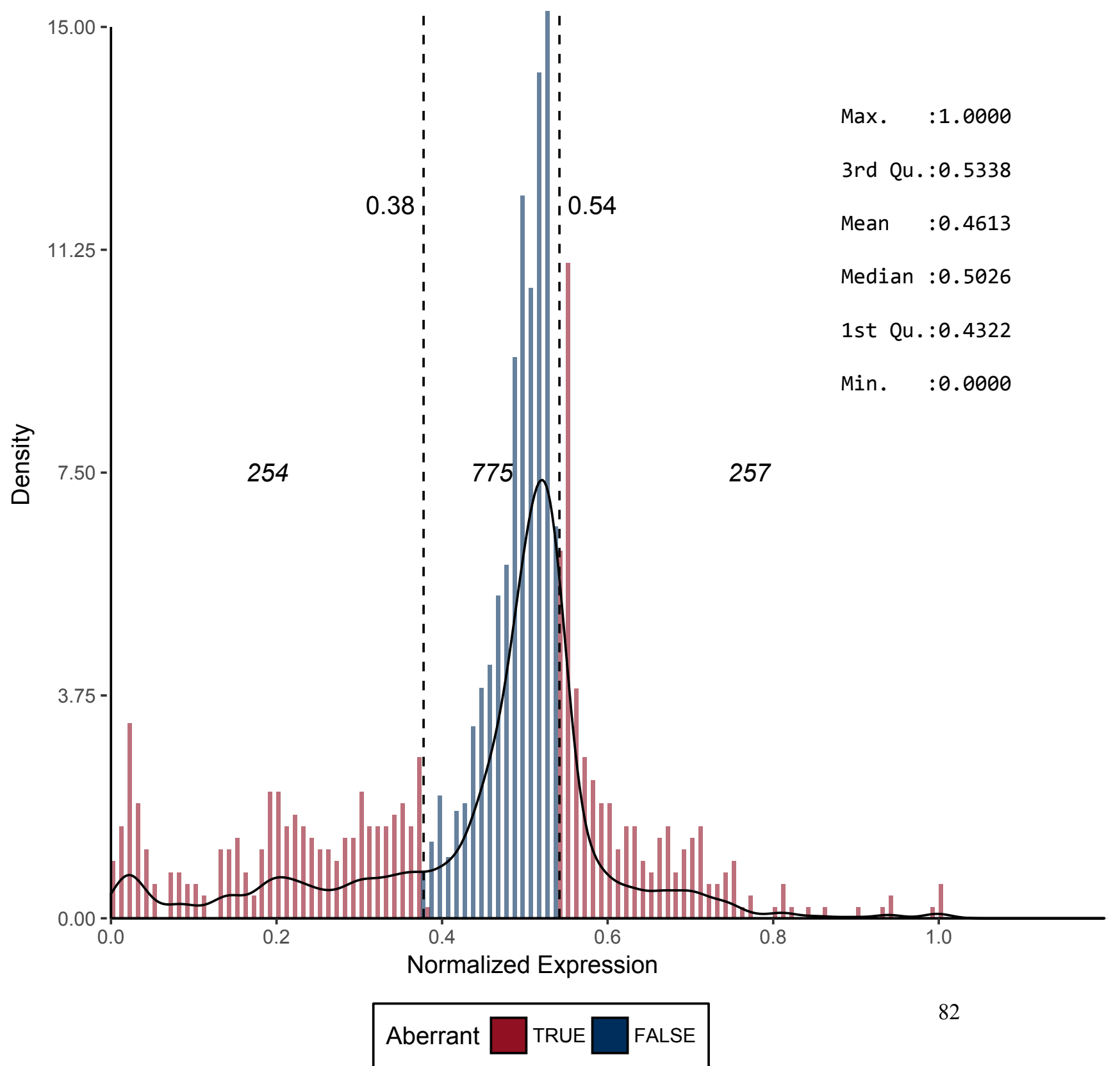


Figure 66 - CD57*

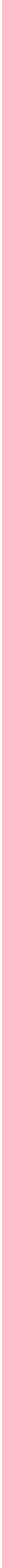


Figure 67 - CD58

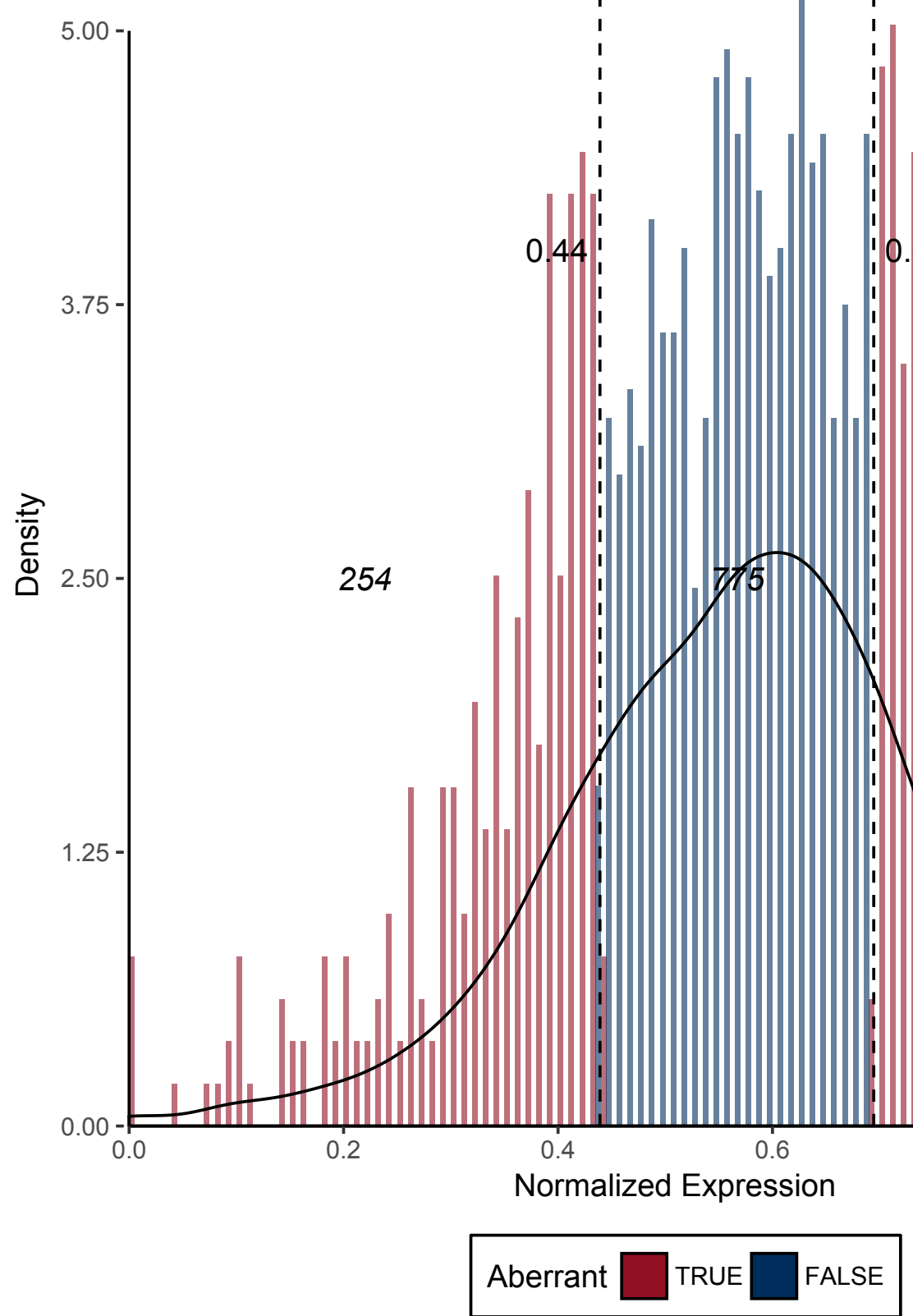

Max. $: 1.0000$

3rd Qu.:0.6744

Mean $\quad: 0.5660$

Median :0.5758

1st Qu.:0.4668

Min. $\quad: 0.0000$ 
Figure 68 - CD59

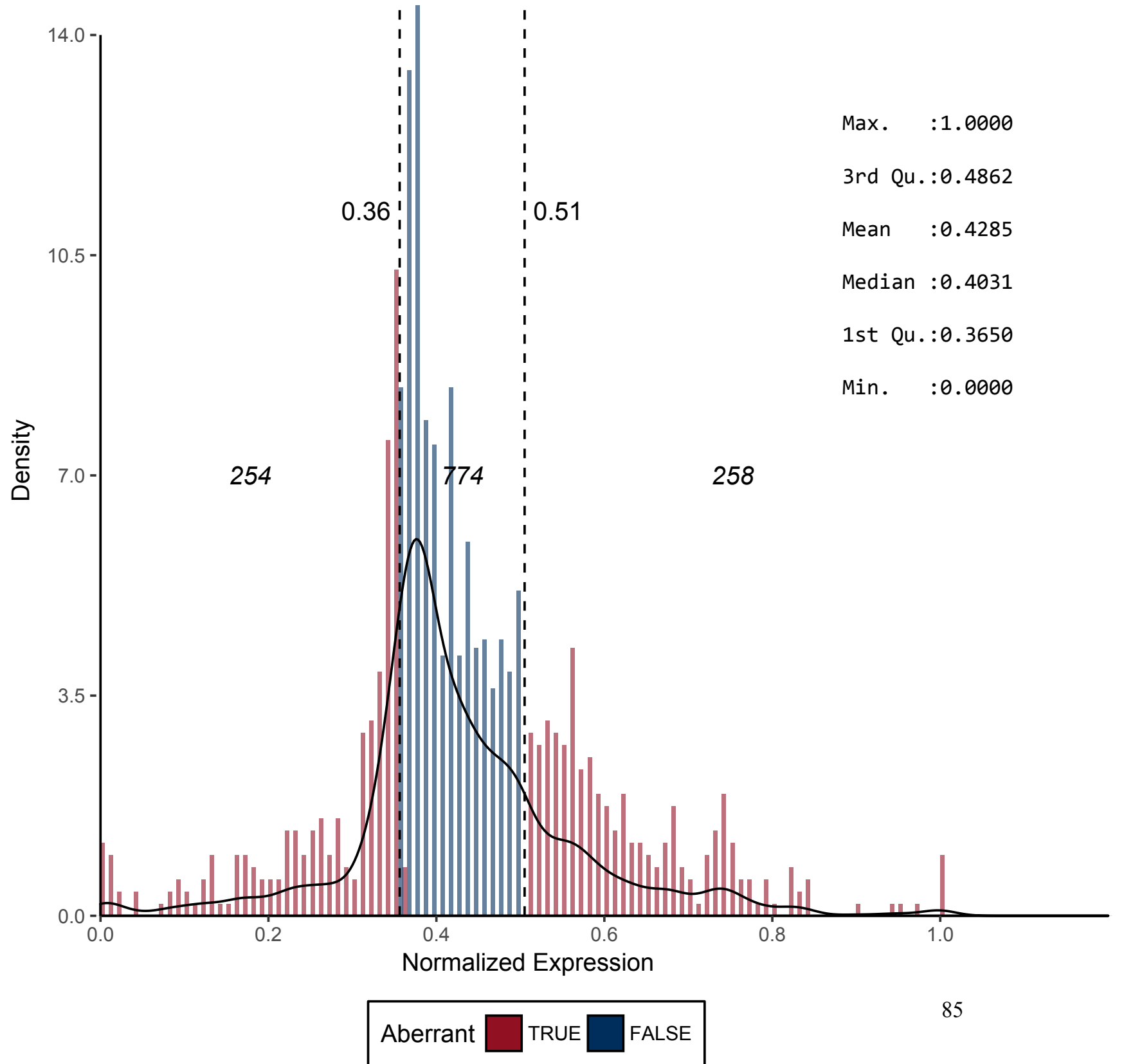


Figure 69 - CD61

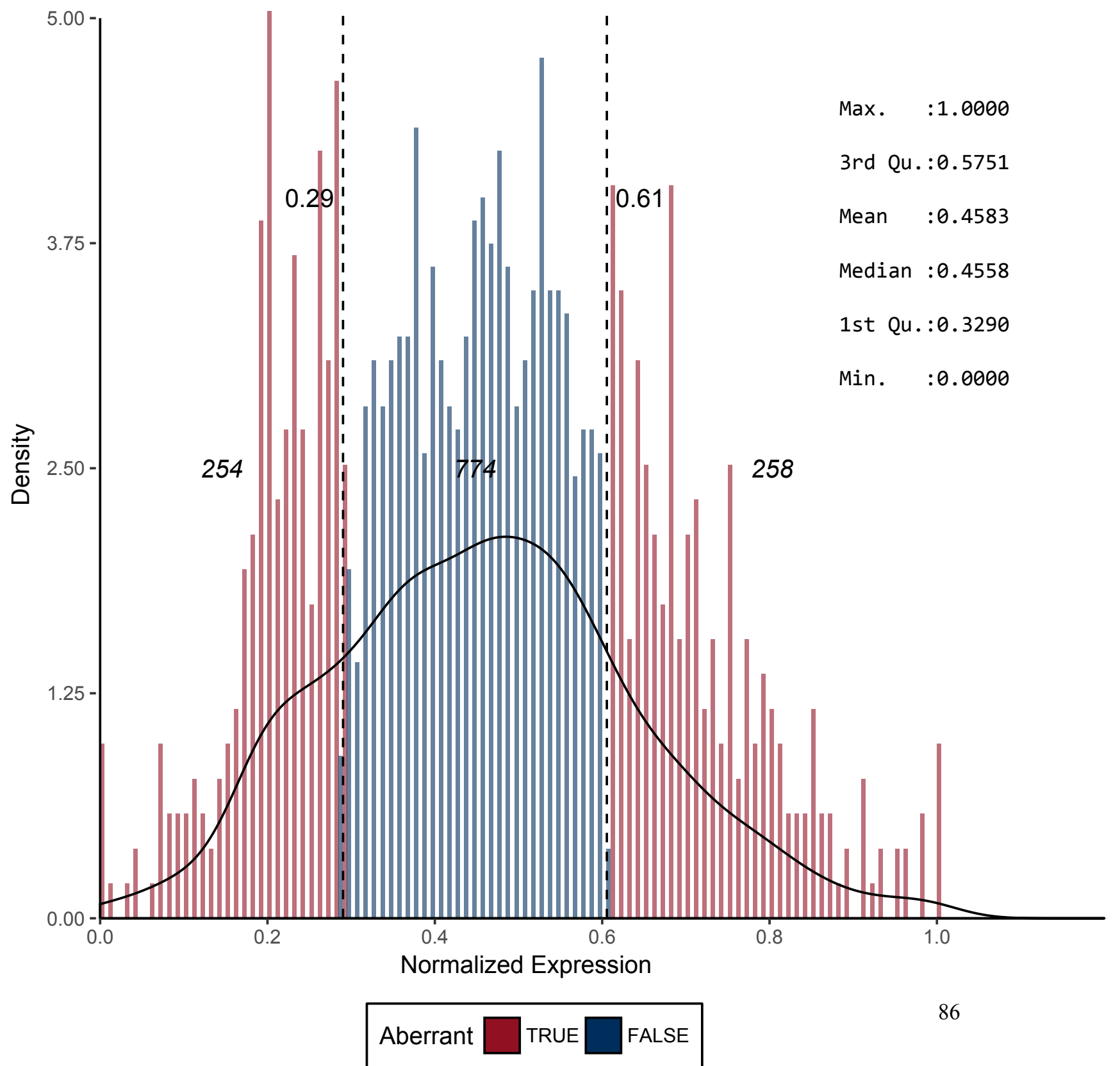


Figure 70 - CD62E*

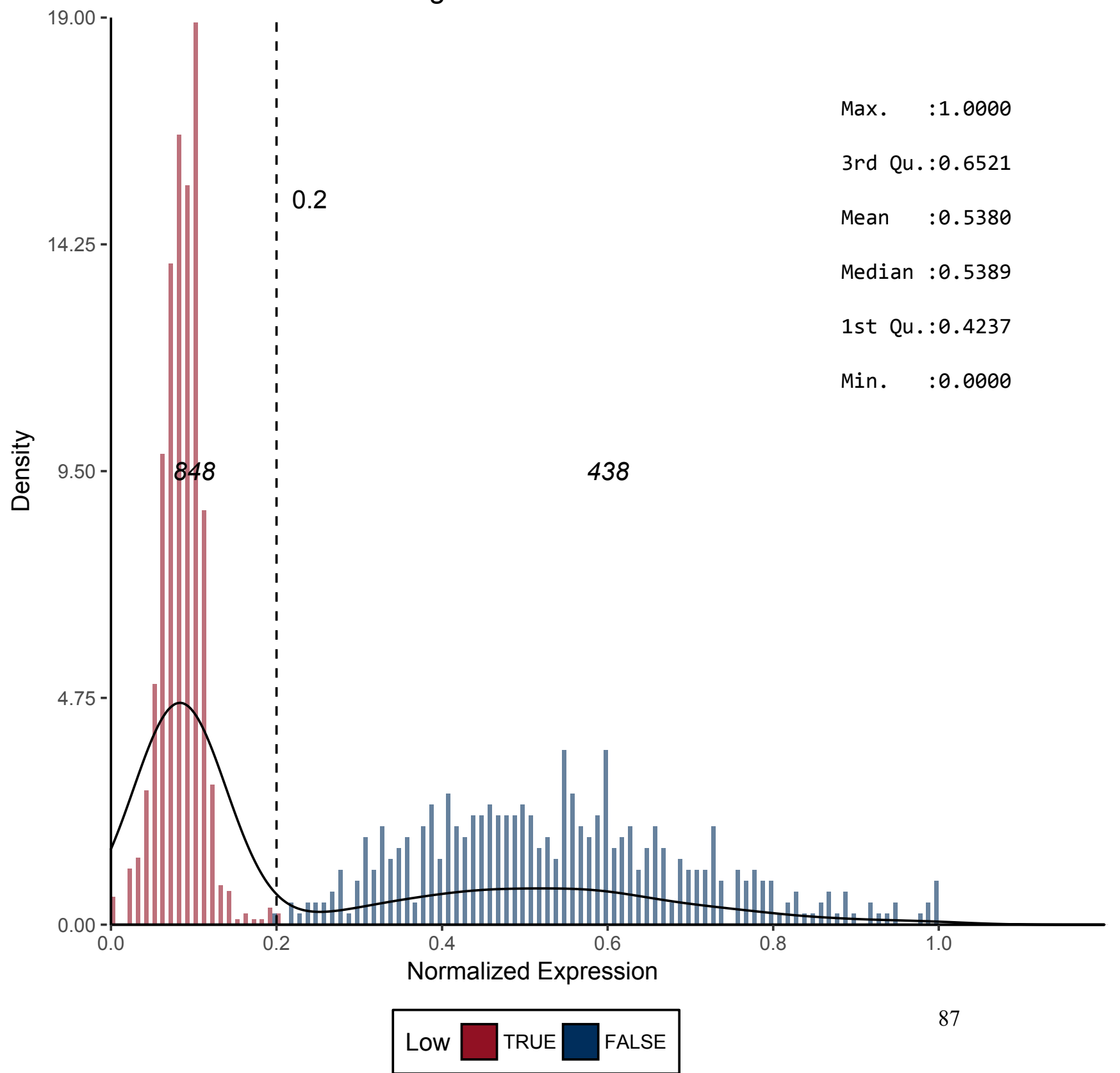


Figure 71 - CD62L*

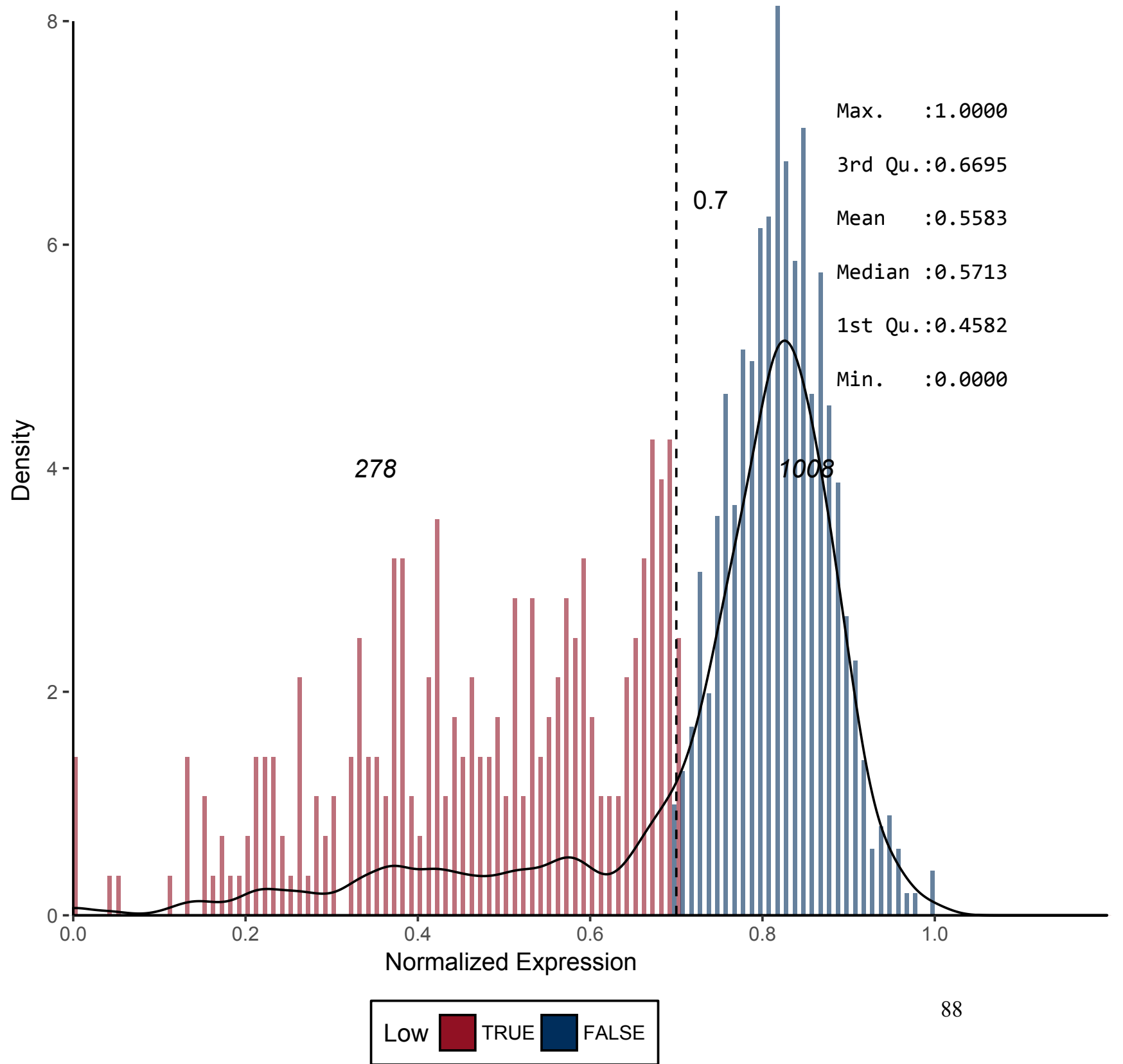


Figure 72 - CD62P

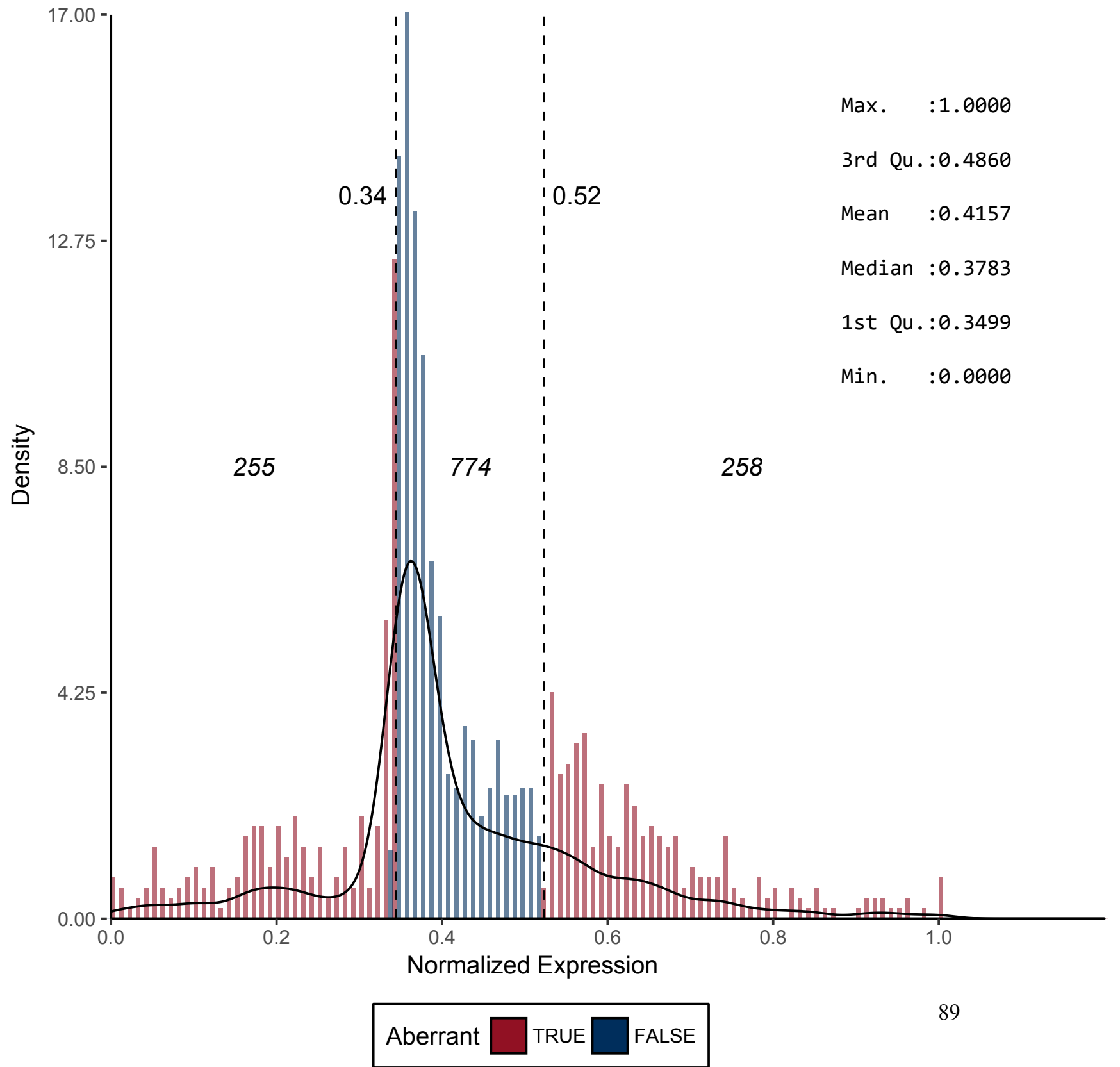


Figure 73 - CD63

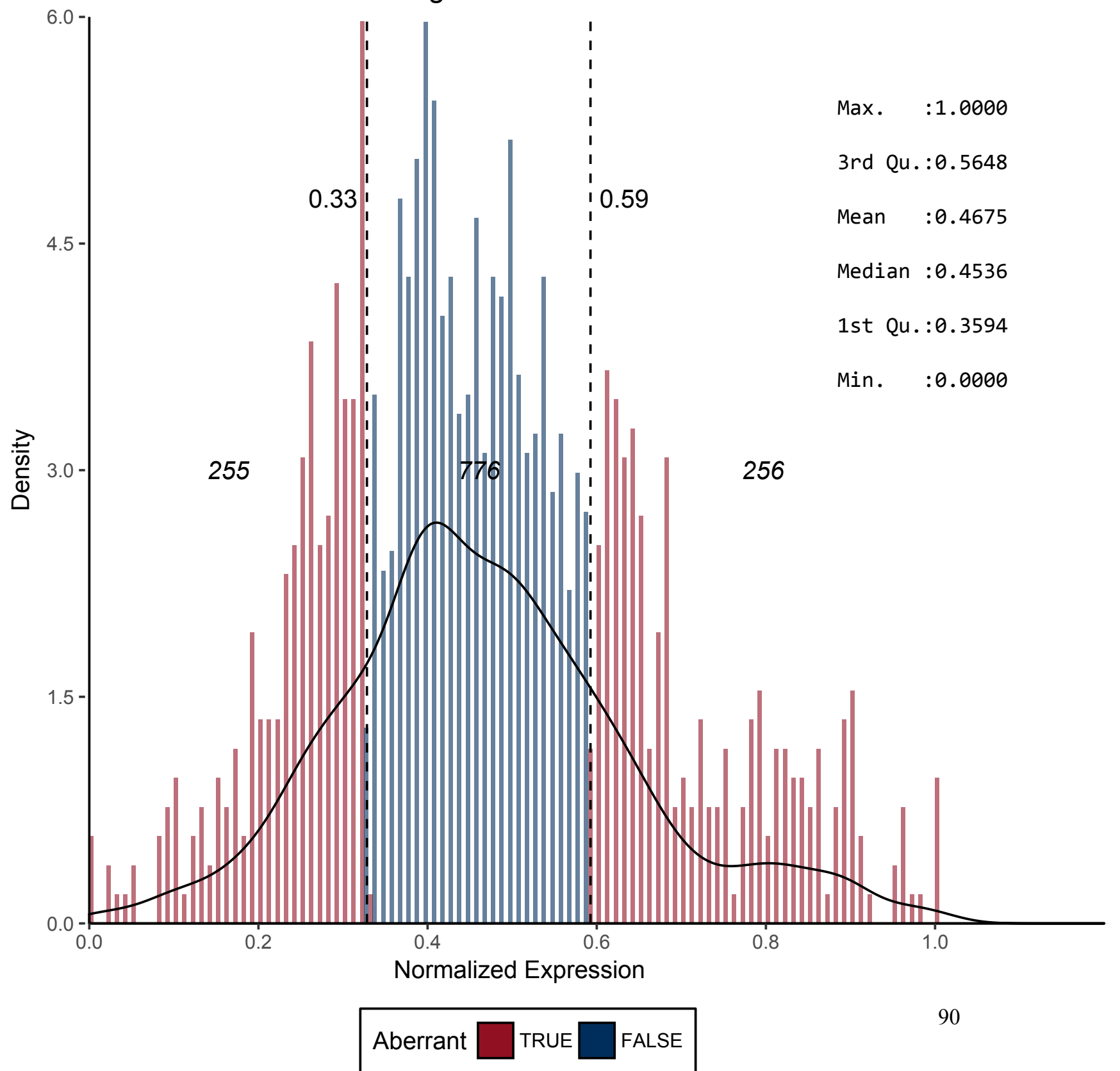


Figure 74 - CD66A

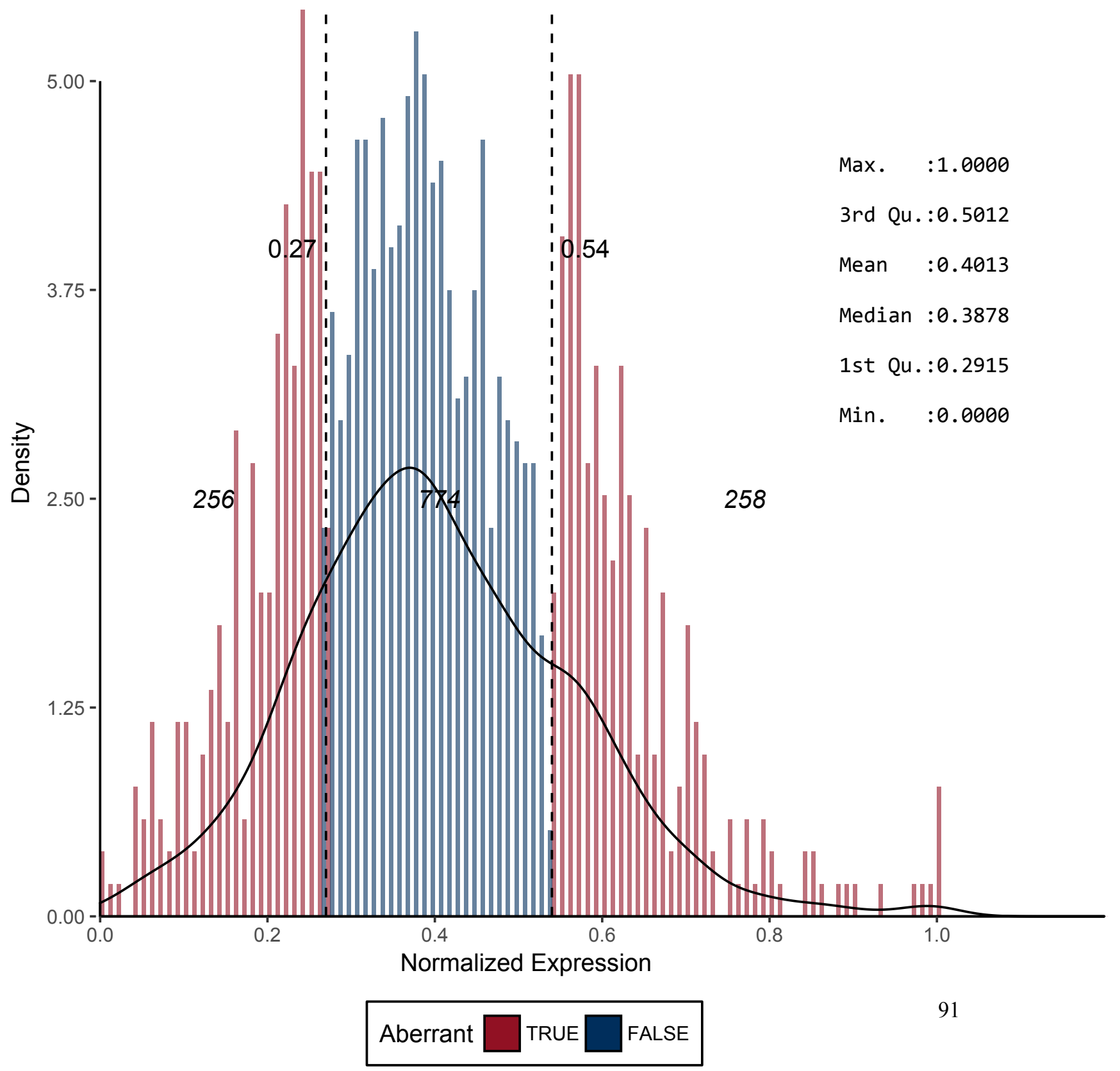


Figure 75 - CD66B

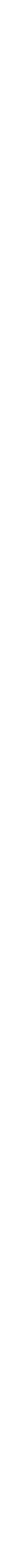


Figure 76 - CD66C

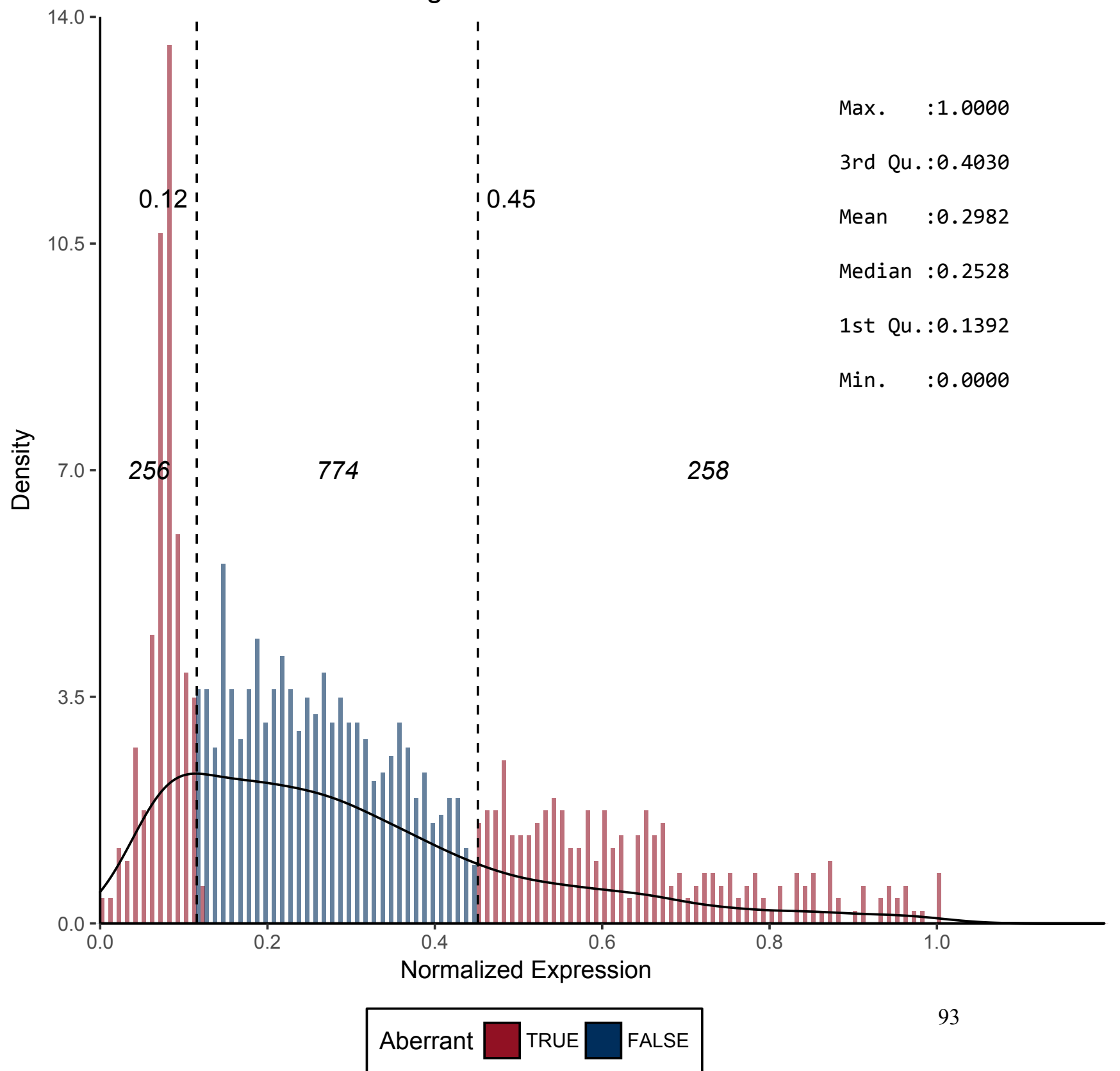


Figure 77 - CD66D

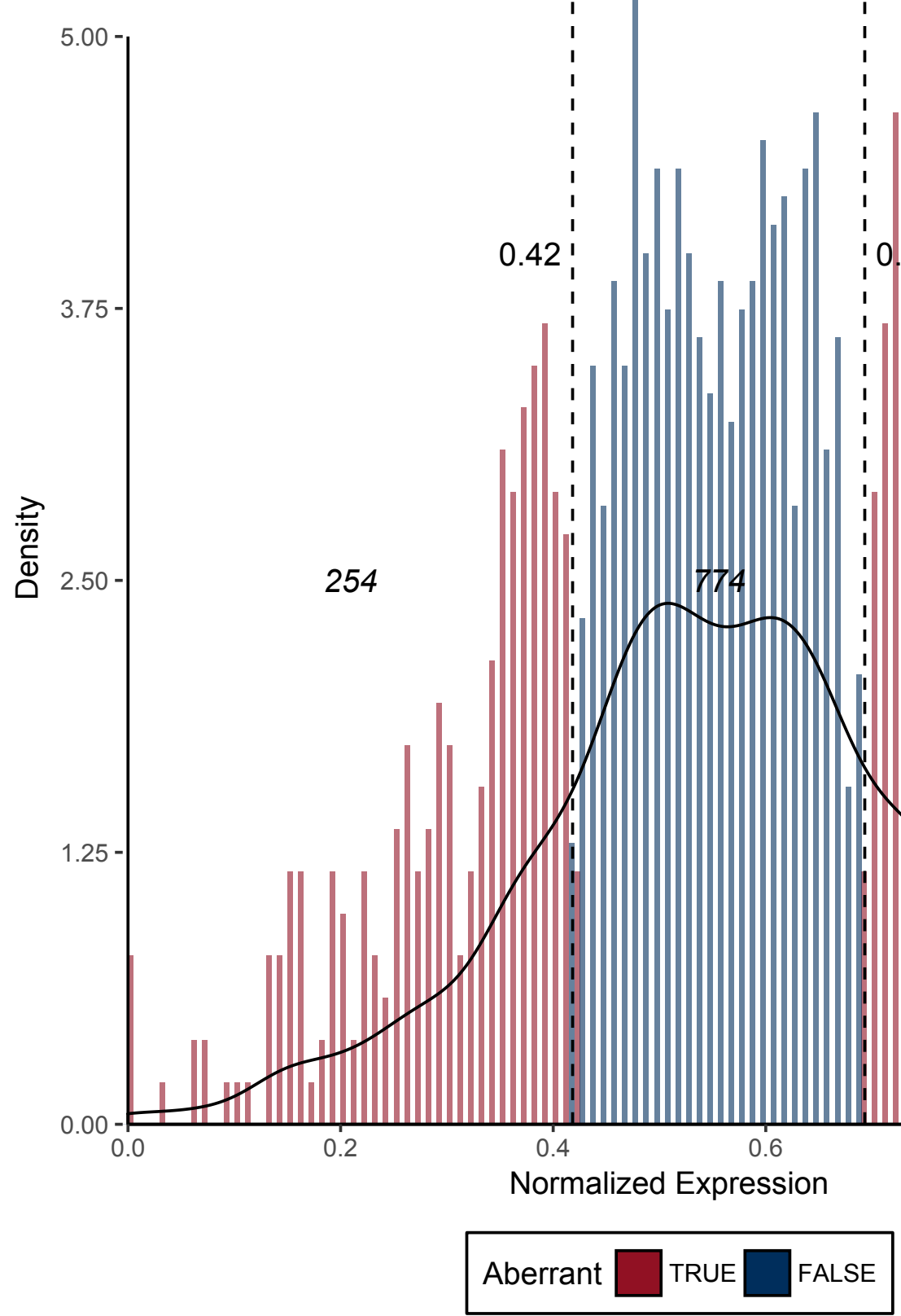

Max. $: 1.0000$

3rd Qu.:0.6591

Mean $\quad: 0.5490$

Median :0.5544

1st Qu.:0.4487

Min. $\quad: 0.0000$

258

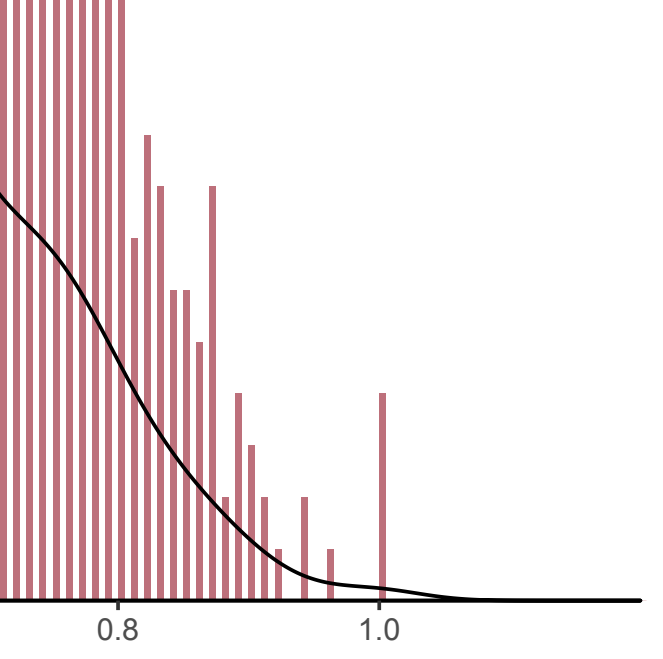


Figure 78 - CD66E*

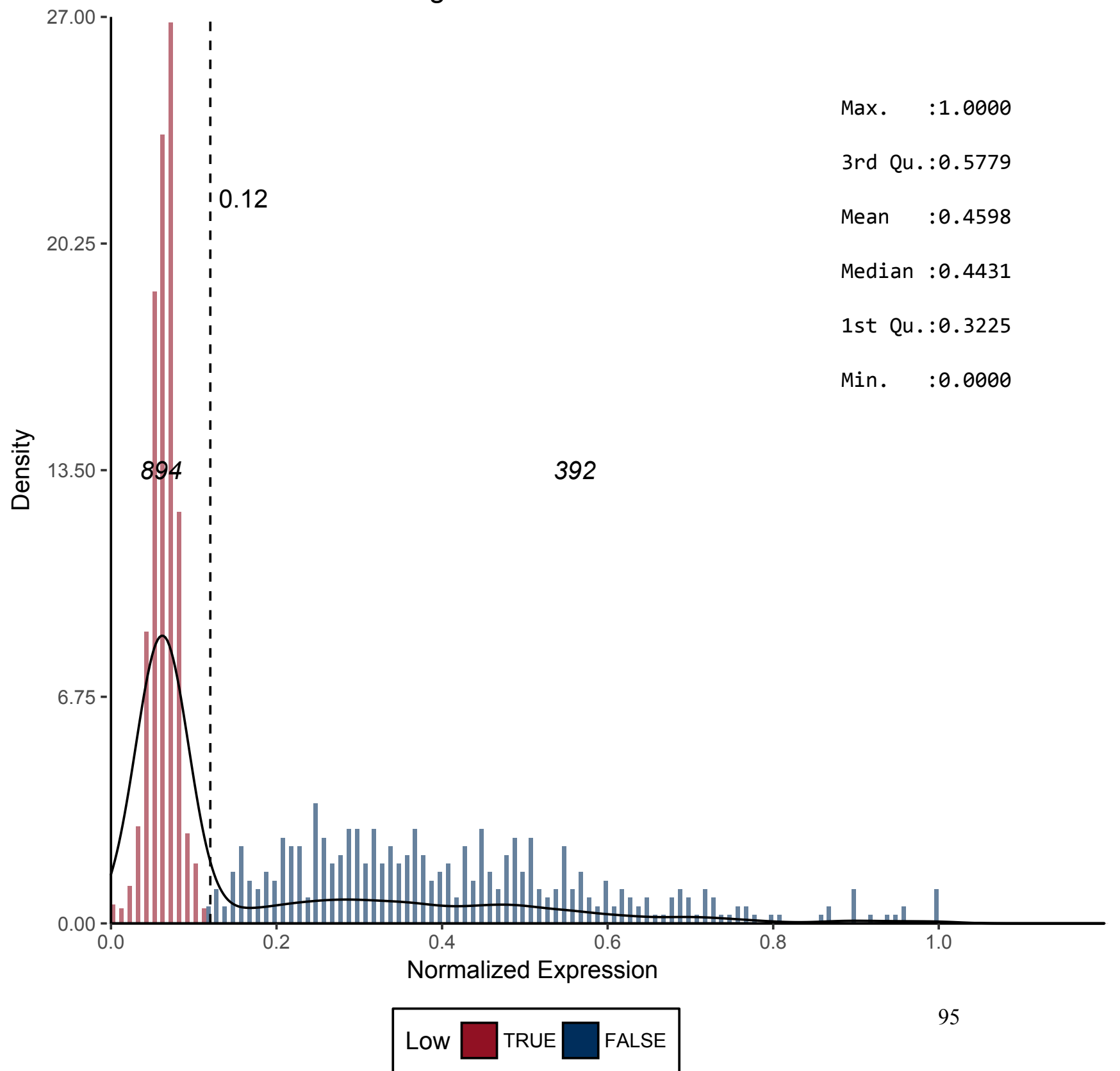


Figure 79 - CD66F*

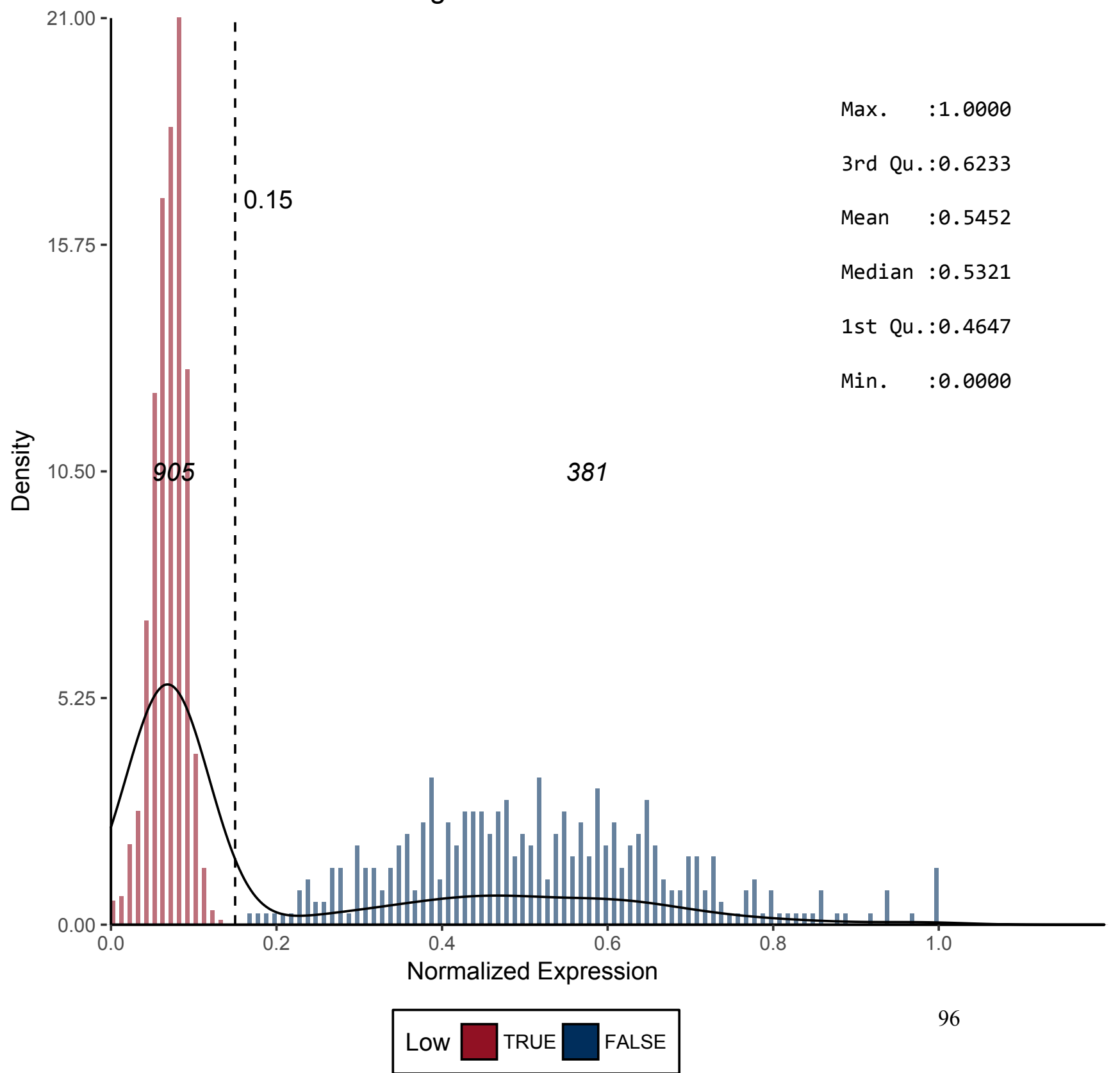


Figure 80 - CD68
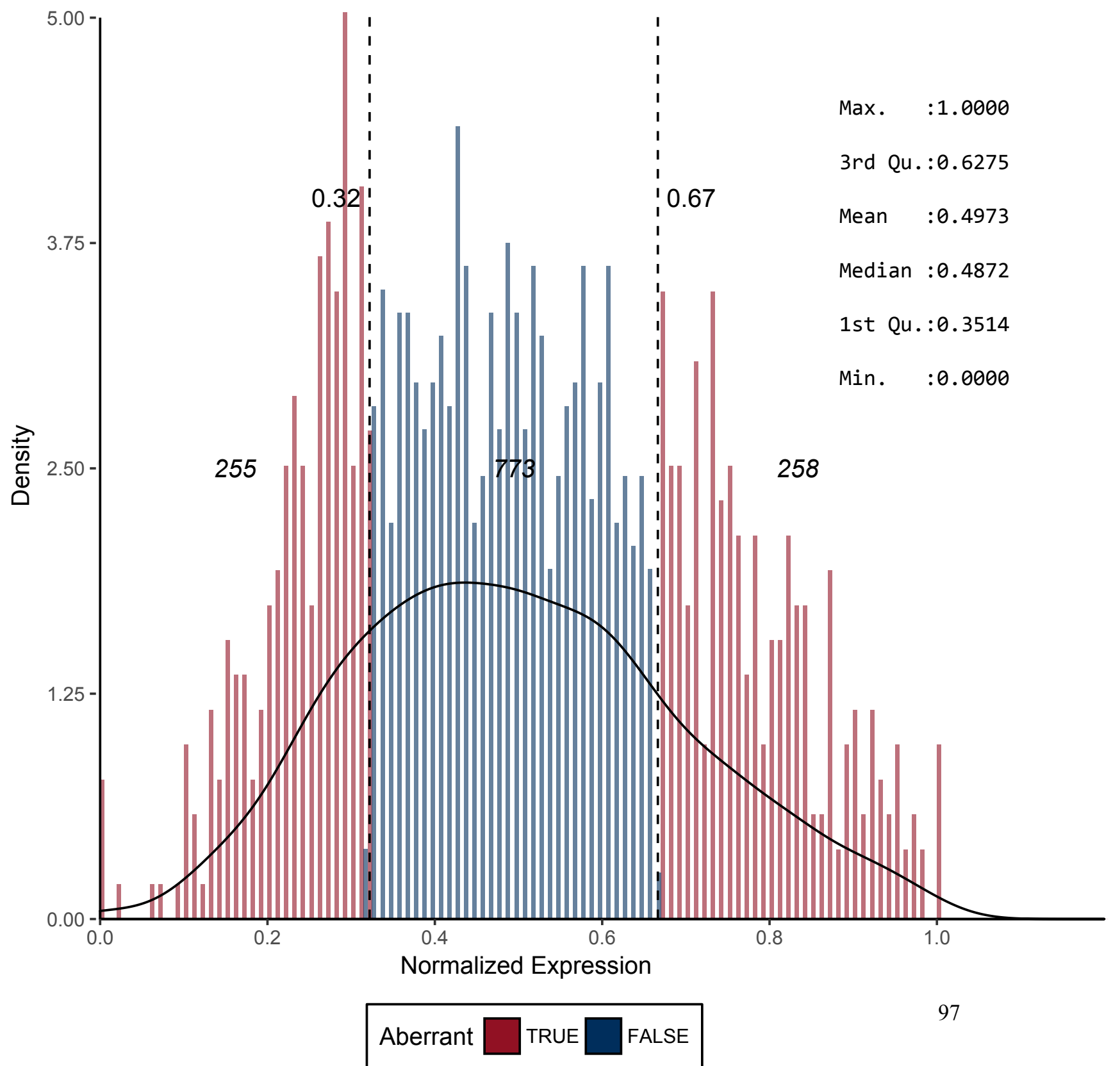
Figure 83 - CD71

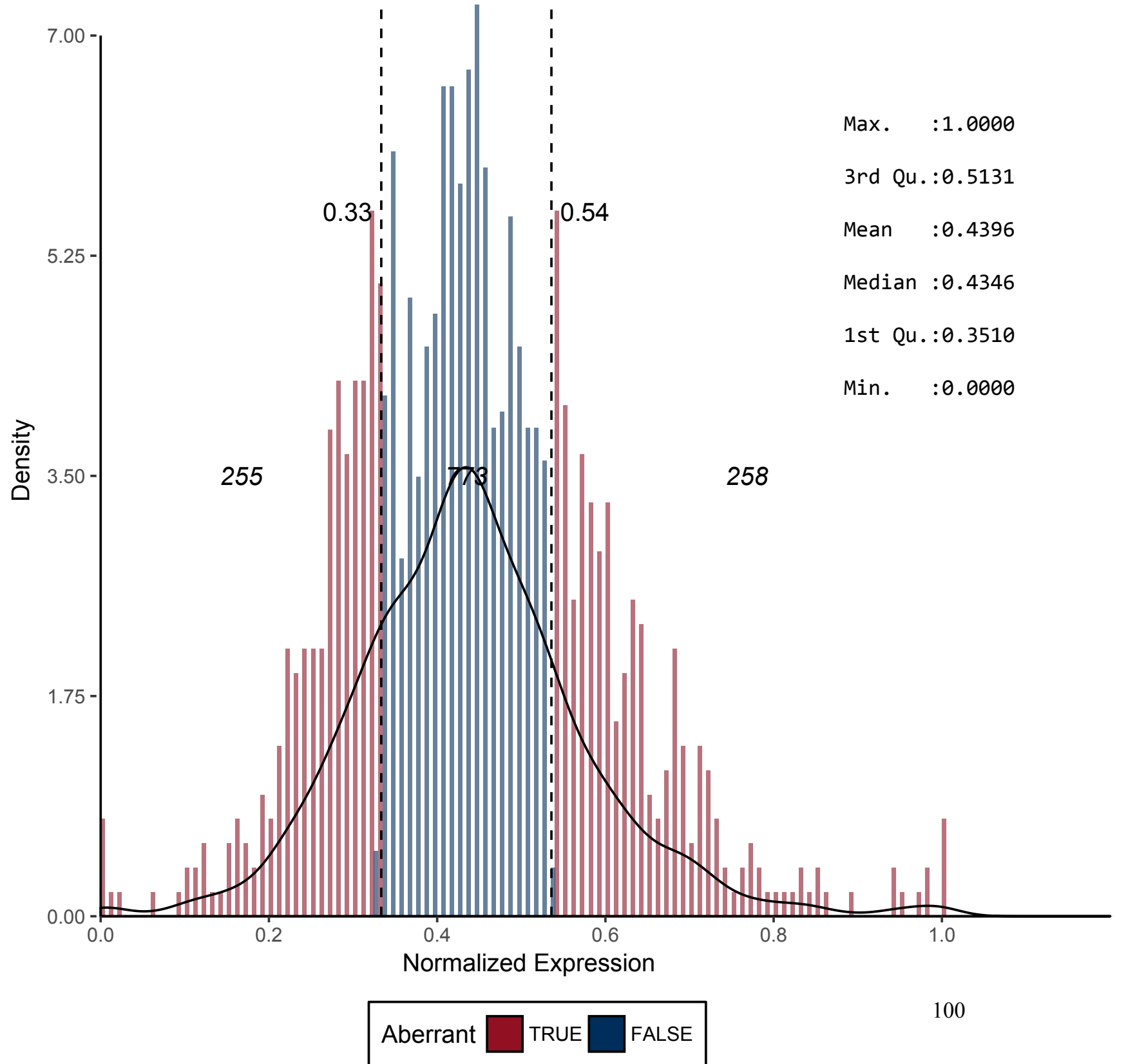


Figure 84 - CD72*

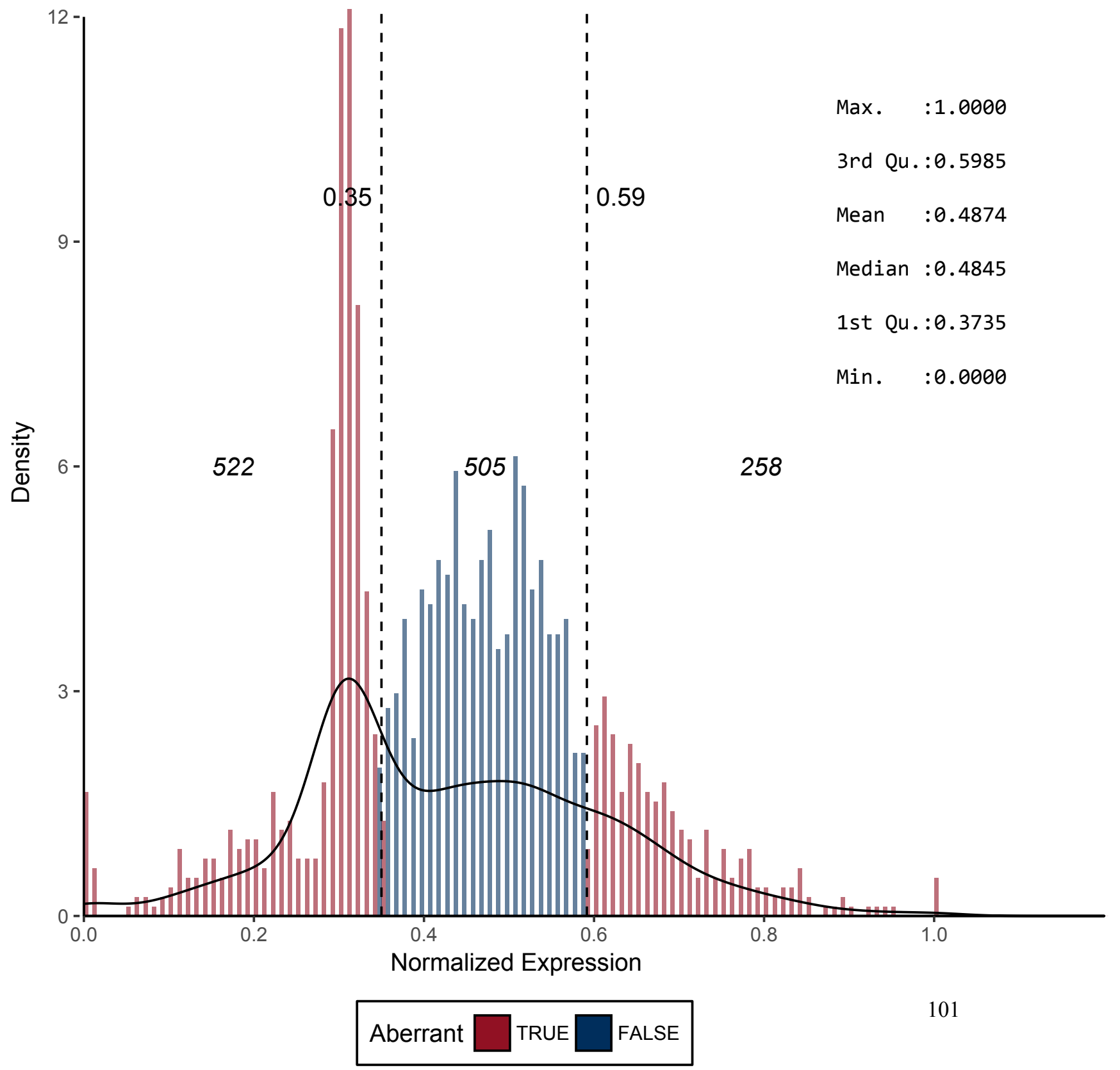


Figure 86 - CD74

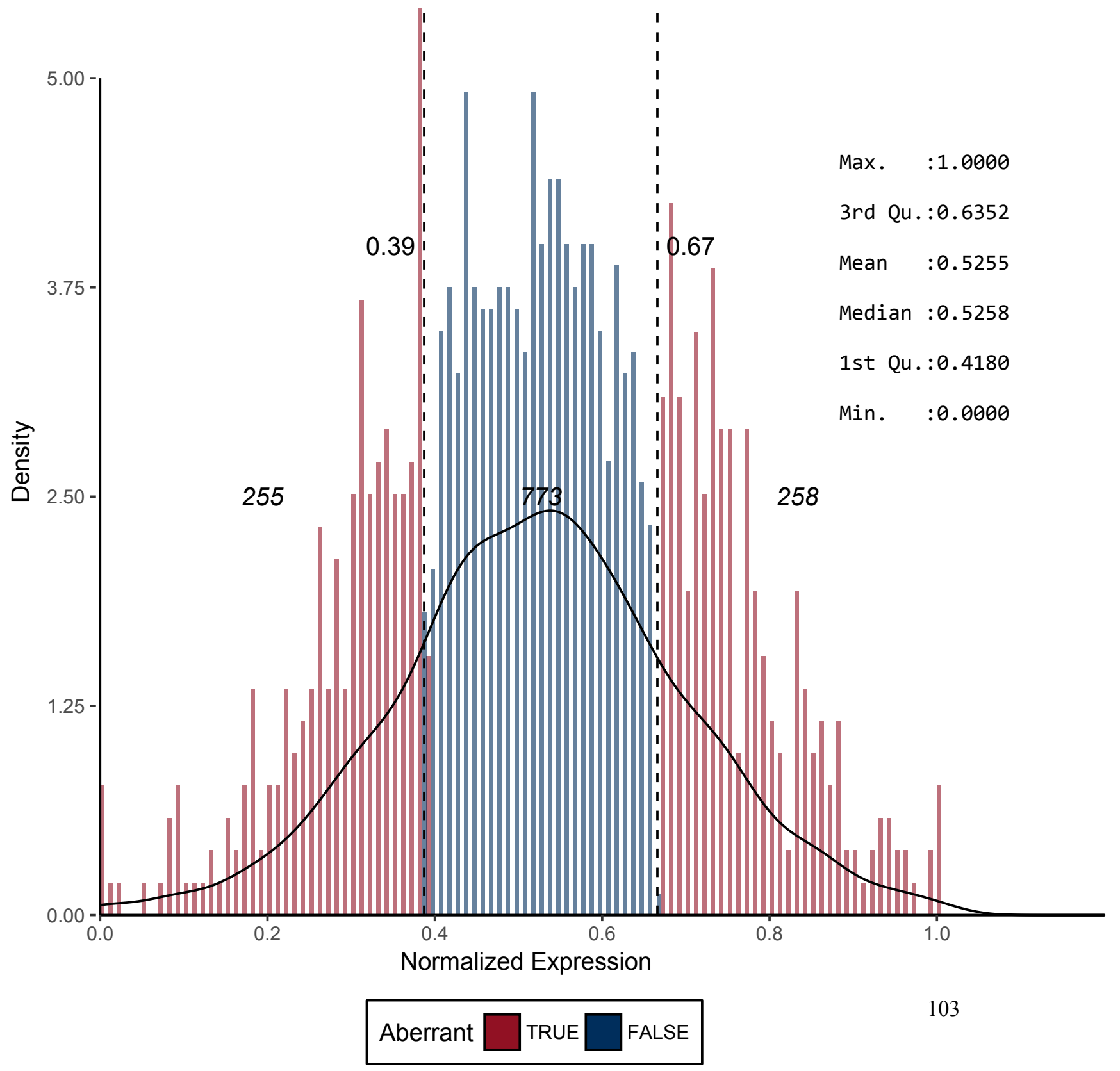


Figure 87 - CD79A
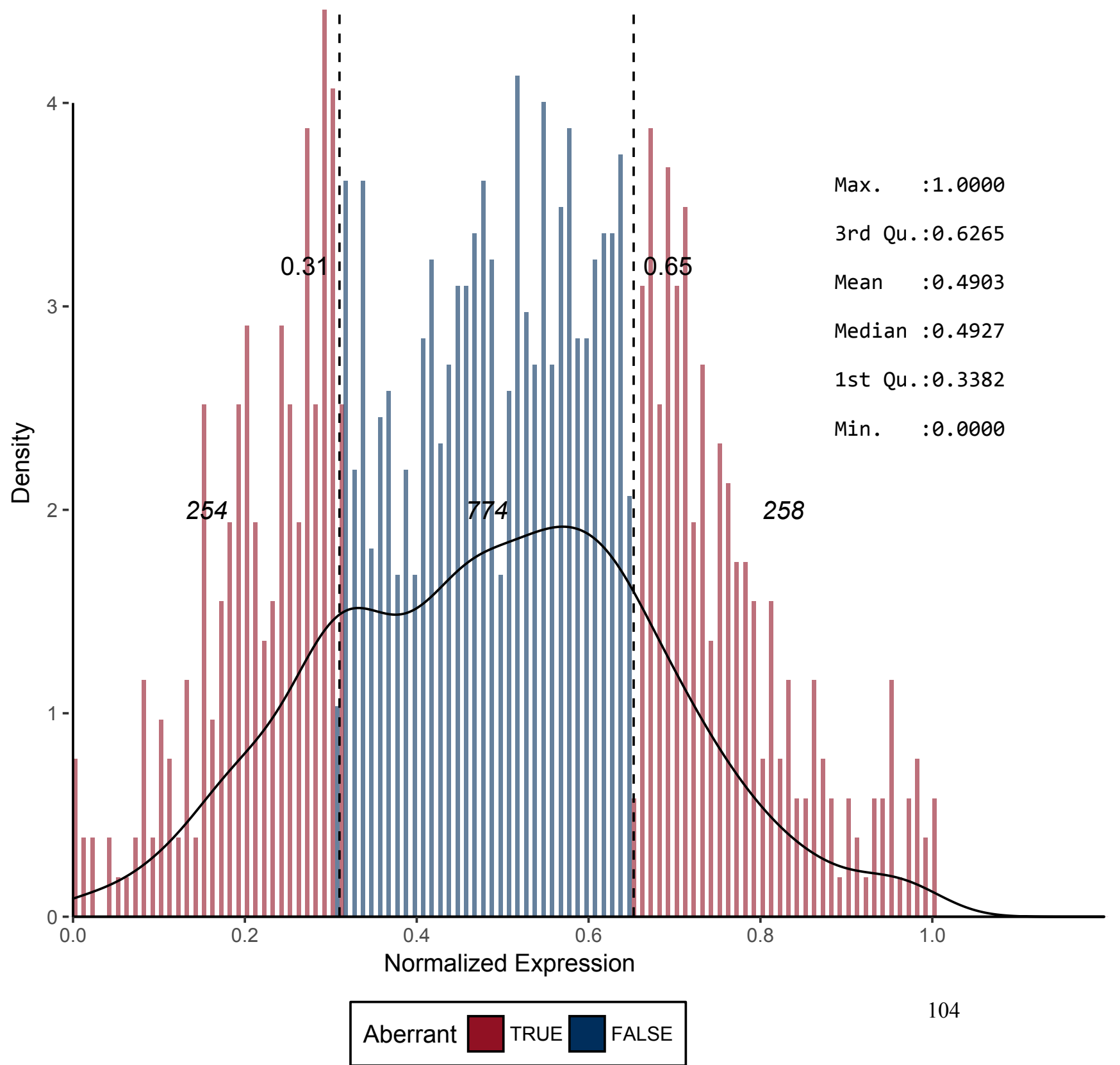
Figure 88 - CD79B

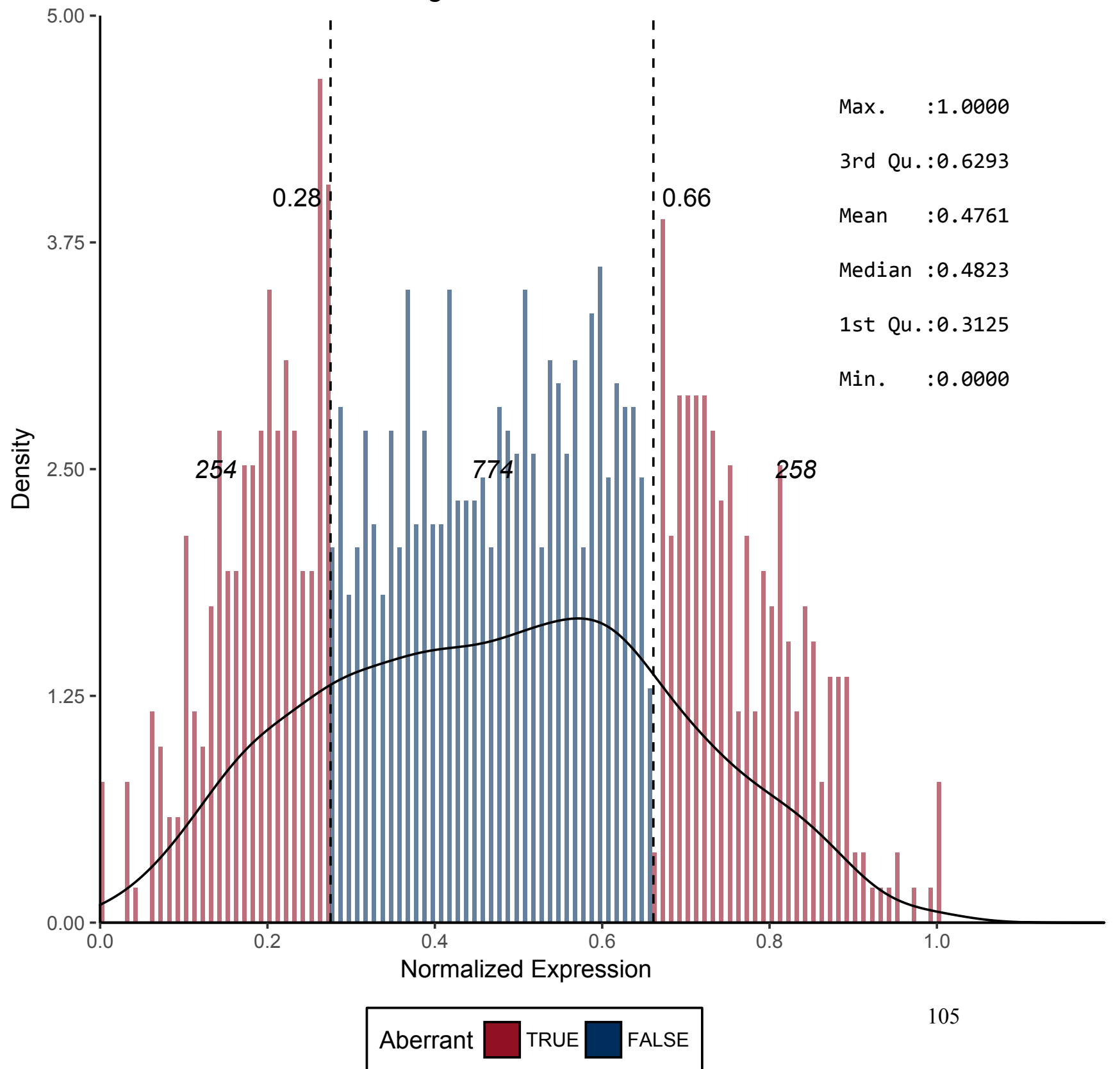


Figure 89 - CD80*

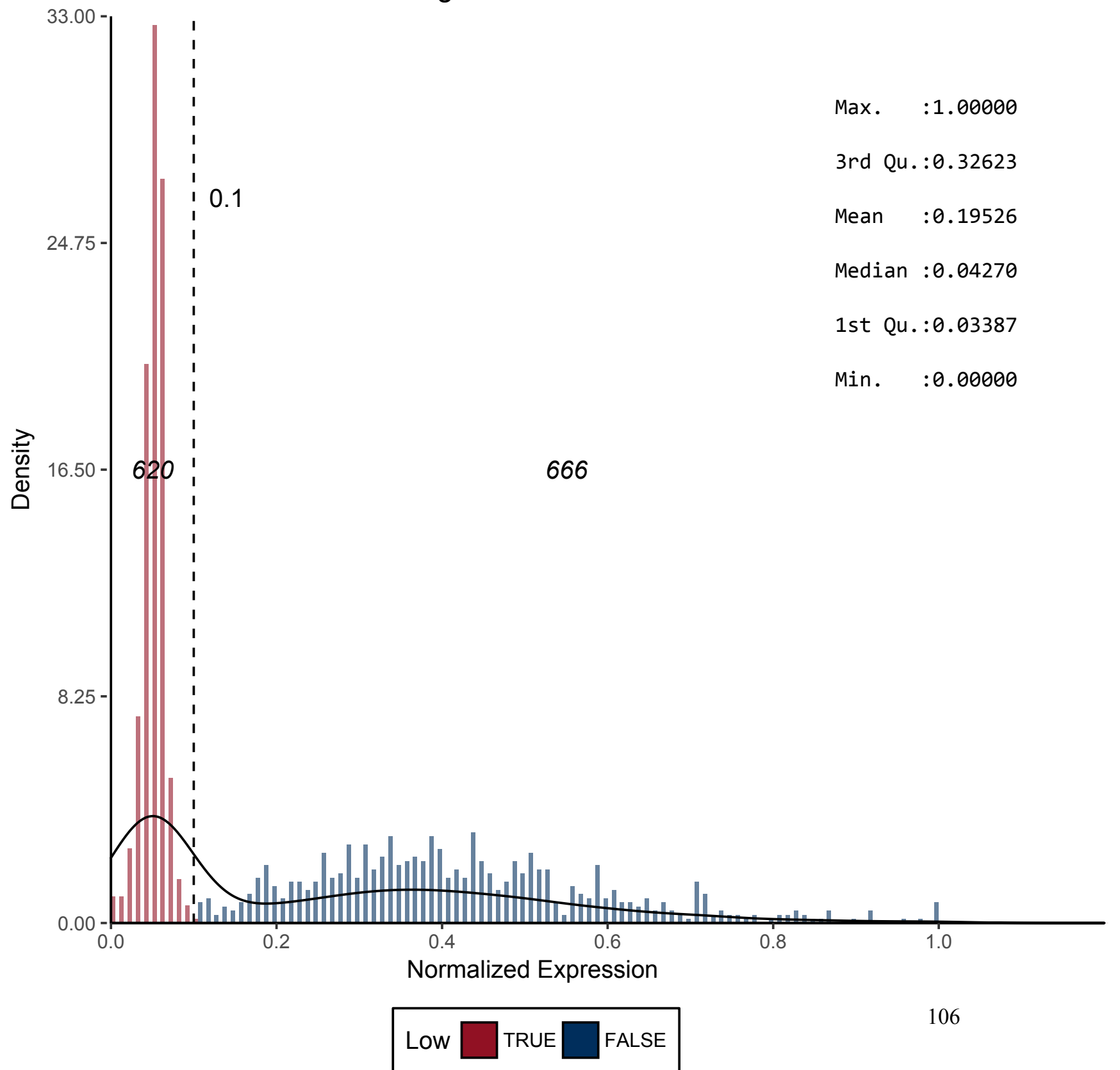


Figure 91 - CD82

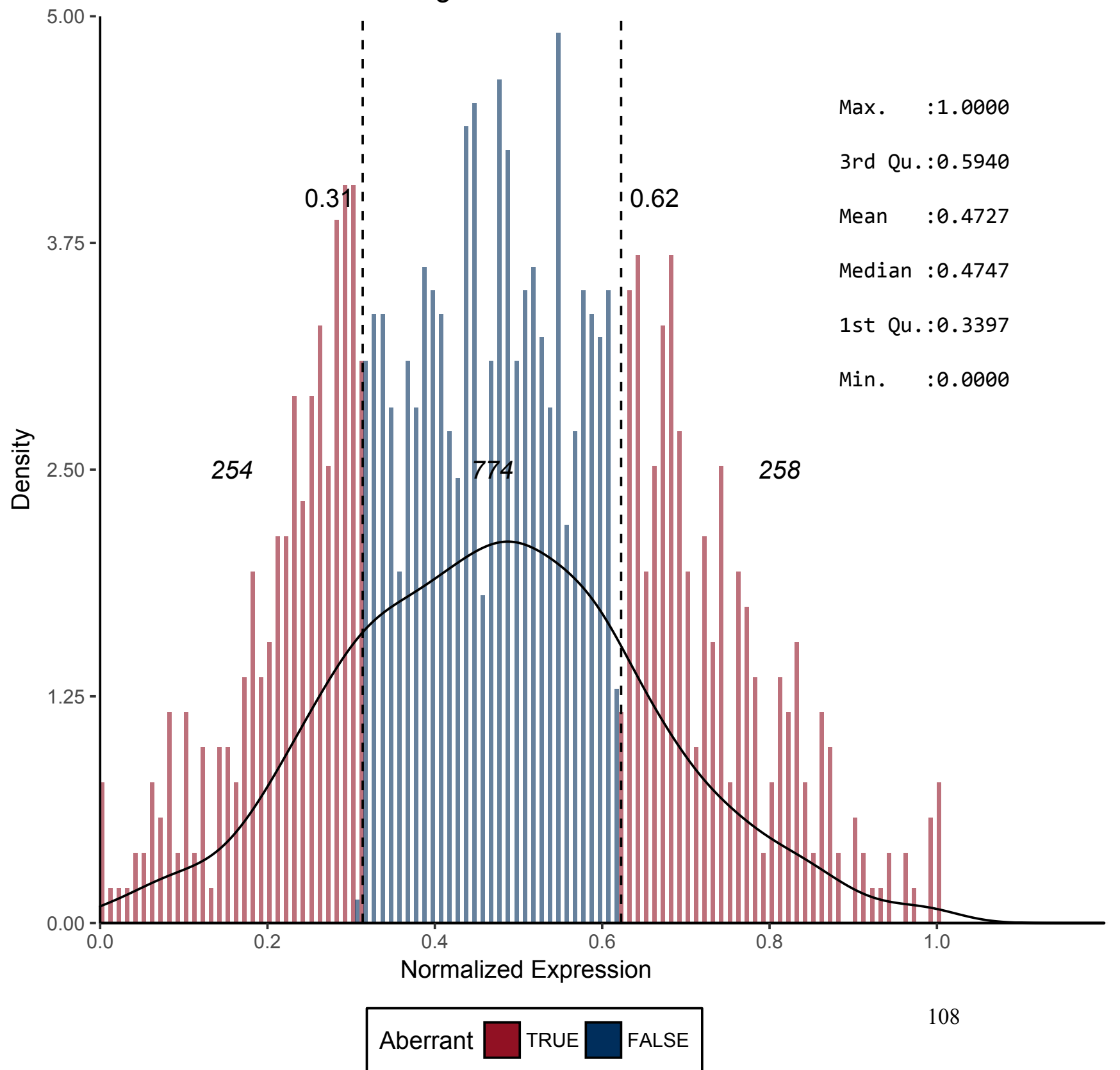


Figure 92 - CD83

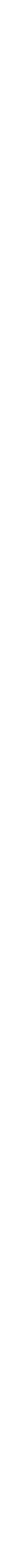


Figure 93 - CD84

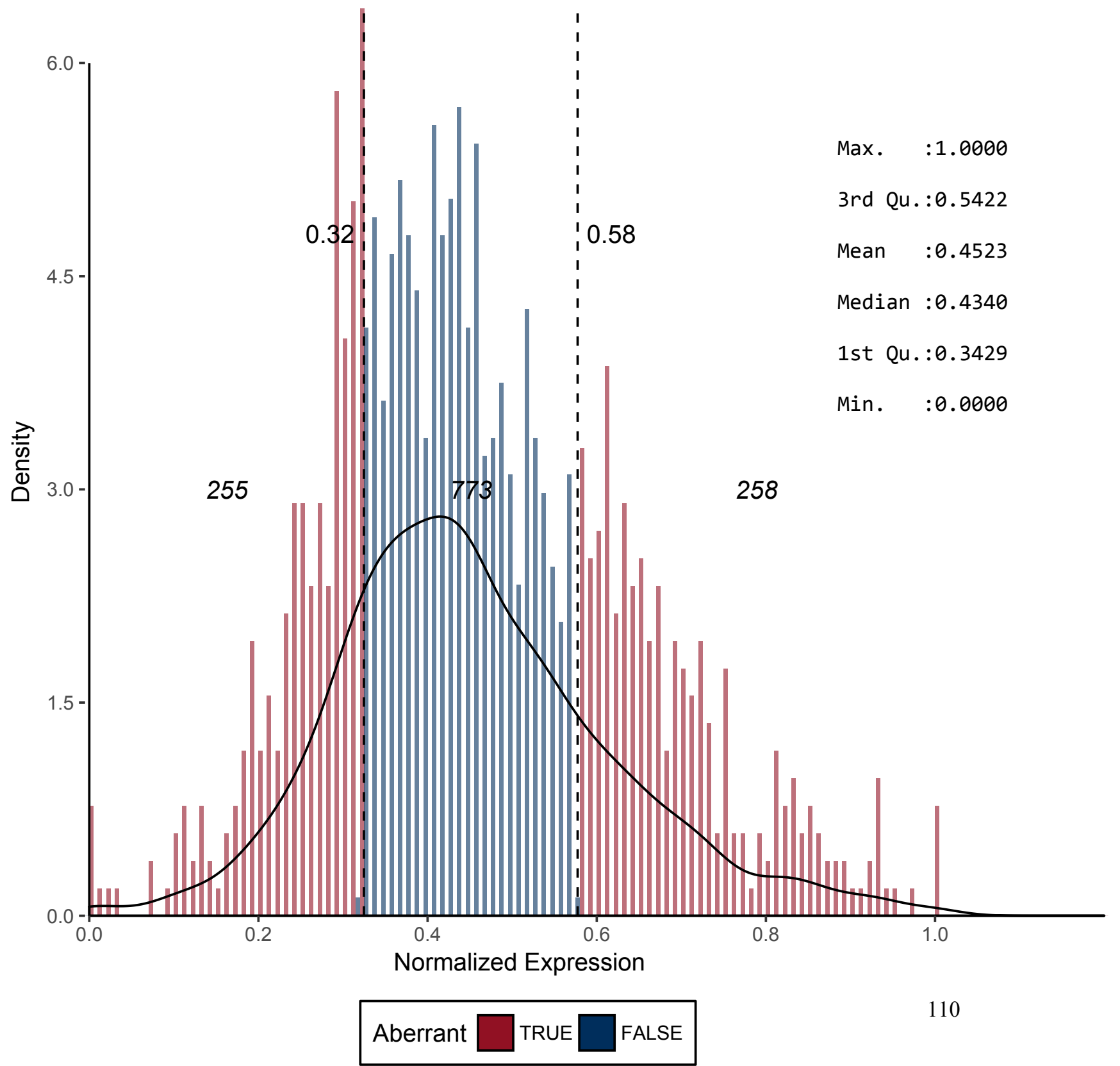


Figure 94 - CD85A

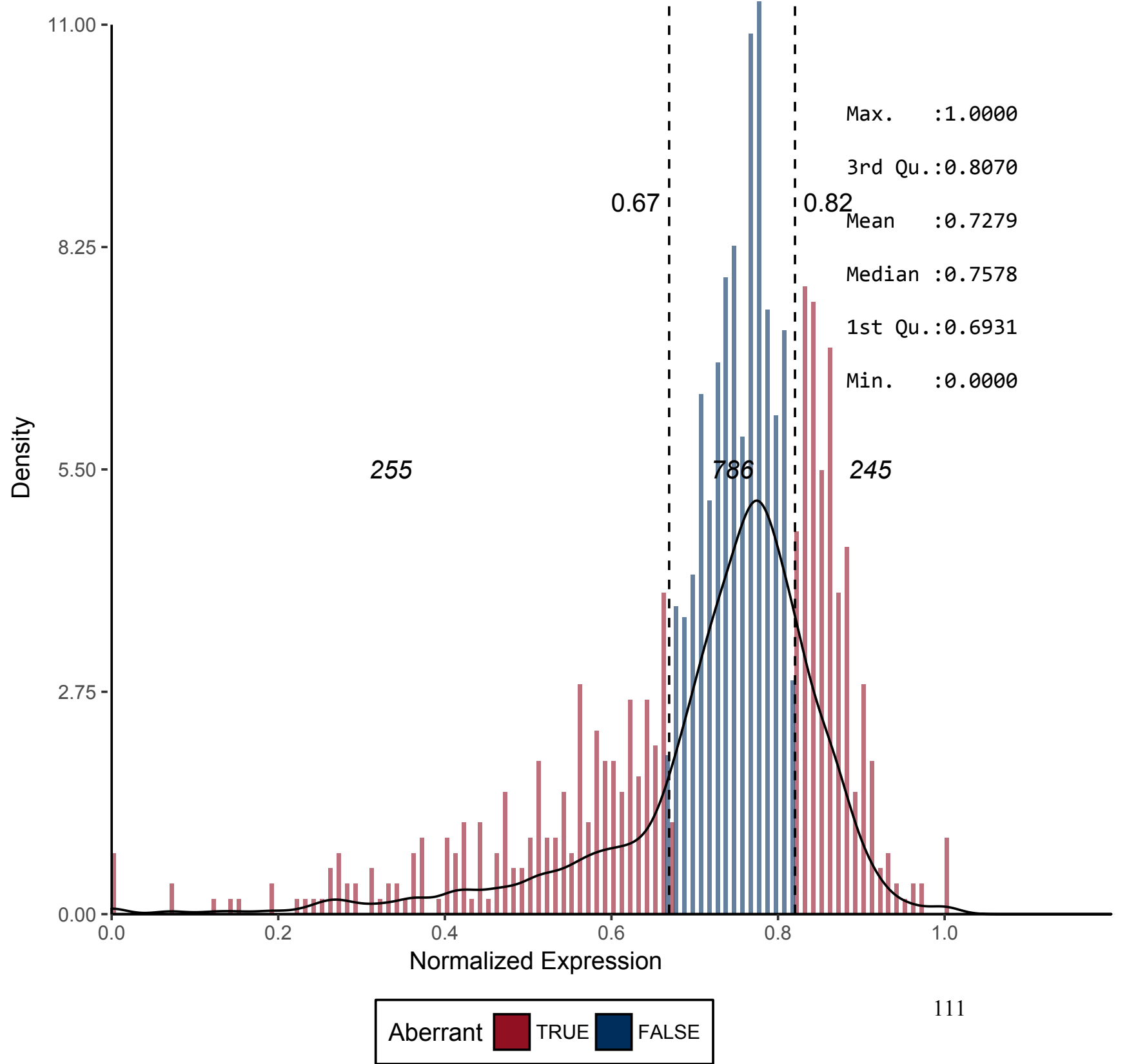


Figure 95 - CD85C*

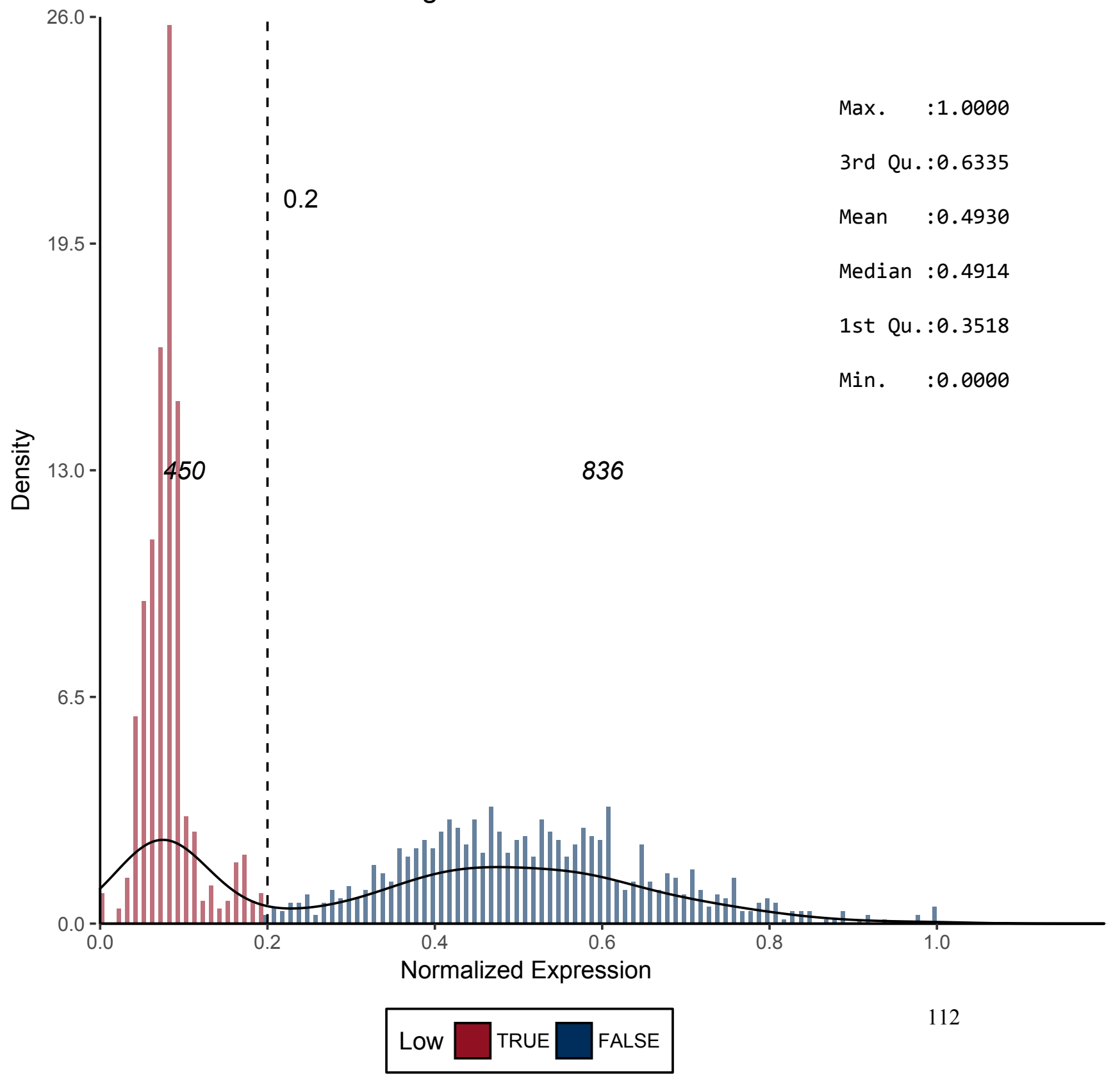


Figure 97 - CD85F

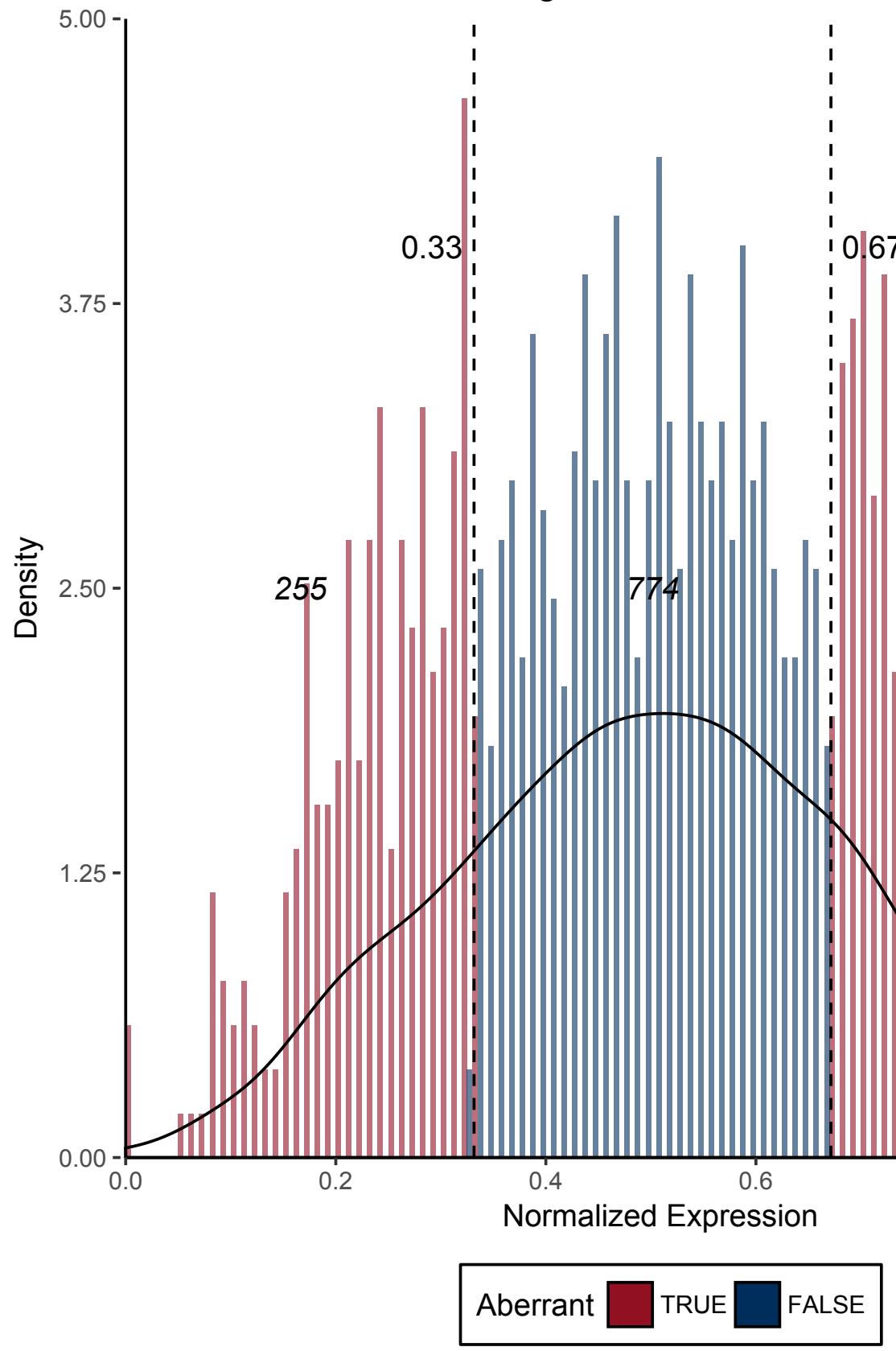

Max. $: 1.0000$

3rd Qu.:0.6397

Mean $\quad: 0.5027$

Median :0.5059

1st Qu.:0.3677

Min. $\quad: 0.0000$ 
Figure 99 - CD85H

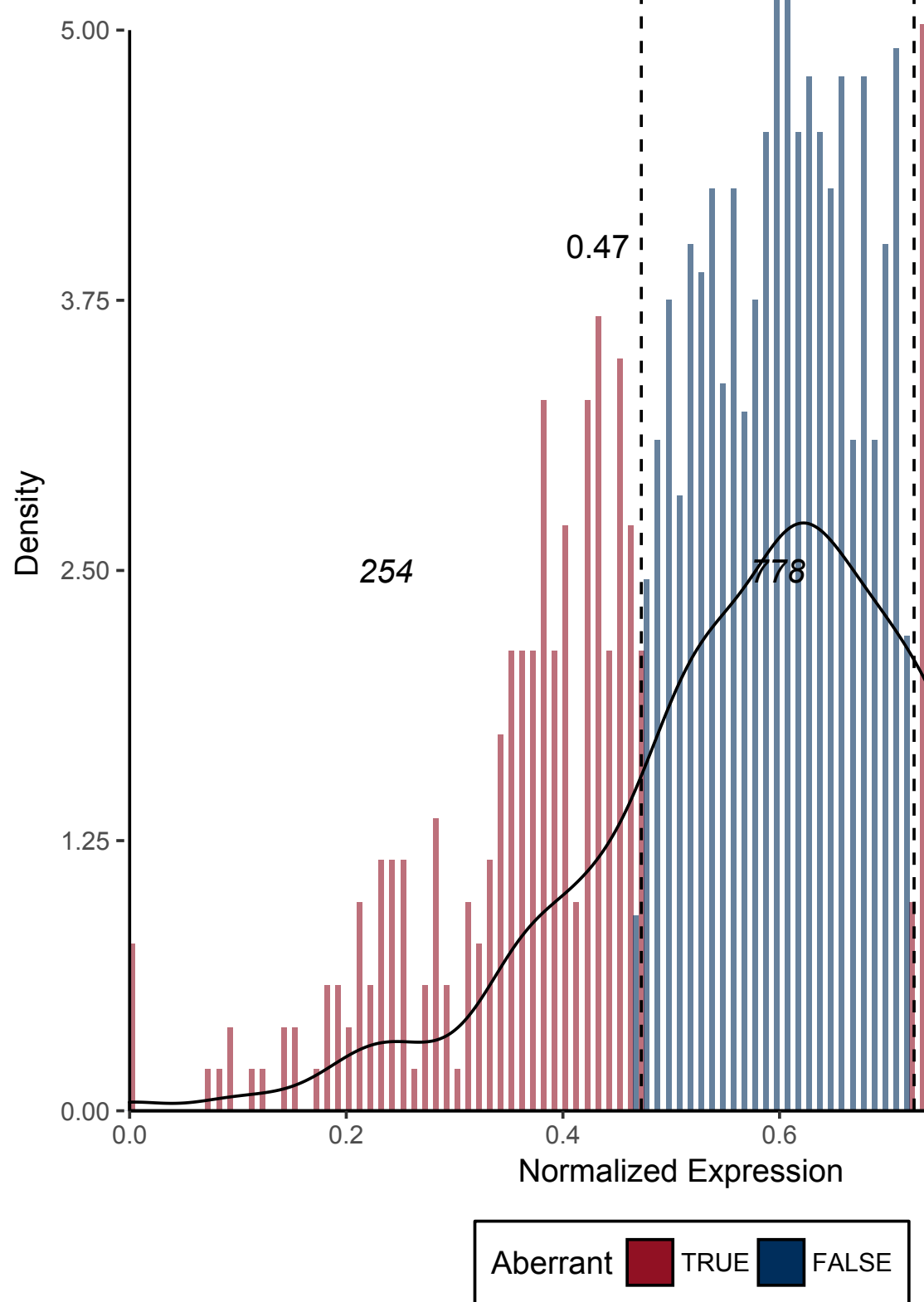

Max. $: 1.0000$

3rd Qu.:0.7014

Mean $\quad: 0.5915$

Median :0.6070

1st Qu.:0.5010

Min. $\quad: 0.0000$

254 
Figure 100 - CD85J

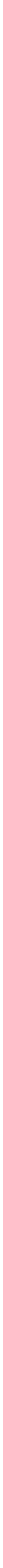


Figure 101 - CD85K

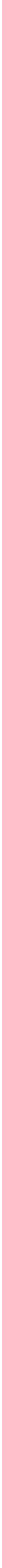


Figure 102 - CD85M*

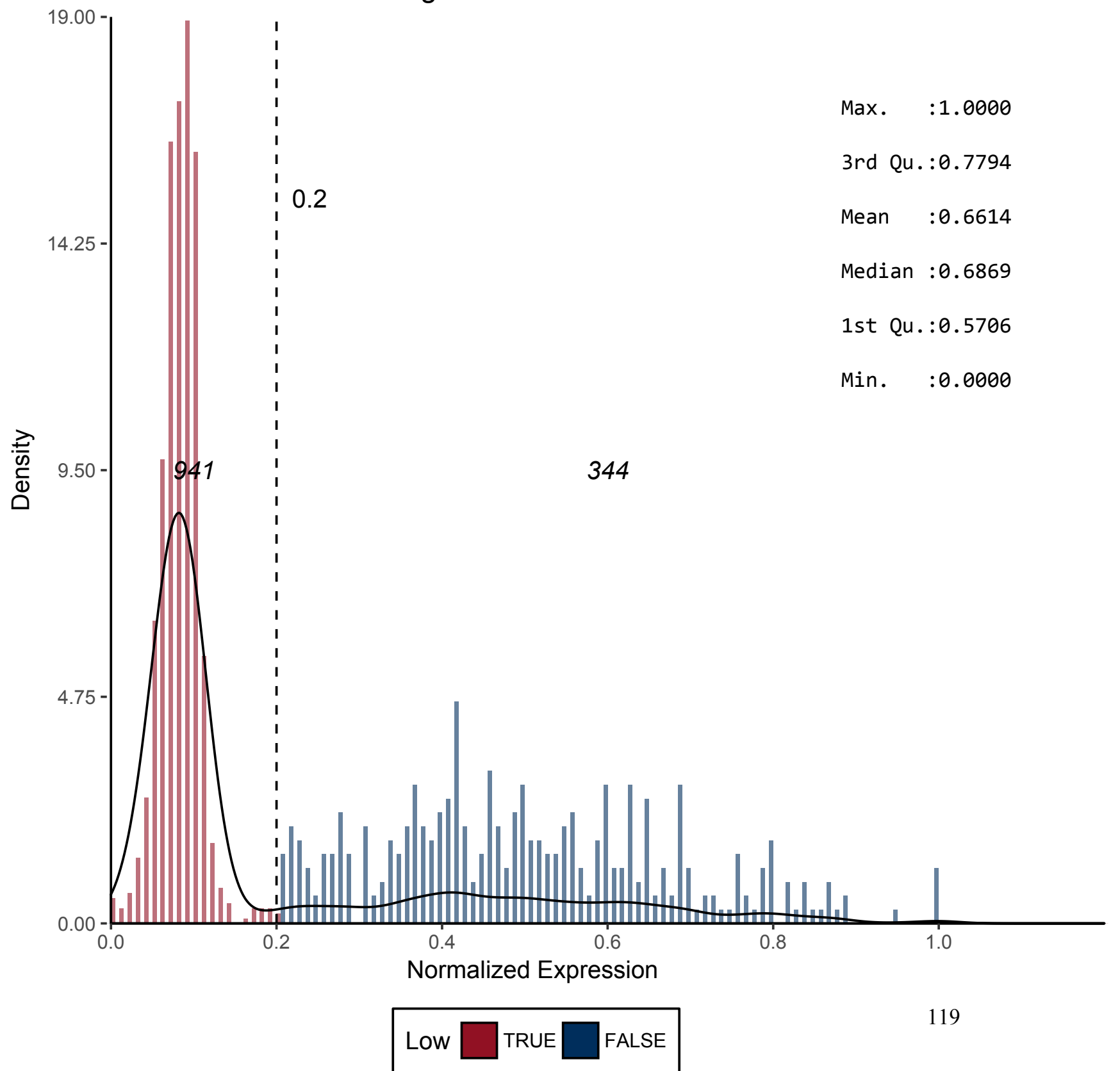


Figure 103 - CD86

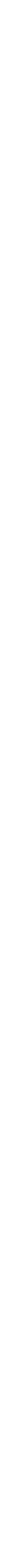


Figure 104 - CD87

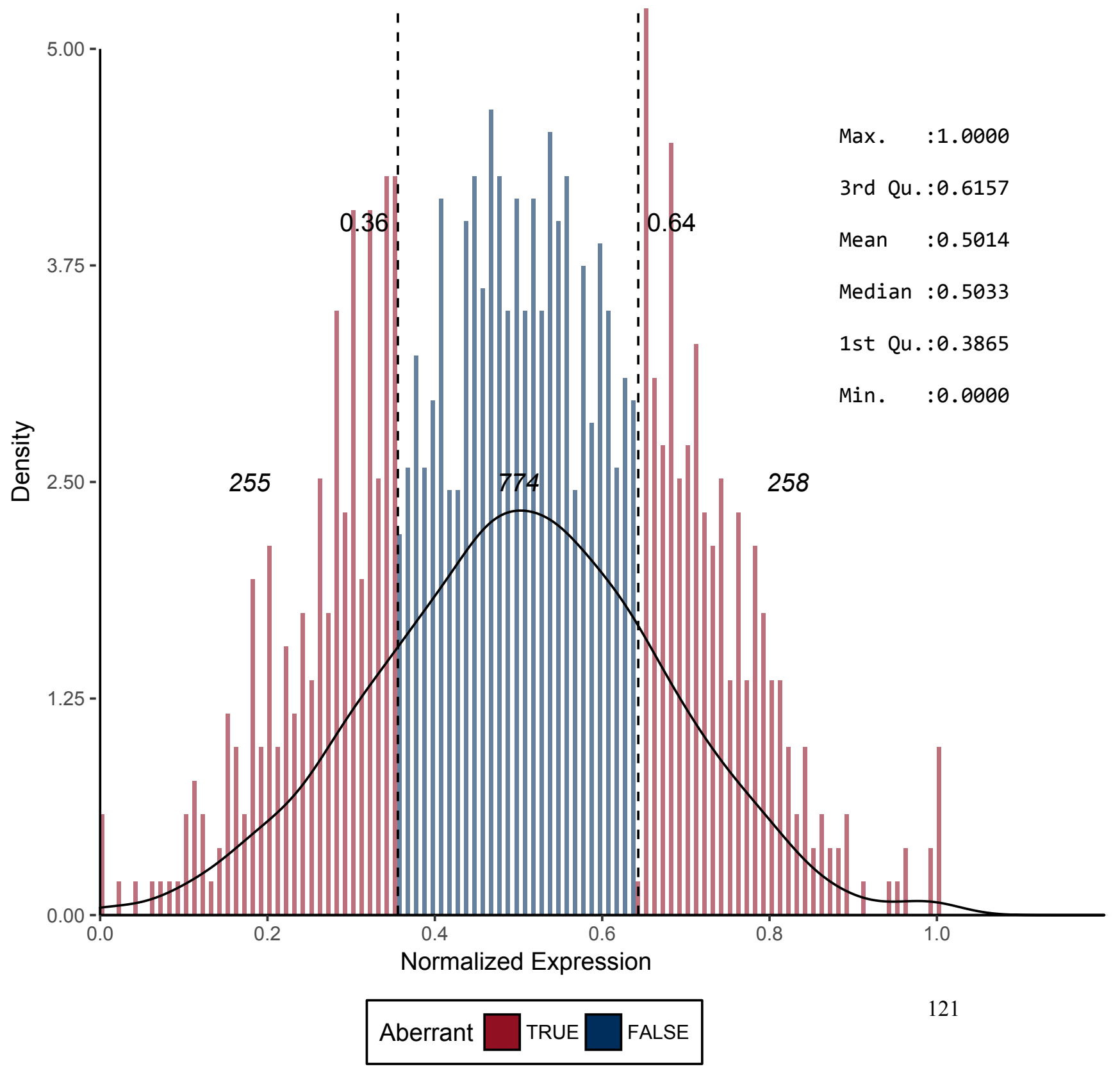


Figure 106 - CD89

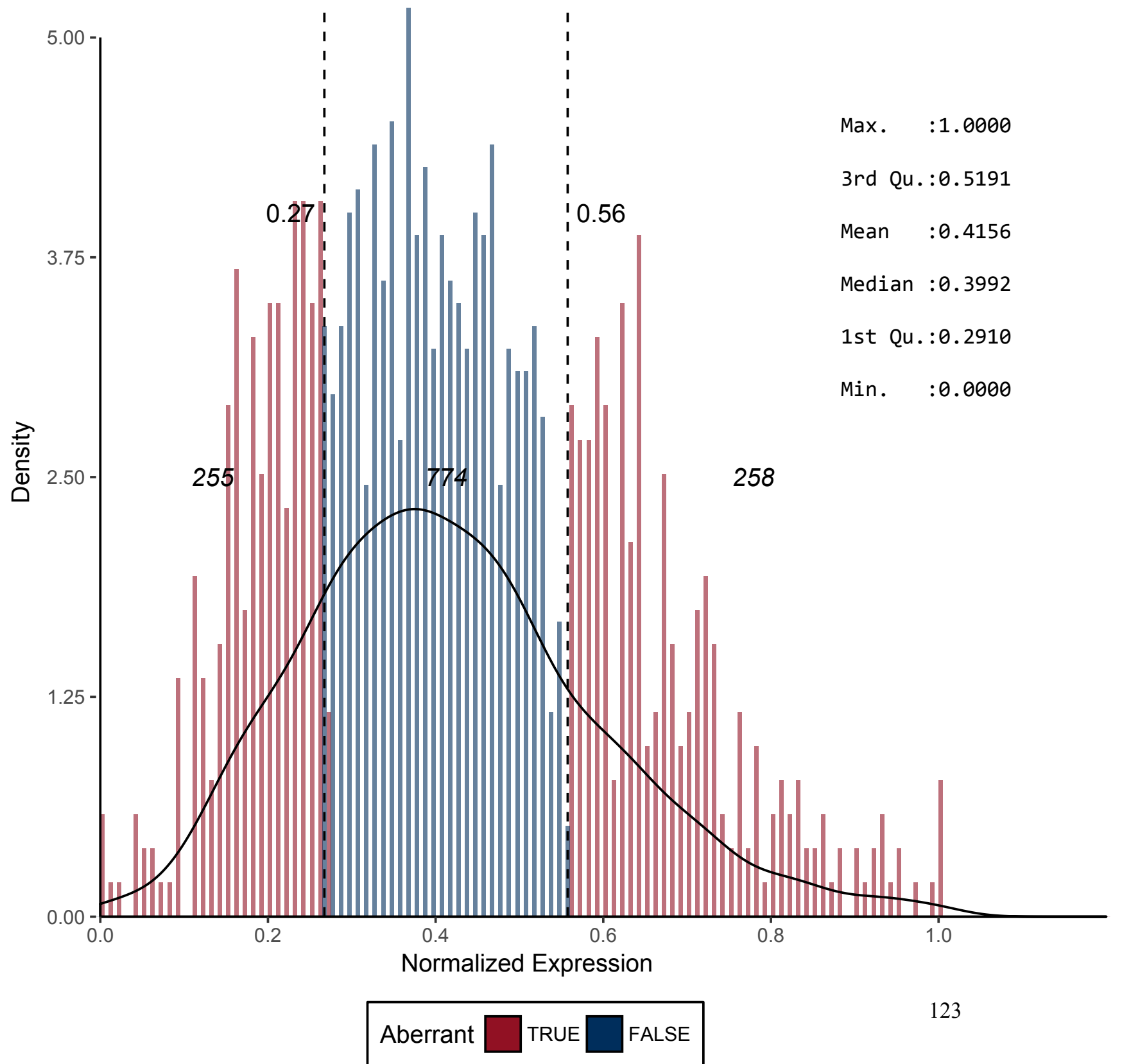


Figure 107 - CD90

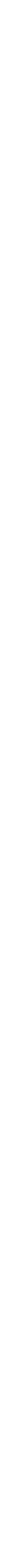


Figure 108 - CD91*

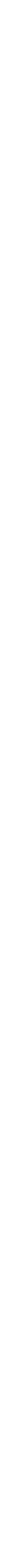


Figure 109 - CD92

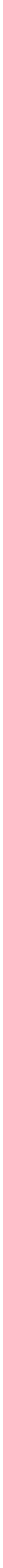


Figure 111 - CD94

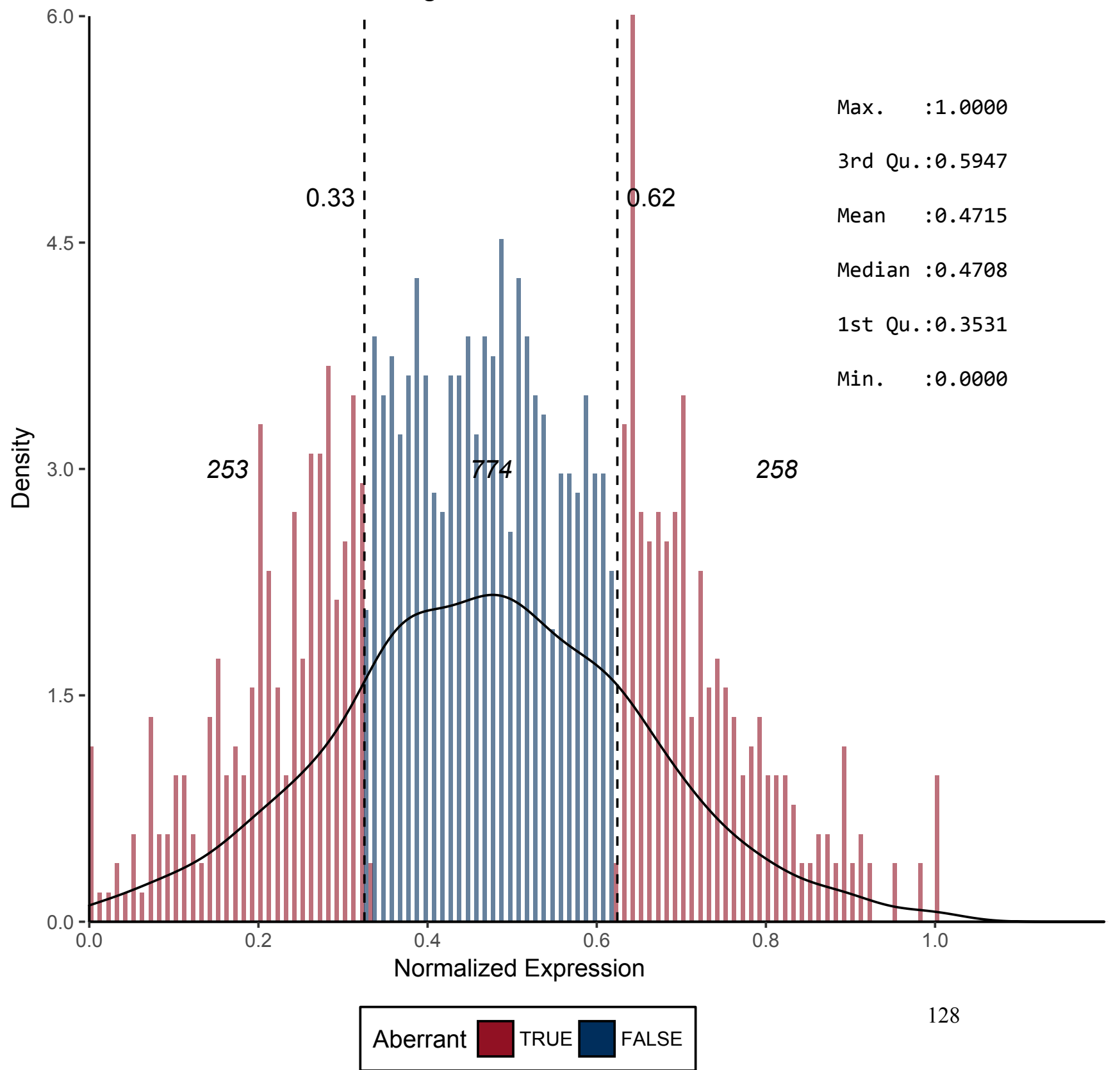


Figure 112 - CD95

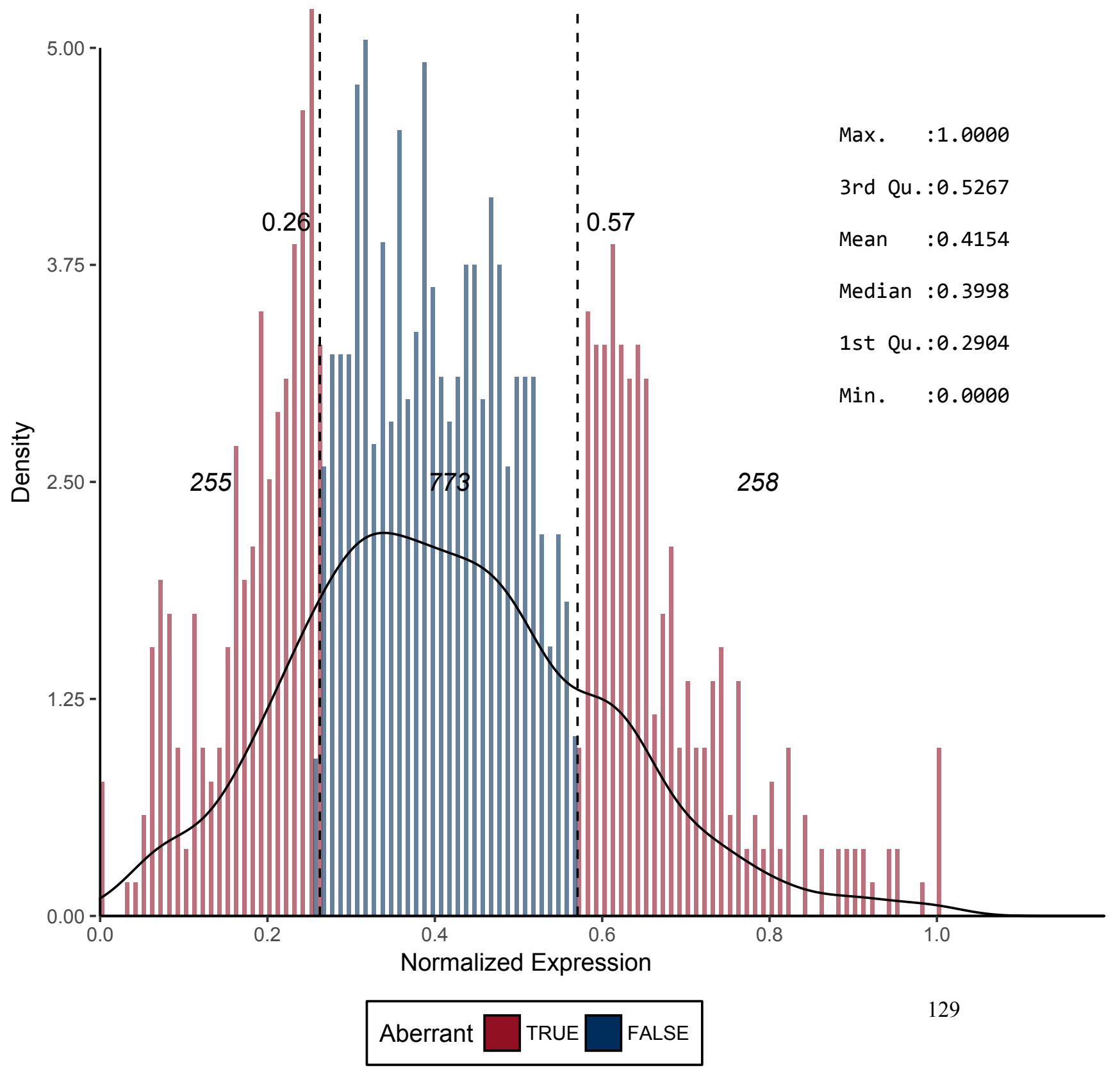


Figure 113 - CD96
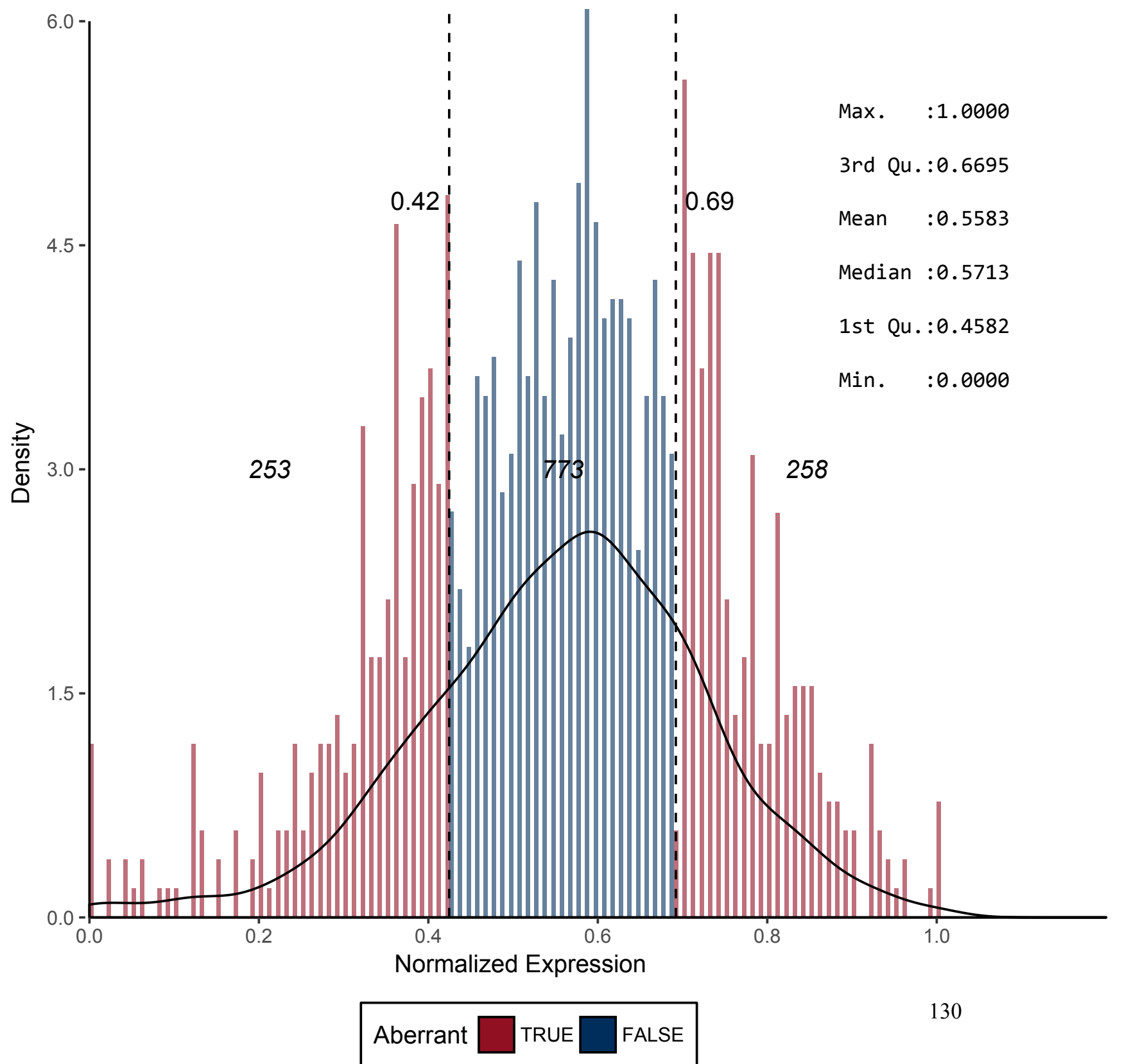
Figure 114 - CD97

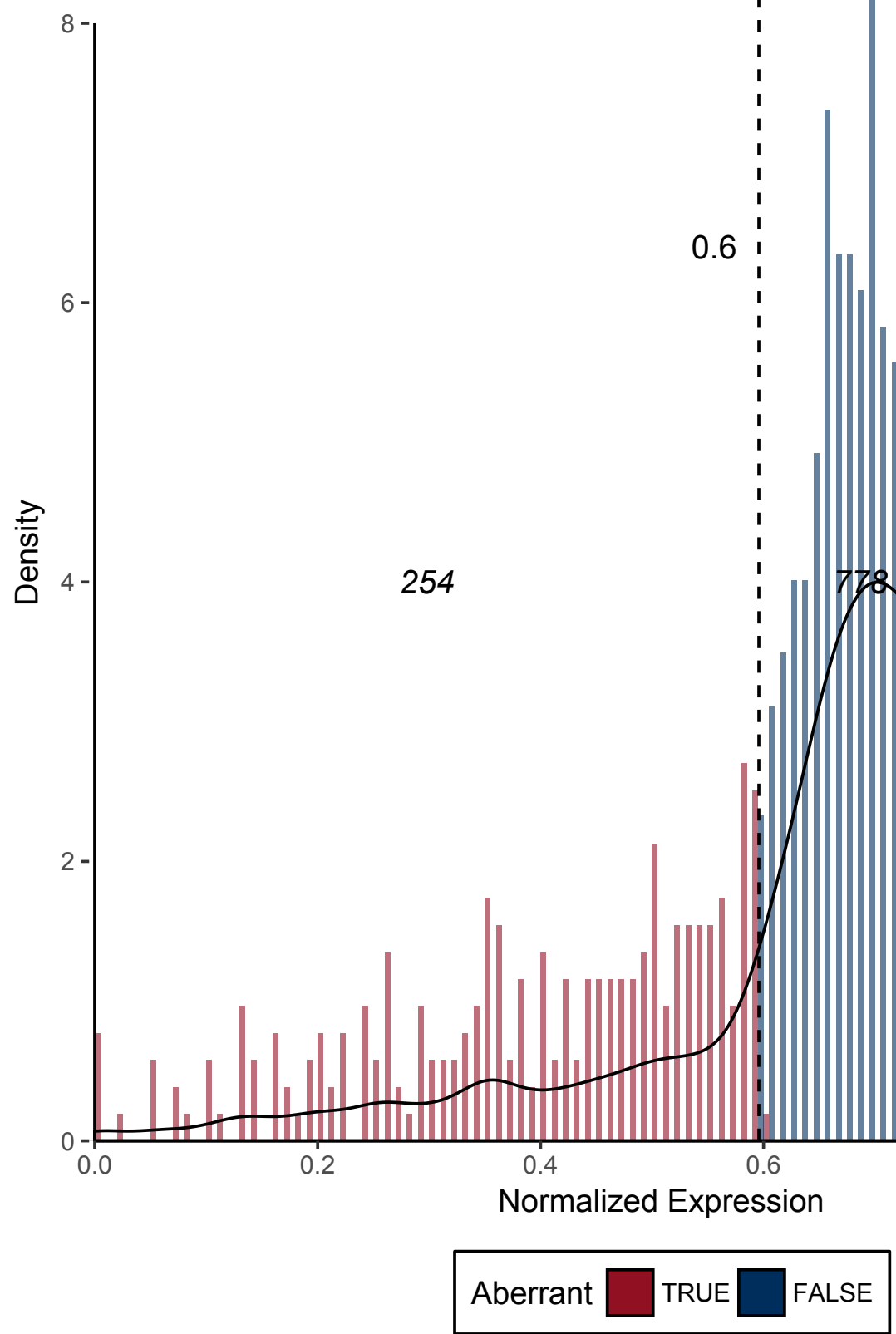

Max. $: 1.0000$

3rd Qu.:0.7619

Mean $\quad: 0.6633$

Median :0.6971

1st Qu.:0.6236

Min. $\quad: 0.0000$

254 
Figure 116 - CD99

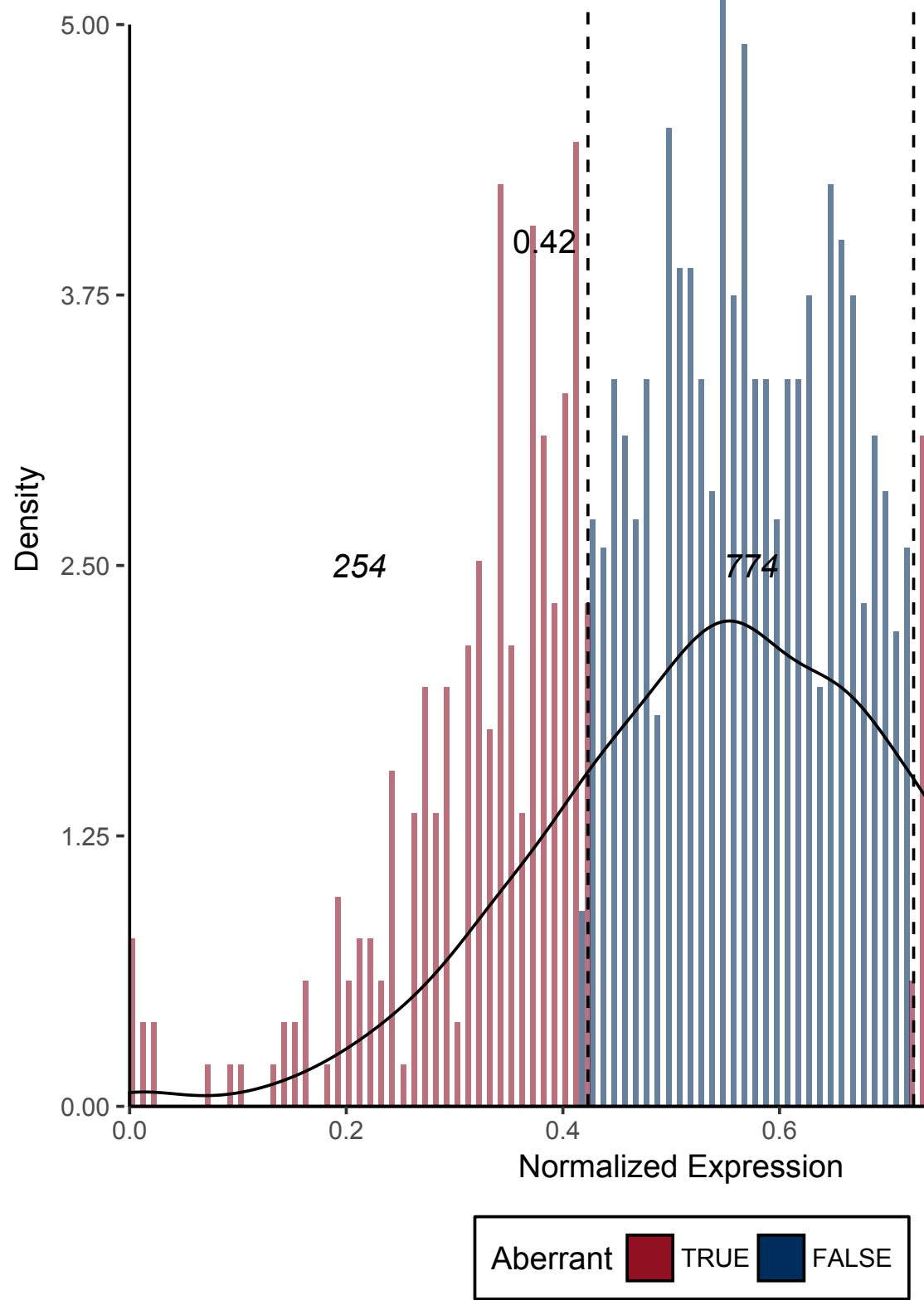

Max. $: 1.0000$

3rd Qu.:0.6912

Mean $\quad: 0.5712$

Median $: 0.5680$

1st Qu.:0.4504

Min. $\quad: 0.0000$ 
Figure 117 - CD100

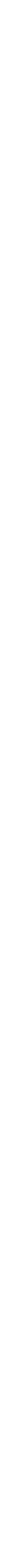


Figure 118 - CD101

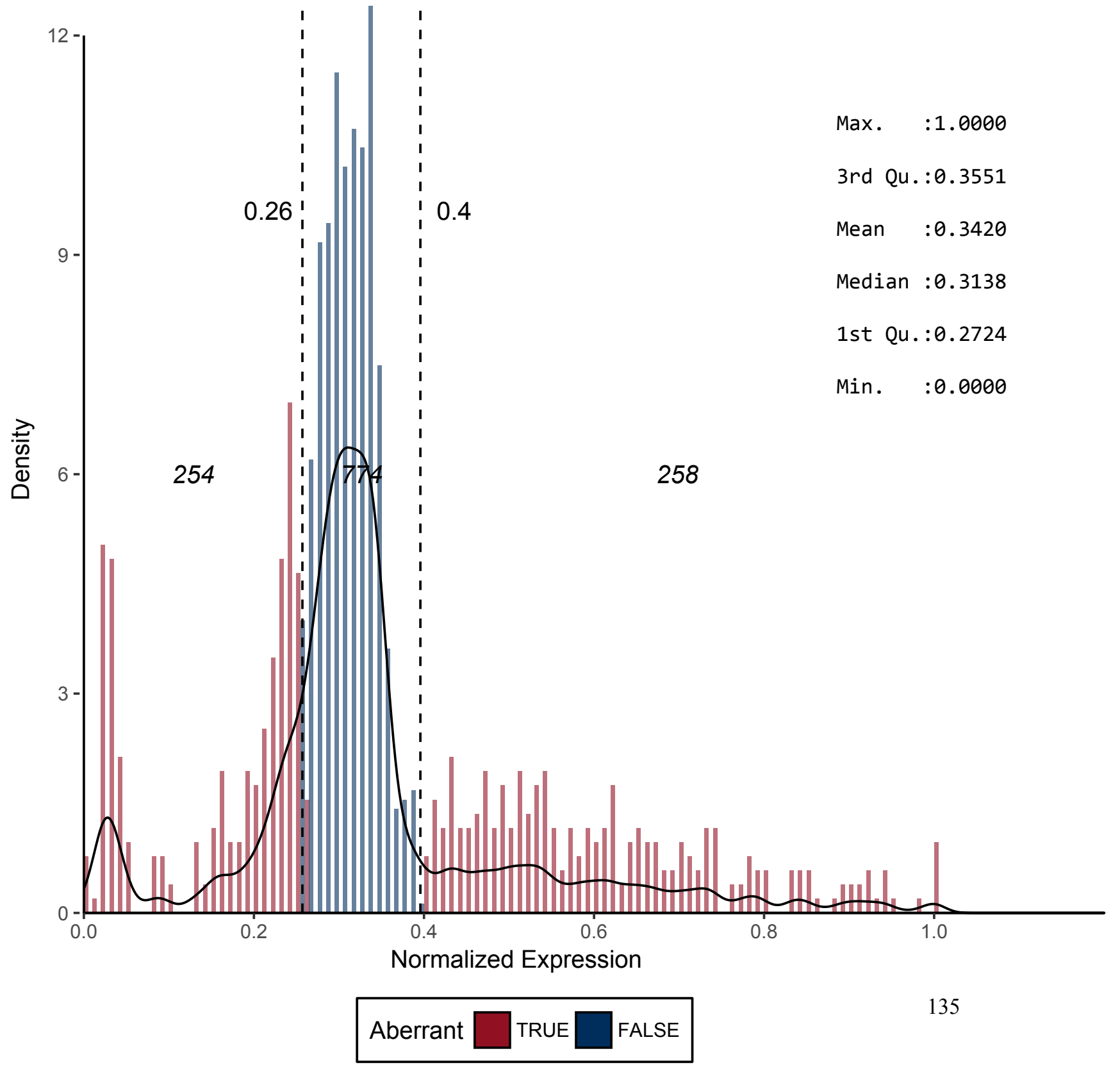


Figure 120 - CD103

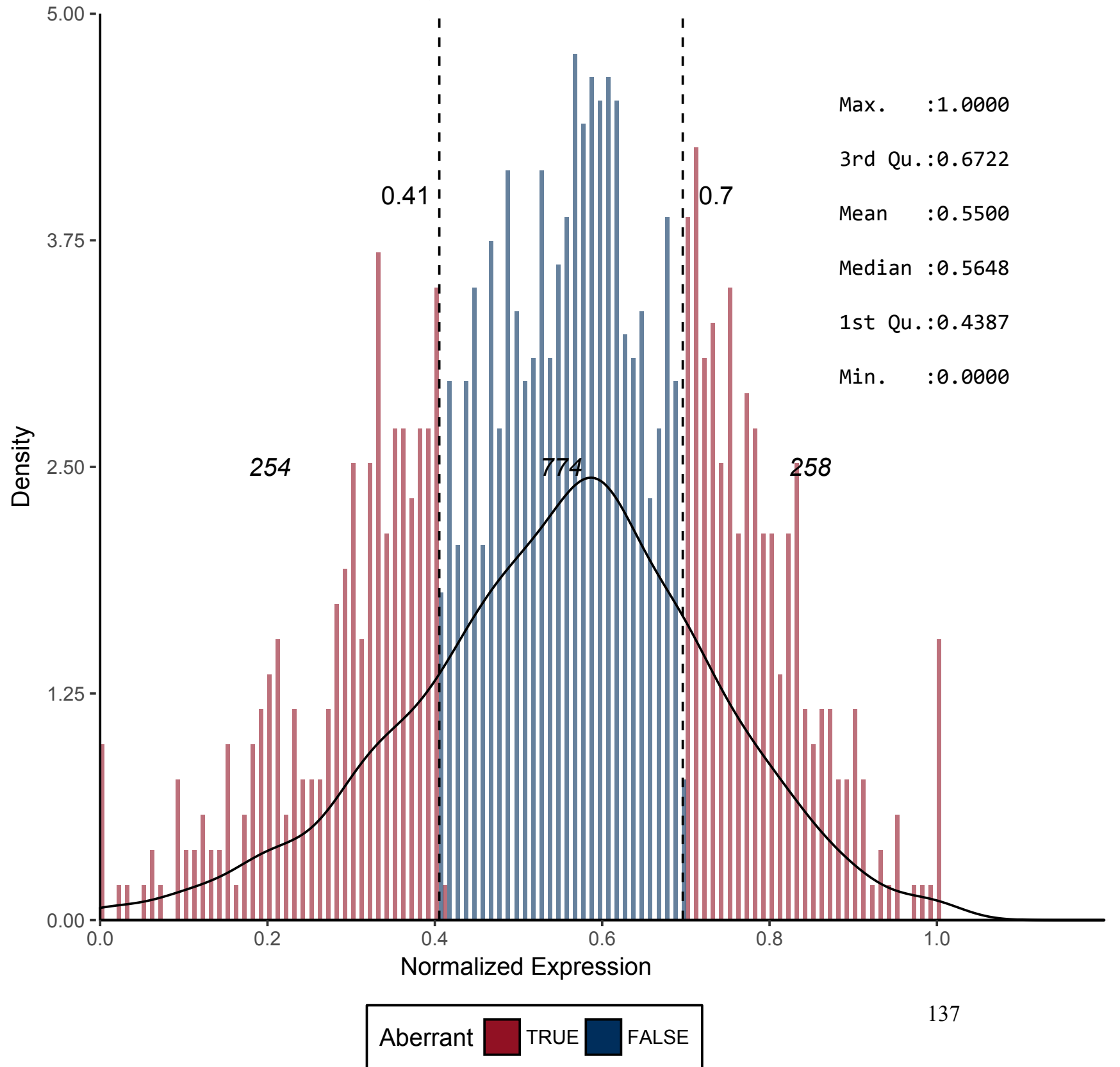


Figure 121 - CD104*

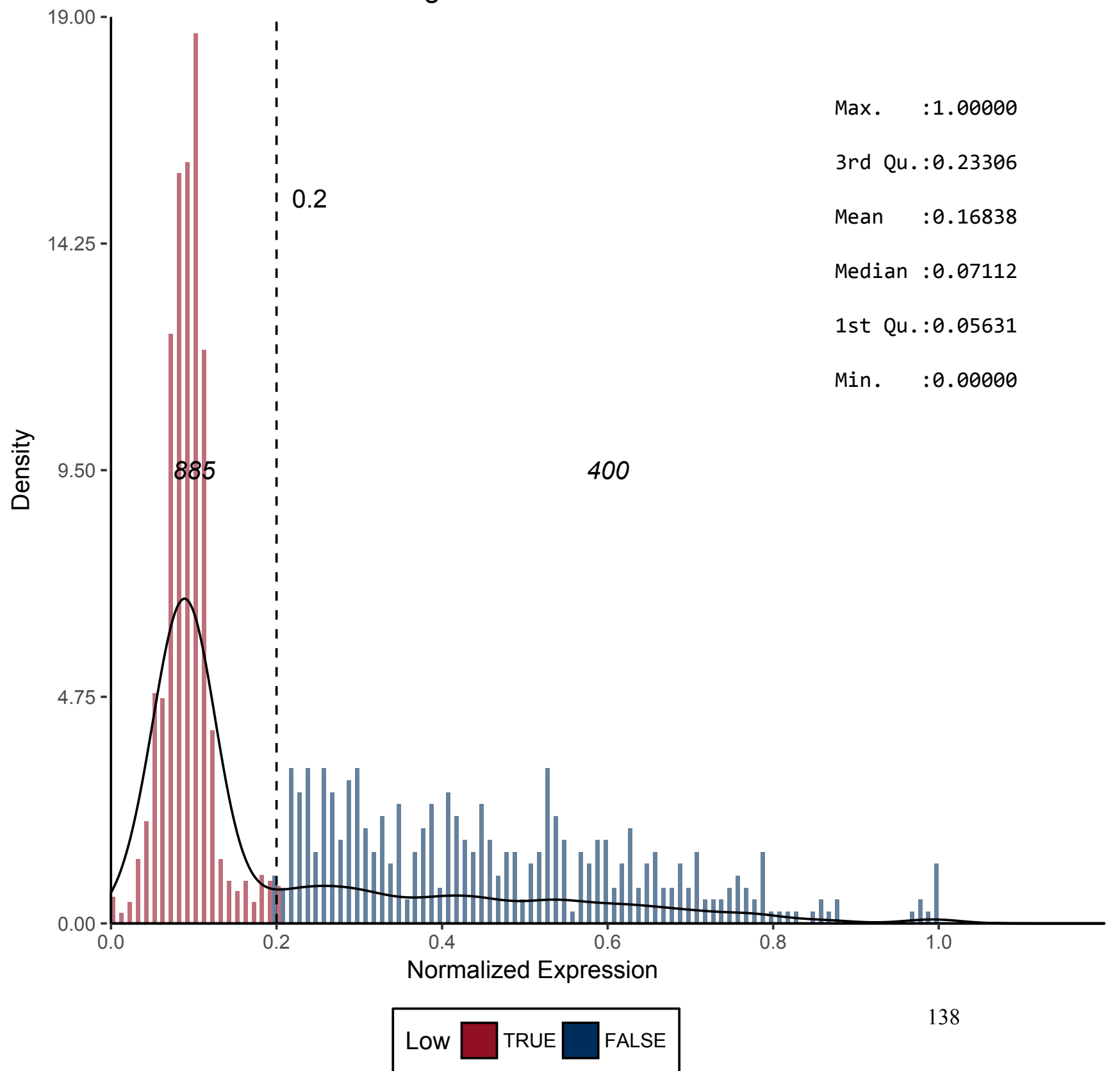


Figure 122 - CD105*

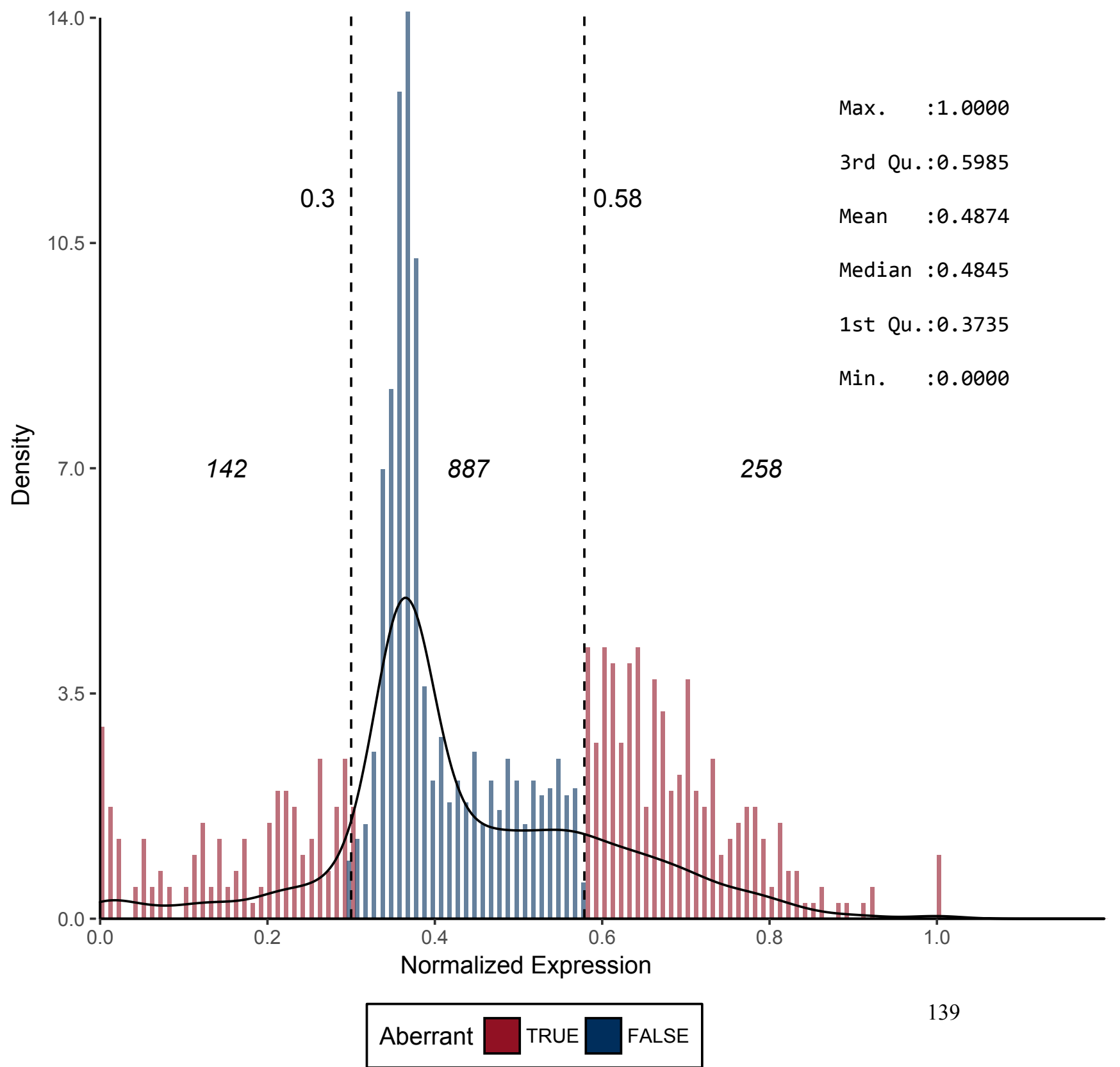


Figure 123 - CD106*

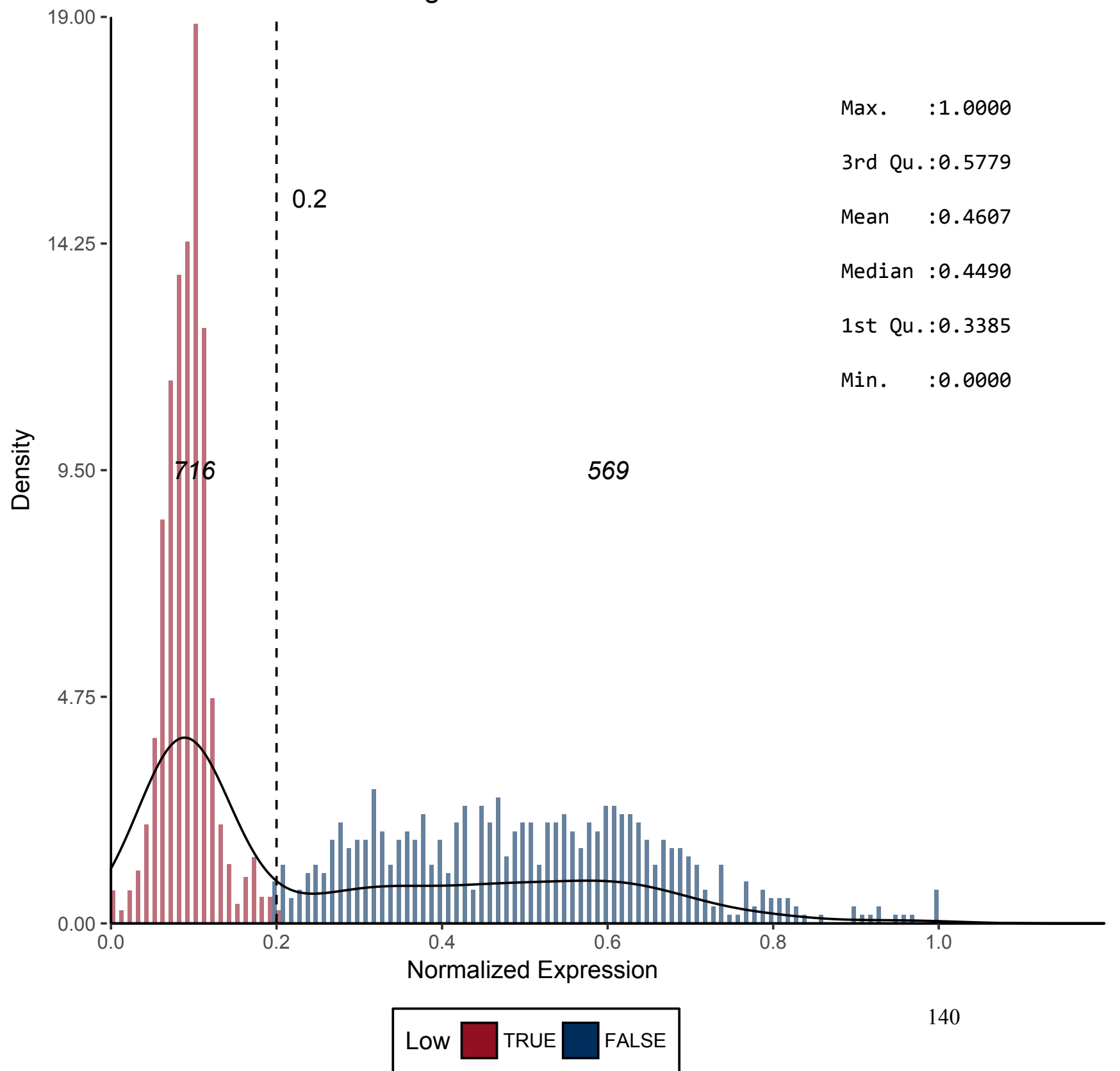


Figure 126 - CD108*

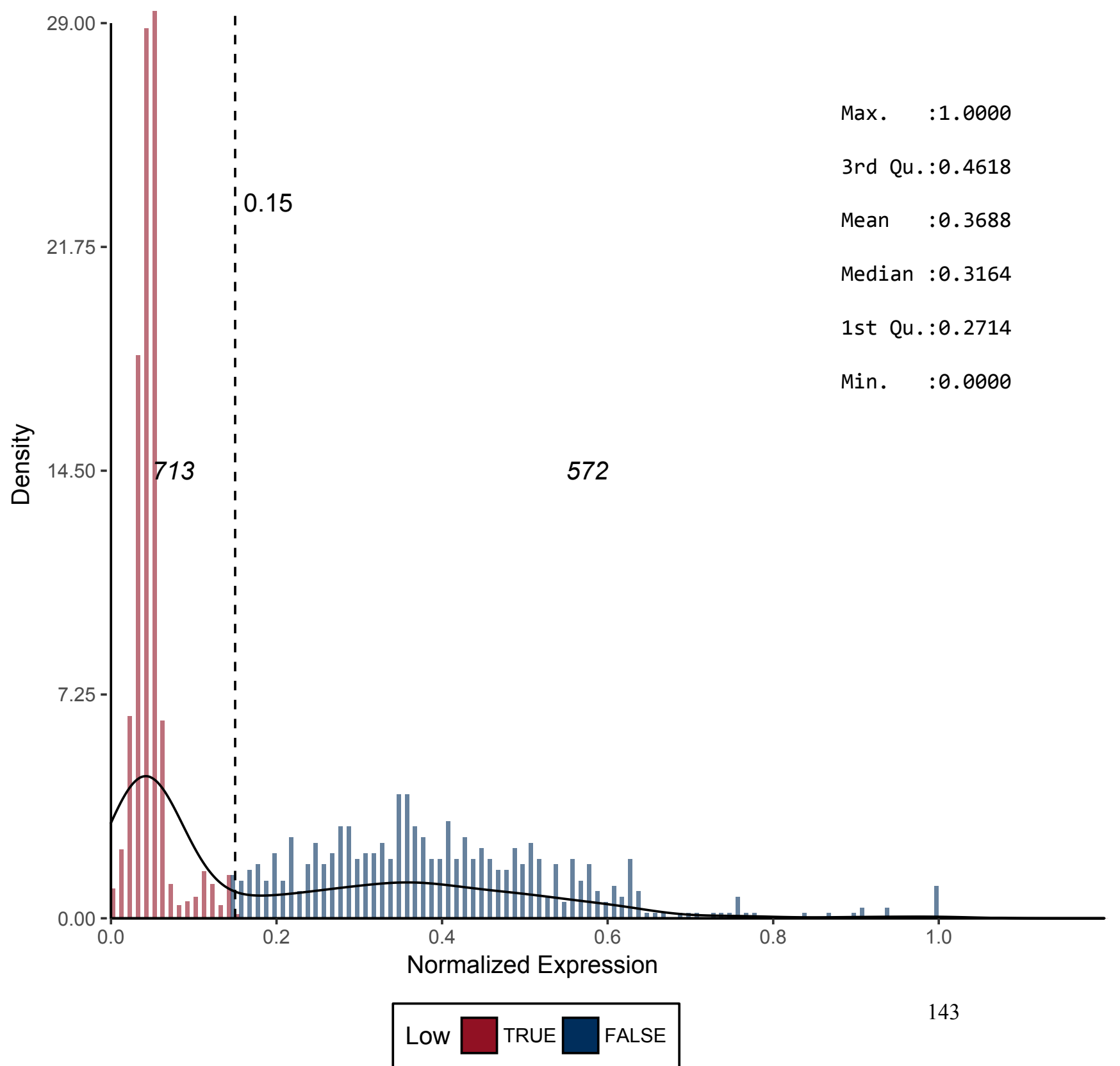


Figure 127 - CD109*

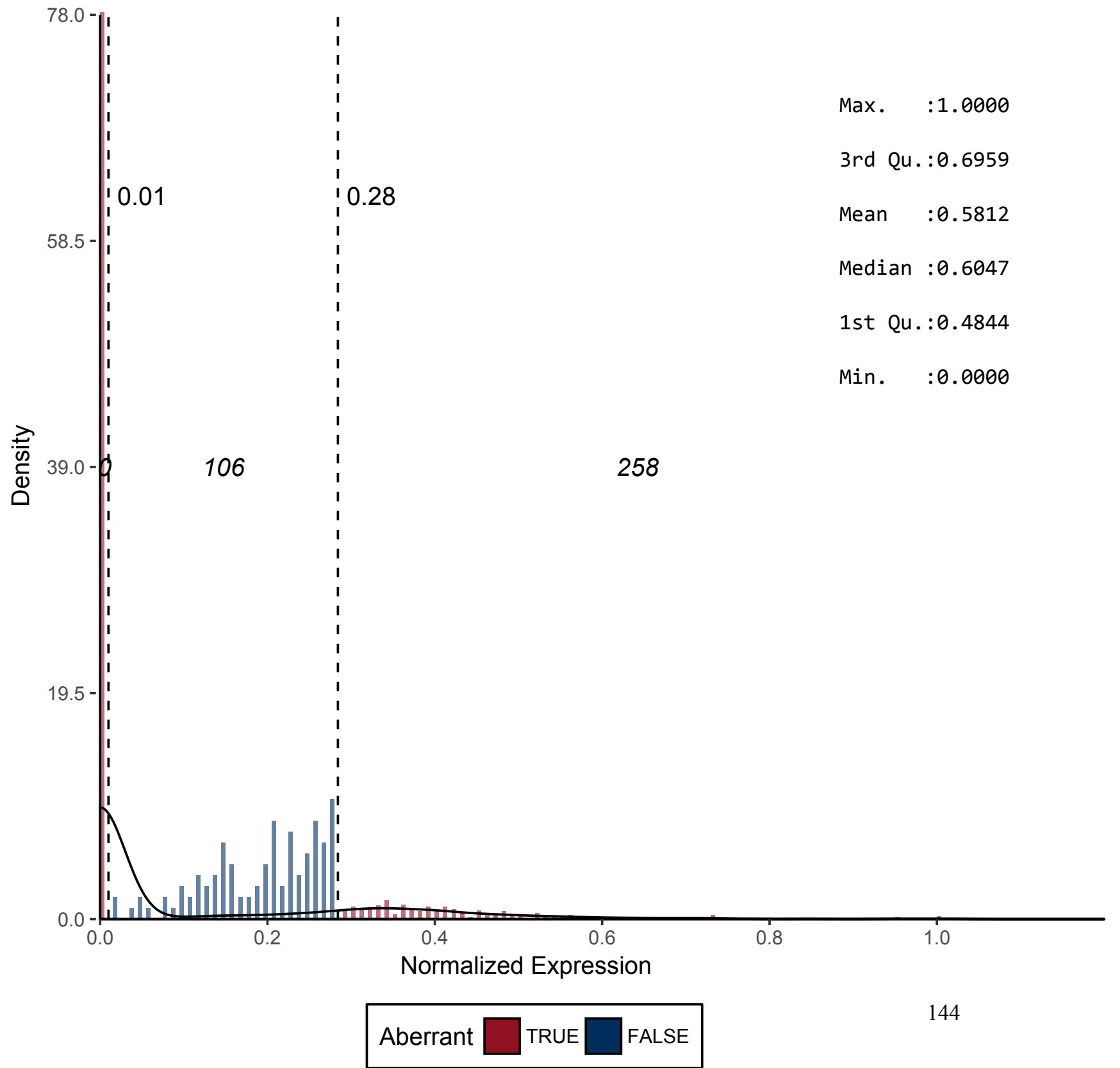


Figure 128 - CD110
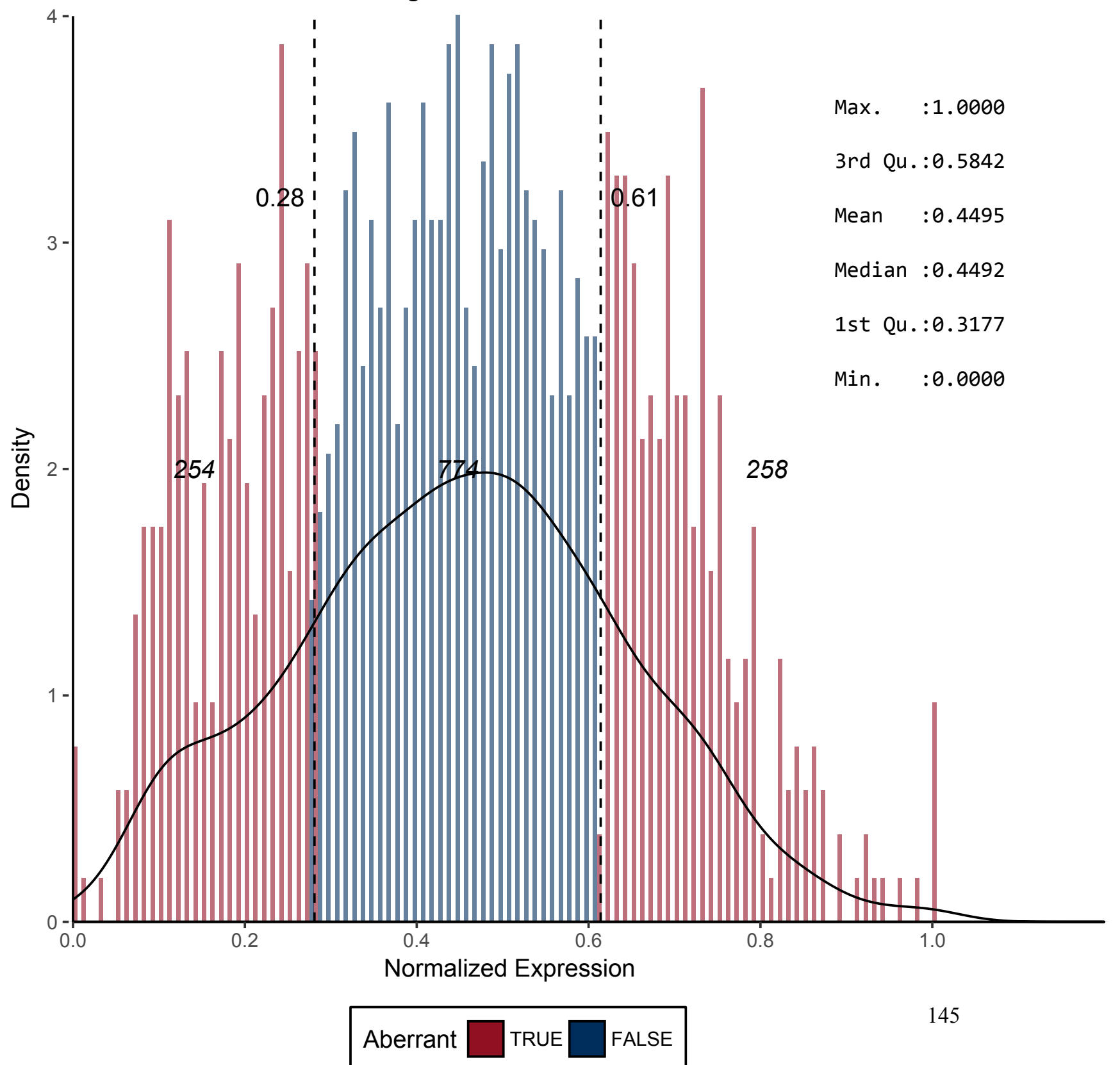
Figure 129 - CD111*

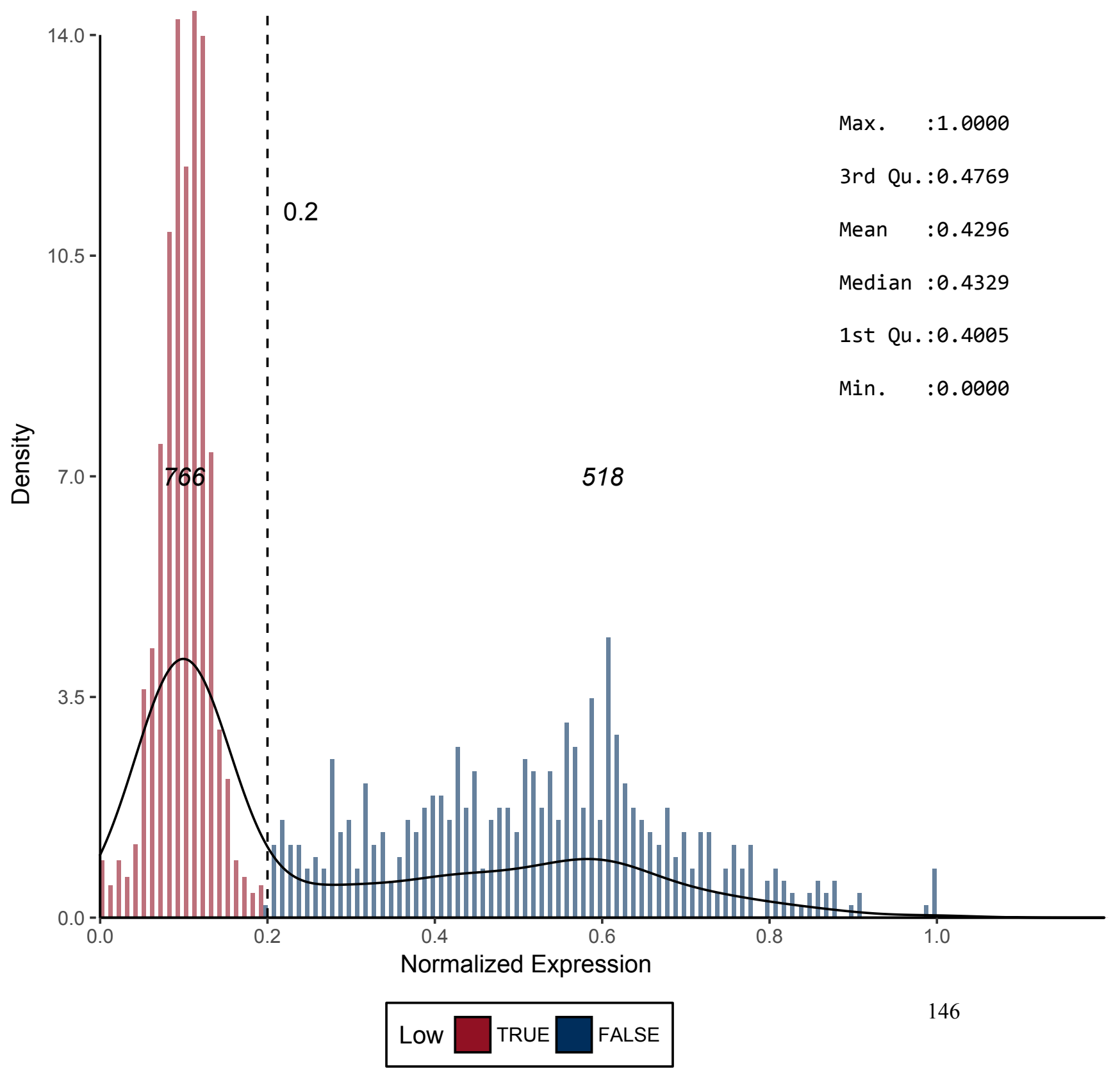


Figure 130 - CD112

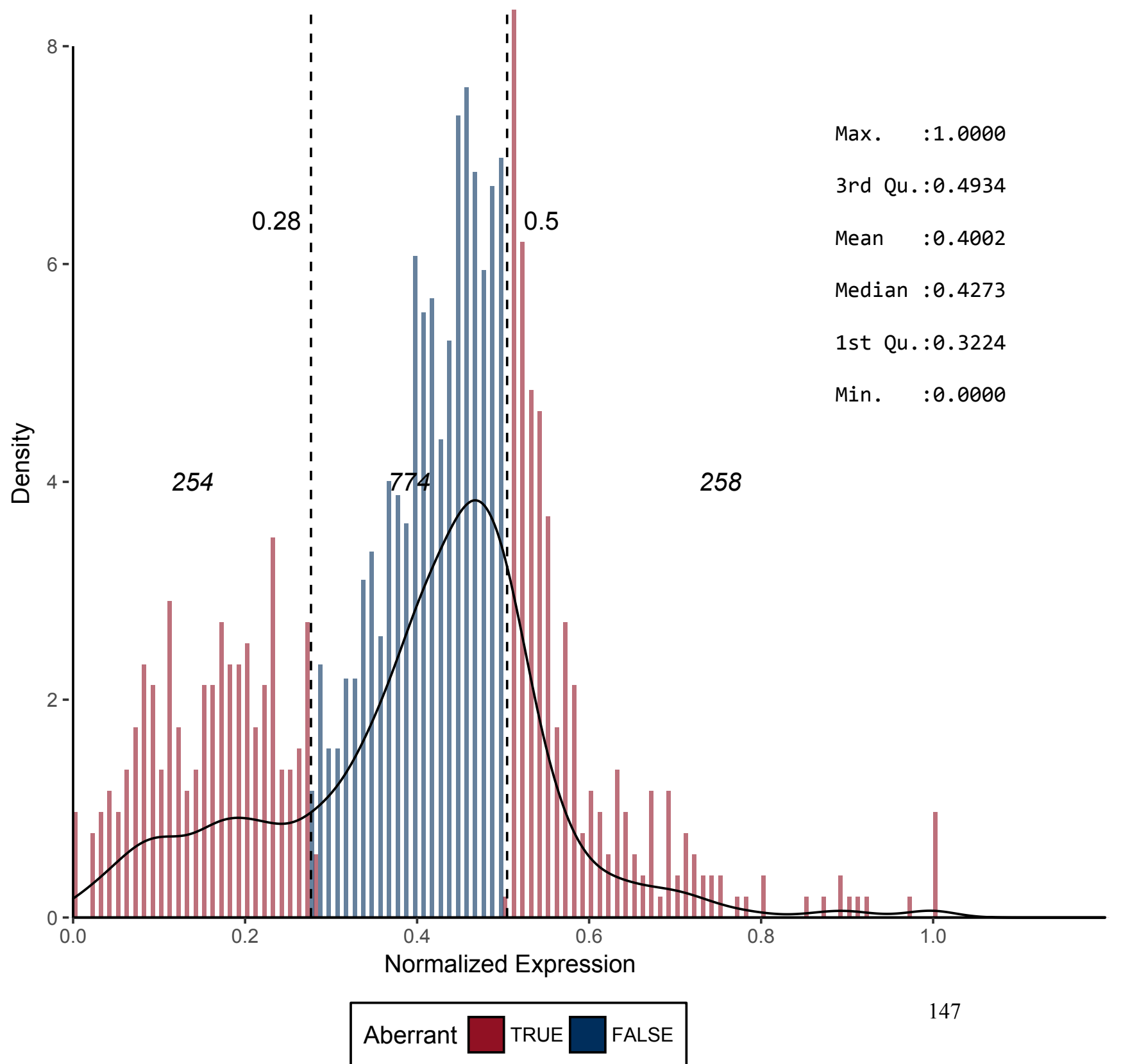


Figure 131 - CD113*

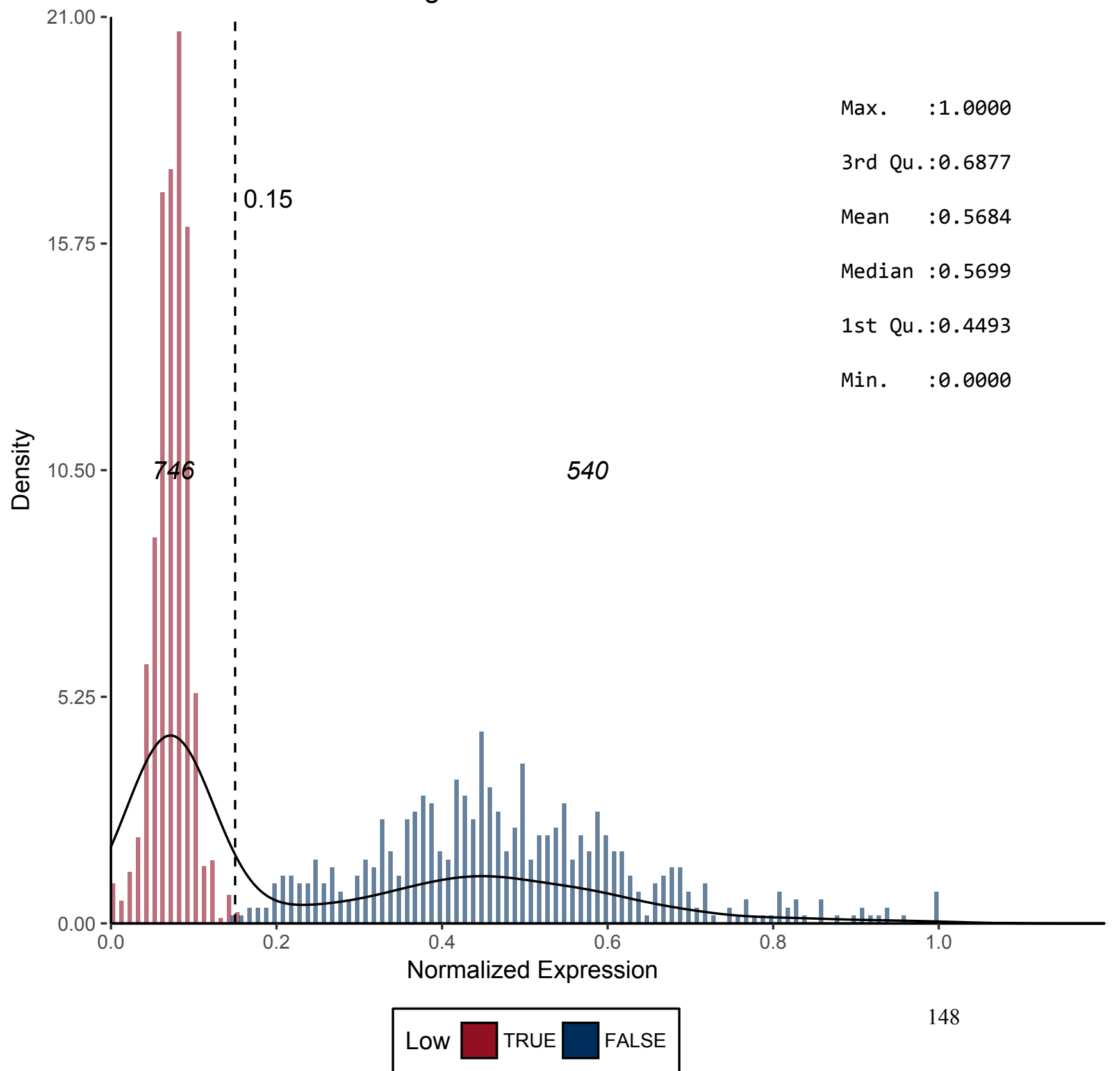


Figure 132 - CD114

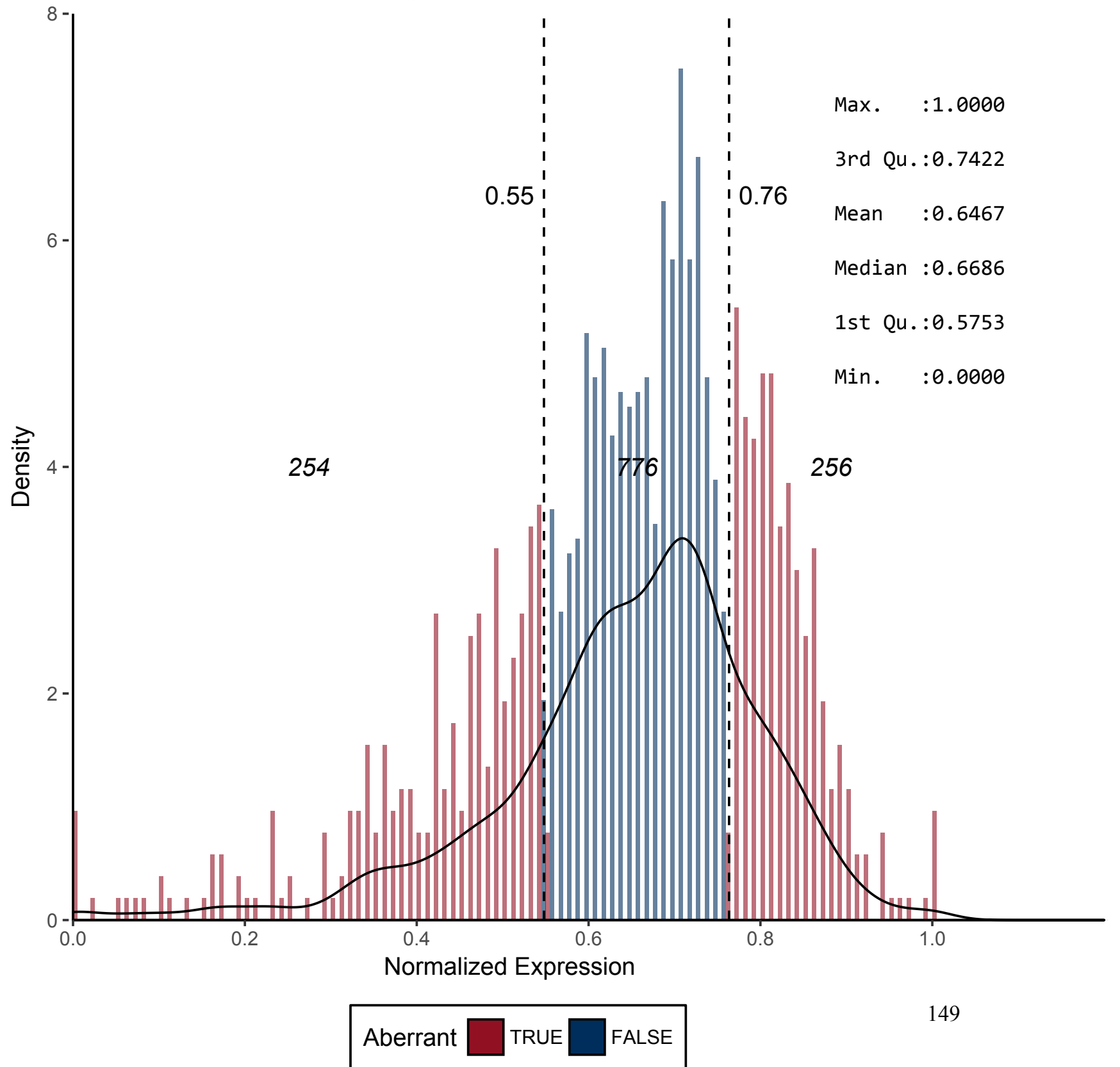


Figure 135 - CD117

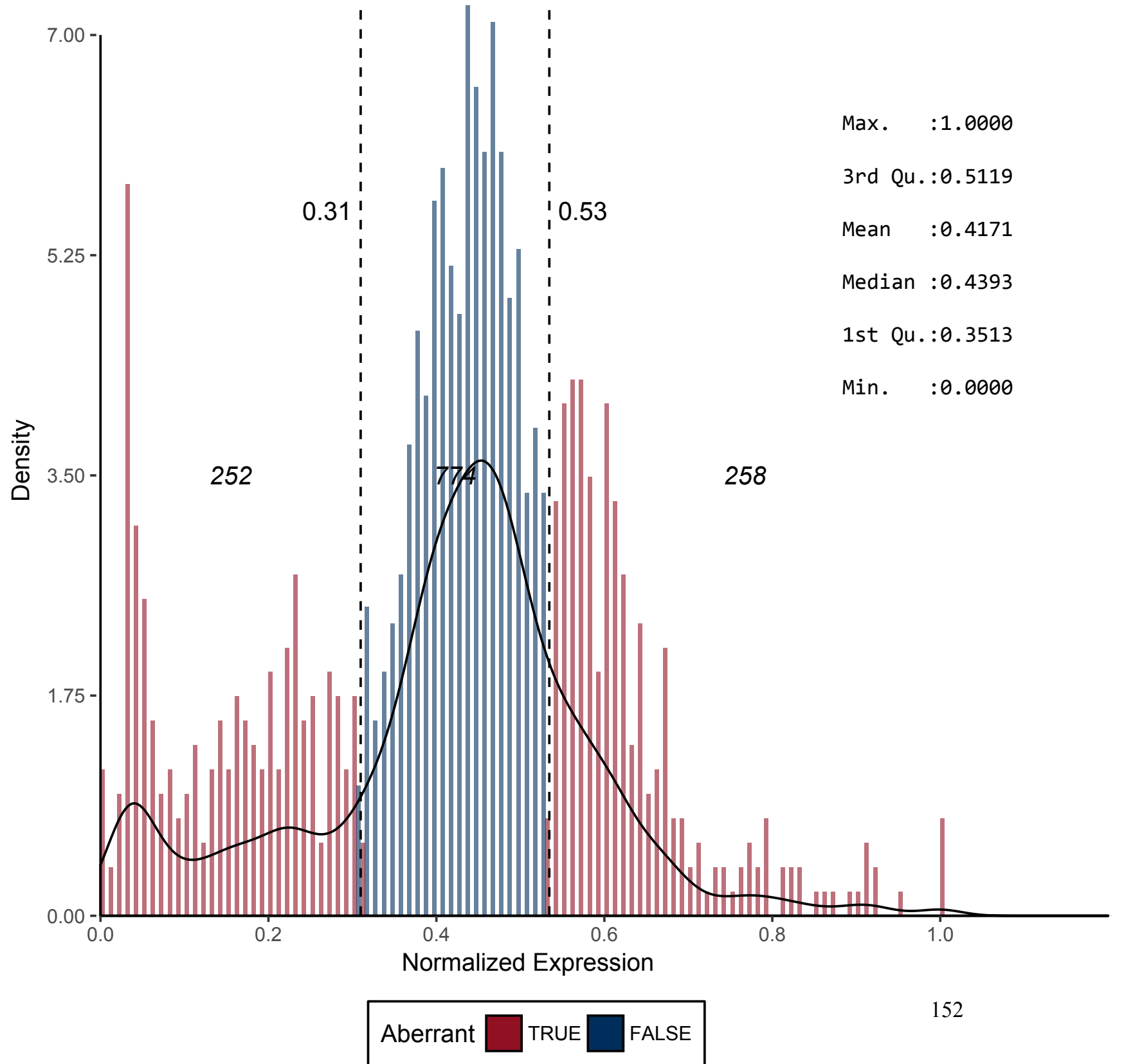


Figure 136 - CD118*

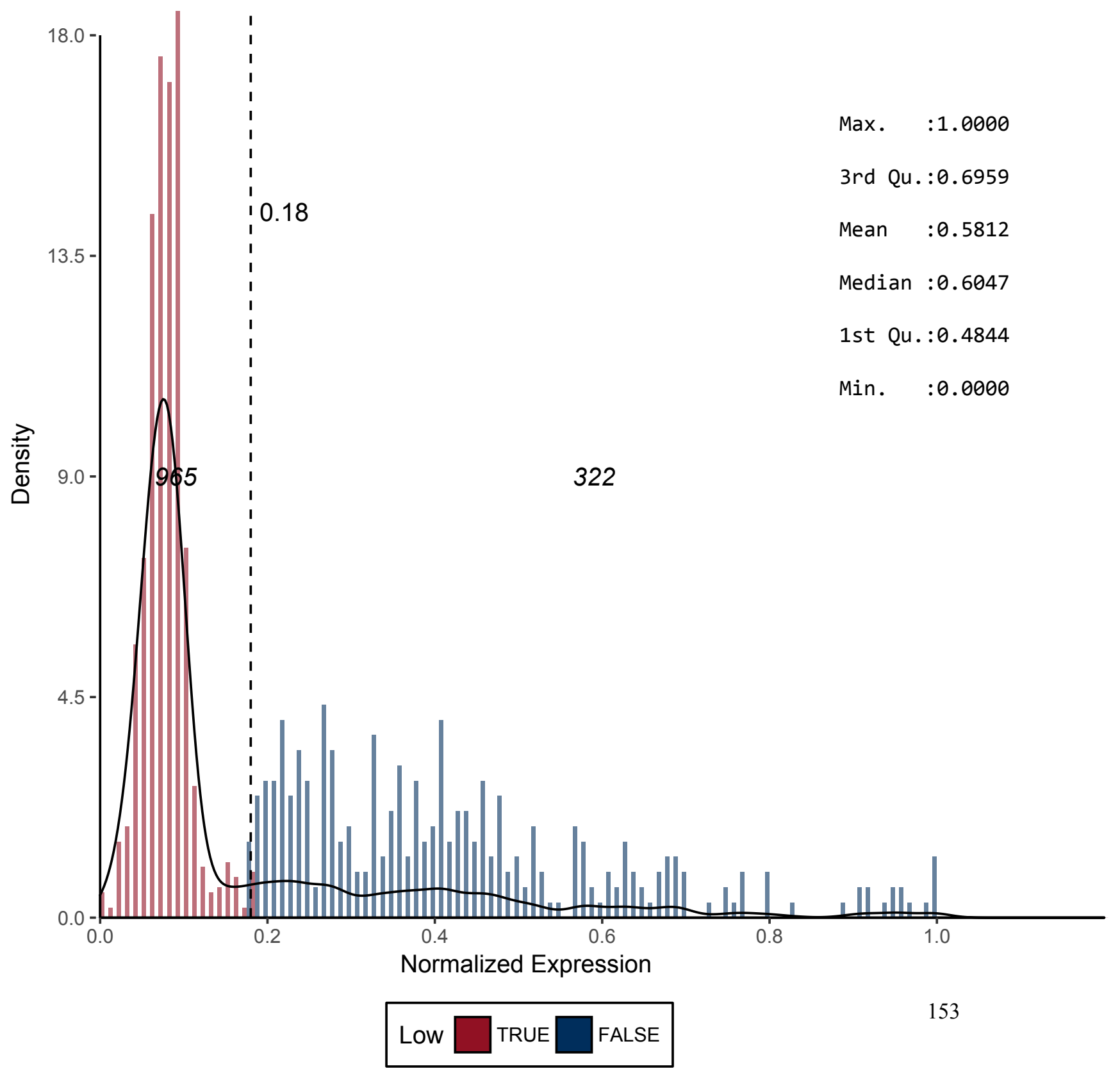


Figure 137 - CD119

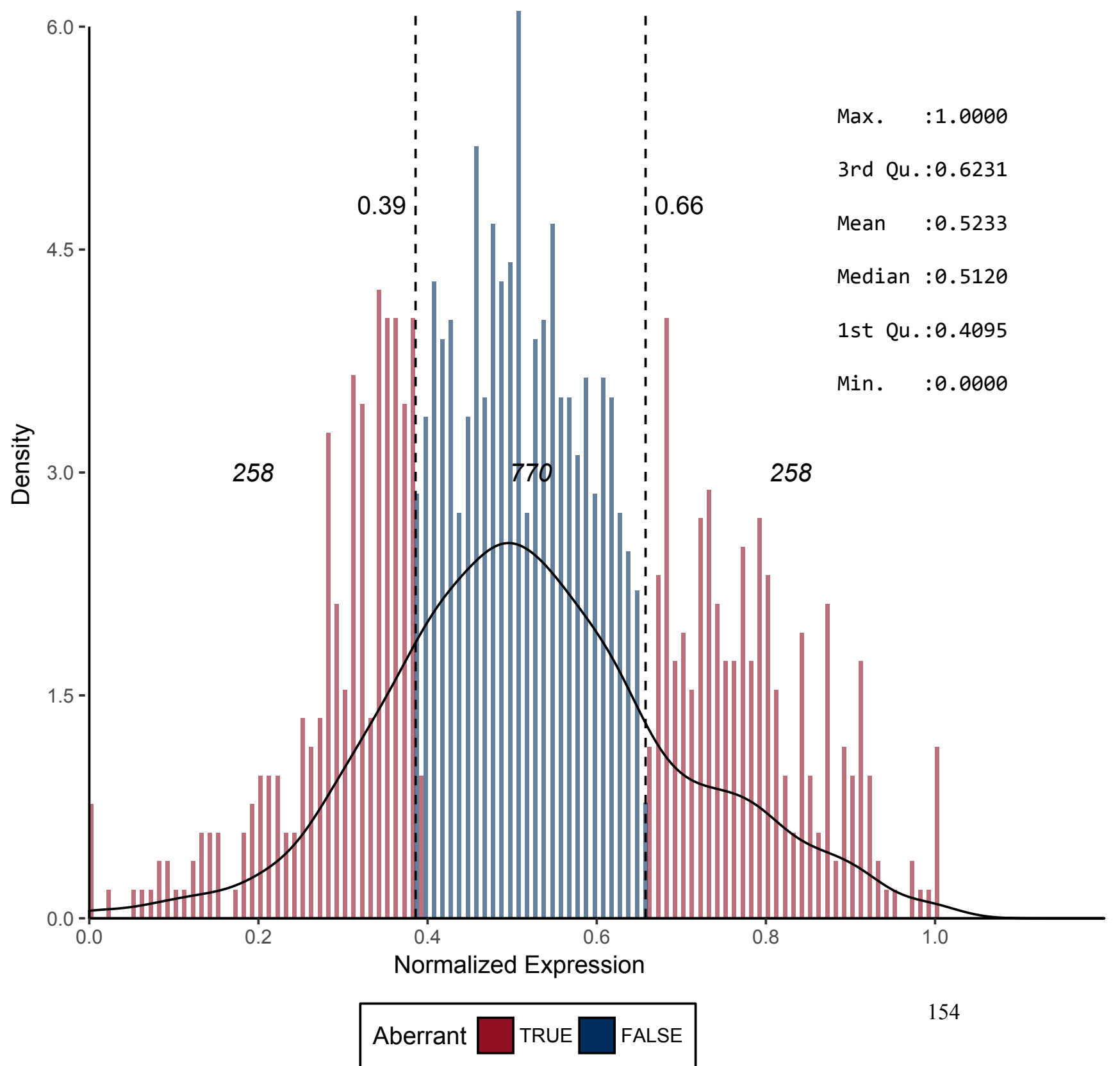


Figure 139 - CD120B

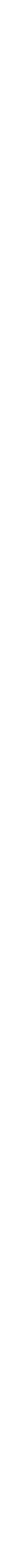


Figure 140 - CD121A*

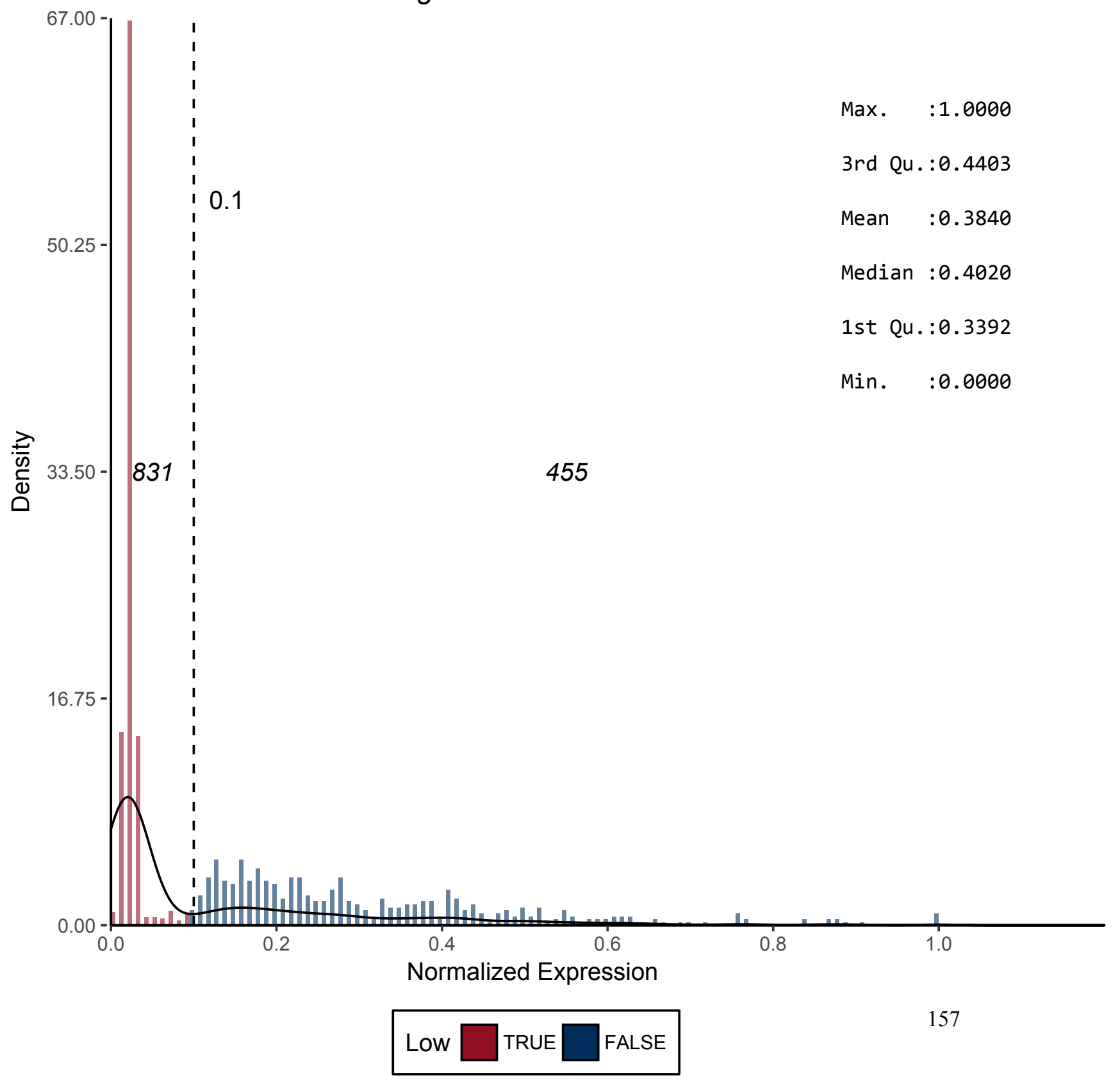


Figure 141 - CD121B

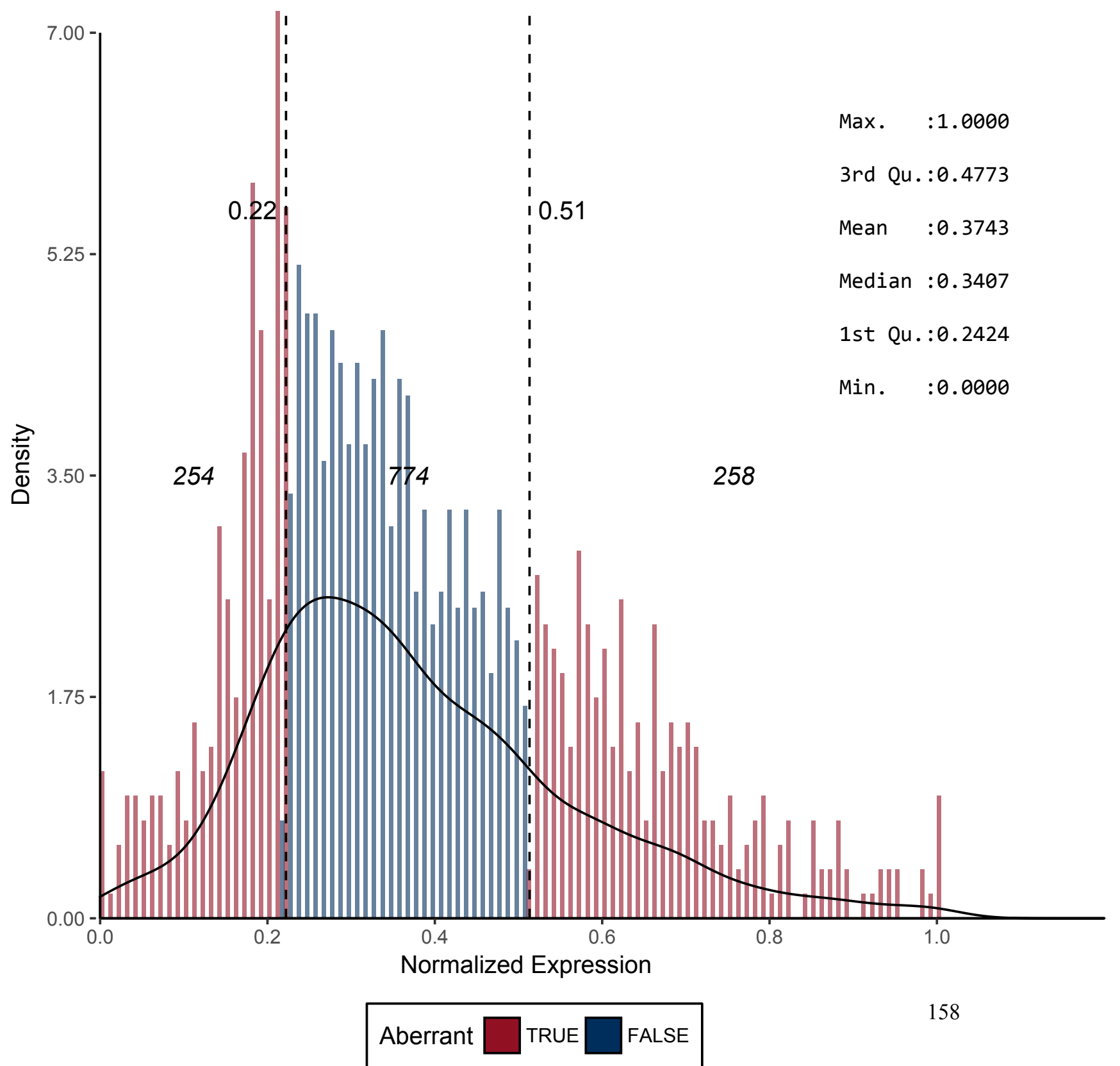


Figure 142 - CD122
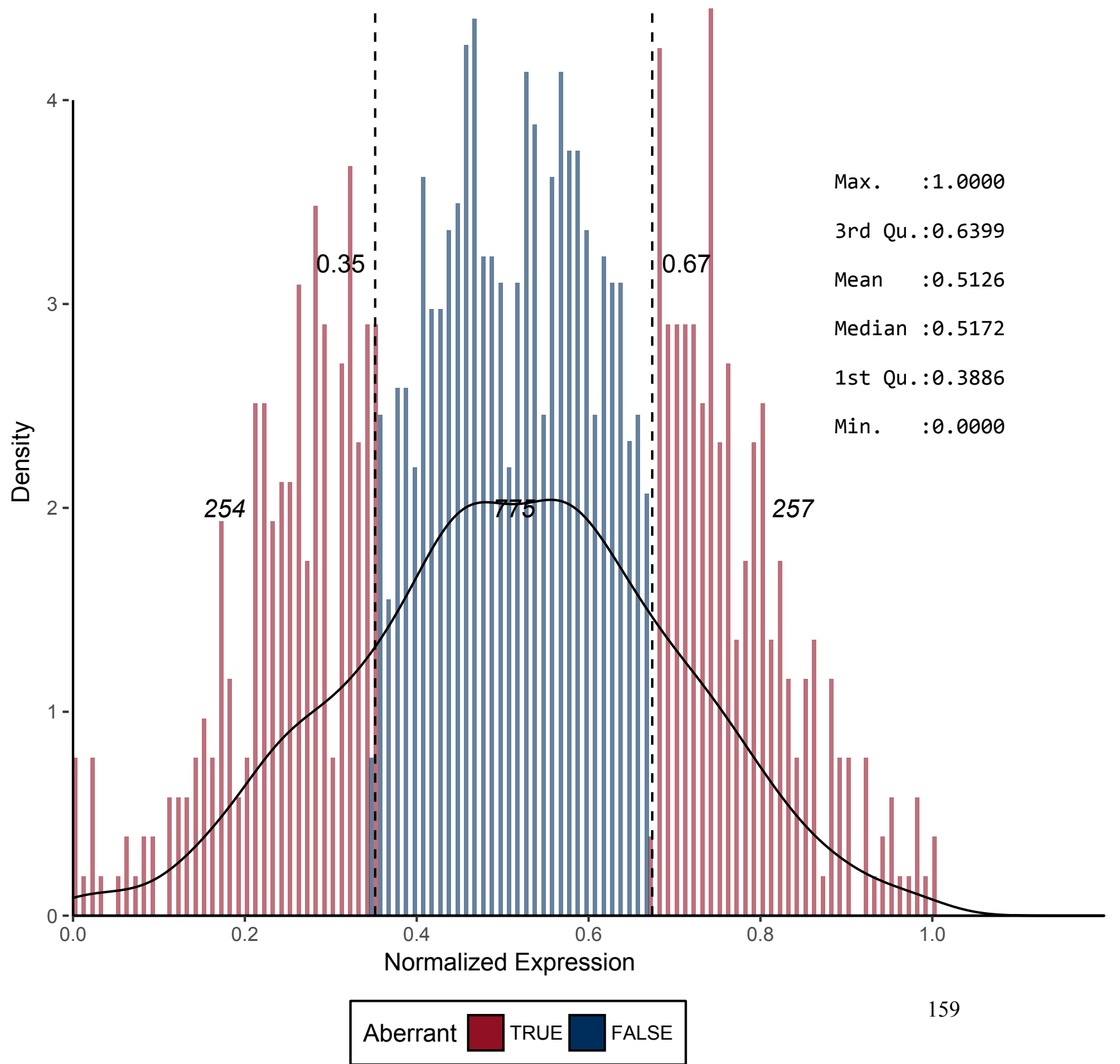
Figure 143 - CD123

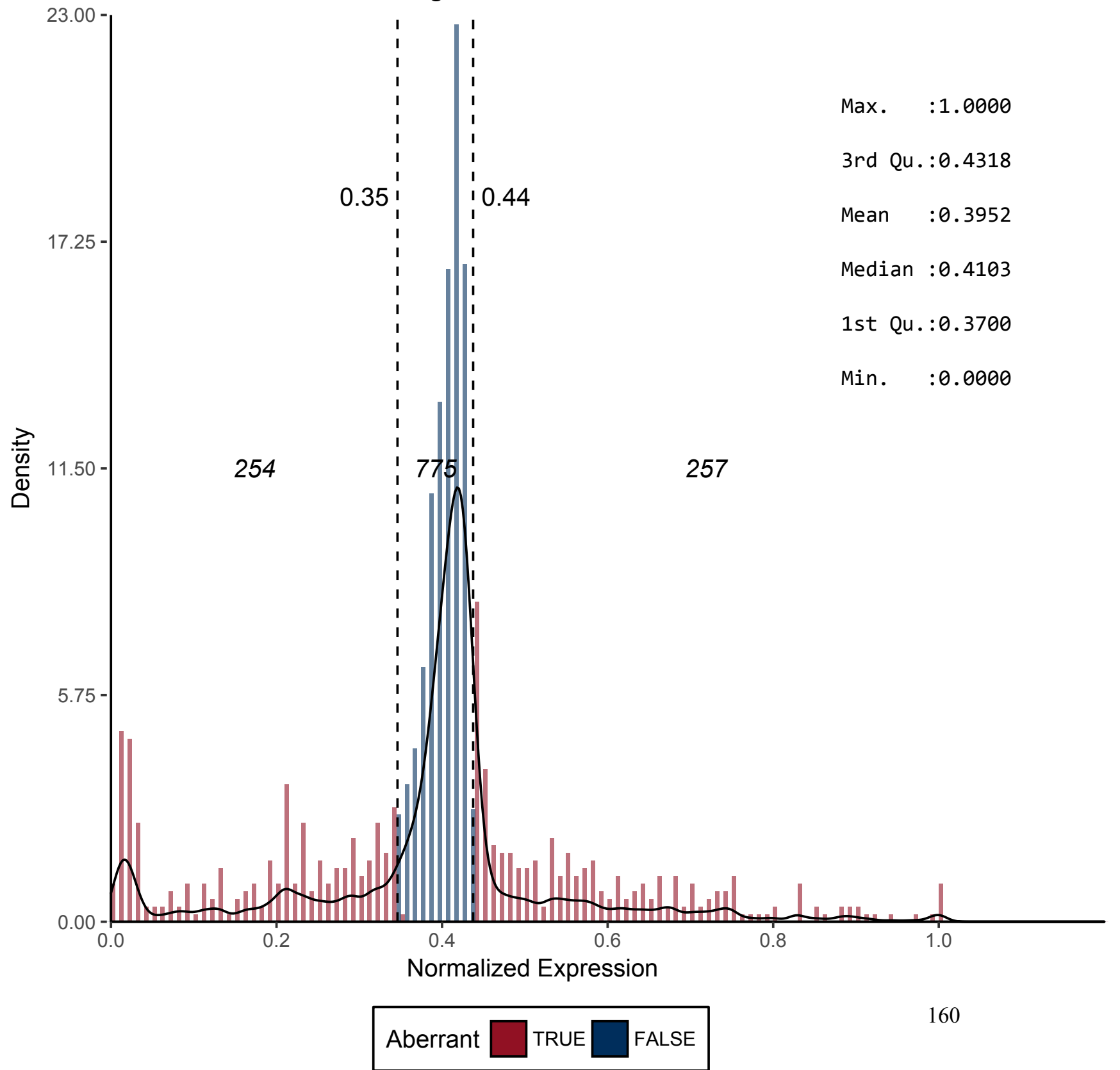


Figure 144 - CD124

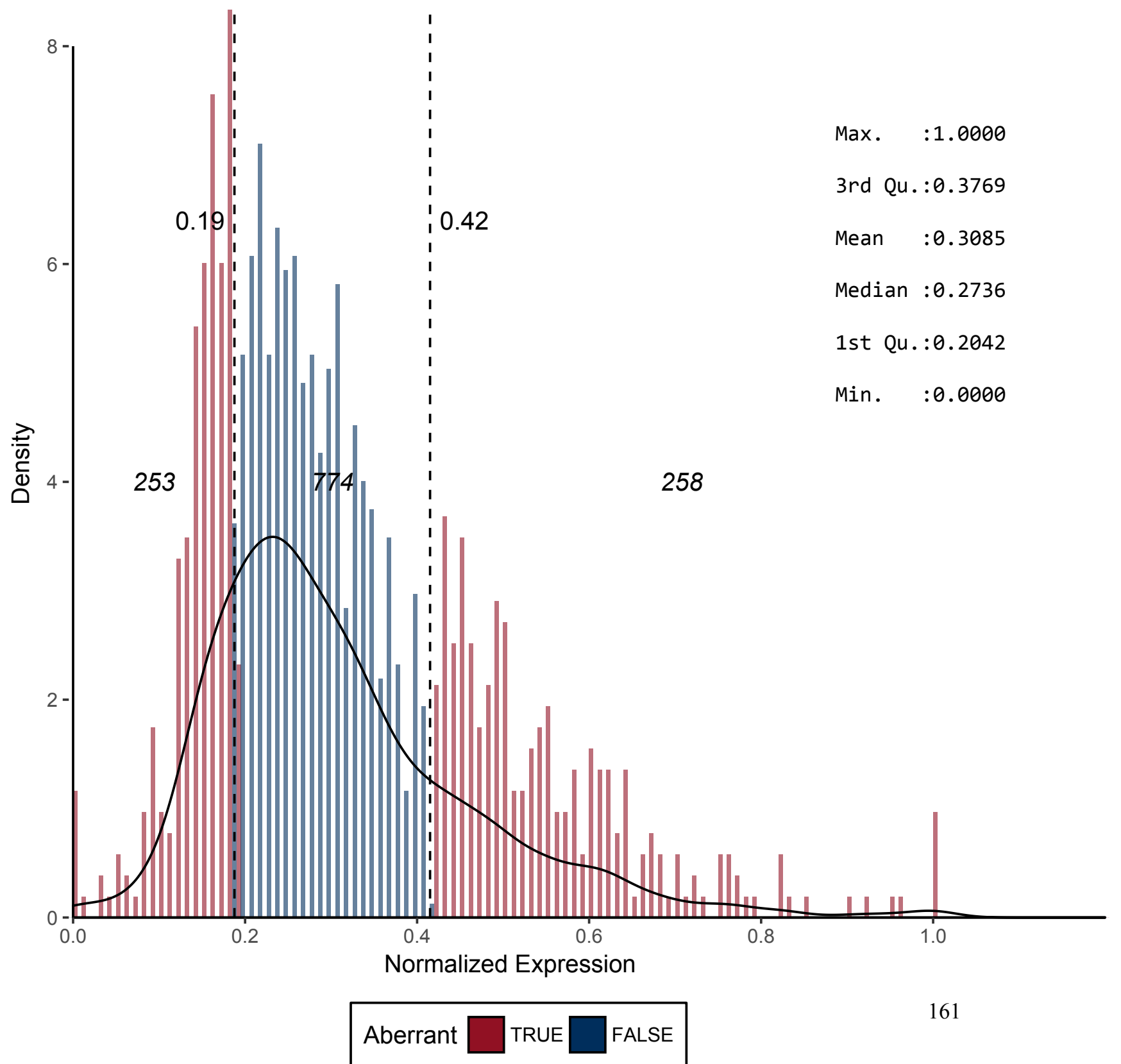


Figure 145 - CD125

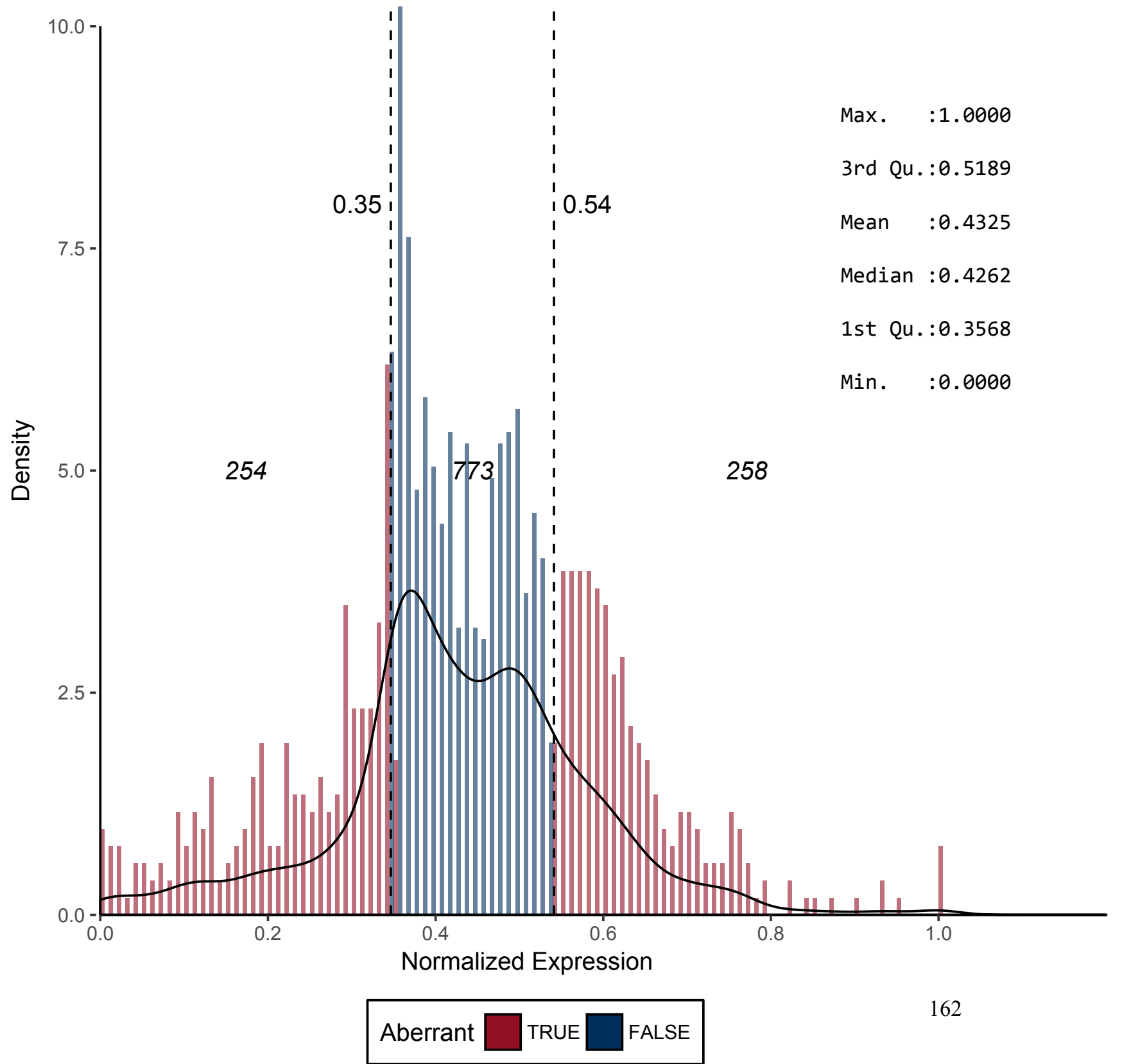


Figure 146 - CD126

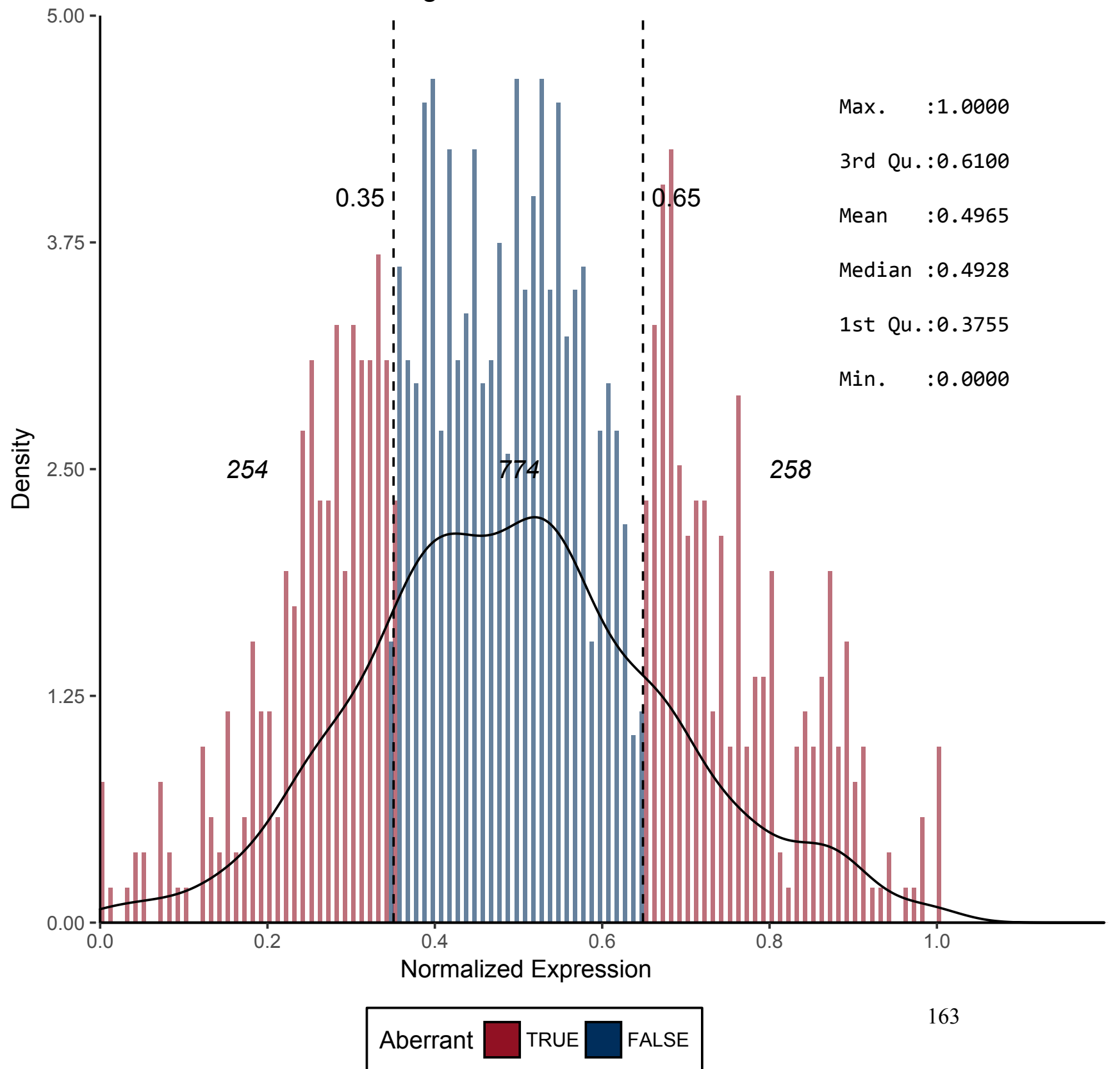


Figure 147 - CD127

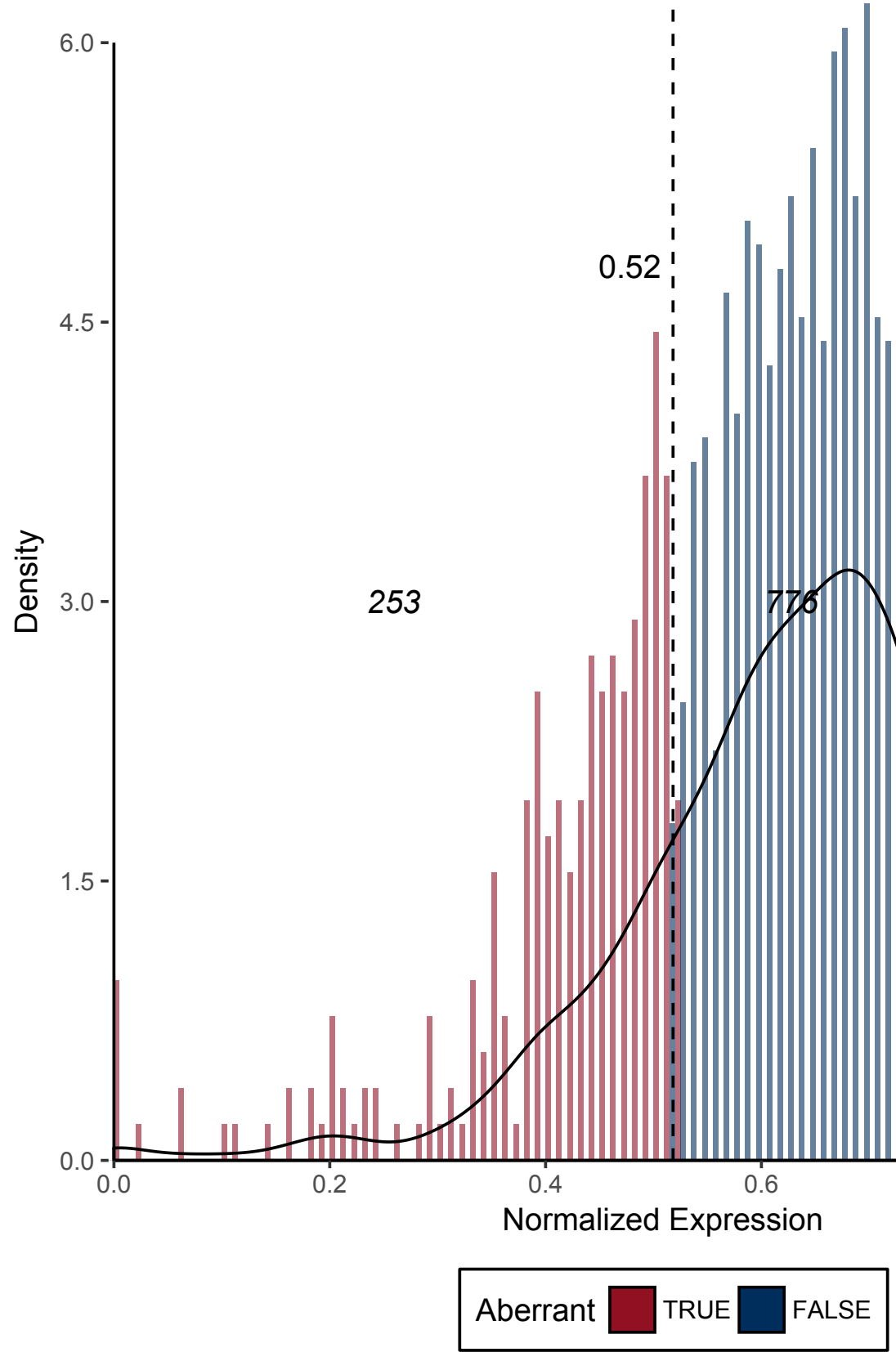

Max. $: 1.0000$

3rd Qu.:0.7205

Mean $: 0.6247$

Median :0.6411

1st Qu.:0.5461

Min. : :0.0000

256

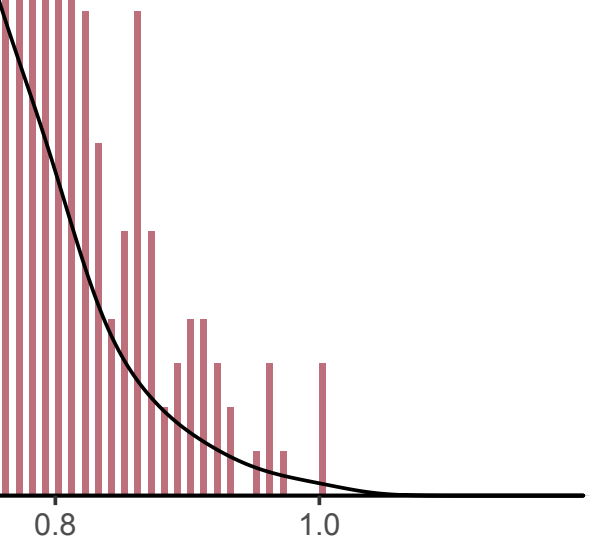


Figure 149 - CD131

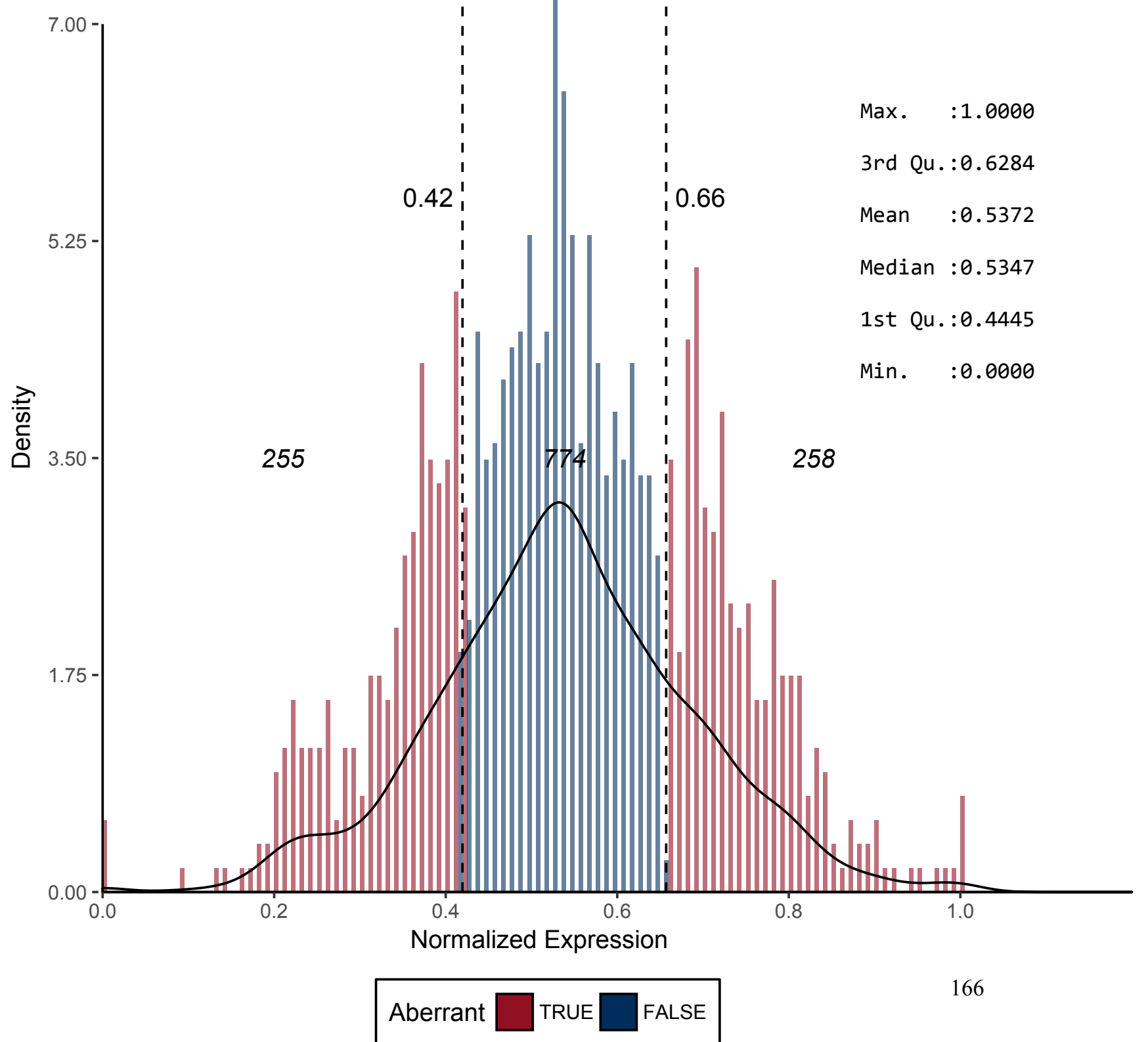


Figure 150 - CD132

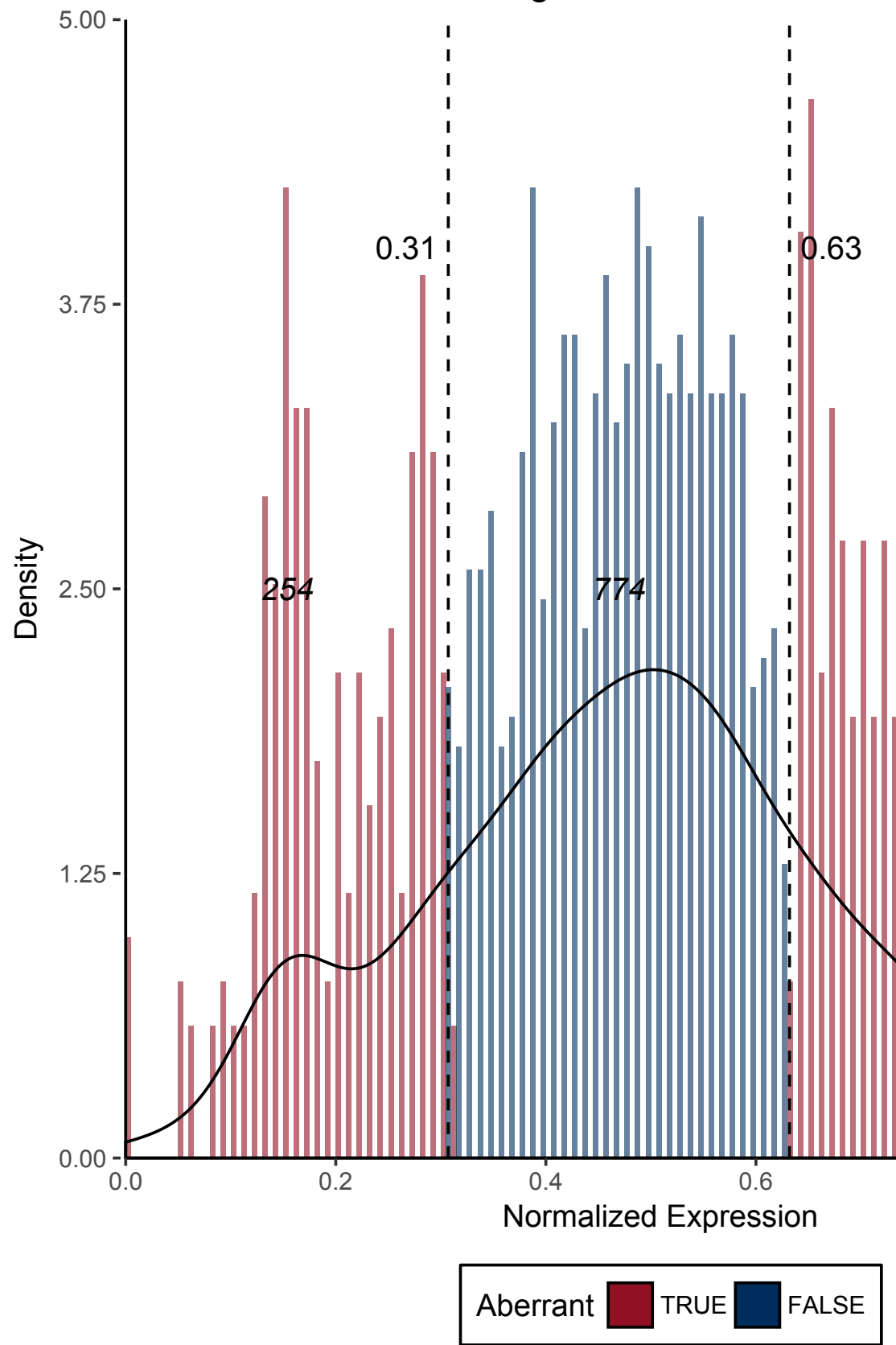

Max. $: 1.0000$

3rd Qu.:0.5944

Mean $\quad: 0.4704$

Median :0.4780

1st Qu.:0.3418

Min. $\quad: 0.0000$

\section{8}


Figure 151 - CD133*

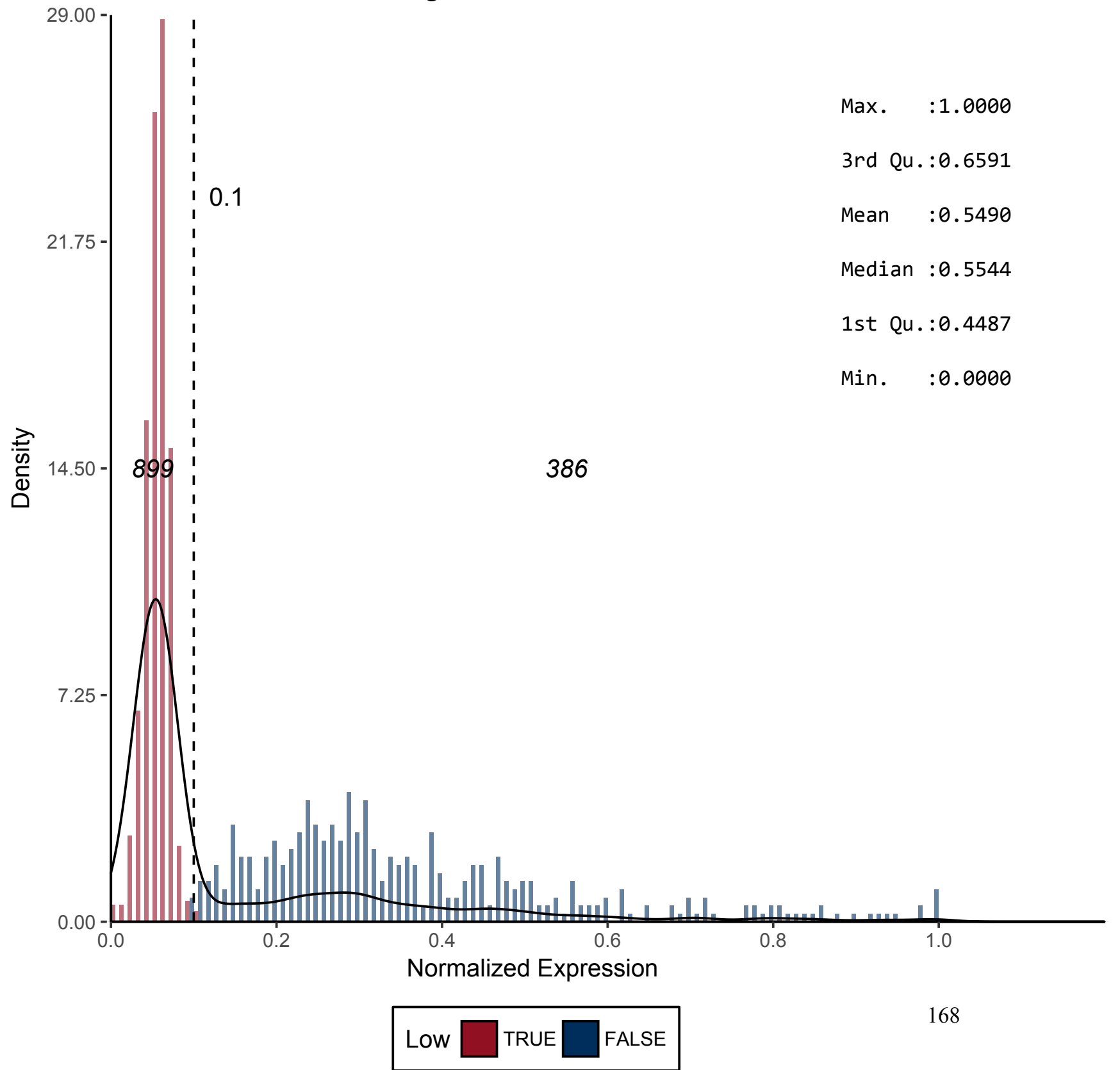


Figure 152 - CD134
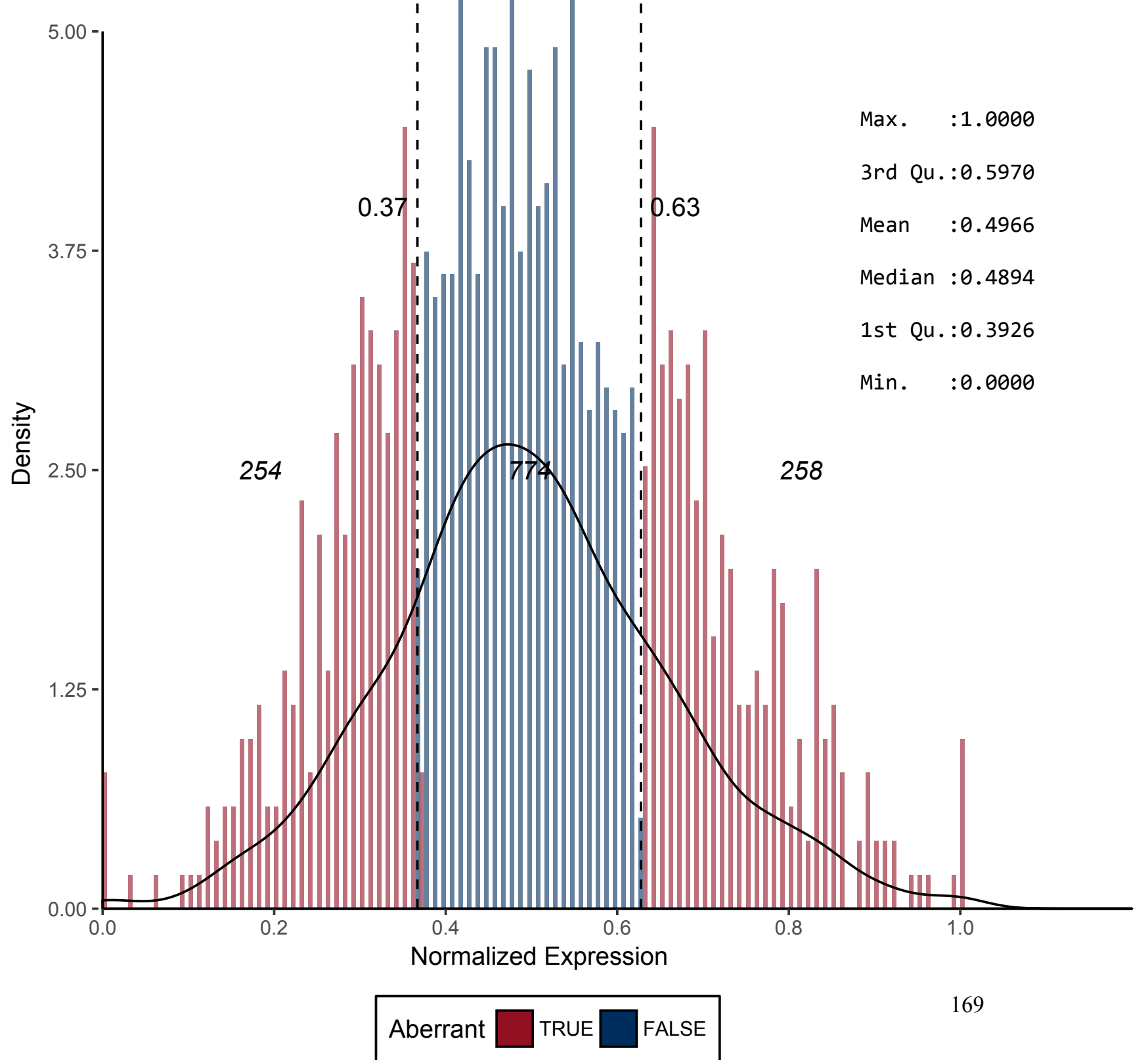
Figure 153 - CD135*

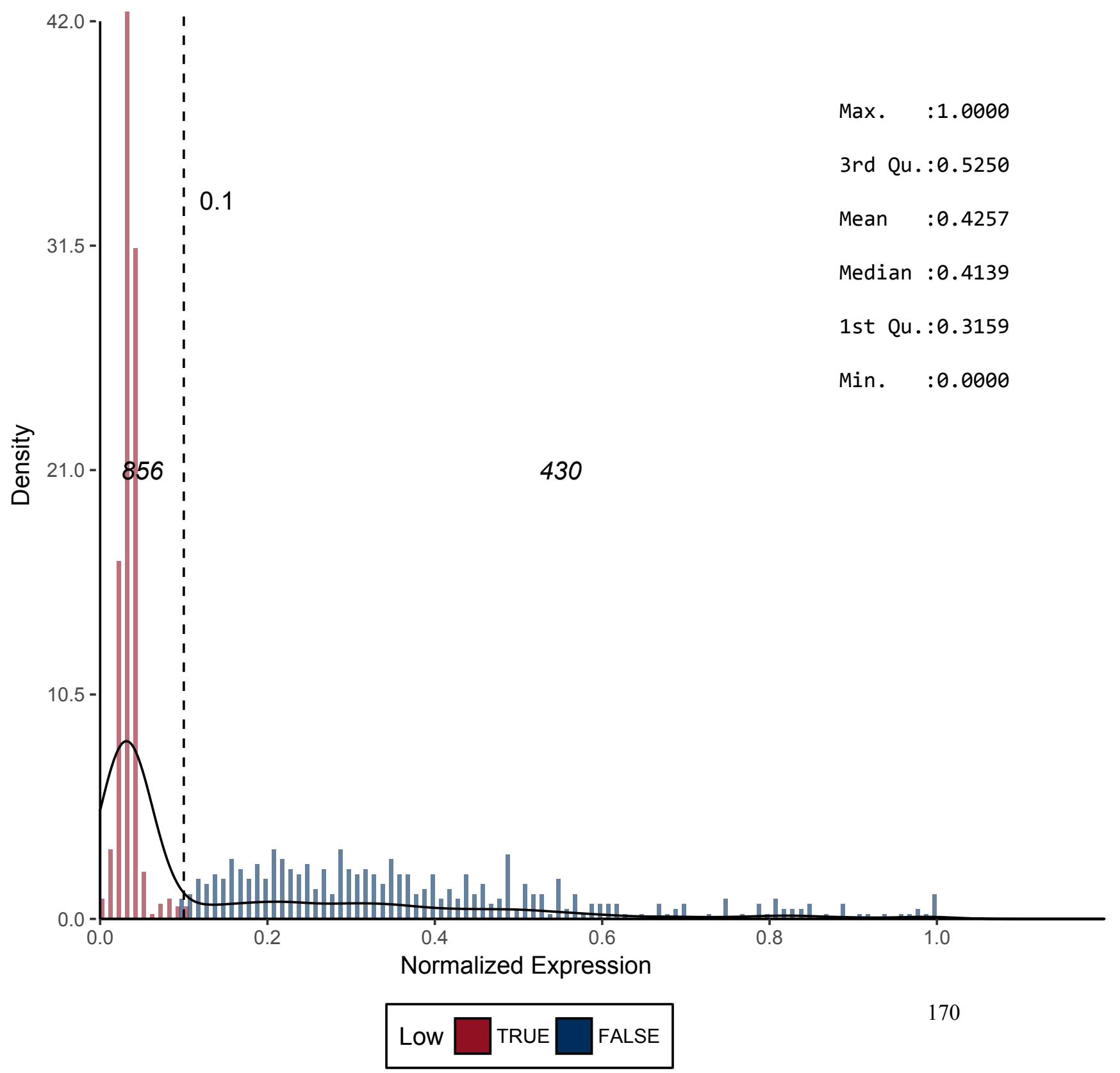


Figure 154 - CD136*

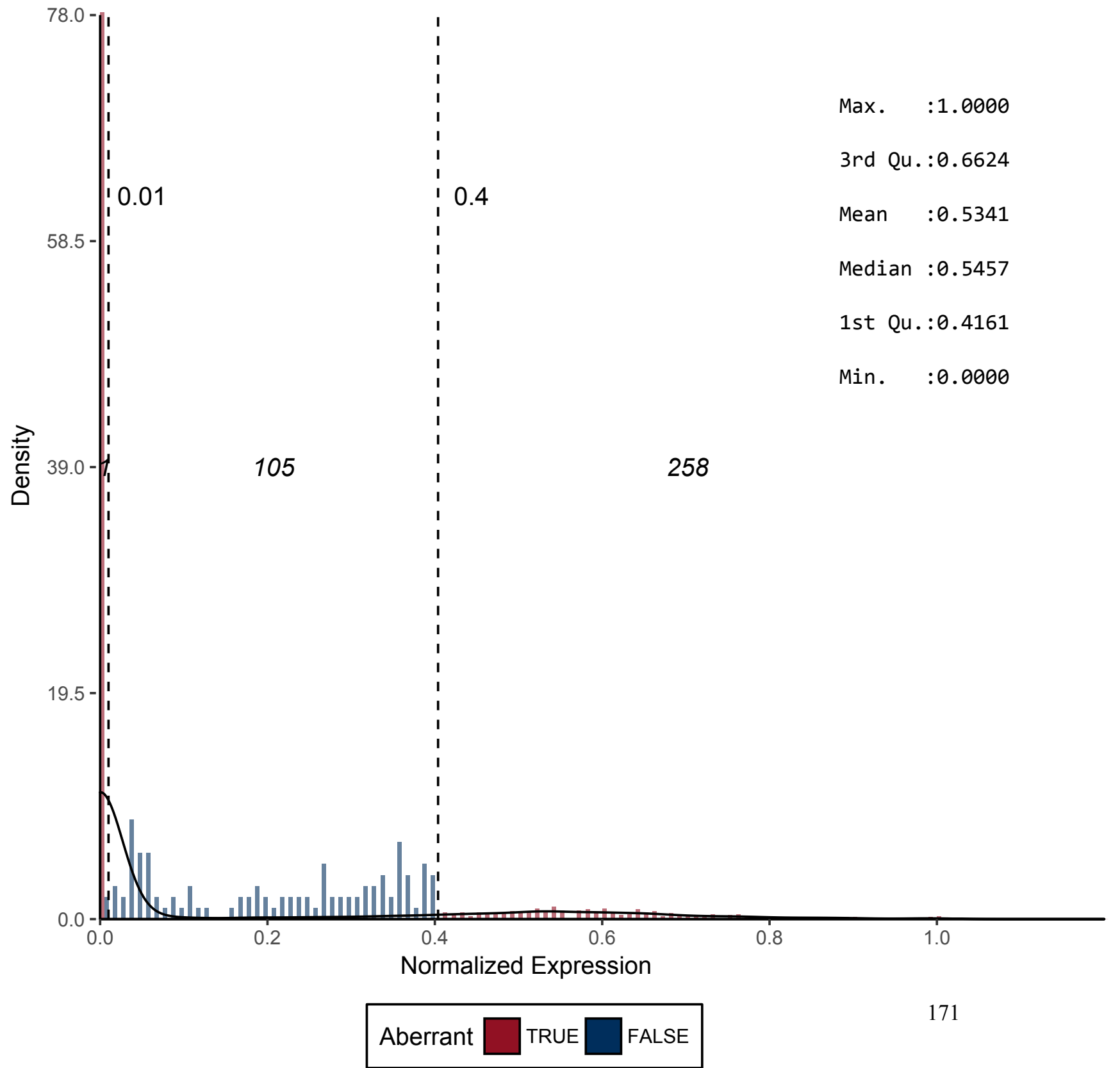


Figure 155 - CD137

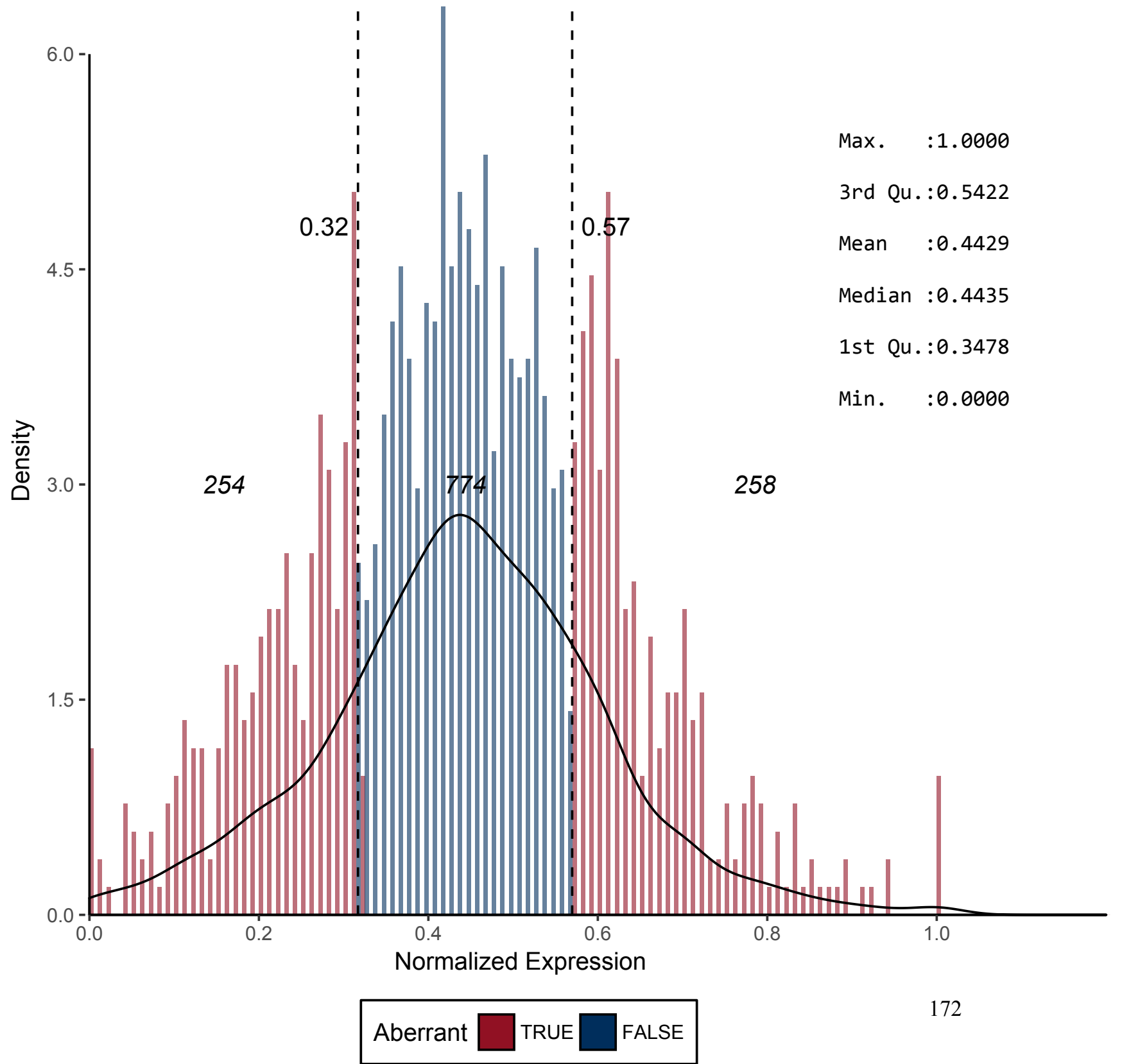


Figure 156 - CD138

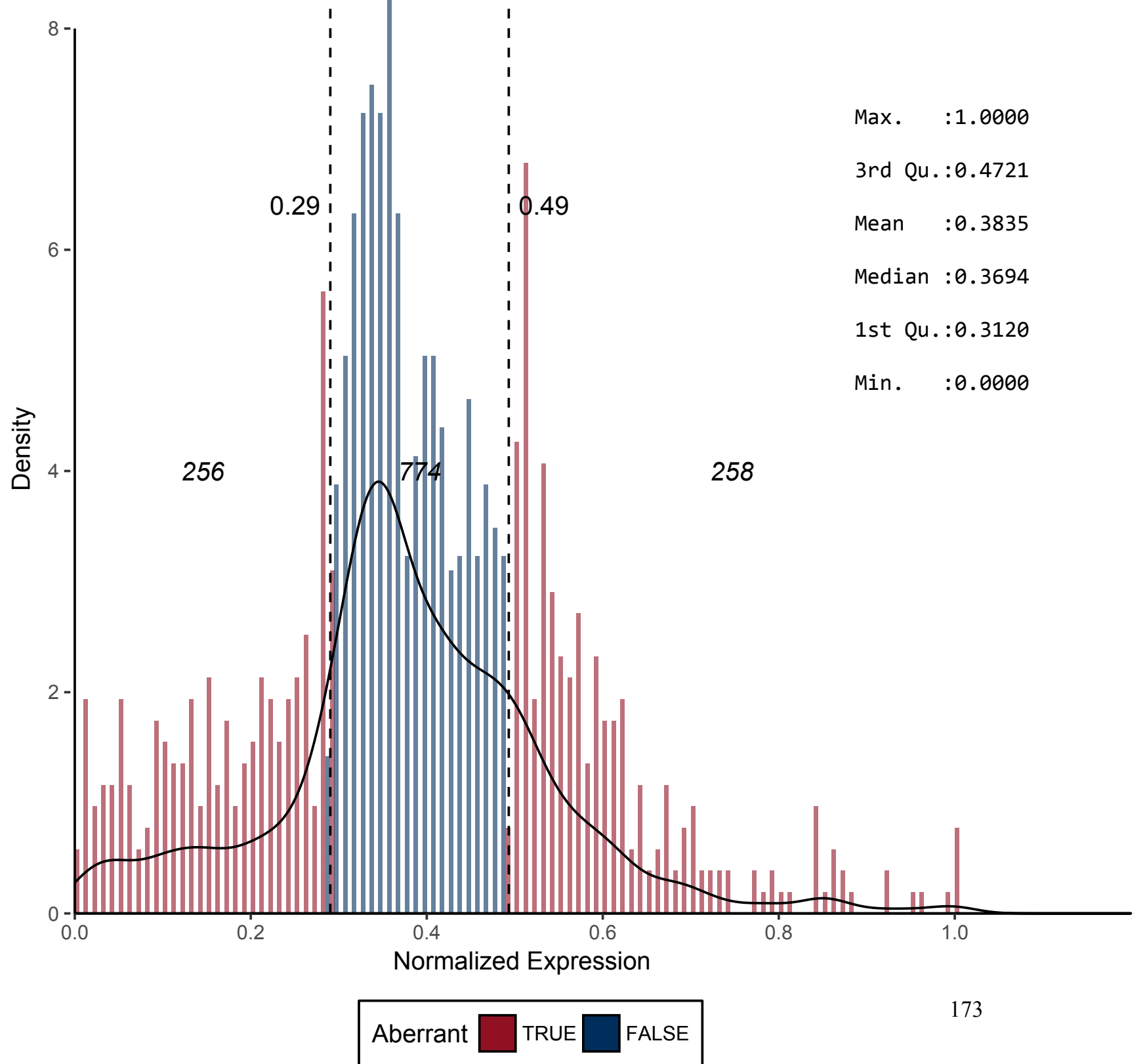


Figure 158 - CD140B

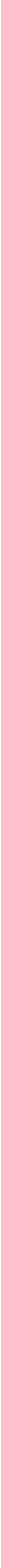


Figure 159 - CD141

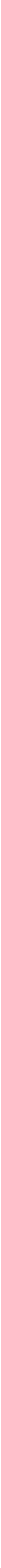


Figure 160 - CD142*

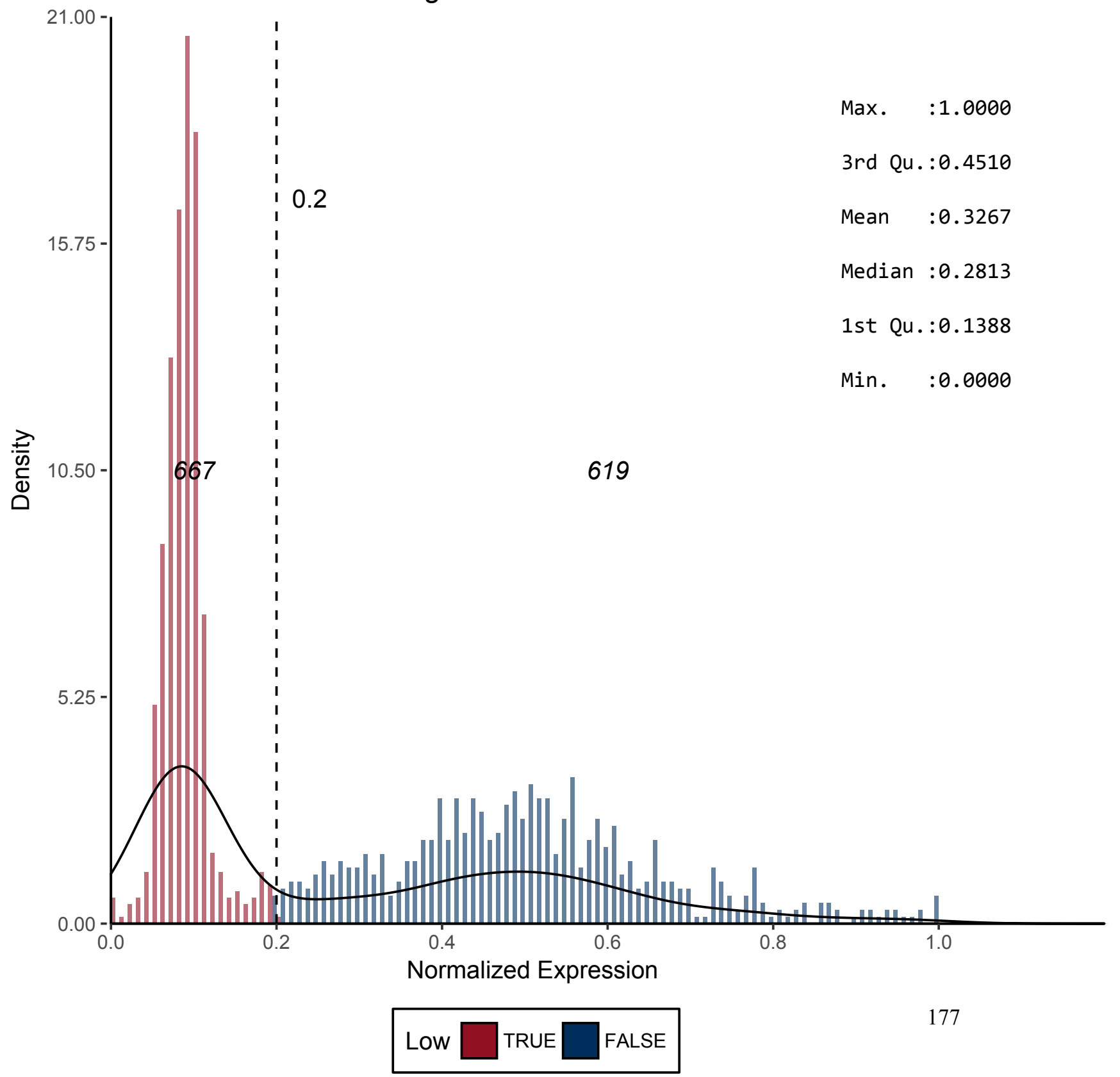


Figure 161 - CD143*

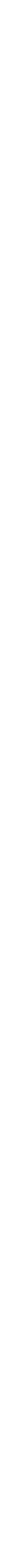


Figure 162 - CD144*

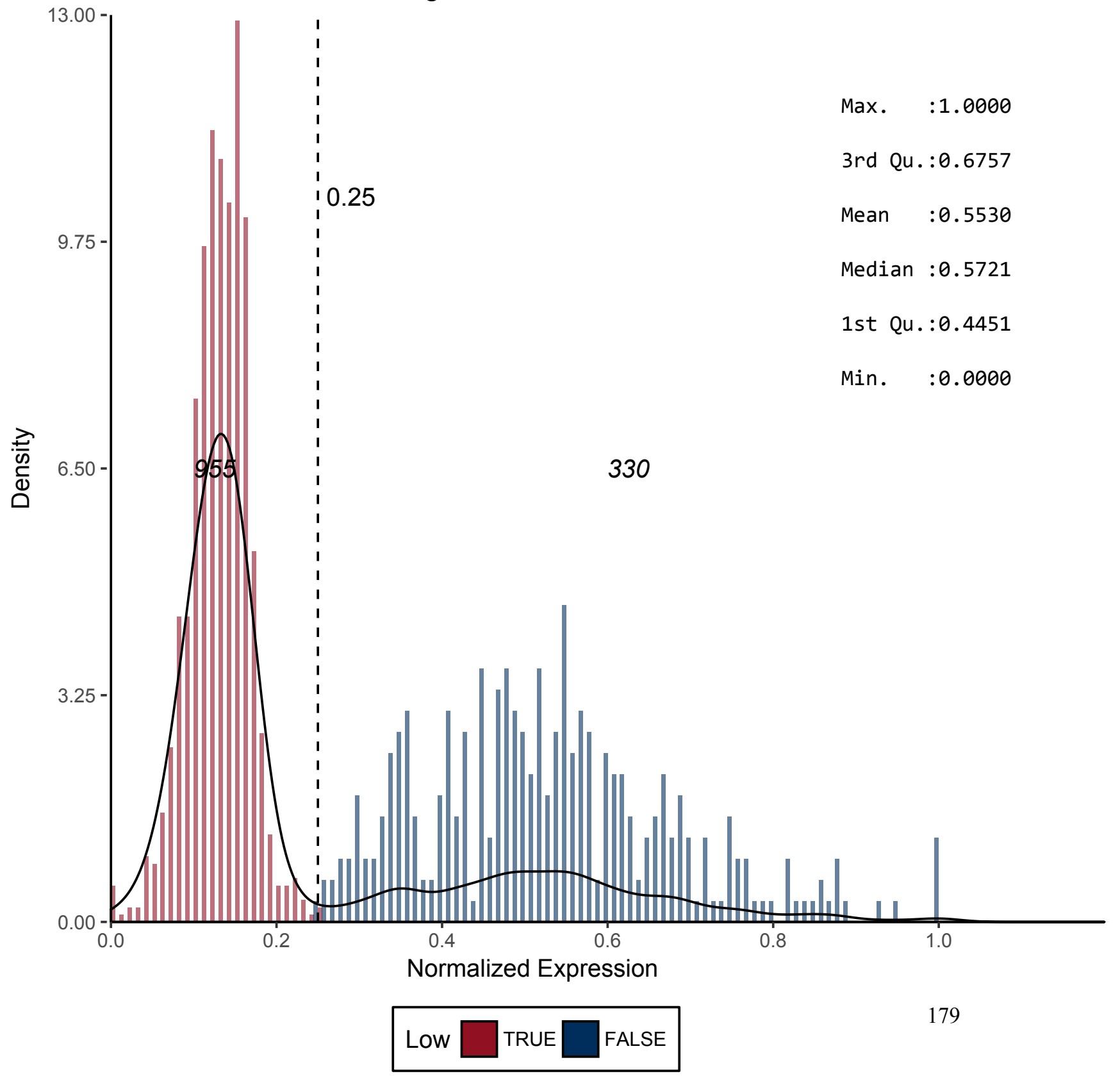


Figure 163 - CD146*

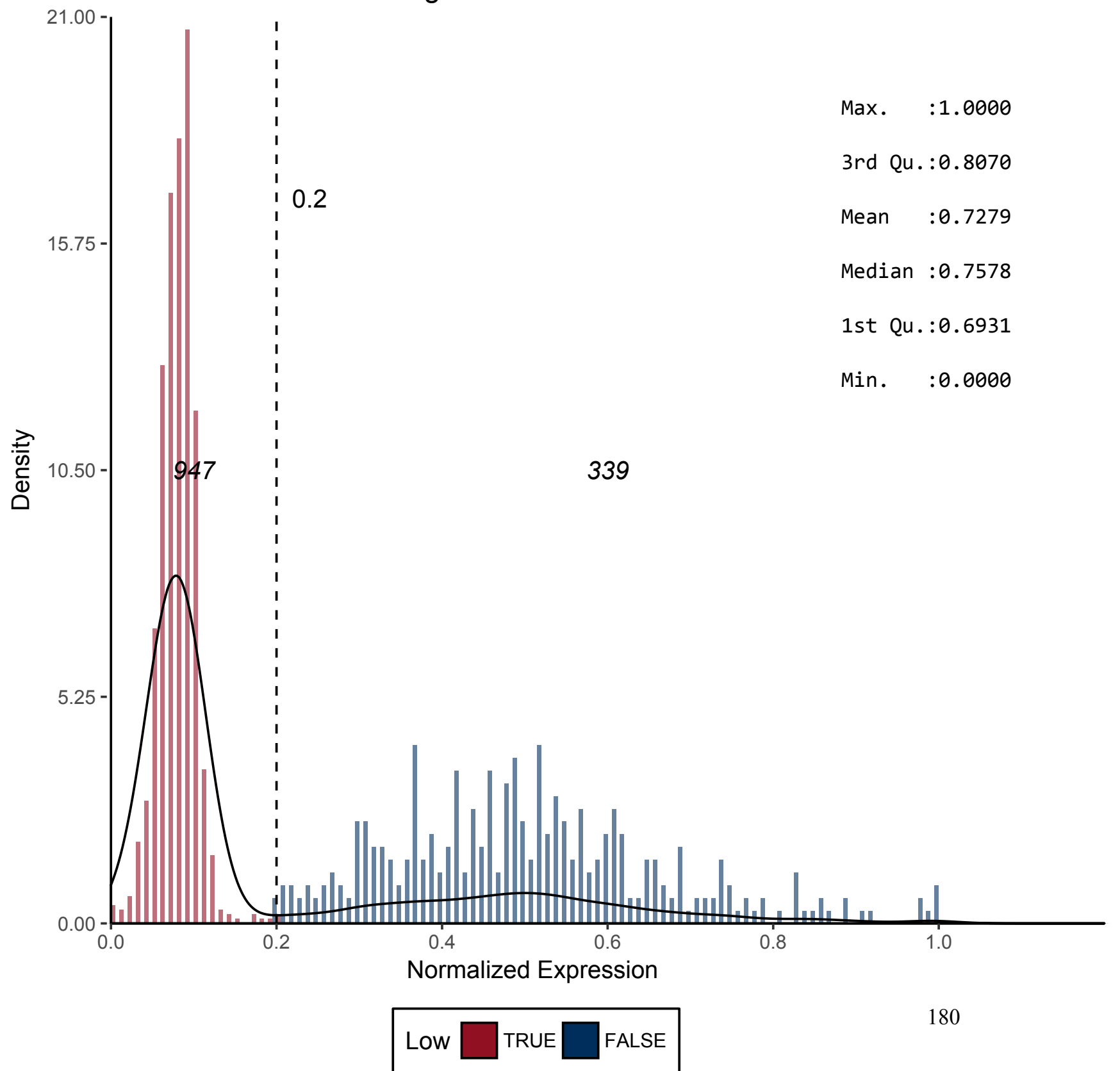


Figure 166 - CD150

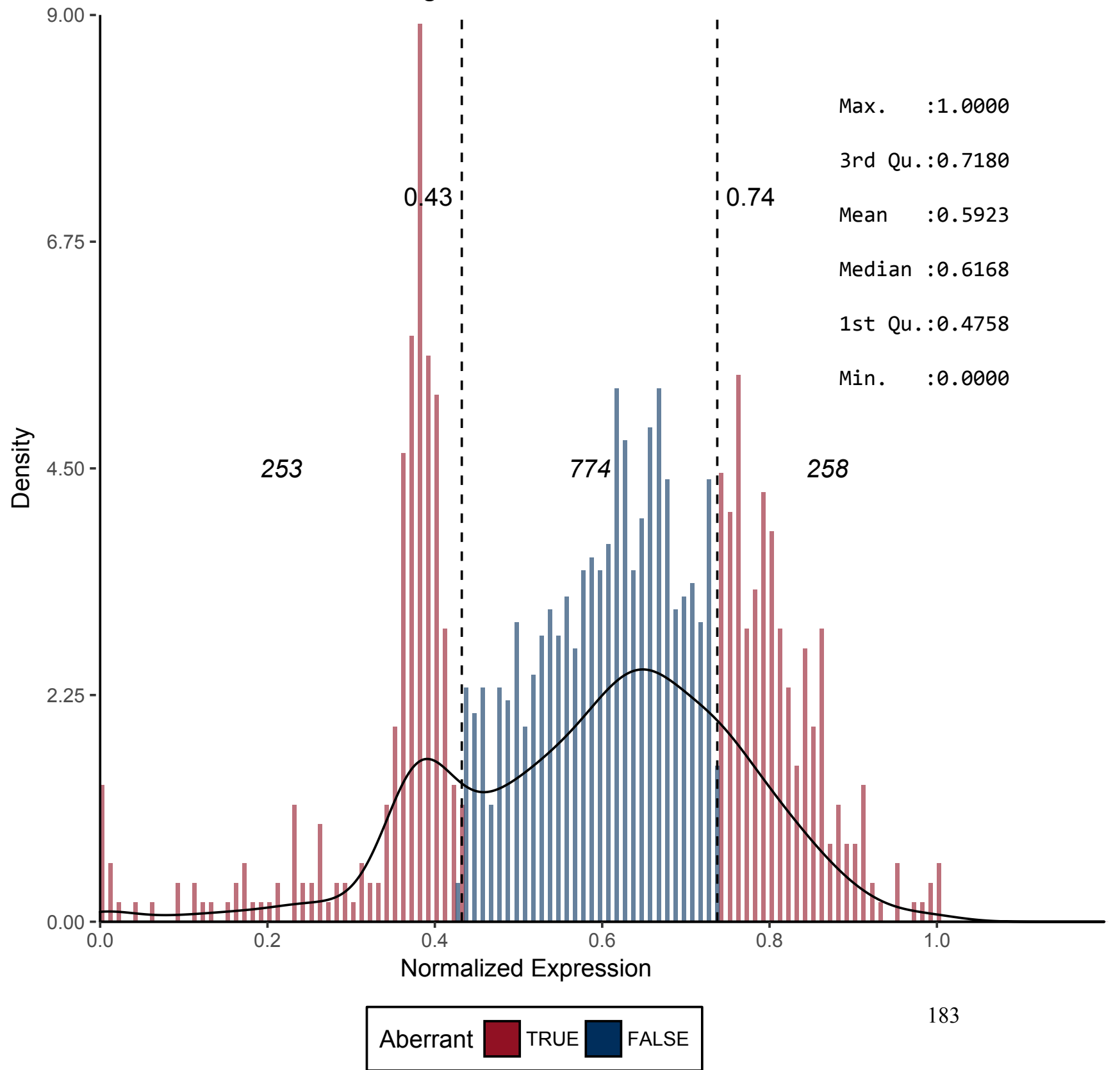


Figure 167 - CD151

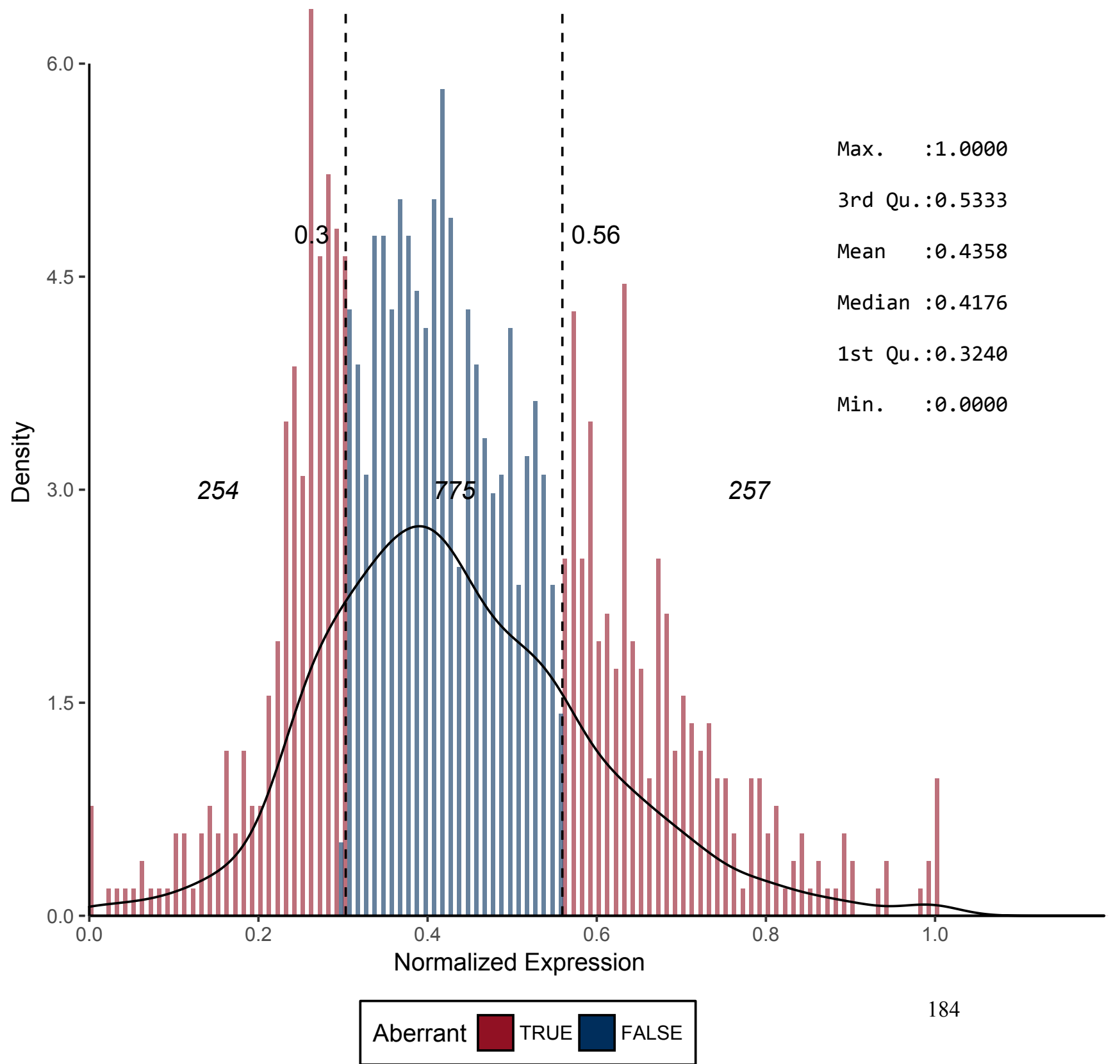


Figure 168 - CD152

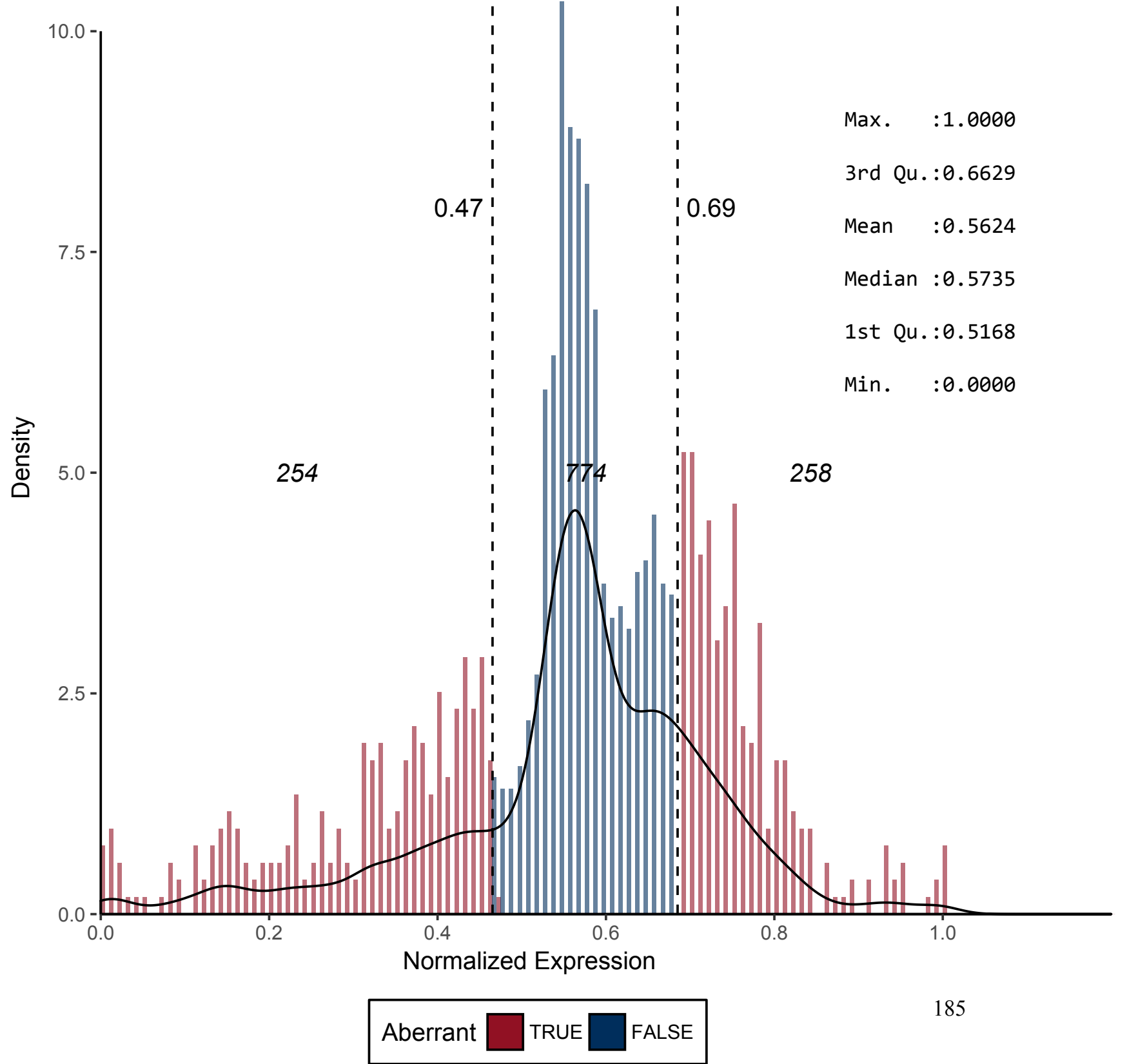


Figure 169 - CD153

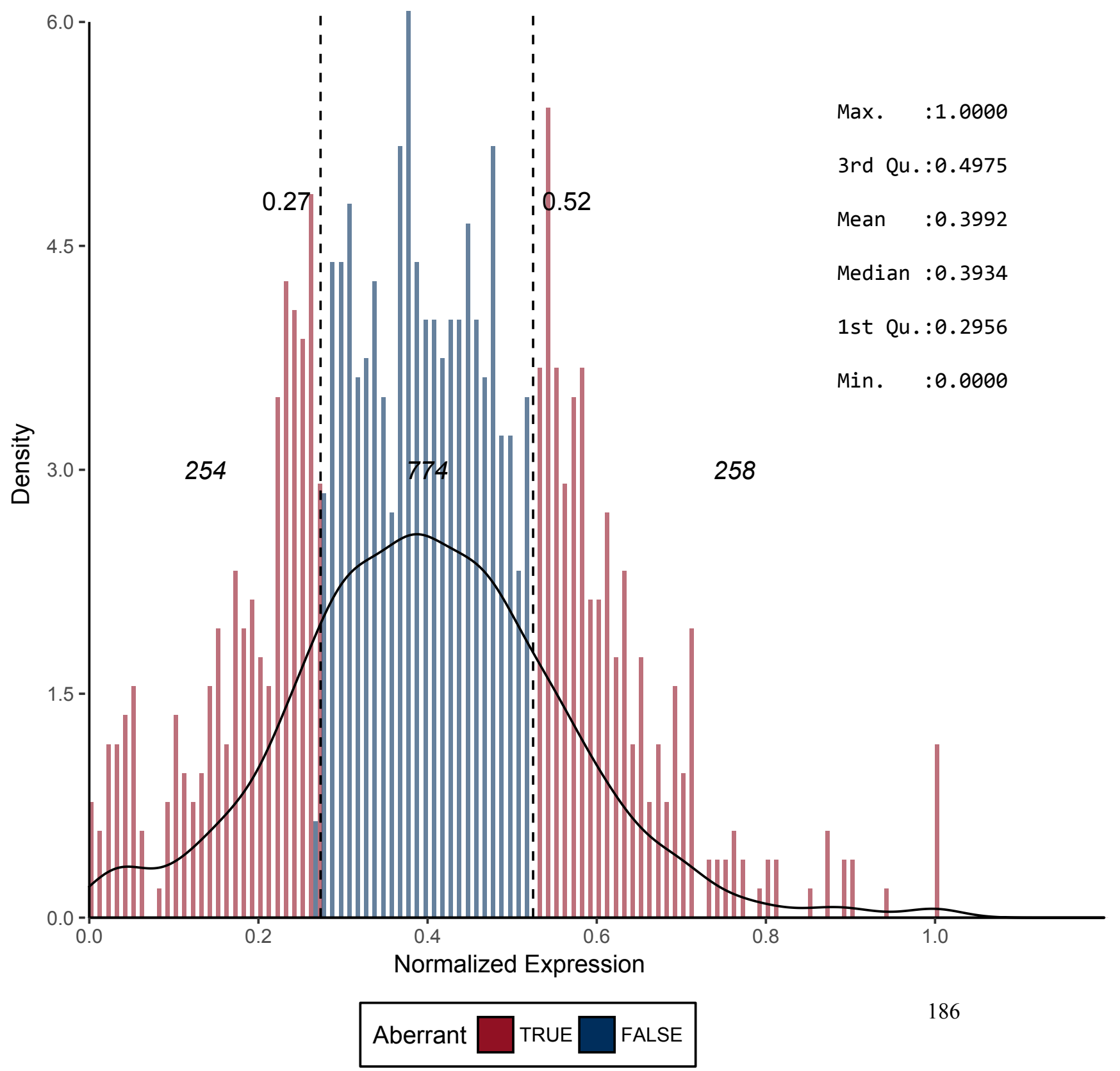


Figure 170 - CD154*

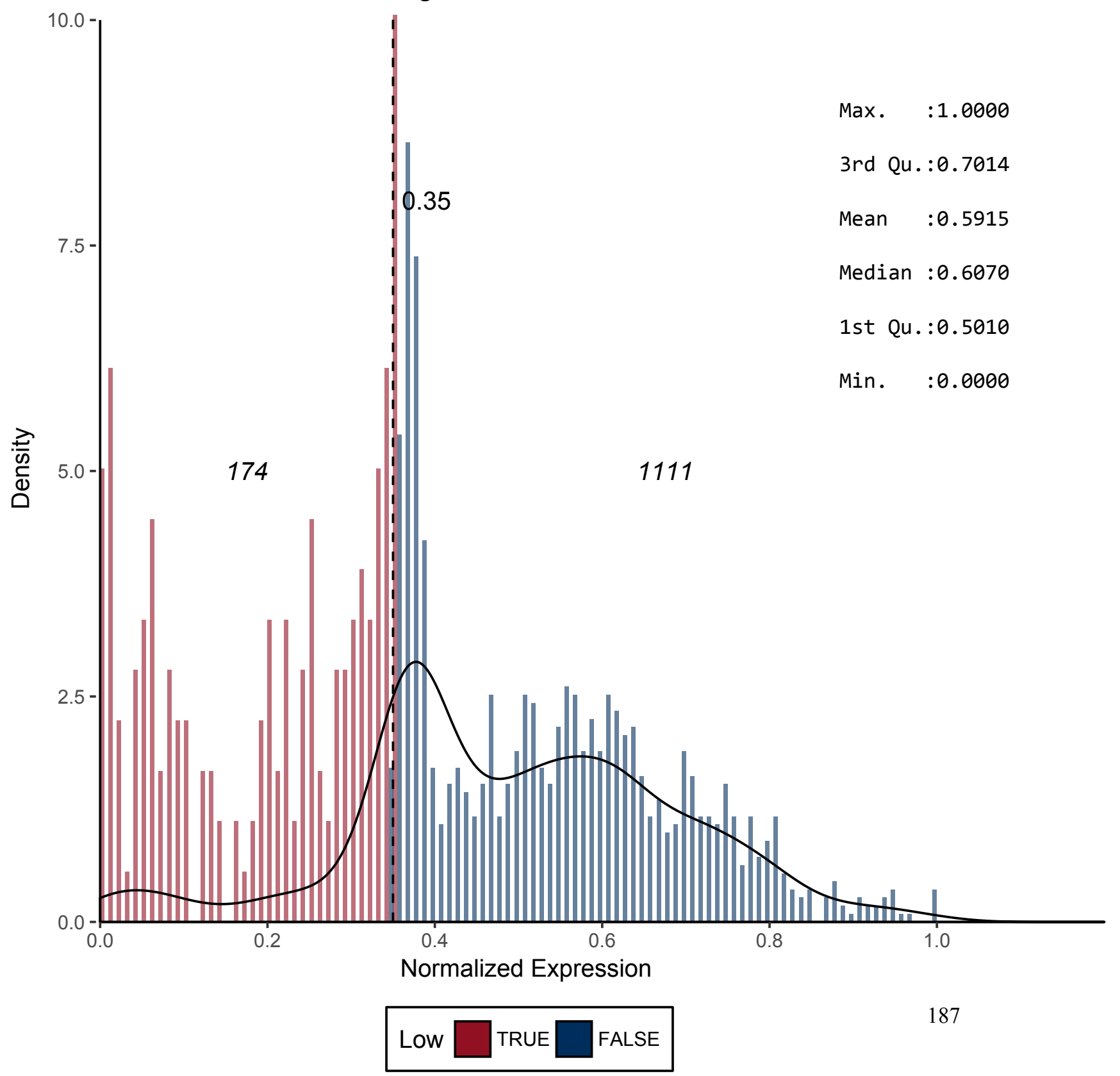


Figure 171 - CD155

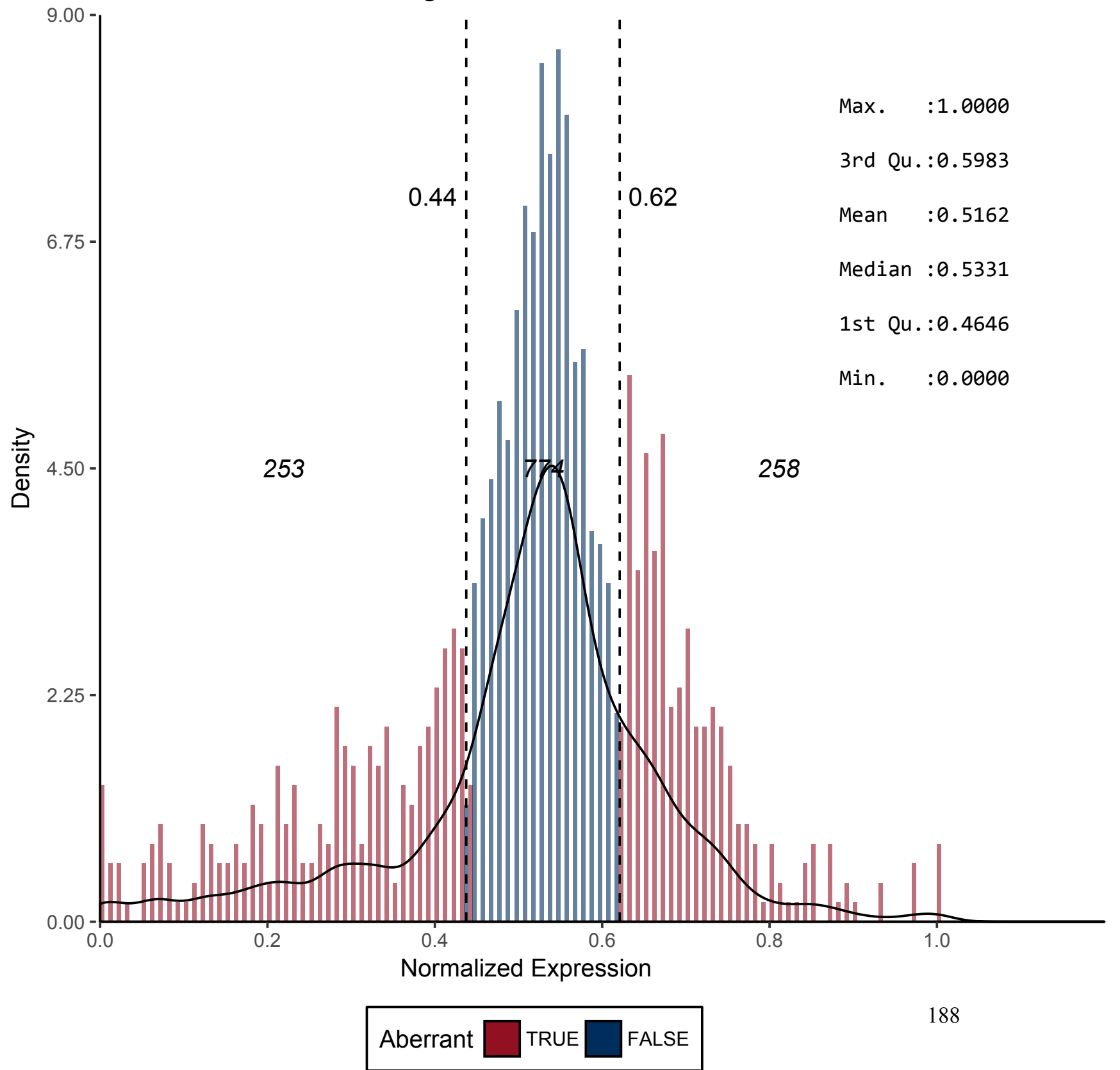


Figure 173 - CD156C

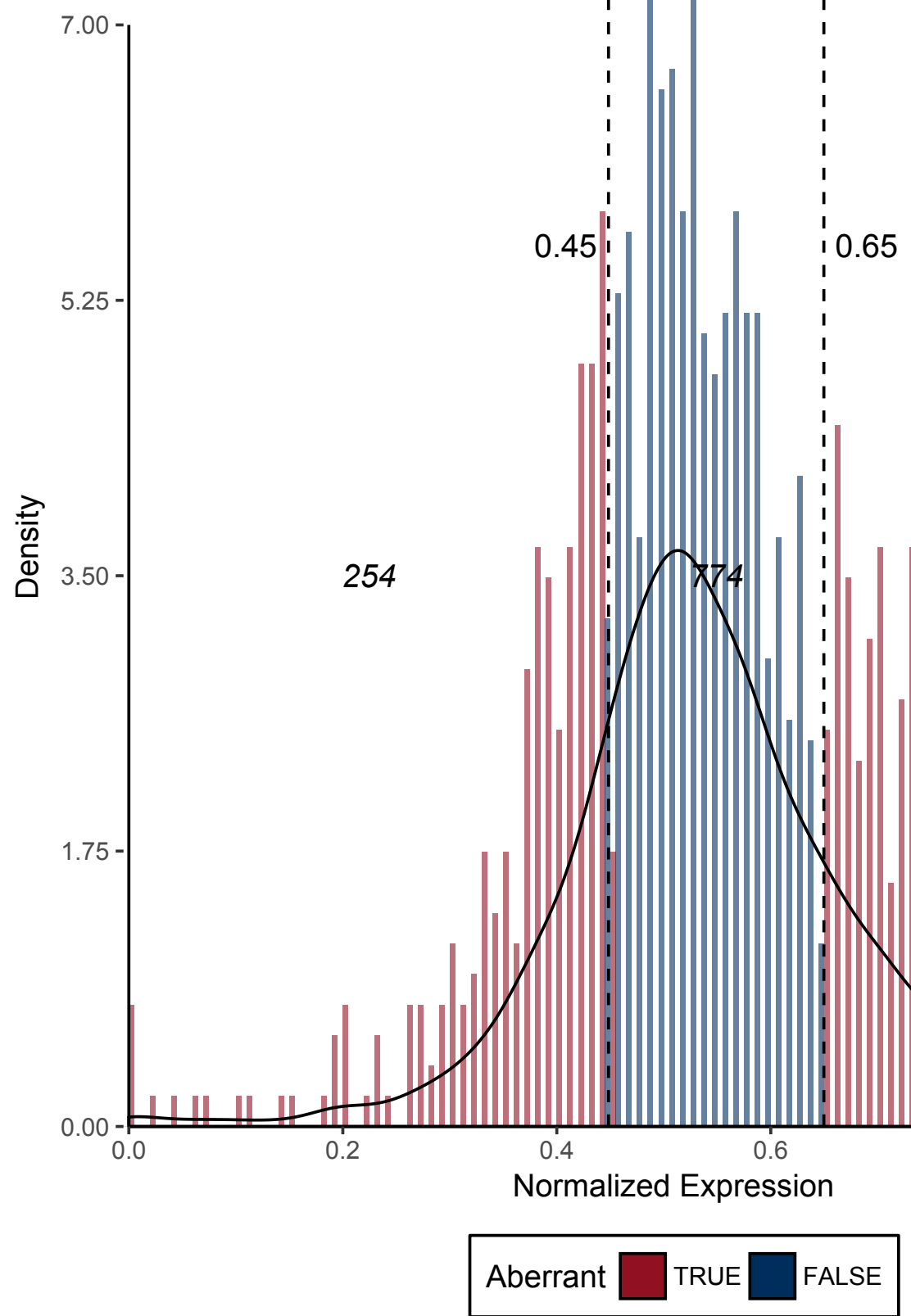

Max. $: 1.0000$

3rd Qu.:0.6233

Mean $\quad: 0.5452$

Median :0.5321

1st Qu.:0.4647

Min. $\quad: 0.0000$

258

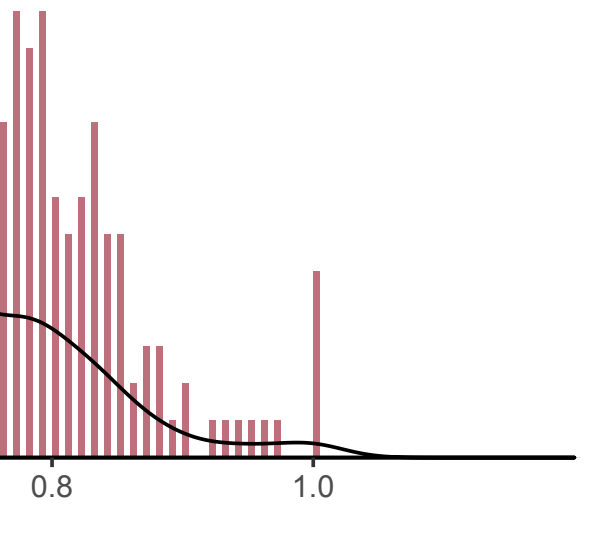


Figure 176 - CD158I*

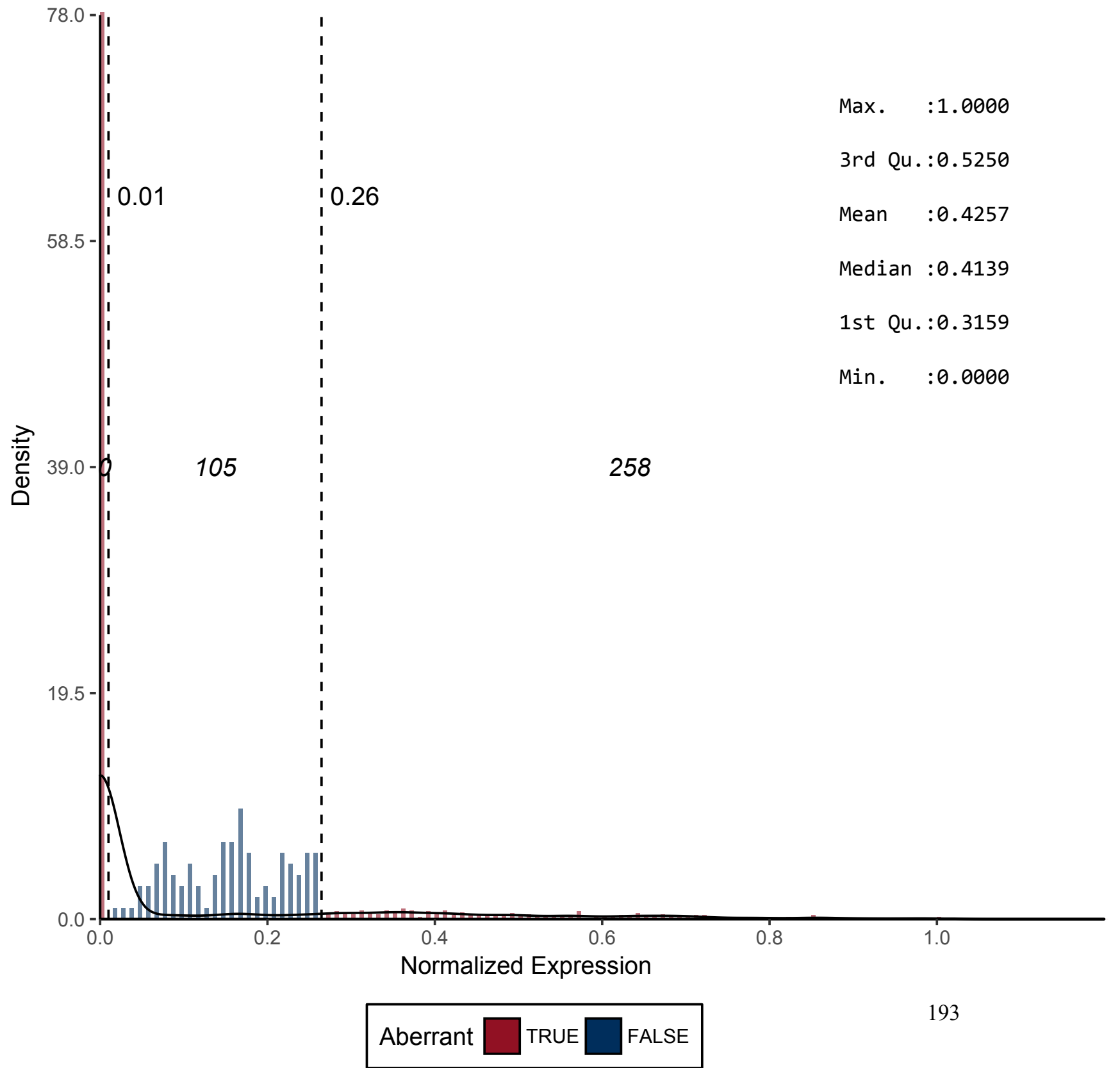


Figure 177 - CD158K

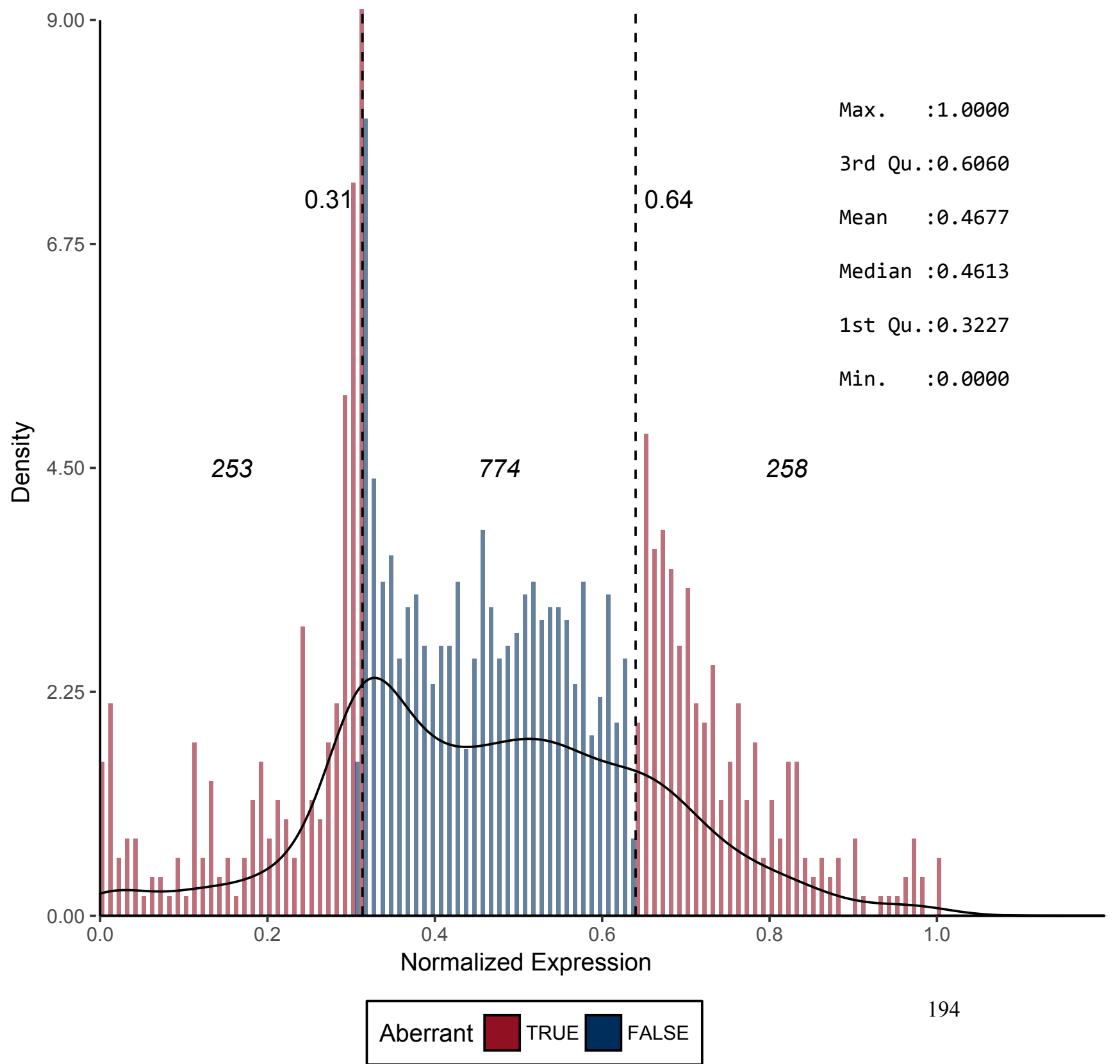


Figure 178 - CD160

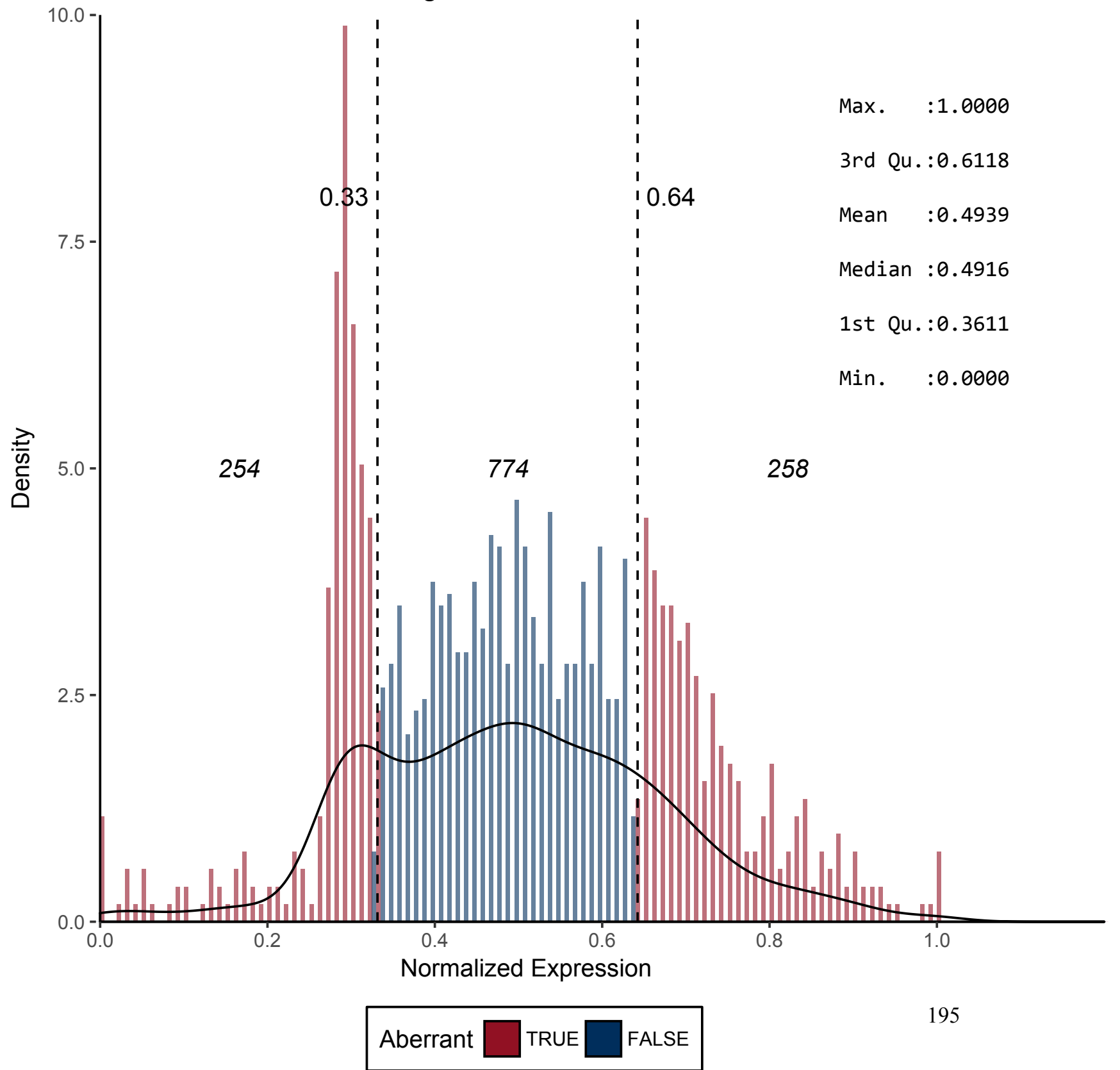


Figure 180 - CD162

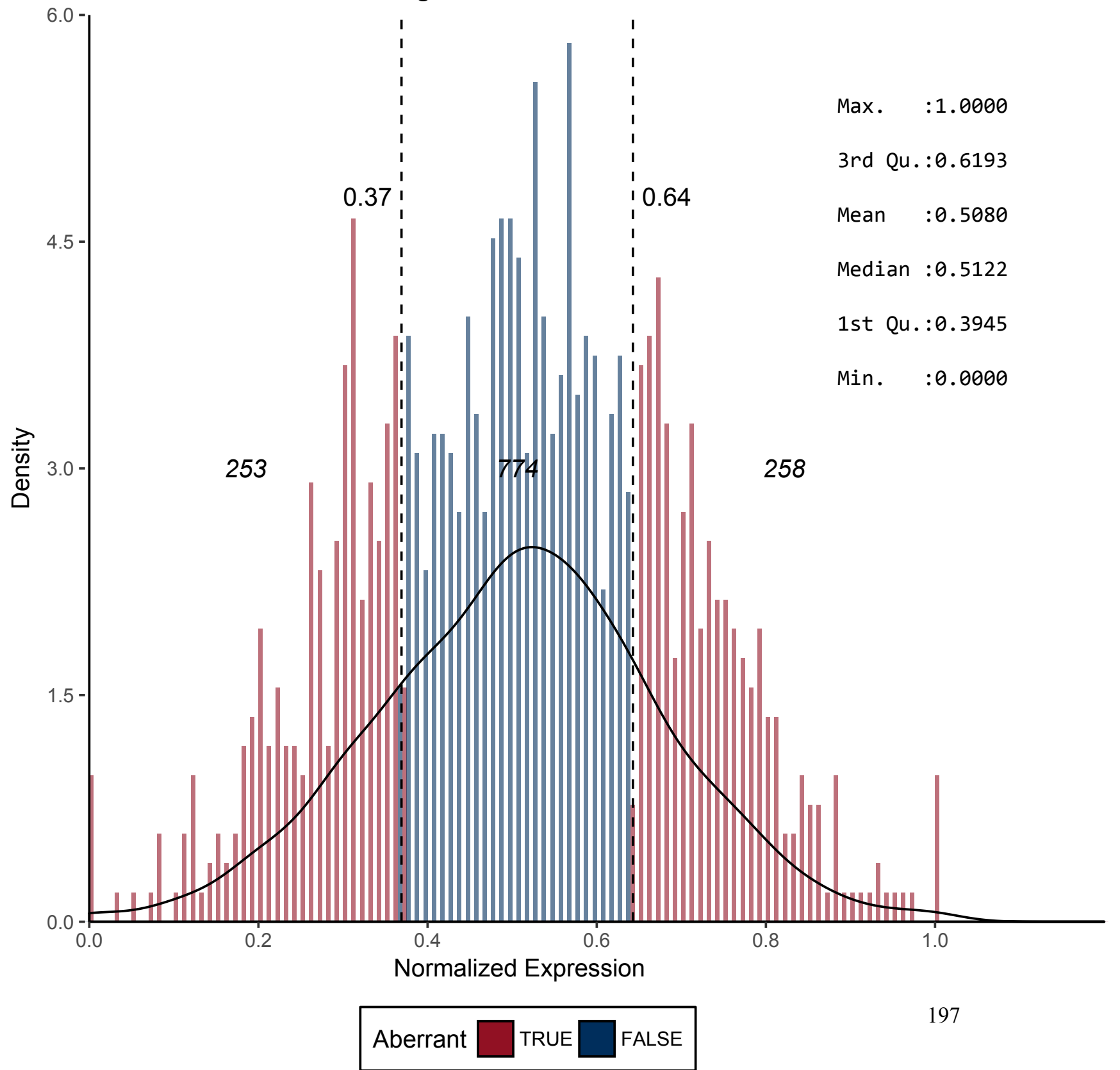


Figure 181 - CD163

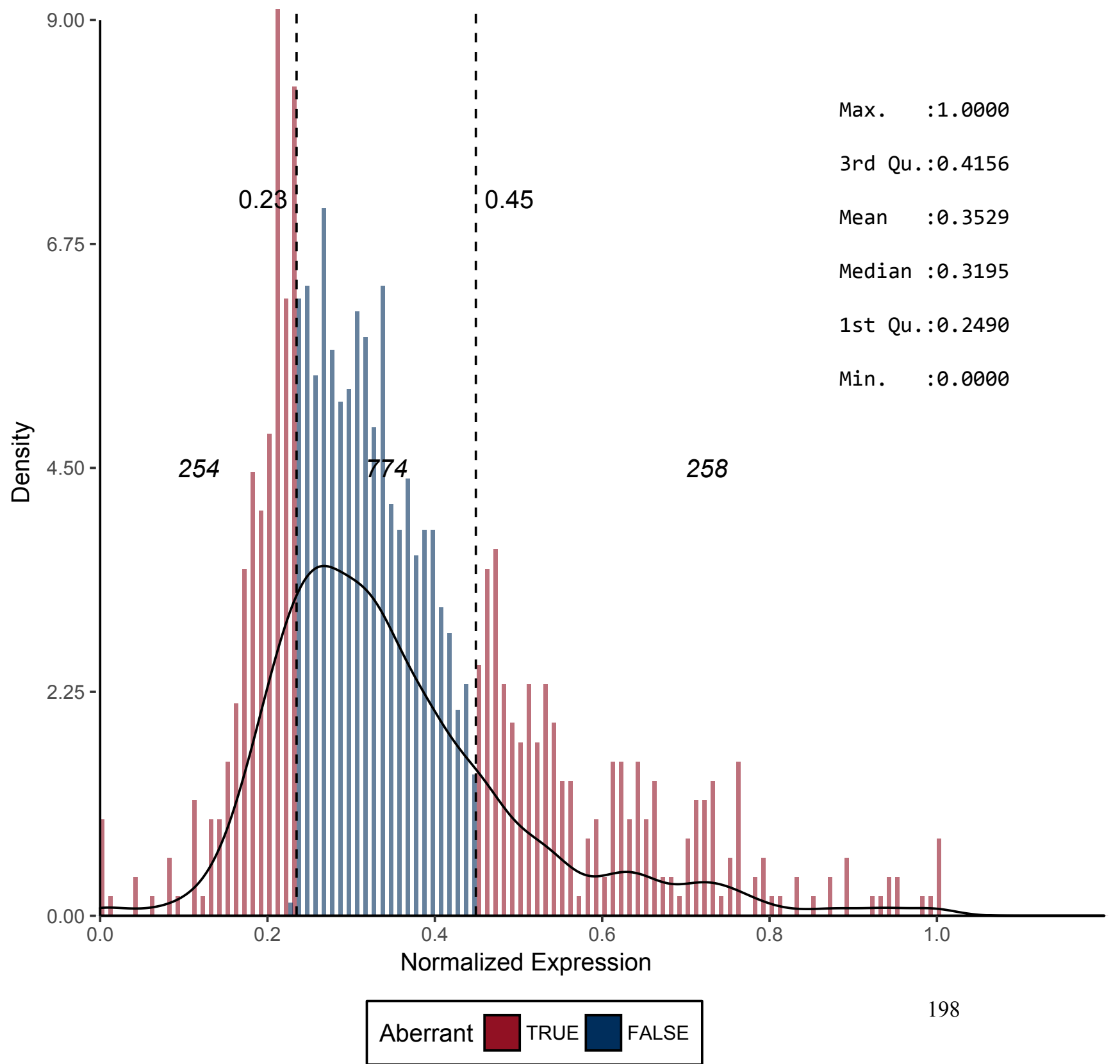


Figure 182 - CD164

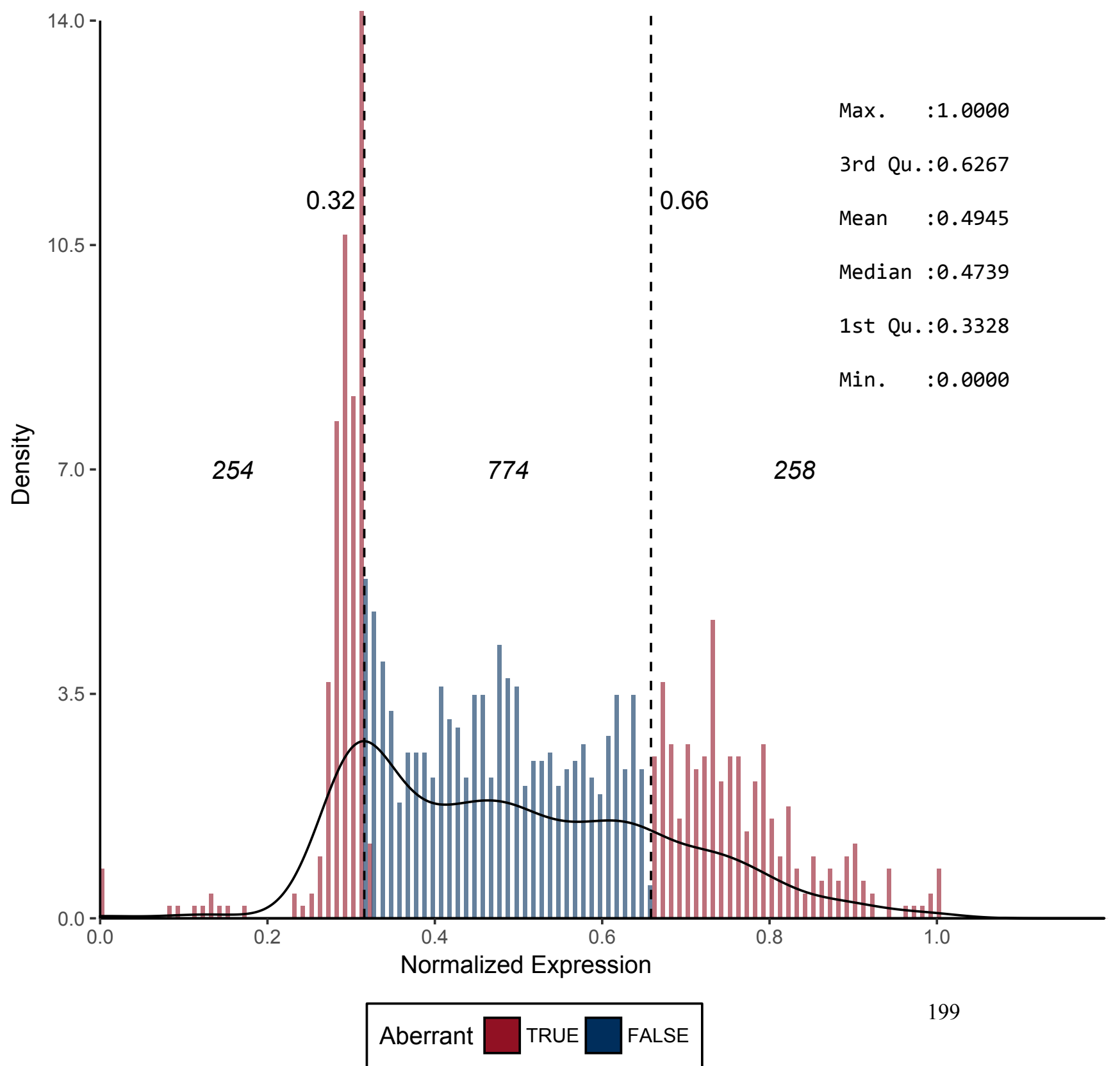


Figure 183 - CD166

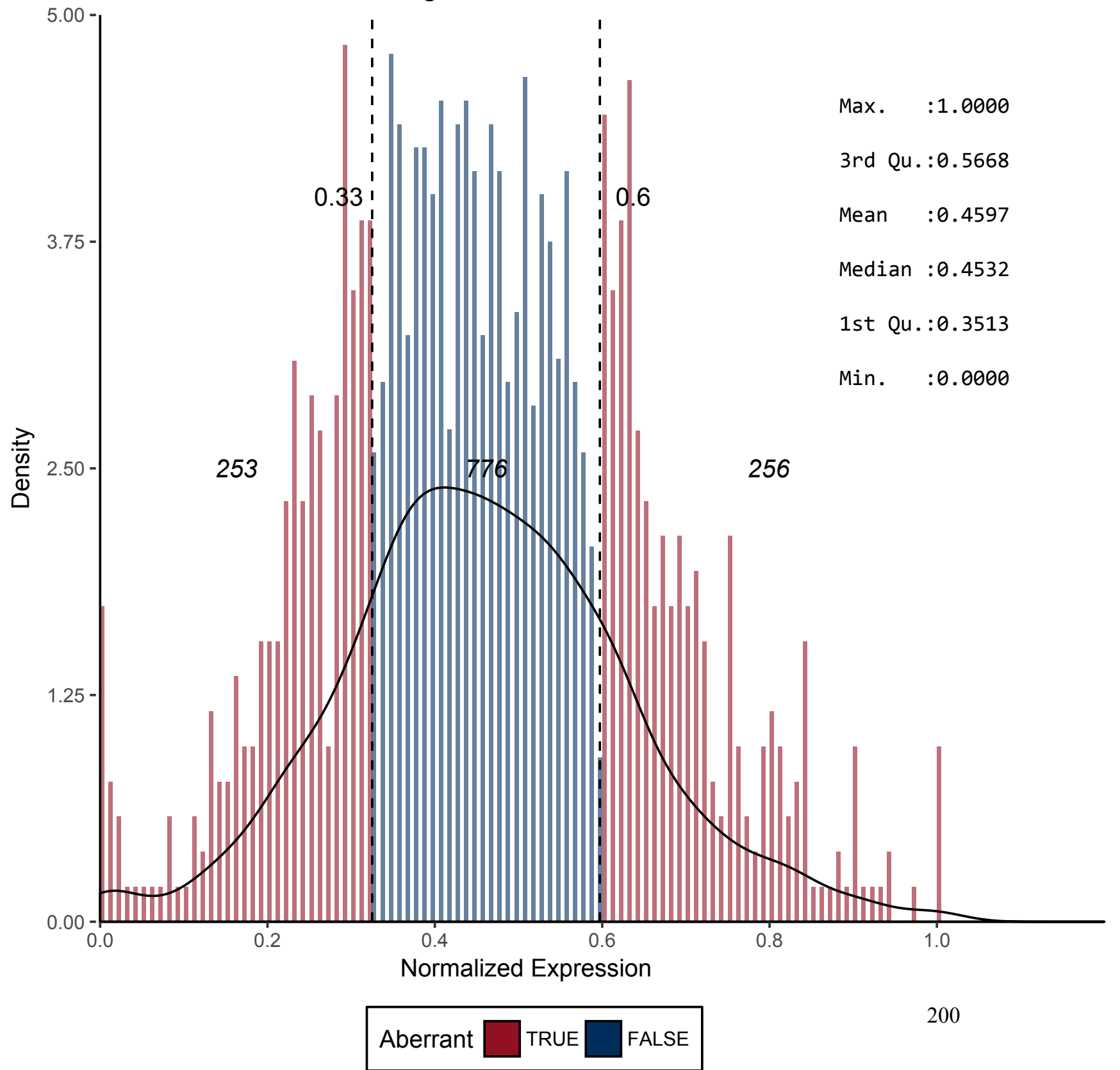


Figure 184 - CD167A

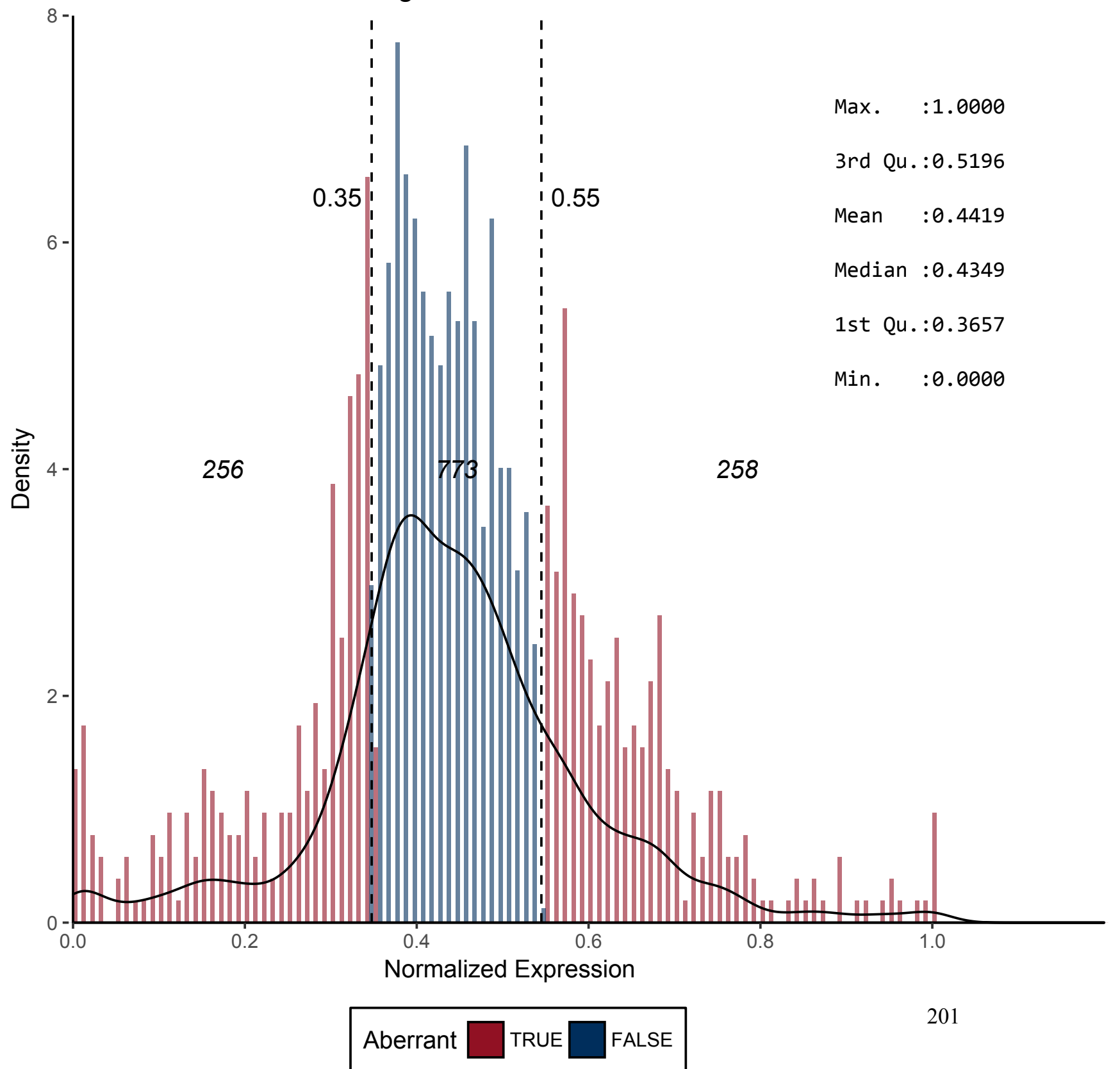


Figure 185 - CD167B*

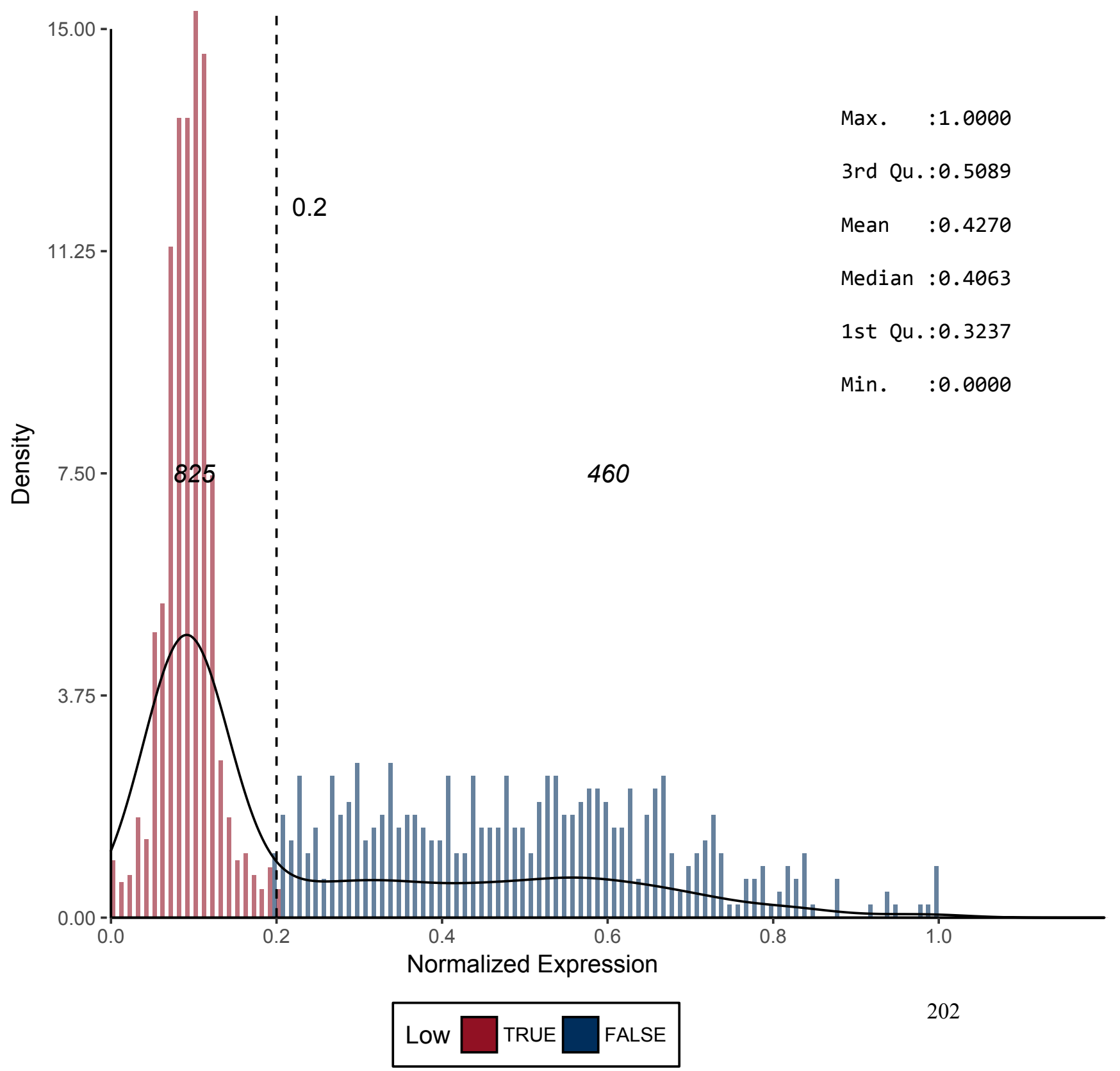


Figure 186 - CD168*

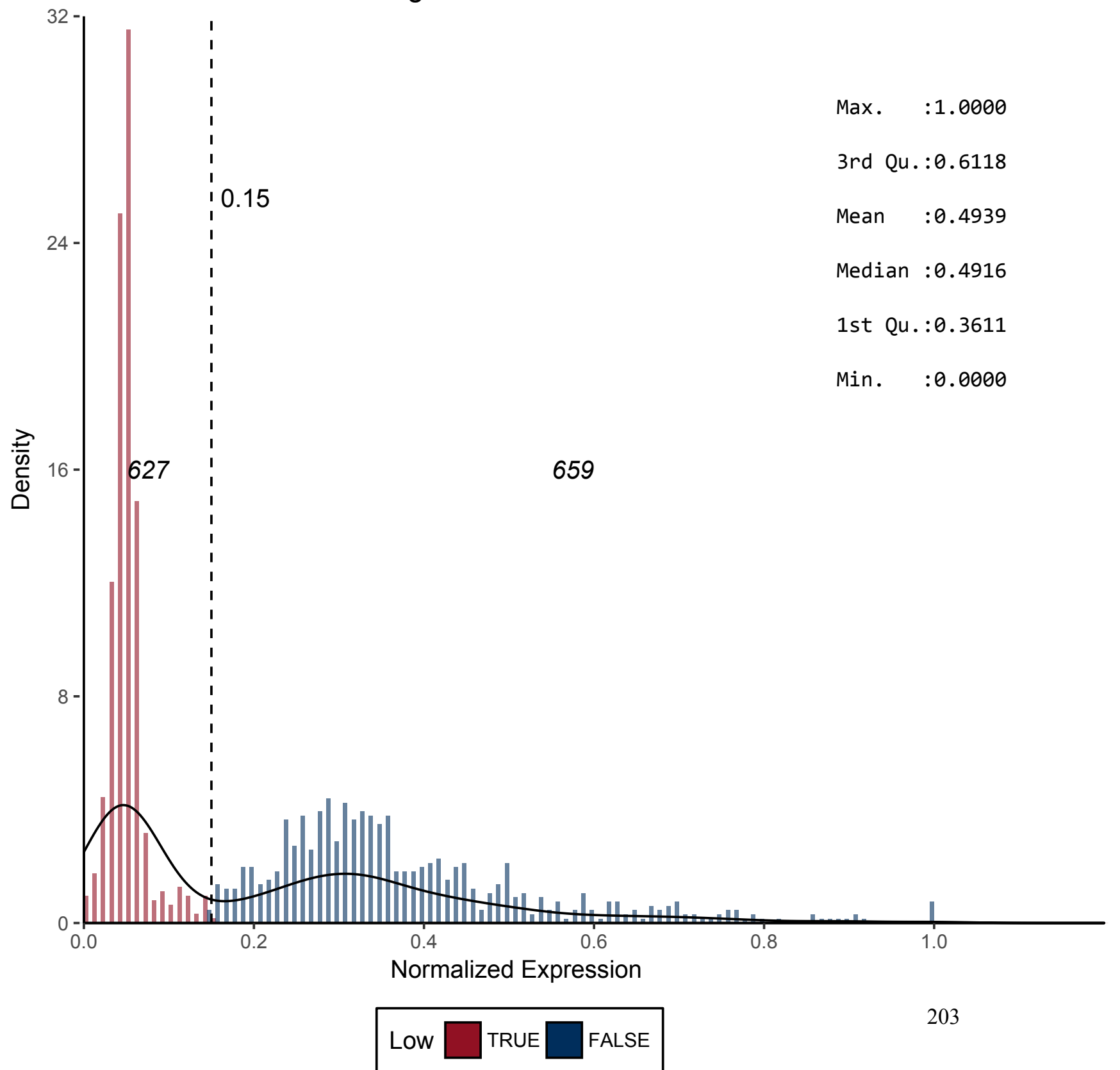


Figure 188 - CD170

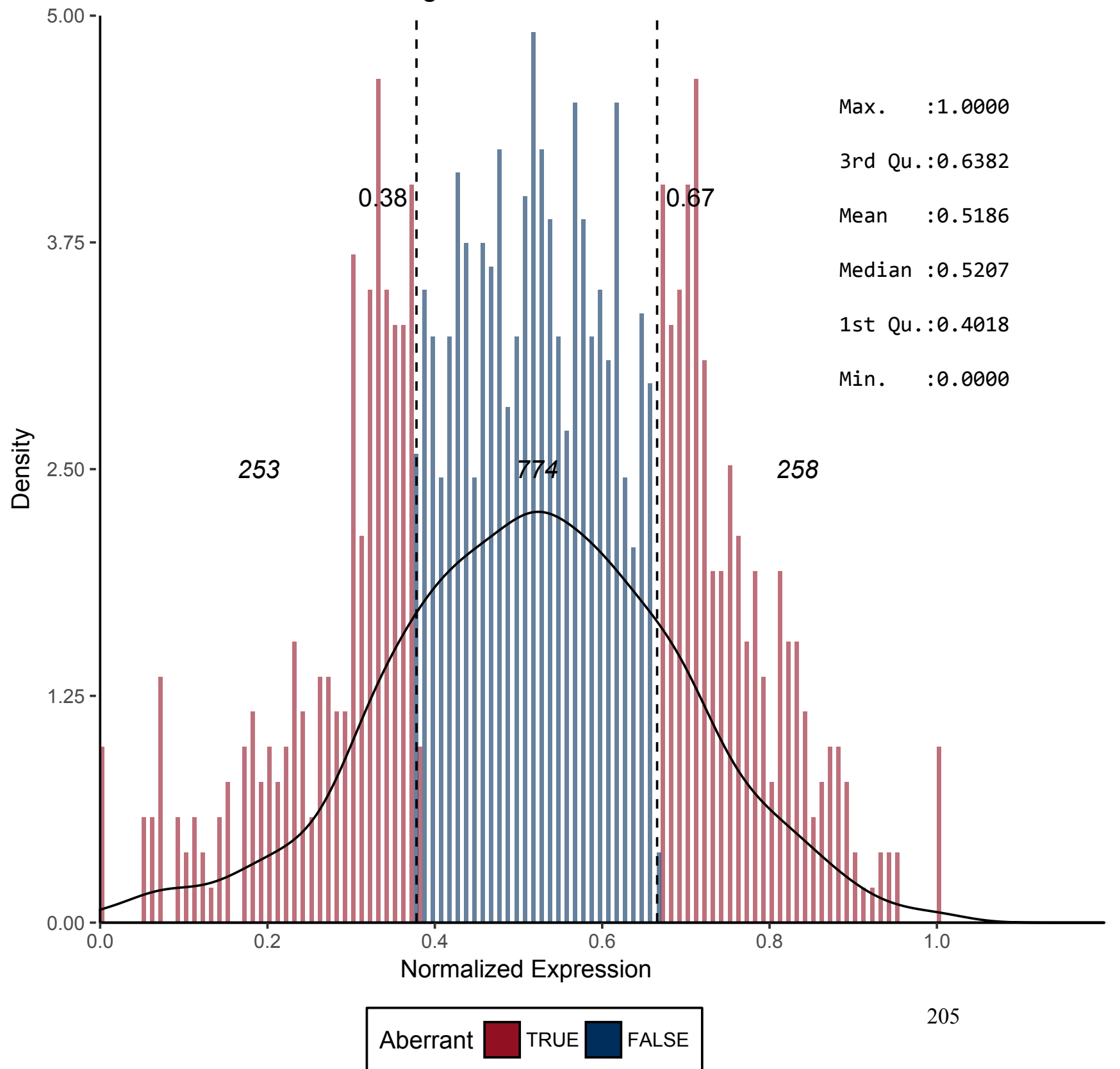


Figure 189 - CD171*

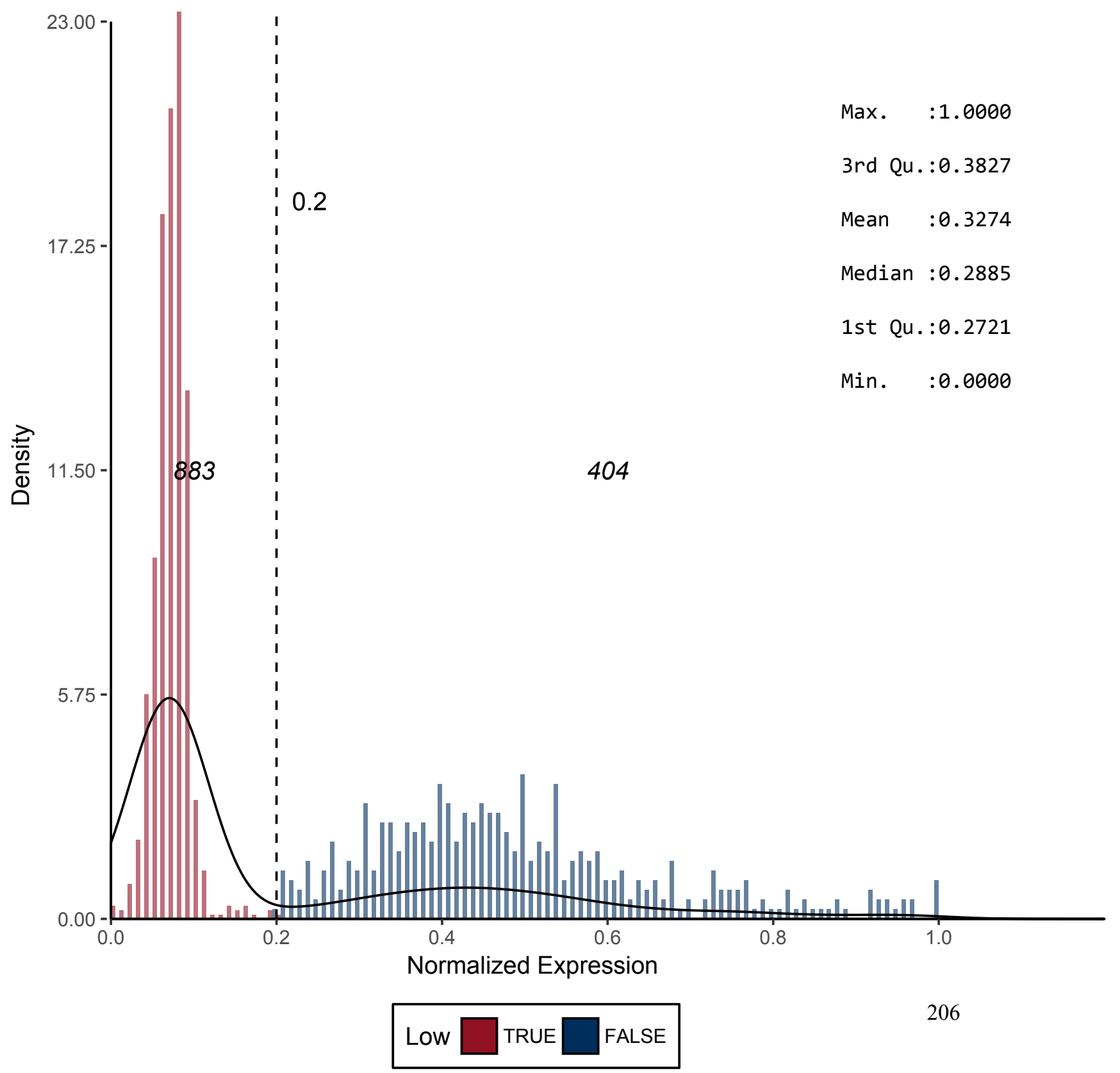


Figure 190 - CD172A

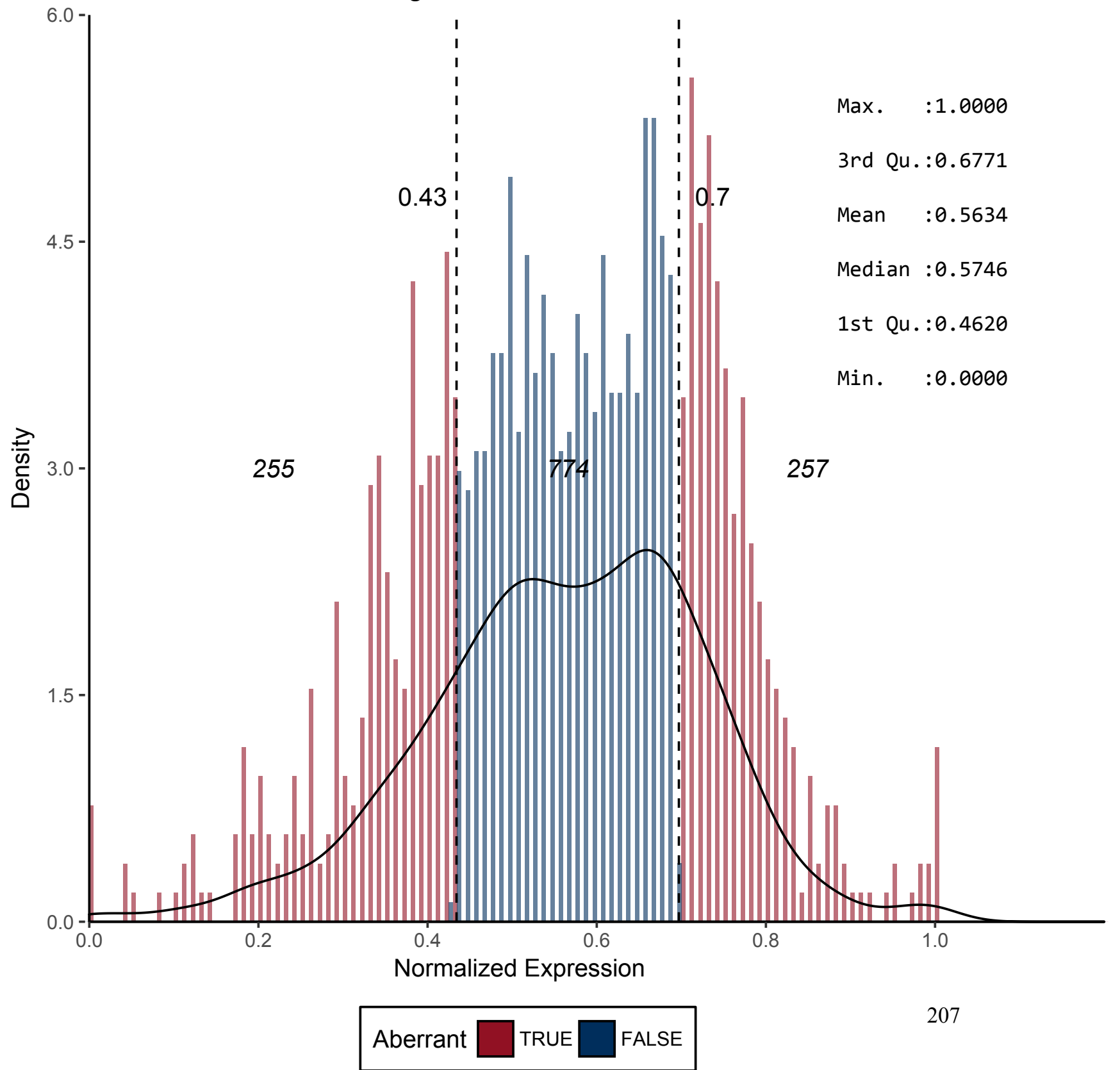


Figure 191 - CD172G

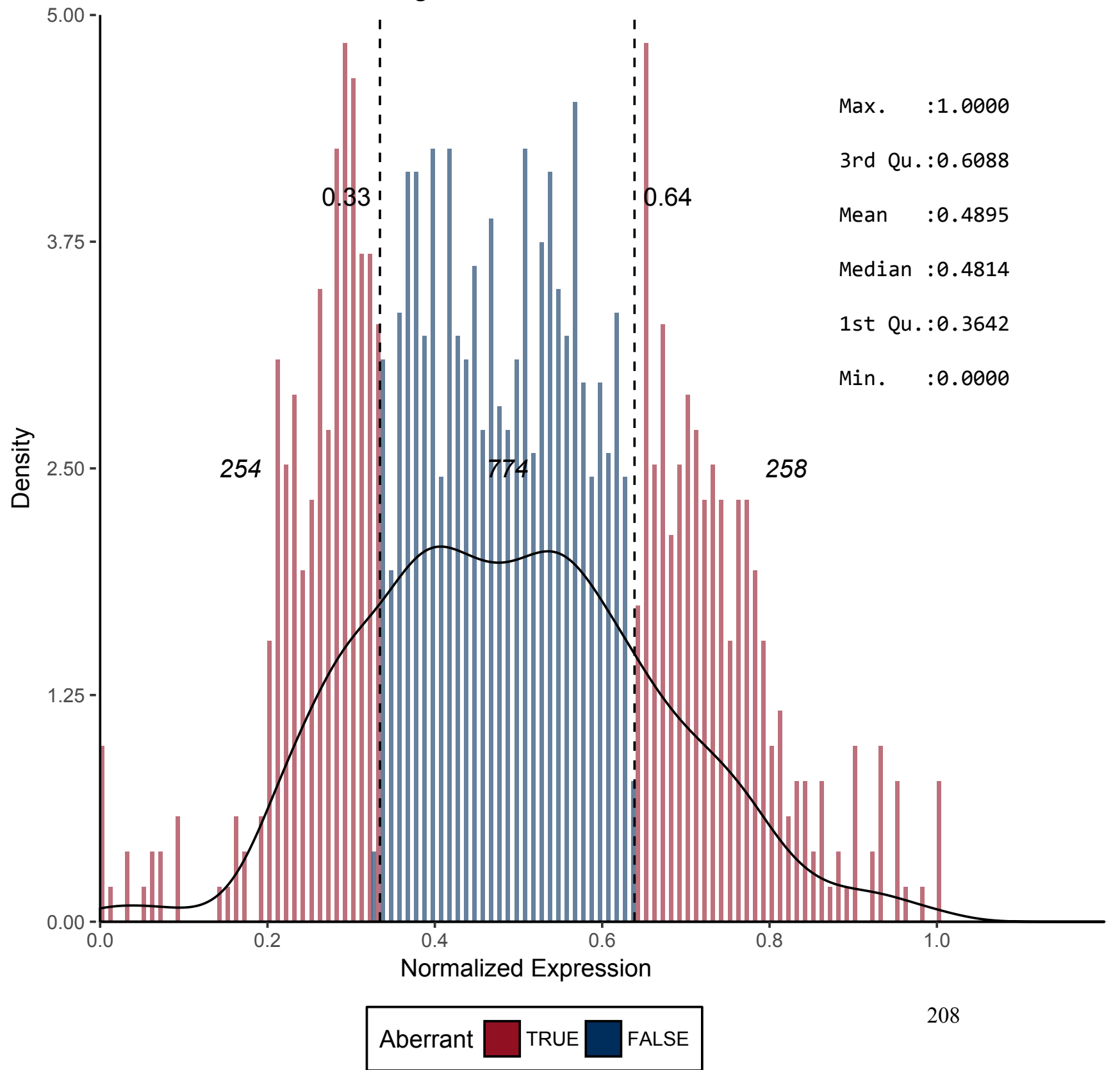


Figure 192 - CD174*

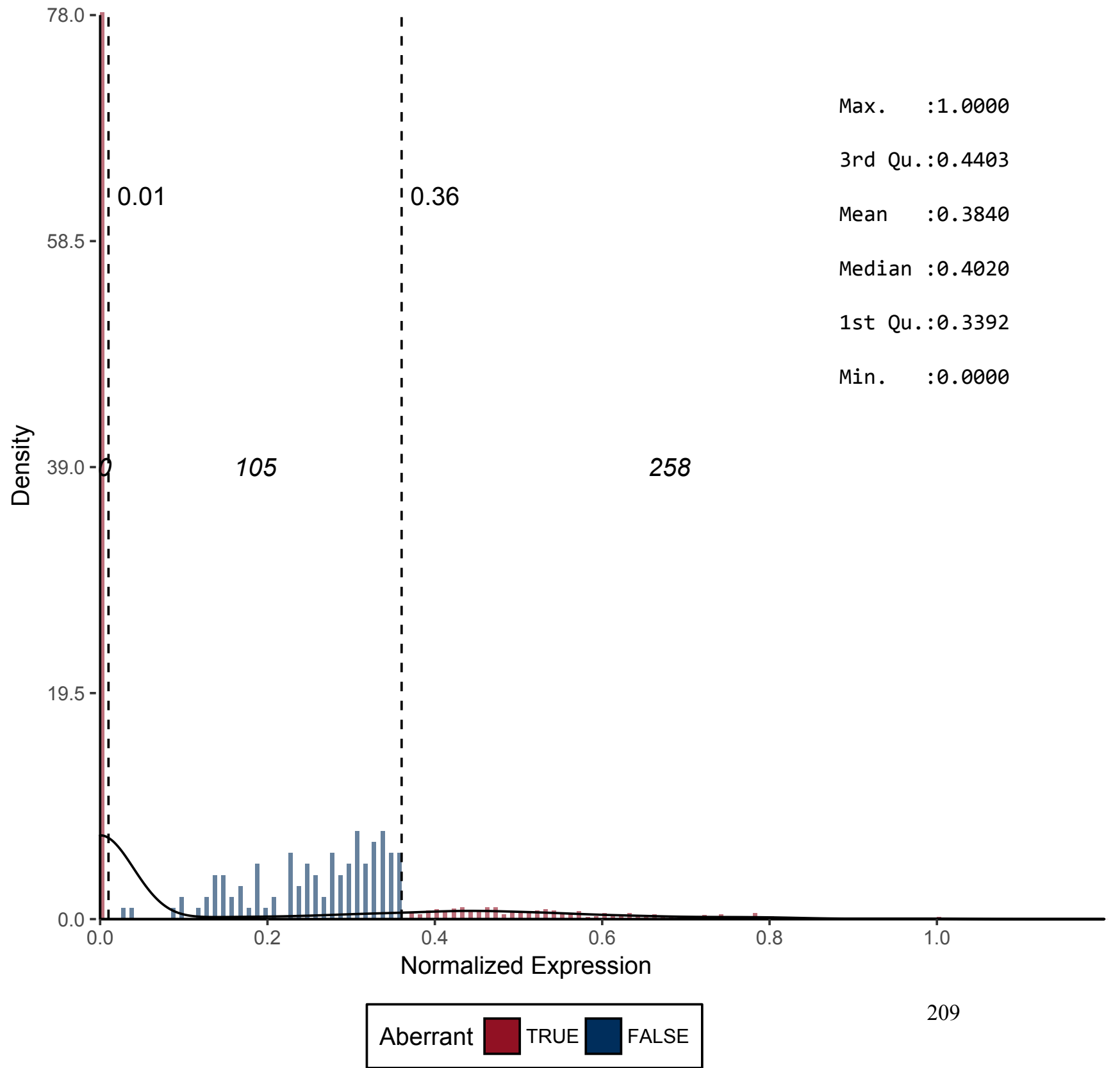


Figure 195 - CD179A*

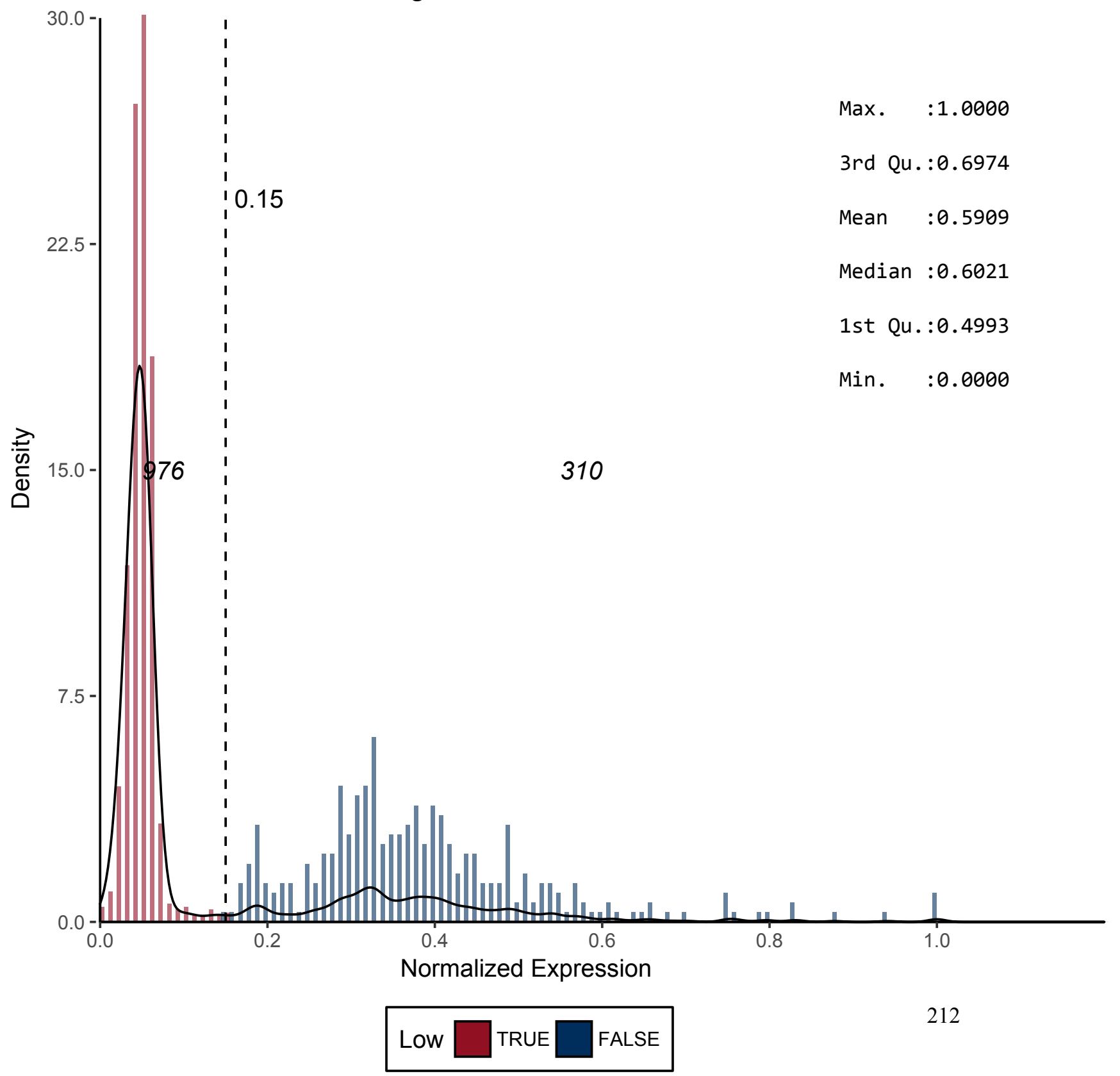


Figure 196 - CD179B

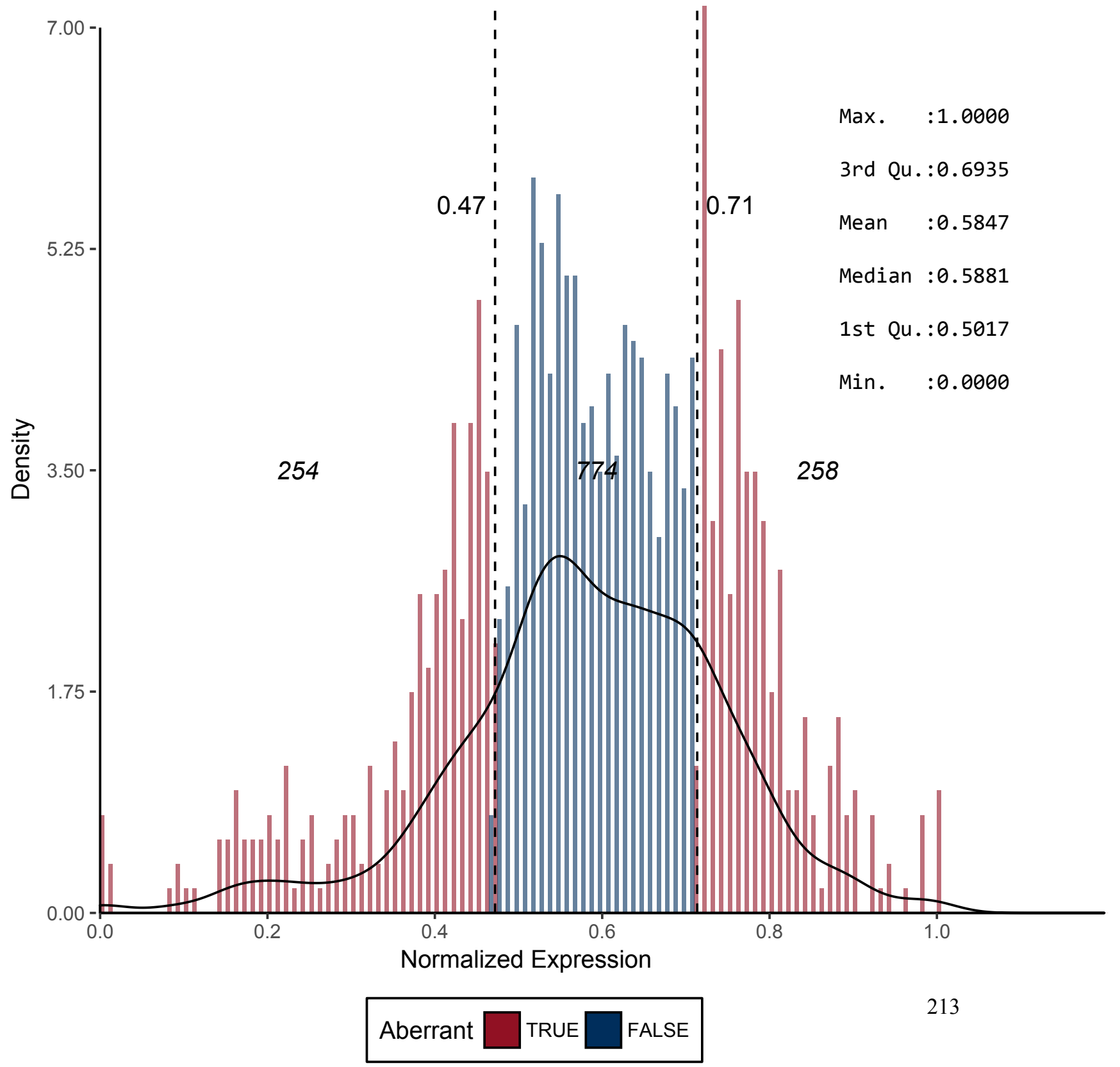


Figure 197 - CD180*

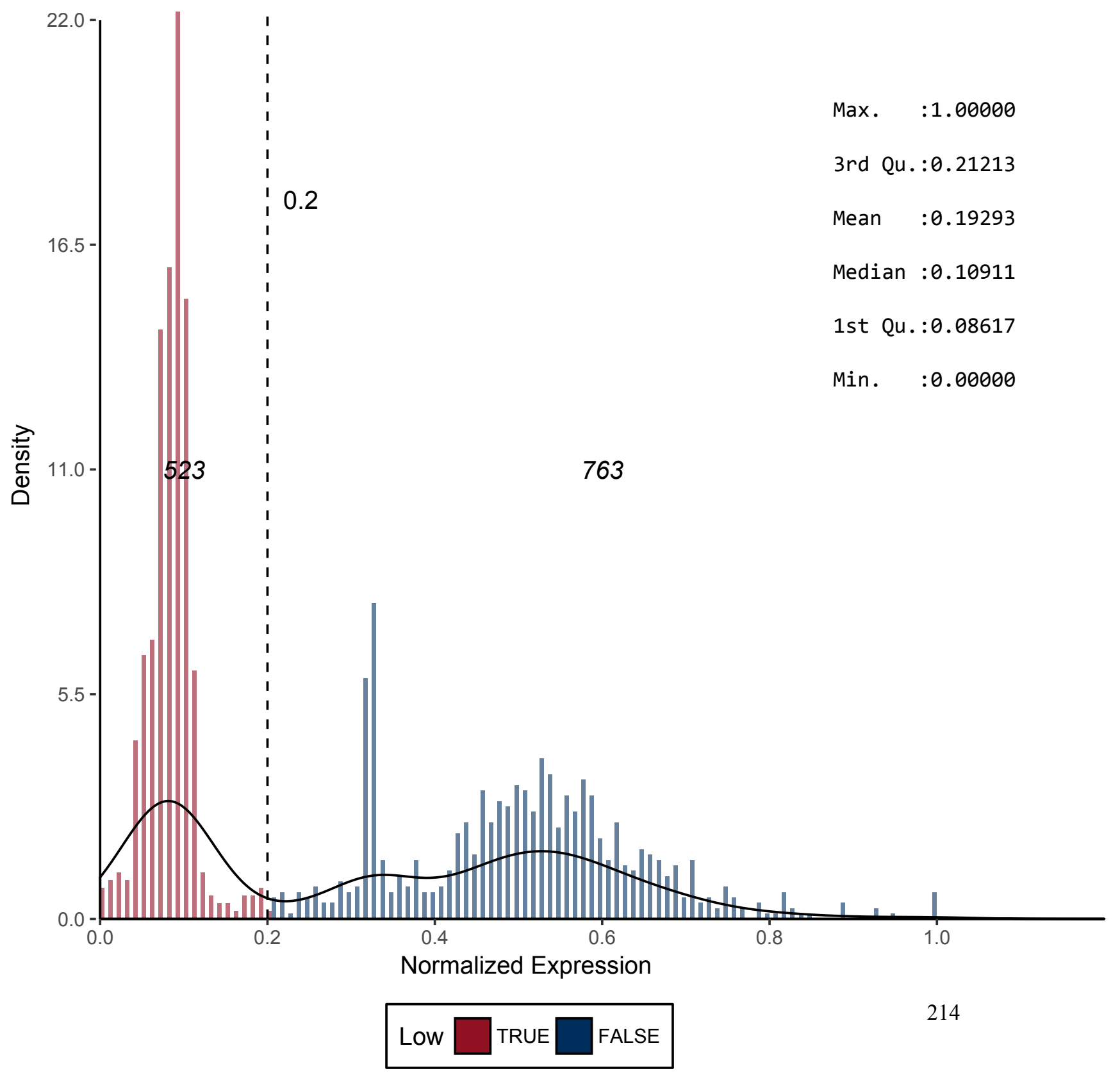


Figure 198 - CD181

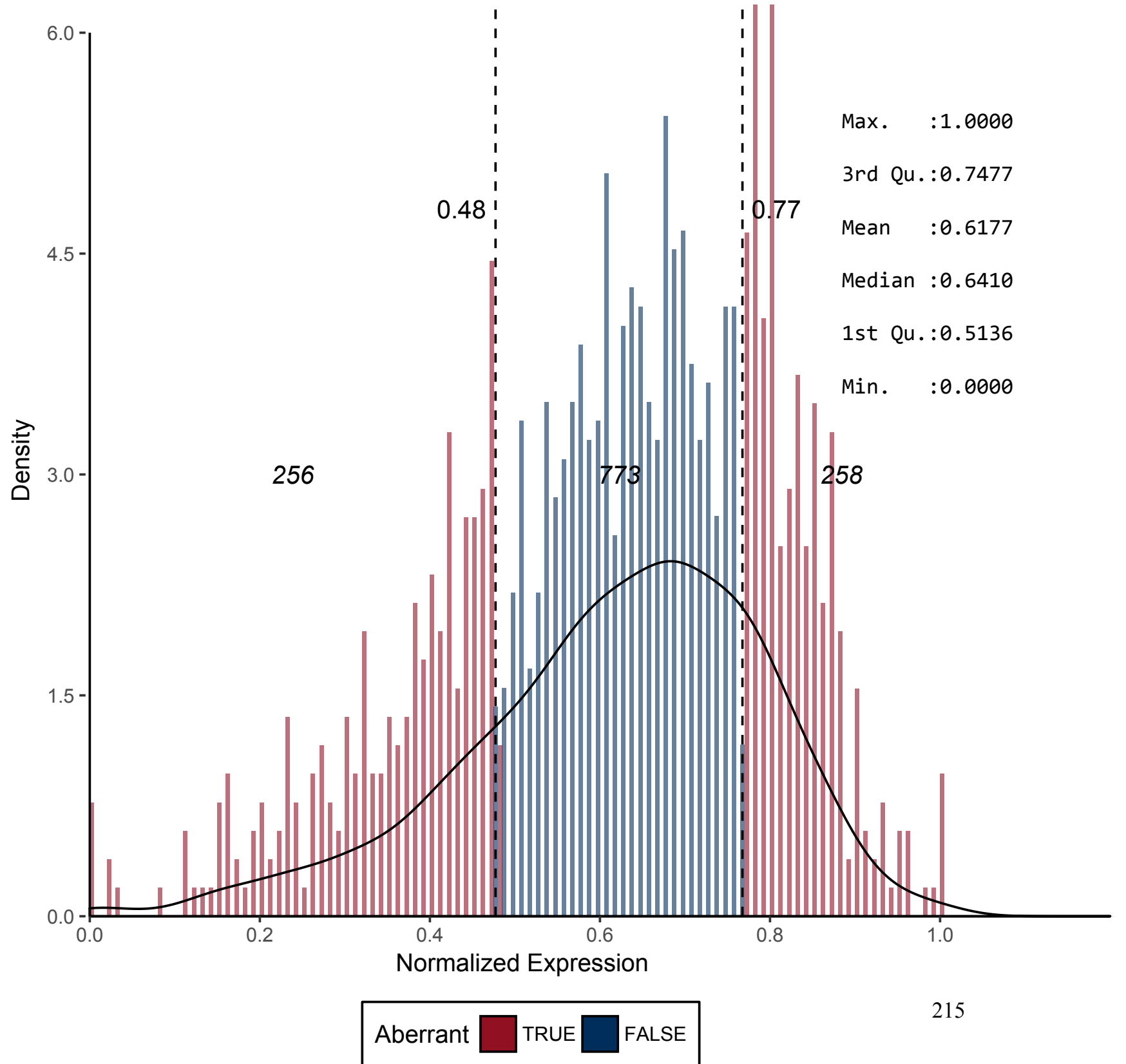


Figure 199 - CD182

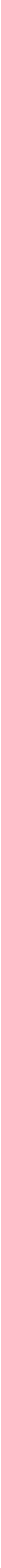


Figure 200 - CD183

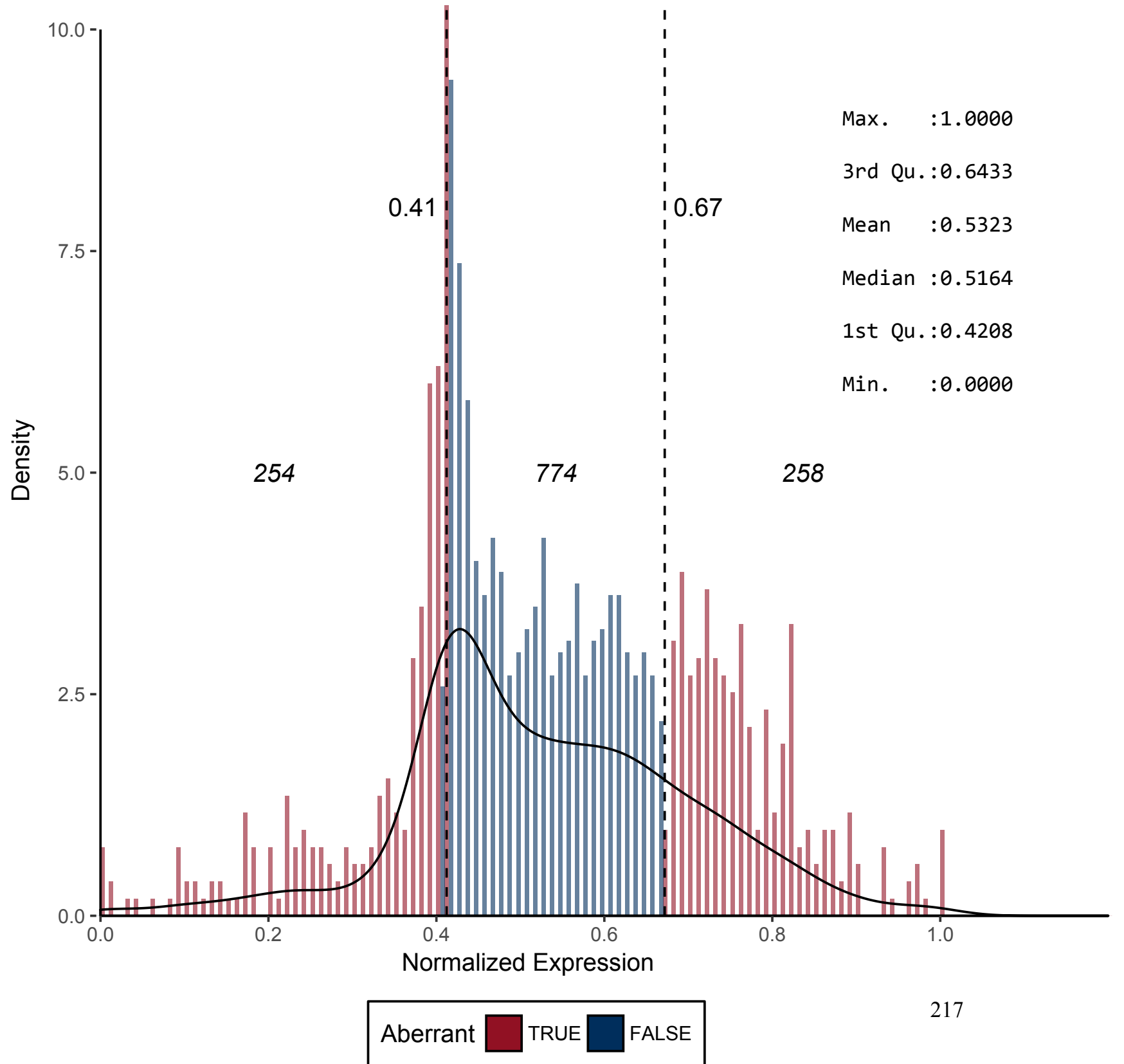


Figure 201 - CD184

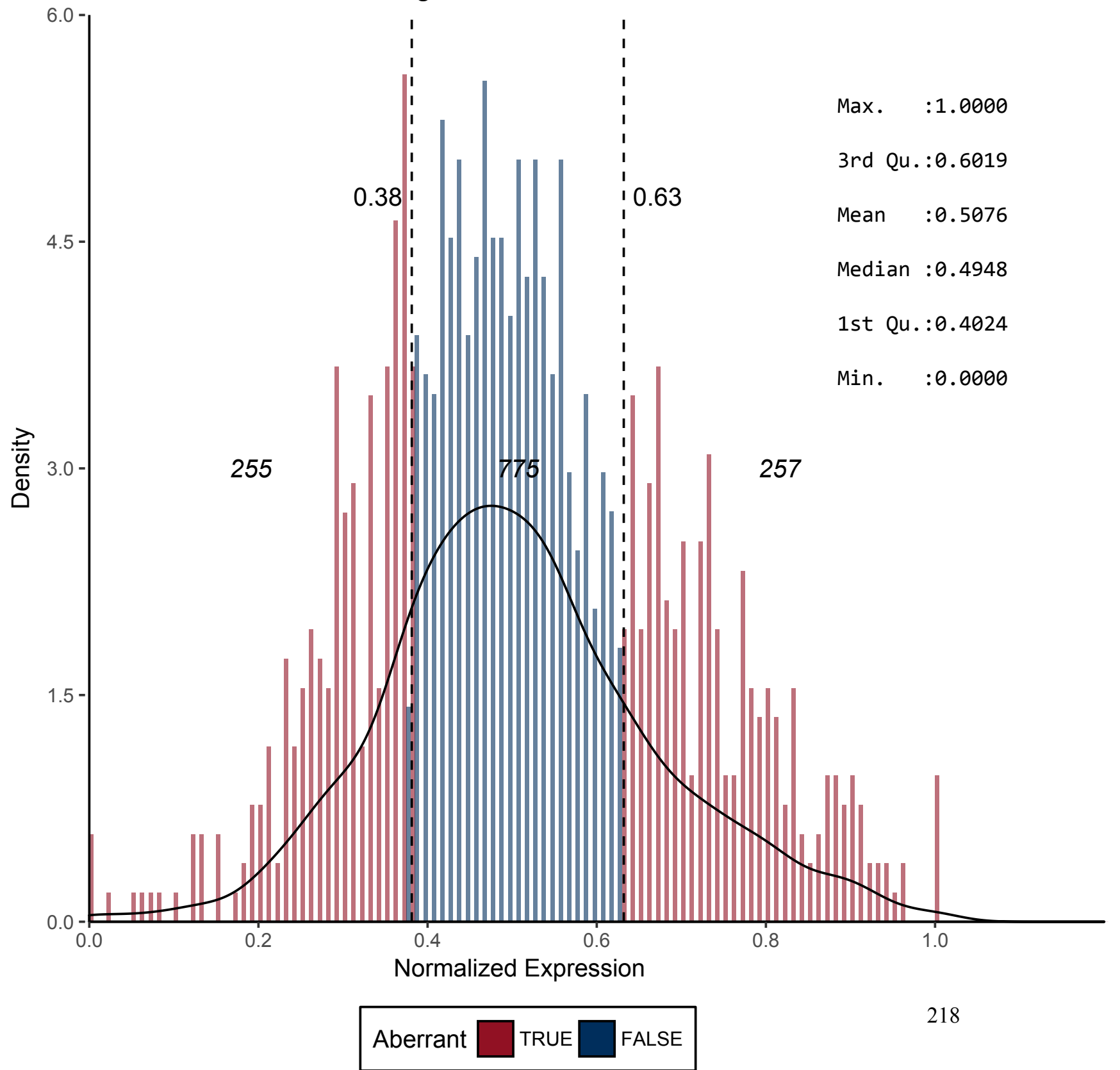


Figure 202 - CD185

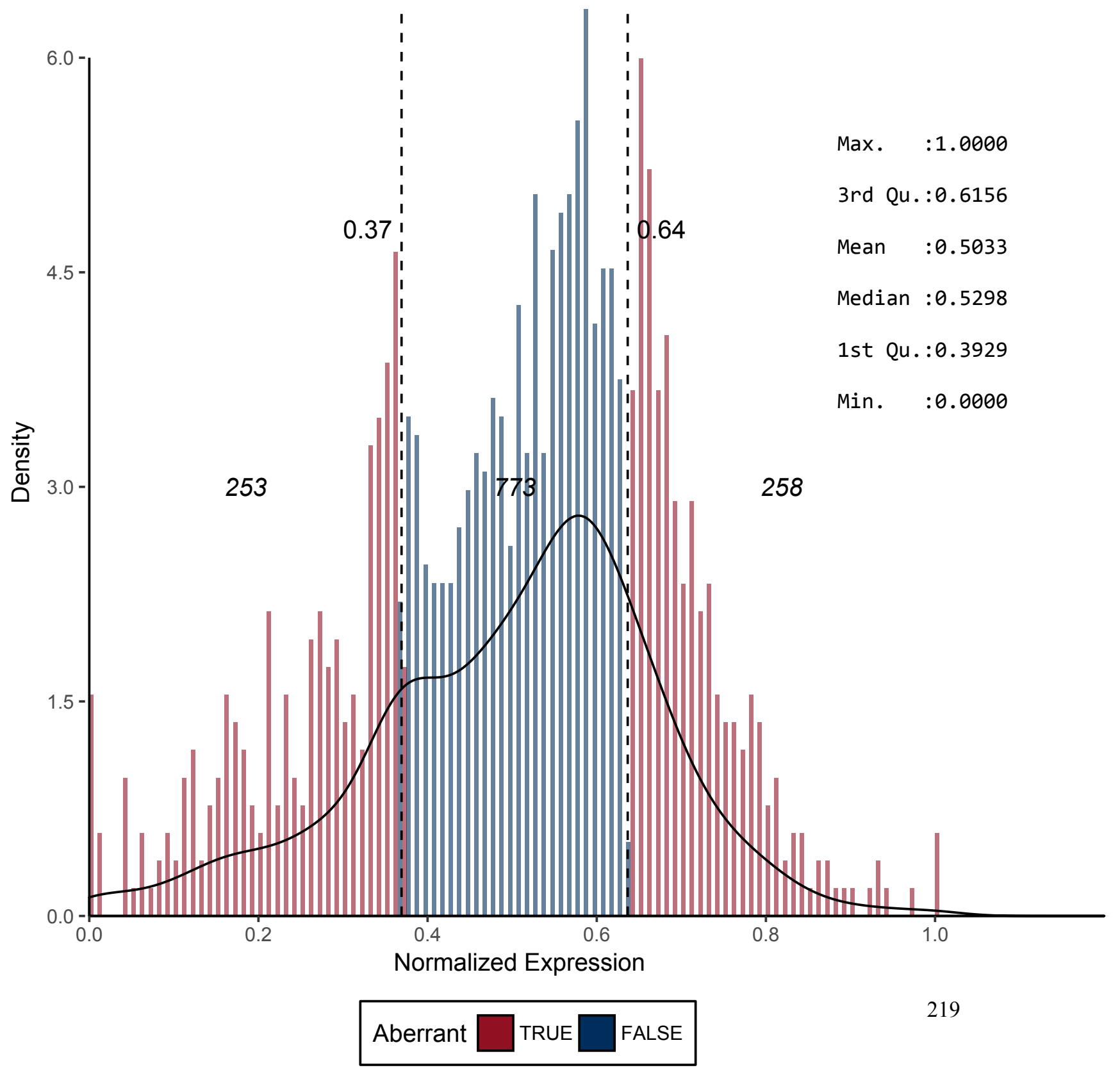


Figure 203 - CD186

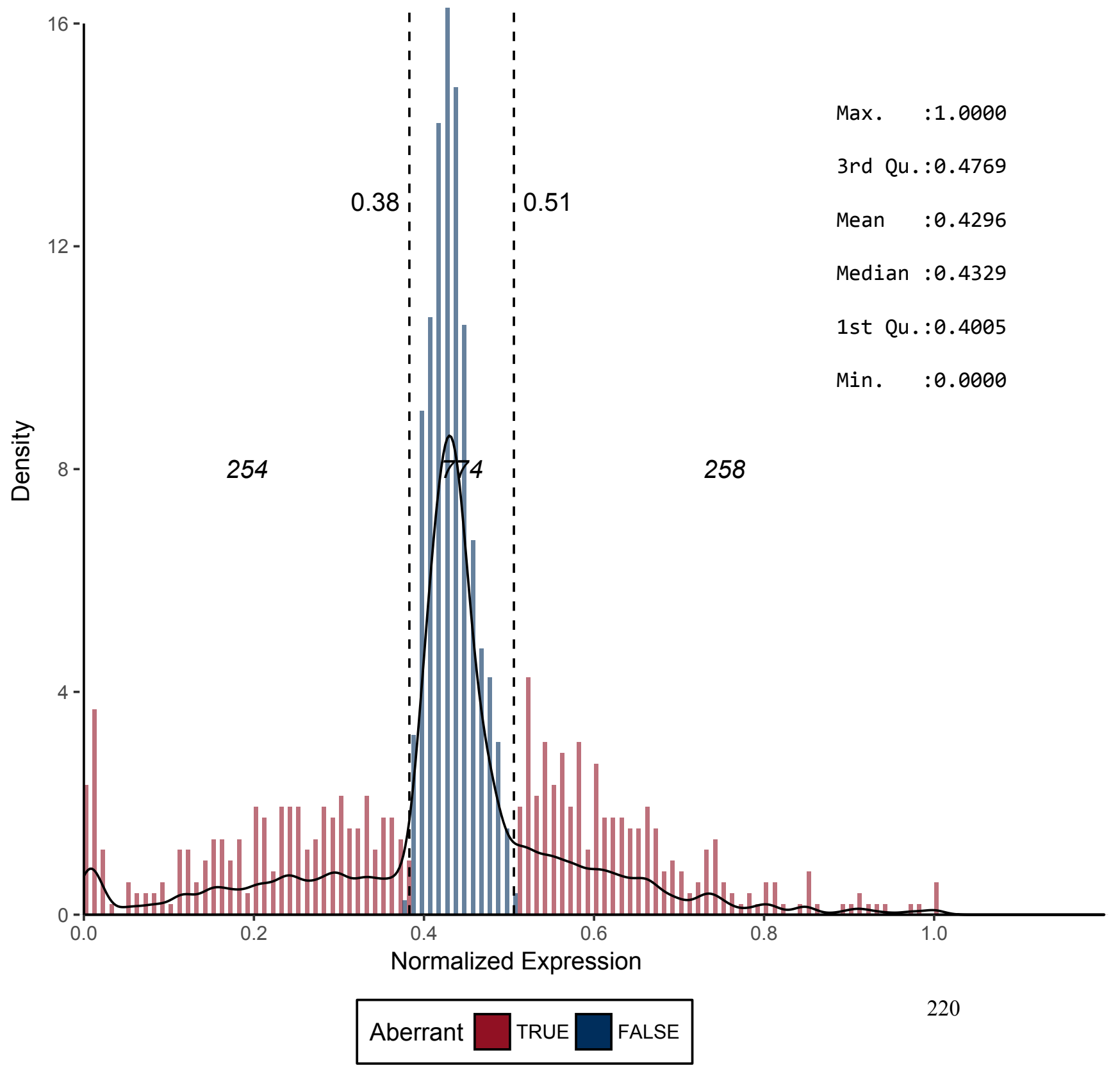


Figure 204 - CD191

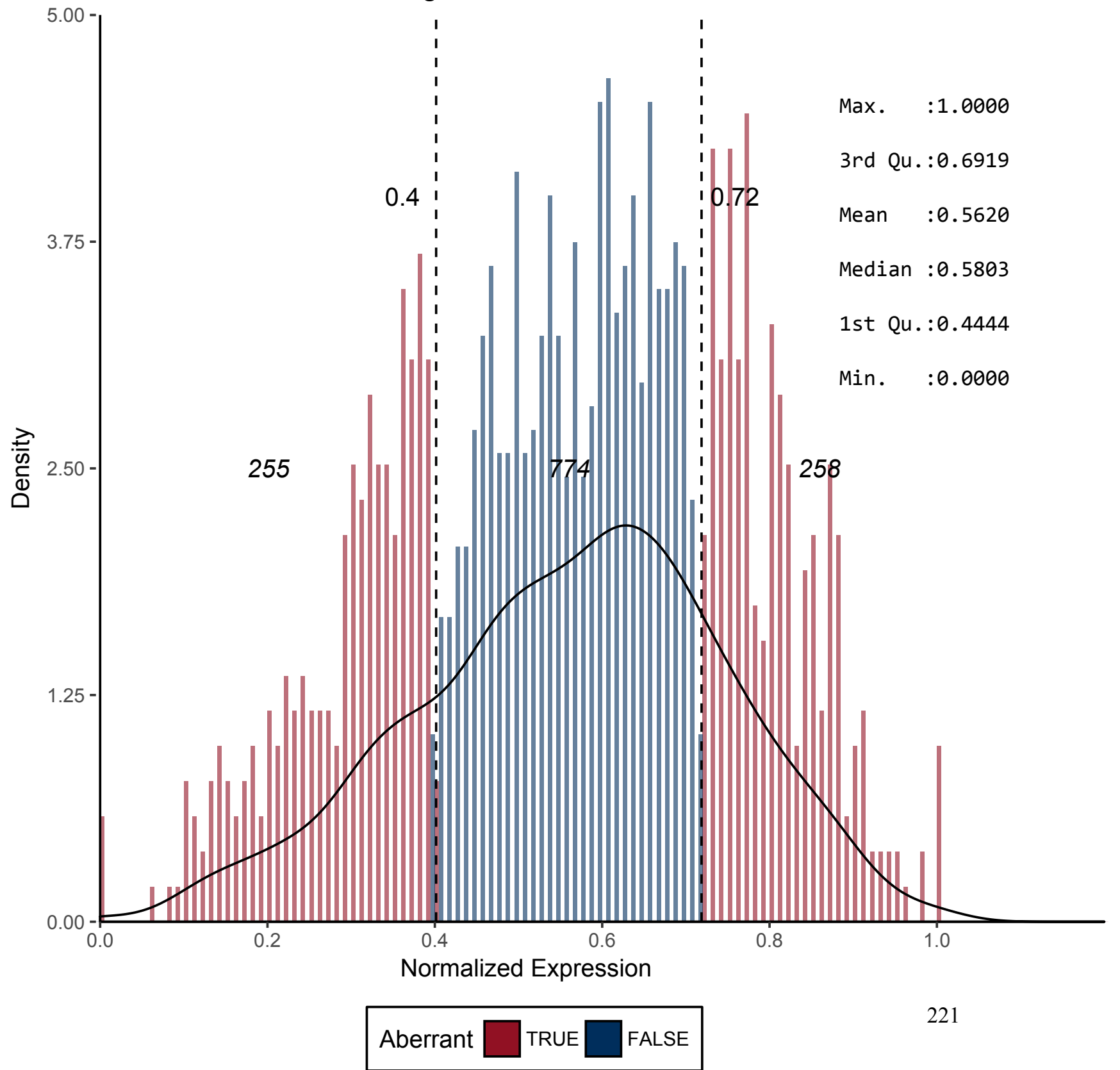


Figure 205 - CD193

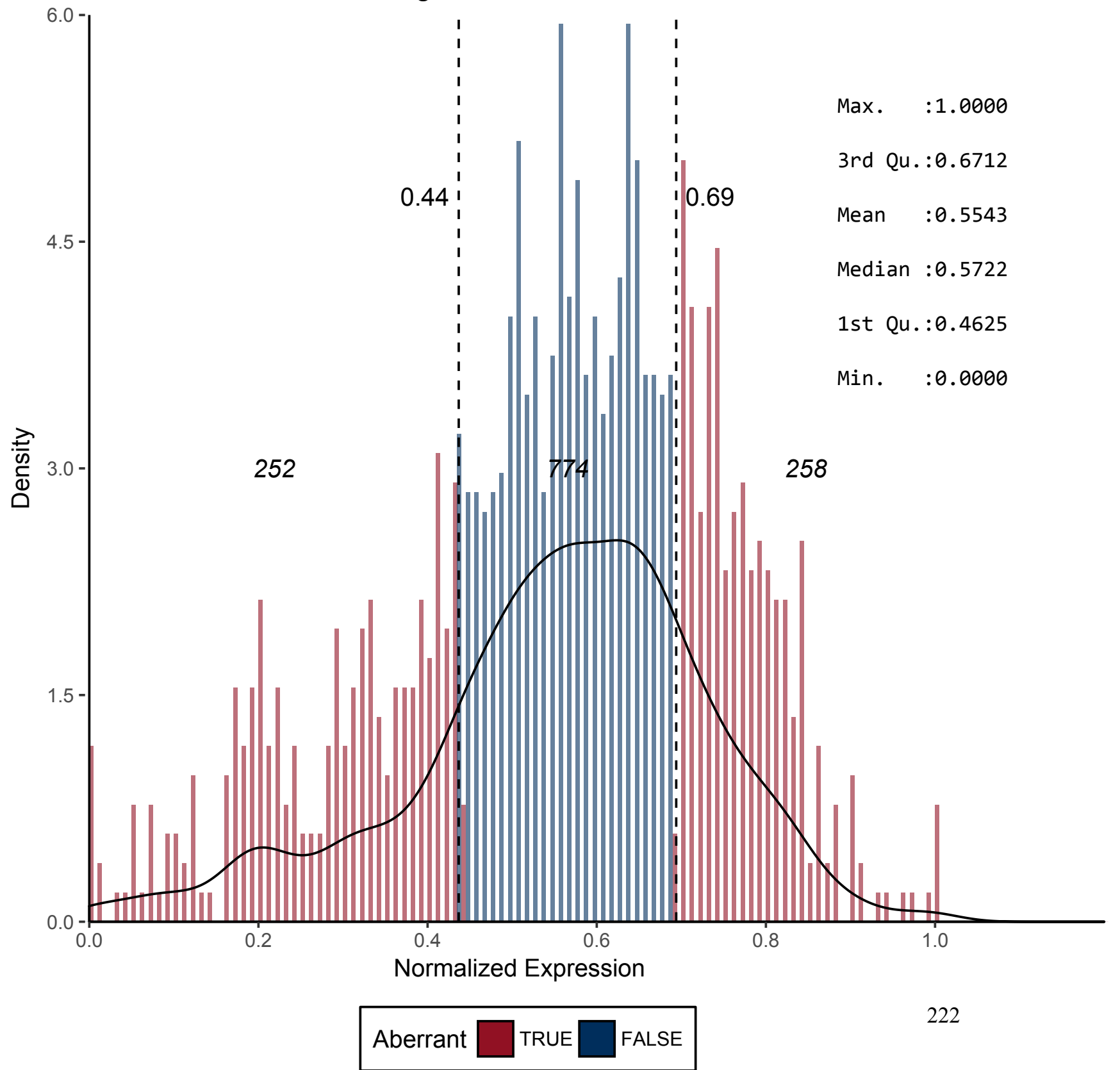


Figure 206 - CD194*

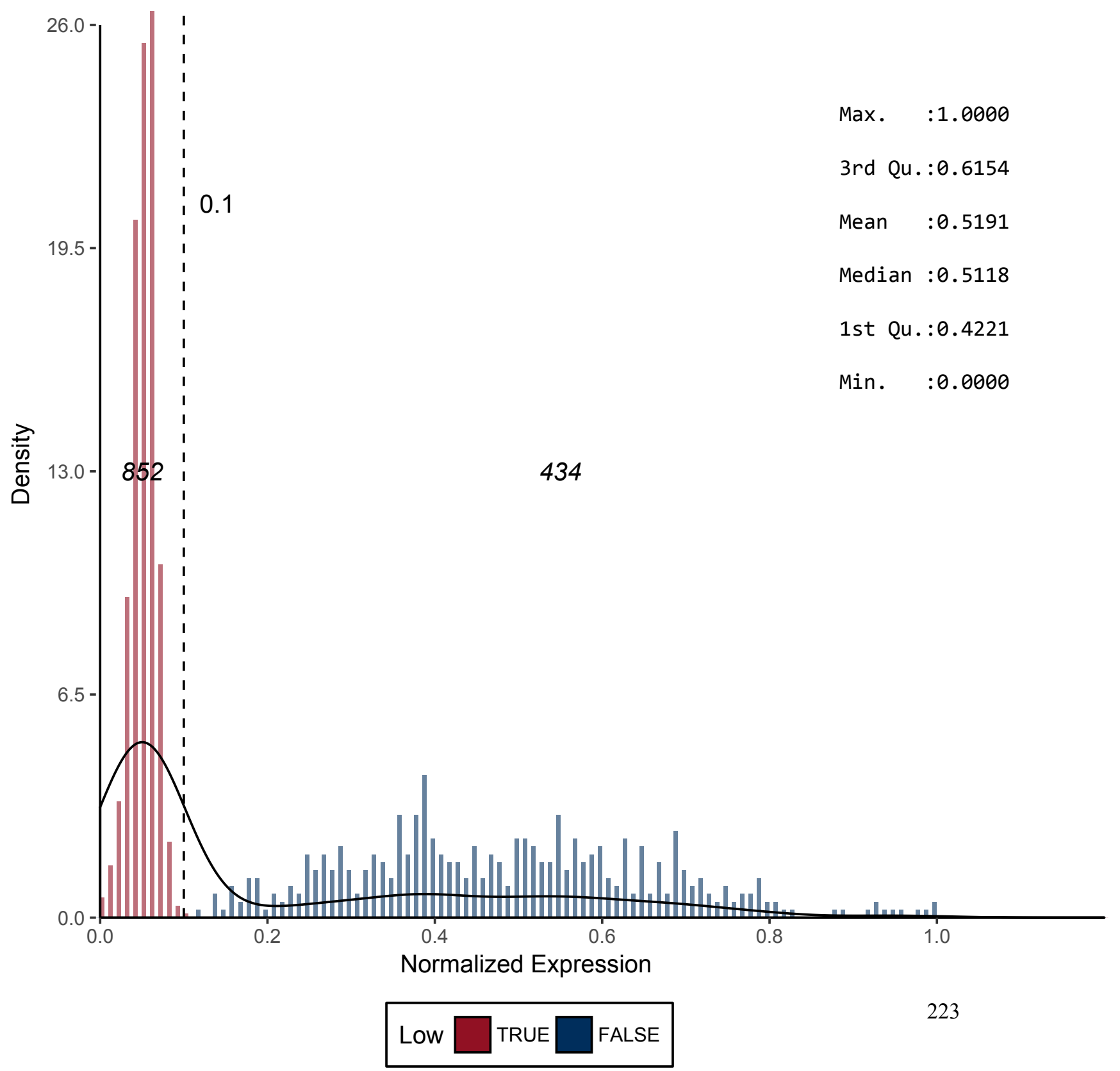


Figure 207 - CD195

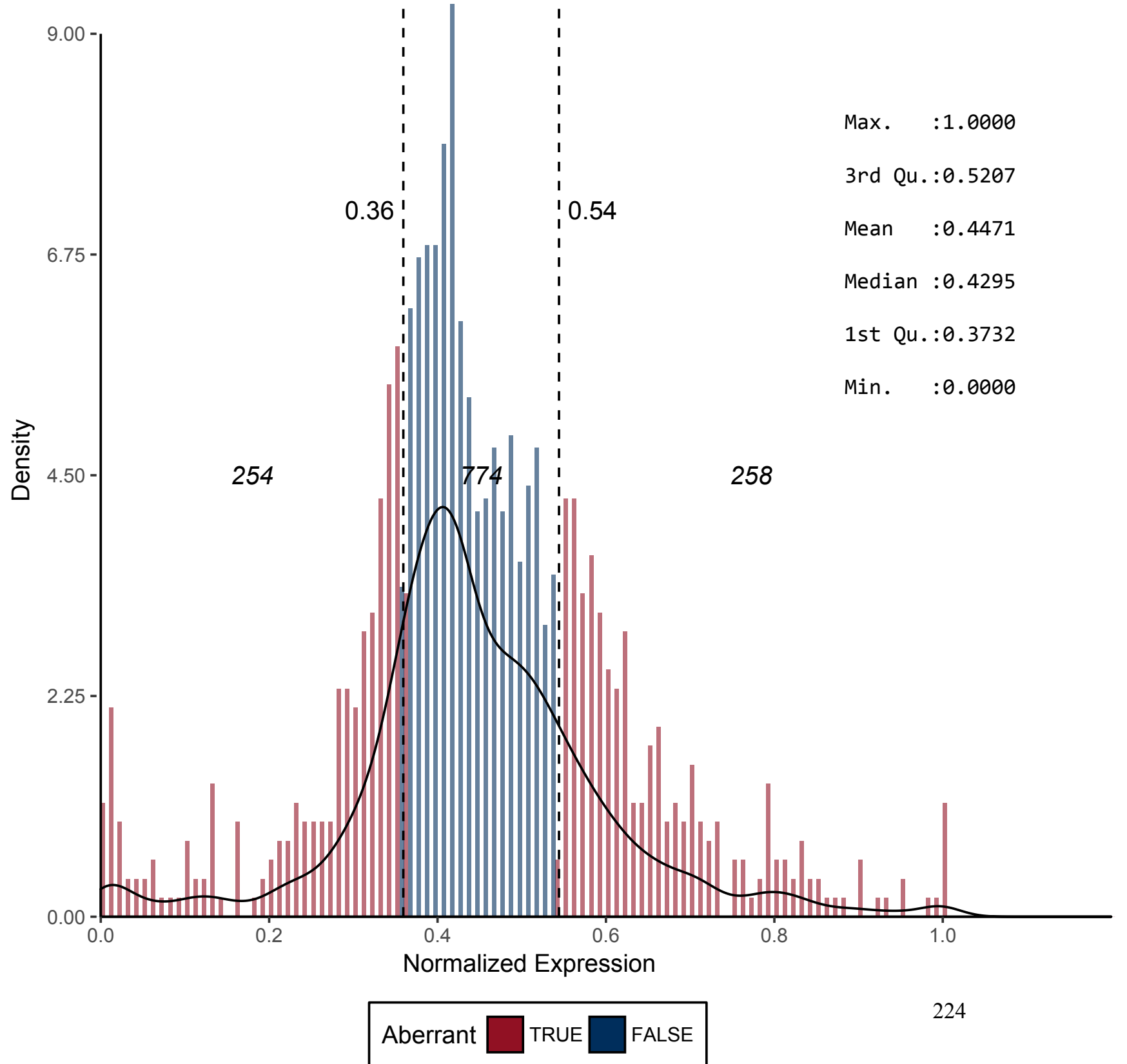


Figure 208 - CD196

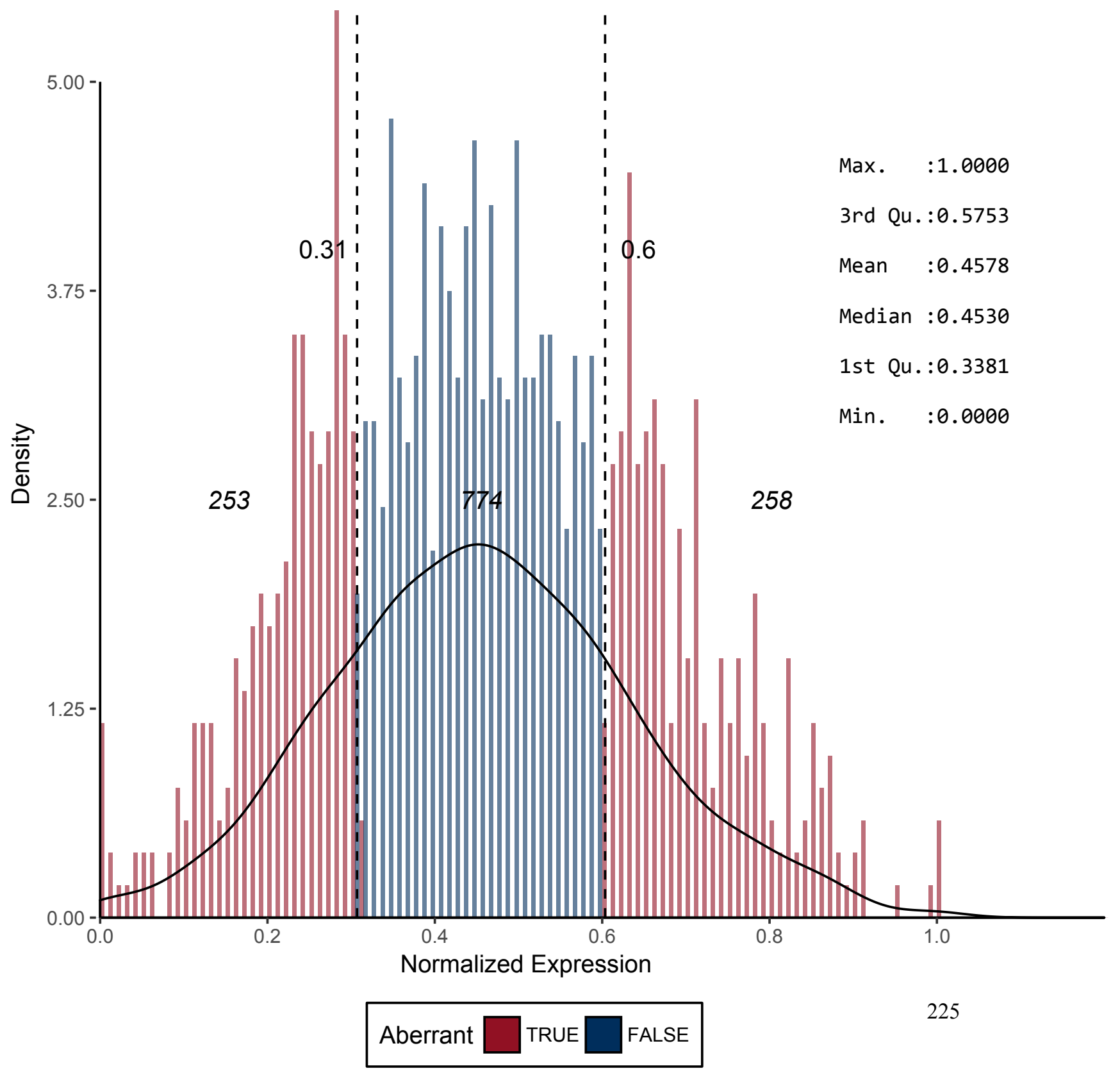


Figure 210 - CD199

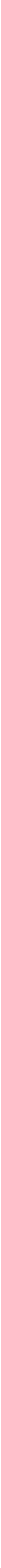


Figure 211 - CD200*

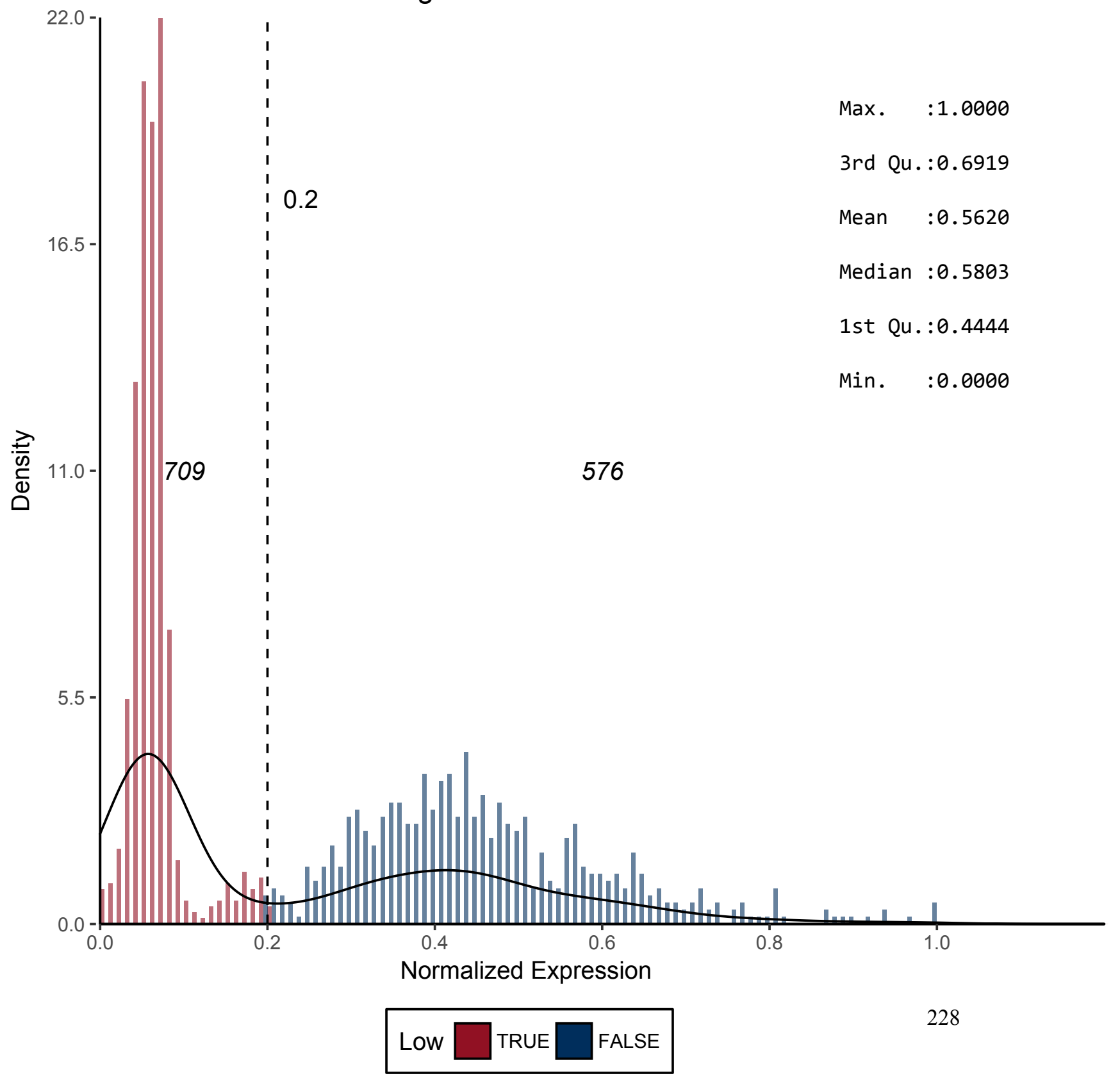


Figure 212 - CD201

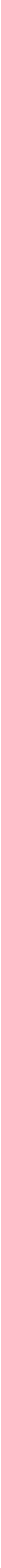


Figure 213 - CD202B*

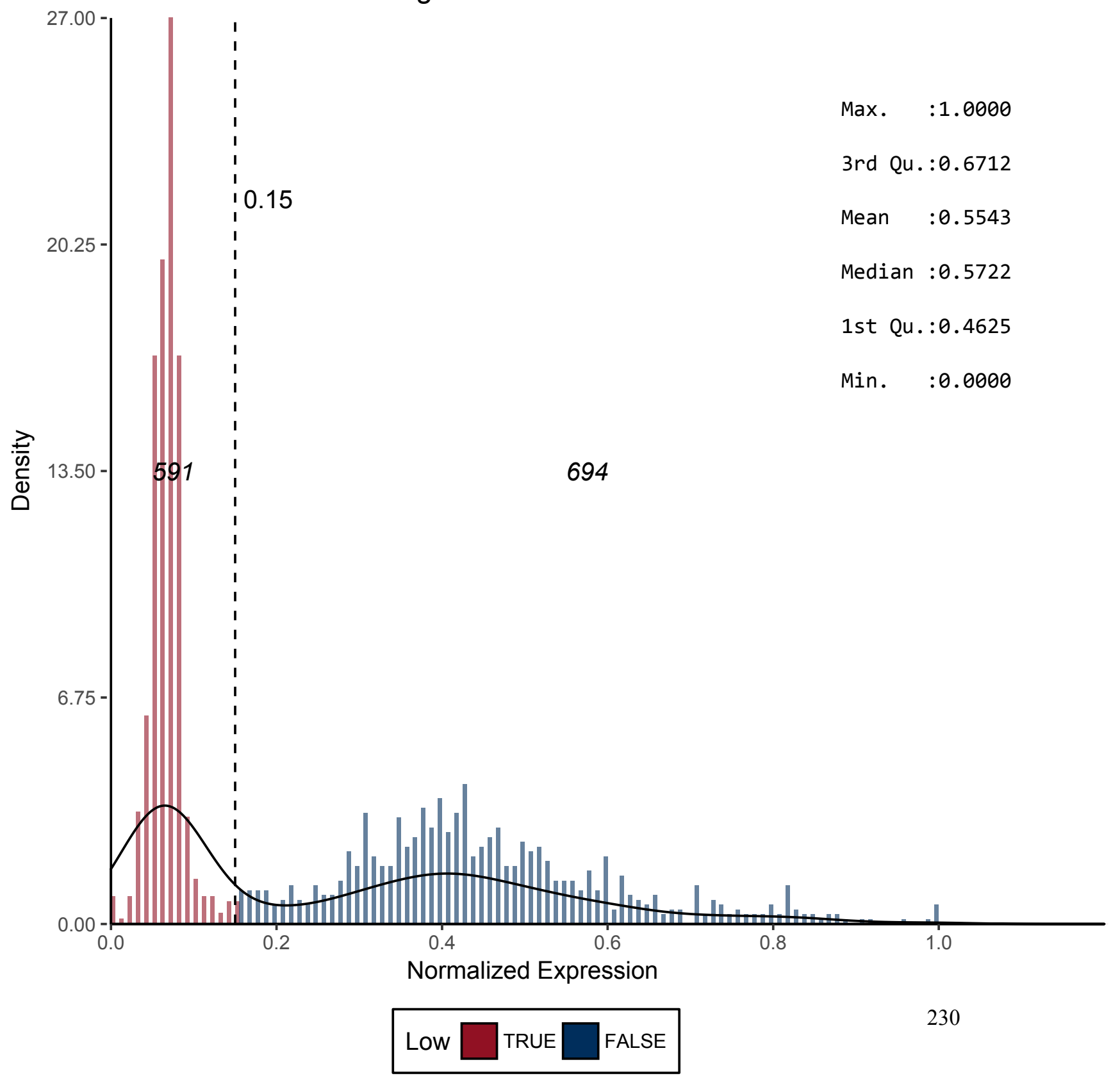


Figure 214 - CD203C*

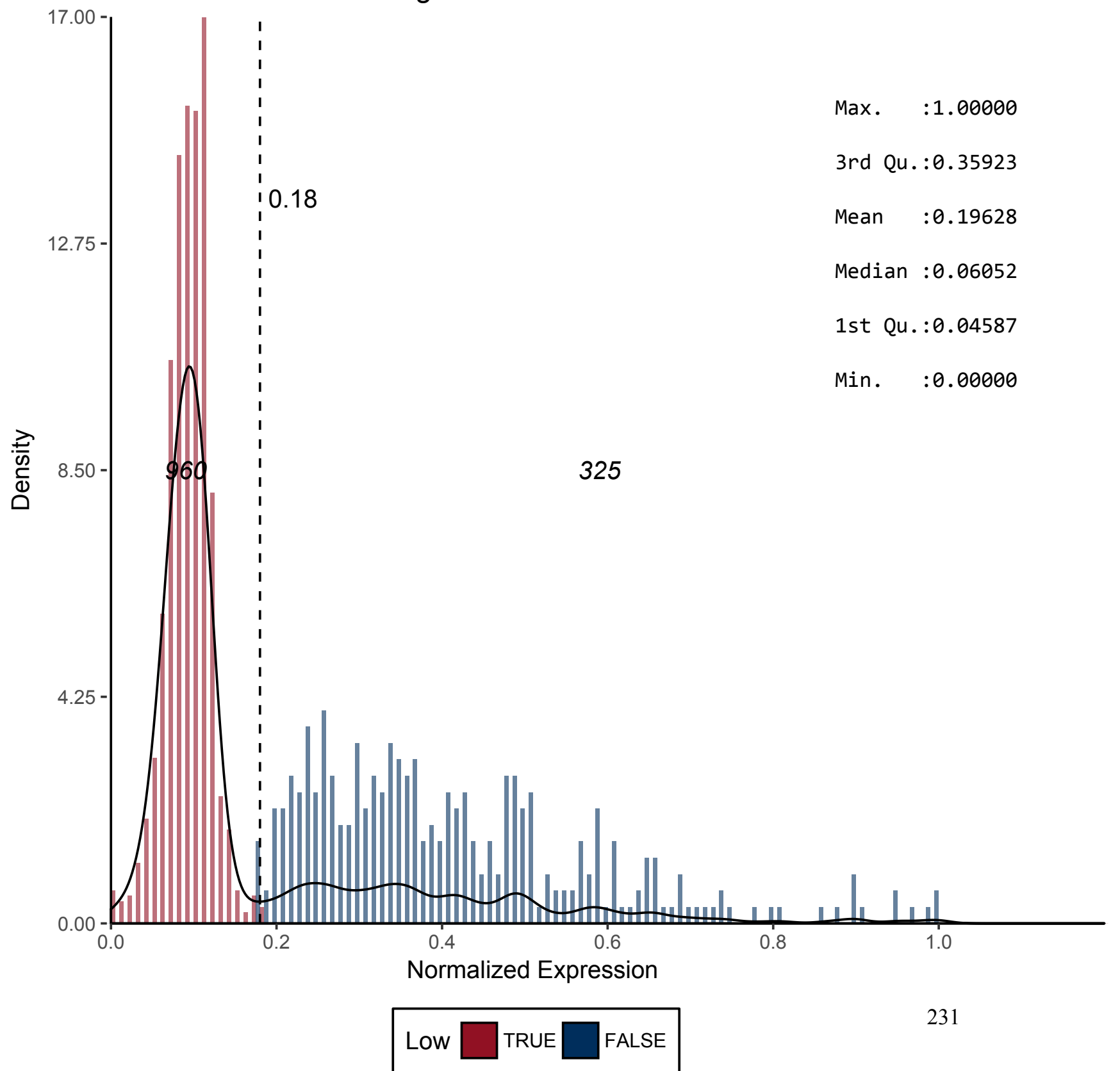


Figure 215 - CD204*

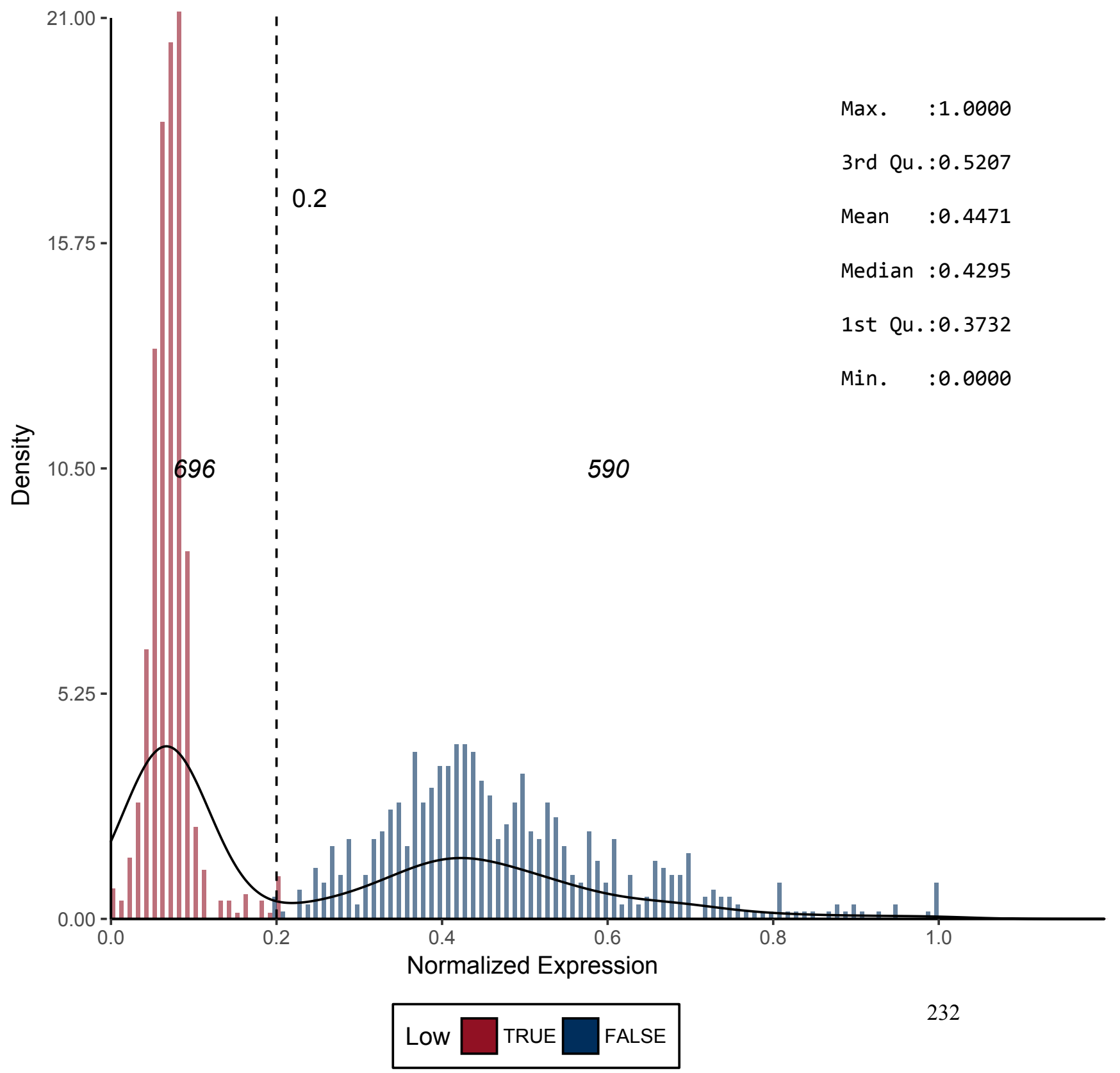


Figure 217 - CD206*

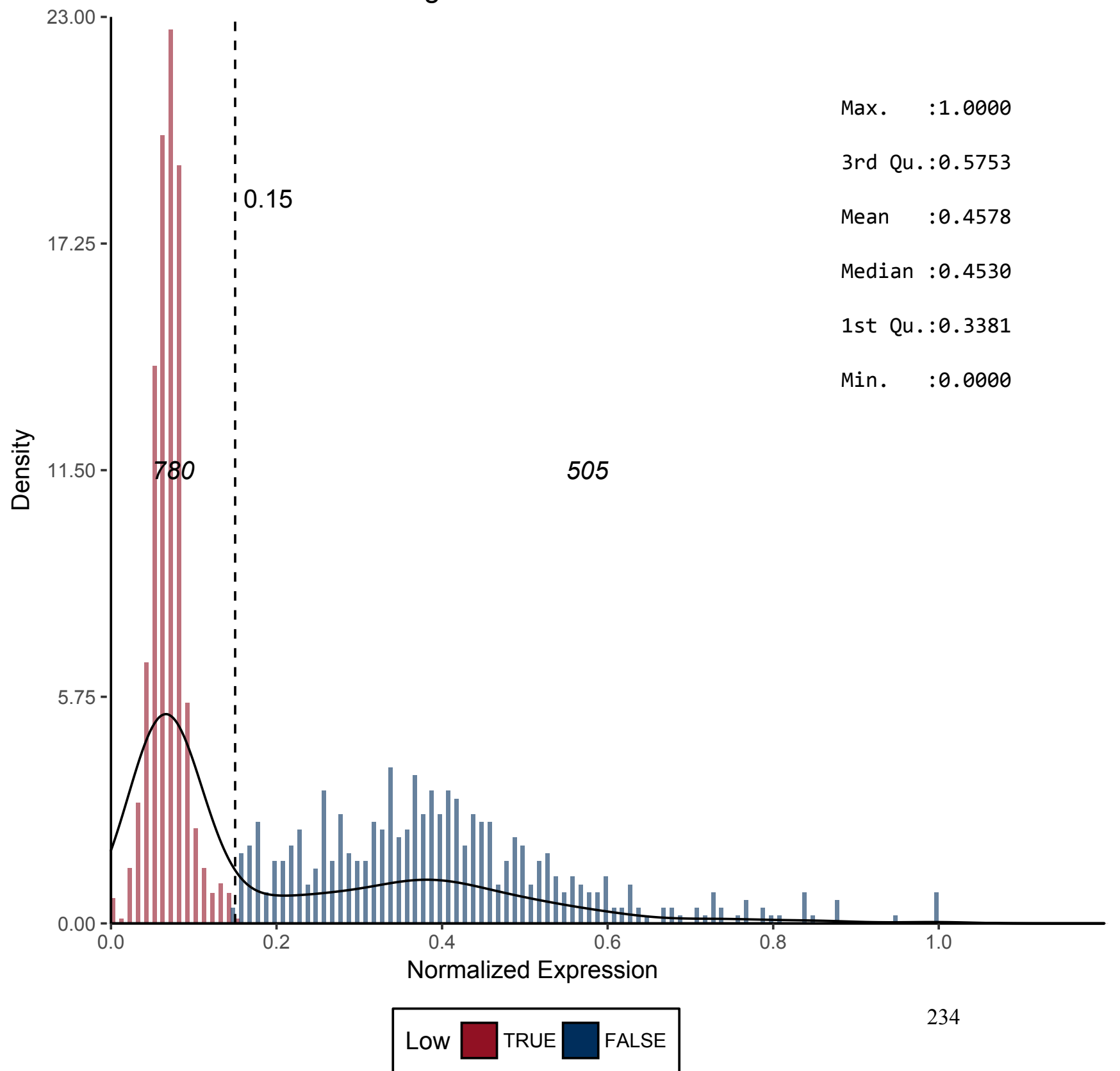


Figure 218 - CD207*

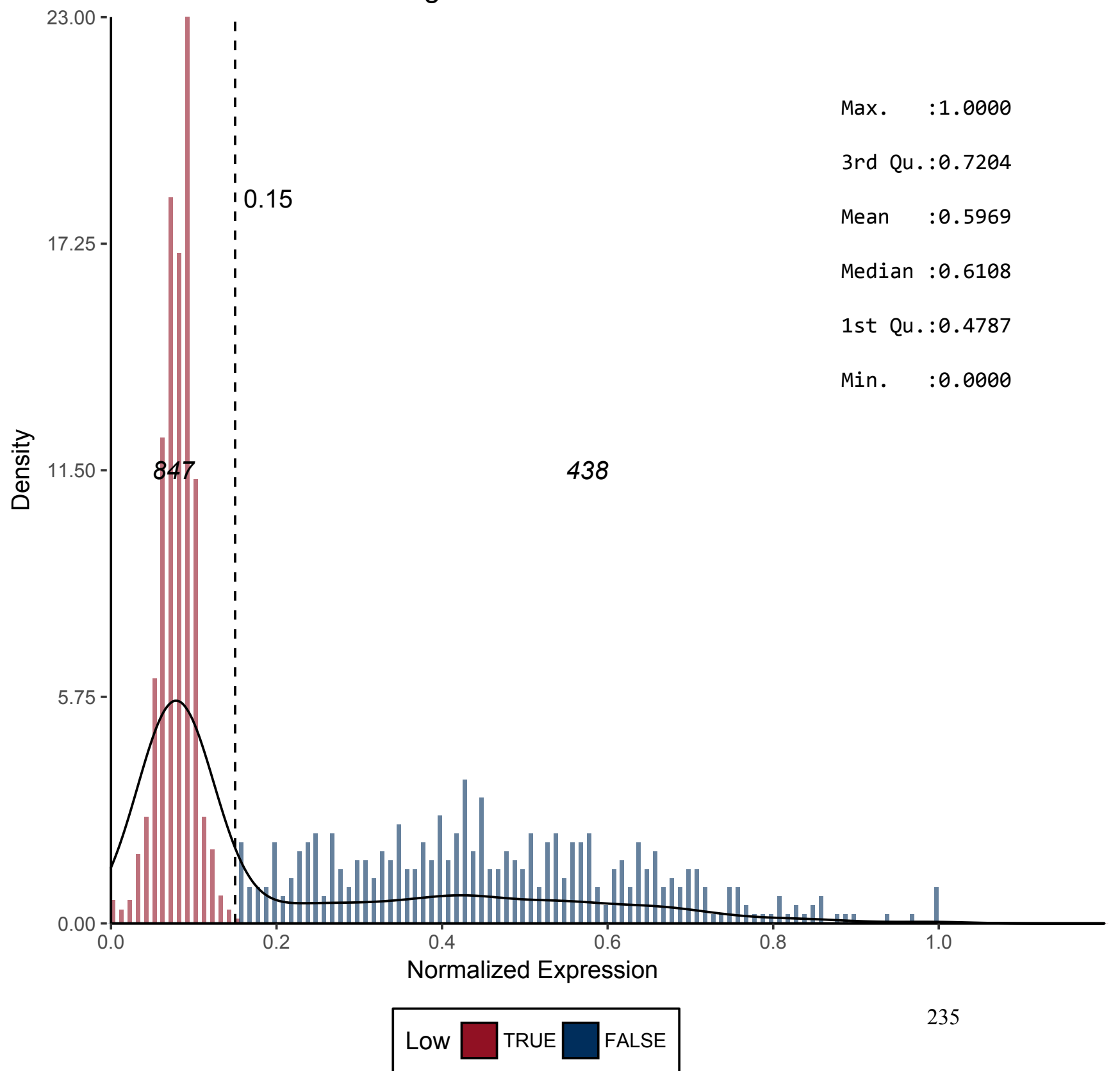


Figure 219 - CD208

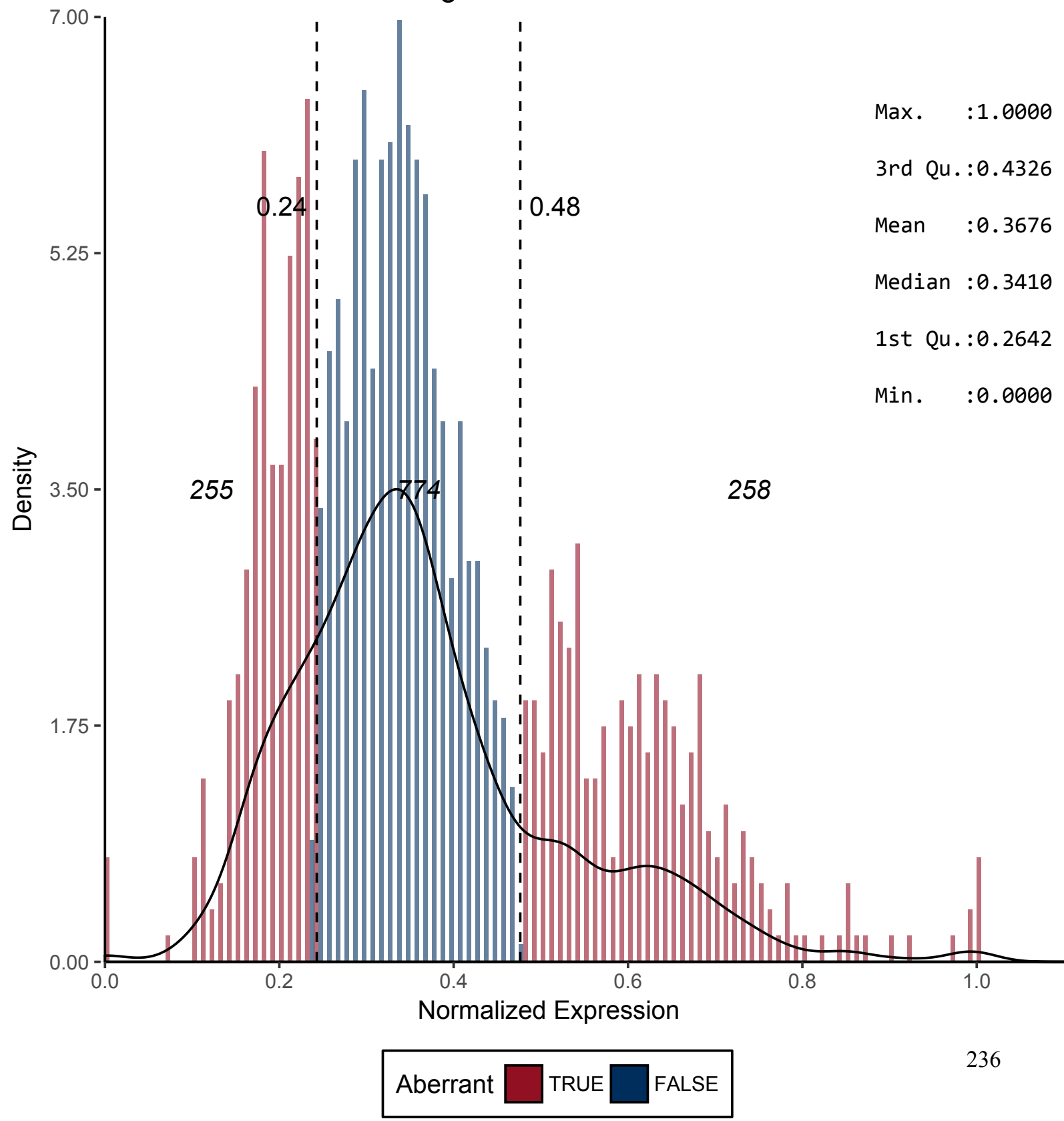


Figure 221 - CD210

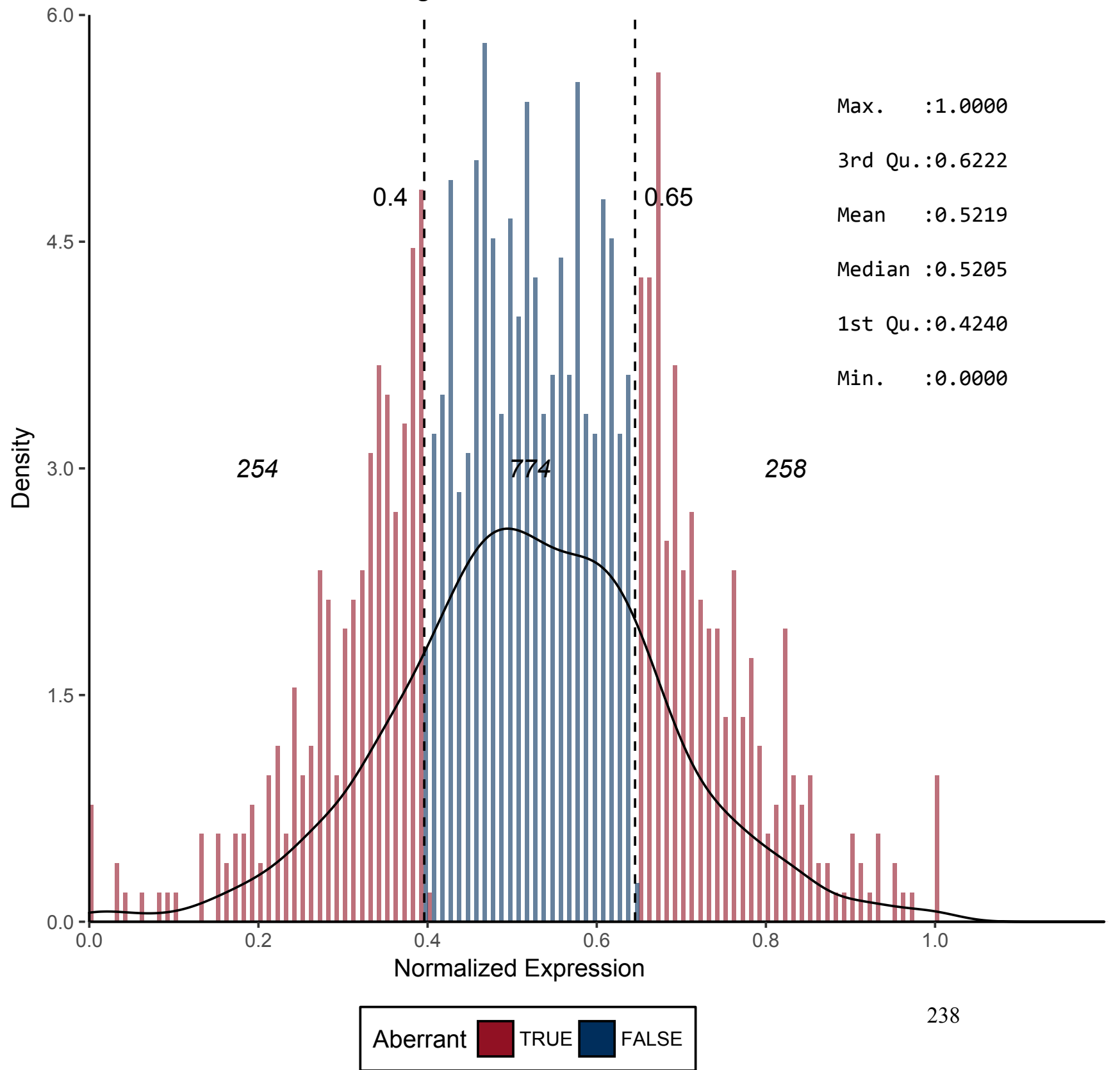


Figure 222 - CDW210B

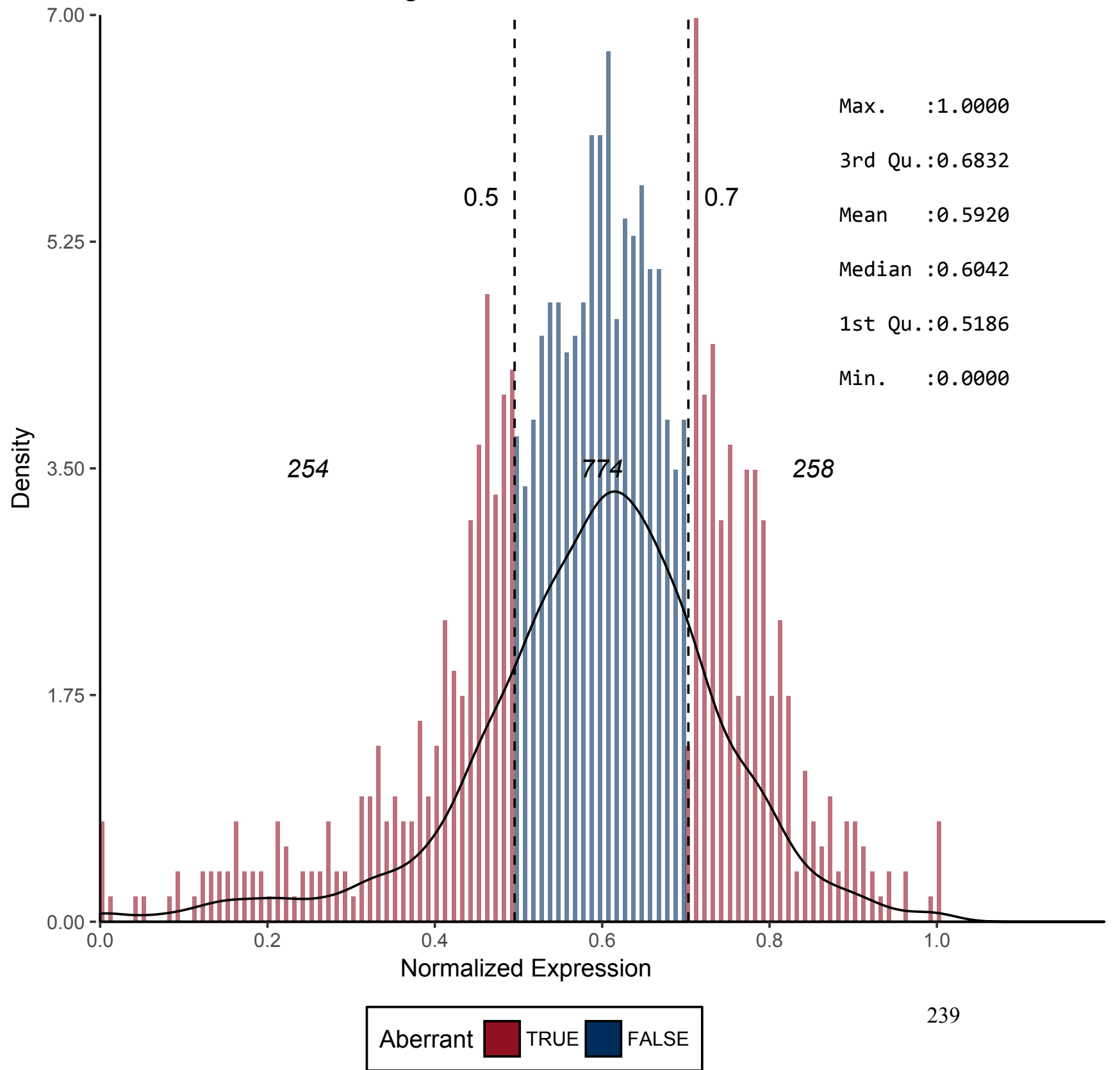


Figure 223 - CD212

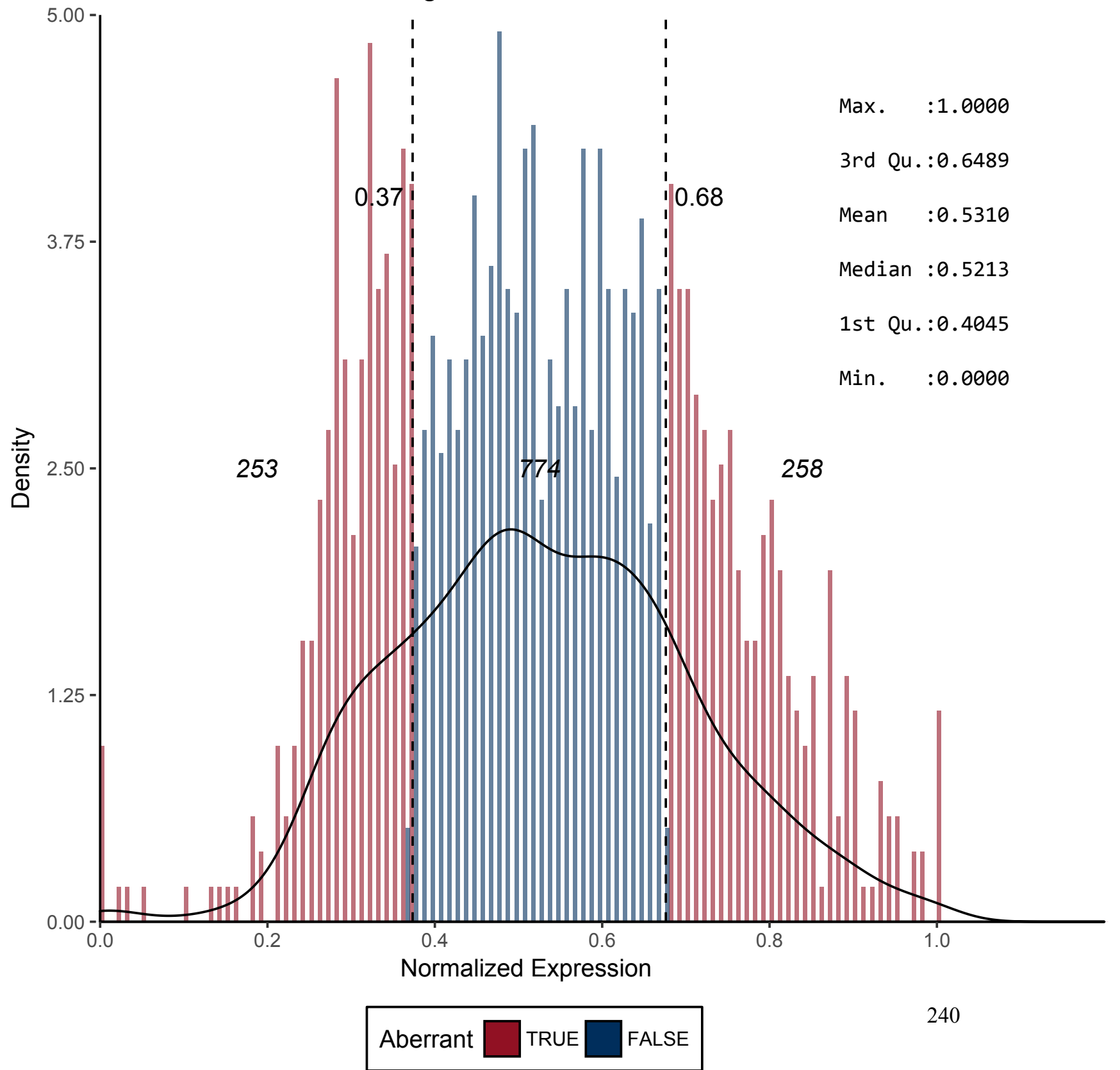


Figure 224 - CD213A1
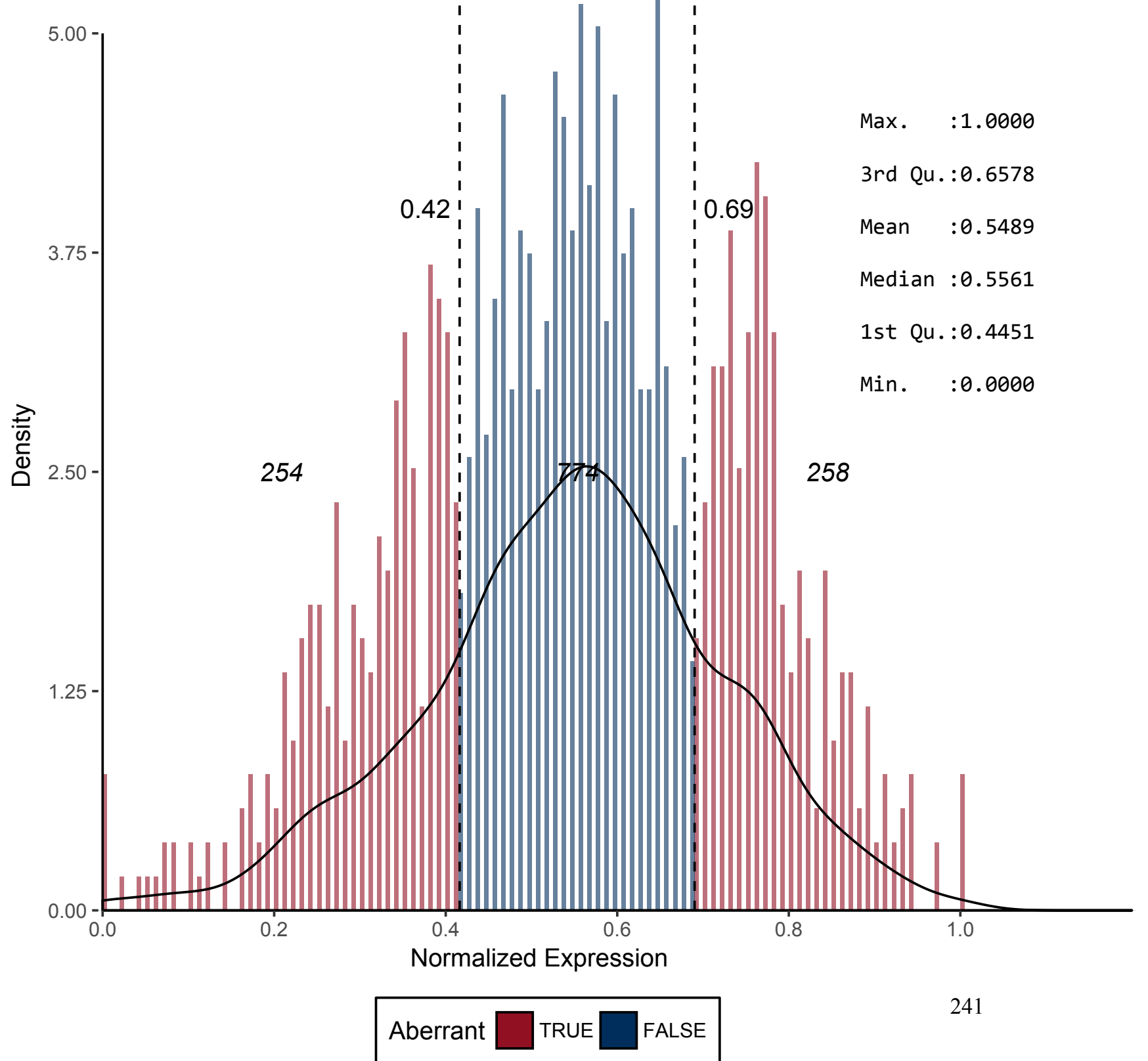
Figure 225 - CD213A2*

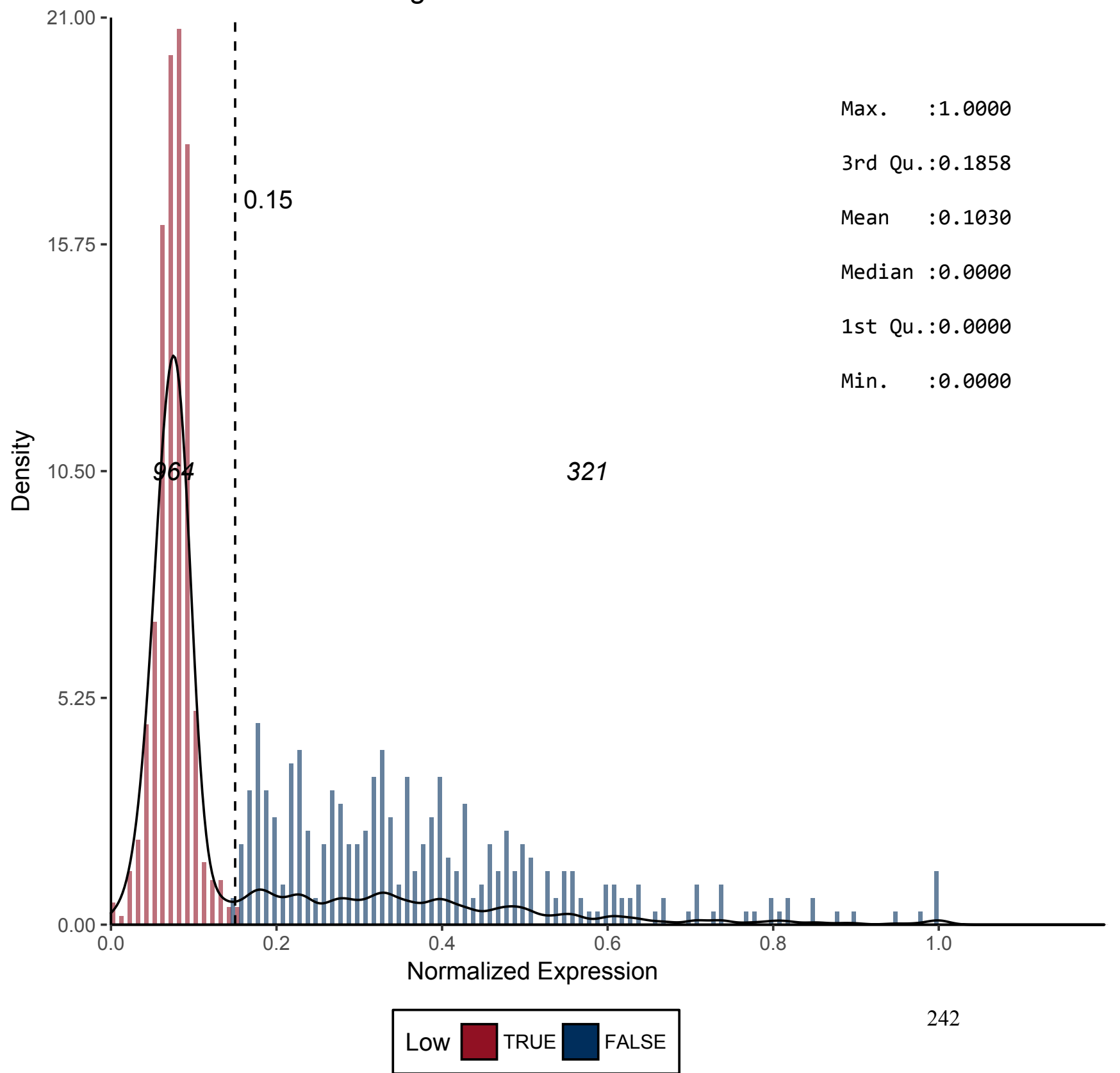


Figure 226 - CD215*

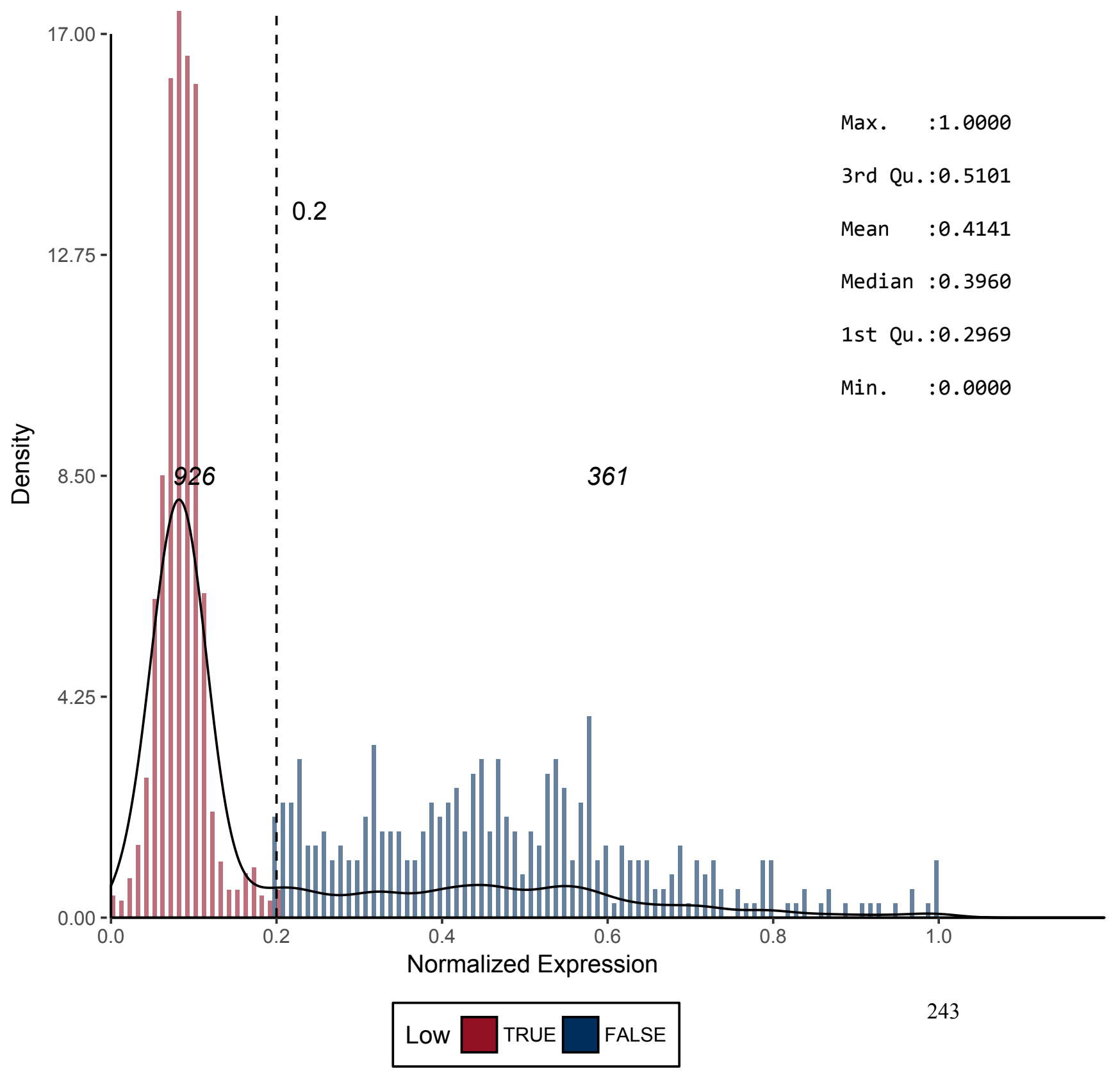


Figure 227 - CD217A

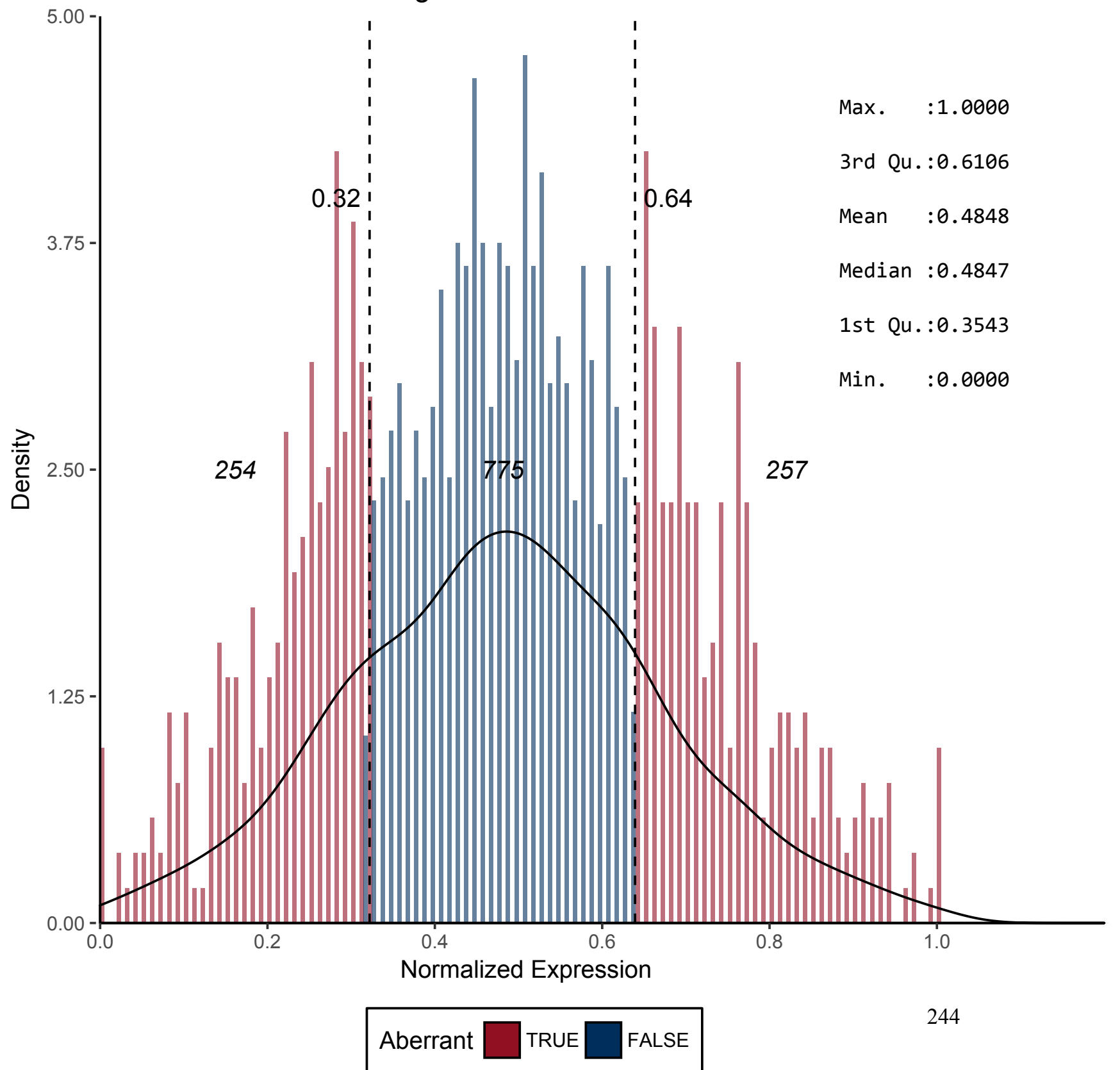


Figure 229 - CD218B

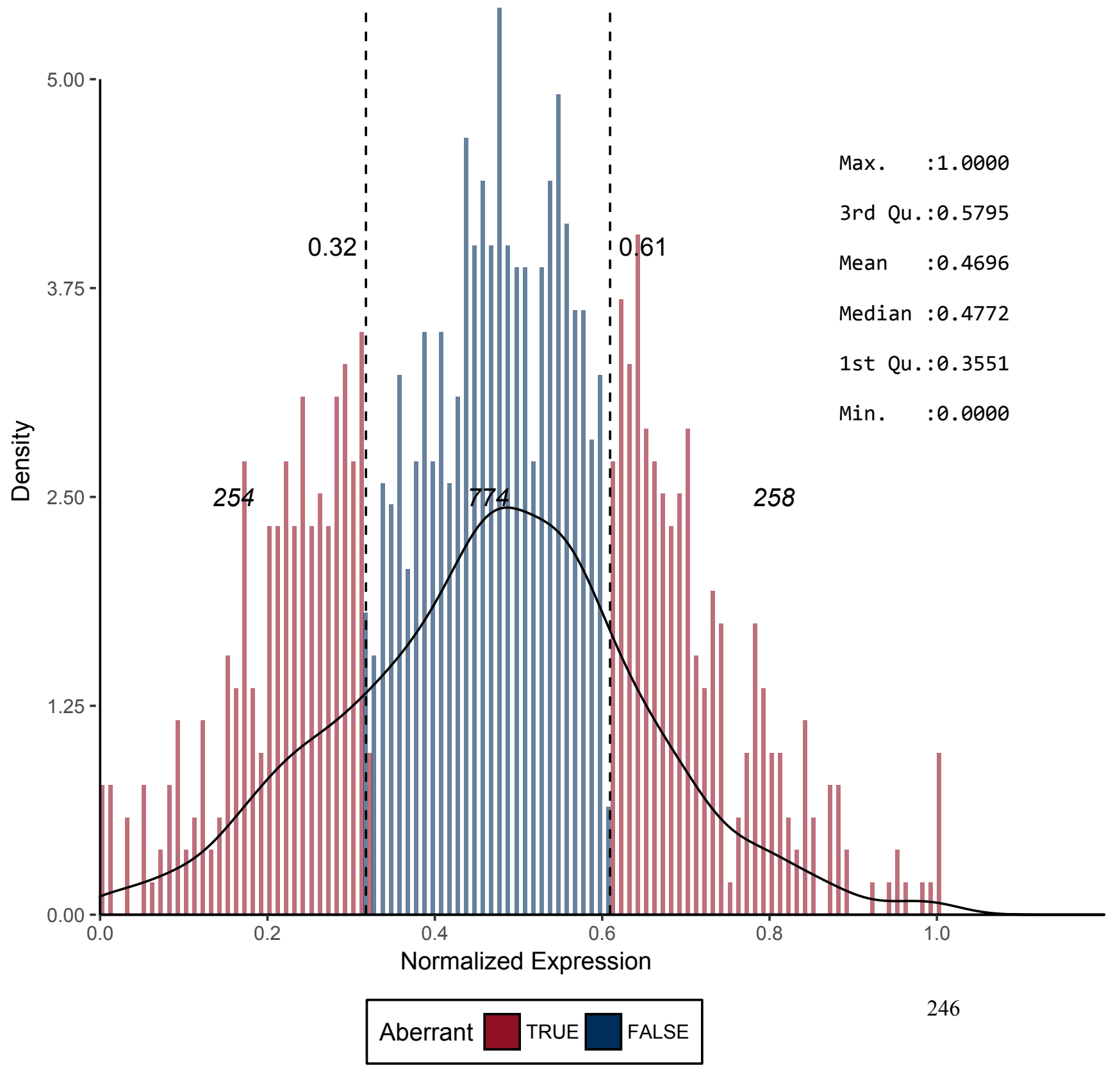


Figure 230 - CD220*

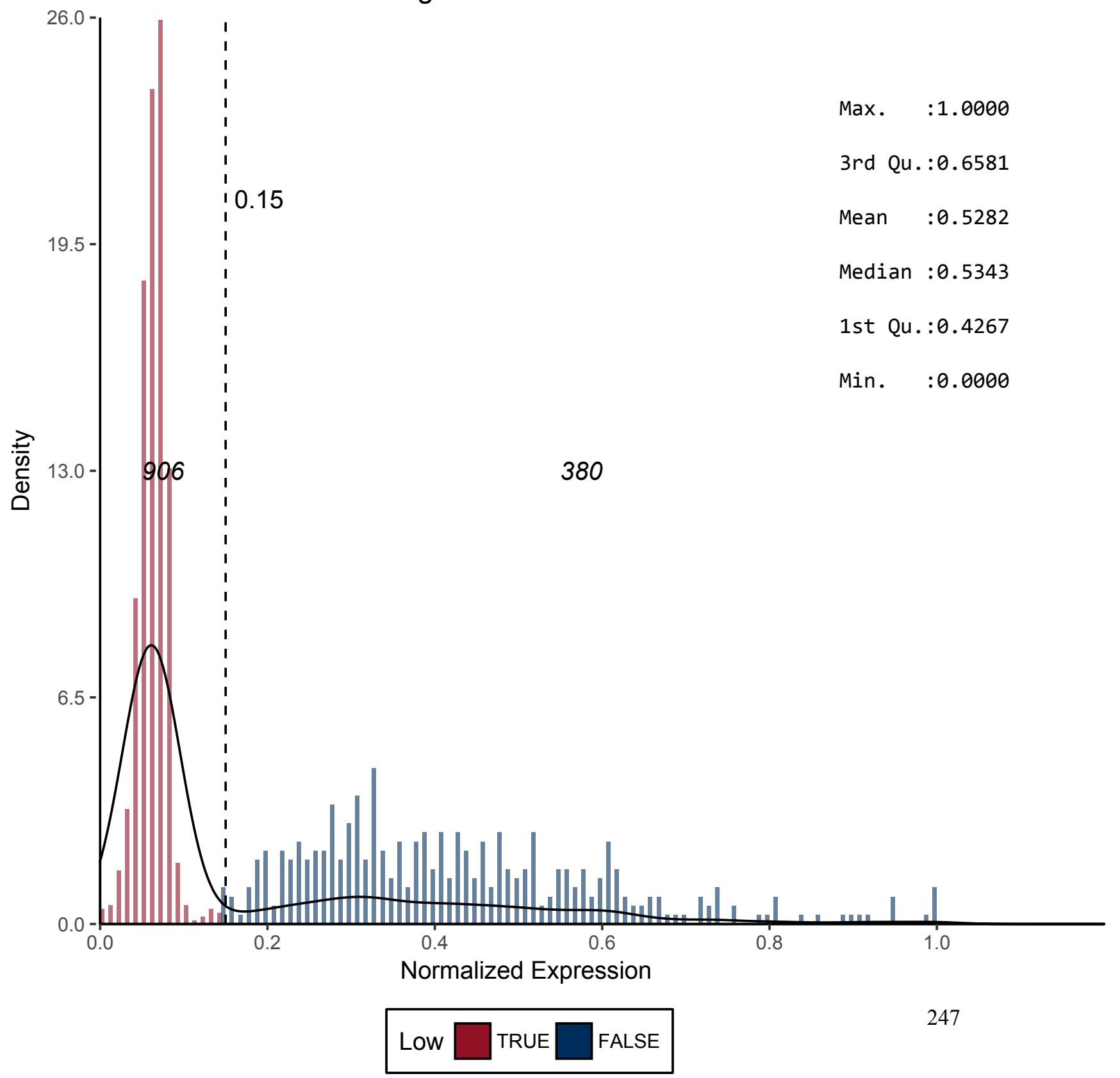


Figure 232 - CD222

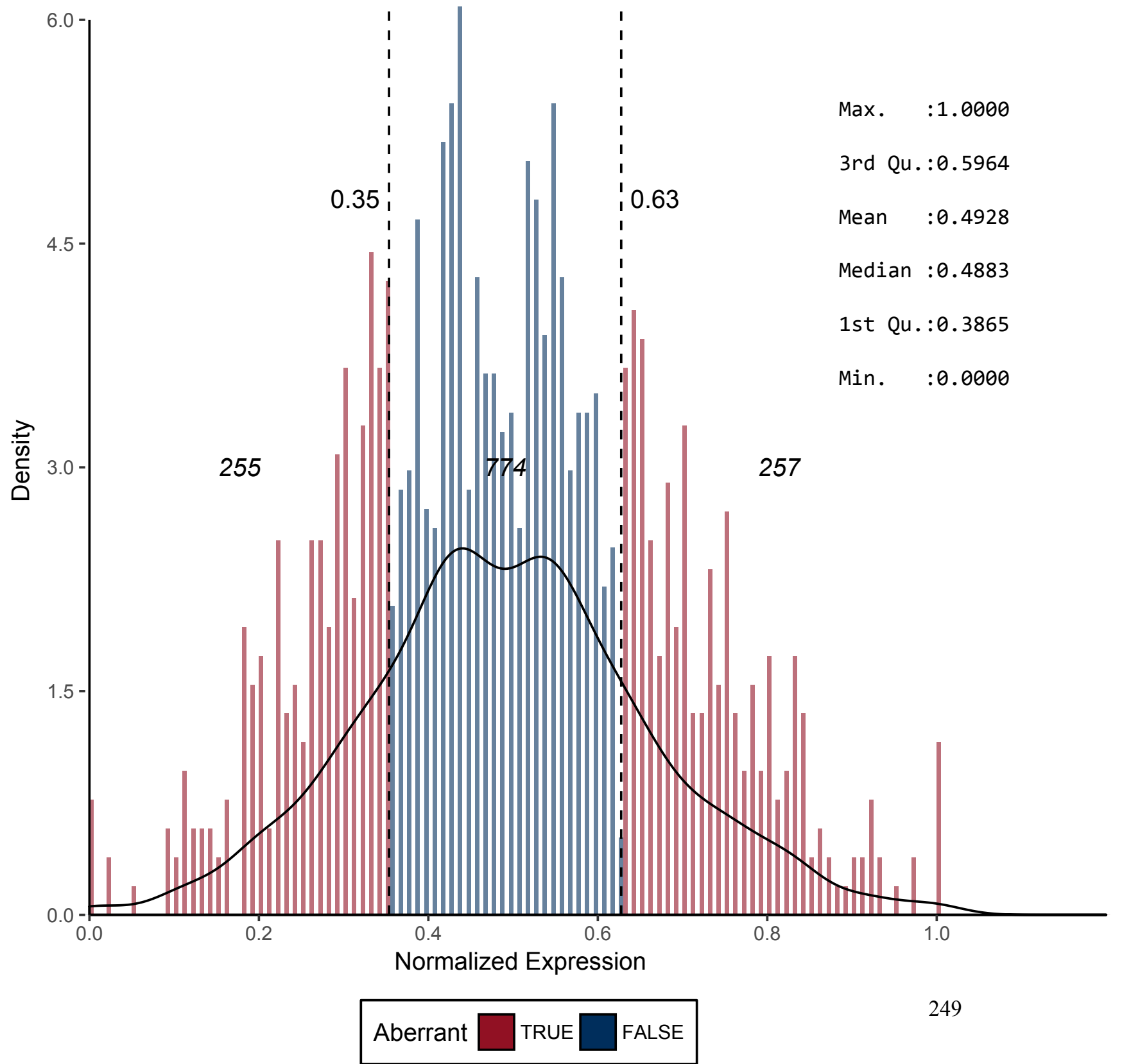


Figure 233 - CD223

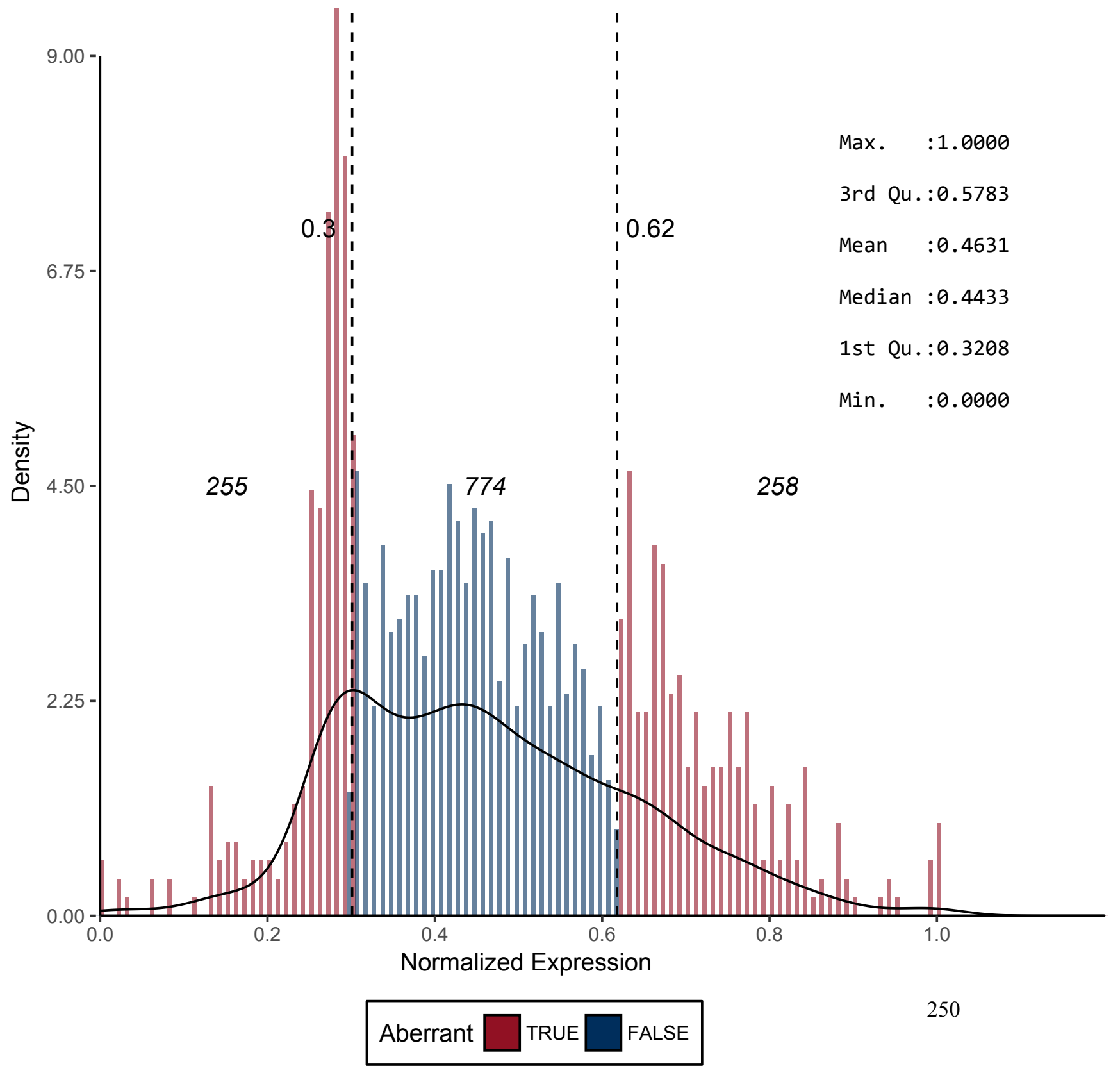


Figure 235 - CD226

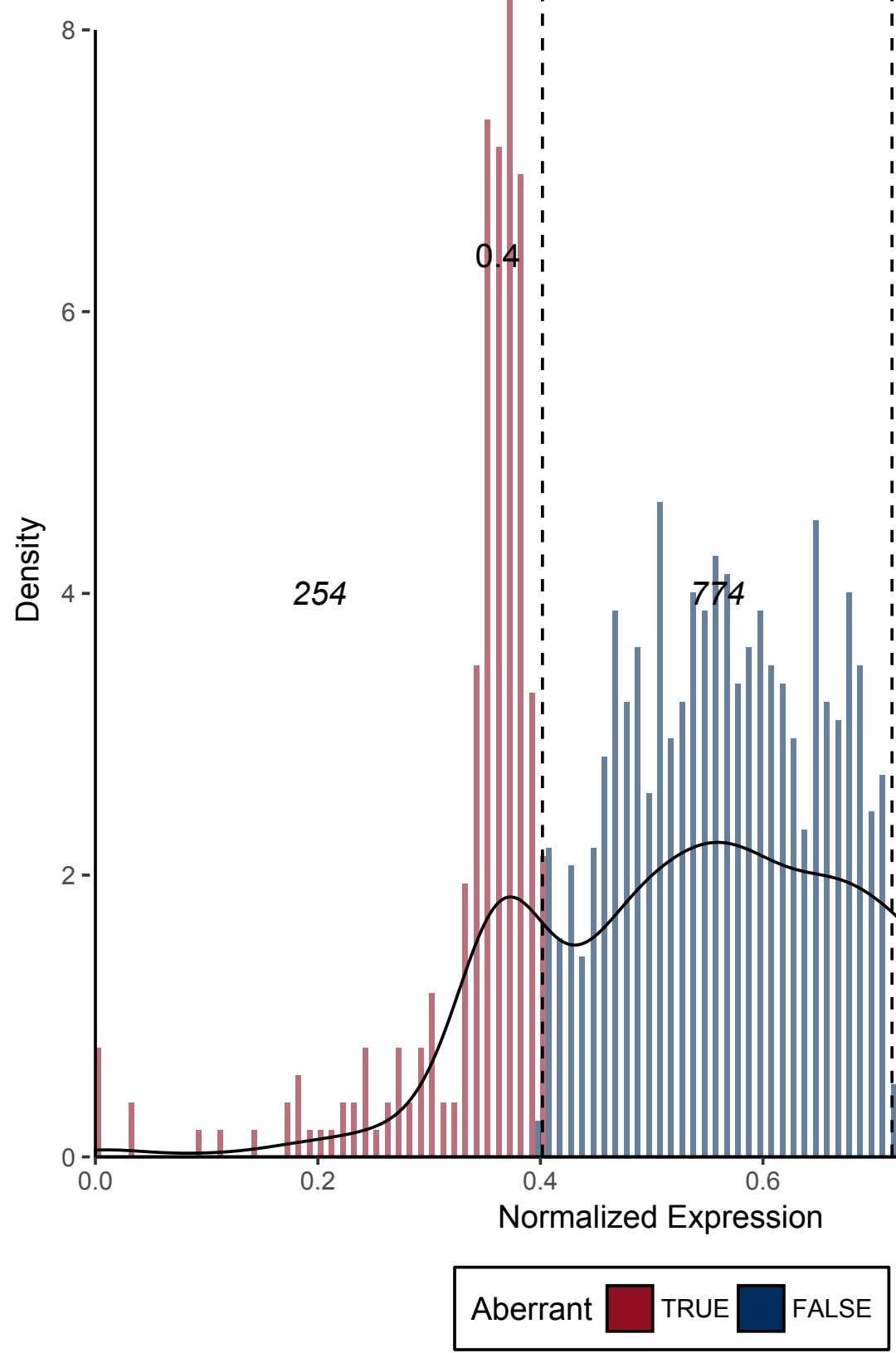

Max. $: 1.0000$

3rd Qu.:0.6877

Mean $\quad: 0.5684$

Median :0.5699

1st Qu.:0.4493

Min. $\quad: 0.0000$

258 
Figure 237 - CD228*

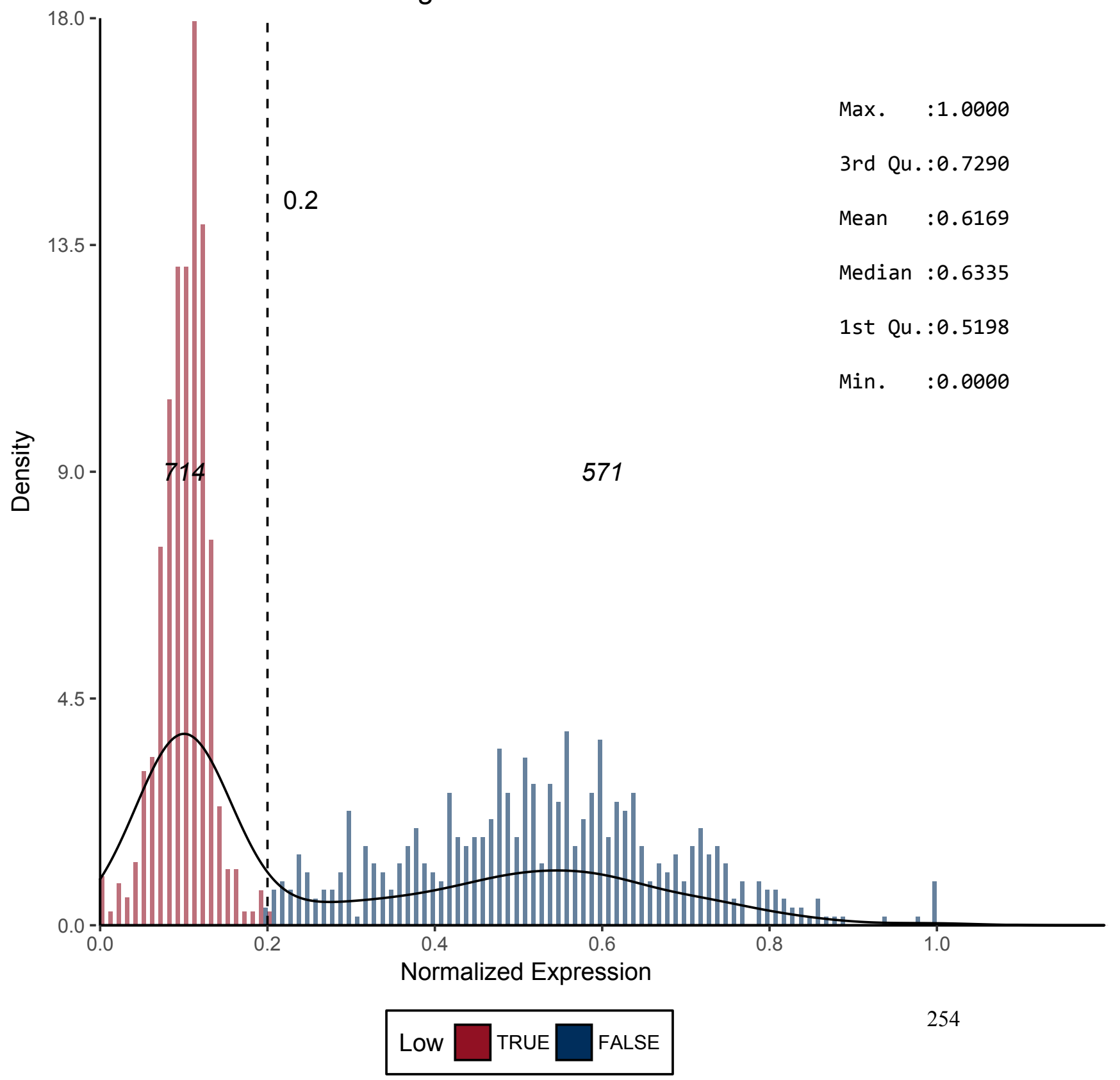


Figure 238 - CD229

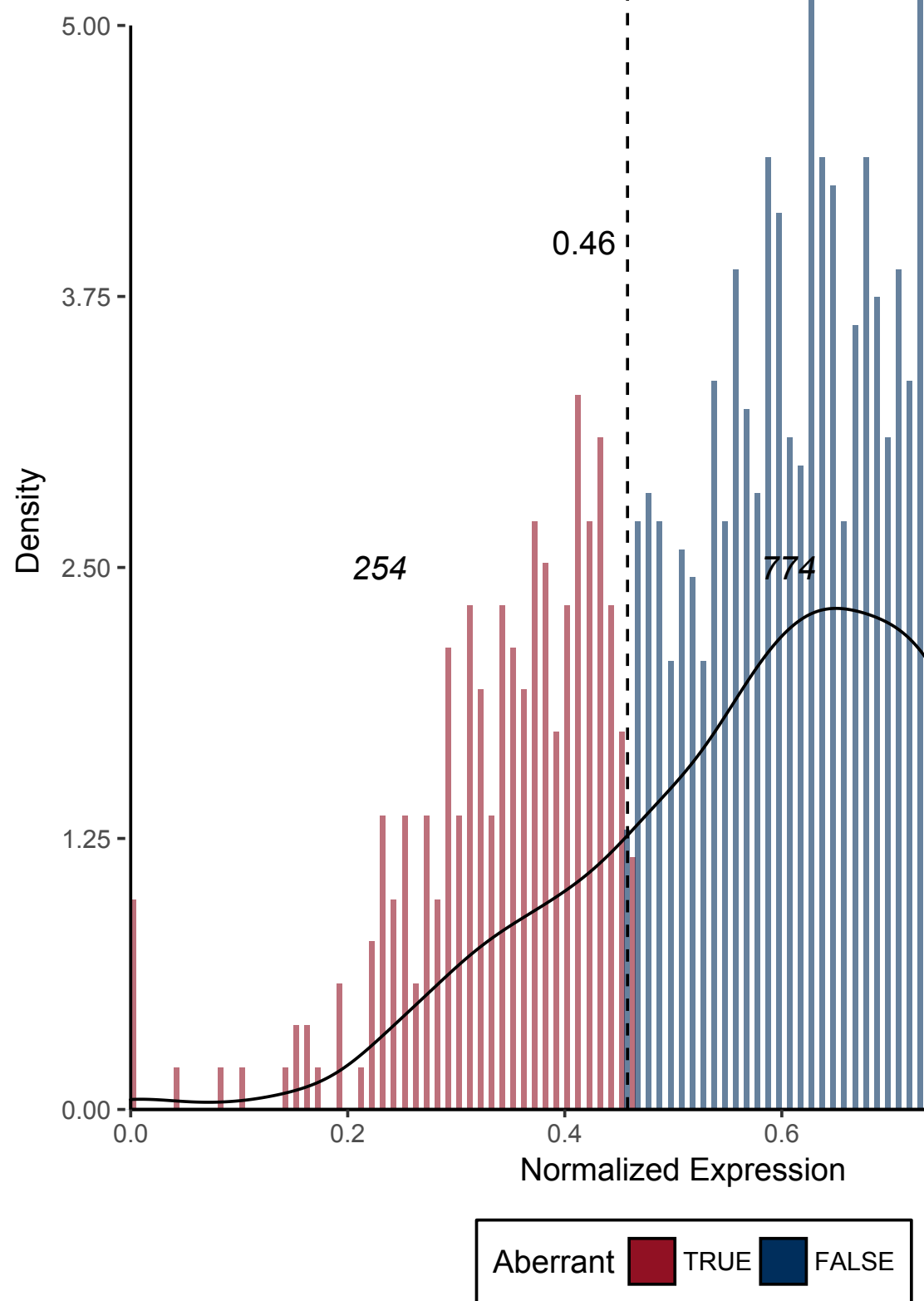

Max. $: 1.0000$

3rd Qu.:0.7315

Mean $\quad: 0.6065$

Median :0.6255

1st Qu.:0.4910

Min. $\quad: 0.0000$

258

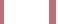


Figure 240 - CD231*

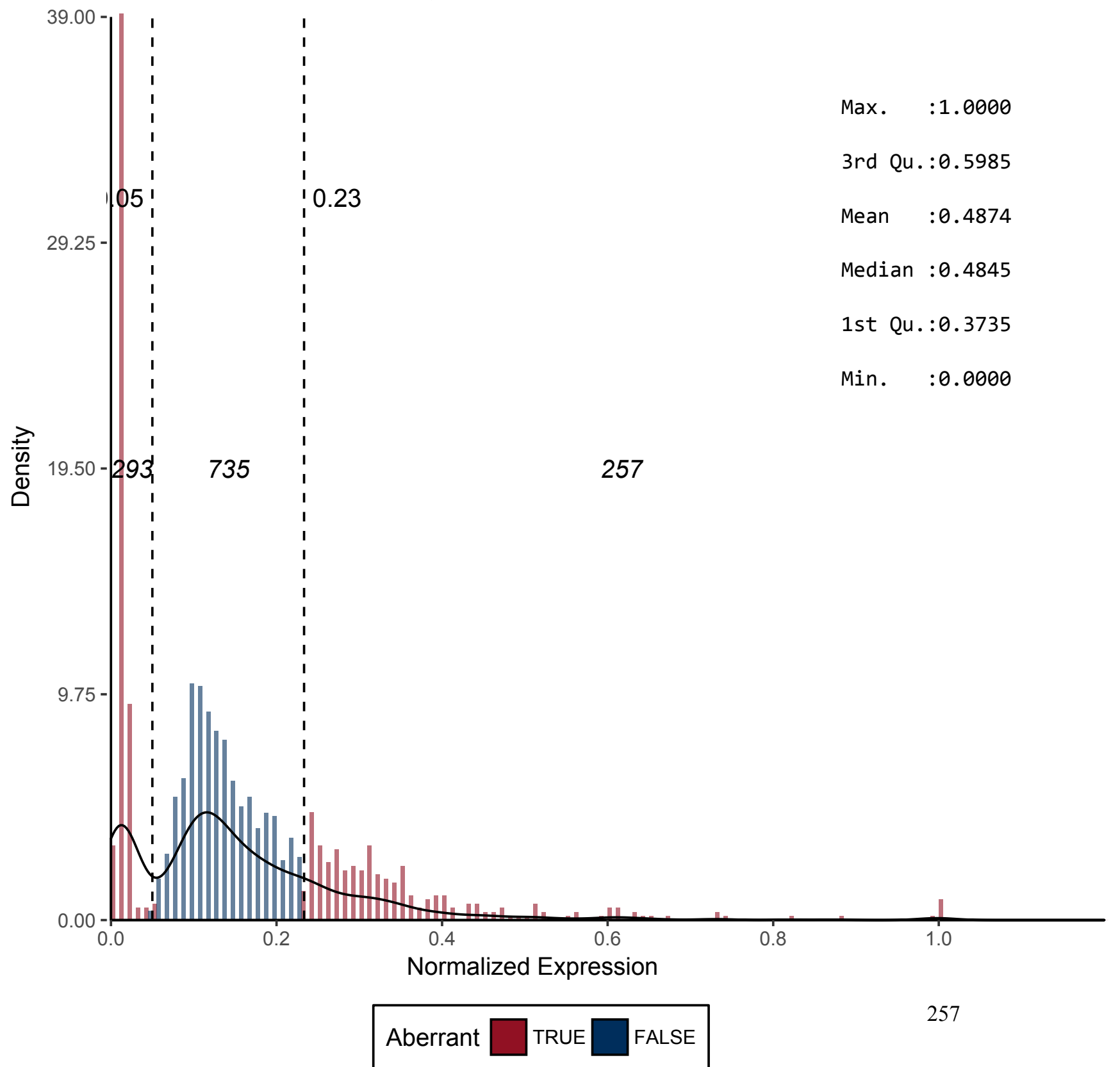


Figure 241 - CD232

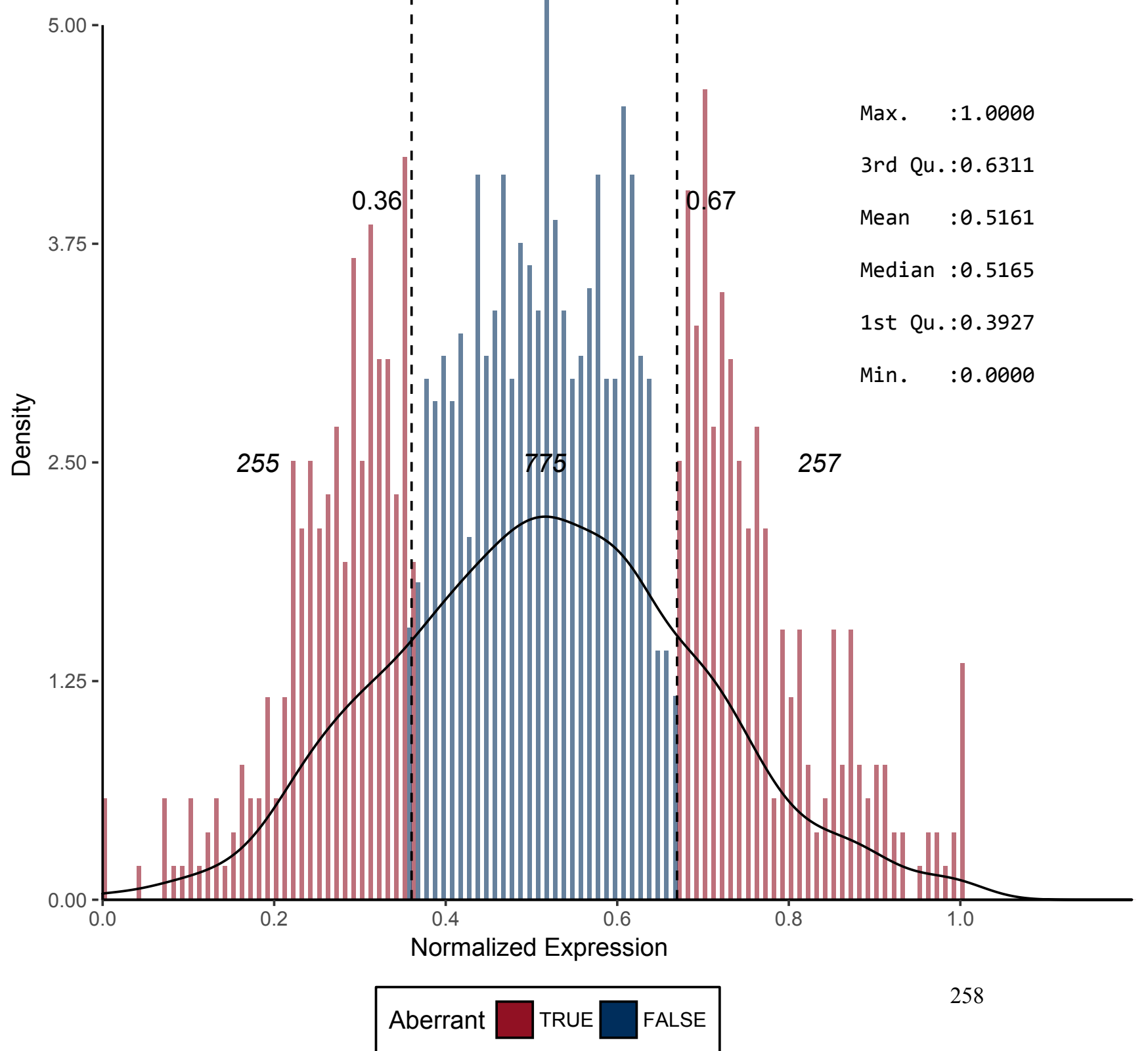


Figure 243 - CD234

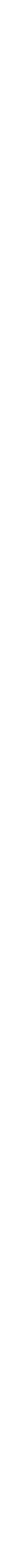


Figure 244 - CD235A*

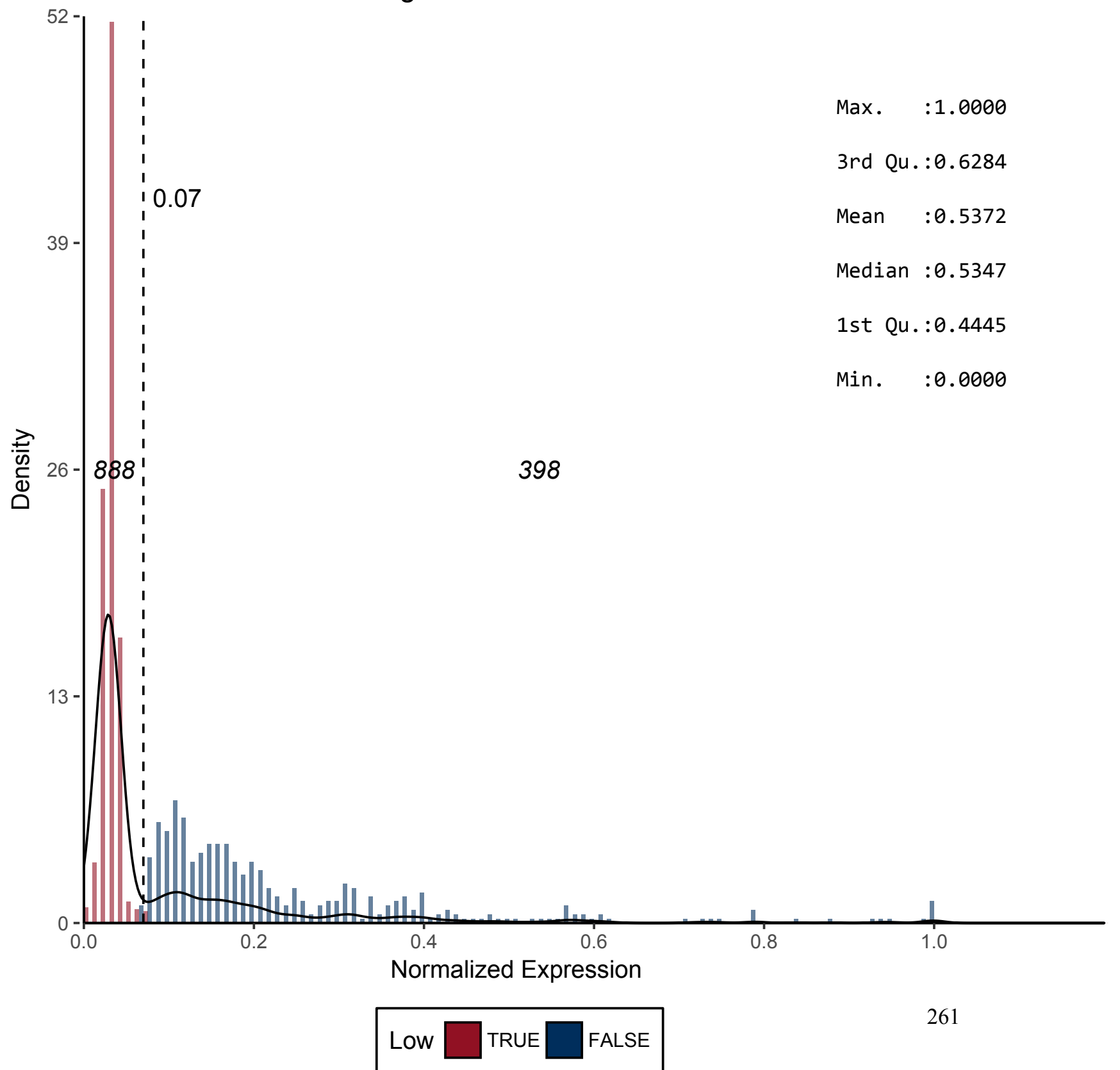


Figure 245 - CD235B

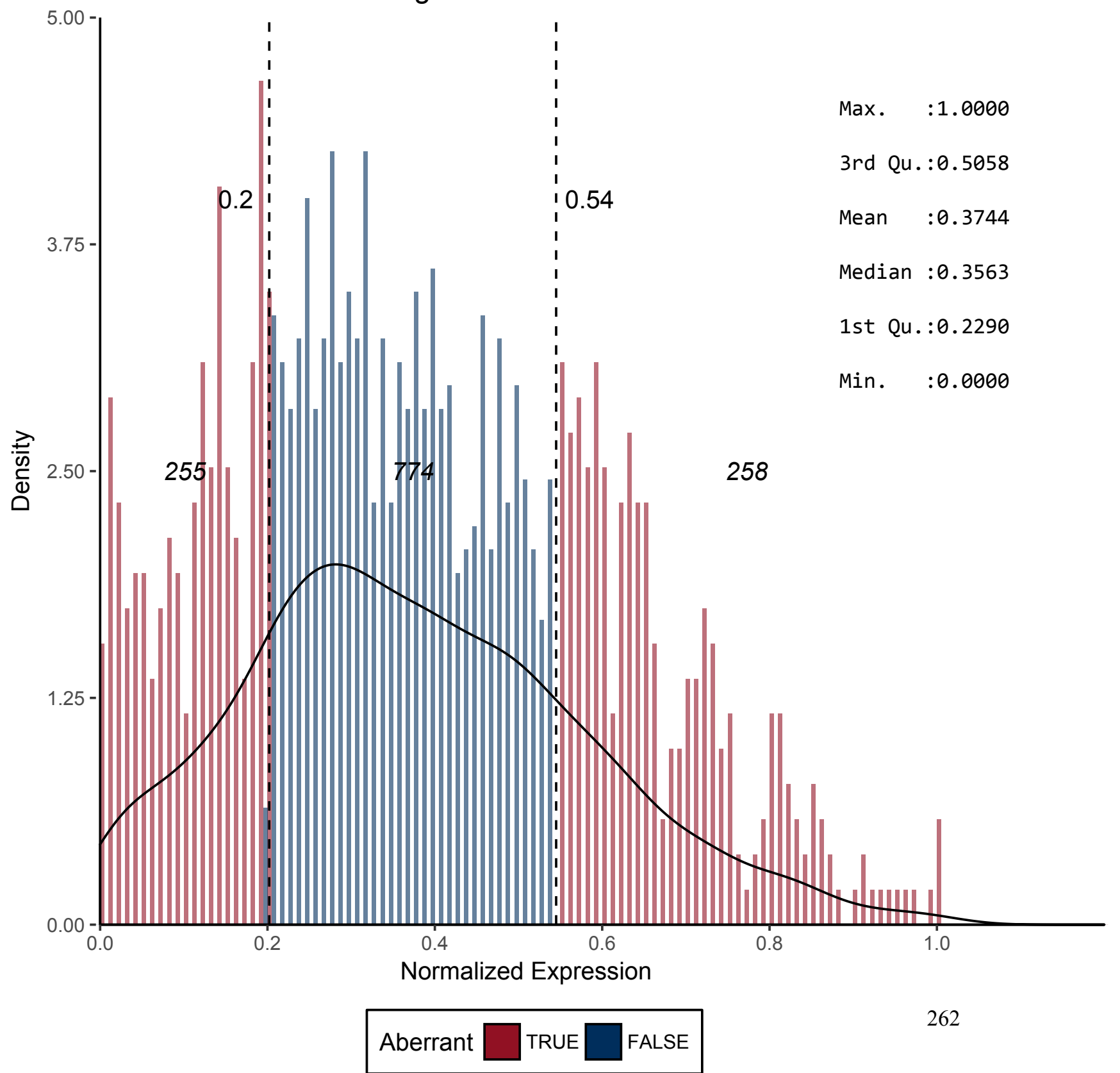


Figure 247 - CD238

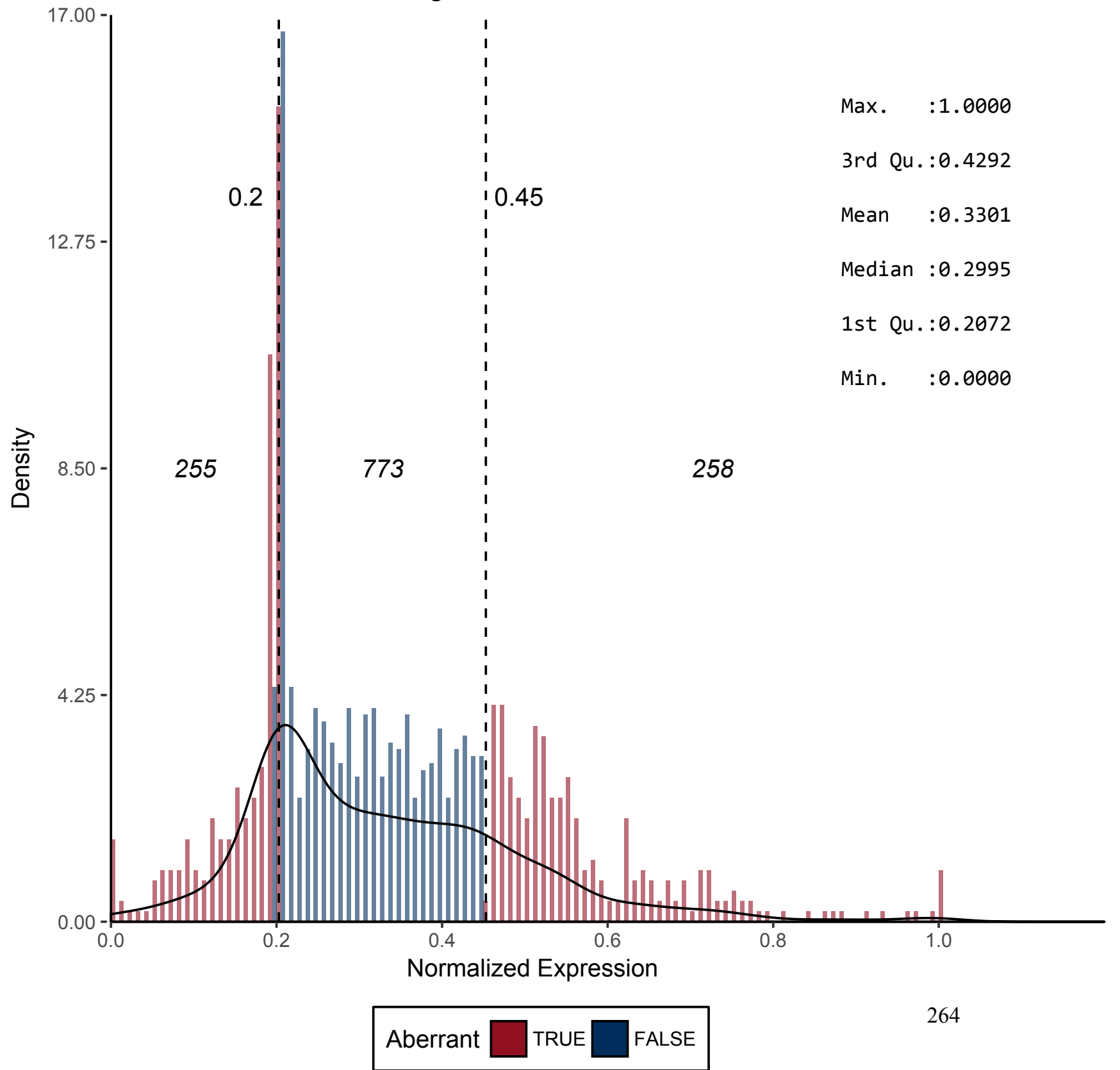


Figure 248 - CD239*

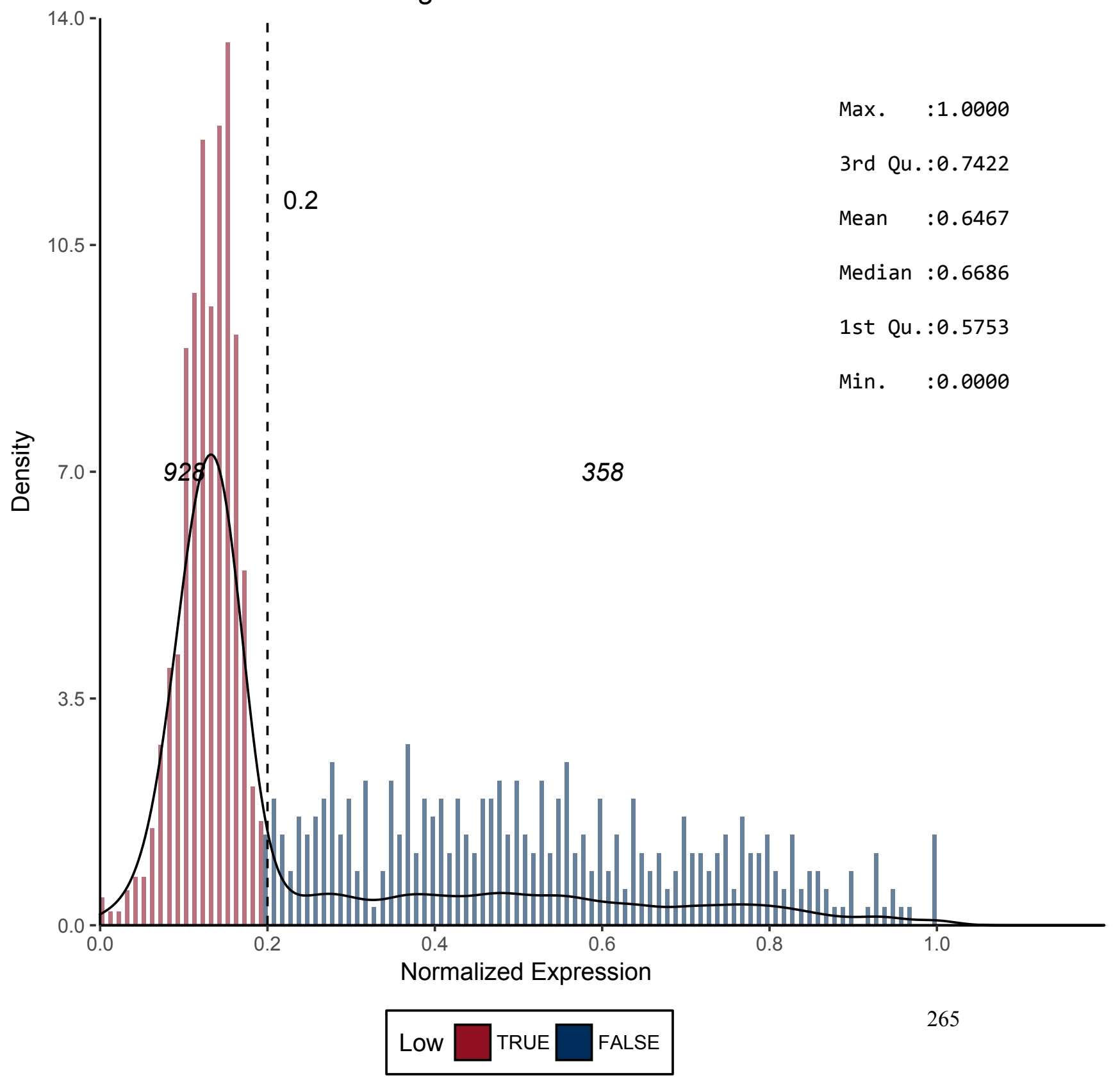


Figure 250 - CD240D*

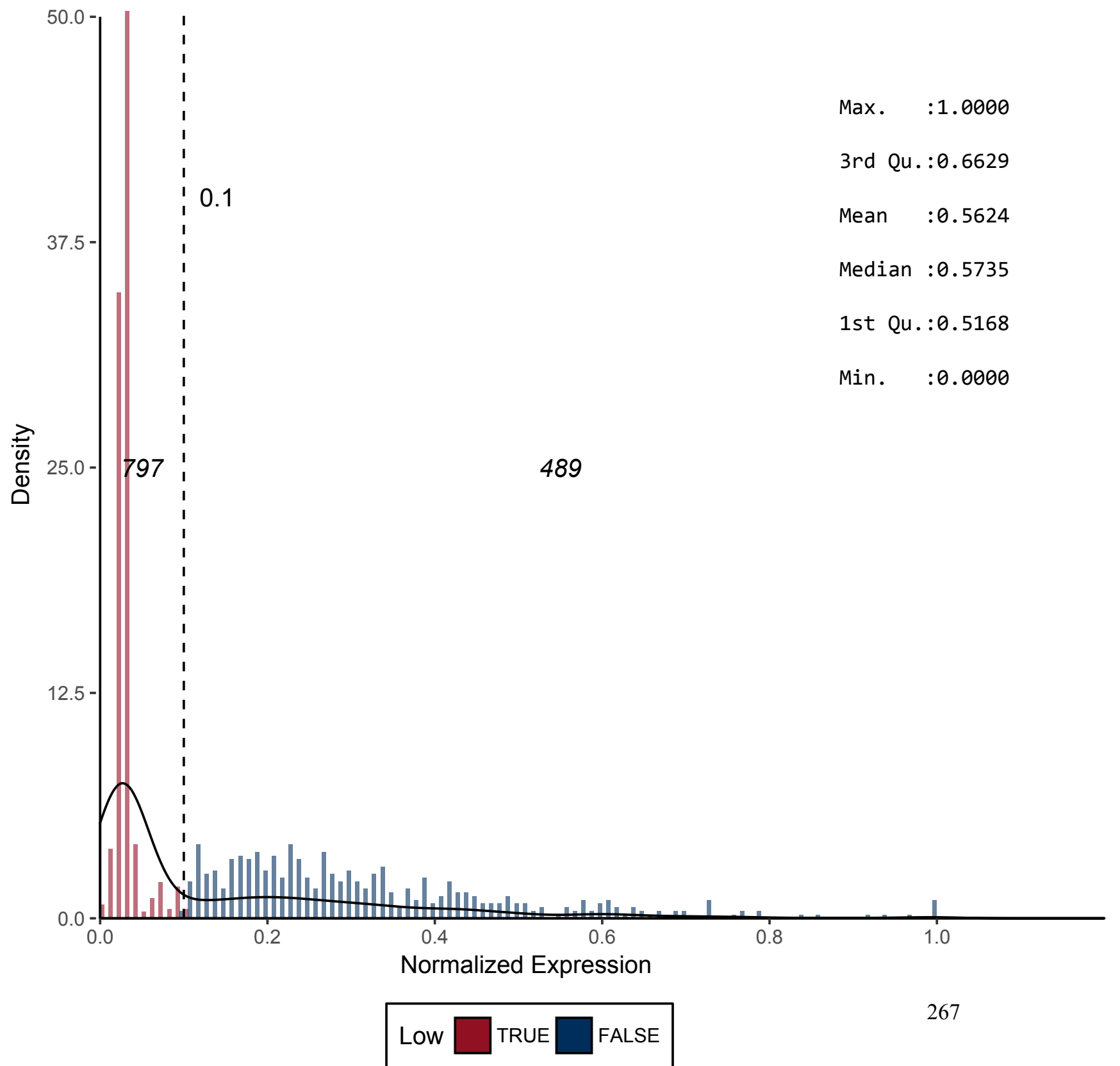


Figure 251 - CD241*

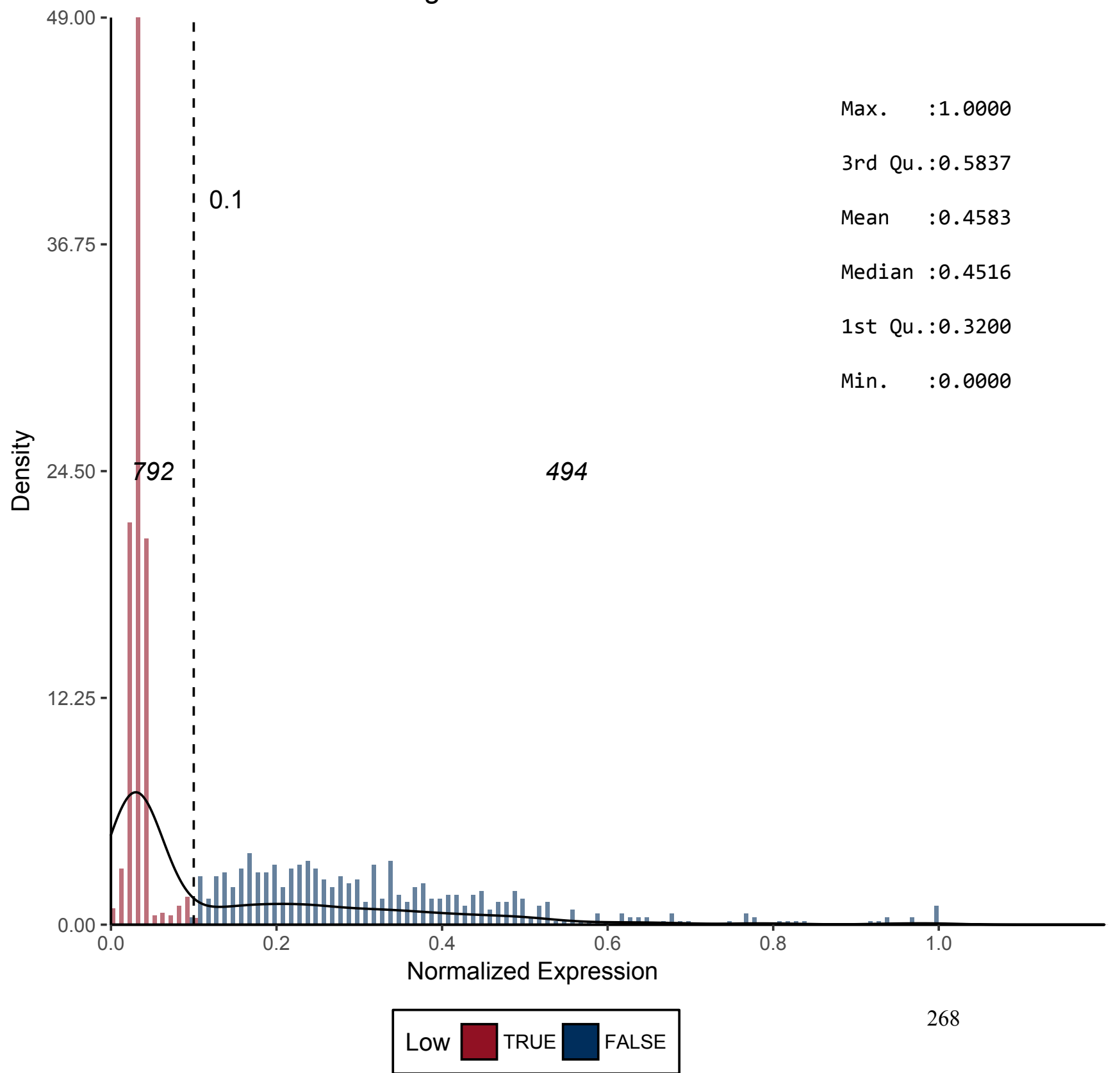


Figure 253 - CD243

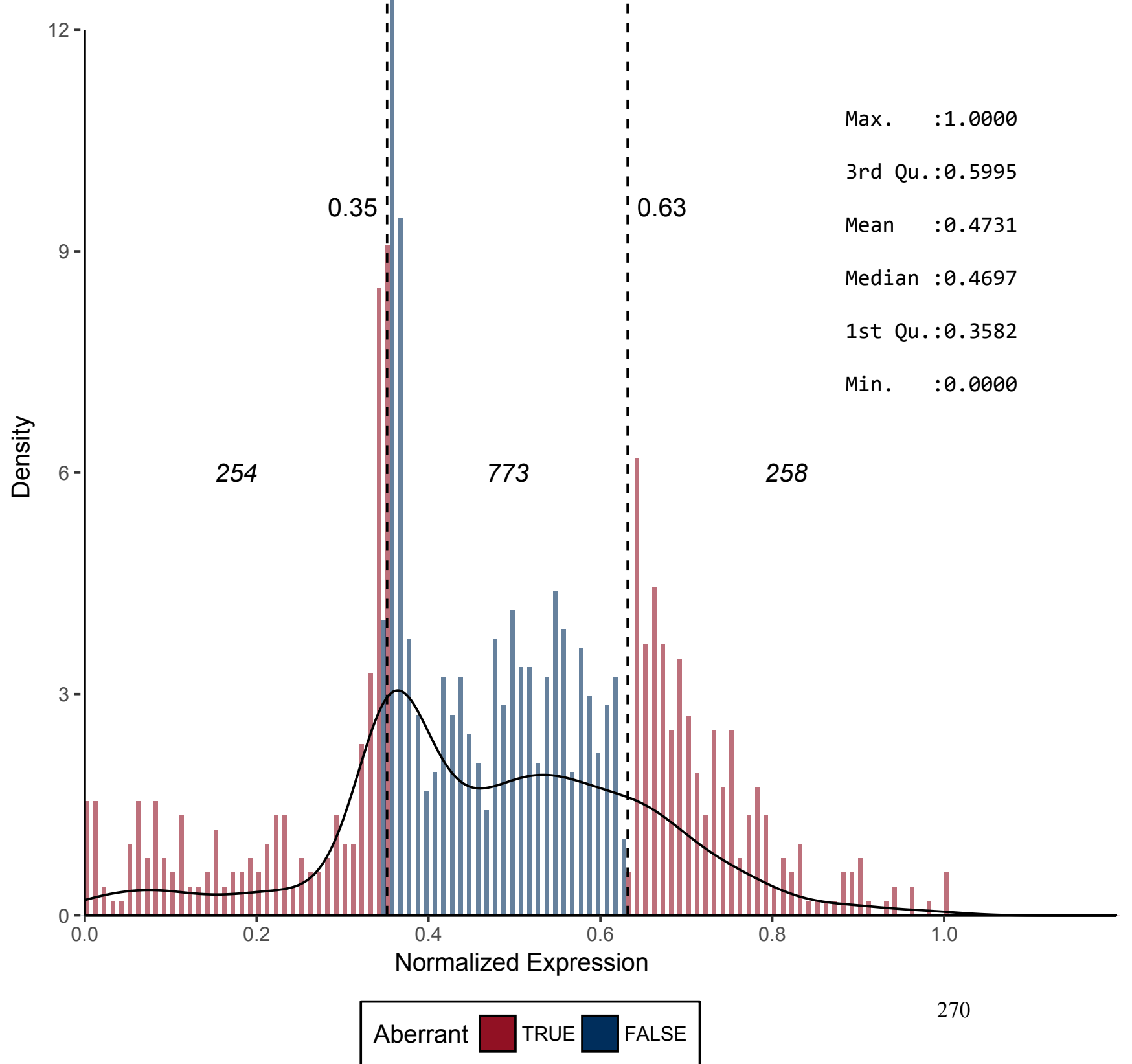


Figure 254 - CD244

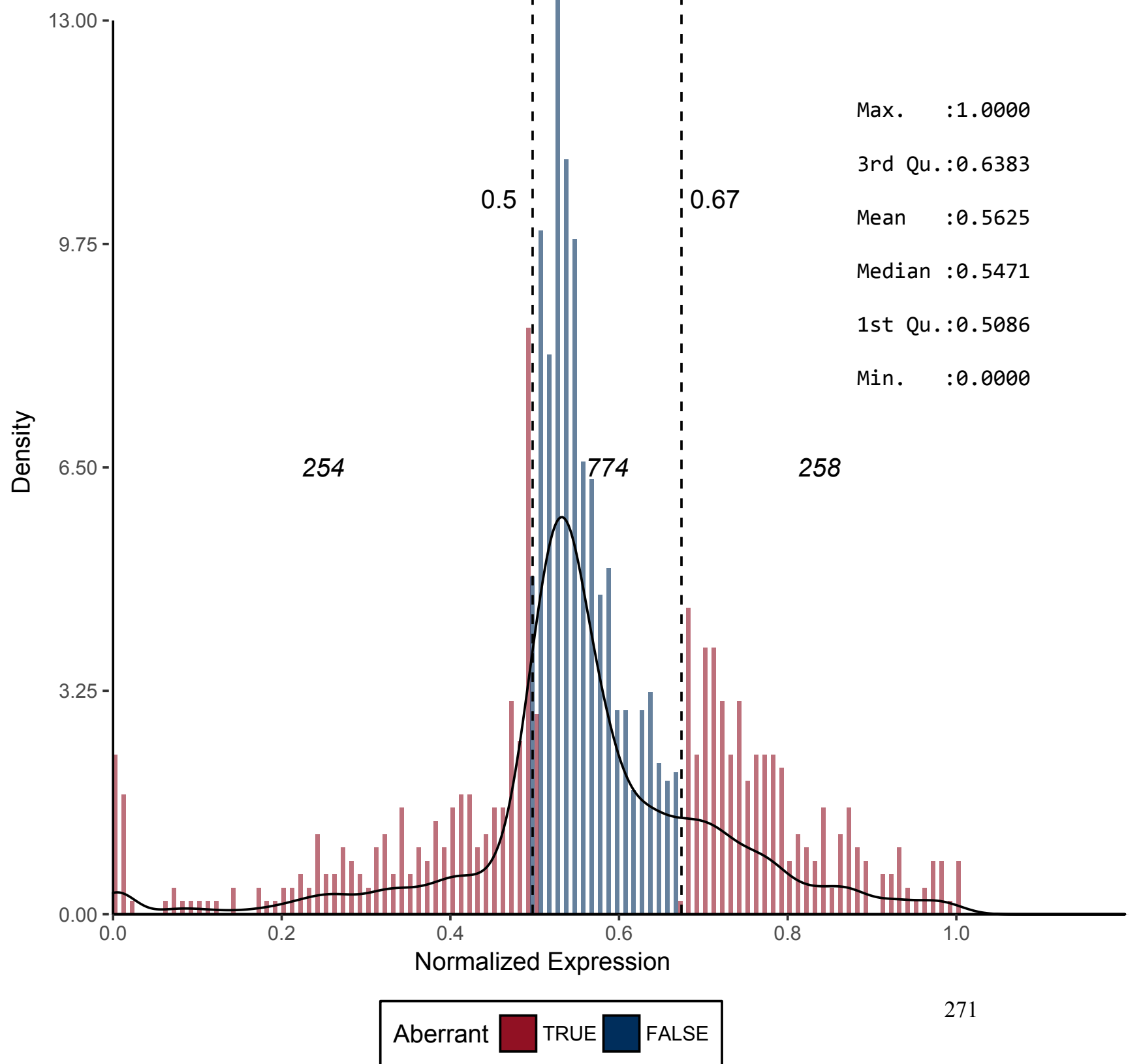


Figure 255 - CD246*

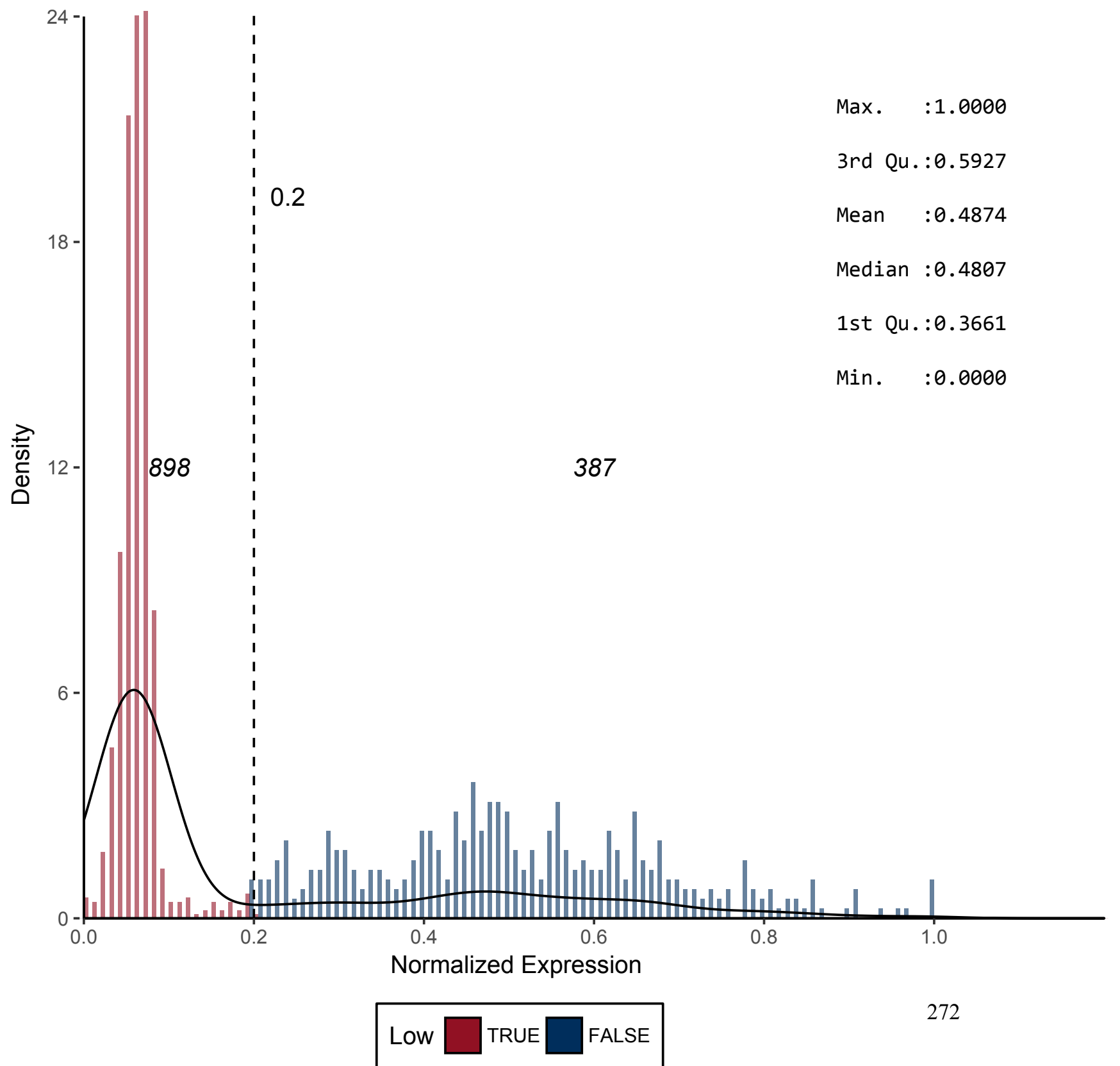


Figure 256 - CD247

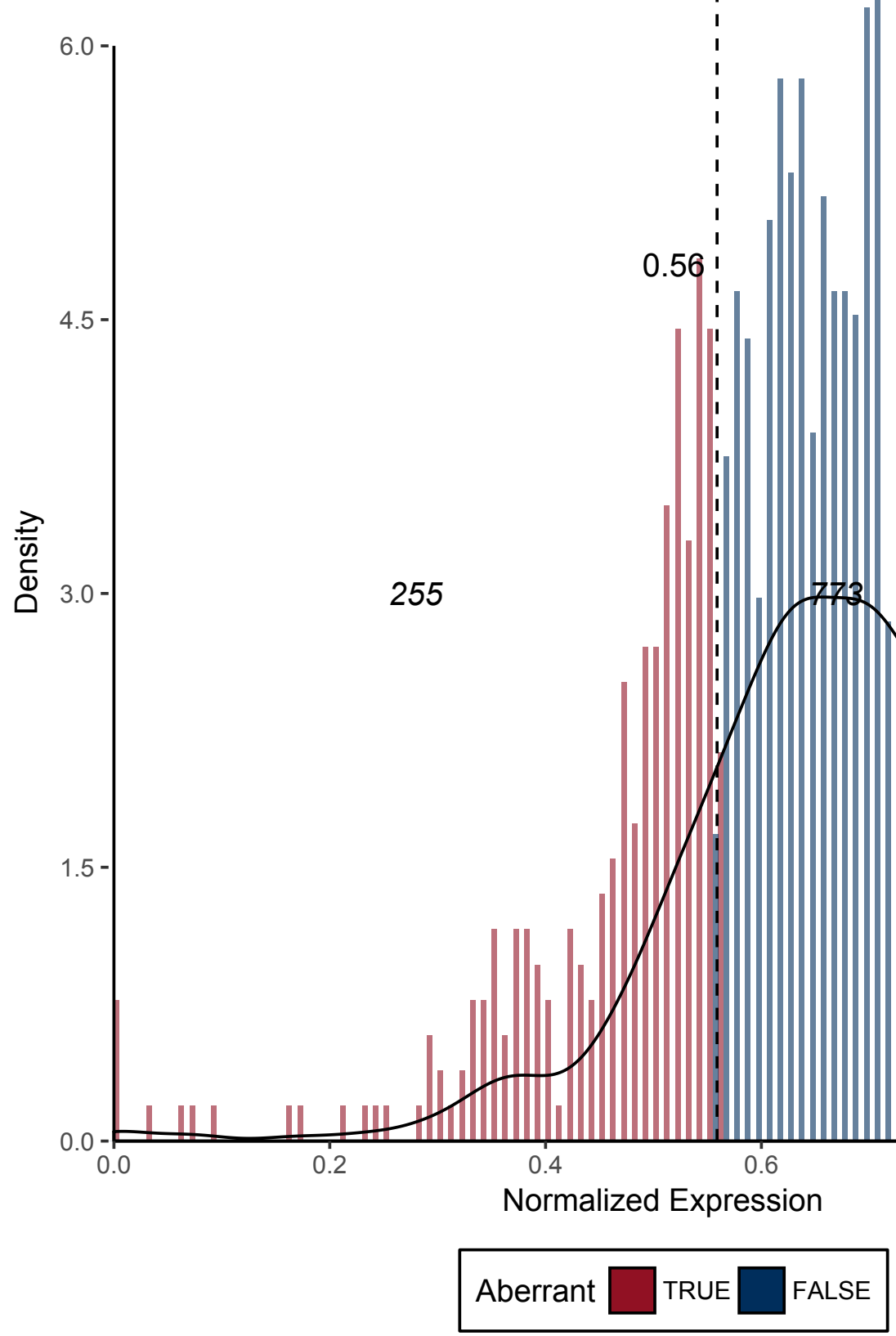

Max. $: 1.0000$

3rd Qu.:0.7547

Mean :0.6598

Median :0.6672

1st Qu.:0.5804

Min. $\quad: 0.0000$

258 
Figure 257 - CD248

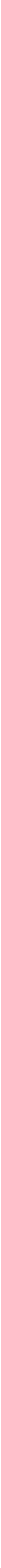


Figure 258 - CD249*

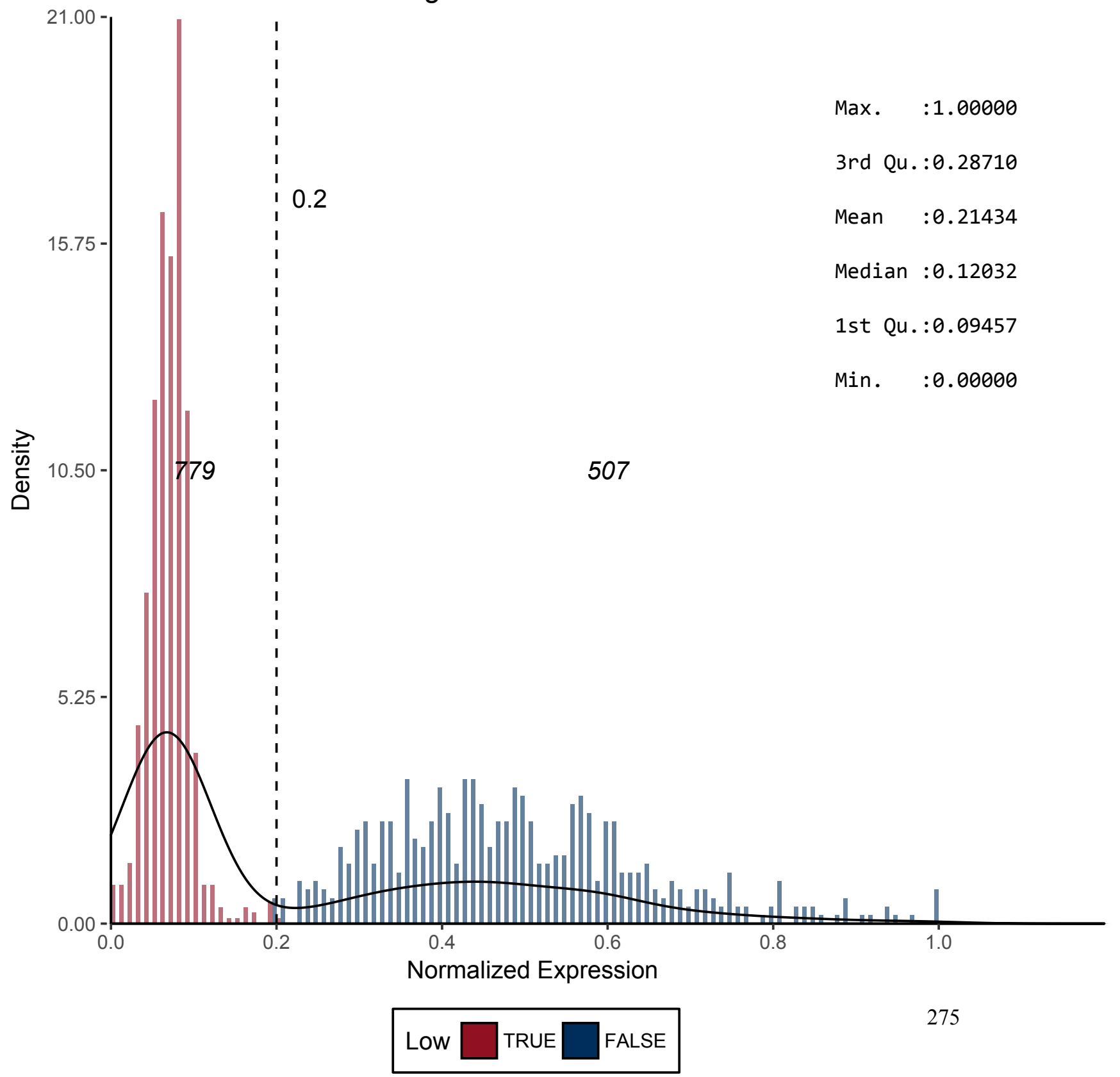


Figure 259 - CD252

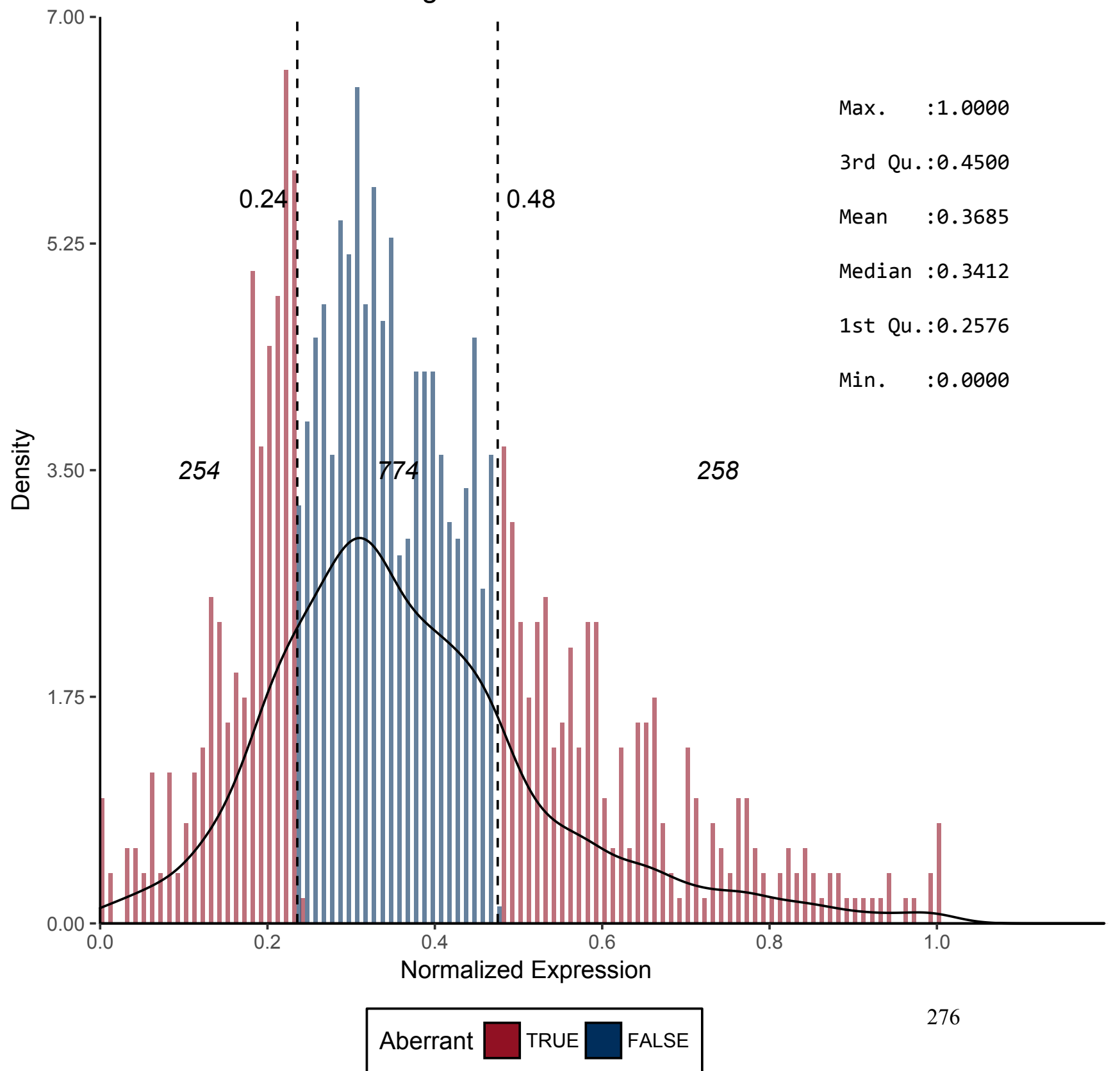


Figure 260 - CD253

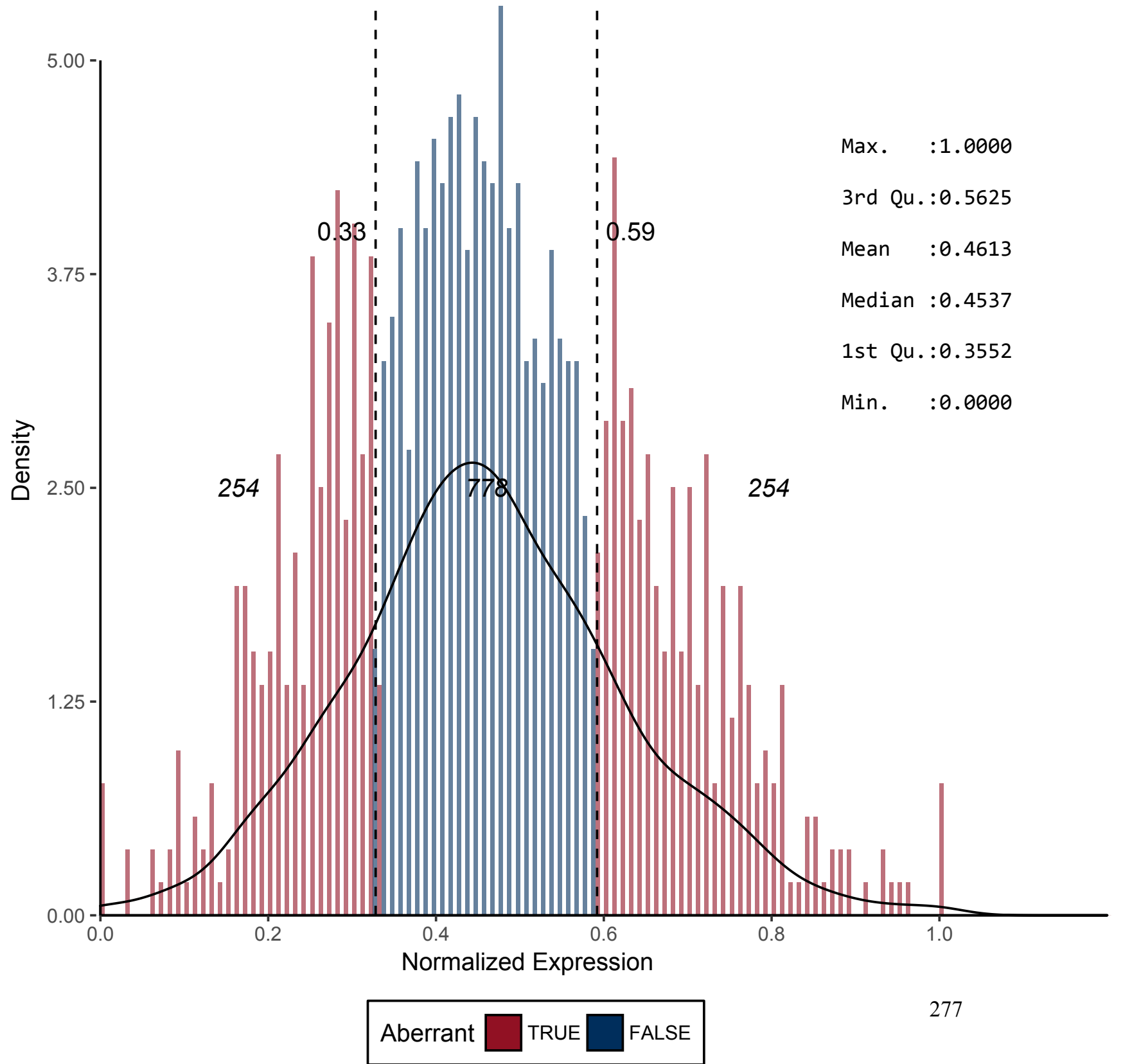


Figure 261 - CD254*

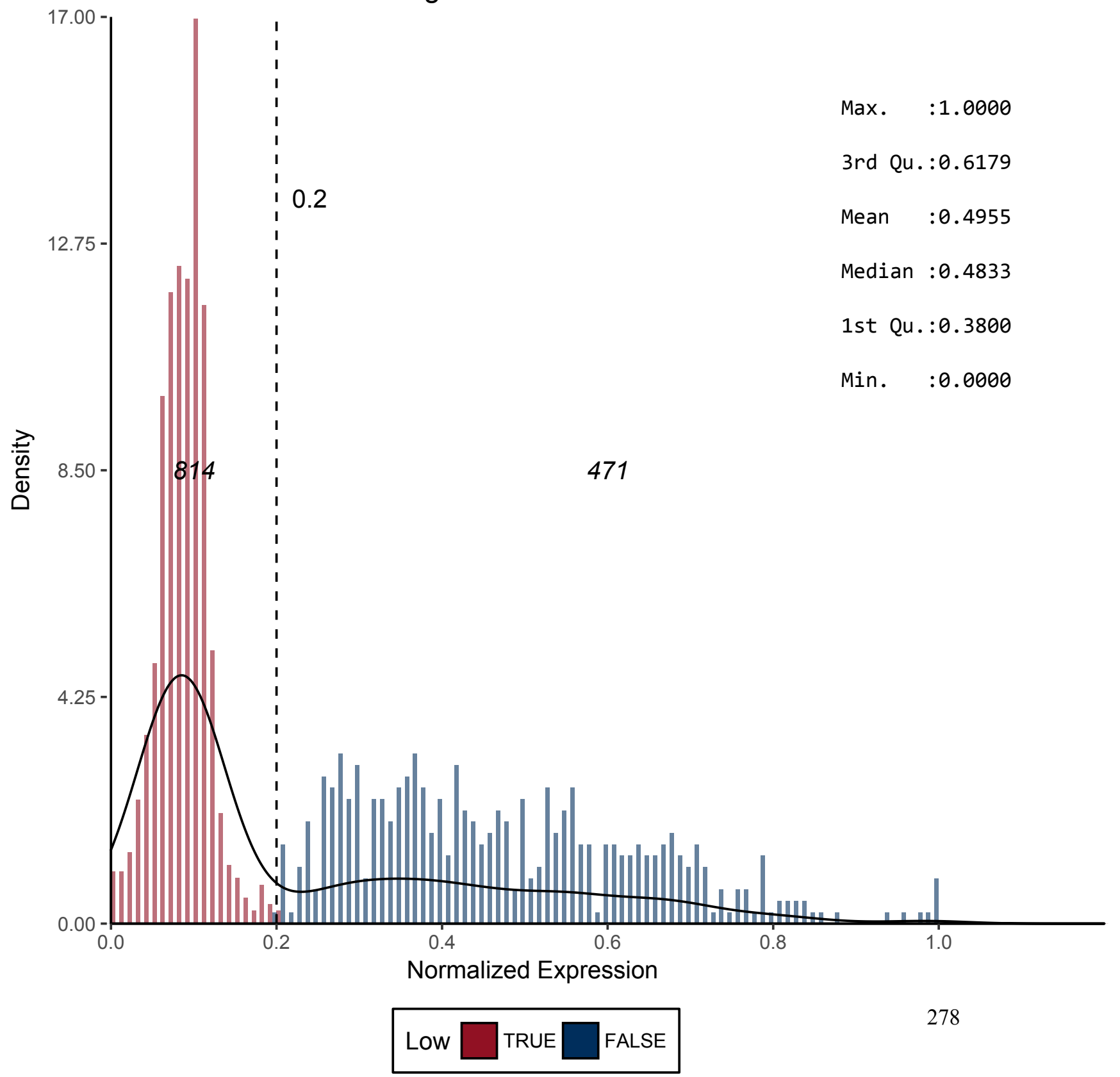


Figure 262 - CD257

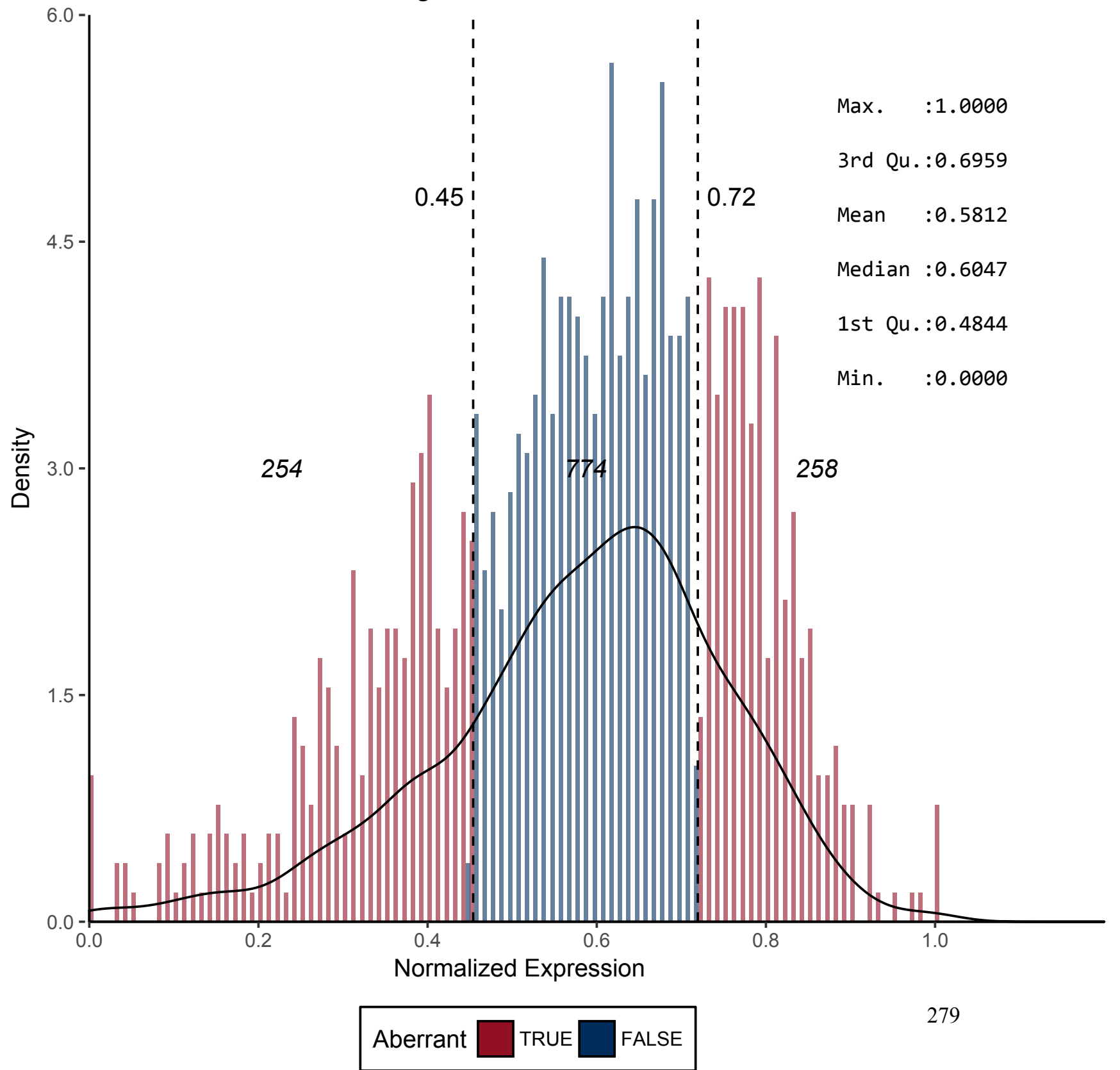


Figure 264 - CD262

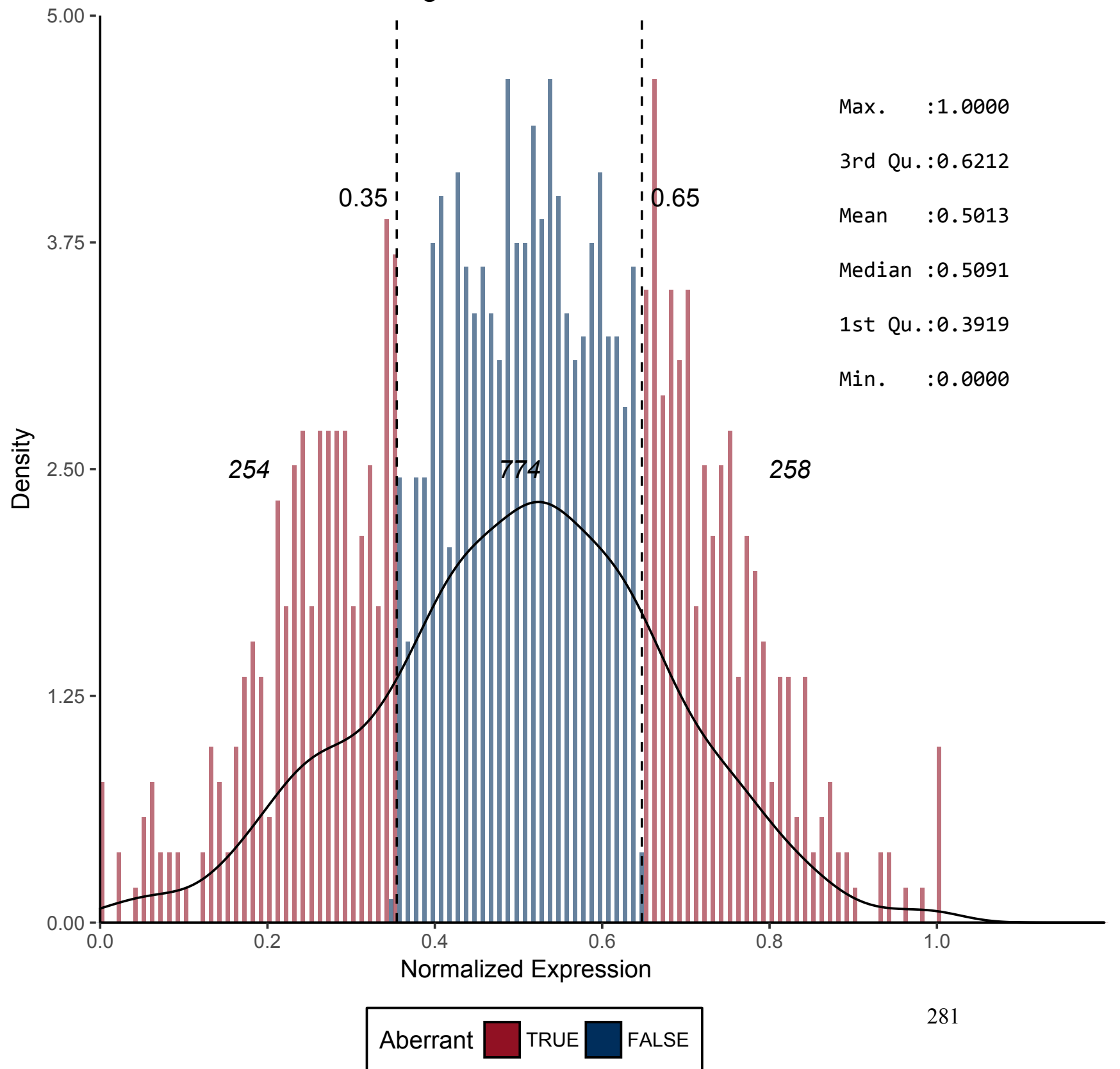


Figure 265 - CD263

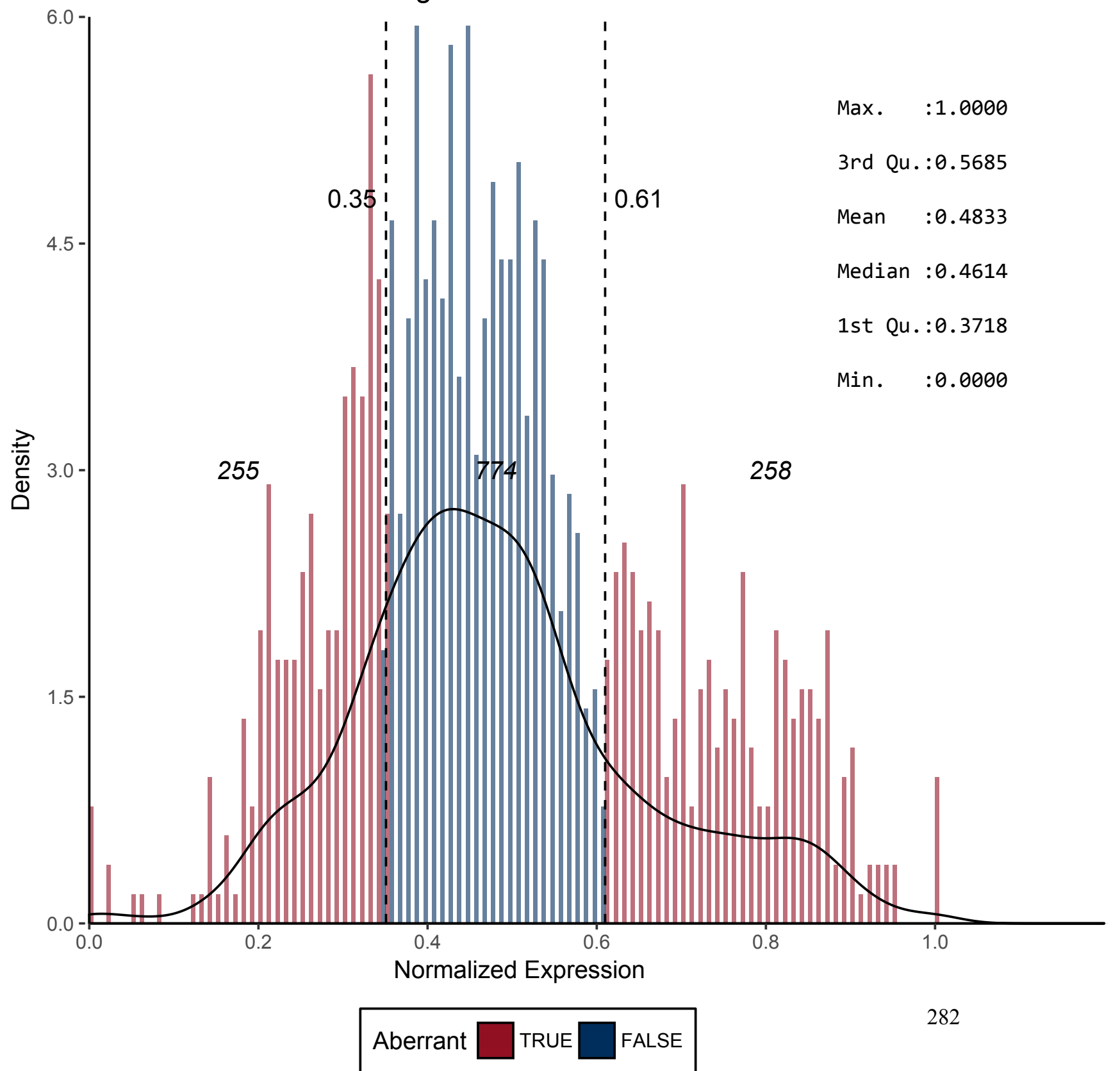


Figure 266 - CD264*

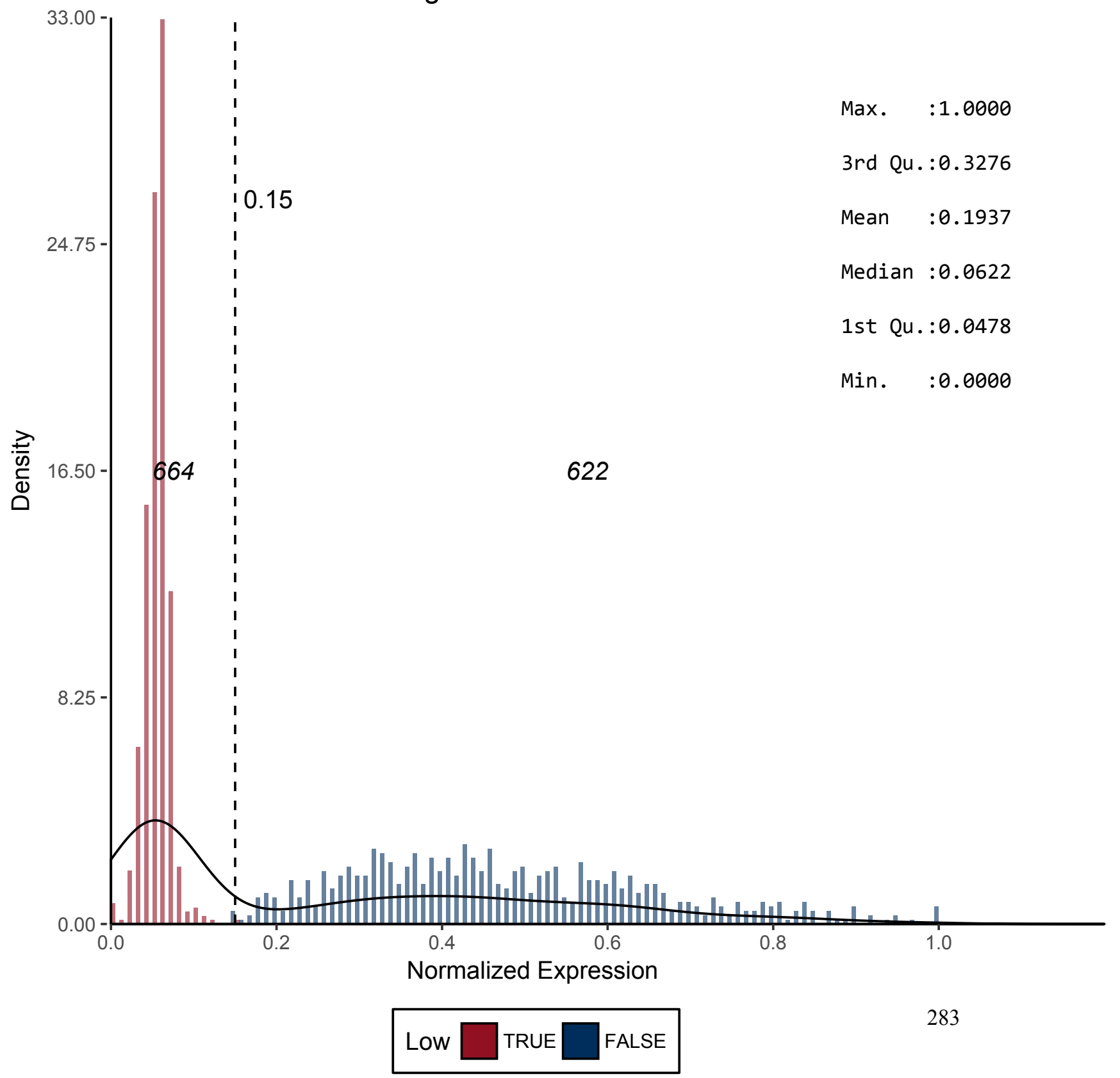


Figure 267 - CD265*

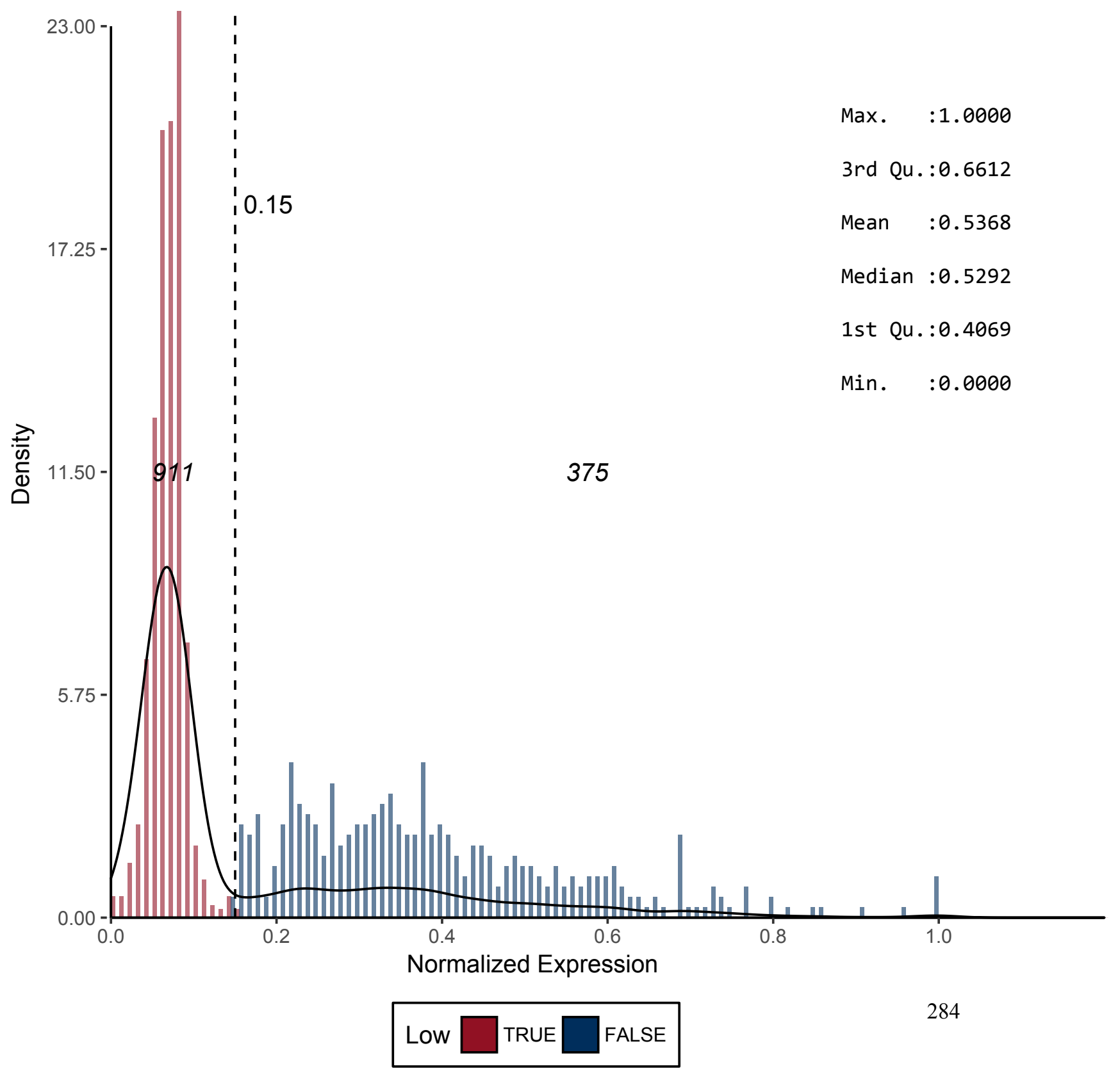


Figure 268 - CD266

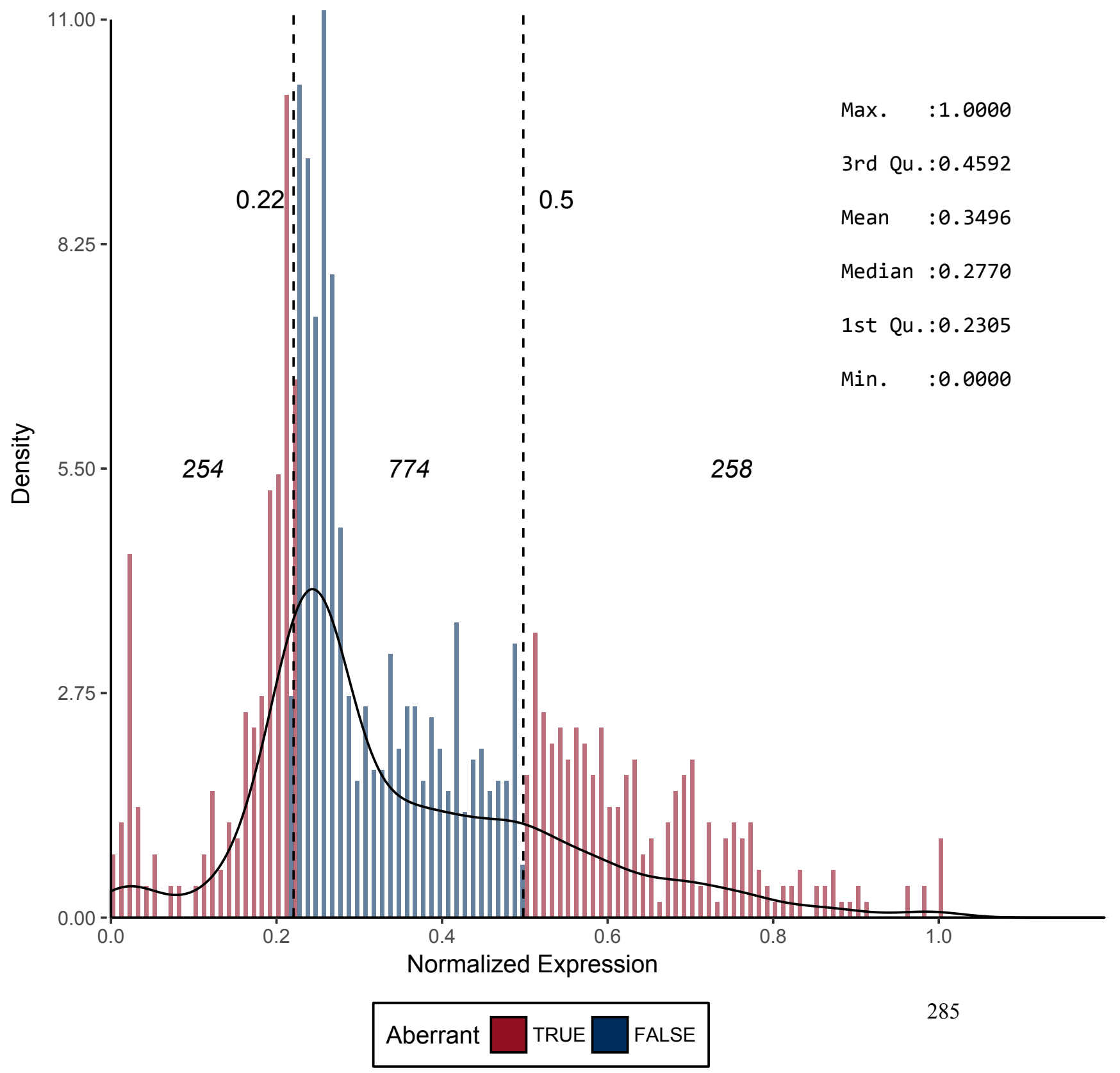


Figure 269 - CD267

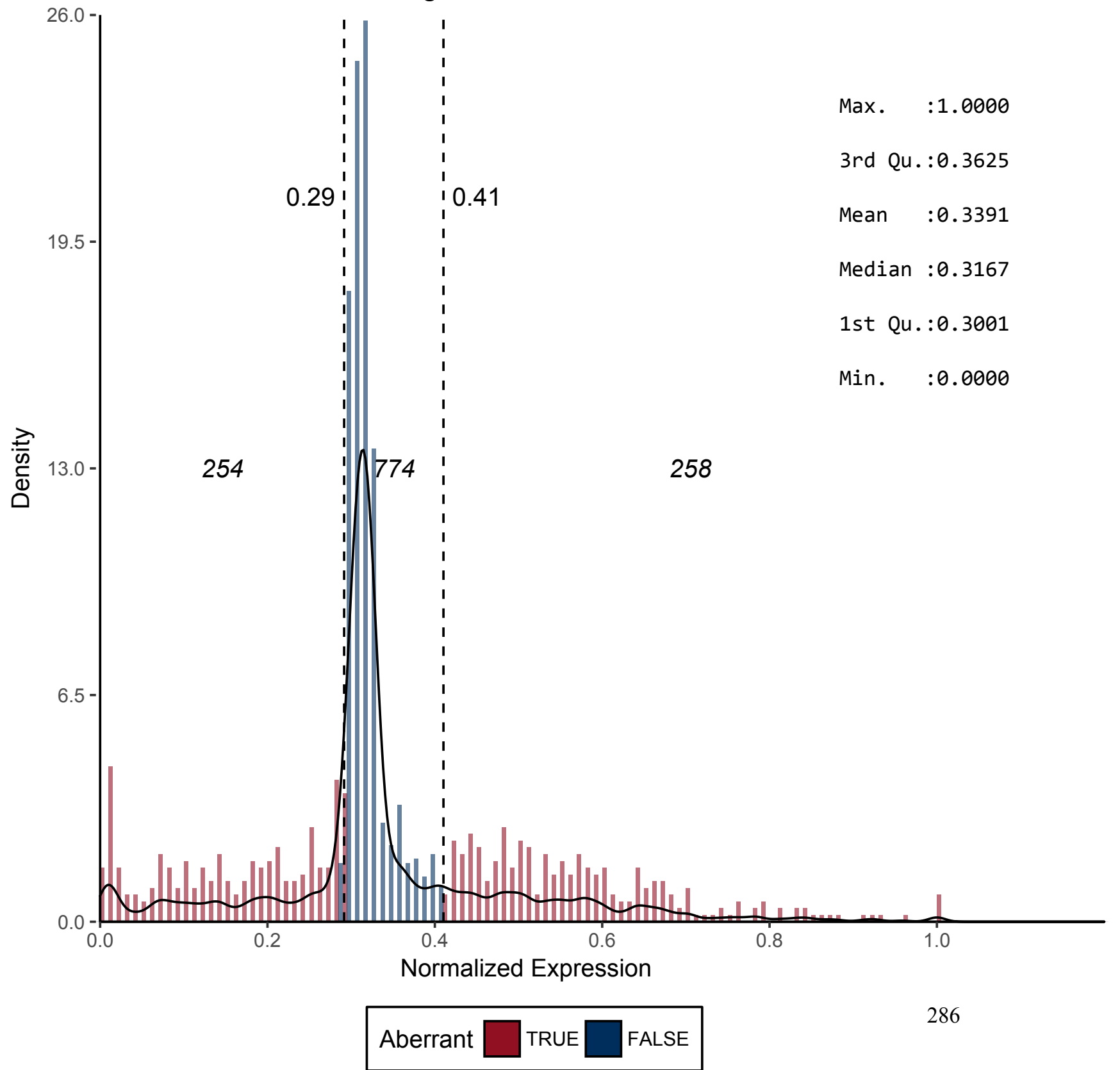


Figure 270 - CD269

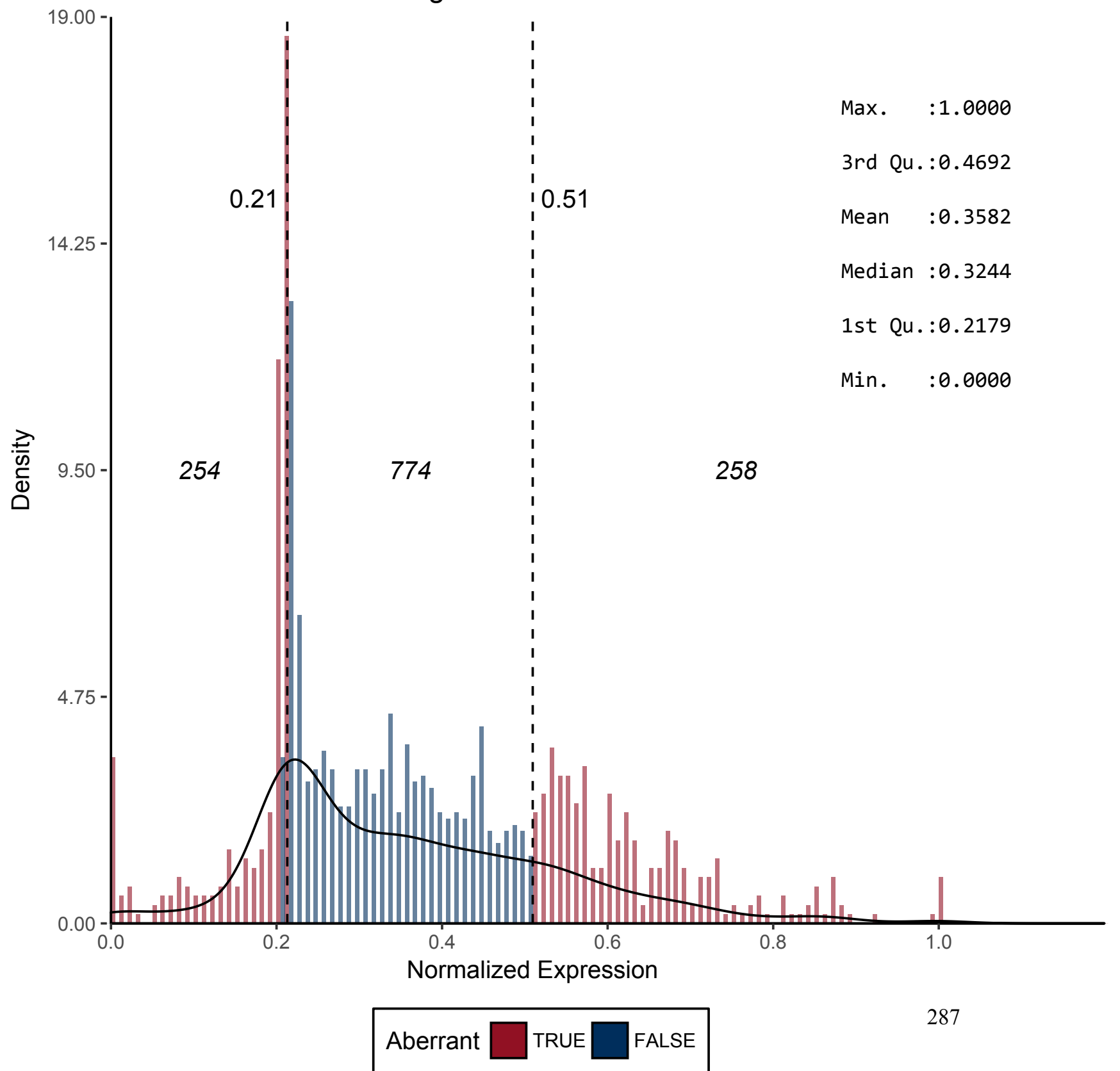


Figure 272 - CD271*

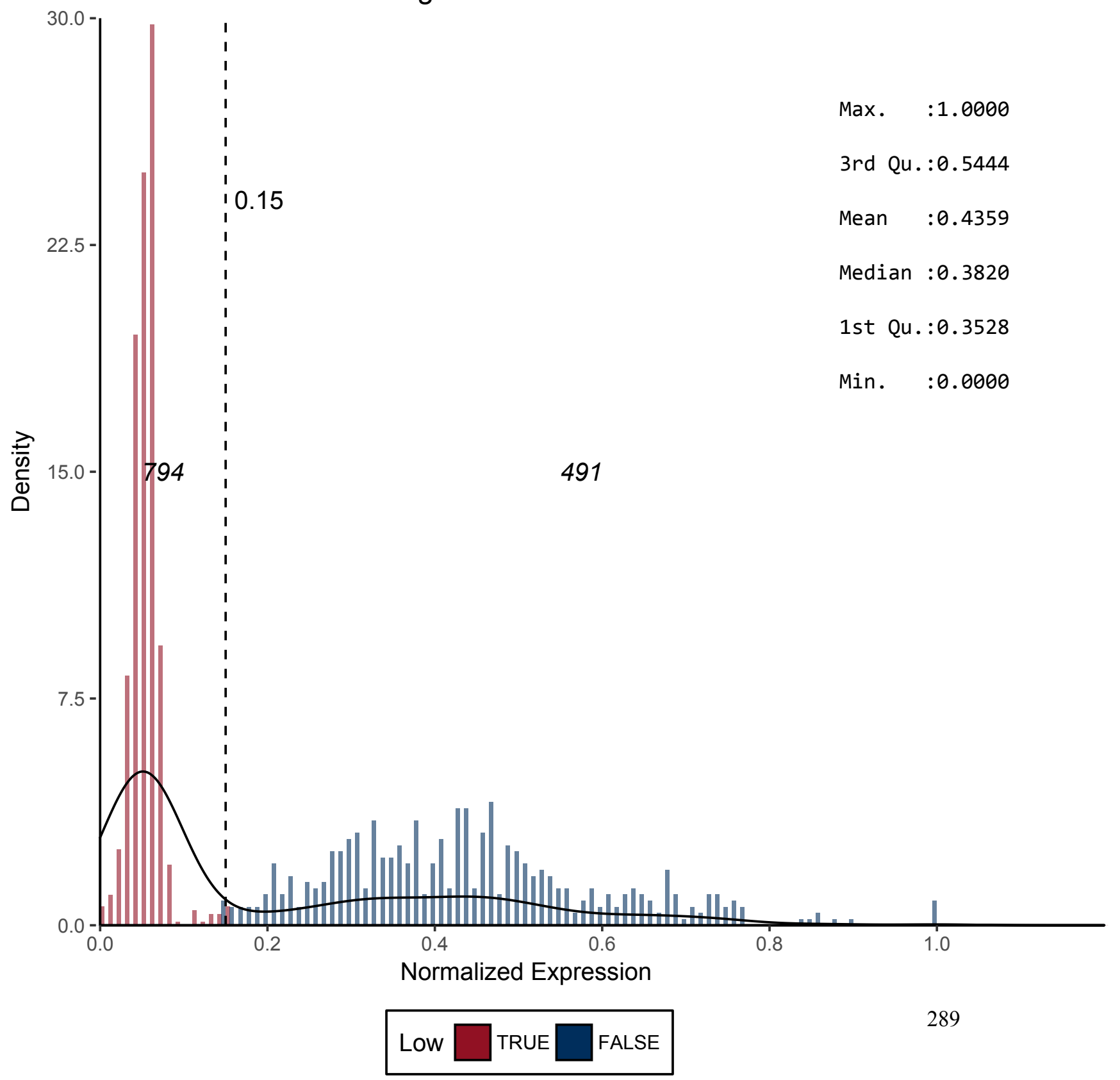


Figure 273 - CD272

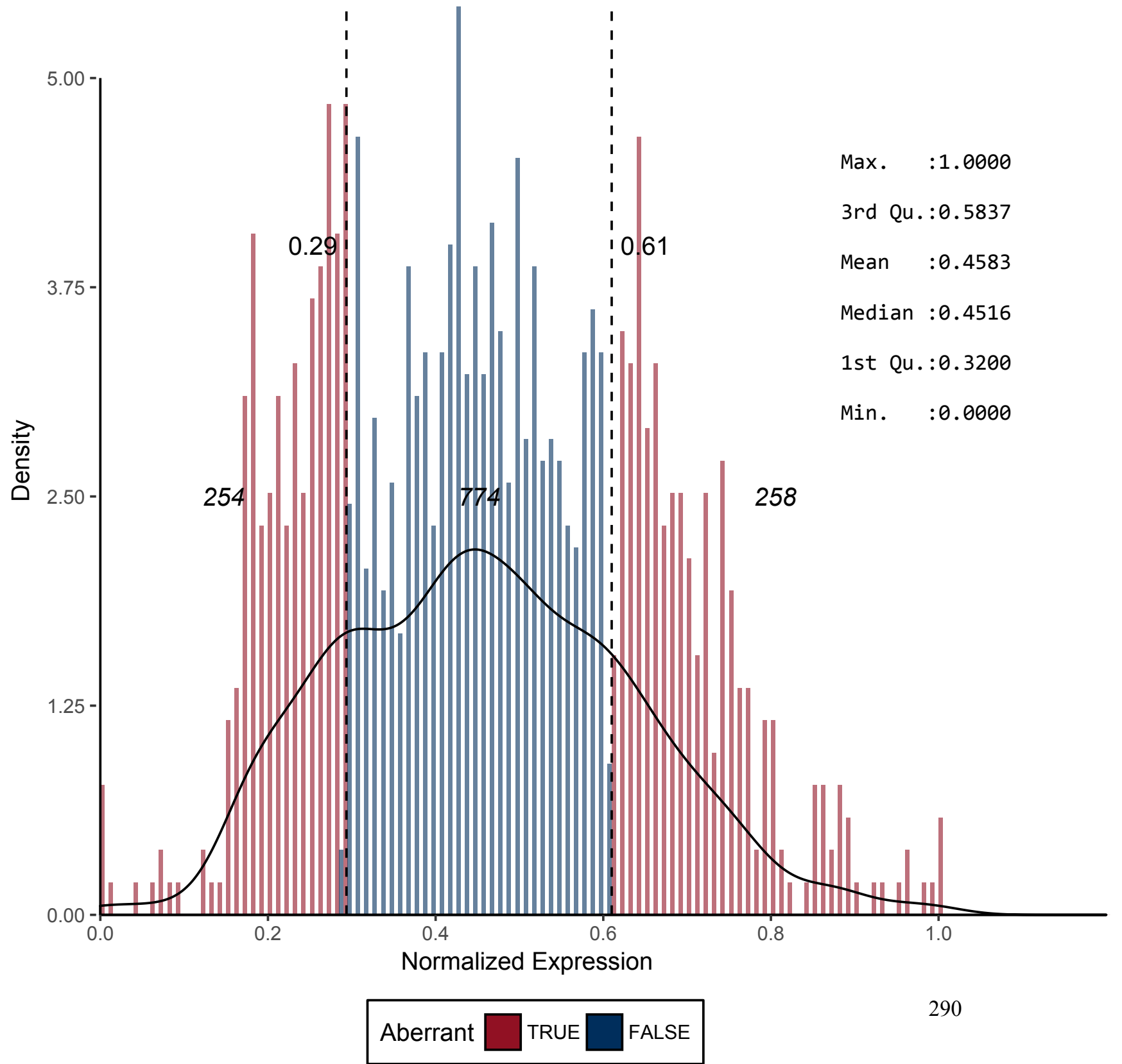


Figure 274 - CD273*

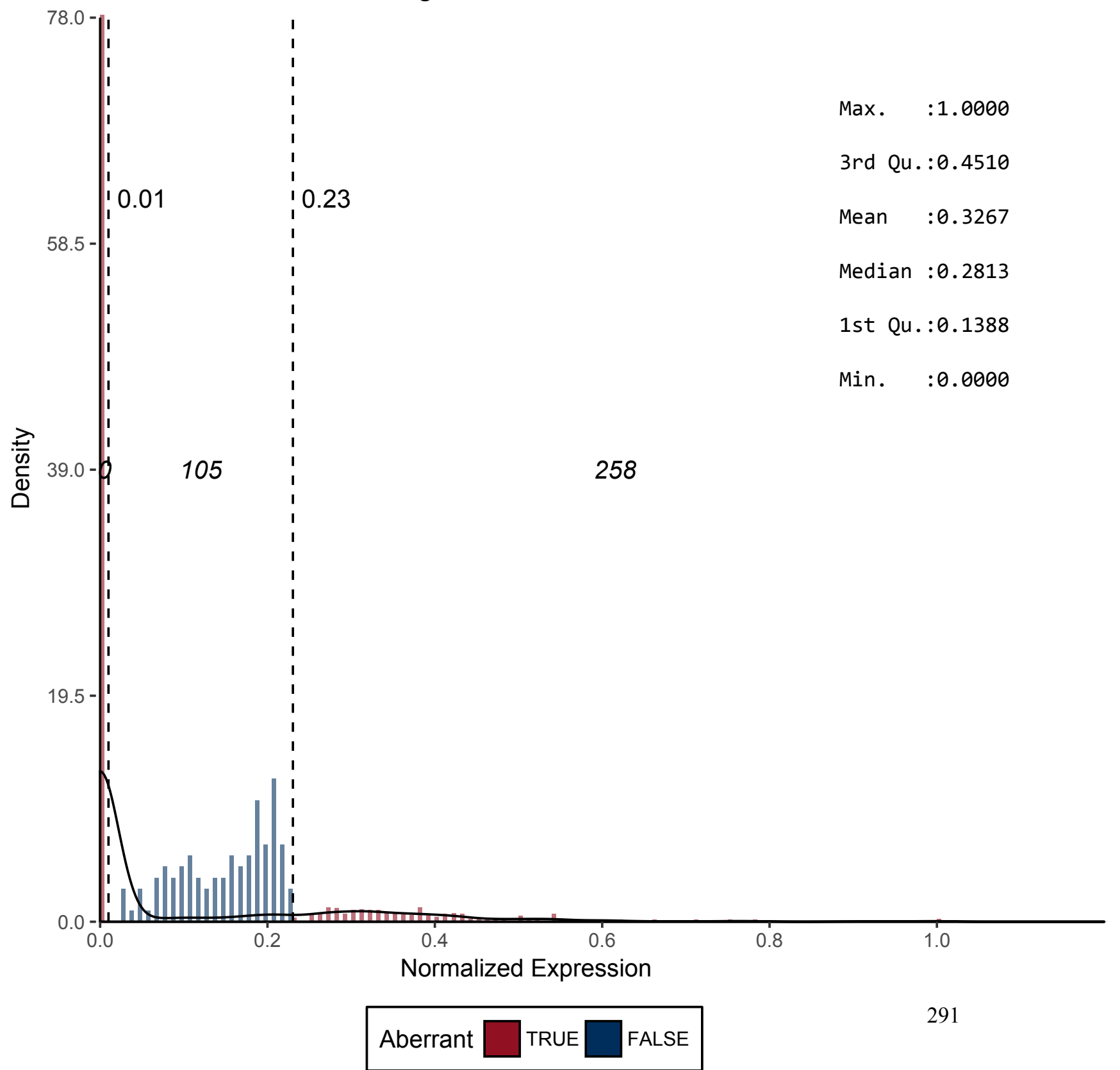


Figure 276 - CD276*

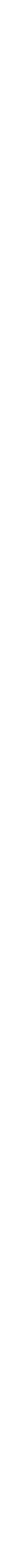


Figure 277 - CD277

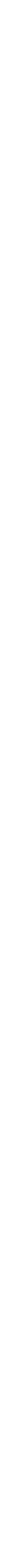


Figure 278 - CD278

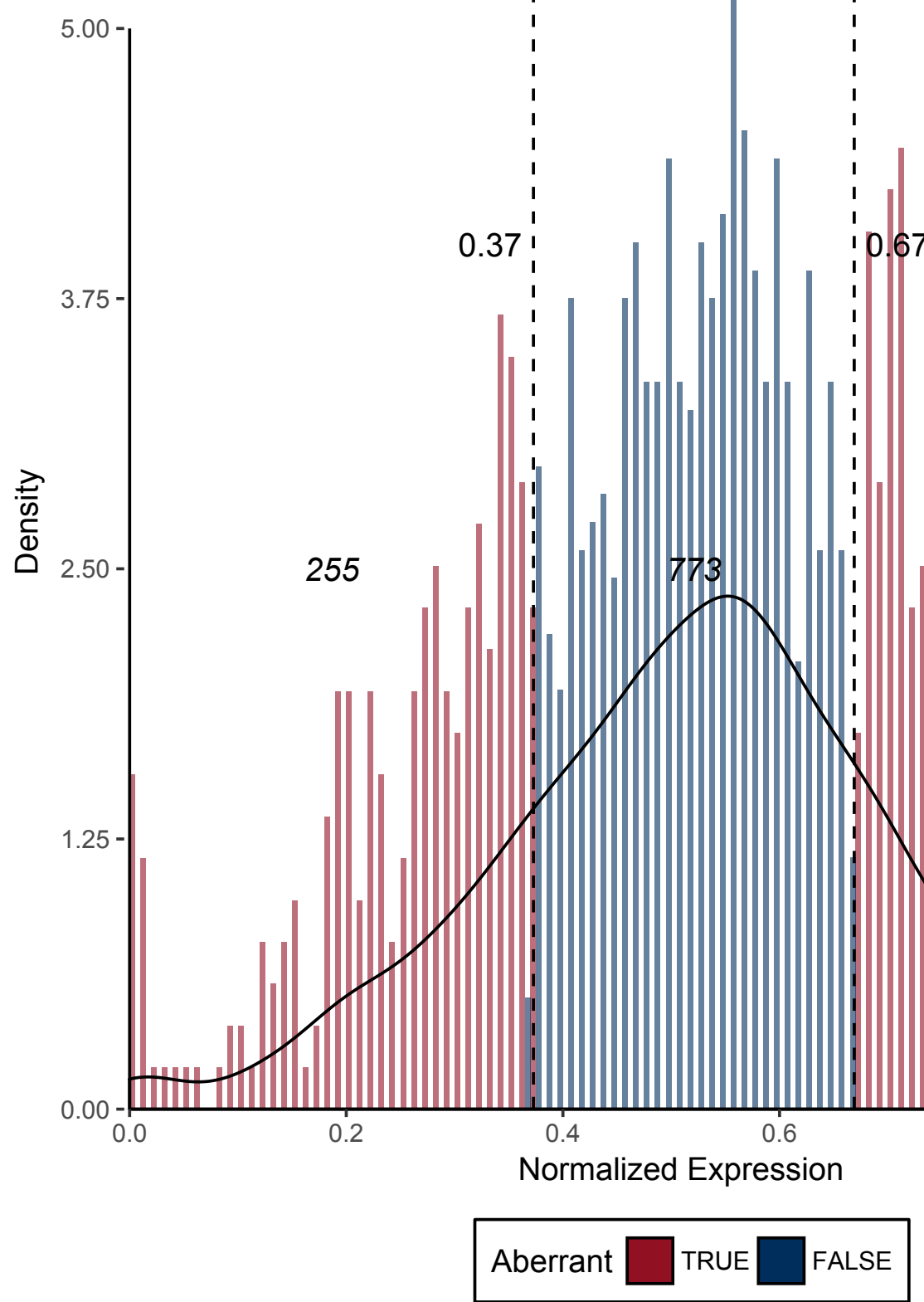

Max. $: 1.0000$ 3rd Qu.:0.6423

Mean $\quad: 0.5204$

Median :0.5303

1st Qu.:0.4065

Min. $\quad: 0.0000$ 
Figure 279 - CD279*

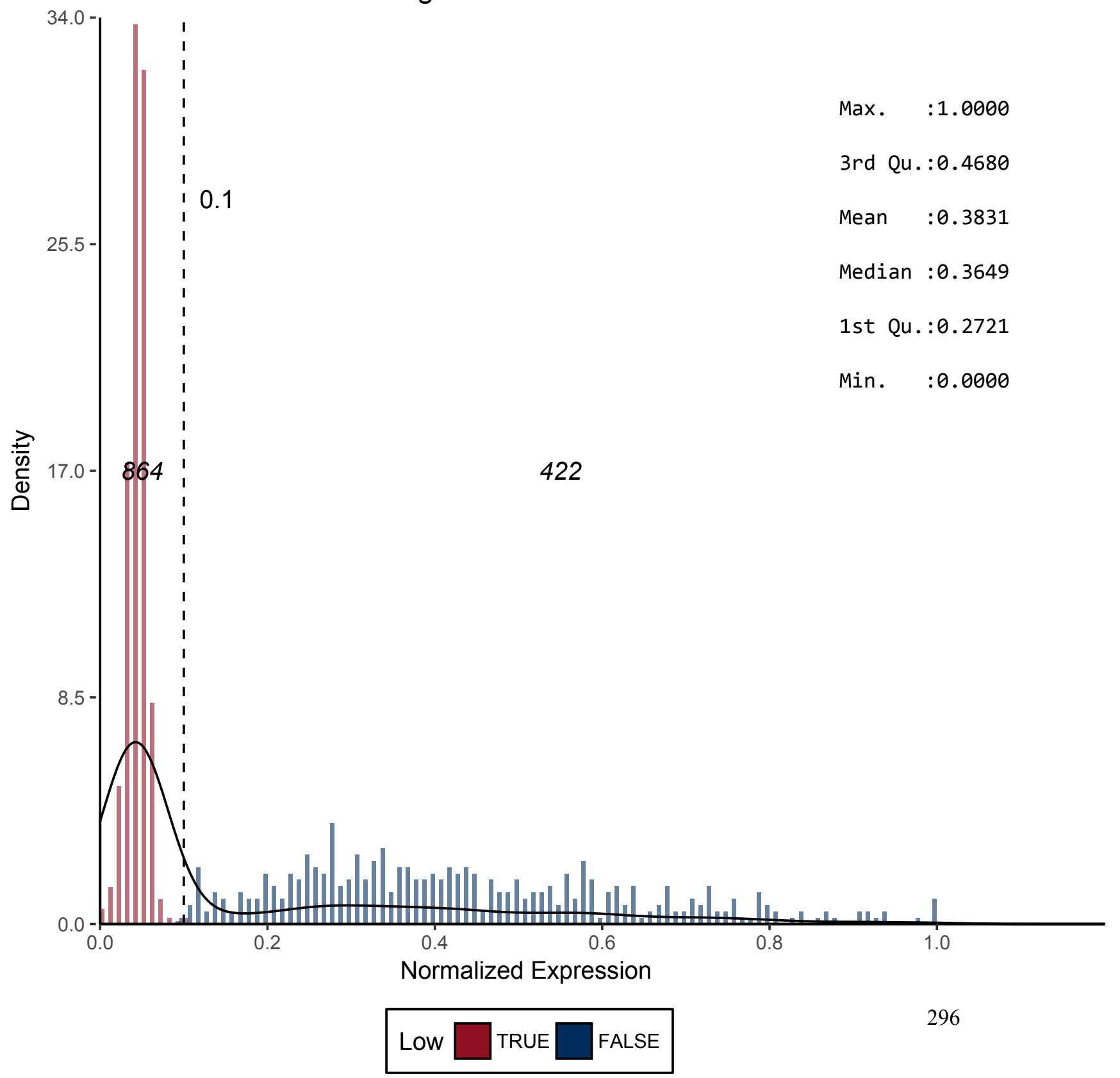


Figure 280 - CD280*

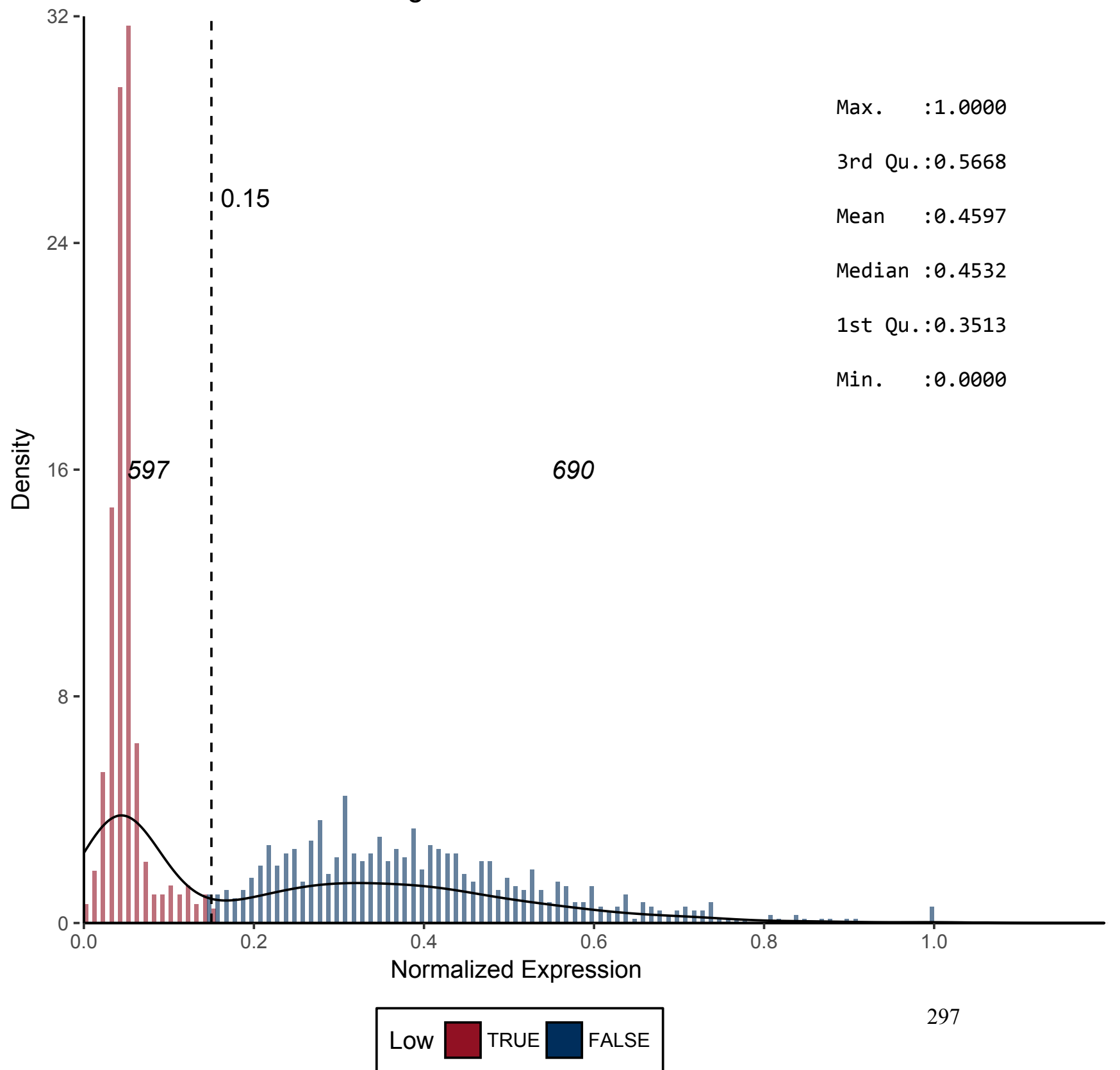


Figure 281 - CD281

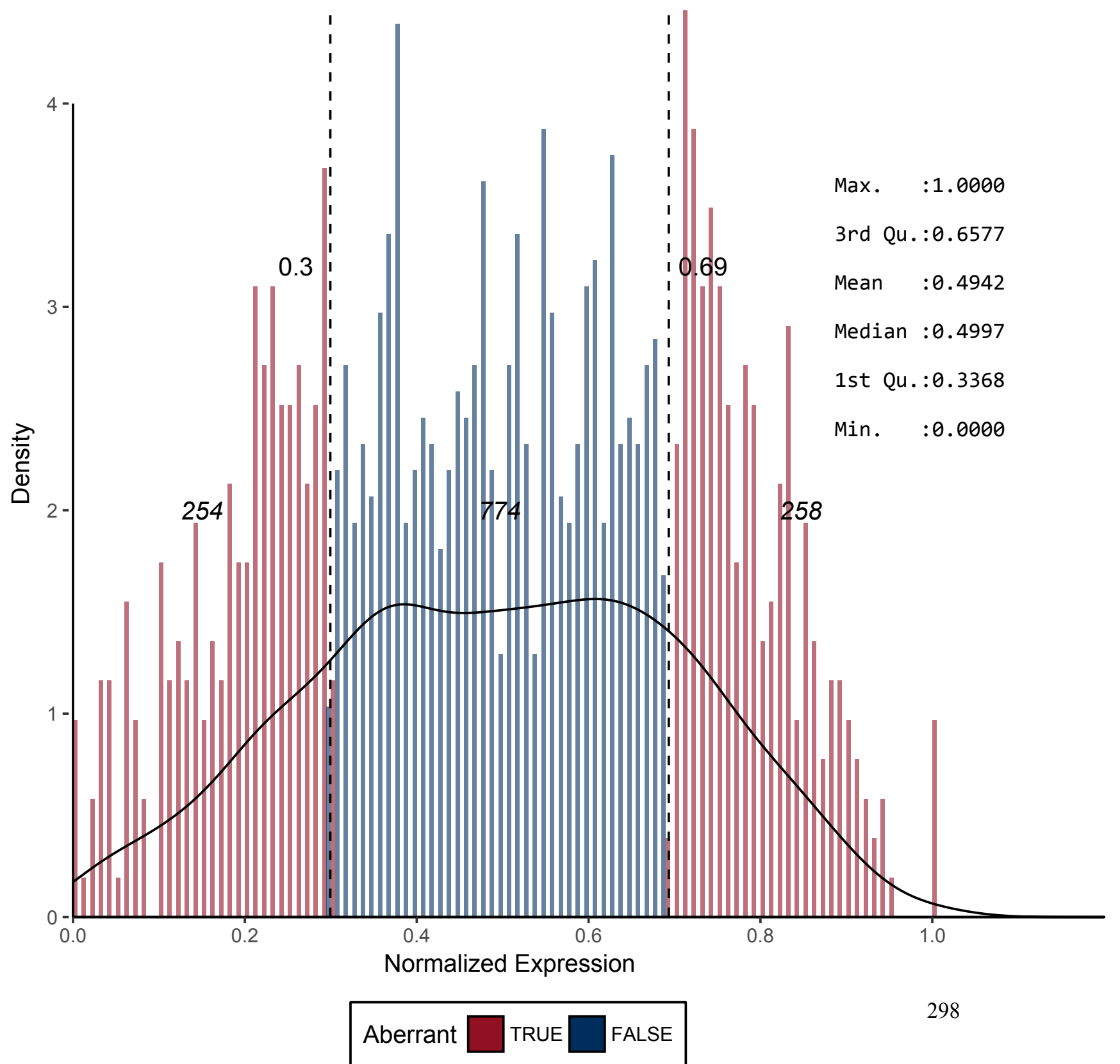


Figure 284 - CD284
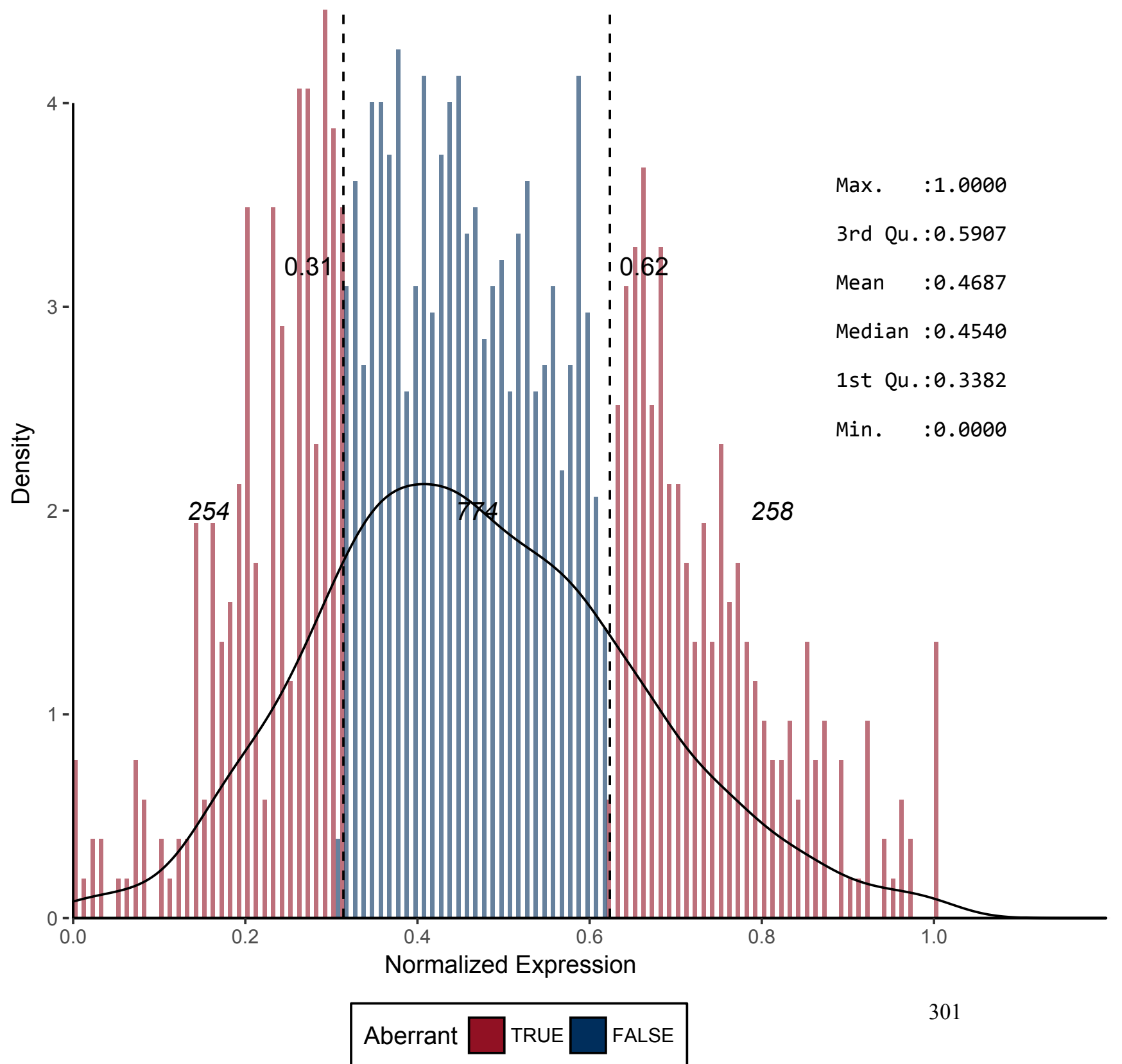
Figure 285 - CD286

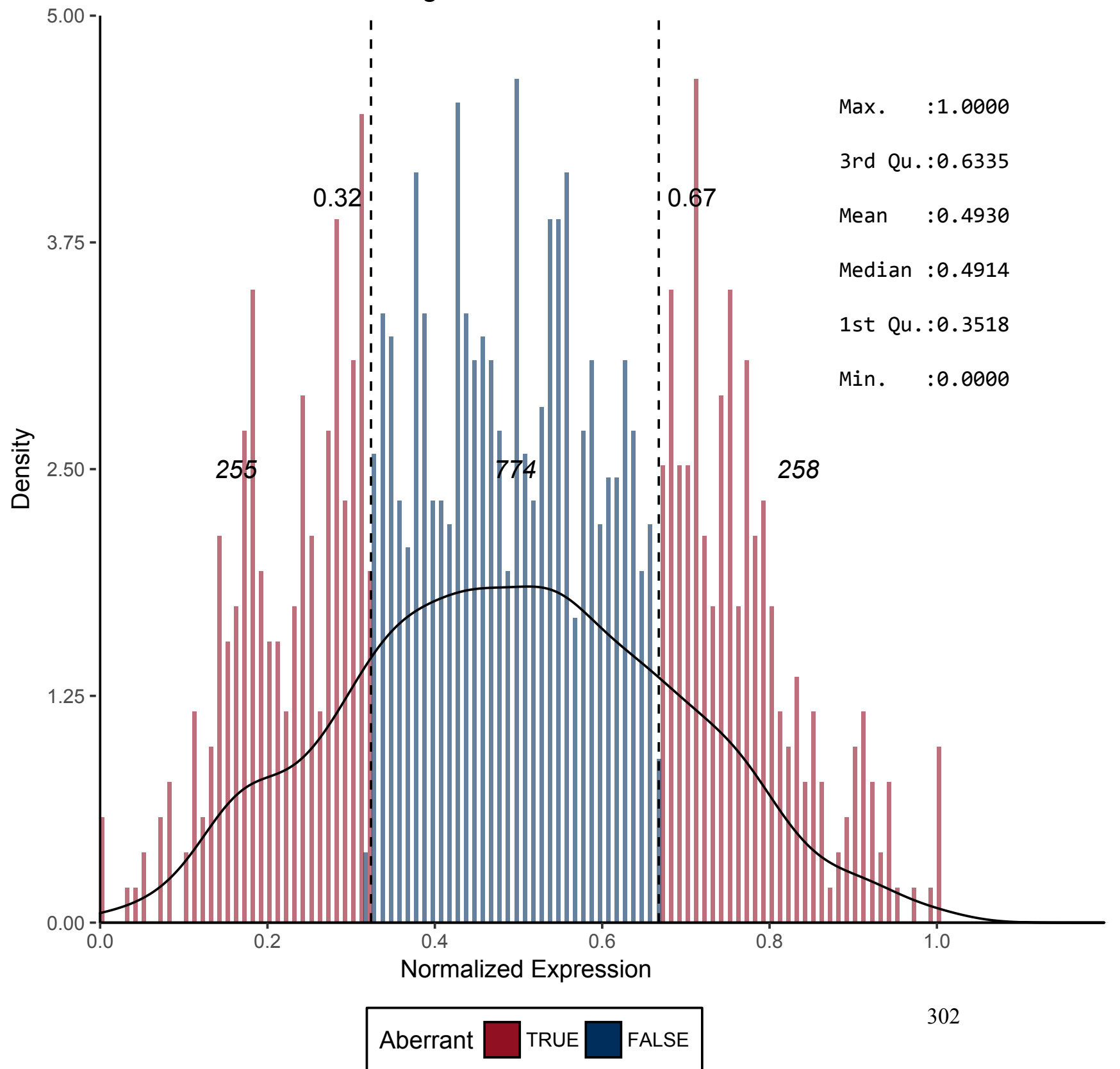


Figure 287 - CD289

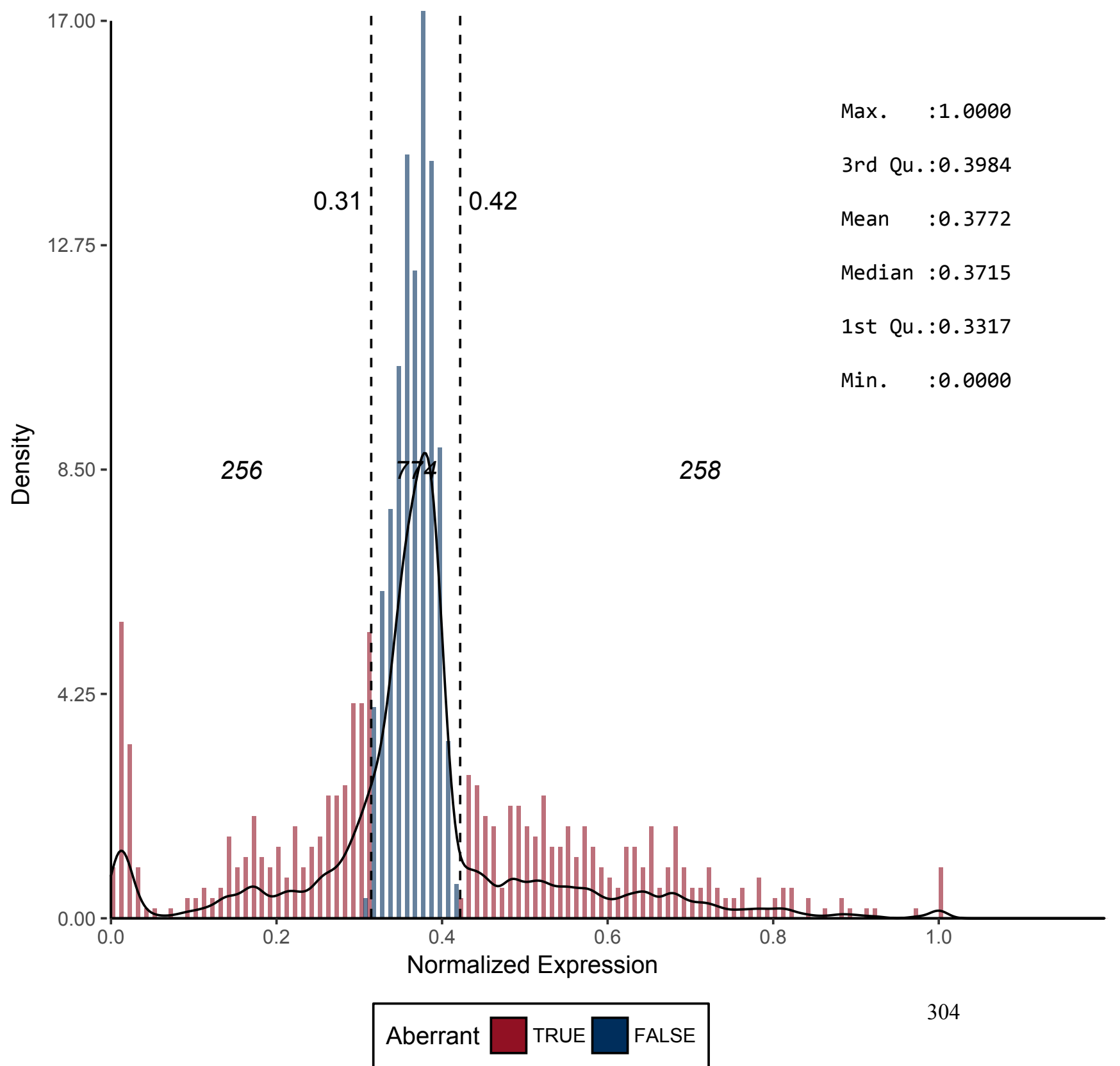


Figure 288 - CD290

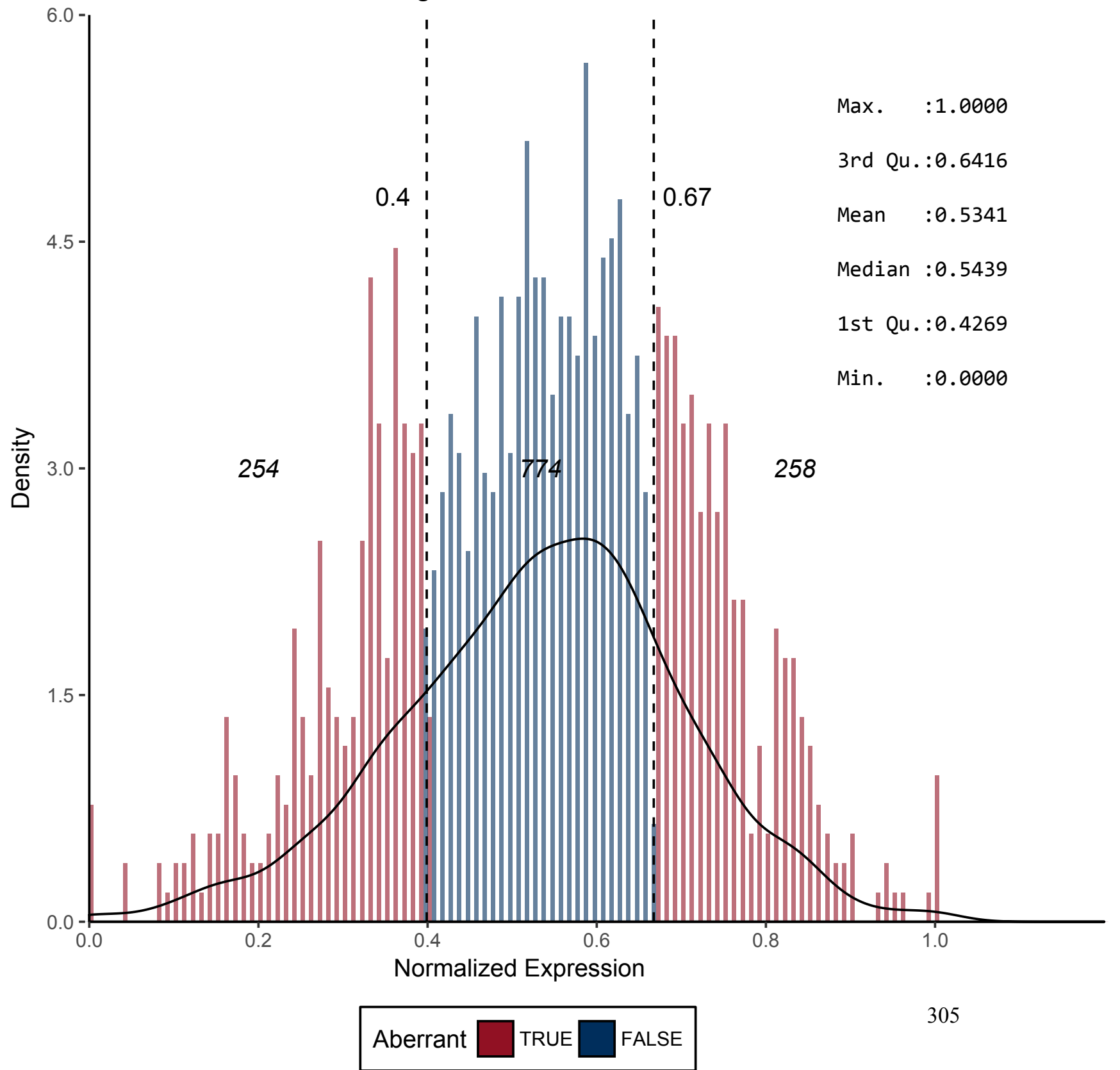


Figure 289 - CD292*

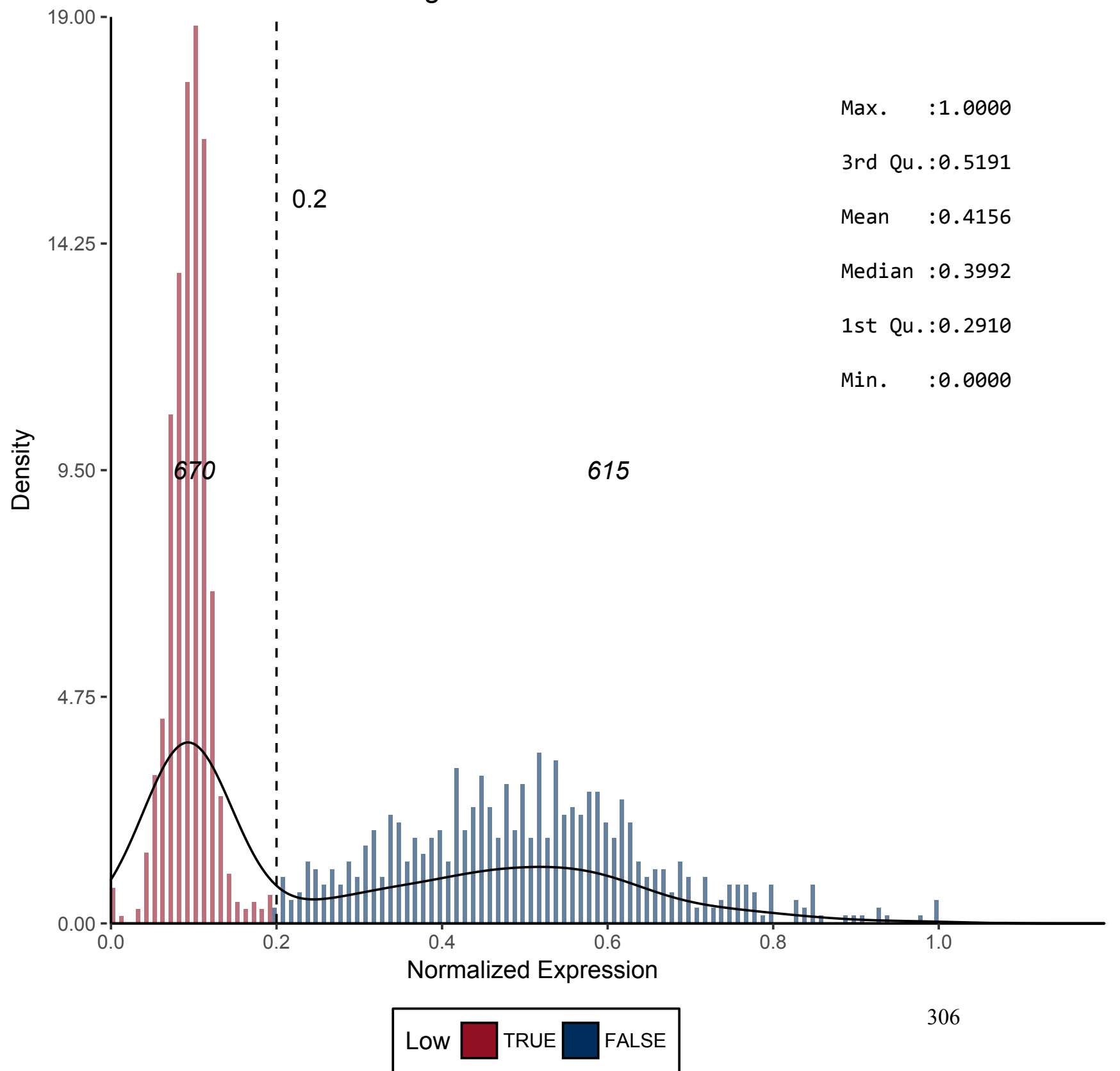


Figure 290 - CDW293*

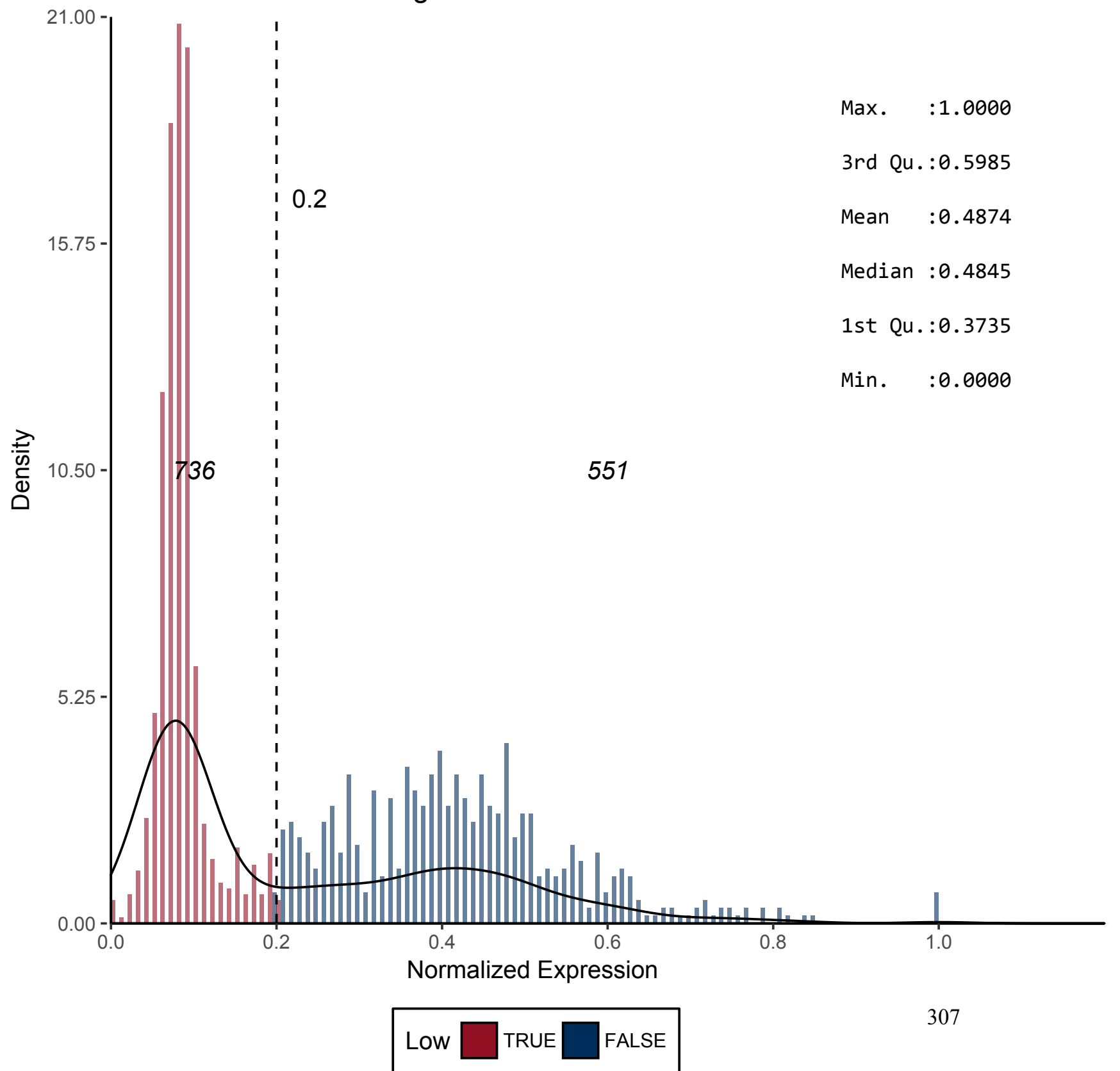


Figure 291 - CD294

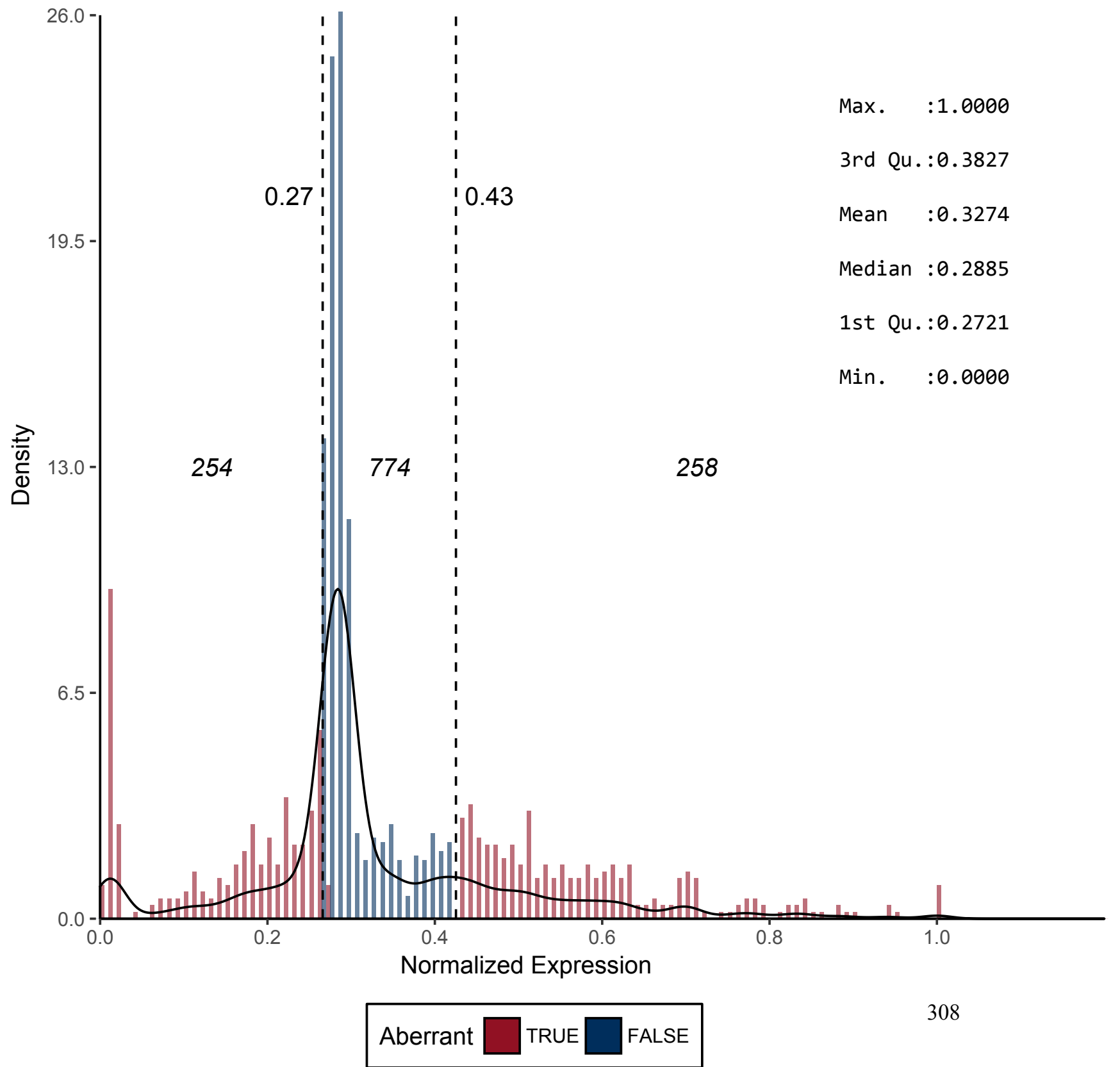


Figure 292 - CD295

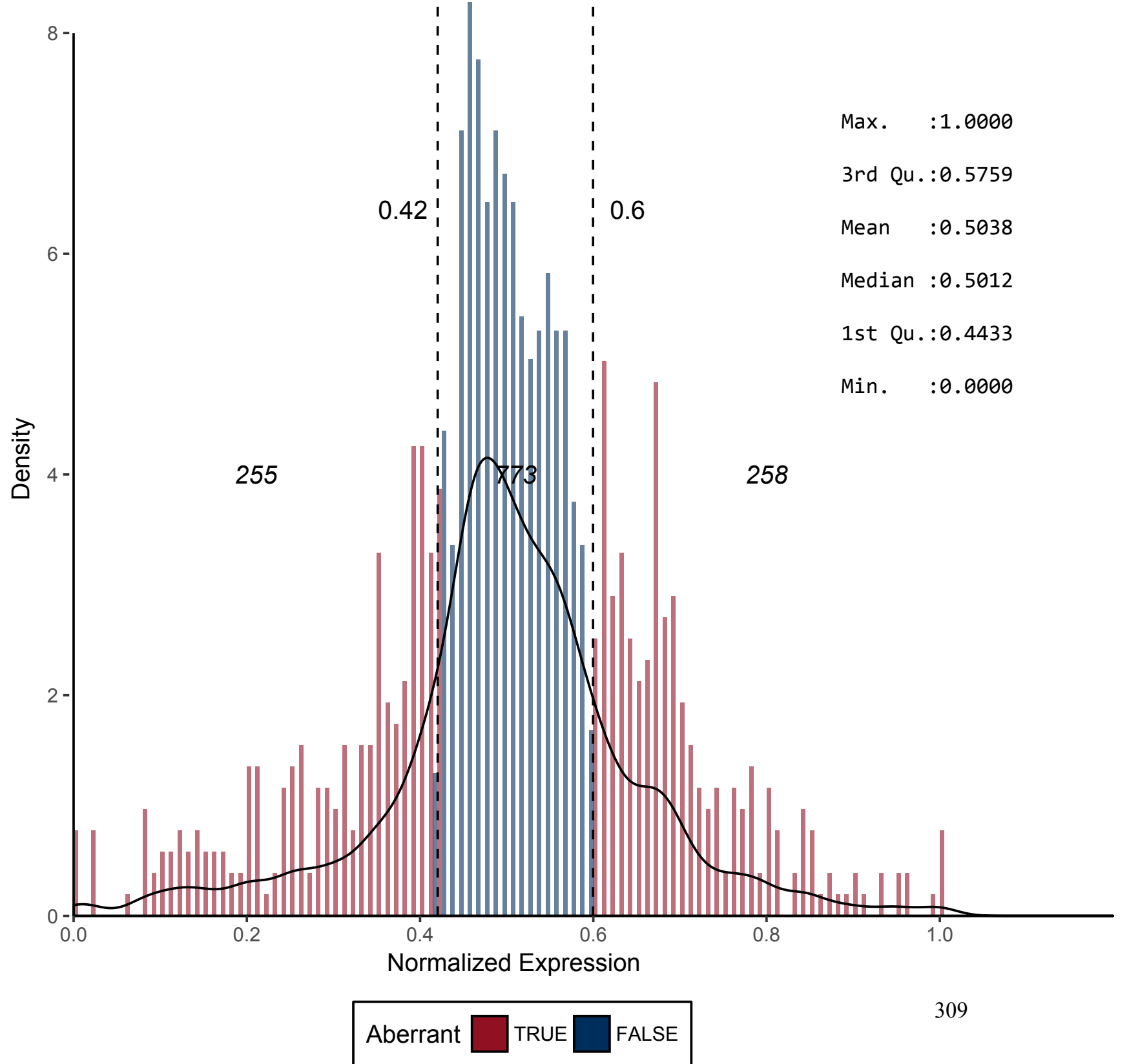


Figure 293 - CD296

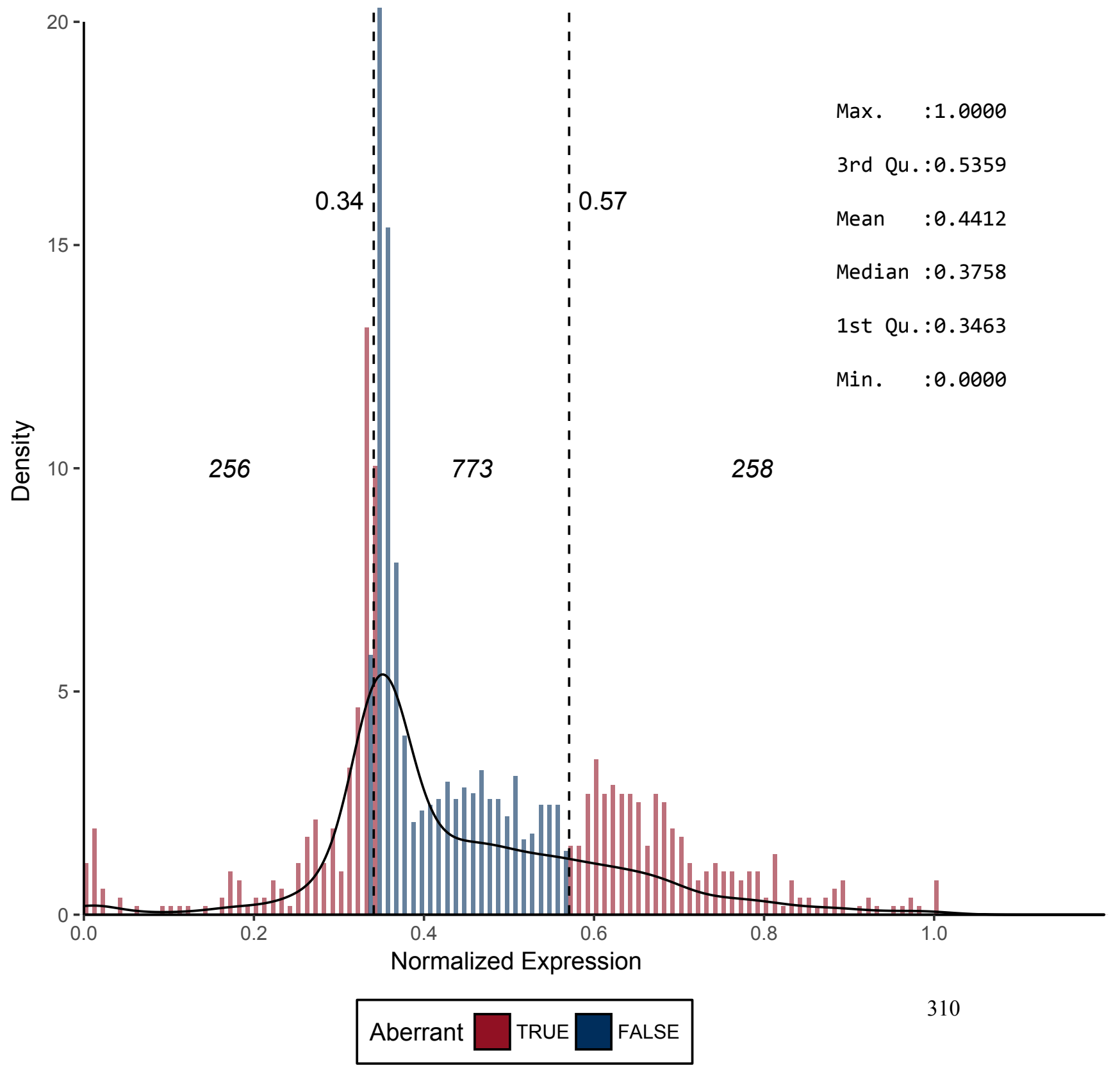


Figure 294 - CD297*

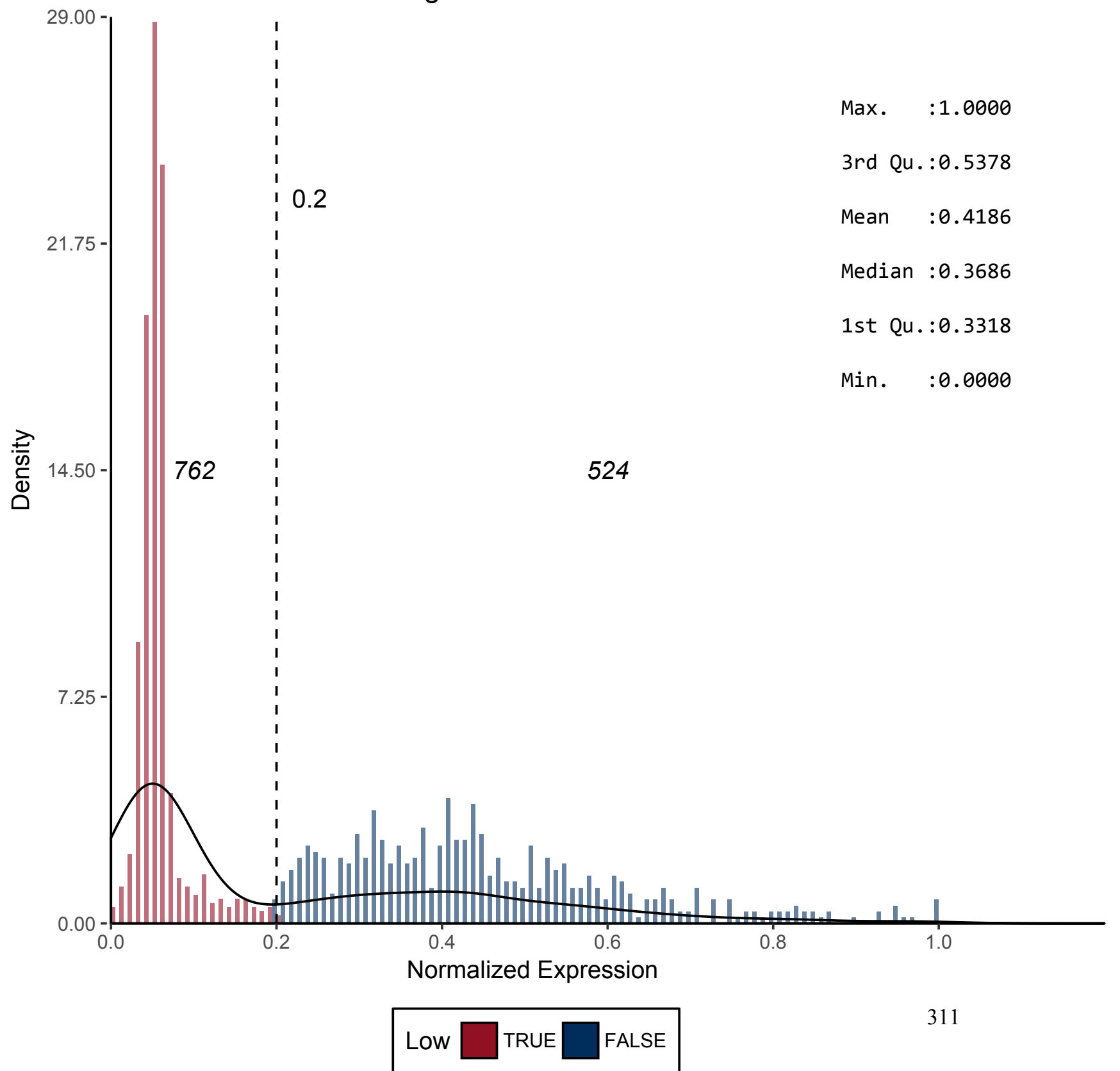


Figure 295 - CD298

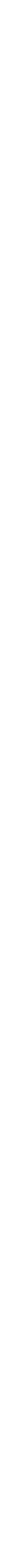


Figure 296 - CD299*

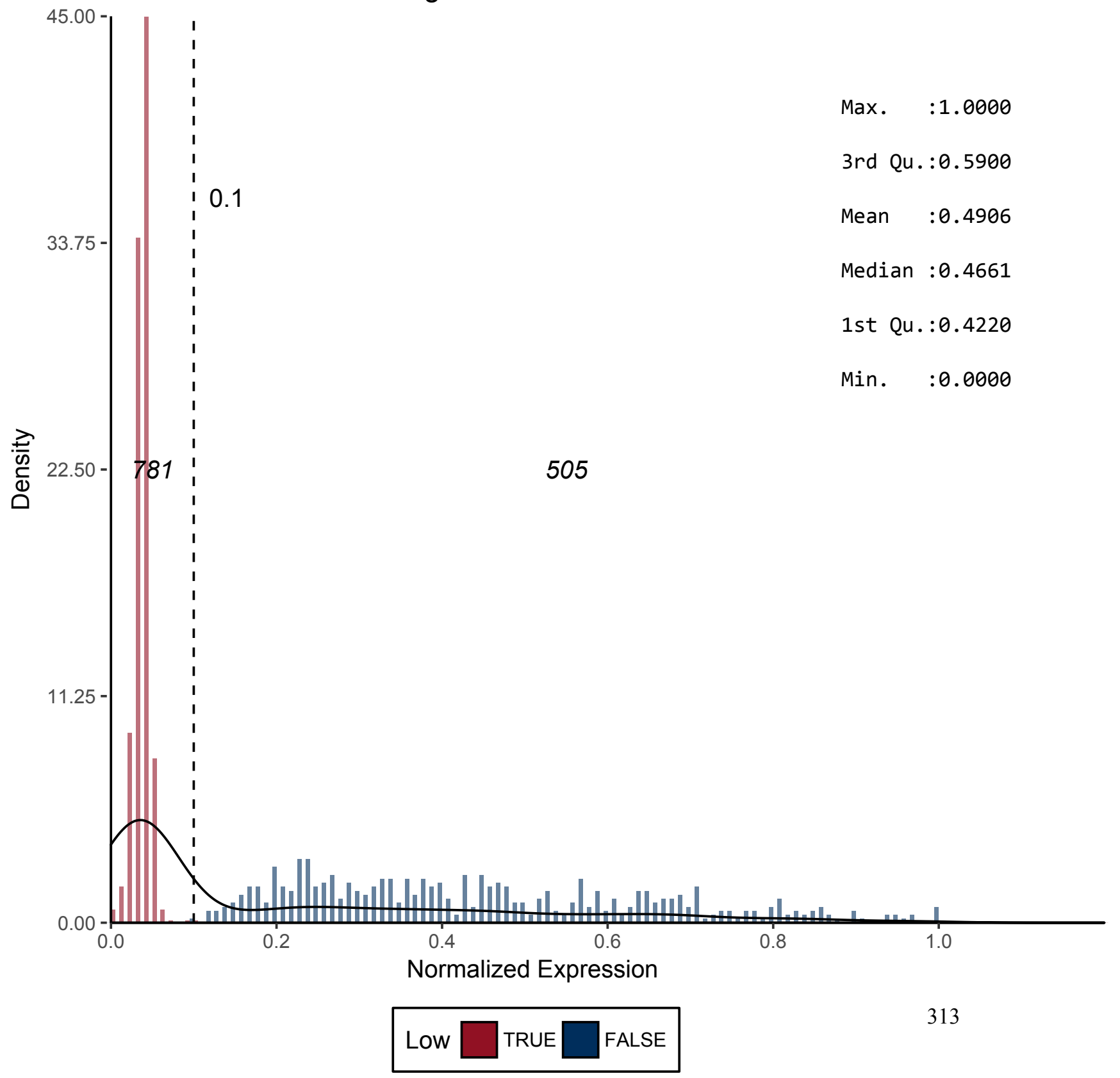


Figure 297 - CD300A

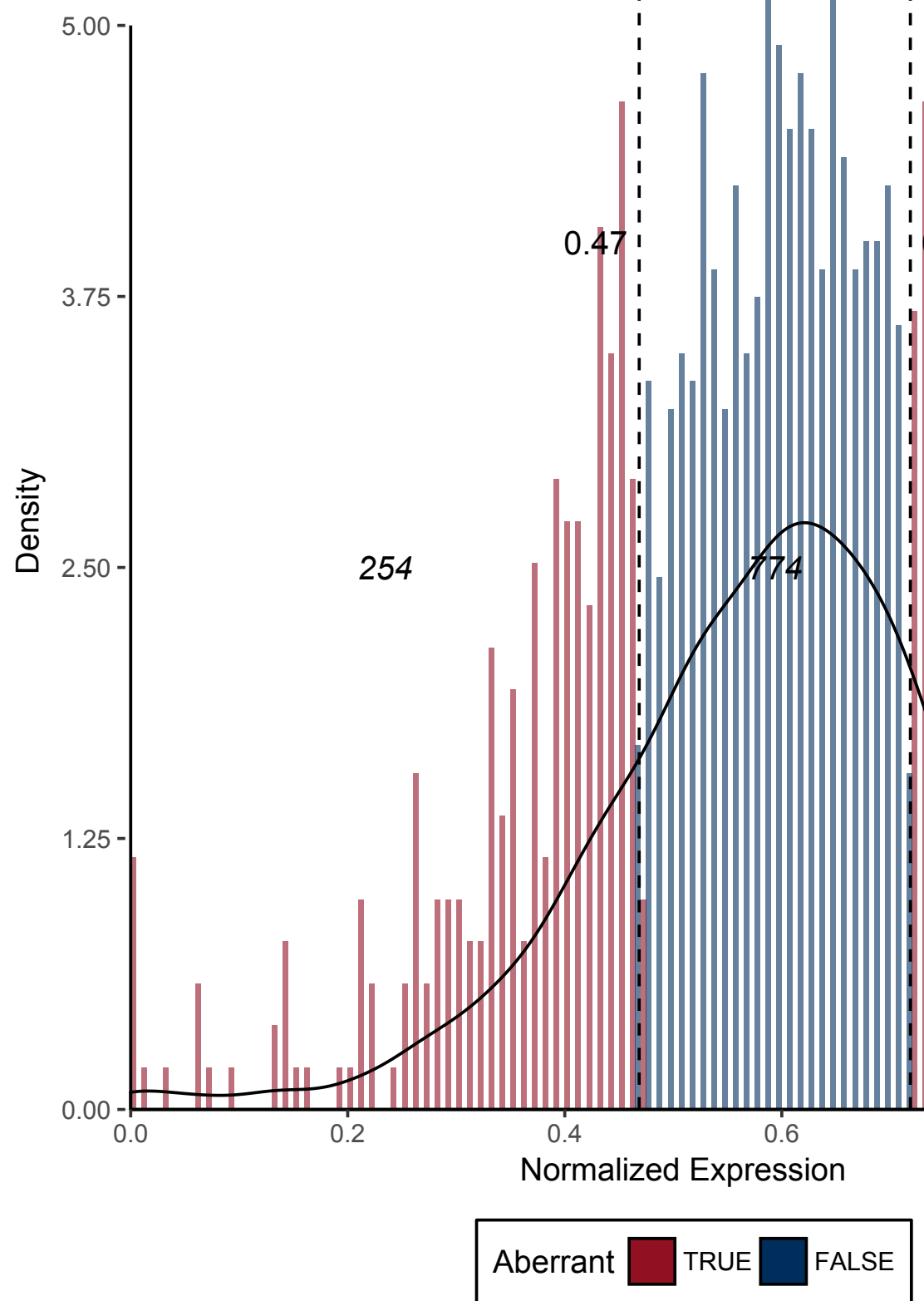

Max. $: 1.0000$

3rd Qu.:0.6974

Mean $\quad: 0.5909$

Median :0.6021

1st Qu.:0.4993

Min. $\quad: 0.0000$ 
Figure 298 - CD300C

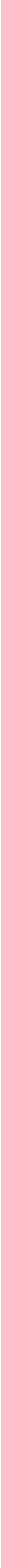


Figure 299 - CD301

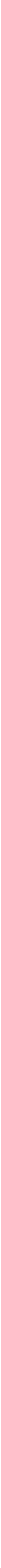


Figure 300 - CD304*

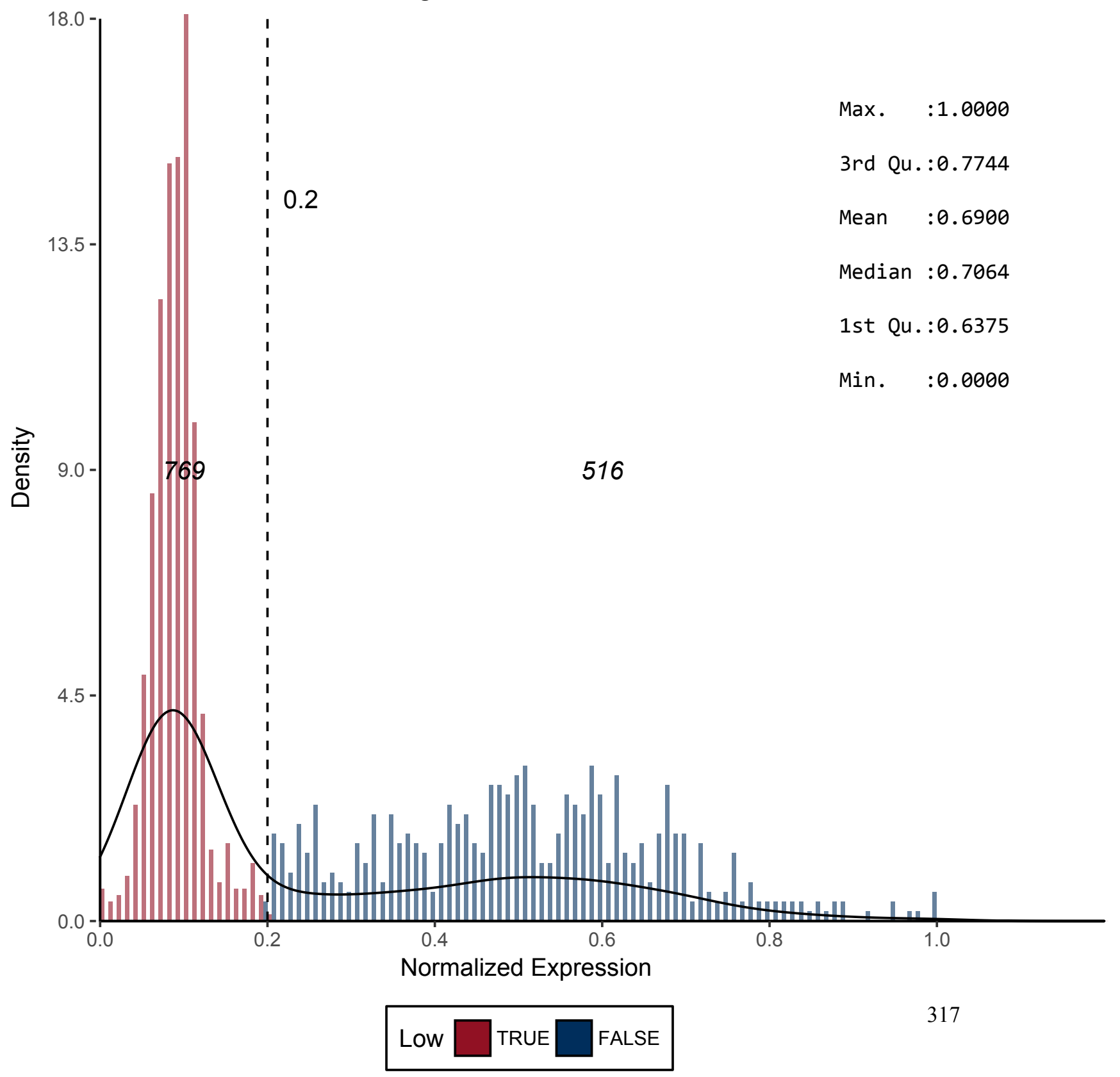


Figure 301 - CD305

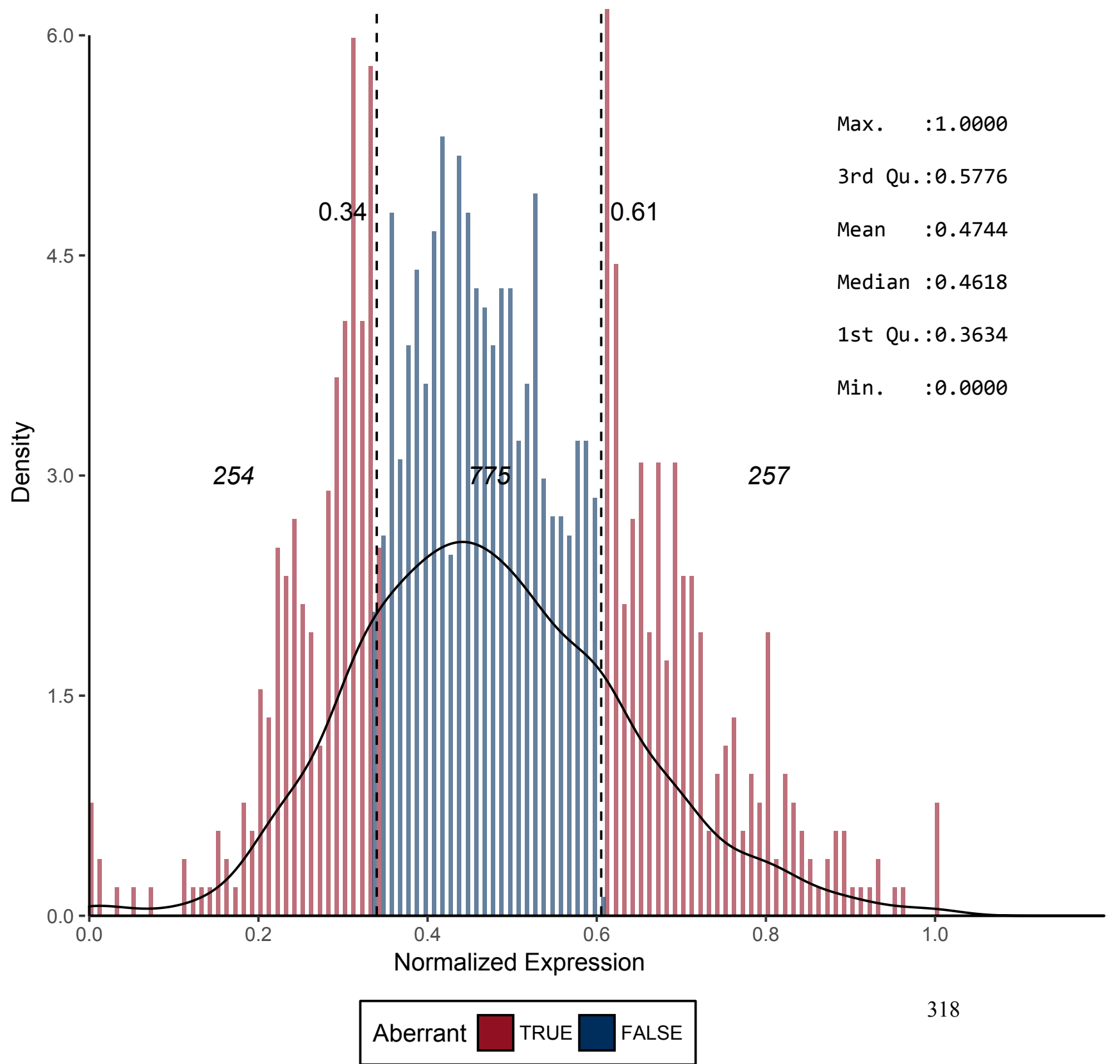


Figure 302 - CD306

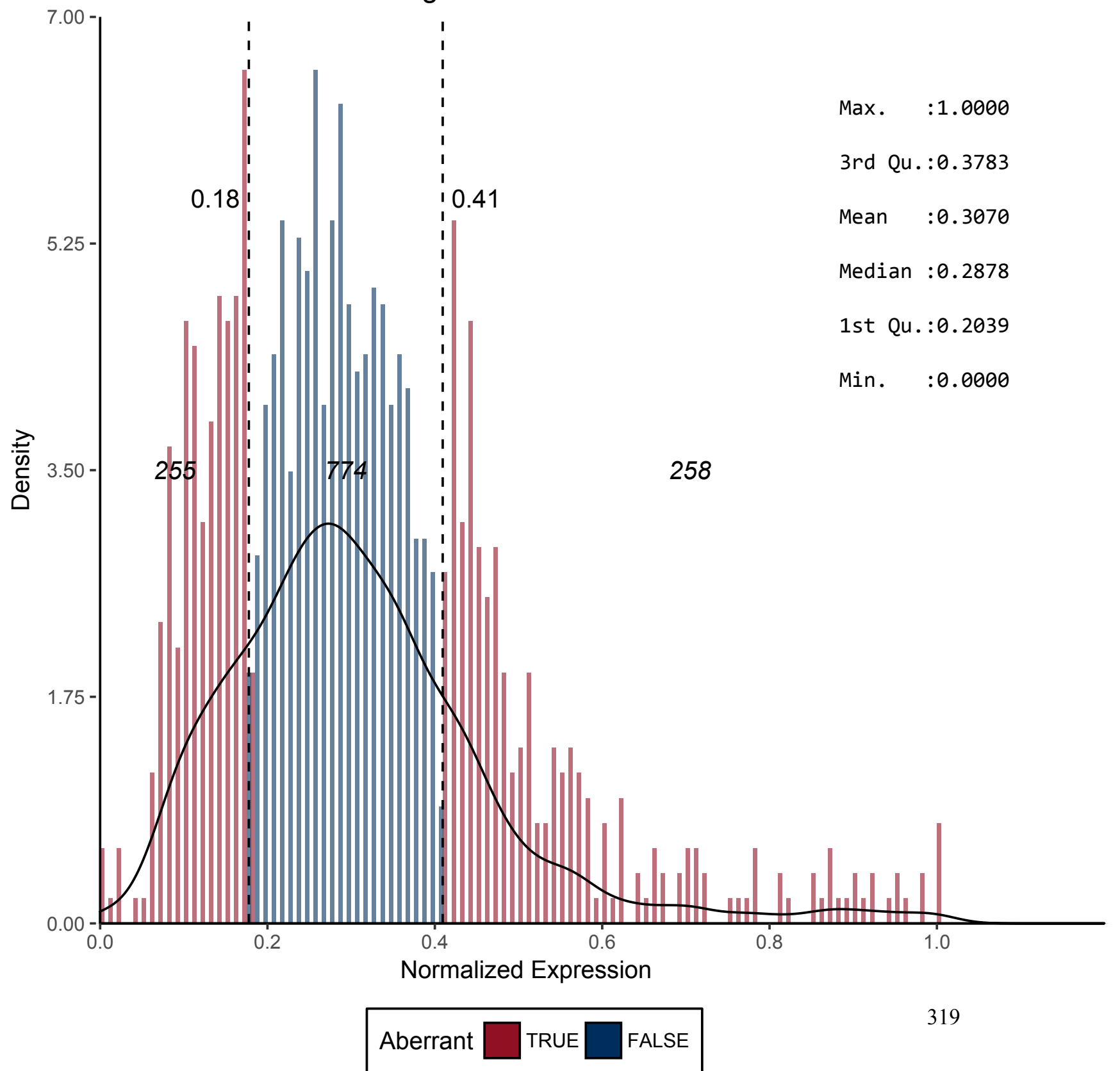


Figure 303 - CD307A*

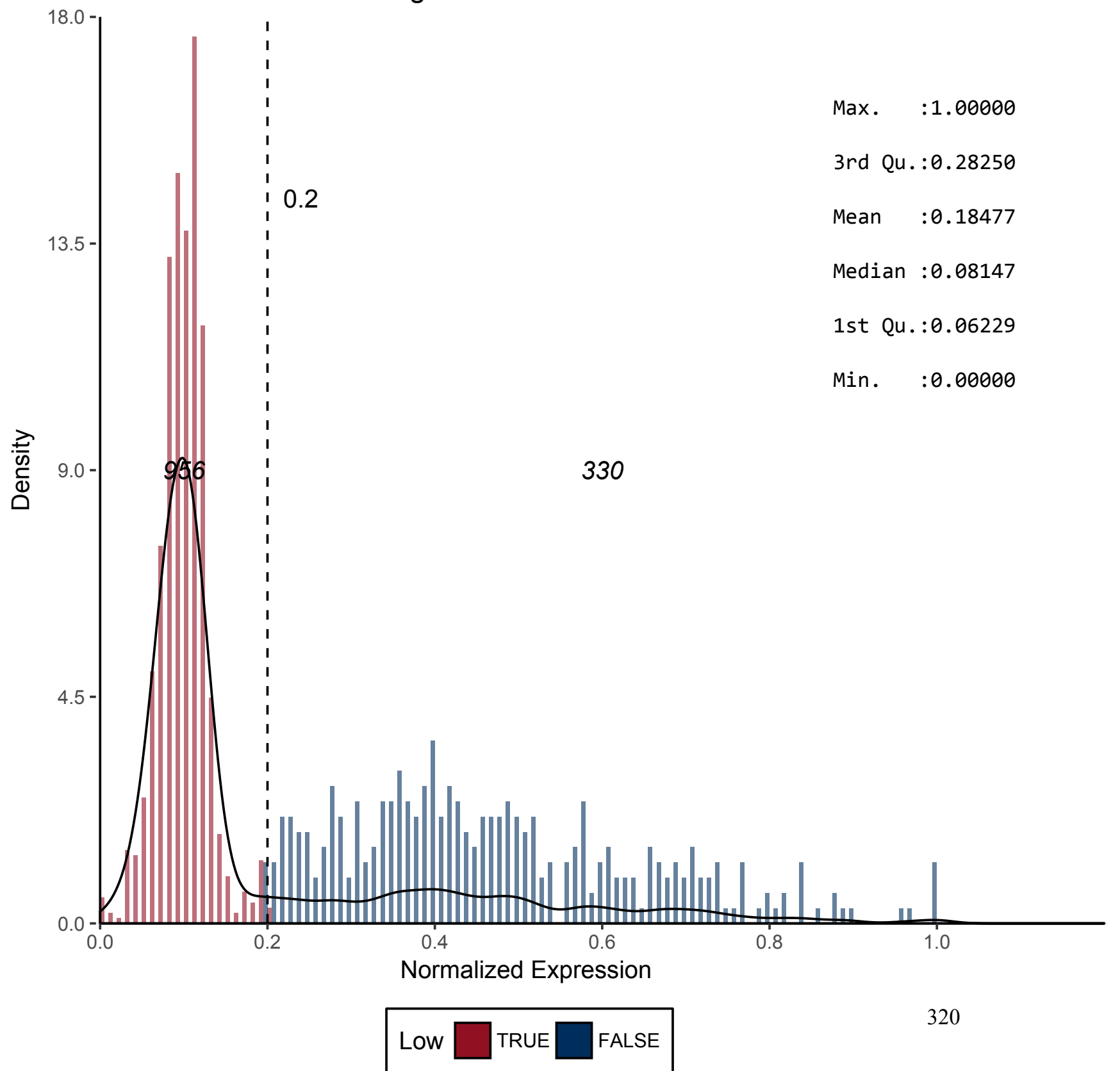


Figure 304 - CD307B

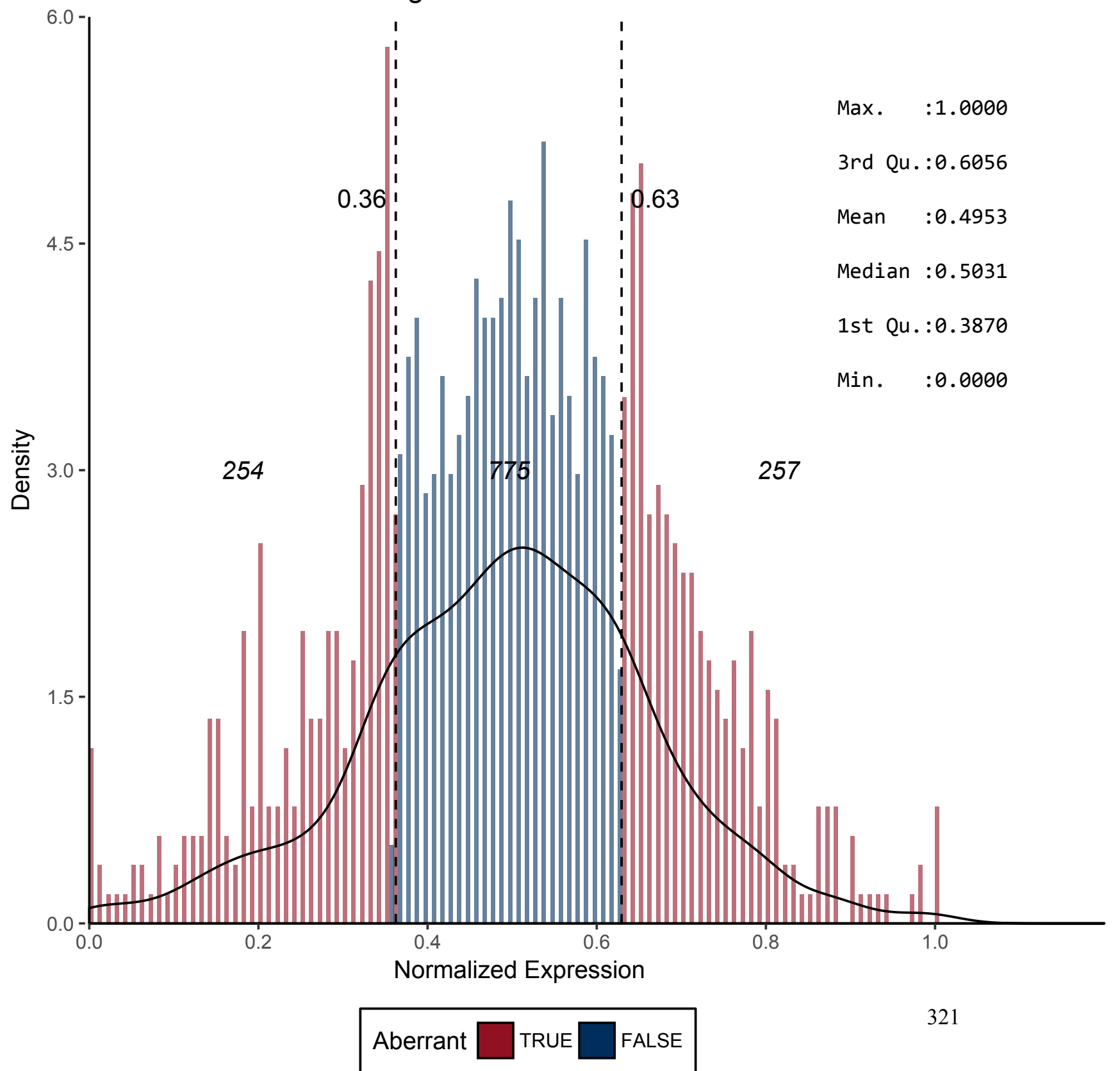


Figure 305 - CD307C

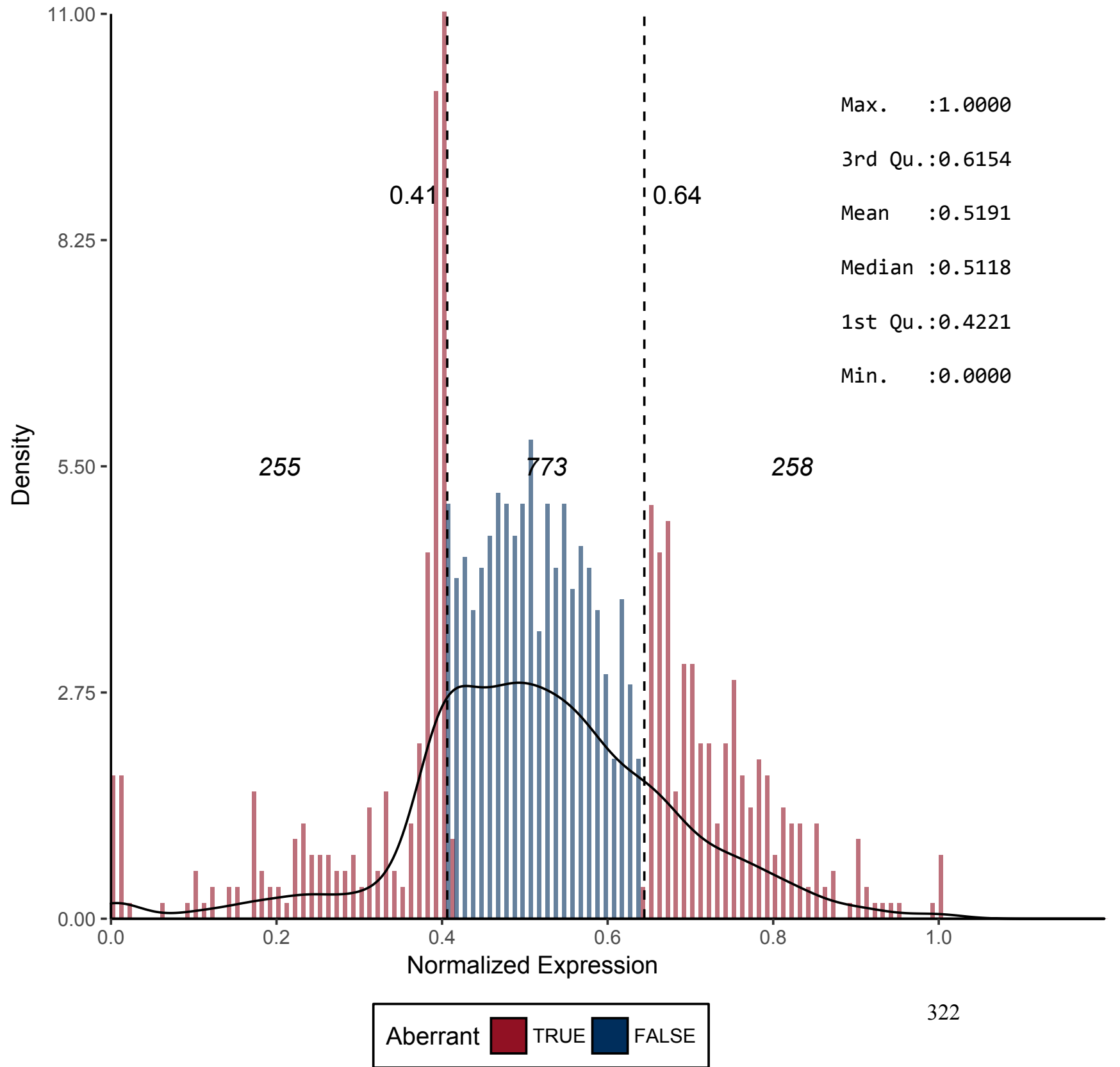


Figure 307 - CD307E

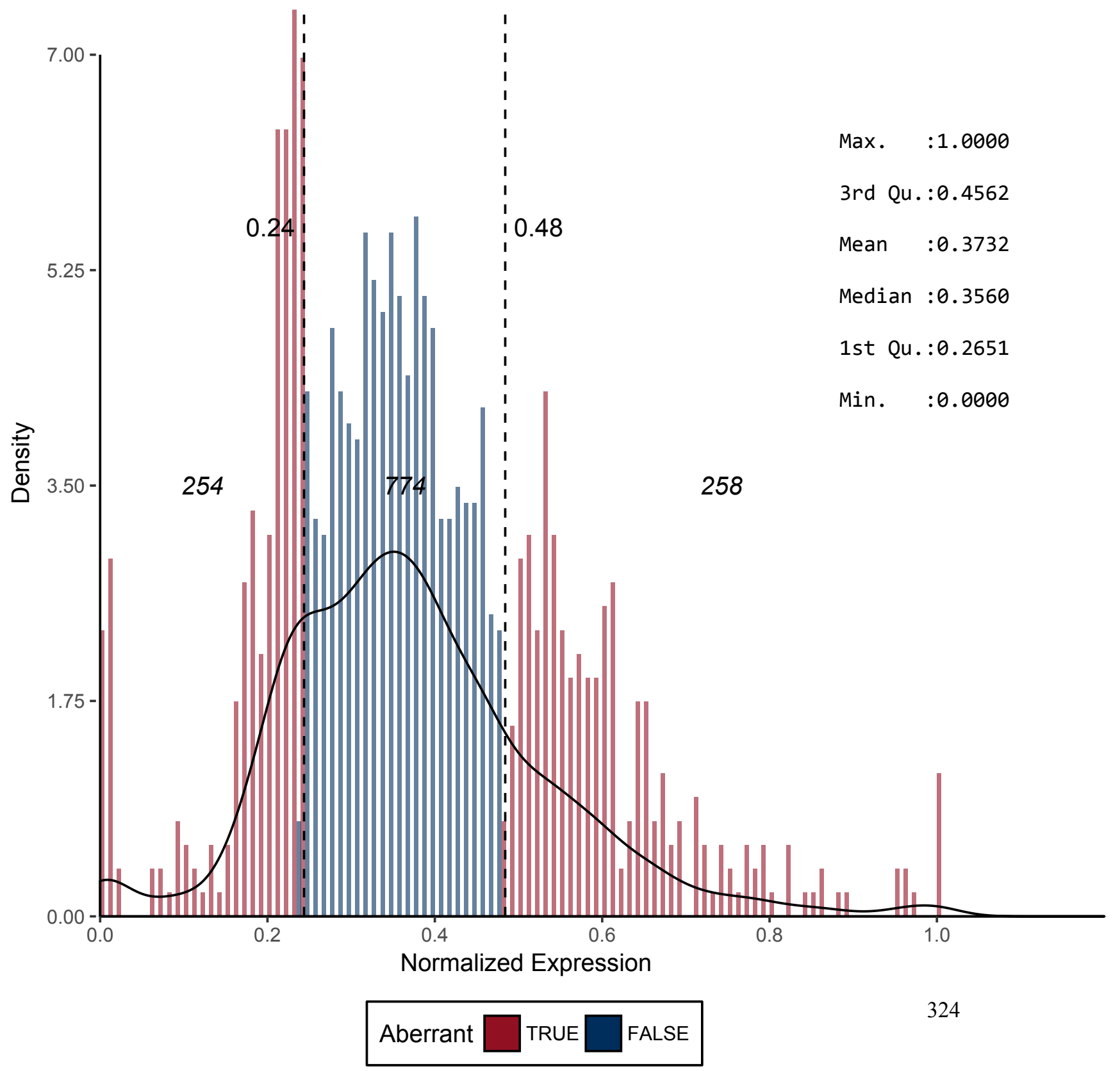


Figure 308 - CD308

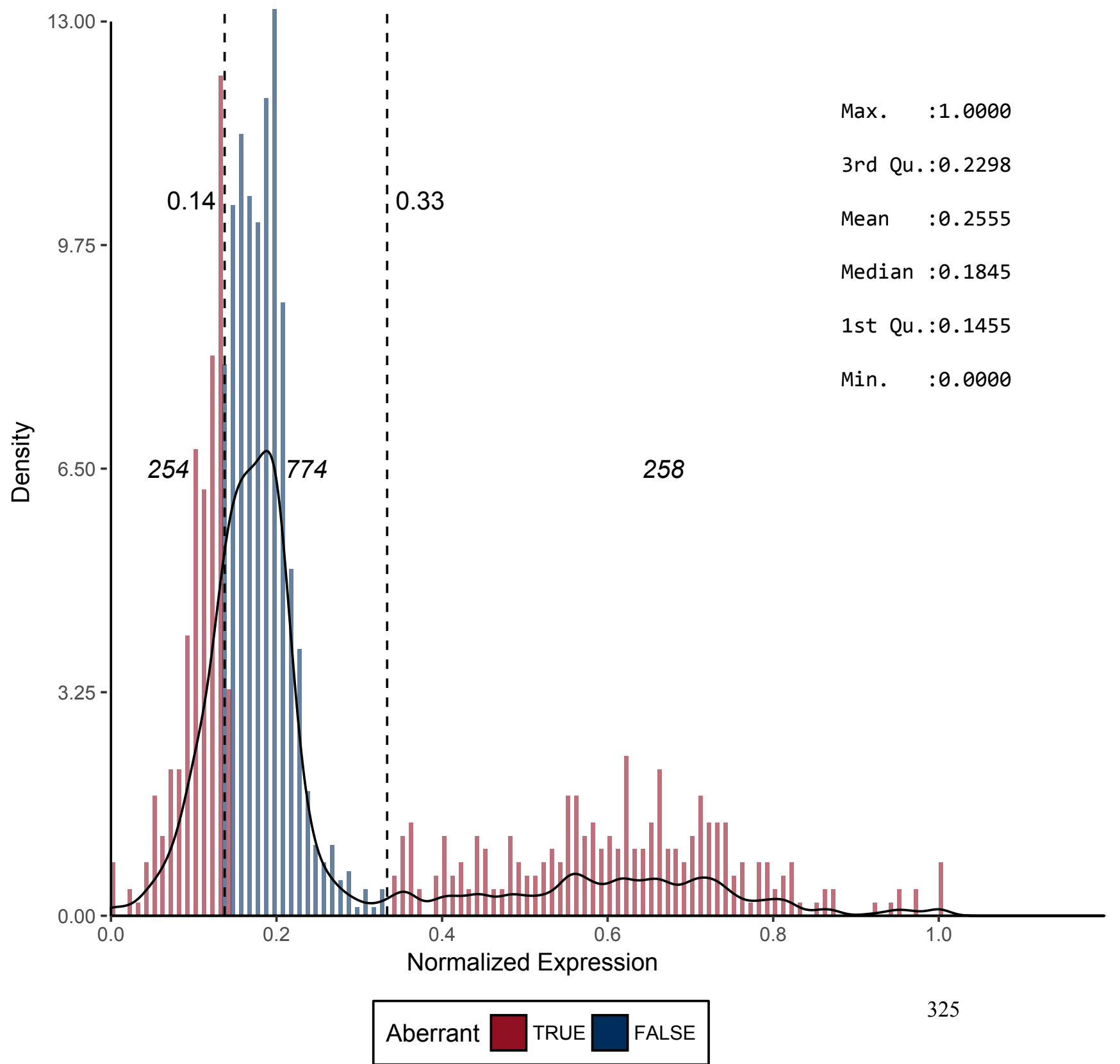


Figure 309 - CD309*

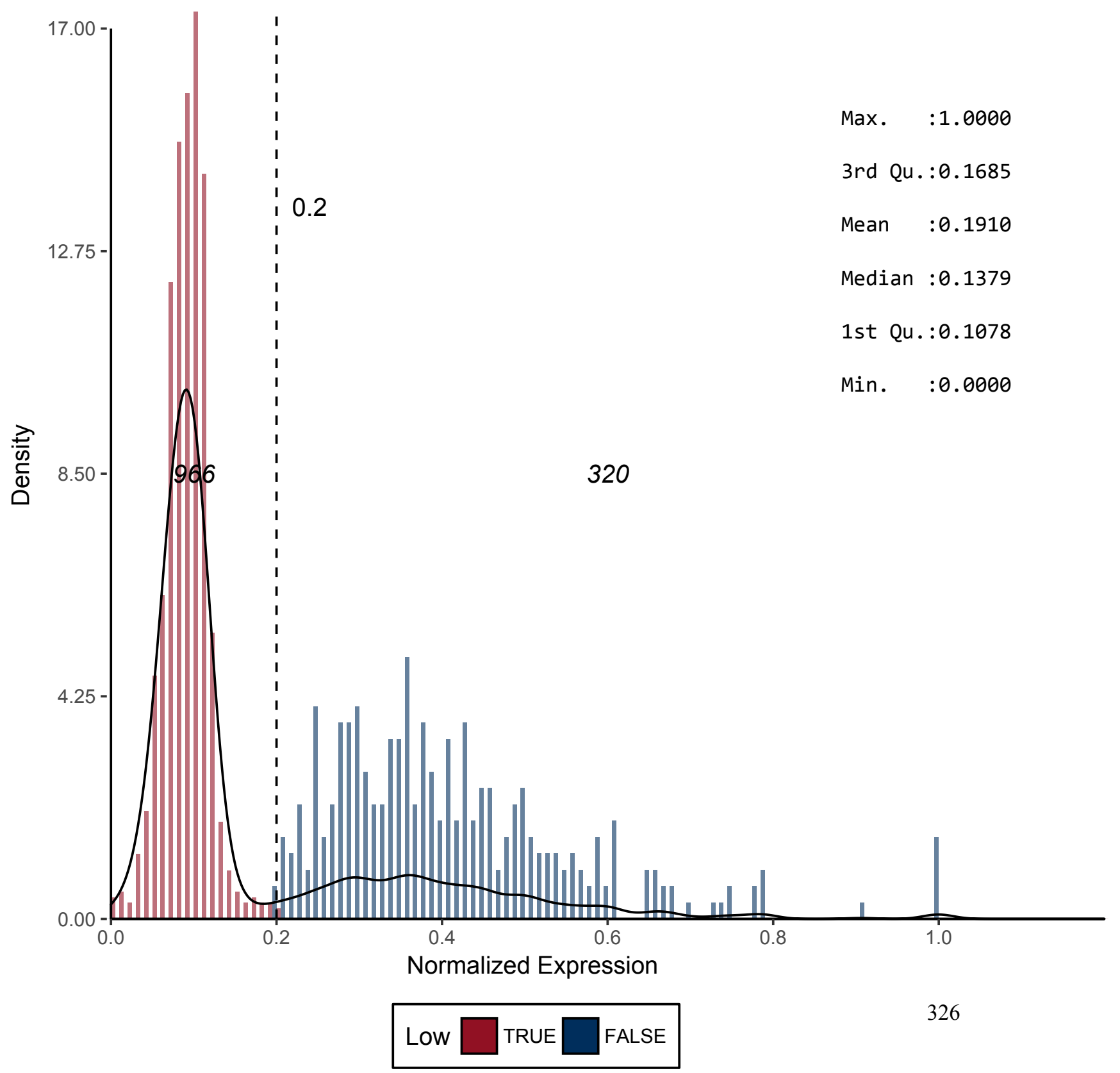


Figure 310 - CD312

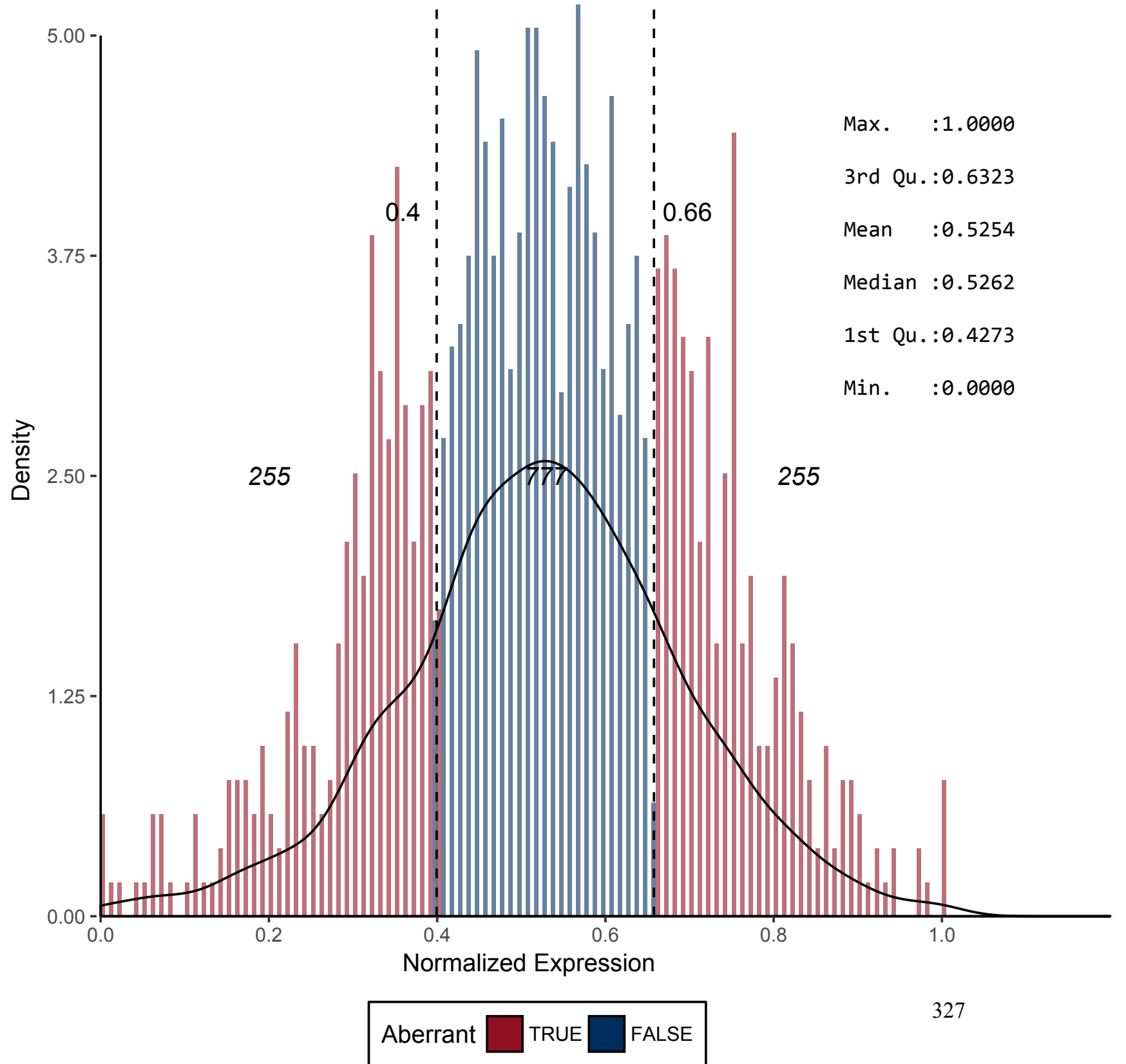


Figure 311 - CD315*

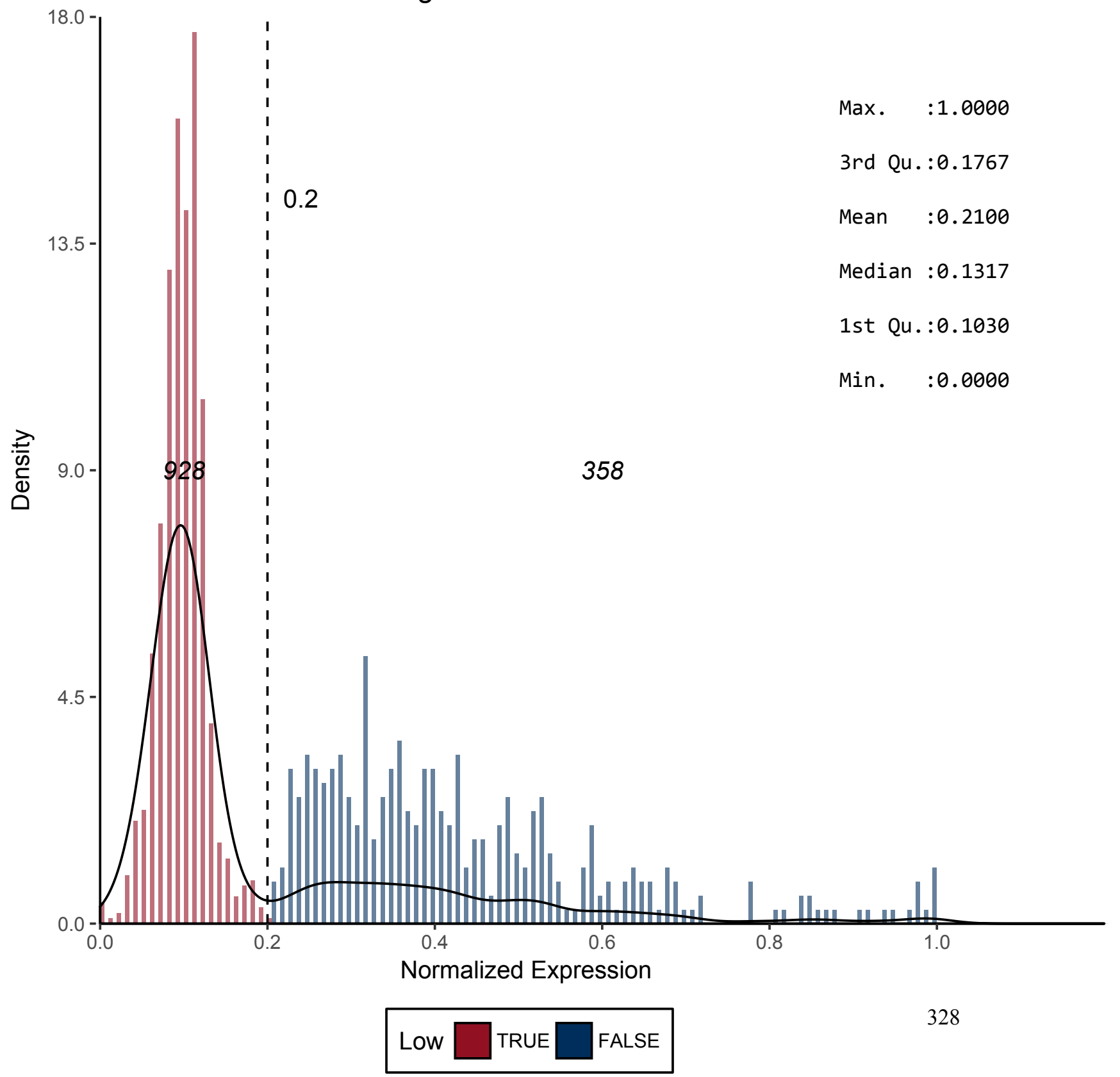


Figure 312 - CD316*

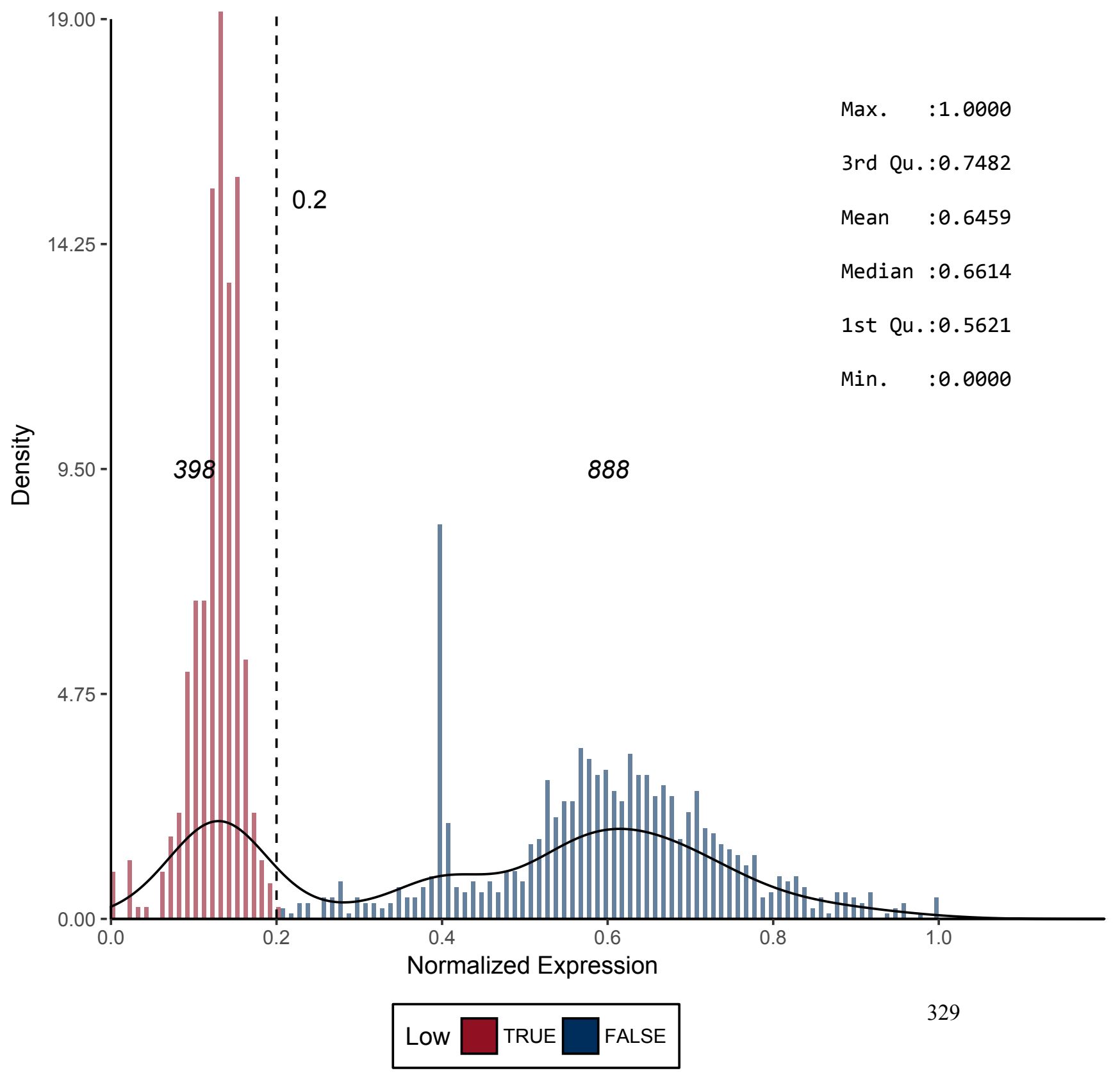


Figure 313 - CD317

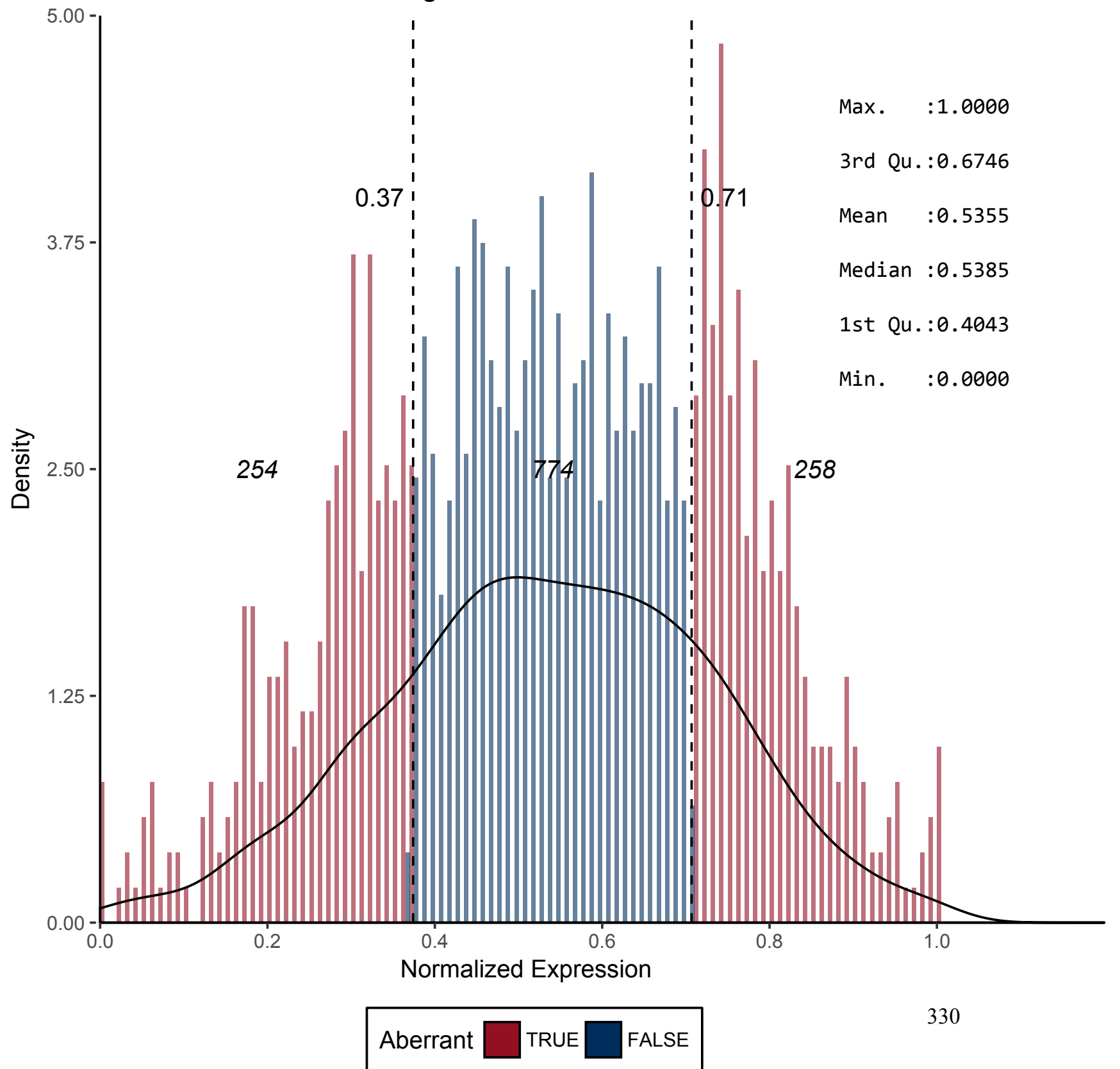


Figure 314 - CD318*

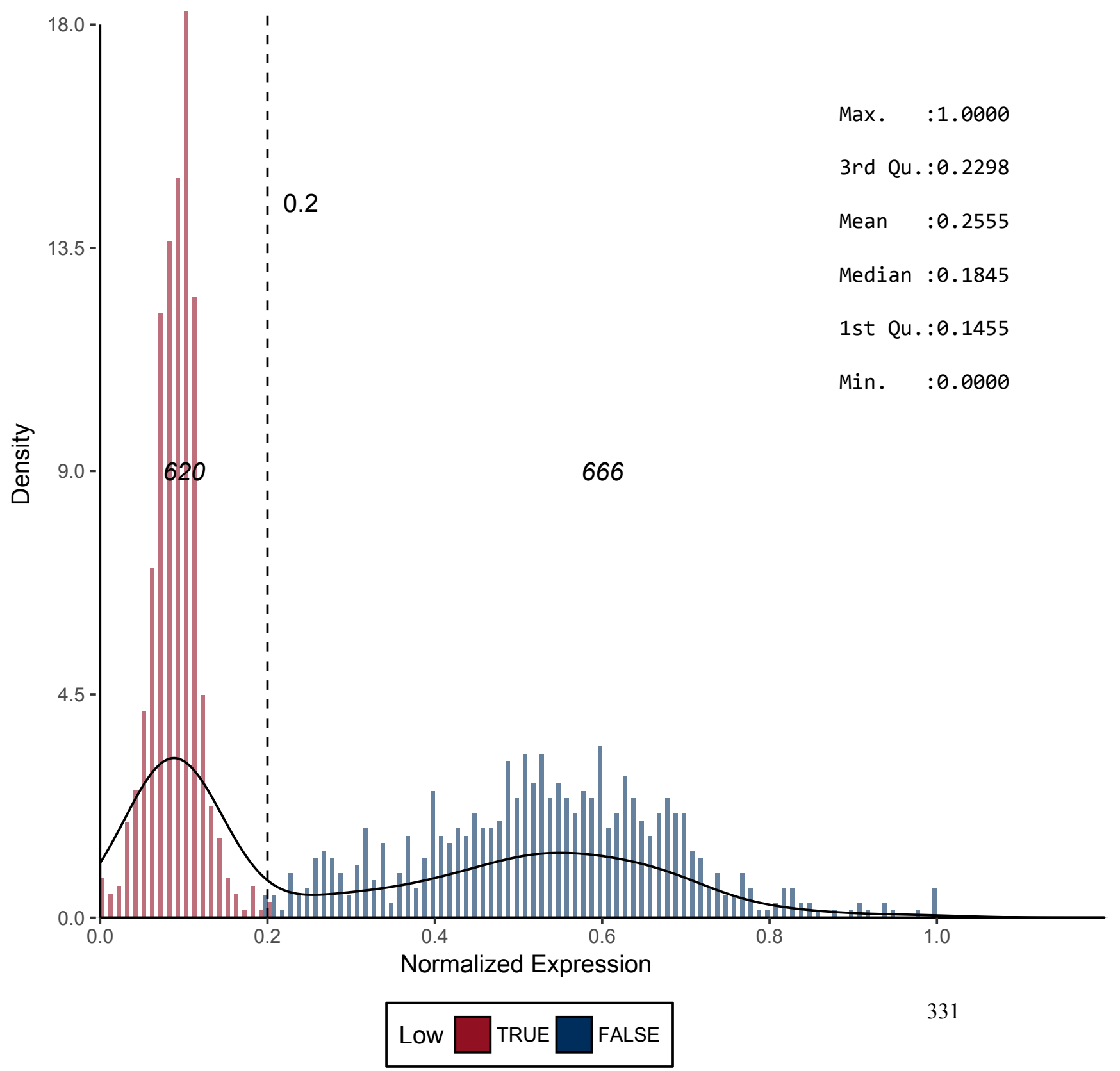


Figure 315 - CD319

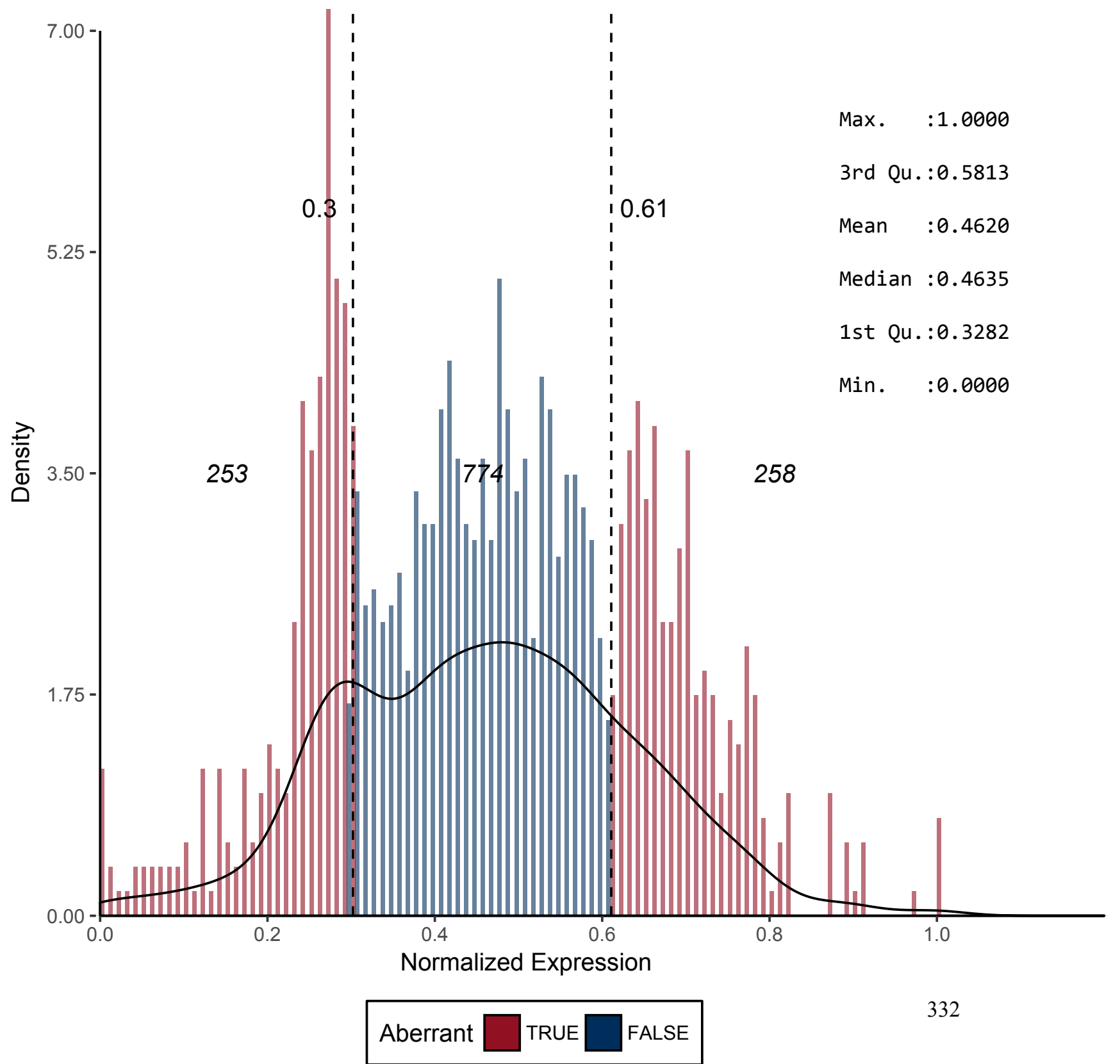


Figure 317 - CD321

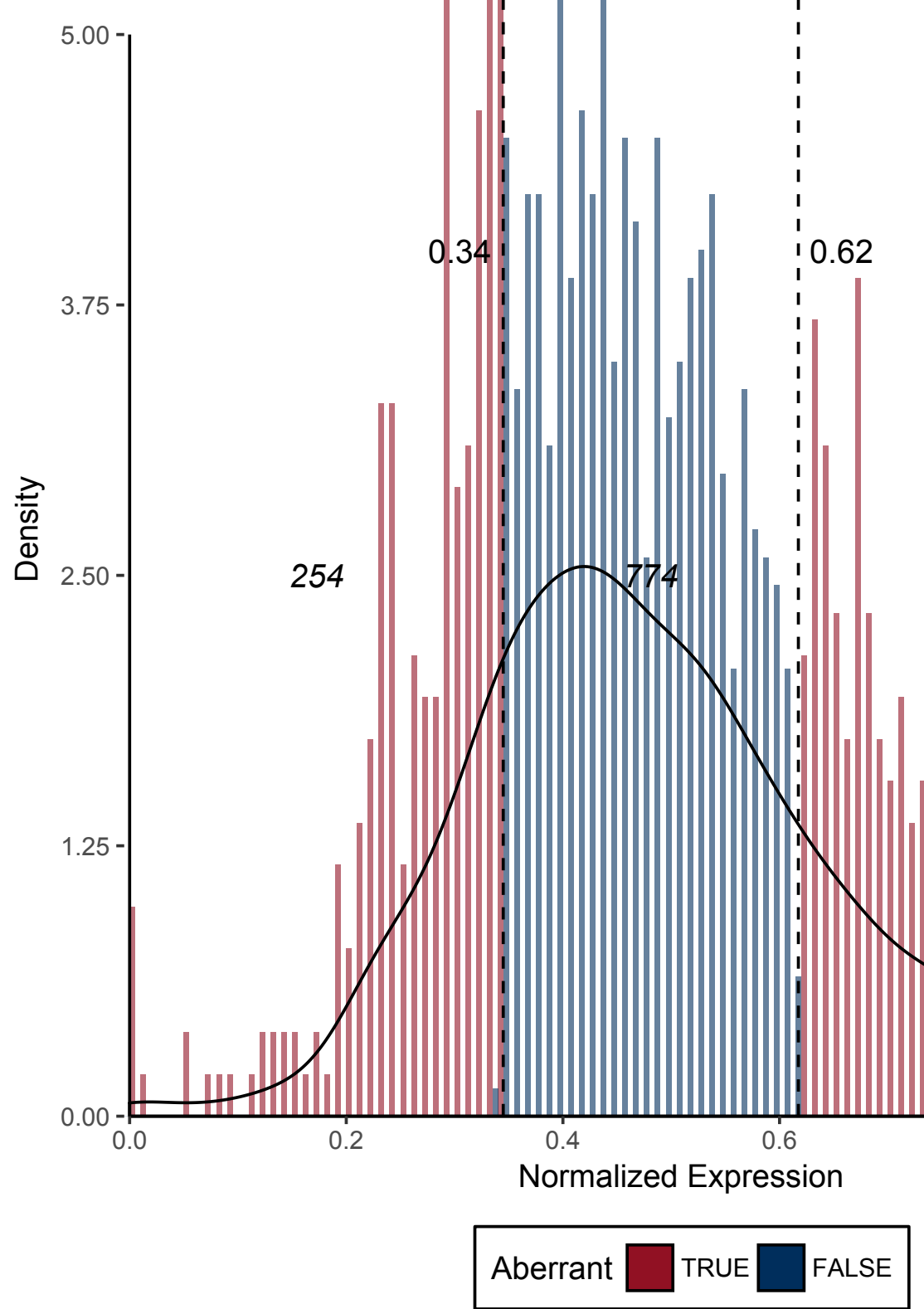

Max. $: 1.0000$

3rd Qu.:0.5838

Mean :0.4825

Median :0.4622

1st Qu.:0.3659

Min. $\quad: 0.0000$

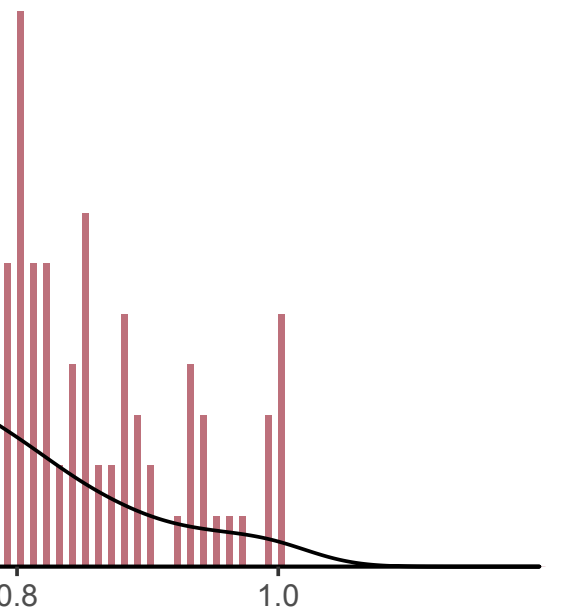


Figure 318 - CD322*

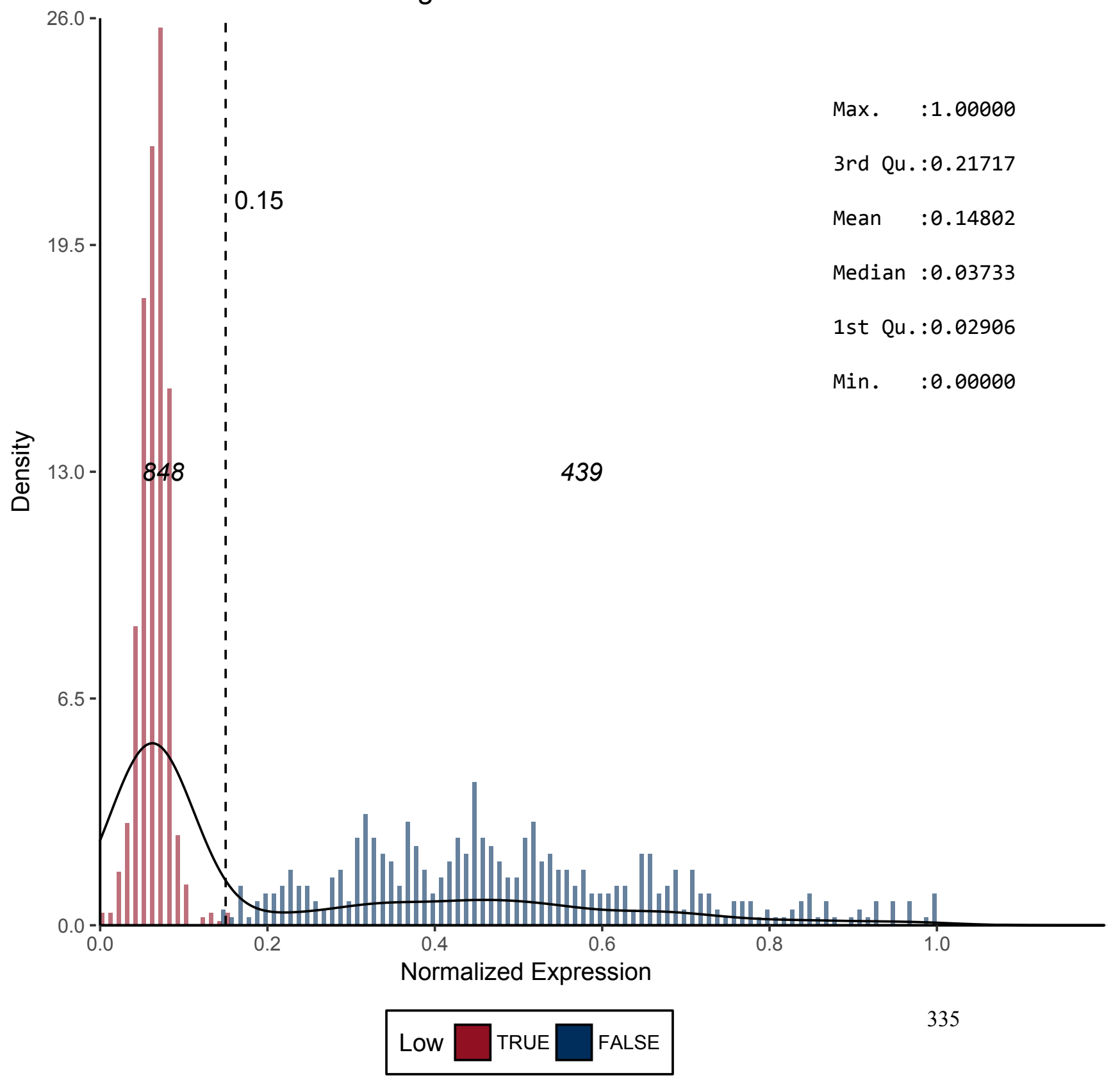


Figure 320 - CD325

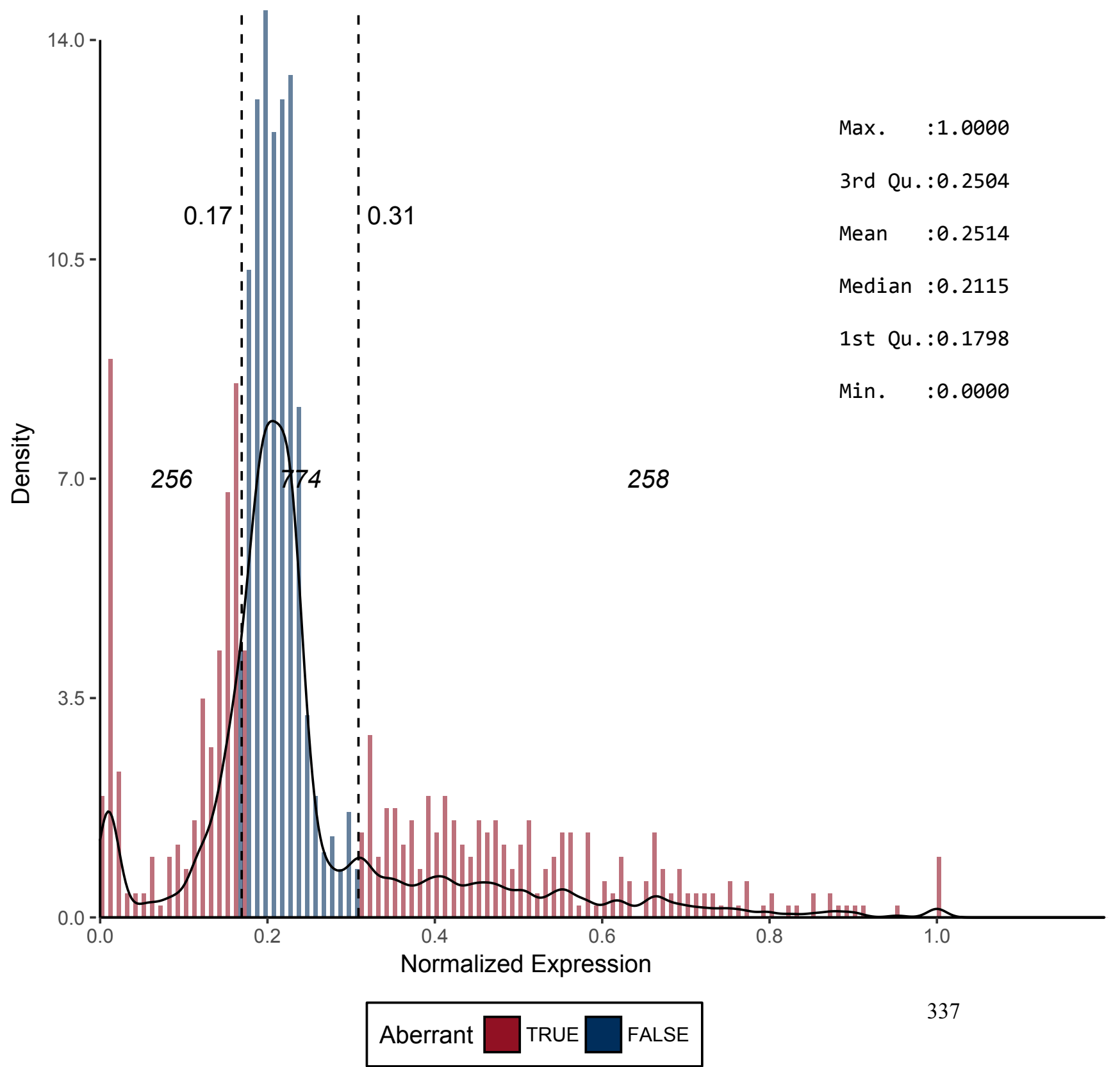


Figure 322 - CD327

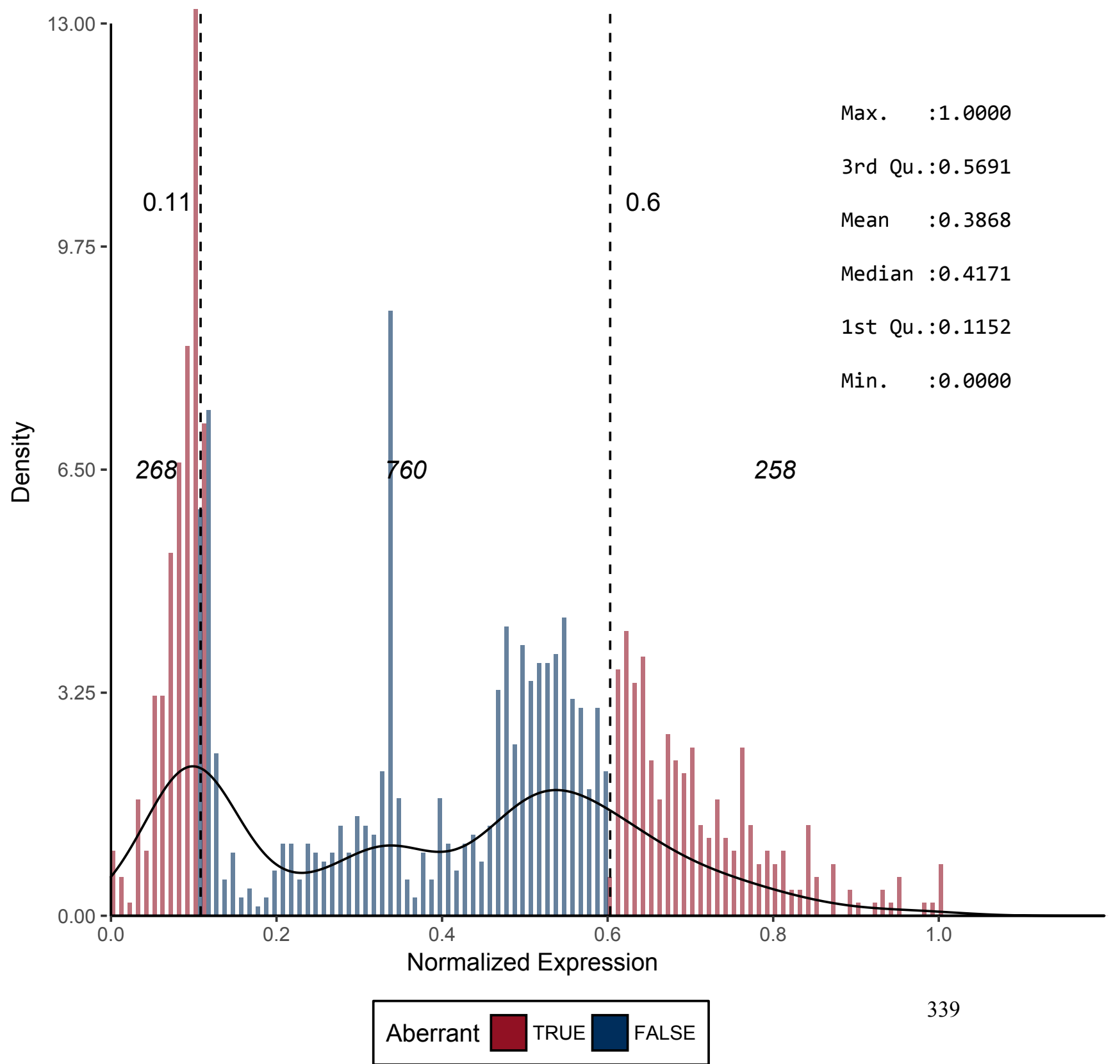


Figure 324 - CD329

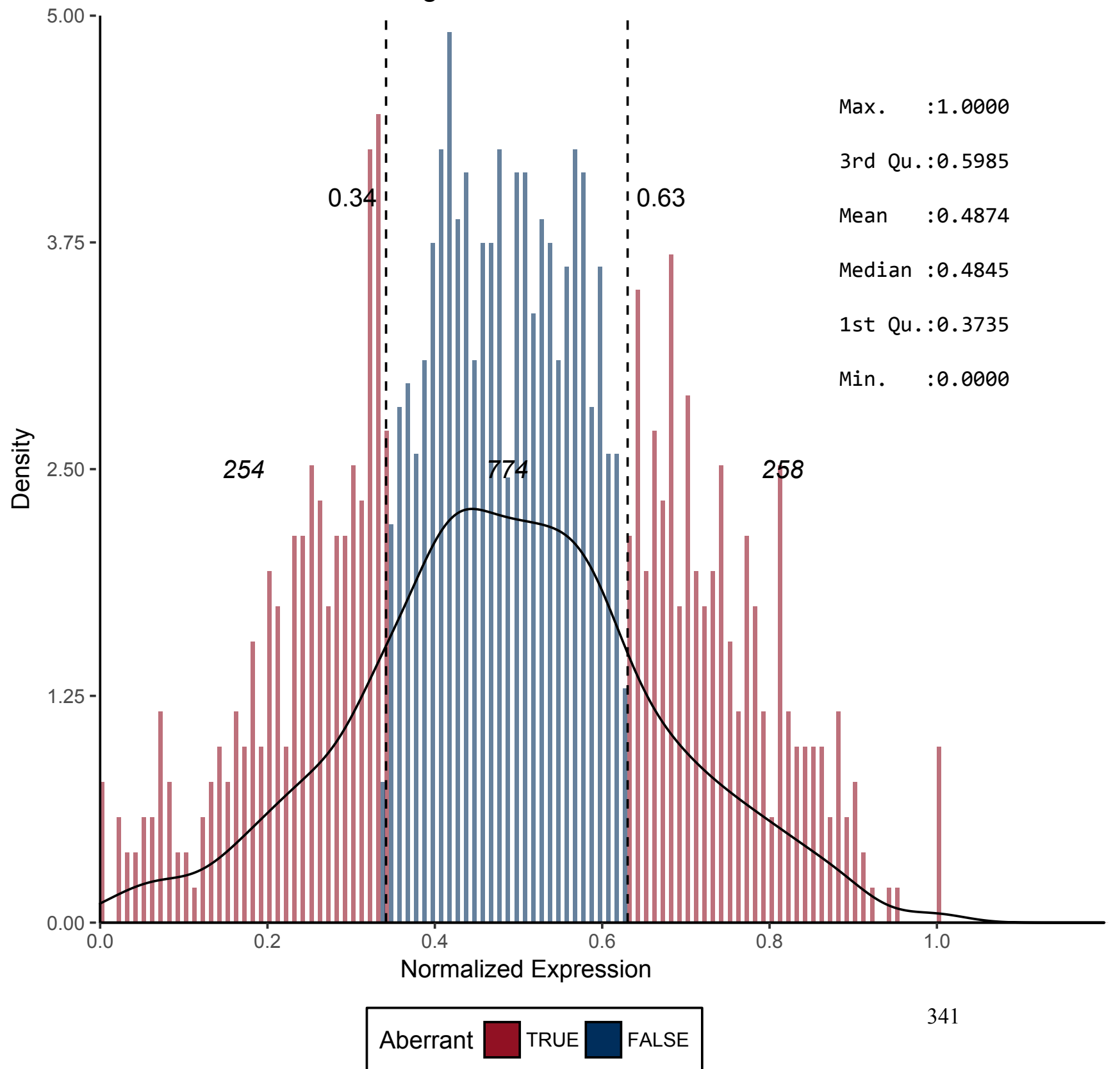


Figure 325 - CD331*

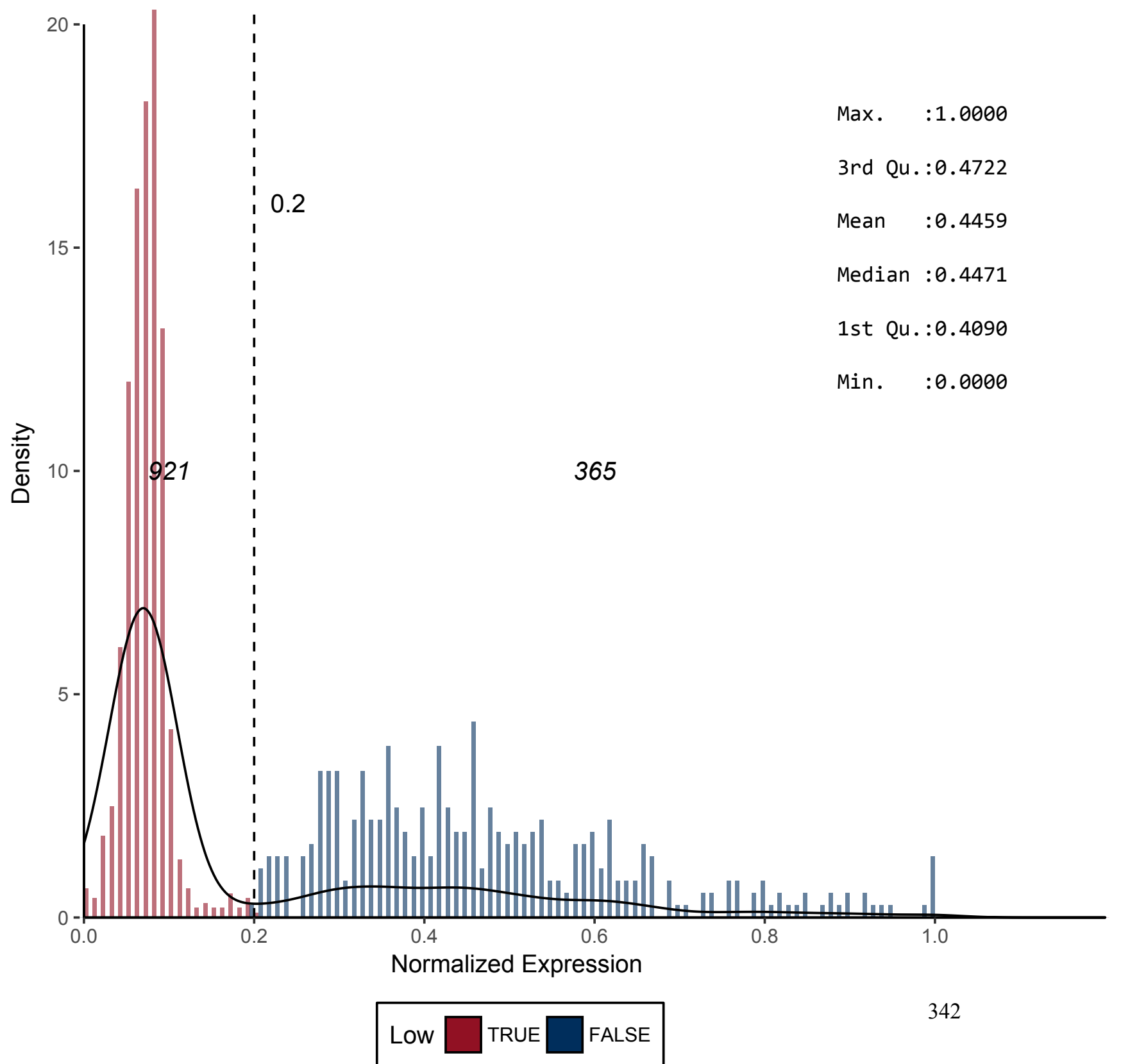


Figure 328 - CD334*

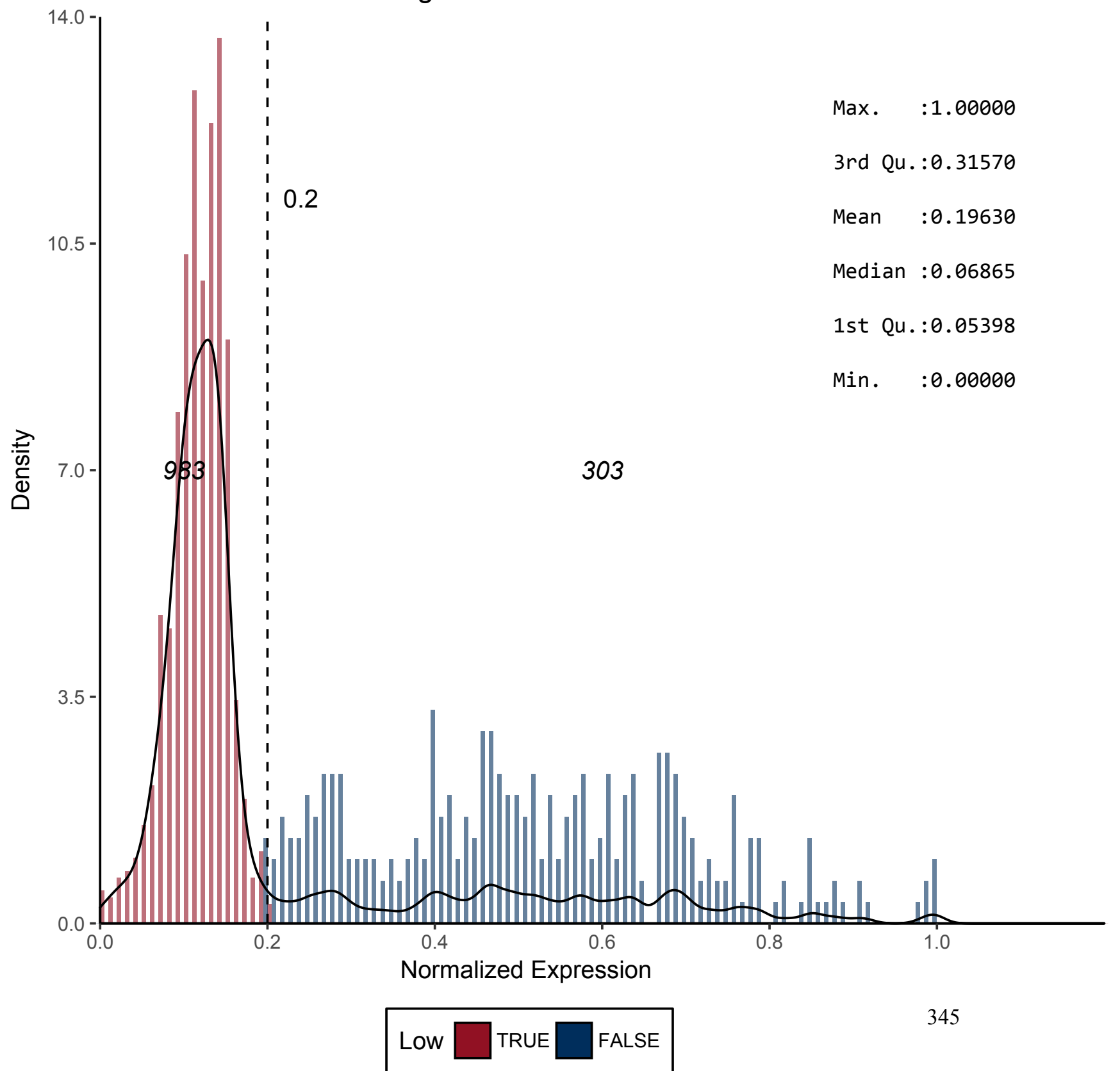


Figure 329 - CD335*

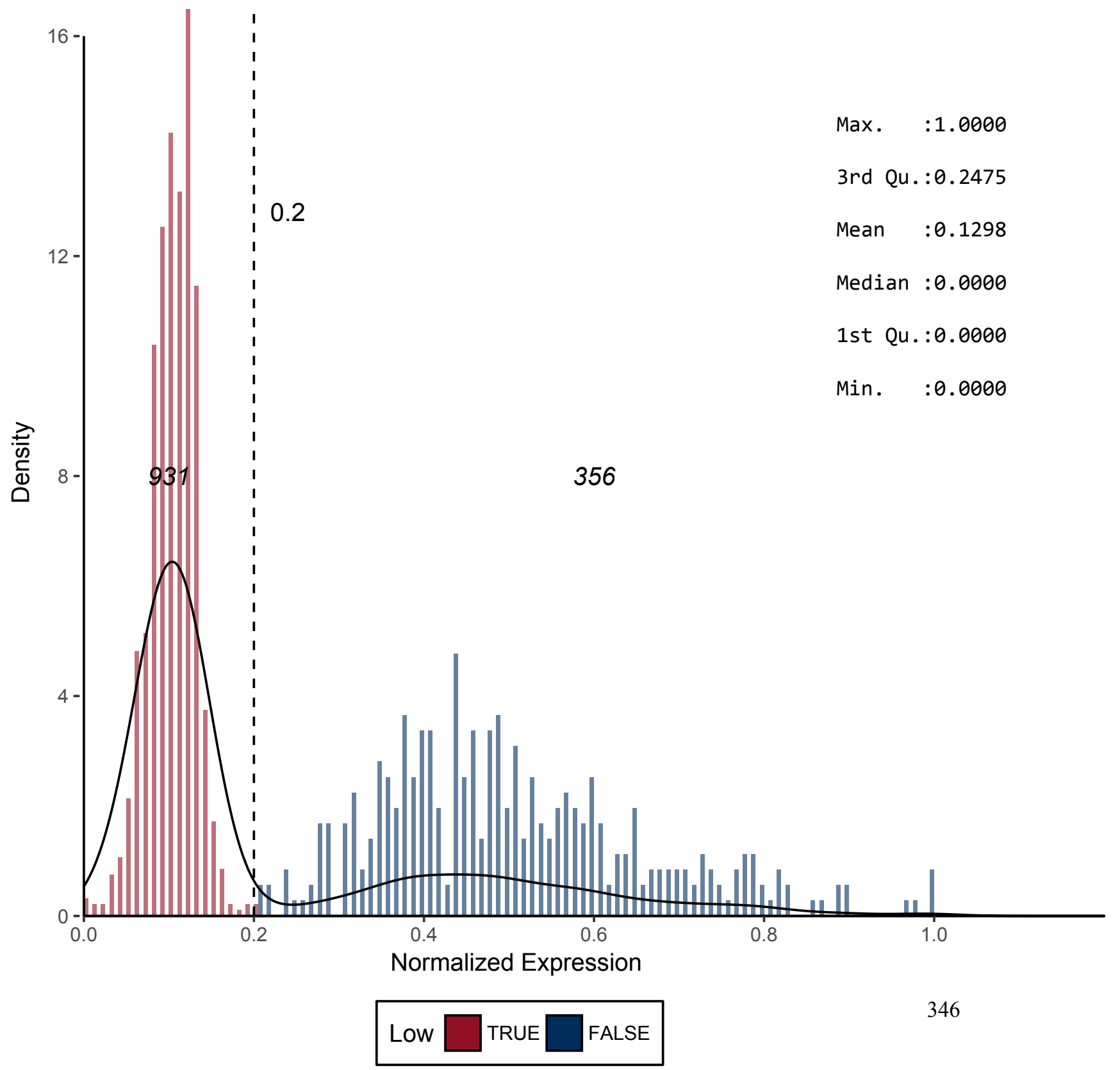


Figure 330 - CD336*

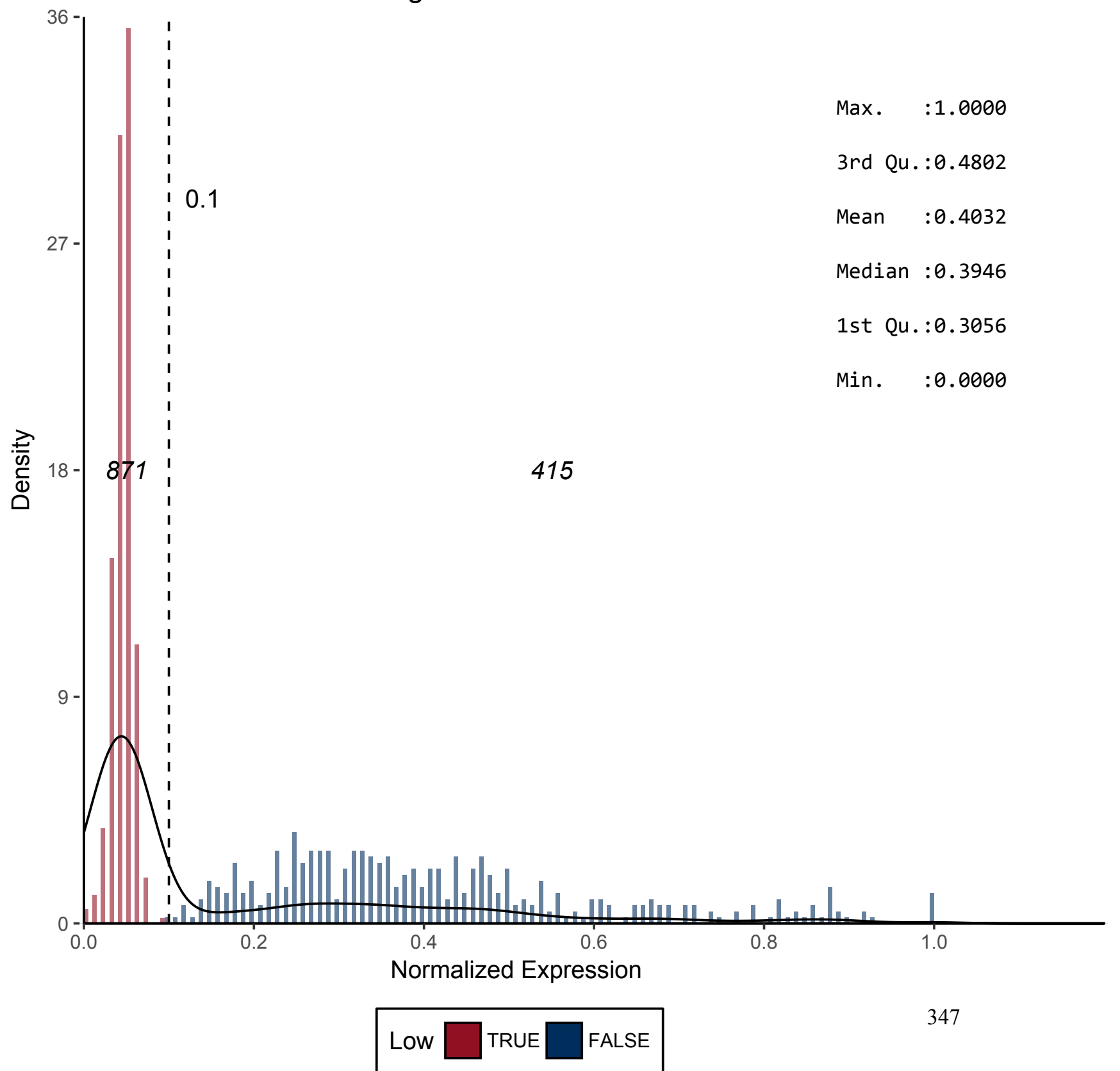


Figure 331 - CD337

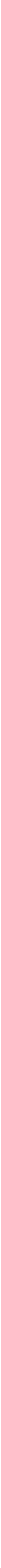


Figure 332 - CD338*

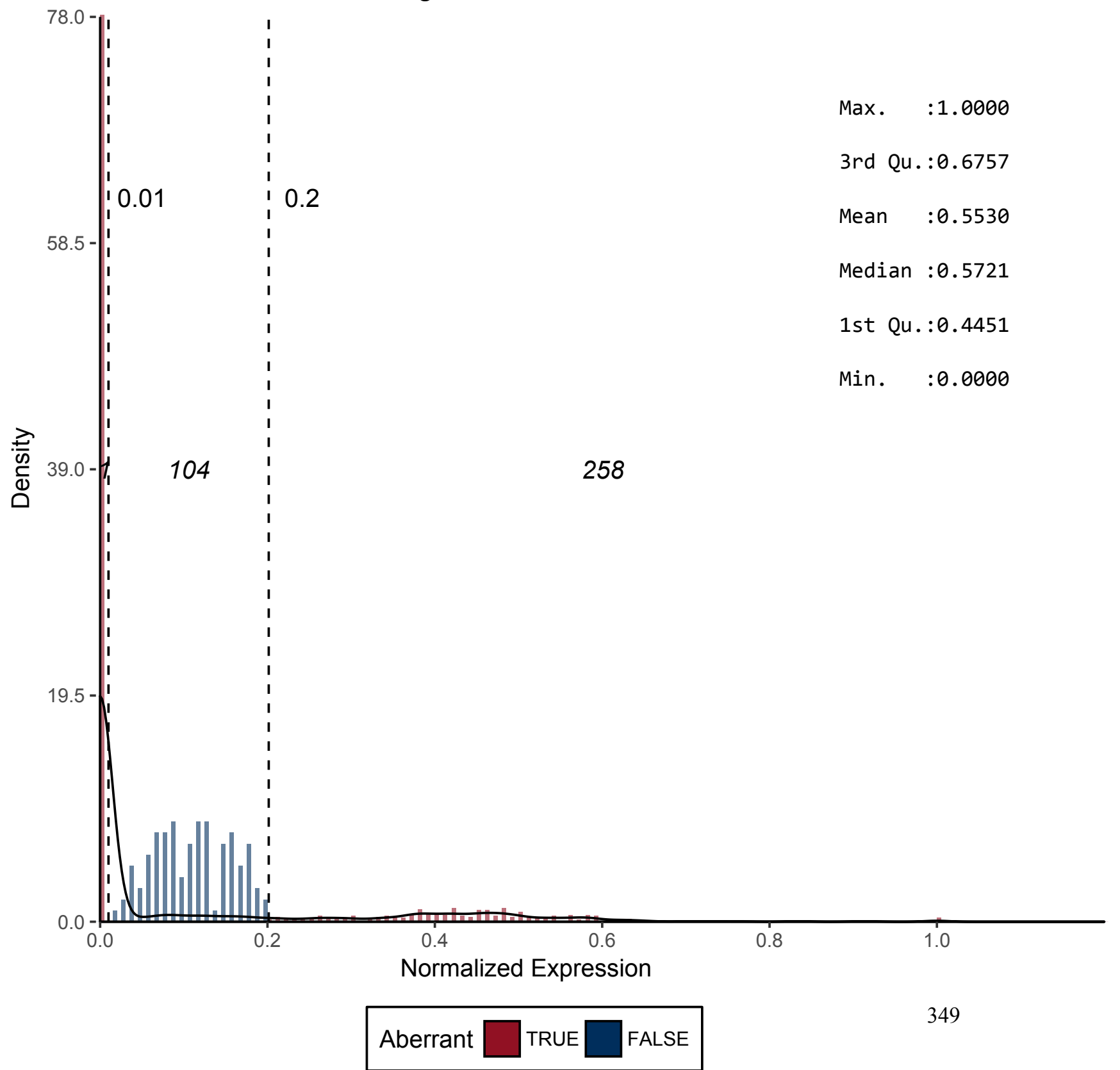


Figure 333 - CD339*

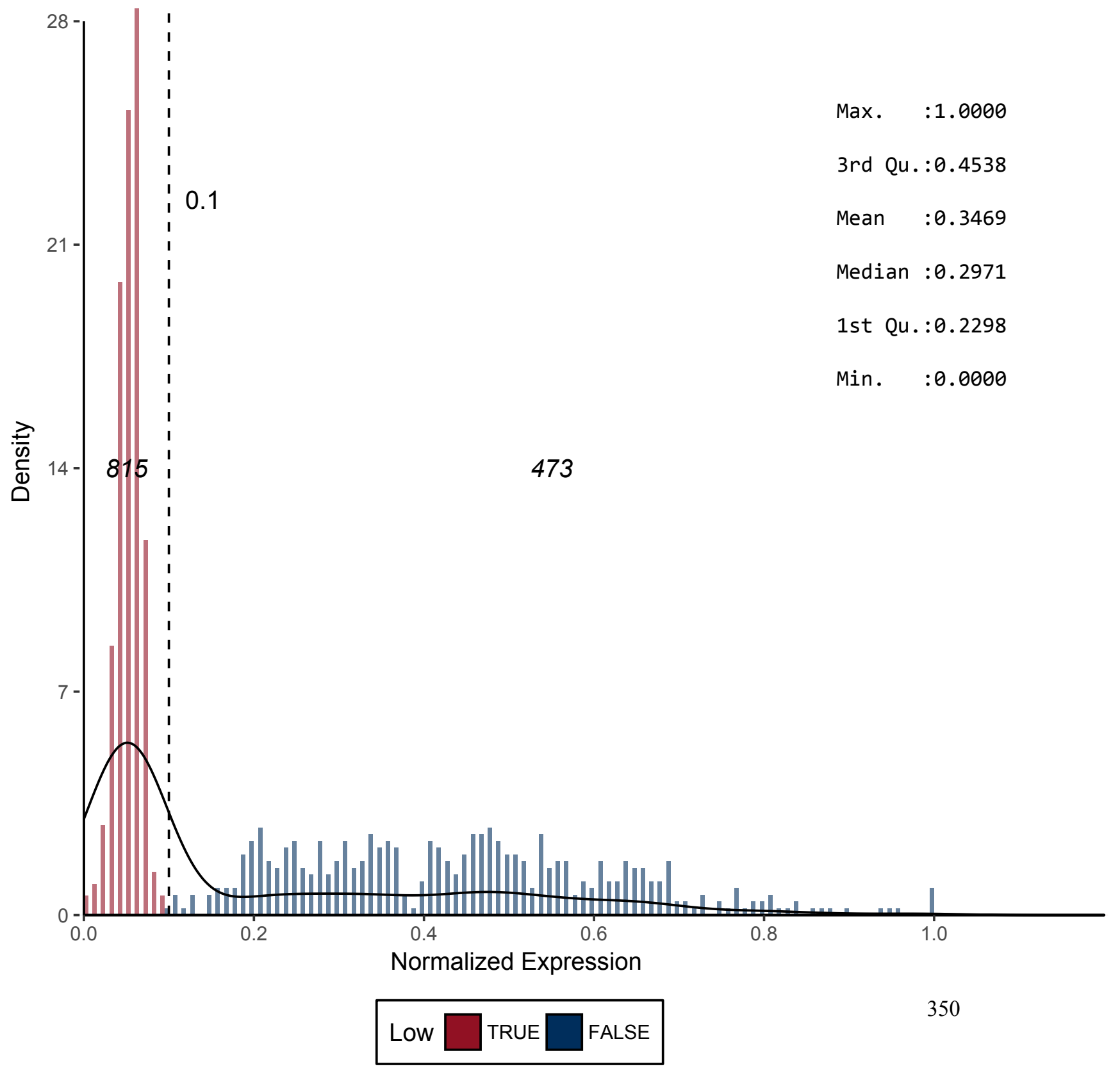


Figure 334 - CD340

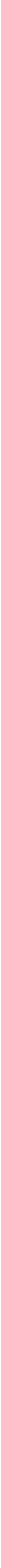


Figure 335 - CD344*

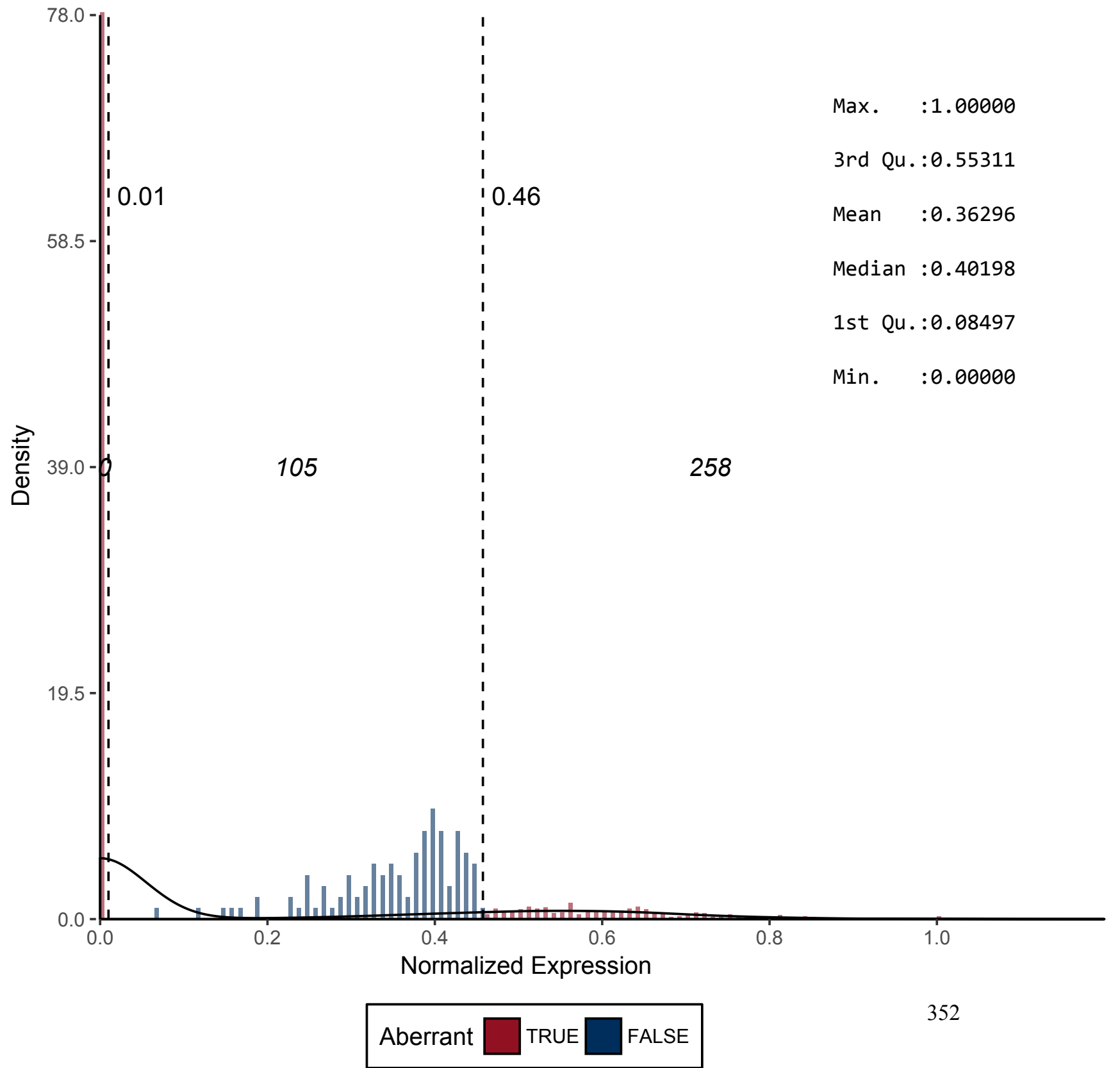


Figure 338 - CD351

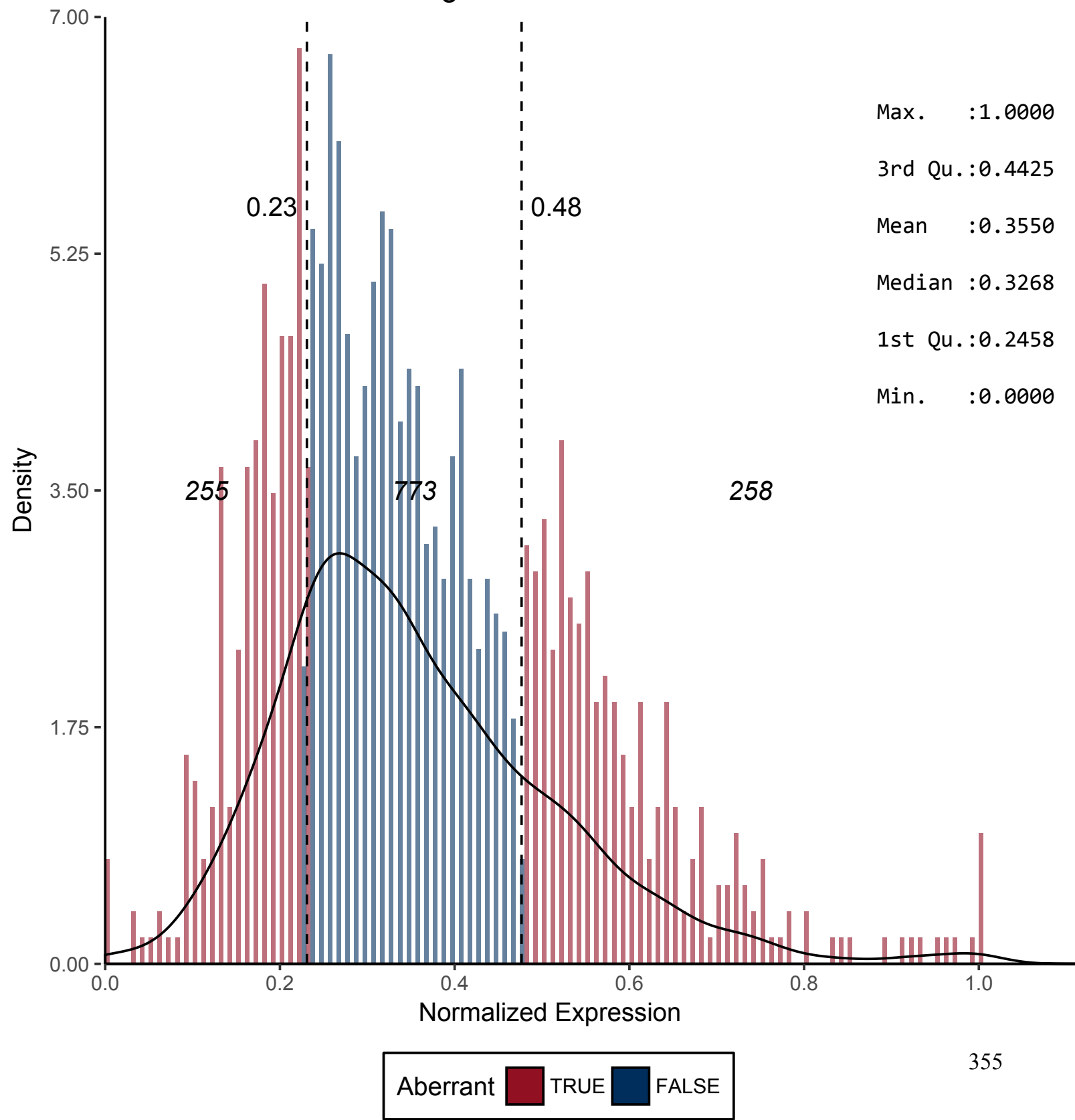


Figure 339 - CD353*

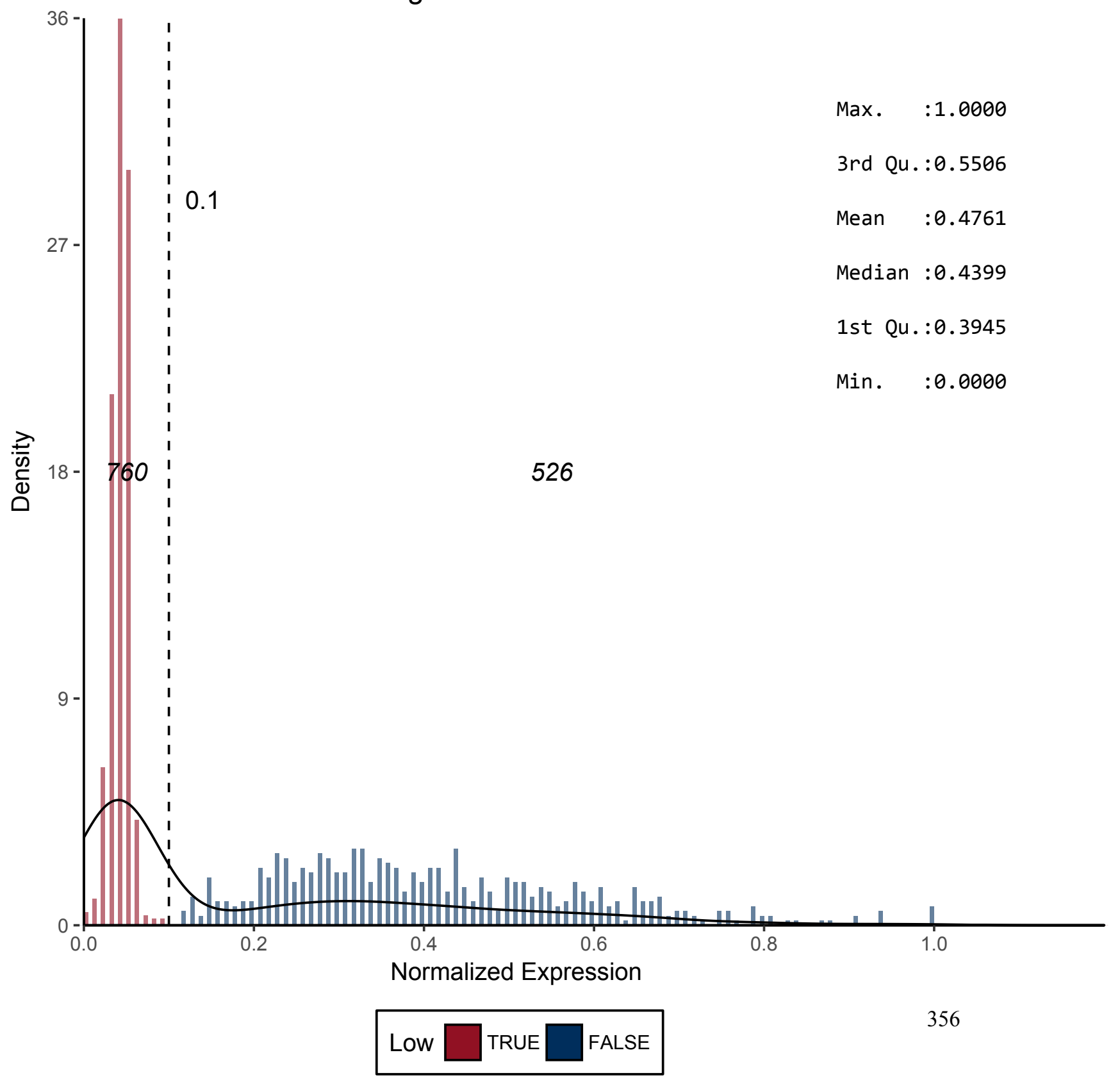


Figure 340 - CD354

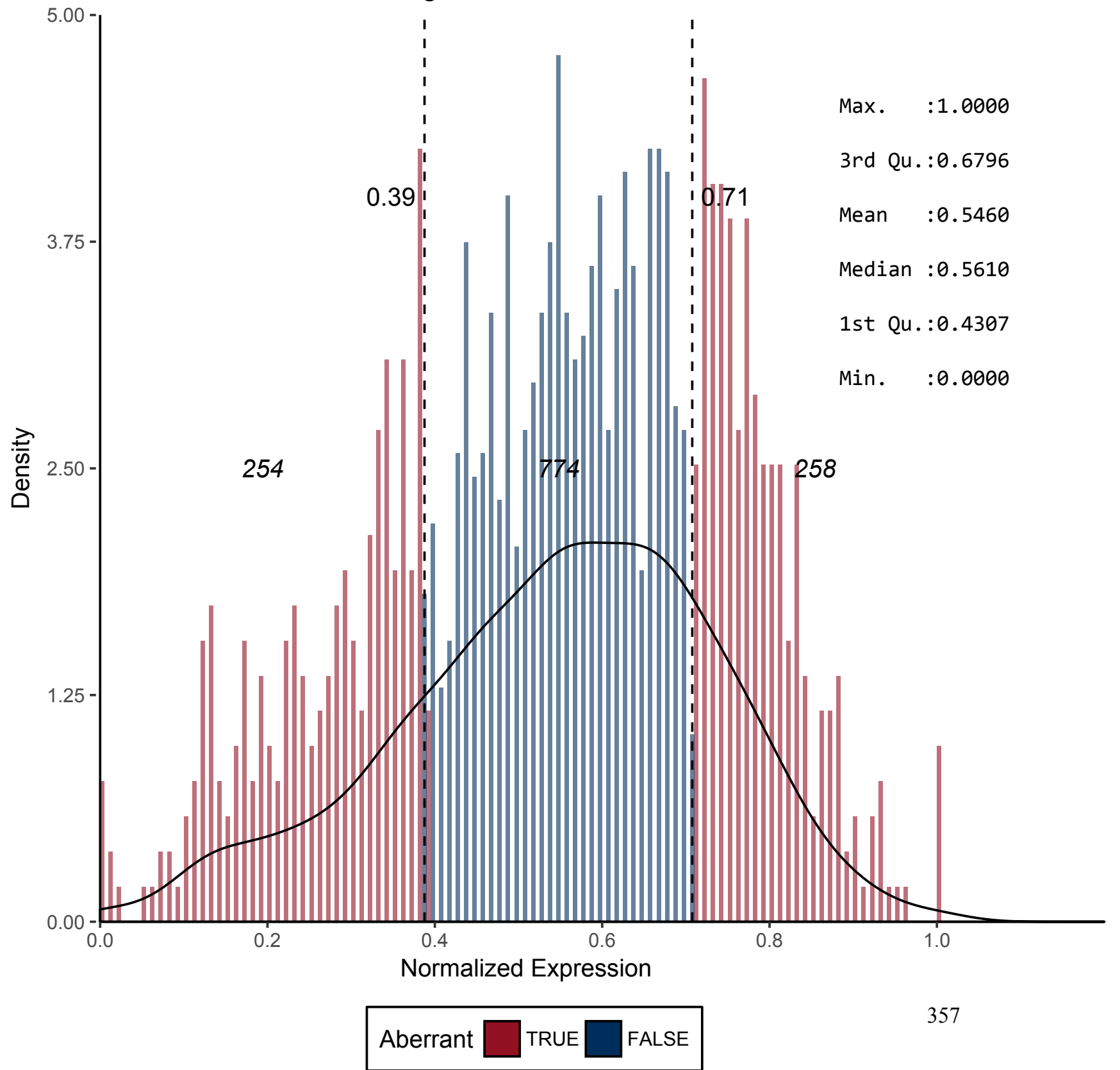


Figure 341 - CD355*

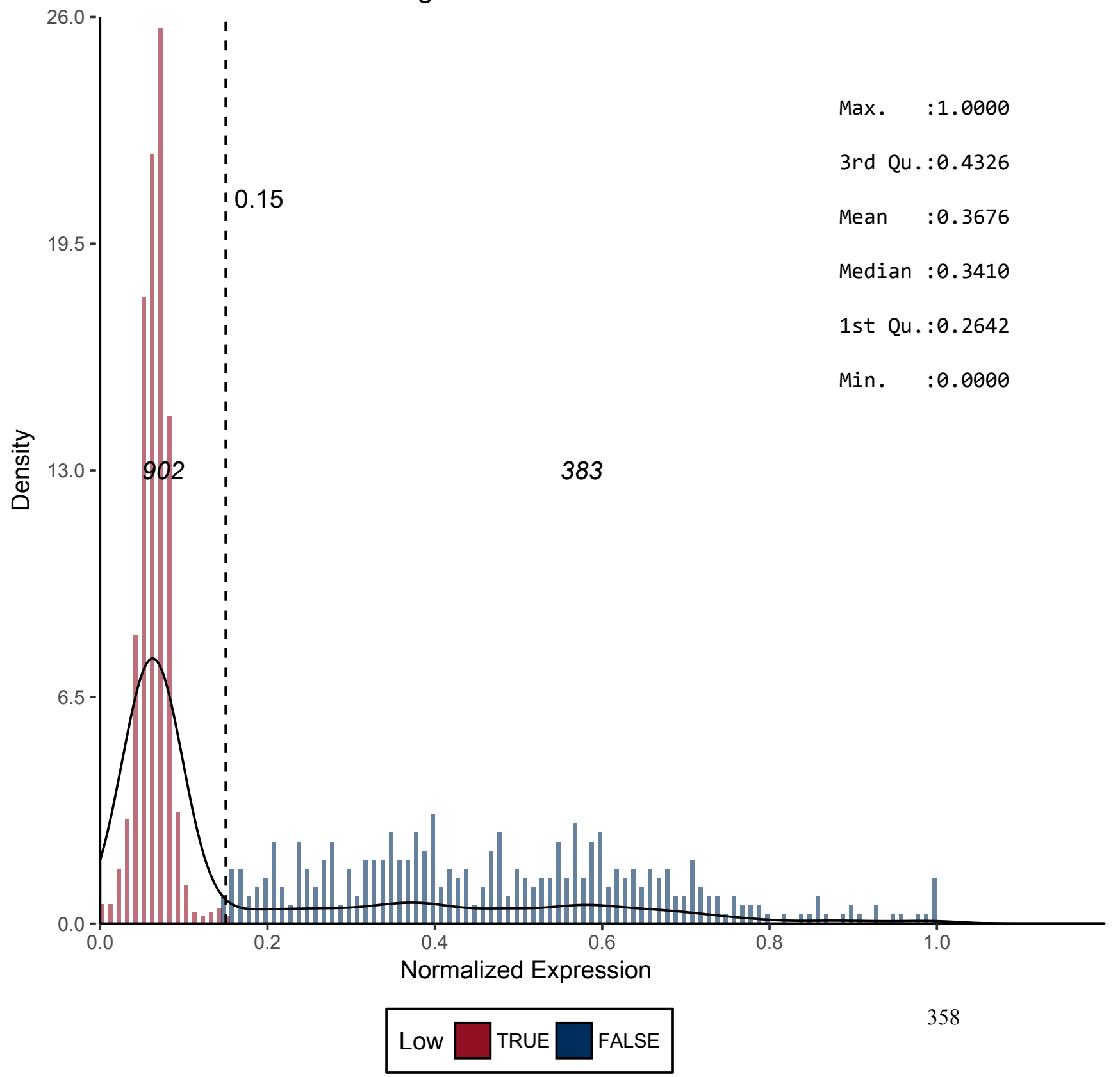


Figure 343 - CD358

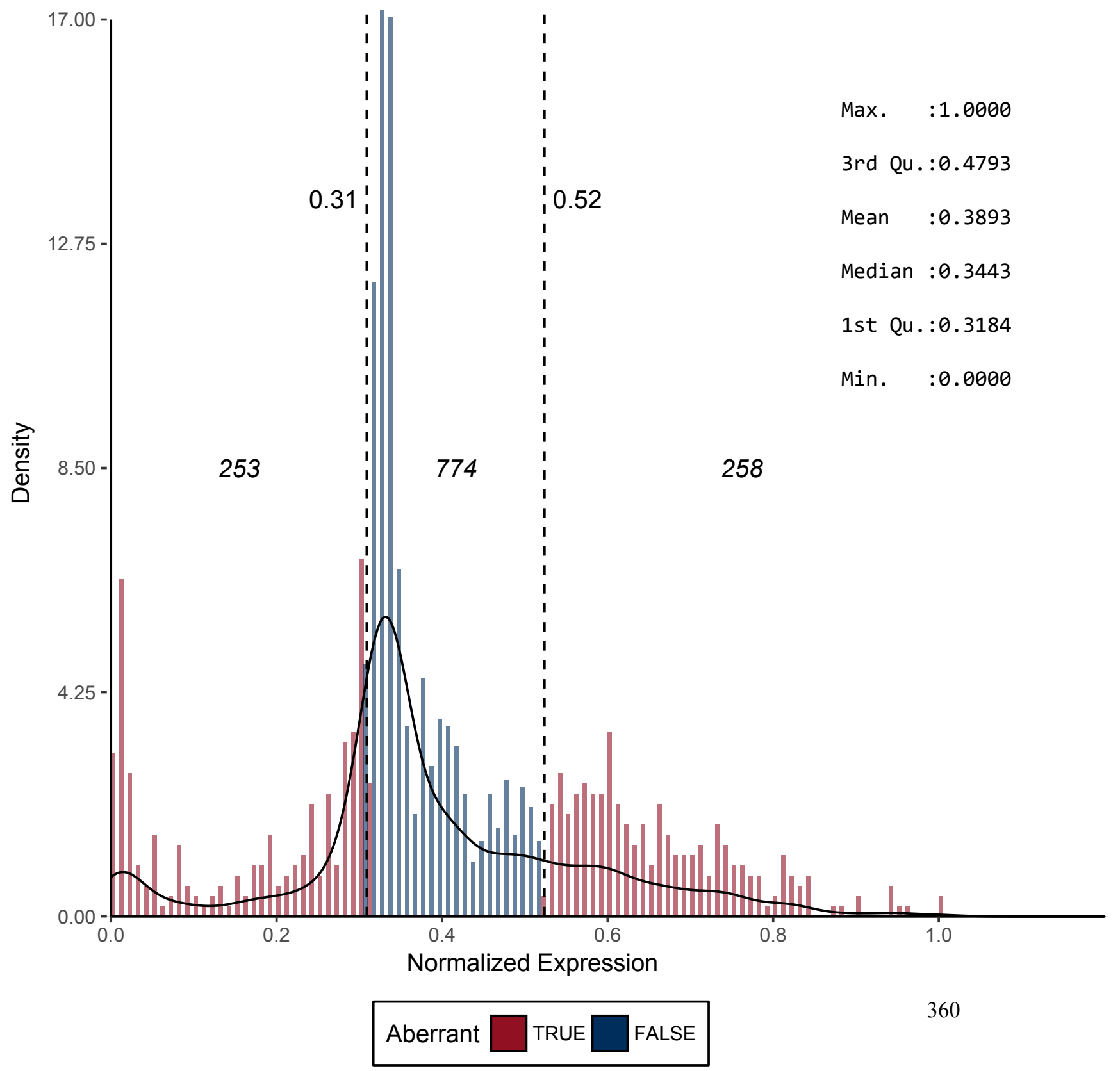


Figure 344 - CD360

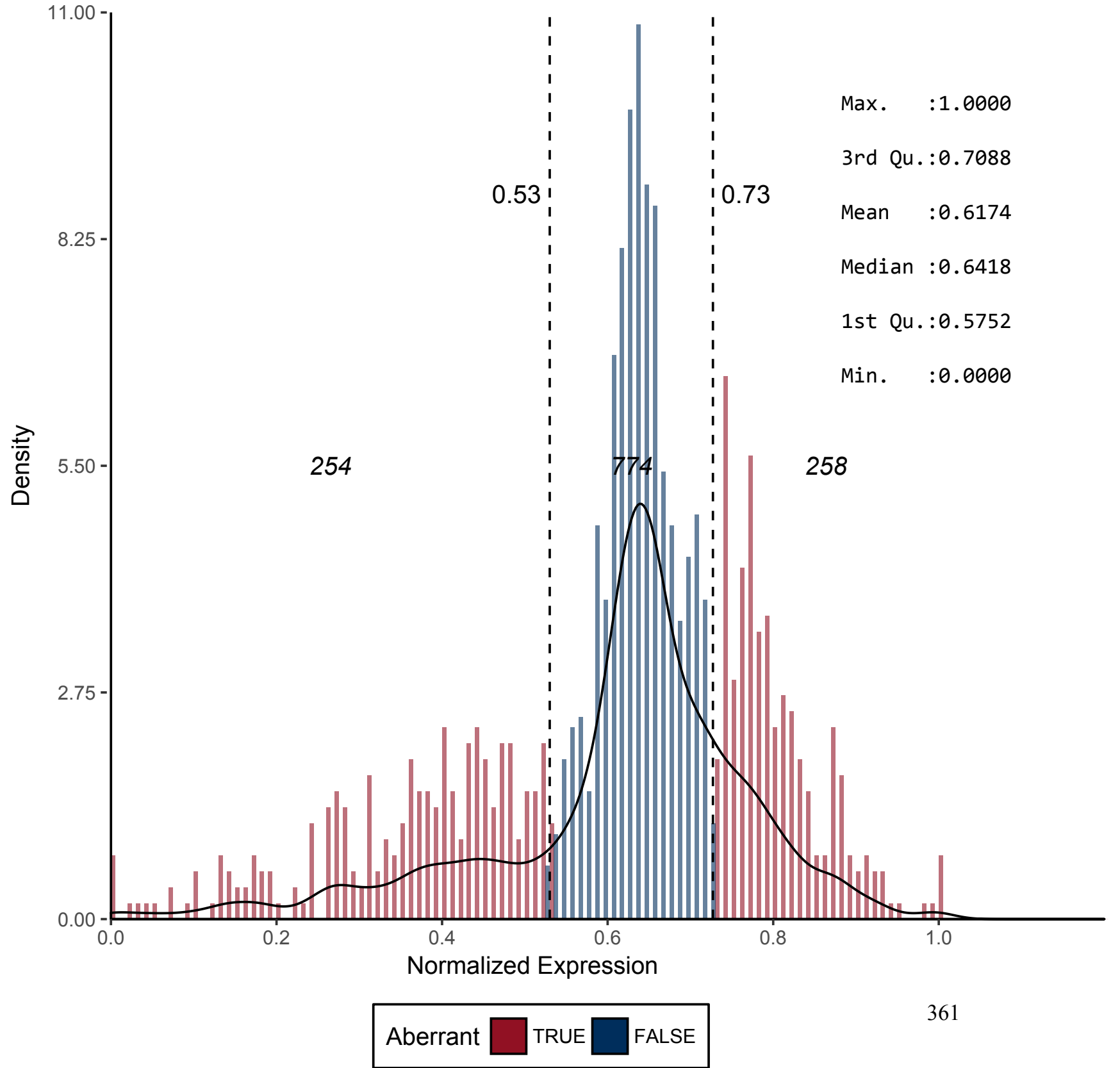


Figure 345 - CD361

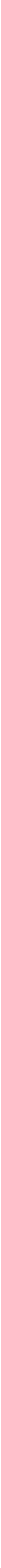


Figure 346 - CD362

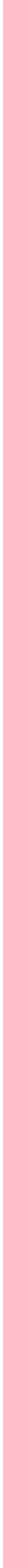


Figure 348 - CD364

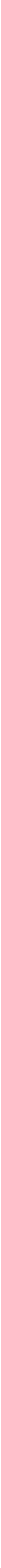


Figure 349 - CD366

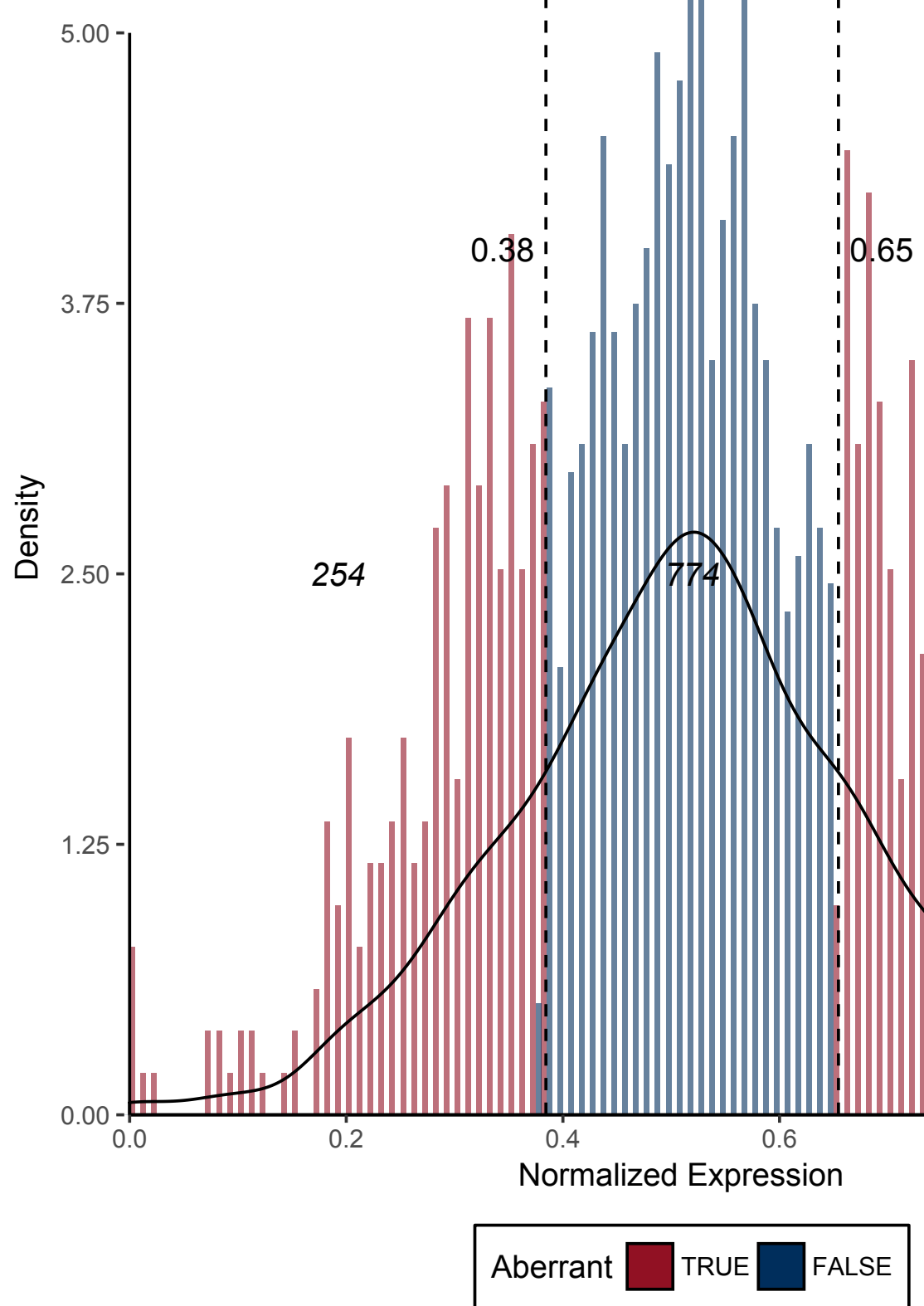

Max. $: 1.0000$

3rd Qu.:0.6249

Mean $\quad: 0.5167$

Median :0.5178

1st Qu.:0.4126

Min. $: 0.0000$

\section{8}


Figure 351 - CD369

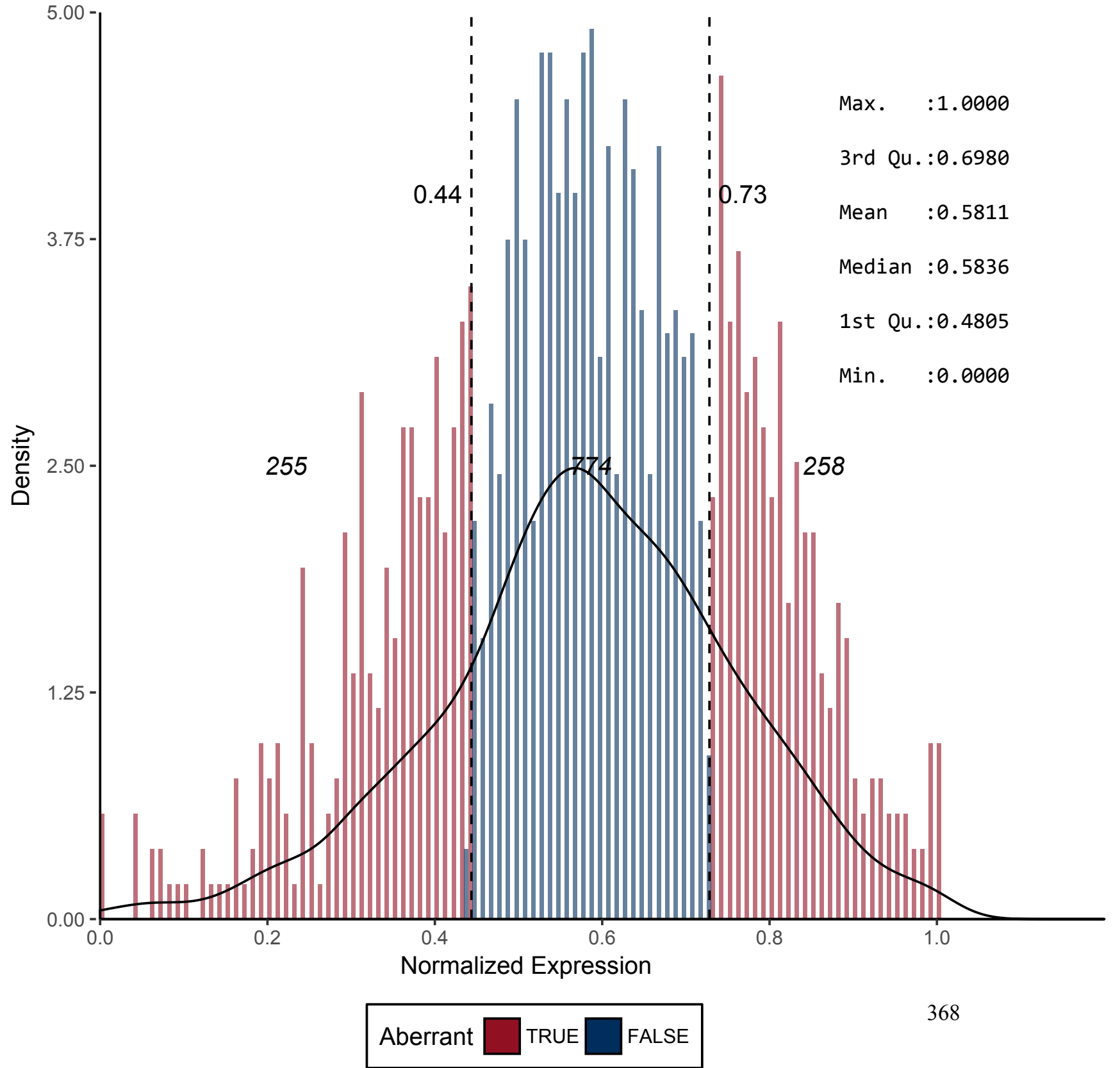

